\title{
A Compilation of Internship Reports
}

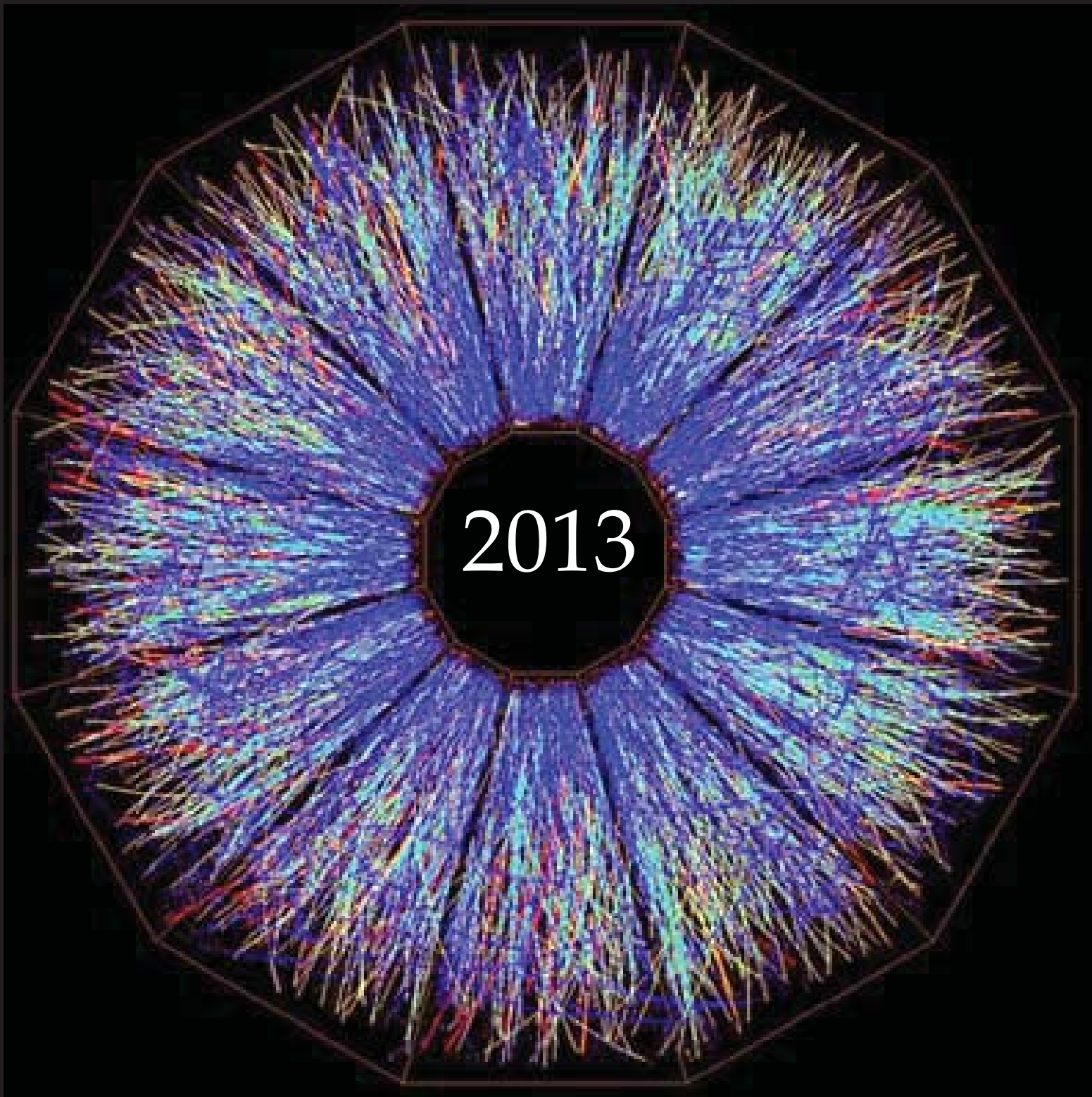

Prepared for The Office of Educational Programs Brookhaven National Laboratory Office of Educational Programs 
Notice: This manuscript has been authored by employees of Brookhaven Science Associates, LLC under Contract No. DE-AC02-98CH10886 with the U.S. Department of Energy. The publisher by accepting the manuscript for publication acknowledges that the United States Government retains a non-exclusive, paid-up, irrevocable, world-wide license to publish or reproduce the published form of this manuscript, or allow others to do so, for United States Government purposes.

\section{DISCLAIMER}

This report was prepared as an account of work sponsored by an agency of the United States

Government. Neither the United States Government nor any agency thereof, nor any of their employees, nor any of their contractors, subcontractors, or their employees, makes any warranty, express or implied, or assumes any legal liability or responsibility for the accuracy, completeness, or any third party's use or the results of such use of any information, apparatus, product, or process disclosed, or represents that its use would not infringe privately owned rights. Reference herein to any specific commercial product, process, or service by trade name, trademark, manufacturer, or otherwise, does not necessarily constitute or imply its endorsement, recommendation, or favoring by the United States Government or any agency thereof or its contractors or subcontractors. The views and opinions of authors expressed herein do not necessarily state or reflect those of the United States Government or any agency thereof.

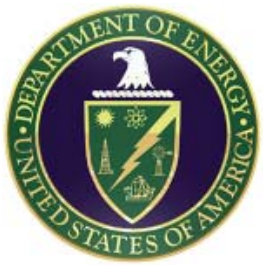




\section{Table of Contents}

Brookhaven Atmospheric Tracer Sampler: Urban Atmospheric Dispersion Study . . . . . . . 9 Adedoyin Akingba, Matthew B. Mayers, Jessica Saintibert, Marie Lamothe, Ilia Vardishvili, Lezou Dali

Standardizing climate model metadata and producing visualizations for user

discoverability in the Department of Energy's long term climate data archive . . . . 11 Neil Aram

Optimizing the target geometry and material type of the long-baseline neutrino

experiment beam line. . . . . . . . . . . . . . . . . . . . . . . . . . 14

Catherine Ayuso

The Subway-Surface Airflow Exchange Study. . . . . . . . . . . . . . . . . . . . 21 Adam Batley

Analysis of continuous long-term cloud records from climatically-significant global sites . . 23 Shannon Baxter

Analysis of soil temperatures in the Long Island Solar Farm (LISF) and its impact

on the local fauna and flora . . . . . . . . . . . . . . . . . 26

Ricky Bhola

Studies in calorimetry using silicon photomultipliers, and tracking using gas

electron multipliers for the super-PHENIX upgrade experiment . . . . . . . . . . . 29 Marie Blatnik

Analysis and fabrication of carbon micro-ribbon argets for proton polarimetry

studies at RHIC . . . . . . . . . . . . . . . . . . . . . . . . 35

Kevin Bogaert

Acoustic identification of Chiroptera species in eastern Suffolk County and

Brookhaven National Laboratory using full spectrum call analysis.. . . . . . . . . . 39

Jessica Bristol

Characterization of Room-Temperature Radiation Detectors. . . . . . . . . . . . . . . . 44 Trevor Chan

Determination of thickness for laser ablation plasma generation on a sputtered target . . . . 47 David Cinquegrani

Electrochemical and spectrophotometric studies of conjugated polymers . . . . . . . . 50 Altramiese Tippie and Tiera Corey

Detection of hydroxymethylcytosine in human cells exposed to ionizing radiation . . . . 57 Benjamin Daniels

Mapping Iron-based Fischer-Tropsch Synthesis Catalysts Using X-ray

Computed Microtomography . . . . . . . . . . . . . . . . . . . 61

Ryan Davella

Protein Crystallography Database programming, analysis, and modification . . . . . . . 65 Kyle Davis

Waveform digitizing electronics for time-of-flight detectors . . . . . . . . . . . . . 67 Olivia Dickens 
A performance study of the micro-channel plate photomultiplier tube . . . . . . . . . 70 Kahlil Dixon

Analysis of cloud observations to determine its impact on solar panel output. . . . . . . . 72 Alison Egbon

In silico metabolic control analysis of Brassica napus seeds based on

dynamic kinetic modeling . . . . . . . . . . . . . . . . . . 75

Michael Galazka

Reducing Hazardous Waste at Brookhaven National Laboratory . . . . . . . . . . . . . 81

Kenneth Goodman

Comparison of measured and calculated light scattering by aerosol particles

over the eastern North Pacific Ocean . . . . . . . . . . . . . . . . . . . . . . 84

Michelle Gostic

Expression, purification, and characterization of the Extender Module

of PolyKetide Synthase $(\mathrm{PKS}) \ldots \ldots$. . . . . . . . . . . . . . . . 86

Joshua Greenough

Optimization of perfluorocarbon tracer data analysis using Visual Basic . . . . . . . . 88

Nathan Gubser

Development and Application of "On-the-fly" Scanning for Fast XRF

Imaging and Microspectroscopy $\ldots \ldots \ldots 90$

Chris Guerrero

The Role of $\mathrm{Cu} / \mathrm{CeO} 2$ Nanospheres in the Water-Gas Shift reaction:

An X-ray Diffraction and Transmission Electron Microscopy study . . . . . . . . 92

Ilana Heckler

Investigation of Air Temperatures on the Long Island Solar Farm. . . . . . . . . . . . 95

Lauren Herrera

Redesigning an events $\&$ issues database to allow for statistical analysis and

accident prediction . . . . . . . . . . . . . . . . . . . 98

Daniel Hirsh

Using perfluorocarbon tracers to prevent chemical warfare . . . . . . . . . . . 102

Richard Houanche

Beam emittance measurements in the transfer line to RHIC at BNL . . . . . . . . . . . . 104

J. Huang

Systems Biology Markup Language formulation of a dynamic kinetic model

and its uses in quantitative and computational metabolic modeling . . . . . . . . 108

Mark $V$. Jensen

Characterization of new Hamamatsu Multi-Pixel Photon Counters and the AdvanSiD

8x8 Silicon Photomultiplier array for use in positron emission tomography. . . . . . 113

Ankit Johari

Monte-Carlo simulation of a slant-hole collimator in a trans-rectal gamma camera . . . . . 115 Mark Kamuda

Assessment of 5-hydroxymethylcytosine in the DNA of human skin fibroblasts

exposed to ionizing radiation . . . . . . . . . . . . . . . 118

Lucia Kolodiuk and Katherine Z. Sanidad 
Design and Installation of Oxygen Deficiency Sensors . . . . . . . . . . . . . . . . . 122 Kevin Korol

The use of mark-recapture techniques to study migration and population

densities of Odonate species on a utility scale solar farm . . . . . . . . . . . . . 124 Sarah Langan

Multigap resistive plate chamber for fast timing . . . . . . . . . . . . . . . . 130

Nathaniel Lashley-Colthirst

Experimental gas configuration management. . . . . . . . . . . . . . . . . 132

Raynold Lewis

Analysis and comparison of atmospheric aerosol concentrations measured

using two instruments over the eastern North Pacific Ocean . . . . . . . . . . . . . 135

Danielle Mallon

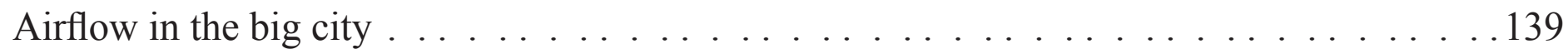

Mauro Mancilla

Oxigraf monitor installation.

Aaron Mohammed

Supporting the Standards-Based Management System staff on key initiatives . . . . . . . 147

Brittany Morgan

Vibrational analysis at Brookhaven National Lab ground sites and on existing x-y stages . .150 Scott Oldham

The study of the carbon monoxide oxidation reaction using copper-titania catalysts . . . . . 154 Naa Adokaley Pappoe

Design proposals for the Linac central malfunction user interface . . . . . . . . . . . . . 159

Divas Persaud

Charge-Coupled Device Calibration Methods: The Photon Transfer Curve . . . . . . . . . . 162

Kevin Powell

Tick population analysis and use of 4-poster devices

by white-tailed deer (Odocoileus virginianus $)$. . . . . . . . . . . . . . . . 165

Lucas Price

Hot-pressing and physical properties characterizations of polycrystalline

specimens of dysprosium titanate and dysprosium hafnate for elasticity studies

at extreme conditions. . . . . . . . . . . . . . . . . . . 170

Brandon Rhymer

An Analysis of the mPower Small Modular Reactor Design . . . . . . . . . . . . . . . 172

Jonathan Rolland

Magnetic shaping of laser ablation plasma . . . . . . . . . . . . . . . . 176

Mark Romanelli

Control of the beam orbit in the NSLS-II storage ring injection straight section $\ldots \ldots$. . 179

Evgeniya Rubin

Brookhaven atmospheric tracer sampler: Urban atmospheric dispersion study . . . . . . . . 182

Jessica Saintibert, Adedoyin Akingba, Lezou Dali, Ilia Vardishvili,

Marie Lamothe, Matthew B. Mayers 
Human machine interfaces for the Access Controls Security system

Linear Accelerator upgrade . . . . . . . . . . . . . . . . . . . . . . . . . . . . . . 184

Fidel Salgado

Digital multi-meter cable and powered magnet testing; creating datasets for tracking

and monitoring one-wire sensors at the National Synchrotron Light Source II . . . . 190

Pavel Salnikov

Phosphorylation in the phosphoenolpyruvate branch point: the extension

of a dynamic model of Brassica napus seeds. . . . . . . . . . . . . . . . . . . 193

Bradley T. Salvato

Linac early symptoms detection software. . . . . . . . . . . . . . . . . . . . . . 198

Kevin Sanders

Determining the optimum carbon stripping foil for use in cancer radiotherapy . . . . . . 201

Rachel Sandler

Differences between carbon and proton beam radiotherapy . . . . . . . . . . . . . 206

Rachel Sandler

Evaluation of DNA double-strand break repair kinetics in cells exposed

to low dose charged particle irradiation and vorinostat treatment . . . . . . . . . . . 209

Katherine Z. Sanidad

Cobalt Molybdenum Nitride Nanosheet as an efficient catalyst

for producing hydrogen from water . . . . . . . . . . . . . . . 213

Andrea Sarmiento

Synthesis and characterization of Tungsten Carbide/Nitride on Graphene

as a Hydrogen evolution electrocatalyst

Jonathan Schneider

Analysis of the perfluorocarbon tracer compounds used in the New York City

Subway-Surface Airflow Exchange program using gas chromatography

with electron-capture and thermal conductivity detection. . . . . . . . . . . . . 222

Mackenzie Shipley

Android application for Olog, a logbook service for accelerator operations . . . . . . . . .231

Michael Skinner

Urban Atmospheric Tracer Sampling: Samples throughout NYC . . . . . . . . . . . . . . 232

Nicholas Smith

From massive magnets to molten glass: My summer reporting on science

at Brookhaven National Laboratory . . . . . . . . . . . . . . . . . . . . . . . 234

Nicholas St. Fleur

Electrochemical and spectrophotometric studies of conjugated polymers . . . . . . . 235

Altramiese Tippie and Tiera Corey

Growth of tropical cumulus congestus clouds . . . . . . . . . . . . . . . . . . . . . 242

Katherine Towey

Value engineering report: Brookhaven National Laboratory Alternatives

to existing strontium-90 groundwater treatment system $\ldots \ldots$. . . . . . . . 245

Rebecca Winter 
Categorization and Statistical Analysis of Fast Solar Transients. . . . . . . . . . . . . . . 252 Jovani Withers

Bio-oil material compatibility. . . . . . . . . . . . . . . . . . . . . 255 Michael Worek

A study of muon injection efficiency improvements involving the electrostatic focusing quadrupoles in the Muon g-2 Experiment . . . . . . . . . . . . . . . . . . . 259 David $W u$

Phase shift full bridge power converter analysis and test fixture design $\ldots \ldots \ldots$. . . . . 263 Man Zhang

Regulation of triacylglycerol biosynthesis and storage in plant vegetative tissues . . . . . .267 Elizabeth Zipf

Appendix. . . . . . . . . . . . . . . . . . . . . . . 270

List of interns, their colleges or univerisities, and location in this compendium . . . . . 273 
viii Office of Educational Programs, 2012 Compilation of Internship Reports 


\title{
Brookhaven Atmospheric Tracer Sampler: Urban Atmospheric Dispersion Study
}

\author{
Adedoyin Akingba \\ Department of Biology, State University of New York at Potsdam, Potsdam NY, 13676 \\ Matthew B. Mayers \\ Department of Human Ecology, Cornell University, Ithaca NY, 14850-2824 \\ Jessica Saintibert \\ Department of Environmental Science, Tallahassee Community College, Tallahasee, F1 32304 \\ Marie Lamothe \\ Department of Engineering Science, Suffolk Community College, Selden, NY 11784 \\ Ilia Vardishvili \\ Department of Electrical Engineering, Stony Brook University, Stony Brook, NY 11790 \\ Lezou Dali \\ Department of Electrical Engineering, , Stony Brook University, Stony Brook, NY 11790 \\ John Heiser \\ Department of Environmental Sciences, Brookhaven National Laboratory, Upton NY 11973
}

\section{ABSTRACT}

The New York City Police Department requested that Brookhaven National Laboratory (BNL) develop an air flow survey for the Tristate Area. The survey was named The Subway Surface Air- Flow Exchange project which produced a detailed map of air flow patterns between the surface and subway systems to assist against chemical terrorism in a large populated city. In this survey, a chemical (Perflurocarbon (PFT)) was dispersed on surface and below in the subway. The liquid imitated any chemical that could be released accidentally or intentionally within a city. The PFT tracers being used are odorless, tasteless and non-radioactive, thus the population will not be affected in any way by their presence. Air samplers developed by BNL and Intel. were used to record flow rates of PFTs. The city was divided into three sections labeled as Intensive Operation Period (IOP) 1, IOP 2 and IOP 3. These areas were surveyed during a period of three days, which were selected by weather and wind conditions. The Brookhaven Atmospheric Trace Samplers (BATS) and Black Box Sampler (BBS) units were assembled by our specialized team in the laboratory for this specific study. The air sampling units were then distributed throughout the city to their respective IOP locations; as the PFTs were released within the city; and monitored by different groups. The data collected from this survey will provide the Department of Energy and Homeland Security with information for the New York Police Department's (NYPD) chemical counterterrorism unit. The information will help the NYPD better understand how to protect and prepare the city if an incident were to occur. Assisting in this project equipped me with useful experience in field and laboratory settings needed to be successful within scientific research professions.

\section{PROJECT}

New York City is one of the most populated cities in the world; therefore, it is more susceptible to terrorist attacks. Brookhaven National Laboratory (BNL) and the Department of Homeland Security collaborated to conduct an air flow stud. The Subway-Surface Air Flow Exchange (S-SAFE), in New York City to investigate air flow throughout its subway systems and surface areas. Studies similar to this have been conducted in the subway systems in Boston, and Washington, D.C.1 this will be the largest dispersion study to date. The air was sampled using air sampling devices known as (Black Box Samplers (BBS)) and (Brookhaven Atmospheric Tracer Samplers (BATS) about 200 of these sampling devices were used during the study, and sample dispensers units were used, which dispersed perflurocarbons at "select subway and street-level locations over three, non-consecutive days in July."1 The perflurocarbon tracers (PFTs) were the liquid of choice due to it harmless, odorless, and colorless properties. PFTs have been used in medical applications like eye surgeries and within artificial breathing systems."1 This project was highly contingent upon the weather conditions; meteorologists were assisting BNL with monitoring the weather patterns throughout the week. The results of this project provided the NYPD and the Department of Homeland Security with detailed statistics to execute a proper emergency response in the event a chemical toxin is released in New York City. Its sensitive nature requires that the data collected in this experiment be confidential from the public.

The Subway Surface Air Flow Exchange is, a \$3.4 million project funded by the Department of Homeland Security and run by BNL. The (S-SAFE) project took a couple of years to prepare. Our team worked diligently to prepare and monitor the equipment used within the project. The two instruments essential to the project were the (BATS) and (BBS) air sampling units. Both were tested and modified in preparation for S-SAFE stud. In addition, several other pieces of equipment were prepared that were beneficial to the project. The S-SAFE project was strategically spread across a grid of the city, each section of the city survey was identified as an Intensive Operation Period (IOP) and during survey days; which were randomly selected according to the weather; more interns were hired to aid in the process of monitoring the air samplers. Each team logged onto the BATS Wi-Fi to check flow rates within given increments of time, that ranged from 15, 30 and last 60 minute intervals The BBS air sampling unit were monitored differently, a light would show which tube was sampling within each interval. On IOP days, the NYPD had specific locations where teams would pick up and, later, dropped off the BATS and BBS air samplers. During each IOP day the air samplers were programmed to sampled air for 6 hours. For completion of the 
S-SAFE project, we performed other tasked such as fixing the wires, checking CATS tubes inside the BATS, placing guard tubes in the BBS, which were used to filter the air. Thereafter several cable wires and bungee cords were made to aid in holding the BATS and BBS securely in places while placed in their respective IOP locations. Also, we were assigned to make wooden strips to hold the guard tubes and the Capillary Absorption Tubes (CATS) inside each BBS. Wood strips were prepared by a mentor. Then elastic strips were fasten to the wood strips and numbered according to its respective BBS. Holes were drilled in several hundred plastic caps and attached to the apparatus. These plastic caps were used to connect the CATS tubes and the guard tubes. We were also assigned to charge the BATS for use during selected IOP days.

We were briefed that the motherboard controlling the sampling units contained four microprocessors, which enabled the samplers to have a GPS location, Wifi capability, and storage space for the specific program to collect the data. The circuit layout of the sampler contained a custom circuit board with throughhole and surface mount technology. The circuitry also contains four power shifter and other small components similar to capacitors, diodes, transistors, and resistors. The power shifters make it possible to switch between different valves within the BATS units; therefore, data can be collected. The switching system design of the units is built in a way that it is power conserving and environmentally efficient. They have a mass flow meter and pump controller, which resulted in accurate and precise flow rates over an operational range from 0 to $450 \mathrm{sccm}$. The perfluorocarbon traces was collected through the BATS using an absorbent called ambersorb. With this technology, we are also able to synchronize new schedules and programs wirelessly and download the data logging of each individual sampler. Furthermore, to power the units, they are equipped with $24 \mathrm{~V}$ rechargeable batteries and a small $3 \mathrm{~V}$ battery to power the GPS tracking. Everything on the BATS units is up-to-date technology. The Black Box Samplers units function in a slightly different way, the brain is simpler circuitry and is not equipped with most of the tools that the BATS units have. Since the technology is older, it had less sufficient data and more troubleshooting problems.

This study, expected to help the New York Police Department and other agencies to refine their emergency evacuation plans, was a success. Were confident that if a terrorist act were to result in the release of a dangerous gas that could harm citizens, that the city's first responders, should have an idea whether to shelter people, or in a case of evacuation, know what evacuation routes to take. Although the study focuses more on a potential terrorist attack; the result will help with determining the dispersion possibilities of other inhalational gases, such as smoke or fumes from an industrial accident or a chemical spill. These results will help ensure America's security and prosperity through environmental means

\section{ACKNOWLEDGEMENTS}

This project was supported in part by the U.S. Department of Energy, Office of Science, Office of Workforce Development for Teachers and Scientists (WDTS) under the Science Undergraduate Laboratory Internships Program (SULI)

\section{REFERENCES}

1. BNL Newsroom | "New York City Police Department and Brookhaven Lab to Conduct Airflow Study In New York City Streets and Subways This Summer."

2. BNL Newsroom | New York City Police Department and Brookhaven Lab to Conduct Airflow Study In New York City Streets and Subways This Summer. Brook Haven National Lab, 24 Apr. 2013. Web. 28 July 2013. 


\title{
Standardizing climate model metadata and producing visualizations for user discoverability in the Department of Energy's long term climate data archive
}

\author{
Neil Aram \\ Computer and Electrical Engineering, Binghamton University, Binghamton, NY 13902
}

\begin{abstract}
Climate analysis and modeling provides knowledge about the current state of our climate, and how it may continue to change. Short-term weather forecasting and long-term climate modeling depend upon having sufficient atmospheric data. The U.S. Department of Energy's Atmospheric Radiation Measurement (ARM) program provides this service to scientists globally. ARM has many permanent and mobile sites around the world which gather atmospheric data, as well as an External Data Center (XDC) which receives data from other institutions and stores it within its database. To ensure that users can easily find and use data, ARM imposes requirements which must be met before it is available to users. Some import guidelines include: being in netCDF file format, having descriptive, non-redundant variable names, and ensuring all measurement variables are functions of time. These guidelines ensure that users can locate specific, relevant data within the ARM archives. It also enables the use of powerful utilities to analyze data. I have been working on standardizing Rapid Refresh (RAP) climate model data, as well as creating contour plots. These plots can help users identify macroscopic trends in data, as well as indicate data quality. I have also created time-series plots of precipitable water vapor retrieved from multiple instruments. These are useful for identifying time domain trends, and doing simple, effective comparisons between datasets. We successfully used the Python programming language to create utilities which can plot a variety of different data sets.
\end{abstract}

\section{THE ARM FACILITY}

The Atmospheric Radiation Measurement (ARM) climate research facility is a national user facility which provides global climate data to research scientists worldwide. It has many permanent and mobile sites around the world which gather a wide variety of atmospheric data for storage in the ARM archive. It is one of the largest long-term climate data archives, containing over 20 years of observational climate data from around the world. ${ }^{1} \mathrm{ARM}$ also has an external data center (XDC) tasked with acquiring data-streams produced by other institutions, as well as processing and archiving them. ${ }^{2}$ With such a wealth of climate data, an effective user interface is essential to ensure that users may locate data which is relevant to their research. Figure one shows the data discovery tool which ARM users utilize to find and download data from the ARM archive. Specifically, it is being used to find precipitable water vapor measurements take in Cape Cod, MA. In addition to being accessible, all data on the archive should be un- derstandable as well. To achieve this, ARM has compiled a standards document which outlines requirements for data to become publicly available on the ARM archive. Once standardized, utilities exist which will take an input data stream and produce value added products. These products may be variables derived from existing measurements, or data quality information. ${ }^{3}$ Our goal in this project was to create software utilities which standardized data files, as well as produced plots of the data. These utilities are intended to make more data eligible for inclusion into the archive, as well as produce visuals to help users find relevant data.

NetCDF is a self-describing data format, typically used for scientific data, and it is the standard format for ARM data files. Several tools were used for the process of standardizing data files. The model data being standardized begins in gridded binary (GRIB) format. Therefore, it must be converted into netCDF, as well as modified and plotted once in netCDF format. The Python programming language was selected as the primary tool for working with netCDF data files due to its netCDF and plotting libraries. It is also platform independent, as well as free for academic use. NCAR command language (NCL) as well as netCDF operator (NCO) utilities were used to convert files from GRIB to netCDF and do some minor file modification respectively.

The primary data used in this project is from the Rapid Refresh (RAP) numerical weather prediction model. It is a shortterm weather forecasting model which is released hourly and provides hourly data for the next eighteen hours. ${ }^{4}$ The RAP model is run at the National Centers for Environmental Prediction (NCEP) and is formatted according to NCEP standards. Standardizing the RAP model data begins by converting it from GRIB format into netCDF using NCL. Following that, NCO utilities are used to add time variables to the data. This includes a base time and time_offset variable which are necessary for concatenating or splitting data files. The final step in standardizing the RAP data involved creating a mapping of variable names as well as organizing attributes. Our goal was to have concise, non-redundant variable names, which bridged conventions between the observational data community and the numerical weather prediction community. This process involved "decoding" the variable names assigned by the NCL utility with the help of NCEPs GRIB tables. Once the data conforms to all necessary ARM guidelines, it may be submitted for inclusion into the ARM archive.

In addition to standardizing data so it may become available to users, this project aimed to produce visuals which aid users in finding relevant data. This was done using the matplotlib library for Python as well as its basemap toolkit for plotting model data on a map projection. Creating contour plots of RAP model data first requires extracting the desired variable data, such as pressure, temperature, or wind speed. After this, a range and reso- 


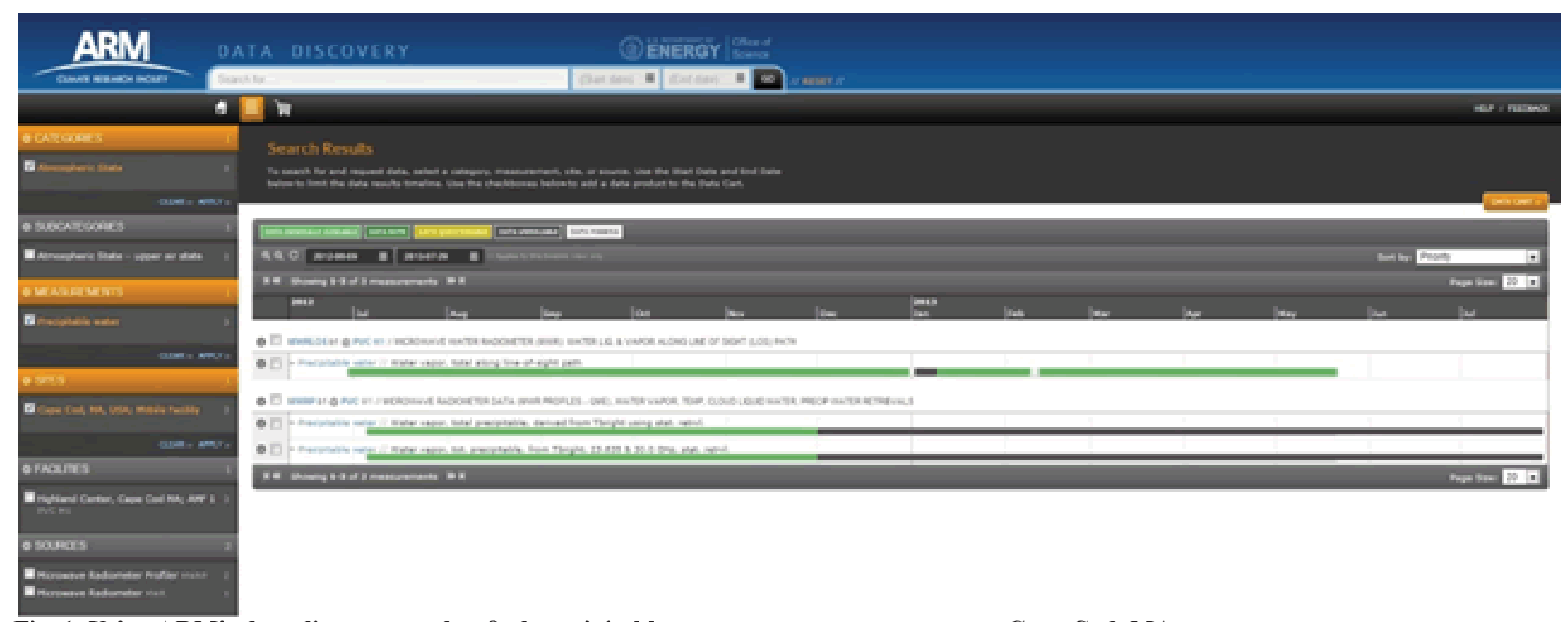

Fig. 1. Using ARM's data discovery tool to find precipitable water vapor measurements at Cape Cod, MA.

lution must be selected for the contour lines, as well as the geographic area the plot will show. The Python code was intended to allow for easy manipulation of these parameters by users. This was achieved quite easily with help from the effective interface provided by the basemap toolkit.

\section{VIZUALIZATIONS}

Figure 2 is an excellent example of how plots may quickly provide information about macroscopic data trends. It is immediately apparent that there are extremely high precipitable water levels off the east coast of the U.S., which were caused by Hurricane Sandy. Considering that these plots coincide with what we expect of a hurricane, they can be indications of reliable data to a degree. They may also help a user decide if this data is relevant to their research without having to download the data and do any analysis themselves. This not only saves the user valuable time, it may also reduce stress on the archive by preventing data retrieval for preliminary analysis. A scientists looking for data in which the hurricane is in a particular location may do so simply by viewing plots like these of prospective data. Compiling multiple of these plots for consecutive hours can provide temporal as well as spatial information.

The time series plot in figure 3 compares precipitable water vapor in Cape Cod, MA from three sources: measured directly by the microwave radiometer (MWR), calculated by the ECMWF numerical weather prediction model, and derived from measured quantities from the Cimel sun photometer (CSPHOT). PWV was derived from specific humidity and pressure at 50 vertical levels using the following equation: ${ }^{4}$

$$
\mathrm{PWV}=\frac{1}{g} \sum q_{i}\left(p_{i}-p_{i-1}\right)
$$

Comparing a measurement from multiple sources in this fashion can be a very good indication of data quality. With more instruments being compared, it is possible to tell if any of them produce data which varies significantly from the rest, and may be unreliable. A plot like this is also useful for verifying the validity of a derived quantity, by comparing it to reliable observational data. Figure 4 shows a more interesting case. The high time resolution MWR data is being compared to the low resolution EC-

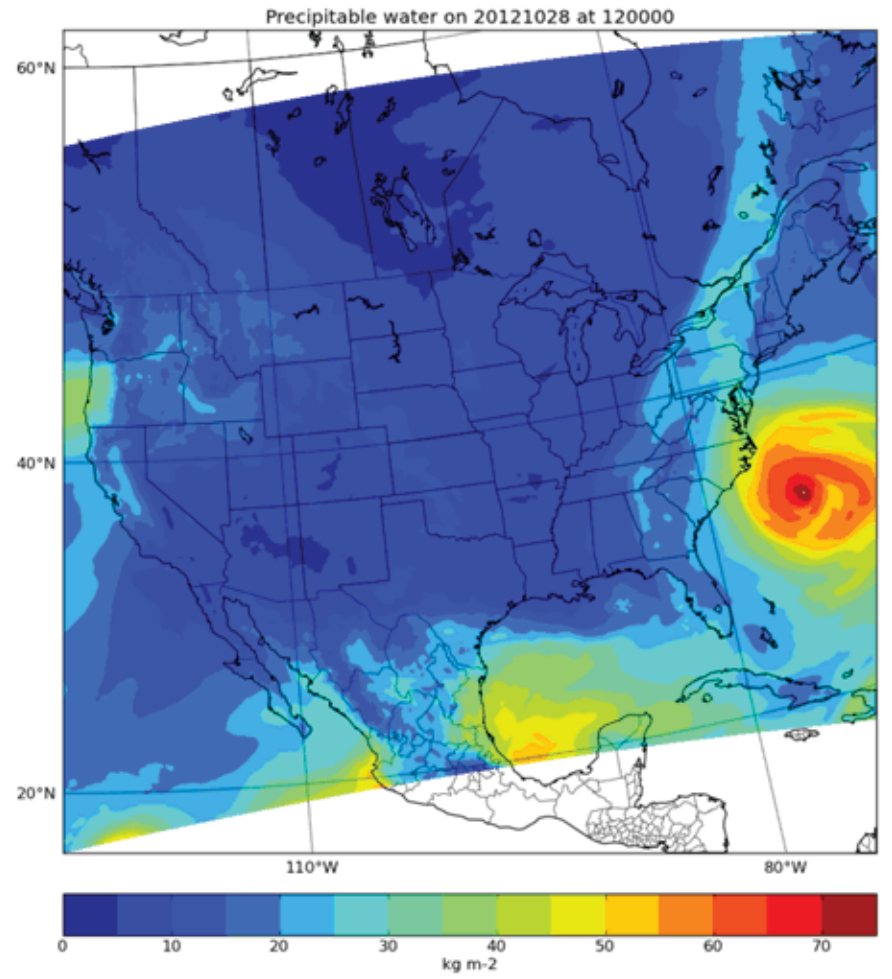

Figure 2. Precipitable water vapor from RAP weather prediction model for 12:00 on 10/28/2013.

MWF data. These data capture the arrival of the outer bands of Hurricane sandy in Cape Cod, which causes many spikes in the high resolution MWR data. Although the ECMWF data does not reproduce these spikes, it matches the trend of the MWR

\section{CONCLUSION}

The figures above serve to demonstrate how helpful visuals can be in gaining qualitative information about a dataset. Users already have the capability to search for data by measurement, location, date, and many other metadata. Implementing a system which allows users to plot and compare data in a similar fashion as these figures would allow them to make their selection based on data characteristics which they interpret from these figures. 


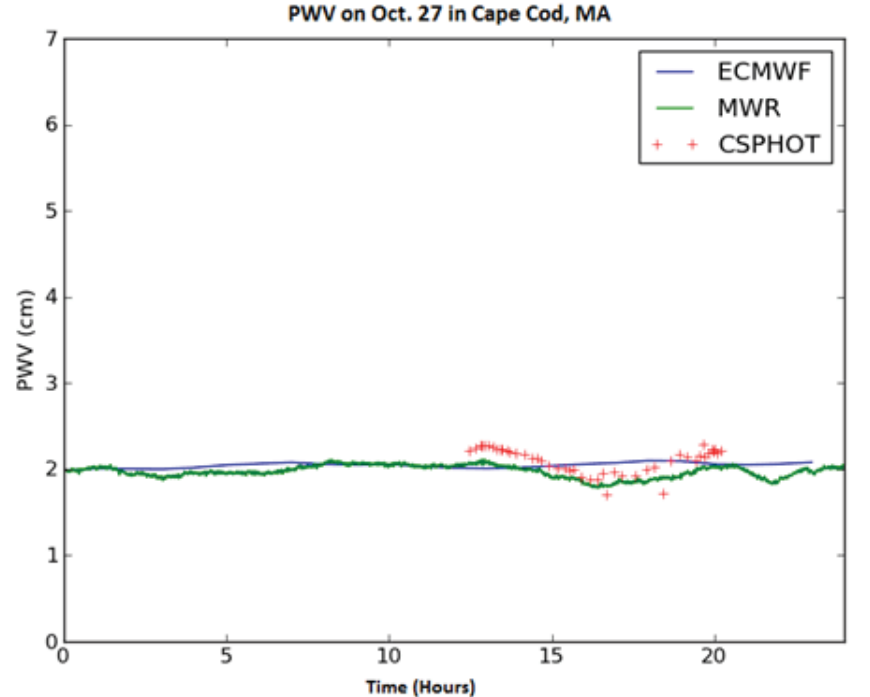

Figure 3. Precipitable water vapor from multiple instruments on 10/27/2013, in Cape Cod, MA.

\section{ACKNOWLEDGEMENTS}

I would like to thank my mentor, Laurie Gregory, as well as Dr. Richard Wagener for their guidance throughout this project. This project was supported in part by the U.S. Department of Energy, Office of Science, Office of Workforce Development for Teachers and Scientists (WDTS) under the Science Undergraduate Laboratory Internships Program (SULI)

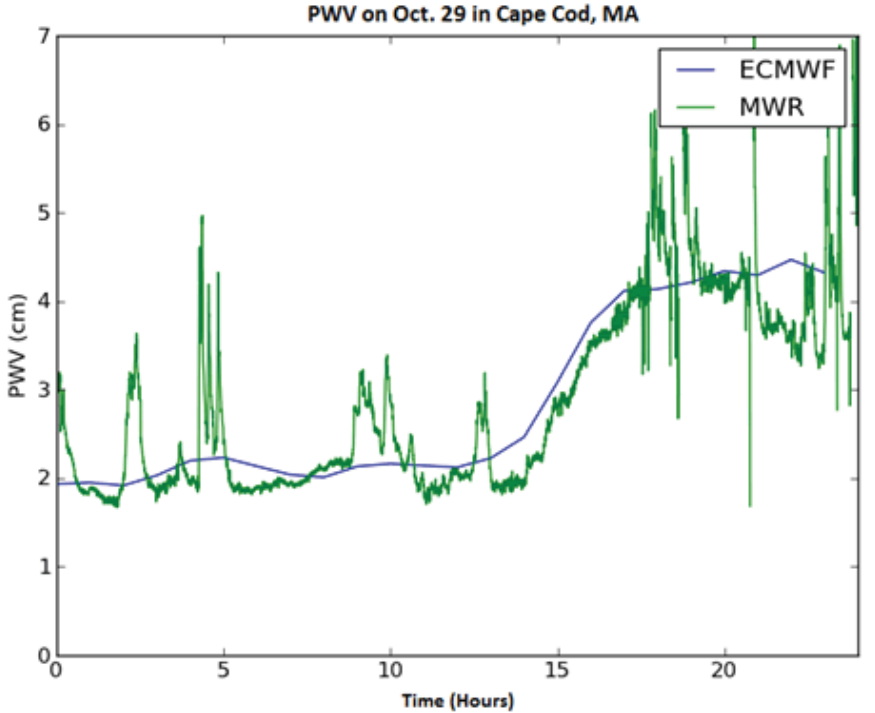

Figure 4. Precipitable water vapor from multiple instruments on 10/29/2013, in Cape Cod, MA.

\section{REFERENCES}

${ }^{1}$ James Mather, Jimmy Voyles. March 2013, THE ARM CLIMATE RESEARCH FACILITY, American Meteorological Society.

${ }^{2}$ External Data Center, Department of Energy, August 1 2013, $<\mathrm{http} / / / \mathrm{www} . \operatorname{arm} . g o v / \mathrm{xdc}>$

${ }^{3}$ ARM Organization. Department of Energy, August 12013. $<$ http://www.arm.gov/about/organization>.

${ }^{4}$ Rapid Refresh (RAP),National Oceanic and Atmospheric Administration, August 1 2013. <http://rapidrefresh.noaa. gov/> 


\title{
Optimizing the target geometry and material type of the long-baseline neutrino experiment beam line
}

\author{
Catherine Ayuso \\ Florida International University, Miami, FL 33174 \\ Zeynep Isvan \\ Brookhaven National Laboratory, Upton, NY 11973 August 9, 2013
}

\begin{abstract}
Neutrinos are neutral particles in the Standard Model of particle physics that, although very abundant in quantity around us, have yet to be fully understood. We currently know of three types, or flavors, of neutrinos, which have been observed to change from one flavor to another upon traveling a distance. This quantum mechanical phenomenon is known as neutrino oscillations and can be parameterized by three mixing angles $(\theta 12, \theta 23$ and $\theta 13)$, the difference in the squares of the neutrino masses $(\Delta \mathrm{m} 221, \Delta \mathrm{m} 232)$ and the CP-violating phase $\left(\delta_{C P}\right)$. The Long-baseline Neutrino Experiment (LBNE), a project proposed by several laboratories and research groups nationwide, including Brookhaven National Laboratory (BNL), seeks to measure the $\mathrm{CP}$-violating phase $\left(\delta_{C P}\right)$, and to resolve the order of the masses, known as the mass-hierarchy problem. Plans of developing a beam line to accurately measure these parameters have initiated at BNL by running exploratory simulations seeking to determine the most optimal and technically feasible beam design. In this study, we evaluate a variety of simulated beam lines by varying the target material, geometry and size. Results show that the most optimal target design is a beryllium cylindrical target of $96 \mathrm{~cm}$ in length. With this configuration, neutrino flux, in the $v_{u-}>$ ve oscillated channel, increases for various types of simulated beam lines, particularly in the energy range most sensitive to neutrino oscillations $(0-2 \mathrm{GeV})$. More fine tuning optimizations are on-going on the length and location of this beryllium target. These results and the ones to come will aid in establishing the final design decision for the LBNE beam line. Being a part of the LBNE research group and conducting this study has exposed me to various methods of conducting optimization studies in high energy physics and to the programs used in these studies, such as particle simulator program, Geant4 and beam line visualizer, Heprapp. I have also become more comfortable using various types of computer languages most commonly used in experimental physics such as python and $\mathrm{C}++$. This experience has also helped solidify my research graduate school preferences for next year.
\end{abstract}

\section{Introduction}

\section{A. The Standard Model}

Neutrino physics is gravitating towards the forefront of experimental science primarily because of the challenges recent experimentally demonstrated characteristics of neutrinos seem to pose to the Standard Model of Physics (SM). In the Standard Model, both neutrinos and antineutrinos are massless [1]. However, with the discovery of neutrino oscillations, a phenomenon predicted by Italian nuclear physicist, Bruno Pontecorvo, shown through atmospheric experiments by SuperKamiokande and confirmed by other particle physics projects studying neutrinos such as Daya Bay and MINOS, it is now known that at least two of the three active neutrinos must be massive in order for these flavor transformations to take place [1] [4] [5]. If not for a complete reworking of the SM, these findings at least call for an extension to its framework that includes non-zero masses and their ranking; a task that several research groups and laboratories, including BNL, are interested in being a part of.

\section{B. CP Violation}

Besides challenging the framework of the Standard Model of Physics, further exploration of this oscillation phenomenon may point to a mechanism that not only gives neutrinos mass but allows for the possibility of explaining the matter-antimatter asymmetry of the universe. Arguments based on cosmology and particle physics conclude that the universe contained equal amounts of particles and anti-particles right after the Bing Bang, yet we now observe a very obvious asymmetry, with virtually no anti-particles present [2].

One of the most compelling responses to this particle asymmetry is the proposal of matter and anti-matter being intrinsically different which in turn, makes them behave and evolve differently. This difference in behavior would be a violation of $\mathrm{CP}$ (Charge-parity) invariance [1]. While laboratory experiments have demonstrated $\mathrm{CP}$ violation in quark mixing due to a phase factor similar to that of the phase factor found in neutrino mixing $\left(\delta_{\mathrm{CP}}\right)$, the contribution of this violation to the matter-antimatter asymmetry we observe today is not a significant one [2]. Consequently, the search continues for other violators of this charge-parity invariance and experimental and theoretical discoveries of the past decade, such as the observation of different neutrino mass states, point at the neutrino as a likely candidate.

The violation of $\mathrm{CP}$ in any physical system arises from the effects of complex phase factors in quantum mechanical amplitudes. In neutrino physics, these phase factors are found in the leptonic mixing matrix,

$$
\begin{aligned}
U & =\left(\begin{array}{lll}
U_{e 1} & U_{e 2} & U_{\epsilon 3} \\
U_{\mu 1} & U_{\mu 2} & U_{\mu 3} \\
U_{\tau 1} & U_{\tau 2} & U_{\tau 3}
\end{array}\right) \\
& =\left(\begin{array}{ccc}
1 & 0 & 0 \\
0 & c_{23} & s_{23} \\
0 & -s_{23} & c_{23}
\end{array}\right)\left(\begin{array}{ccc}
c_{13} & 0 & s_{13} e^{-i \delta} \\
0 & 1 & 0 \\
-s_{13} e^{i \delta} & 0 & c_{13}
\end{array}\right)\left(\begin{array}{ccc}
c_{12} & s_{12} & 0 \\
-s_{12} & c_{12} & 0 \\
0 & 0 & 1
\end{array}\right) \\
& =\left(\begin{array}{ccc}
c_{12} c_{13} & s_{12} c_{13} & s_{13} e^{-i \delta} \\
-s_{12} c_{23}-c_{12} s_{23} s_{13} e^{i \delta} & c_{12} c_{23}-s_{12} s_{23} s_{13} e^{i \delta} & s_{23} c_{13} \\
s_{12} s_{23}-c_{12} c_{23} s_{13} e^{i \delta} & -c_{12} s_{23}-s_{12} c_{23} s_{13} e^{i \delta} & c_{23} c_{13}
\end{array}\right)
\end{aligned}
$$

a matrix which contains information about the strength of neutrino mixing [1] [6].

LBNE aims to precisely measure this phase factor by exploring various cases of neutrino oscillations [7]. If shown to be 
non-zero, this finding will be directly linked to the matter- antimatter asymmetry of observed today and may ultimately explain why the universe evolved the way it did.

\section{Neutrinos and Neutrino Oscillations}

The exploration of this phenomenon requires preliminary understanding of what these neutrino flavors are, how they are defined and how we're able to detect their oscillations. We currently know of three different flavors of neutrinos: the electron neutrino, $v e$, the muon neutrino, $v \mu$, and the tau neutrino, $v \tau$, and each are coupled through the weak interaction to a flavor corresponding charged lepton [3]. When the carrier of the weak force (W boson) decays into a charged lepton and a neutrino, the flavor of the neutrino is always the same as the flavor of the lepton. Inversely, when a neutrino collides with a target and produces a charged lepton, the resulting lepton will be of the same flavor as the interacting neutrino [2]. If we know the original flavor of the neutrino, observing its flavor oscillation upon traveling a distance becomes a relatively straightforward task. Once the flavor is identified and the neutrino is allowed to propagate, the neutrino must interact with a target to produce a lepton; if the lepton flavor is different from the original flavor of the neutrino, then the neutrino oscillated flavors before interacting with the target.

The reason for these oscillations is deeply rooted in quantum mechanical principles. It is due to each neutrino existing at a different superposition of three quantum mechanical mass eigenstates, where each state has a corresponding mass value only marginally different from the others [6]. In other words, each weak type neutrino of the known flavors, is a precise but different mixture of the three neutrinos of definite mass. Because within each flavor state are three different mass-types, as the neutrino propagates, the phases of the heavier ones lag behind while the lighter ones pull ahead. These slightly different velocities are a result of the mass and energy dependency of a relativistic particle's velocity observed in the following equation [3]:

$$
v=c\left(1-\frac{\left[\frac{m c^{2}}{E}\right]^{2}}{2}+\ldots\right)
$$

These differences in velocity result in a changing mixture of mass states which in turn may yield a different flavor state.

In matrix notation, this relationship between flavor and mass eigenstates is express as follows:

$$
\left(\begin{array}{l}
v_{e} \\
v_{\mu} \\
v_{\tau}
\end{array}\right)=U\left(\begin{array}{l}
v_{1} \\
v_{2} \\
v_{3}
\end{array}\right)
$$

where $U$, is the leptonic mixing matrix define in (1) [1].

Generally, a neutrino created at $\mathrm{t}=0$ in a weak flavor $\alpha$ can be expressed as a sum of mass eigenstates $i$, where $i=1,2,3 \ldots$ :

$$
\left|v_{\alpha}(0)\right\rangle=\sum_{i} U_{\alpha i}\left|v_{i}\right\rangle
$$

Mass eigenstates are eigenstates of the Hamiltonian and evolve through time as such while the neutrino propagates:

$$
\begin{aligned}
& \text { where }\left|v_{\alpha}(t)\right\rangle=\sum_{i} U_{\alpha i} e^{i p_{i} \cdot x}\left|v_{i}\right\rangle \\
& \qquad p_{i} \cdot x=E_{i} t-\mathrm{p} \cdot \mathrm{x} \\
& =t \sqrt{|p|^{2}+m_{i}^{2}}-\mathrm{p} \cdot \mathrm{x}
\end{aligned}
$$

with $E_{i}$ and $m_{i}$ representing the energy and mass of the $i$ th mass eigenstate [1]. Moreover, the assumption, $t=L$ and $\mathbf{p} \cdot \mathrm{x}=|\mathbf{p}| \mathrm{L}$, can be made for utra-relativistic neutrinos because $m_{i}<<E_{i}[3]$. This simplifies the term within the exponent in (5) to:

$$
\begin{aligned}
& p i \cdot x=|p| L\left(1+\frac{m_{i}^{2}}{2|p|^{2}}\right)=|p| L \\
& =\frac{m_{i} L}{2 E}
\end{aligned}
$$

To understand how the parameters governing neutrino oscillations come into play, we consider the case of muon neutrino survival after traveling a distance $\mathrm{L}$ in the following two- flavor approximation:

$$
\begin{gathered}
\left(\begin{array}{c}
v_{\mu} \\
v_{\tau}
\end{array}\right)=\left(\begin{array}{c}
\cos \theta \sin \theta \\
-\sin \theta \cos \theta
\end{array}\right)\left(\begin{array}{c}
v_{2} \\
v_{3}
\end{array}\right) \\
|\nu(0)\rangle=\left|\nu_{\mu}\right\rangle=\cos \theta\left|v_{2}\right\rangle+\sin \theta\left|v_{3}\right\rangle \\
|\nu(L)\rangle=\left|\nu_{\mu}\right\rangle=\cos \theta \exp \left(-i \frac{m_{2}^{2} L}{2 E}\right)\left|\nu_{2}\right\rangle+\sin \theta \exp \left(-i \frac{m_{3}^{2} L}{2 E}\right)\left|\nu_{3}\right\rangle
\end{gathered}
$$

The probability that the neutrino will be detected with the same flavor after traveling a distance $\mathrm{L}$ is given by:

$$
\begin{aligned}
P_{\nu_{\mu} \rightarrow \nu_{\mu}} & =\left|\left\langle\nu_{\mu} \mid \nu(L)\right\rangle\right|^{2}=\left|\cos ^{2} \theta \mathrm{e}^{-\frac{i m_{2}^{2} L}{2 E}}+\sin ^{2} \theta \mathrm{e}^{-\frac{i m_{3}^{2} L}{2 E}}\right|^{2} \\
& =1-2 \cos ^{2} \theta \sin ^{2} \theta\left[1-\cos \left(\frac{\Delta m_{23}^{2} L}{2 E}\right)\right] \\
& =1-\sin ^{2} 2 \theta\left[2 \sin ^{2}\left(\frac{\Delta m_{23}^{2} L}{4 E}\right)\right] .
\end{aligned}
$$

From the resulting probability equation we see, the emergence of one of the mixing angles, $\theta_{23}$, and the term of the mass difference betweem $\mathrm{m}_{2}$ and $\mathrm{m}_{3}$ squared, $\Delta \mathrm{m}_{23}^{2}$. The remaining parameters, including phase $\delta_{C P}$, can be obtained similarly, by considering other neutrino mixing cases. For example, in the more complete three-flavor case, the probability of electron neutrino appearance is parameterized as [1]:

$P\left(v_{\mu} \rightarrow v_{\mathrm{e}}\right) \cong \sin ^{2} 2 \theta_{13} \mathrm{~T}_{1}-\alpha \sin 2 \theta_{13} \mathrm{~T}_{2}+\alpha \sin 2 \theta_{13} \mathrm{~T}_{2}+\alpha^{2} \mathrm{~T}_{4}$ where the parameters $\mathrm{T}_{\mathrm{n}}$ are calculated as follows:

$$
T_{1}=\sin ^{2} \theta_{23} \frac{\sin ^{2}[1-x] \Delta}{(1-x)^{2}}
$$

$$
\begin{gathered}
T_{2}=\sin \delta \sin 2 \theta_{12} \sin 2 \theta_{23} \sin \Delta \frac{\sin (x \Delta)}{x} \frac{\sin [(1-x)] \Delta}{(1-x)} \\
T_{3}=\cos \delta \sin 2 \theta_{12} \sin 2 \theta_{23} \cos \Delta \frac{\sin (x \Delta)}{x} \frac{\sin [(1-x)] \Delta}{(1-x)} \\
T_{4}=\cos ^{2} \theta_{23} \sin ^{2} \theta_{12} \cos \Delta \frac{\sin ^{2}(x \Delta)}{x^{2}}
\end{gathered}
$$


with,

$$
\begin{gathered}
\Delta \equiv \Delta m_{31}^{2} L / 4 E \\
\alpha \equiv \Delta m_{21}^{2} / \Delta m_{31}^{2} \\
x \equiv 2 \sqrt{2} G_{F} N_{e} E / \Delta m_{31}^{2}
\end{gathered}
$$

By inspection of (11) and (12), we observe the three leading parameters that describe oscillation probabilities: the mixing parameters involving mixing angles, the value of $\delta_{c p}$ and the differences between three masses squared. We characterize and defined them in our simulations as follows:

- The $\sin ^{2} \theta_{i j}$ are the mixing parameters, defined by the mixing angles, $\theta_{i j}$, which determine the maximum probability of conversion and their values are observed experimentally. The values used in the simulation were defined as follows:

\begin{tabular}{|c|r|}
\hline Parameter & \multicolumn{1}{|c|}{ Value } \\
\hline $\sin ^{2} \theta_{13}$ & 0.100 \\
\hline $\sin ^{2} \theta_{13}$ & 1.00 \\
\hline $\sin ^{2} \theta_{13}$ & 0.869 \\
\hline
\end{tabular}

Table 1: Values of mixing parameters used in LBNE simulations [3]

- The CP-violating phase, $\delta_{\mathrm{cp}}$, has yet to be experimentally determined but if observed to be non-zero, this will confirm if neutrino oscillation violates CP symmetry [2]. We alternate the value of this phase from 0 to the maximum value, $\pi / 2$ in our simulations. Because the statistical trend of this study is the same, results shown are for $\delta_{\mathrm{cp}}=0$.

$\left|\Delta \mathrm{m}^{2}{ }_{\mathrm{ij}}\right|$ is the difference between the values of the neutrino masses squared, $\left|\mathrm{m}_{\mathrm{i}}^{2}-\mathrm{m}^{2}\right| \cdot[2]$ It has been determined that neutrino masses, $\mathrm{m}_{1}$ and $\mathrm{m}_{2}$, have squared masses with a separation of $\mathrm{m}_{\text {sol }}^{2}$ $=\mathrm{m}^{2} 12=7.59 \times 10-5 \mathrm{eV}^{2}[3]$. The separation between the third definite mass, $\mathrm{m} 3$, and the other two is $\mathrm{m}_{\text {atm }}^{2}=2.32 \times 10^{-3} \mathrm{eV}^{2}$, about thirty times larger [3]. The order of masses remains to be discovered. While we know from solar experiments that $\mathrm{m}_{1}<\mathrm{m}_{2}$, we do not know whether $\mathrm{m}_{3}<\mathrm{m}_{1}$ or $\mathrm{m}_{3}>\mathrm{m}_{1}$. If the $\mathrm{m}_{1}-\mathrm{m}_{2}$ pair is at the bottom, the neutrino hierarchy would resemble the charged lepton and quark spectra therefore earning the name of normal hierarchy [2] [7]. If it is at the top, the spectrum would be referred to as an inverted hierarchy [2]. The following is a graphical representation of both possible hierarchies:

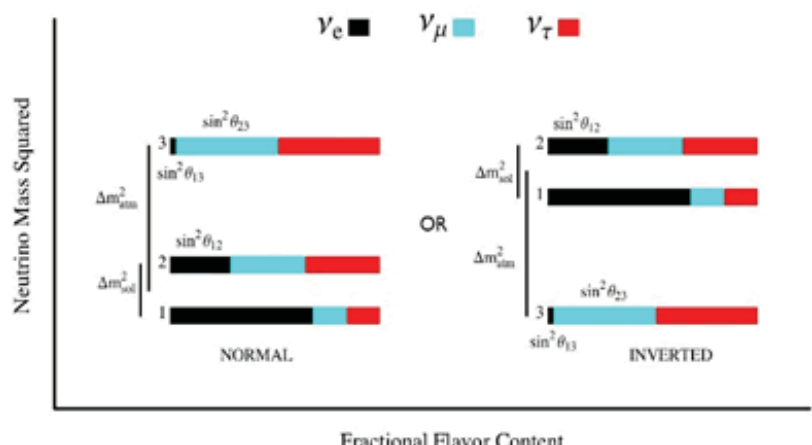

Figure 1: Normal (left) and inverted hierarchies. The colors indicate flavor composition of each mass eigenstate [2].
The varying effects of these leading parameters on oscillation probabilities can be seen graphically in the following plots:
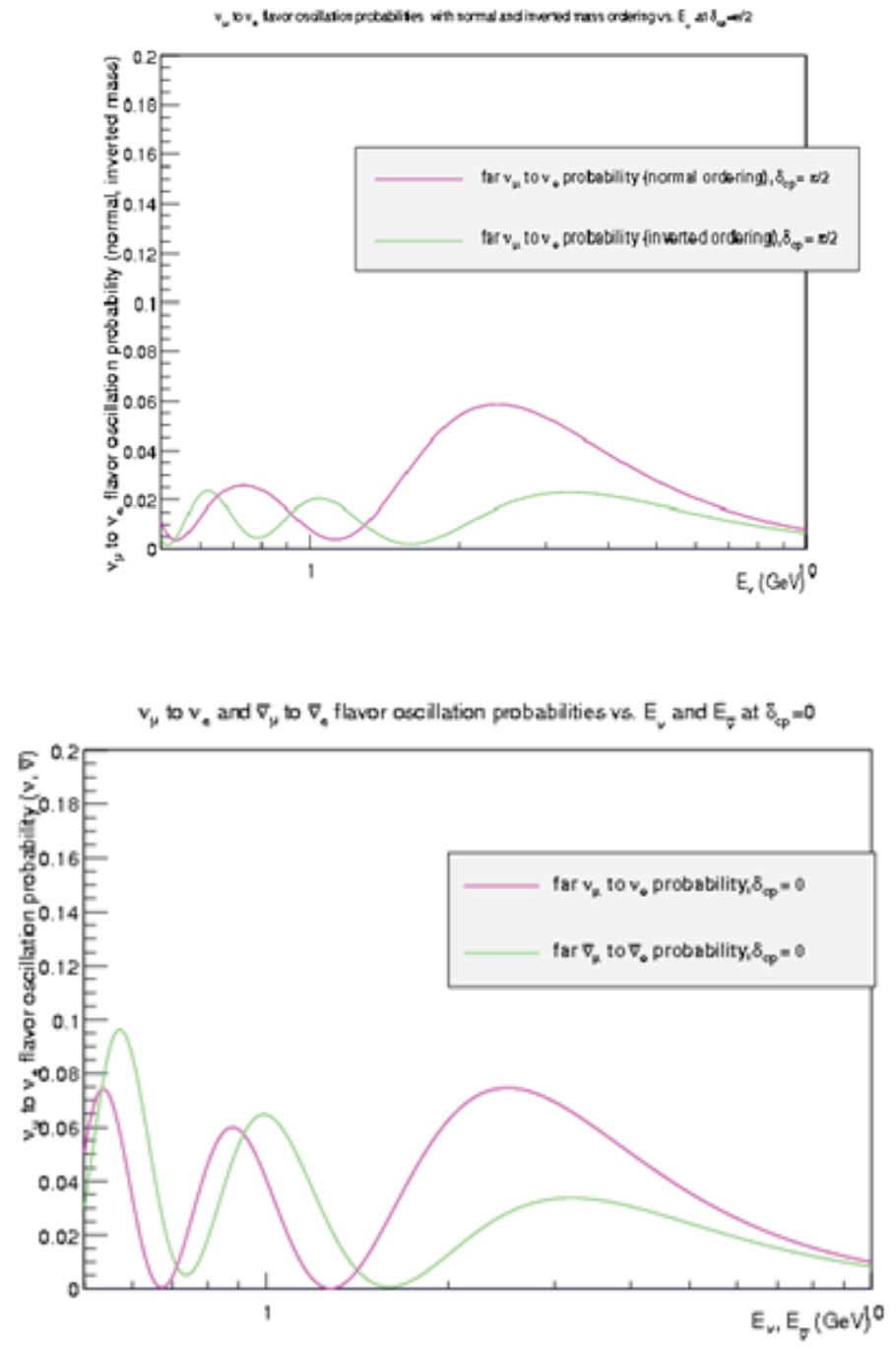

Figure 2: Flavor Oscillation Probabilities for Normal and Inverted Mass Hierarchies (top) and neutrinos and anti-neutrinos (bottom).

In long-baseline experiments, these probability values are determined by comparing the expected number of neutrinos in a given flavor in the absence of oscillation to the observed number of neutrinos in that flavor [8]. Via parameter fitting and equations (11) and (12), these survival and oscillation probability values are utilized to determine the oscillation parameters of interest. For LBNE, these parameters are $\delta_{c p}$ and the mass ordering parameters that will help determine the hierarchy of neutrino masses.

\section{The LBNE Experiment and Beam Line}

The Long-Baseline Neutrino Experiment is designed to make precision measurements of these remaining unknown neutrino oscillation parameters. The experiment consists of a muon neutrino beam generated at Fermilab and directed towards the LBNE Far Detector $1300 \mathrm{~km}$ away South Dakota [8]. The detector can observe both muon neutrinos and electron neutrinos, therefore, LBNE can measure both the survival probability of muon neutrinos and the appearance probability of electron neutrinos. In order to make a muon neutrino beam, $120 \mathrm{GeV}$ protons extracted from the Fermilab Main Injector (NuMI) are incident on an $80 \mathrm{~cm}$ 


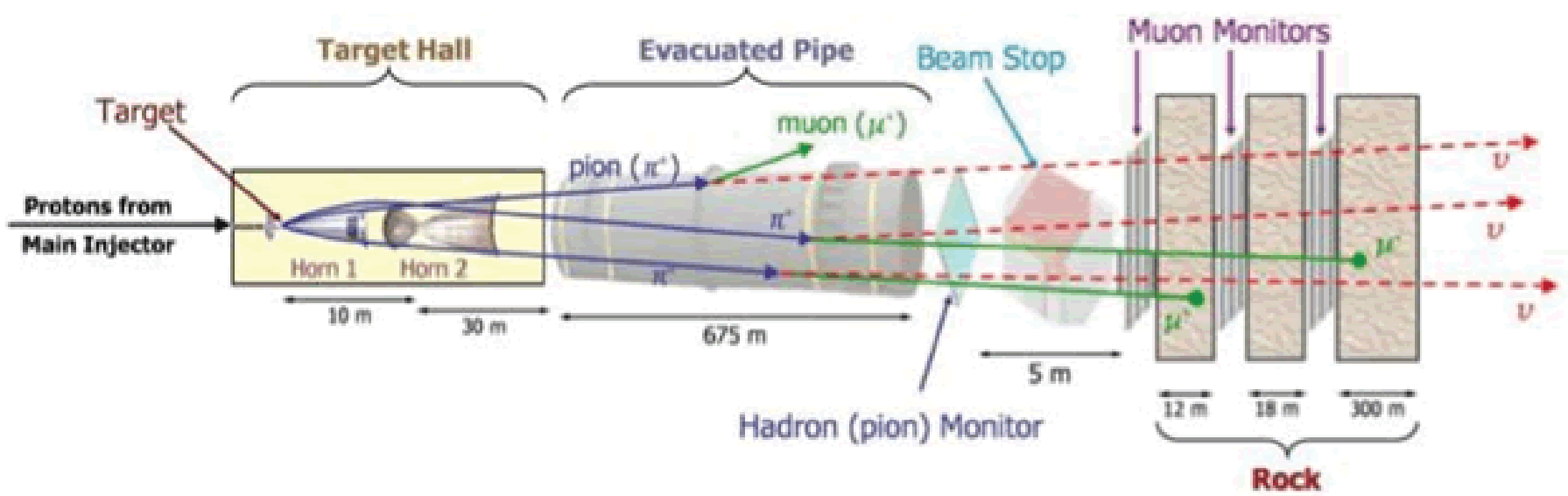

Figure 3: The LBNE beam [10].

long cylindrical graphite target of $6 \mathrm{~mm}$ radius [8]. This produces a secondary beam of pions and kaons that are focused by a pair of magnetic horns of similar horn design to that of the existing NuMI horns [8] [10]. In neutrino mode, the horns focus positively charged mesons. In antineutrino mode, the horn current is reversed to focus negatively charged parents. The focused particles enter a decay pipe where they decay into muons and neutrinos (antineutrinos). Muons and any undecayed hadrons are absorbed by rock, leaving a beam of neutrinos.

Because of the magnitude and costliness of this project, studies using beam simulators must be done to establish the most tech-
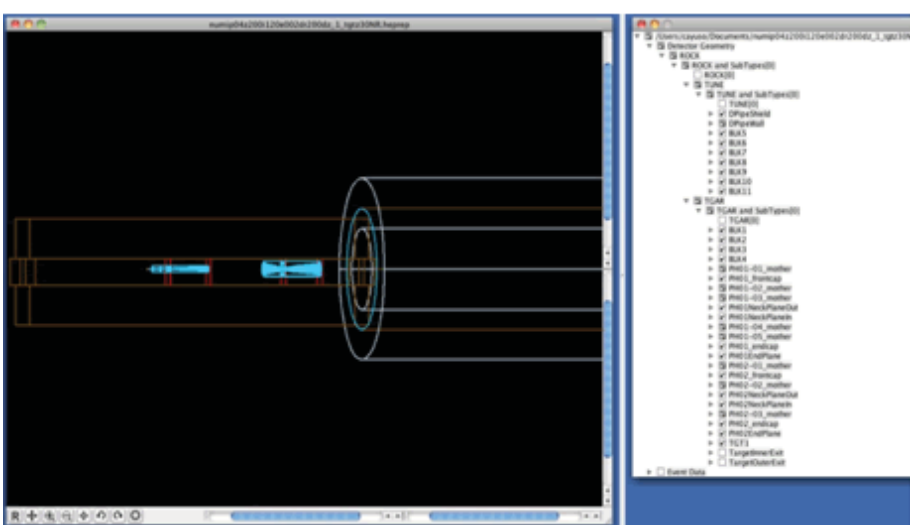

Figure 4. HepRApp window with visualized beam-line (right) and list of beam line parameters.

v CC Events/GeV/1E21 POT vs E, at Far Detector, $\delta_{c p}=0$

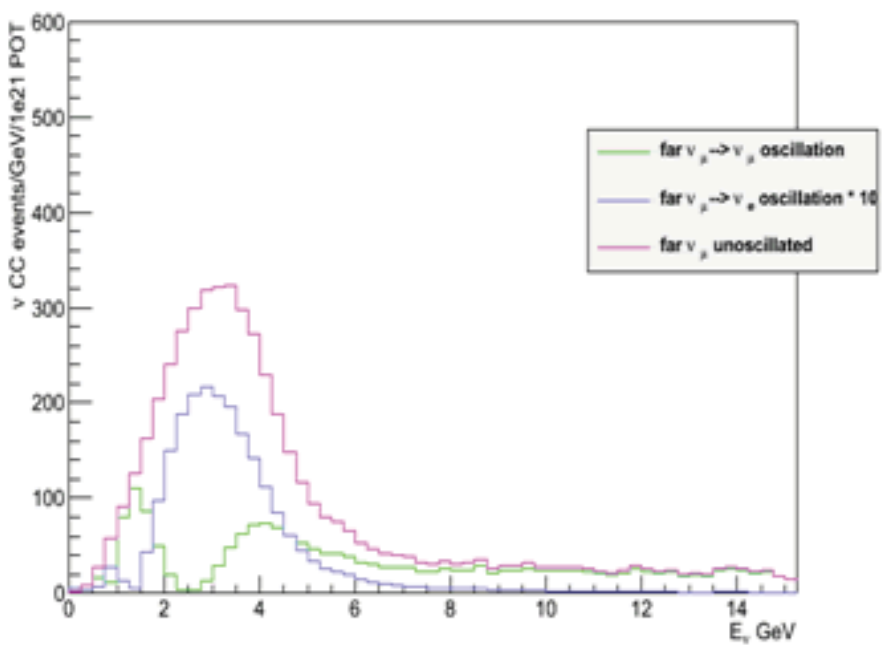

nically feasible and optimal design of the beam. Various studies have narrowed down on a reference design for the beam, but the project is still seeking to compensate for the nearly $30 \%$ loss of statistics from a previous beam model [7]. It is with the aim of increasing neutrino flux in every channel, but more so in the $v_{\mathrm{u}}->$ $v_{e}$ oscillated channel, that this study on the LBNE target was initiated.

\section{Optimizing the LBNE Target Design}

In this study, we sought to improve on statistics in the lower energy spectrum of the neutrino $v_{u}->v_{e}$ oscillated channel by altering the material, geometry and size of the LBNE target. The various beam line configurations were established with the use of input files and visualized using HepRApp, a visualization program used to the sanity-check the configuration before generating simulated data [9] [11].

Once all the geometric overlaps and/or errors were corrected, the input files were simulated using the GEANT4 LBNE simulator, a program developed to simulate the passage of particles through matter [9]. 1e21 protons-on-target were simulated for each configuration. The following parameters were kept set: a horn separation of $6.6 \mathrm{~m}$, and an air filled decay pipe of $200 \mathrm{~m}$ in length and $4 \mathrm{~m}$ in diameter.

The energy and parent particle weight for each neutrino, position of particles upon colliding with the target, as well as other outputs were calculated by the simulator [9] [12]. The output v CC Events/GeV/1E21 POT vs E, at Far Detector, $\delta_{\varphi}=\pi / 2$

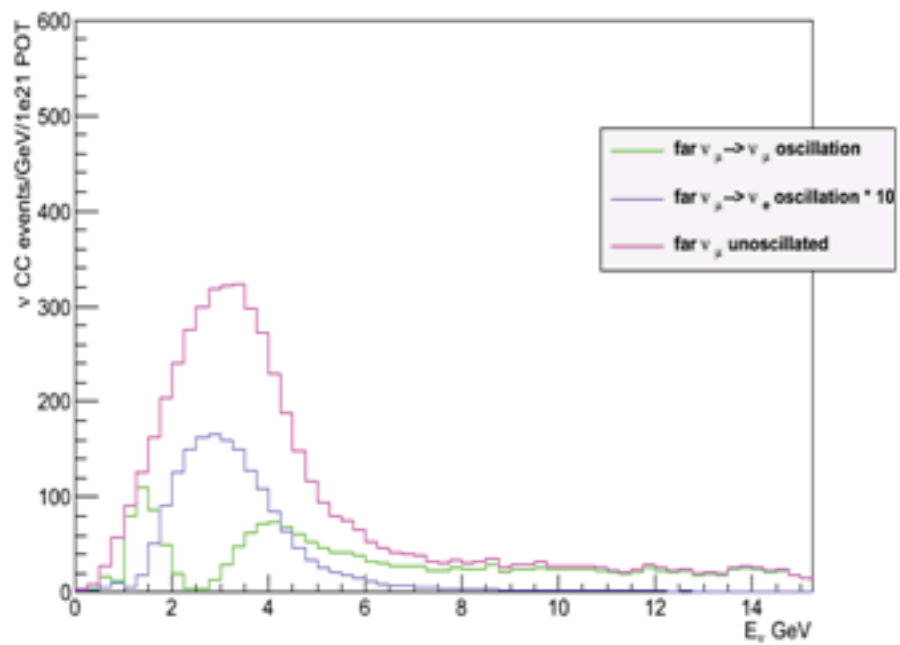

Figure 5: Energy spectrum of oscillated and unoscillated channels for $\delta_{c p}=0, \pi / 2$. 
was analyzed with a PyROOT interface where distribution plots of neutrino events ranging from $0-15 \mathrm{GeVs}$ for $\delta_{\mathrm{cp}}=0, \pi / 2$ were generated [9] [12]. These were visualized as follows:

From these distributions, the integrated number of electron neutrino events was calculated in the $0-15 \mathrm{GeV}$ range as well as the $0-2 \mathrm{GeV}$ range, a region that gives higher sensitivity to oscillation as can be observe in the considerably higher peak in probability in this energy range in the lower plots of Figure 2.

The study conducted this summer consisted of two parts. The first part of the study compared the statistics resulting from using a beryllium target with those resulting from using the nominal beam line target material, graphite. The geometry of the beryllium target was also varied between cylindrical and rectangular. The length (L) of each target type was determined by calculating the interaction length (IL) corresponding to each material and doubling it $(\mathrm{L}=2(\mathrm{IL})=2 *$ nuclear collision length(NCL)/density) . This length also determined the location of each target type with respect to the beginning of horn 1 (h1). The lengths and locations were established to be the following:

- Graphite target: (density: $1.785 \mathrm{~g} / \mathrm{cm}^{\wedge} 3, \mathrm{NCL}: 59.2 \mathrm{~g} / \mathrm{cm}^{\wedge} 2$ ) $96 \mathrm{~cm}$ long, placed at $35 \mathrm{~cm}$ from $\mathrm{h} 1$.

- Beryllium target: (density: $1.848 \mathrm{~g} / \mathrm{cm}^{\wedge} 3$, NCL: $55.3 \mathrm{~g} /$ $\mathrm{cm}^{\wedge}$ 2) $84 \mathrm{~cm}$ long, placed at $23 \mathrm{~cm}$ from $\mathrm{h} 1$.

\section{Results}

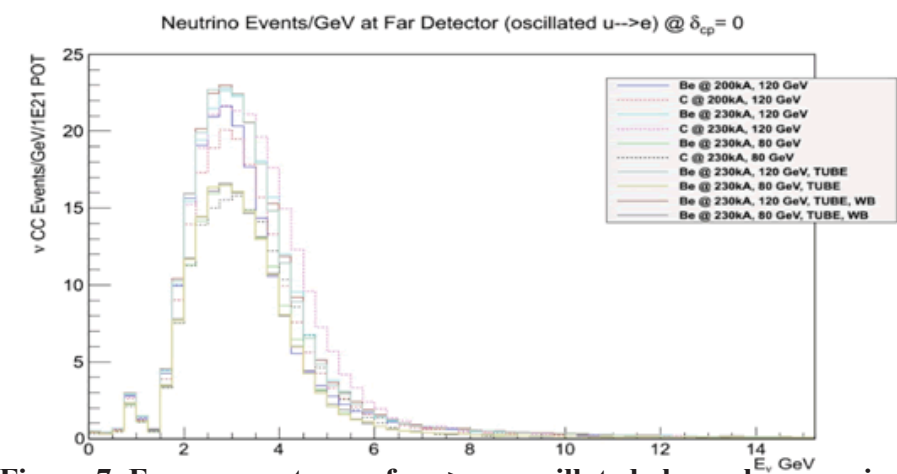

Figure 7. Energy spectrum of vu-> ve oscillated channel comparing geometry and material type of target for specified beam configurations.

\begin{tabular}{|c|c|c|c|}
\hline \multicolumn{4}{|c|}{ Real Integrated Neutrino Events (oscillated u-.>e) @ $\delta_{c \Phi}=0$} \\
\hline Energy Ranges & Configuration & Be & C \\
\hline \multirow{7}{*}{$0-2 \mathrm{GeV}$} & $200 \mathrm{kA}, 120 \mathrm{Gev}, \mathrm{Box}$ & 5.10 & 4.66 \\
\hline & $230 \mathrm{kA}, 120 \mathrm{Gev}, \mathrm{Box}$ & 5.20 & 5.16 \\
\hline & $230 \mathrm{kA}, 80 \mathrm{Gev}, \mathrm{Box}$ & 3.95 & 3.91 \\
\hline & $230 \mathrm{kA}, 120$ Gev, Tube & 5.32 & - \\
\hline & $230 \mathrm{kA}, 80 \mathrm{Gev}$, Tube & 4.05 & - \\
\hline & $230 \mathrm{kA}, 120 \mathrm{Gev}$, Tube,WB & 5.40 & - \\
\hline & $230 \mathrm{kA}, 80$ Gev, Tube,WB & 4.06 & - \\
\hline \multirow{7}{*}{$0-15 \mathrm{GeV}$} & $200 \mathrm{kA} 120 \mathrm{Gev}, \mathrm{Box}$ & 51.52 & 51.70 \\
\hline & $230 \mathrm{kA}, 120 \mathrm{Gev}, \mathrm{Box}$ & 59.17 & 6355 \\
\hline & $230 \mathrm{kA}, 80 \mathrm{Gev}, \mathrm{Box}$ & 41.32 & 43.98 \\
\hline & $230 \mathrm{kA}, 120$ Gev, Tube & 59.09 & - \\
\hline & $230 \mathrm{kA}, 80$ Gev, Tube & 41.02 & \\
\hline & $230 \mathrm{kA}, 120$ Gev, Tube, WB & 59.77 & - \\
\hline & $230 \mathrm{kA} 80$ Gev, Tube,WB & 41.20 & . \\
\hline
\end{tabular}

Table 2 Integrated values for each target materIAl at specified energy ranges of spectrums in Figure 7.

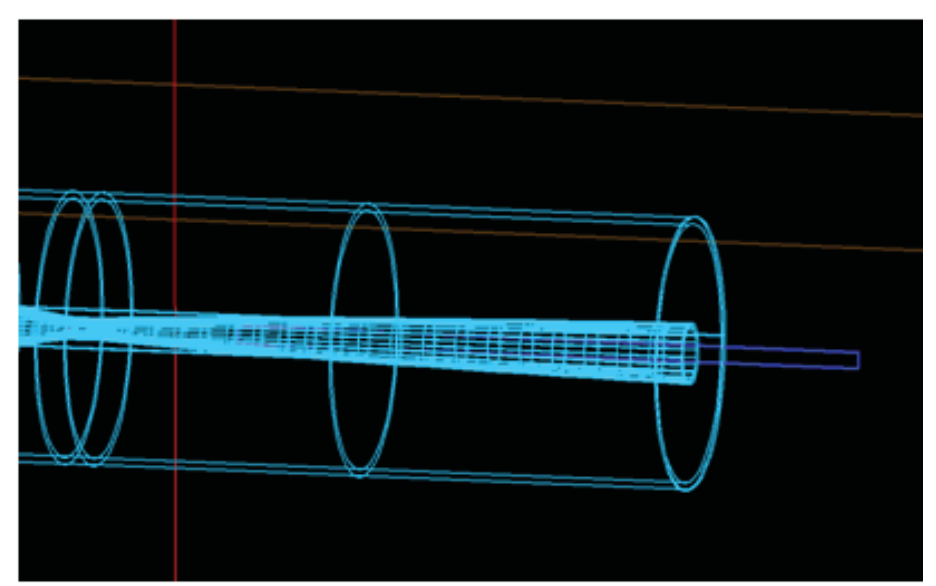

Figure 6. LBNE rectangular target partially inserted within horn 1. Simulations were ran with various horn currents and proton beam intensities and sizes.

The second part of the study sought to optimize target length and compare optimal lengths of each target type by analyzing neutrino flux. Impacts of the focusing effects of the current depending on the location of the target and target length on the flux were also explored. Simulations were ran with a horn current of $230 \mathrm{kA}$ and a proton beam of $120 \mathrm{GeV}$.

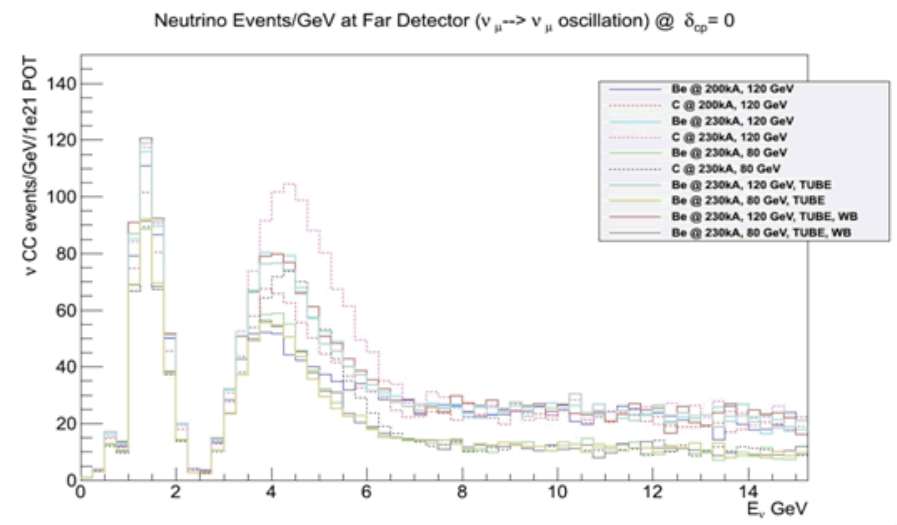

.Figure 8. Energy spectrum of vu-> vu oscillated channel comparing geometry and material type of target for specified beam configurations.

\begin{tabular}{|c|c|c|c|}
\hline \multicolumn{4}{|c|}{ Real Integrated Neutrino Events (oscillated $u-->u$ ) @ $\delta_{\varphi p}=0$} \\
\hline Energy Ranges & Configuration & Be & c \\
\hline \multirow{7}{*}{$0-15 \mathrm{GeV}$} & $200 \mathrm{kA}, 120 \mathrm{Gev}, \mathrm{Box}$ & 441.71 & 443.77 \\
\hline & $230 \mathrm{kA}, 120 \mathrm{Gev}$, Box & 502.53 & 565.35 \\
\hline & $230 \mathrm{kA}, 80 \mathrm{Gev}, \mathrm{Box}$ & 303.17 & 342.38 \\
\hline & $230 \mathrm{kA}, 120 \mathrm{Gev}$, Tube & 500.14 & - \\
\hline & $230 \mathrm{kA}, 80 \mathrm{Gev}$, Tube & 302.67 & - \\
\hline & $230 \mathrm{kA} \mid 20 \mathrm{Gev}$, Tube, WB & 510.66 & - \\
\hline & $230 \mathrm{kA}, 80 \mathrm{Gev}$, Tube,WB & 303.76 & . \\
\hline
\end{tabular}

Table 3 Integrated values for each target material at specified energy ranges of spectrums in Figure 8. 

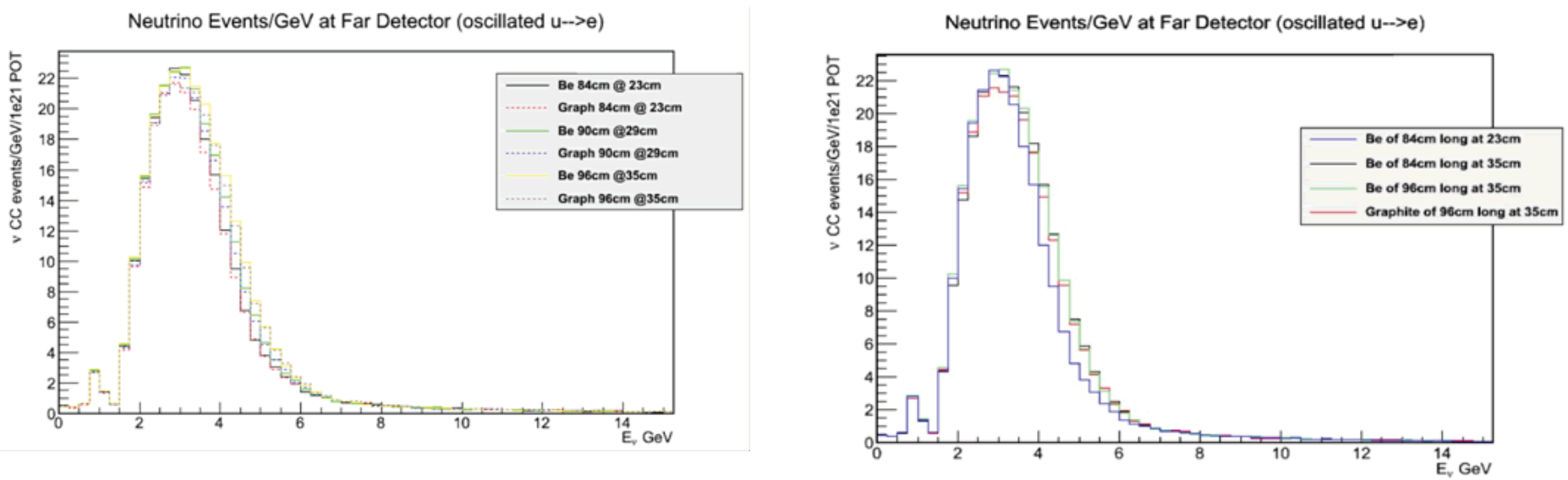

Figure 9. Energy spectrum of vu-> ve oscillated channel comparing equivalent lengths and locations of each target material.

\section{Discussion}

Results conclude that the beryllium target yields a narrower energy distribution than the graphite target. This can be more closely observed in Figure 7. A favoring for the beryllium target in the $0-2 \mathrm{GeV}$ energy range was also noted and can be seen in Table 2 .

- Both in the $80 \mathrm{GeV}$ and $120 \mathrm{GeV}$ beams, we observe a greater number of $v_{\mathrm{e}}$ events in the lower energy ranges for the beryllium target.

- The gain in the number of $v_{e}$ events from using a beryllium target is more significant when the horn current is lower.

In changing the beryllium target geometry from rectangular to cylindrical shape, we observe greater ve events overall for the cylindrical target compared to the same beam line configuration with a rectangular target. The benefits of the beryllium target are not observed in the $v_{u}>>v_{u}$ oscillated channel; a channel which was only visualized to display a complete picture of the effects on all the channels.

Results also conclude that the $96 \mathrm{~cm}$ target gives a larger flux for both materials in both energy ranges, as can be observed in both Figure 9 and Table 4. Table 4 also shows that the longer the target, the higher the flux (for these three lengths). A plateau would be observed if we keep reducing or increasing the length, but for these three values, length (i. e., amount of material) is more important than focusing for flux. The beryllium target yields a larger flux in both energy ranges for all lengths in comparison to the graphite target at the same configuration.

Ultimately, this study concludes that the $96 \mathrm{~cm}$ cylindrical beryllium target is the better choice, giving the better low and high energy flux. This target design optimization study will bring LBNE closer to an optimal beam line design that is expected to resolve, to the highest precision, the value of $\delta_{\mathrm{cp}}$ and the mass hierarchy of neutrinos [7].

\section{References}

[1] Isvan, Zeynep. Antineutrino Oscillations and a Search for Non-Standard Interactions with the MINOS Experiment. Diss. University of Pittsburg, 2012. Print.

[2] Kayser, Boris, and Stephen Parke. "The Neutrinos." LBNE Fermilab. N.p., 09 2009. Web. 20 Nov 2012. $<$ http://lbne.fnal.gov/pdfs/Neutrino-overview-KayserParke.pdf $>$.

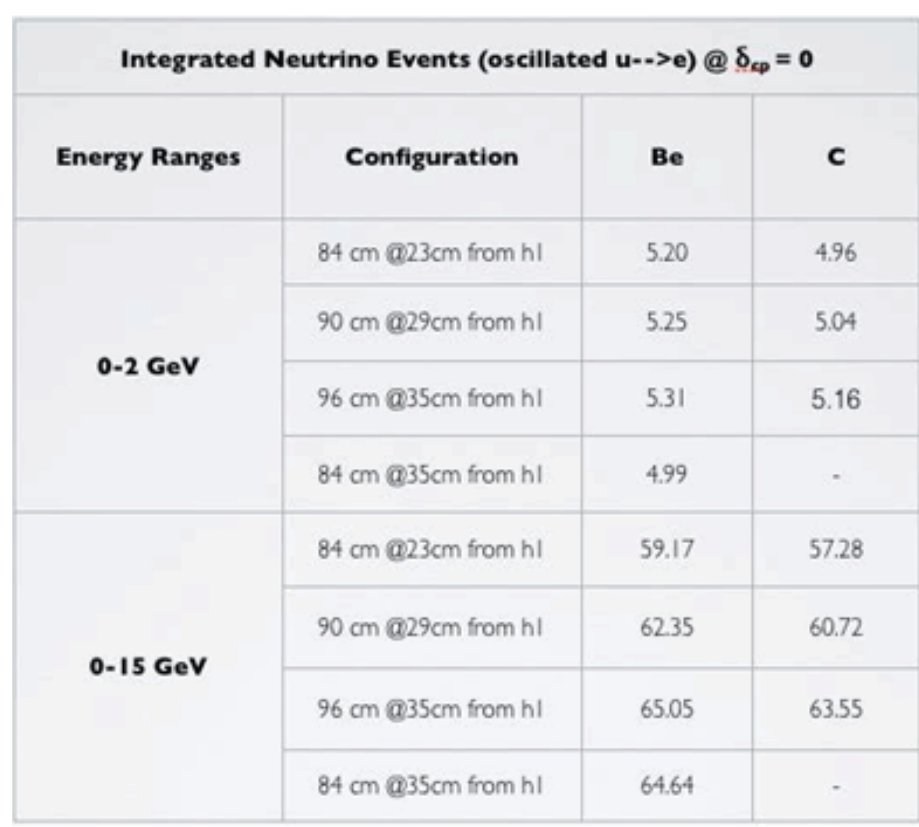

Table 4. Integrated values for each target material at specified energy ranges of spectrums in Figure 9.

[3] K. Nakamura et al. (Particle Data Group), The Review of Particle Physics". J. Phys. G 37, 075021 (2010).

[4] P. Adamson et al. [MINOS Collaboration] Search for the disappearance of muon antineutrinos in the NuMI neutrino beam." FERMILABPUB-11-357-PPD, BNL- 96122 2011-JA, Phys.Rev.D84:071103,2011. arXiv:1108.1509

[5] B. Pontecorvo, "Inverse beta processes and onconservation of lepton charge," Sov.Phys.JETP, vol. 7, pp. 172-173, 1958.

[6] Z. Maki, M. Nakagawa, and S. Sakata, "Remarks on The Unified Model of Elementary Particles," Prog.Theor. Phys., vol. 28, pp. 870-880, 1962.

[7] T. Akiri et al. [LBNE Collaboration] \The 2010 Interim Report of the Long Baseline Neutrino Experiment Collaboration Physics Working Groups" arXiv:1110.6249 
[8] LBNE Collaboration, Conceptual Design Report: Volume I, LBNE-Doc 5235

[9] S.Agostinelli et al., "GEANT4: A Simulation toolkit," Nucl.Instrum.Meth., vol. A506, pp. 250-303, 2003.

[10] K. Anderson et al., FERMILAB DESIGN-1998-01 293 [22] (1998).

[11] J. Allison et. al. "Geant4 Developments and Applications." IEEE Transactions on Nuclear Science 53 No. 1, 270 (2006).

[12] Rene Brun and Fons Rademakers, "ROOT - An Object Oriented Data Analysis Framework", Proceedings AIHENP ' 96 Workshop, Lausanne, Sep. 1996, Nucl. Inst. And Meth. in Phys. Res. A 38981 (1997).

[13] John R. Taylor "An Introduction to Error Analysis: The Study of Uncertainties in Physical Measurements." University Science Books (1982).

\section{ACKNOWLEDGEMENT}

This project was supported in part by the U.S. Department of Energy, Office of Science, Office of Workforce Development for Teachers and Scientists (WDTS) under the Science Undergraduate Laboratory Internships Program (SULI). 


\title{
The Subway-Surface Airflow Exchange Study
}

\author{
Adam Batley \\ John Heiser, Scott Smith, Andrew McMahon, Laurence Millian, and Richard Wilke \\ Environmental Sciences and Sustainable Technology, Brookhaven Nationaql Laboratory,, Upton, NY 11973
}

My internship this summer is focused on the finalization and deployment of the 100 Brookhaven Atmospheric Tracer Samplers units (aka BATS). Preparatory work has been performed to ensure proper operation during the S-SAFE dispersion experiment, scheduled for July 8th-28th, 2013. We will be utilizing 90 additional interns from Manhattan area schools to assist in the experiment. The S-SAFE dispersion experiment will be using Per-fluorocarbon Tracer gas to model how air moves through Manhattan and how subways redistribute air through the city. After a thorough spatial analysis of the collected data, this information will be used to create an airflow modeling program for NYC Emergency Services and First Responders to implement safe emergency staging, route safer evacuation paths, and assist in containment. This will further research into how air flows through a metropolitan area and how airflow is affected by air exchange between the surface and subway tunnels. The S-SAFE experiment is being funded by a $\$ 3.4$ million grant from the U.S. Department of Homeland Security. The study is being performed by Brookhaven National Laboratory with assistance from NYPD Department of Counter Terrorism, Argonne National Laboratory, and Los Alamos National Laboratory. While dispersion studies have been performed before, relatively few have been performed on this scale in a metropolitan area.

Further background information can be found in my previous reports:

- Generating New Brookhaven Atmospheric Tracer Samplers: Fabrication and Revision.

- Brookhaven Atmospheric Tracer Sampler: Assembly and Testing.

- Brookhaven Atmospheric Tracer Sampler: QAQC Testing This report is supplemental to the aforementioned reports.

Over the last year, I have been working with Brookhaven National Laboratory and the Environmental Sciences tracer group to manufacture, test, and troubleshoot the BATS. This summer my work was focused on preparing for the Subway-Surface Airflow Exchange Study (S-SAFE). I have helped assemble BATS units, test for functionality, repair units where necessary, and assisted in the "beta testing" of the samplers until now. The final testing of the samplers took place in June. Our team removed, repaired, or replaced faulty components. I helped perform repairs and firmware updates to the BATS as required. I performed flow testing of the BATS to ensure proper flow, proper logging of data, and proper functionality of the sampler's programmable faculties. With $\sim 98 \%$ of the BATS functioning properly, they were adequately prepared for deployment and sampling.

The goal of my internship, the Tracer Technology Group, and this study is to collect sufficient information in order to create spatial models of airflow and subway/surface air exchange. It was discovered in 2005 during the last urban dispersion project that air in the city can be mass redistributed by the vacuums occurring behind moving subway trains. This redistribution sucks in air from the surface into the subway, where it becomes redistributed to the surface in a new area. While this doesn't affect air quality in Manhattan or the surrounding areas, this proves to be vital information in the event of an accidental or intentional chemical release. These models will be used to assist NYPD, emergency services, and first responders in disaster evacuation and containment of airborne chemicals. To model this airflow, seven different perfluorocarbon tracers are released from different locations on surface level and subway level; perfluorotrimethylcyclohexane $(\mathrm{PTCH})$, ortho-perfluorodimethylcyclohexane (o-PDCH), para-perfluorodimethylcyclohexane (p-PDCH), perfluorodimethylcyclobutane (PDCB), perfluoromethylcyclopentane (PMCP), perfluoromethylcyclohexane $(\mathrm{PMCH})$, perfluoroisopropylcyclohexane (iPPCH).

In preparation for the S-SAFE study I have attended orientations, met with the city student interns to discuss what is required of them, discuss safety, and answer any questions they might have. I have walked various routes with my sub-teamlead, Lezou Dali, to become familiar with the sampler locations, truck drops, and subway stations. I have travelled with the Tracer Technology Group and the collective team of interns into Manhattan to reinstall equipment removed by Metropolitan Transit Authority (MTA), Department of Transportation (DOT), or others.

Deployment days took a lot of planning and preparation to facilitate happen. Each day, around 8am, weather data was collected from a meteorological station in Manhattan and evaluated. By 9am, principle investigators would make a decision to GO or NO-GO the following day. If a GO day was declared, we would depart from BNL to arrive at the Hoyt St residence to prepare the BATS samplers and the Batelle black box samplers (aka BBS) with fresh capilary adsorbsion tubes (CATS), charge the sampler batteries, address any problems that can be fixed, and relax. At 5 am the following day, we would load the samplers and ladders into NYPD vans. The surface teams would report to their destinations by $6 \mathrm{am}$, await the BATS, and hang them on the appropriate poles. The subway teams would claim a BBS and take a subway route to the appropriate stop. The tracer teams would take the tracer units to the designated locations and wait until the release time. All samplers and tracer units had to be in place and switched on before $8 \mathrm{am}$.

The sampling schedule started at $8 \mathrm{am}$. The samplers were programmed to take 12 consecutive samples of differing lengths; one 30 minute background sample, four 15 minute samples, five 30 minute samples, and two 60 minute samples for a total of 6 hours of sampling. The tracers were released after the conclusion of the 30 minute background sample and ceased at 10am.

At $2 \mathrm{pm}$, trucks are deployed to collect the BATS at their respective drop off points. When the trucks return to the Hoyt St residence, they are unloaded and the CATS are removed and replaced with new tubes. The BBS sample racks are removed and 
replaced as well. The de-tubing process usually took until 430pm. After de-tubing, the different groups would be dismissed. Some returned to BNL while some opted to return the next day after some needed rest.

Due to the sensitivity of the samplers and samples, the tracer team never set foot into the Hoyt St residence hall where the surface teams, subway teams, and all of the samplers were located. This eliminated the risk of contamination during the recapping and cataloguing of samples.

The first week of our possible deployment only included one deployment day. Deployments were to never occur on consecutive days. Aside from logistic issues, inclement weather played a large part in the decision to postpone a second deployment until the following week. During the second week, tentative postponing until Wednesday helped avoid an All-Stars Baseball game but subjected us to high temperatures.

During week two, we performed additional separate background sampling by loading samplers and traveling through Manhattan via subway and surface. The second sampling day was performed with a heat advisory. While sampling would still occur, the surface team was not to spend more than 15 minutes outside each hour if the temperatures reached $80^{\circ} \mathrm{F} ; 15$ minutes per two hours if the temperatures rose above $90^{\circ} \mathrm{F}$. Temperatures soared, restricting our rounds to every two hours. Each team was given bottled water and was advised to stay indoors. Most surface team members opted to beat the heat in coffee shops or restaurants.
During week three, we were successful in completing the last sampling day with comfortable weather and good wind conditions. There was a light sprinkle and chance of more rain, but the day remained cloudy and dry. Due to a car accident that damaged a light pole, a sampler went missing as it was transported to Harlem. It was retrieved later, undamaged.

While this experiment is the largest airflow study to date, the information we have collected is still incomplete. While this experiment is extensive in its data, definitive answers are not possible; only projections and calculations. The final results will never be published due to security guidelines, but this does not mean the information collected will never be used. This work is beneficial to national security and environmental science. The future of this experiment lies in how the data is modeled, how the data is used, and how the data can become beneficial to governing agencies while not proliferating the bare-bones results.

This final internship has provided me with a unique experience in field work and helped supplement my varied science background. Leading and communicating team members, delegating tasks, following protocols for equipment and sample handling, and the opportunity to work with a team has helped develop the soft skills I require. While it was difficult to supervise and coach work with my fellow interns, the experience was one to grow on. Overall, these skills might seem unrelated to a "major" or "academic field" but serve a great purpose in the way of experience "in the field" that most fresh graduates do not have outside of an academic environment.

This project was supported in part by the U.S. Department of Energy, Office of Science, Office of Workforce Development for Teachers and Scientists (WDTS) under the Science Undergraduate Laboratory Internships Program (SULI). 


\title{
Analysis of continuous long-term cloud records from climatically-significant global sites
}

\author{
Shannon Baxter \\ Department of Mathematics, SUNY Geneseo, Geneseo, NY 14454 \\ Karen Johnson \\ Environmental Sciences, Brookhaven National Laboratory, Upton, NY 11973
}

\begin{abstract}
The Department of Energy's Atmospheric Radiation Measurement (ARM) Climate Research Facility gathers longterm data from strategically placed, climatically significant sites around the world. These sites are located in diverse climate regions in order to sample a wide range of atmospheric regimes. This data consists of remote sensing observations of various cloud properties, aerosols and atmospheric radiation. The objective of this research project is to analyze the cloud boundary, and other cloud-related data gathered over a 15year period from the various sites around the globe. In my major study, I selected days with primarily single-layer cirrus clouds, to analyze minimum observed cloud reflectivities, as a measure of radar sensitivity. I used Python, a programming language, to create time vs. height images of cloud reflectivity data and minimum reflectivity statistics for each site. These images show the trends of the cirrus cloud reflectivities over the whole range of data collected for a specific site, as well as over individual 24-hour periods for a specific site. The longterm plots enable one to easily see if the data sensitivity changes over time, suggesting radar calibration drifts and possible radar malfunctions. This helps to determine where the data is problematic and where it is valid to use. The single-day images enable one to better understand differences between reflectivity observations when the radar is operating well and when data quality may be compromised. This analysis will help to determine when the radar data is not usable and result in more accurate cloud data, thus making it easier to find and understand changes in cloud properties and weather patterns and facilitate development of more accurate climate and earth system models. This analysis of the cloud radar data has enhanced my knowledge of programming, specifically of Python and has educated me on the usefulness of programming in the scientific world.
\end{abstract}

\section{Introduction}

The goal of ARM is to improve the understanding of cloud properties and the representation of clouds and aerosols in climate and earth system models. In addition, ARM's intention is to reduce the ambiguities in climate and earth system models to aid in the development of sustainable solutions to the energy and climatic challenges that we, as a nation, face [1]. However, it is essential to understand data quality issues before attempting to perform long-term climatic studies. In order to assess these issues, we created a number of different plots that offer insight about the quality of the radar data.

The data we collected came from three ARM sites: Tropical Western Pacific (TWP), Southern Great Plains (SGP) and Northern Slope of Alaska (NSA). Each of these sites have various climate research facilities in different places within that specific region. The specific facilities that we used data from at TWP are
Manus (C1), Nauru (C2) and Darwin (C3). NSA and SGP each only have one climate research facility; referred to as NSAC1 and SGPC1. These sites have very diverse climates, providing information on how climate affects the weather and atmosphere and how clouds differ from one climate and environment to the next. These sites experience environmental and technological issues that can effect the data. For example, SGP has many insects which interfere with the radar and create a lot of "noise" in the data. The instrumentation of the radar also causes issues when they need to be repaired and are not repaired for a while. These issues are what we are trying to filter out of the data by determining when they occurred and when the data is affected by these issues.

\section{Scope and Objectives}

The goal of our study is to characterize the quality of cloud radar reflectivity as a function of time and site, to ultimately provide guidance to ARM data users. This, and several other longterm Millimeter-wavelength Cloud Radar (MMCR) analyses begun as part of this project, will help to determine when the radar data is most reliable and result in more accurate cloud data.

\section{Methods}

In our primary study, days with primarily single-layer cirrus clouds were selected, approximately one per month, in order to analyze the minimum observed cloud reflectivities, as a measure of radar sensitivity. Python, a programming language, was used to compute statistics related to minimum observed reflectivities and create long-term plots of the statistics for each of the five permanent ARM sites. These plots show the trends of daily cirrus cloud minimum detected reflectivities over the whole range of data collected for a specific site. Day-long time vs. height images were also created for each of the cirrus cloud cases. The longterm plots enable one to easily see if the radar sensitivity changes over time and when the radar malfunctions. The single-day plots enable one "zoom in" on a specific day to examine the population of minimum observed reflectivities, and see how the reflectivity changes with time.

\section{Discussion and Results}

Each MMCR has various modes that focus on different portions of the clouds. Figure 1 shows the difference between mode 2 and mode 3 . Mode 2 focuses on cirrus clouds which are generally above $6,000 \mathrm{~m}$ and mode 3 focuses on the clouds as a whole. As you can see, mode 2 was able to detect more of the cirrus cloud. For this reason, we mainly used data from mode 2 of the radar.

Figure 2 is an example of one of the single day plots that were created using mode 2 data. Each of these plots is represented by a single set of data points on the long-term plots for cirrus minimum reflectivity statistics as seen below in figures 3 and 4 . The longterm plots for cirrus minimum reflectivity statistics make it easier to determine exactly when problems with radar occur which en- 
ables one to confidently be able to state where the data is valid to use, unusable, and where one should proceed with caution. The single day plots enable one to view the data for a specific day which may be an outlier on the long-term plot for cirrus minimum reflectivity statistics in order to further investigate the cause for being an outlier.

20080816

sgpmmarmomC1.b1, Cirrus, Mode 2

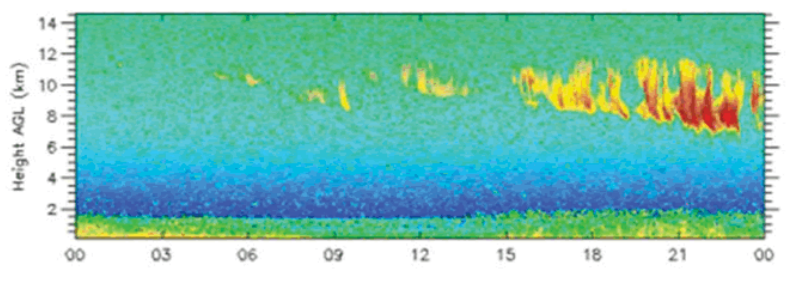

sgpmmcrmomc1.b1, General, Mode 3
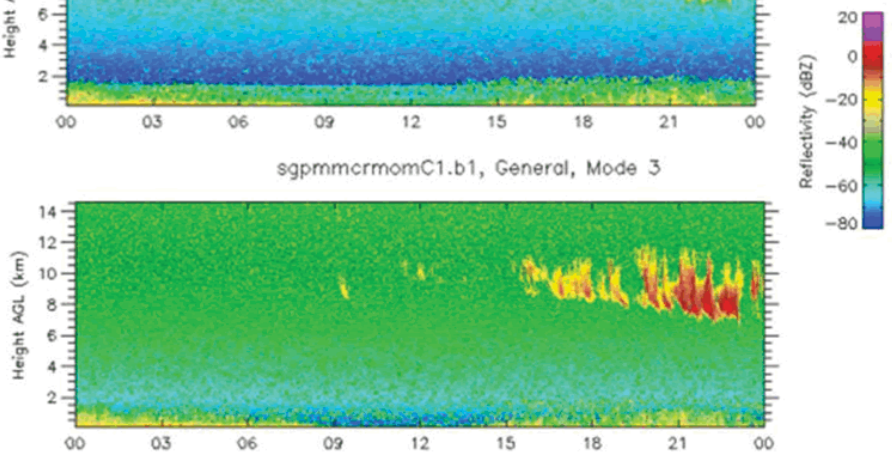

Figure 1. Time vs. height image of cloud reflectivity for one day as observed by the MMCR using mode 2 (top) and mode 3 (bottom).

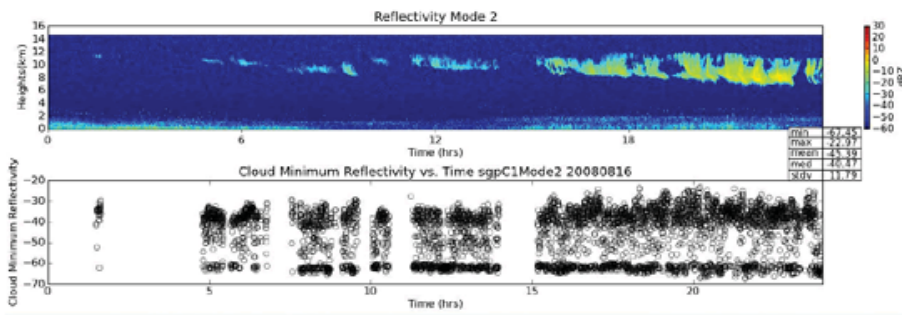

Figure 2. Time vs. height image of reflectivity (top) observed by the MMCR mode 2 at the SGP site. Below that is a plot of minimum cirrus cloud reflectivity, observed at the corresponding time.

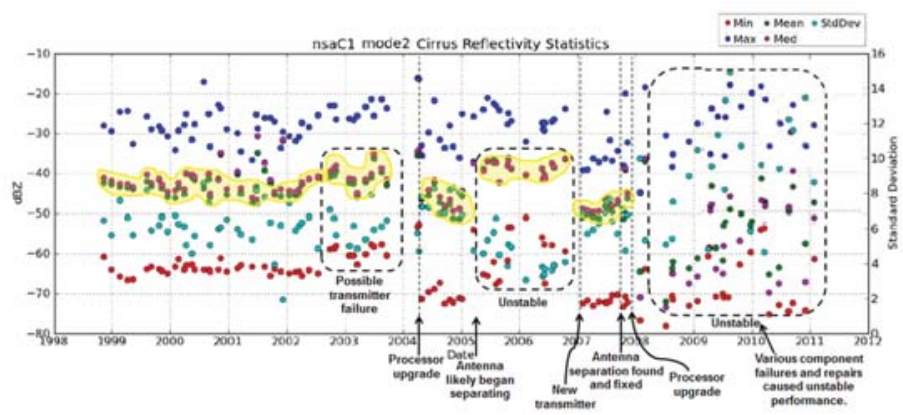

Figure 3. Day-long statistics of cirrus cloud minimum detected reflectivities for NSAC1. Each point represents reflectivity statistics for one day of only those times with single-layer cirrus clouds present. Red points are the minimum value seen, blue points are the maximum of the days' time sequence of minimum reflectivities, green points are the mean reflectivities, purple points are the median and the light blue points are the standard deviation.

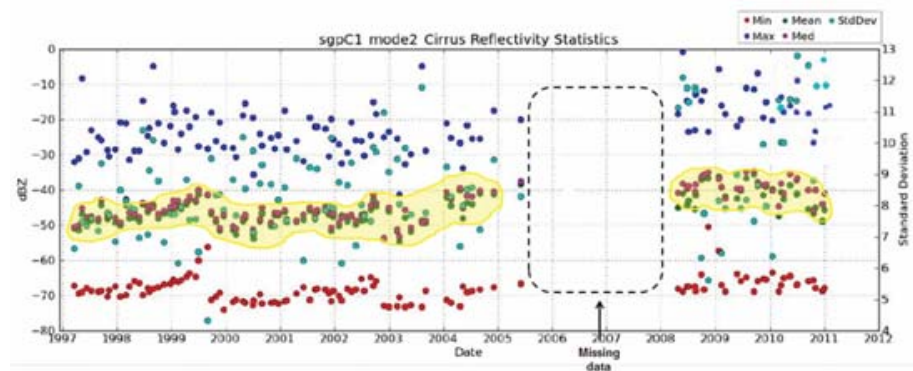

Figure 4. Same description as figure 3, but this is for the SGPC1 site.

Our study has shown that long-term analysis of reflectivity can provide important information on radar data quality. Figures 3 and 4 are long-term plots of cirrus minimum reflectivity statistics for NSA C1 and SGP C1, respectively. As you can see, NSA has many problems with data quality and SGP has much more consistent data trends. These plots have provided strong evidence to when the radar data is unusable. In figure 3 it is easy to see where the data deviates from the general trend, thus enabling one to easily see where issues with instruments and the local environment have rendered the data unusable or potentially less reliable.

The minimum reflectivity statistics plot of NSAC1 mode 2 is an example of the many issues that can occur with radar; resulting in poor data quality. When instruments get old or fail, the data usually deviates from the general trend as can be seen in late 2002 until a processor upgrade in early 2004. After the processer is upgraded, the data returns to the general trend. At NSA there was a problem with an antenna on the radar. The antenna was separating from the radar and no one knows exactly when the antenna began to separate from the radar or how fast it separated. The antenna could have separated at a slow, gradual pace, or could have started separating slowly and then suddenly separated completely. The long-term plot for cirrus minimum reflectivity statistics for NSAC1 mode 2 gives us some insight to when the antenna started separating. As you can see, there is a sudden deviation from the data trend in early 2005 . This is likely when the antenna began separating because after this date, the data is unstable. Putting a new transmitter in place helps the data return to the trend but it isn't until late 2007 that the antenna separation is found and fixed. After 2008, there are various component failures and repairs which caused the highly unstable performance of the radar. The issues with the antenna is a prime example of how being in a harsh climate and isolated location, as the northern slope of Alaska is, can result in much more unusable and less reliable data. This is a great issue that investigators face. Engineers aren't able to visit sites such as NSA as often as they can visit sites such as SGP which is much easier and cheaper to get to. Being in the Southern Great Plains of the United States, engineers can easily go to the site and make repairs. As a result, the data for SGP is much more consistent than that of NSA. 


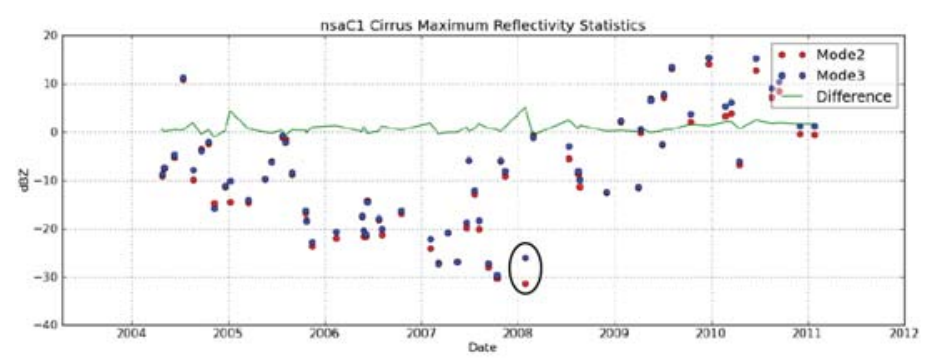

Figure 5. Plot of the maximum reflectivity for modes 3 and 4 and the difference between the modes for NSAC1.

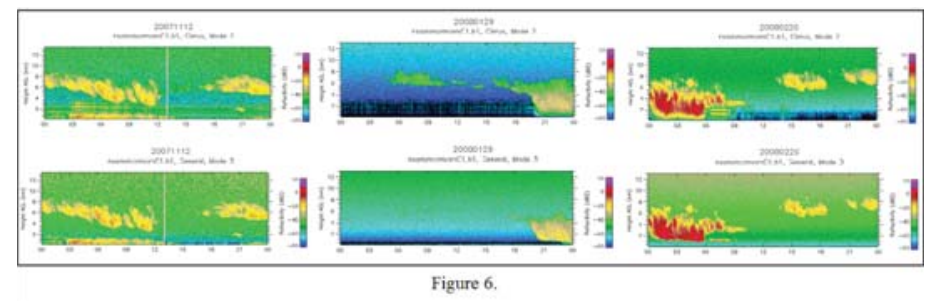

Figure 6. Plots of cloud reflectivity for mode 2 and 3 on specific dates

In order to further investigate the differences between the MMCR modes, I created plots of the maximum reflectivity of the clouds for modes 2 and 3 for each ARM site. The plot consists of the maximum reflectivity for each mode and the difference between the modes on each respective date. This plot shows how consistent the radar is. If a certain day or span of days differs from the average difference value between the two modes, as on 20080129 in figure 5, one could easily assume something went wrong with the radar during that time because on this day, you can see that the difference between the modes suddenly increases greatly. Figure 6 shows plots for a day two months before 20080129 , for 20080129 and for a day a moth after 20080129 . You can see that there is a large difference between the mode 2 and mode 3 plots for 20080129. You can also see that the color of the "background" of the plots is different for 20080129. This is easier seen in the mode 2 plot. This color change indicates that the "background noise" changes for an unknown reason. Both of these issues suggest that the data for this date is not valid. These plots can help to filter out the bad data and add some insight into the different modes of the radar.

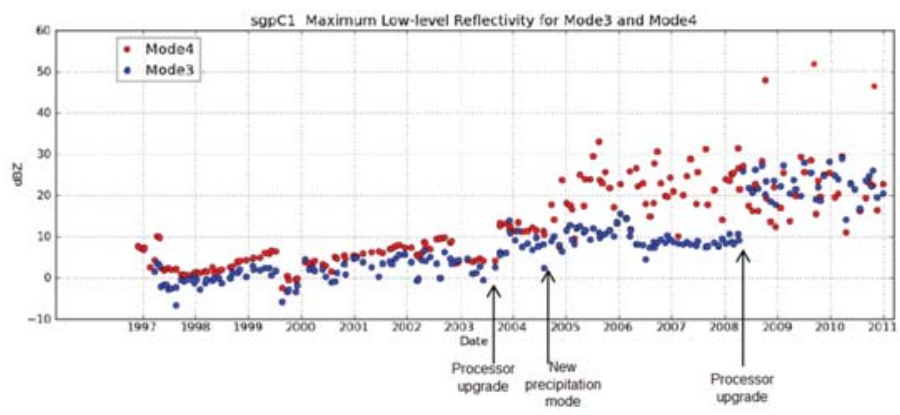

Figure 7. Long-term plot of maximum low-level reflectivity for modes 3 and 4 .

In order to study the performance of the MMCR with respect to signal saturation, we began a long-term study of modes 3 and 4 of the radar. The MMCR is capable of measuring a wide range of backscatter power from cloud droplets. When the backscatter is exceptionally high, as in drizzle or precipitation, it can overwhelm the radar receiver such that the measured reflectivity of the precipitation droplets is inaccurate (too low). To understand how the saturation level changes over time, we selected cases of high reflectivity, or precipitation at the lowest height that the MMCR can accurately measure. Figure 7 is an example of the results for this study for the SGP site. This plot compares the saturation from mode 3 to the saturation from mode 4 . Mode 3 examines the clouds as a whole, whereas mode 4 , known as the 'precipitation' mode, was designed to have the highest level of saturation of all the radar operating modes. As you can see, mode 4 is consistently more capable of measuring higher reflectivities. Therefore, one can conclude that mode 4 is the best mode to utilize when measuring high reflectivity periods. It is also clear that prior to the processor upgrade in late 2004, both modes 3 and 4 were consistently saturated in precipitation situations.

\section{Future Work and Conclusion}

This project analyzes the quality of cloud radar data through various types of plots that represent the data in different ways. We can look at the data over a long span of time, or just examine a specific day that contains questionable radar data. We can also compare the modes of the radar in order to determine whether the data quality changes between the different modes. These plots will help to filter out the bad data in order to have better data quality for ARM data users.

We have created the software framework for several additional long-term studies of MMCR observations to further assess data quality. In addition to continuing the reflectivity saturation analysis we have already begun, we plan to also analyze radar noise power trends, polarimetric differential reflectivity drifts, cirrus cloud maximum reflectivity comparisons between MMCR Modes 2 and 3 and average insect reflectivity at the SGP site all as a function of time. The combination of our completed work and these planned analyses should greatly improve our understanding of epochs of data quality for ARM's long-term cloud radar record.

\section{Acknowledgments}

This project was supported in part by the U.S. Department of Energy, Office of Science, Office of Workforce Development for Teachers and Scientists (WDTS) under the Science Undergraduate Laboratory Internships Program (SULI). I would like to thank Brookhaven National Laboratory, the Office of Educational Programs and my mentor Karen Johnson for the opportunity to take part in the SULI program. Thank you!

\section{References}

1. "ARM Climate Research Facility." ARM Climate

Research Facility. N.p., n.d. Web. 01 Aug. 2013. 


\title{
Analysis of soil temperatures in the Long Island Solar Farm (LISF) and its impact on the local fauna and flora
}

\author{
Ricky Bhola \\ CUNY Hostos Community College, Bronx NY 10451-5323 \\ John Heiser \\ Environmental Sciences Department, Brookhaven National Laboratory, Upton NY 11973
}

\begin{abstract}
The Long Island Solar Farm (LISF) is a 32MW, clean energy generator, producing enough energy to power approximately 4,500 homes annually. The LISF supports the United States Department of Energy's (U.S. DOE) mission of seeking renewable energy and reducing our dependence on fossil fuels. While we seek clean energy alternatives, the LISF sits on a site whose ecology consisted of pine and oak variants, invasive species, and a host of animals. The installation of the solar panels has changed the local environmental conditions. The panels shade the ground in some areas and not others. This can provide microclimates that are beneficial or detrimental to local flora and fauna. This project investigated the temperature variation between the soil (at 18" depth) under the panels to soil in the open walkways between panel rows. Using the data recorded for the year 2012 (March-December), time plots were created to illustrate the differences. It was observed that the temperatures of the soil under the arrays were higher than soil in the walkway for most parts of the year. The mean temperatures were inverted starting in September and continued through winter, that is the temperatures under the array were now lower than in the walkways. December's difference plot indicated that only 8 of 10 collection stations were inverted.
\end{abstract}

\section{Introduction}

As we move into the future, the state of our surrounding environment is of great importance. Surface temperatures are increasing on a global scale, most of which is caused by increased $\mathrm{CO} 2$ in the atmosphere due to the use and burning of fossil fuels 1. Seeking alternate sources of energy such as solar, wind, and hydro are popular choices today. While this venture is in good faith, there may be damages done to ecosystems that were disturbed to facilitate these energy projects. Alternatively, these facilities may provide beneficial conditions for flora and/or fauna (i.e., shade in a desert).

The Long Island Solar Farm (LISF) is a $32 \mathrm{MW}$ solar energy generator, providing enough energy to power approximately 4,500 homes per year. The LISF sits on 200 acres of Brookhaven National Laboratory's (BNL) property and consists of 164,314 solar panels. Prior to development, the LISF area consisted primarily of pine and oak species, most of which were planted between 19341941. Left for approximately 70 years, the area became home for many small plants and animals such as: deer, turkeys, migratory birds, raptors, and a host of invasive plant species 2 .

BNL's Environmental Sciences Department and Environmental Protection Division installed Campbell Scientific Microloggers (CR3000) at 25 locations throughout the LISF. These
Microloggers collect meteorological data such as temperature, relative humidity, and solar irradiance from various sensors located in close proximity to the data collection station.

This paper focuses on soil temperatures for the year 2012 (March- December). Time plots will be created to illustrate the fluctuations in temperature directly under the photovoltaic panels and compared to temperatures for the unobstructed walkway between panels. Differences in temperature occur due to shading that occurs from the panels.

\section{Soil Temperature Trends}

The data used were collected from area 1 of the LISF, which consists of 10 power blocks. 10 minute means were used to create a time plot of the under array temperature and the walkway temperature. The result of this is illustrated in figure 1 .

The maximum and minimum temperature difference (dT) of mean under array and walkway temperatures are approximately 5.78 and $-1.79^{\circ} \mathrm{C}$ respectively. The largest positive difference occurs in the summer while the negative difference occurs in the winter months.

Due to their slight geographic difference in ground elevation and panel height we compared powerblock 8 (PB-8) and powerblock 10 (PB-10) during the month of December. This plot of under array temperature and walkway temperature is illustrated in figure 2.

Figure 2: PB-8 \& PB-10 during the month of December

PB-8 and PB-10 comparison does not validate our yearly plot showing temperature inversions during winter months. According to figure 2, temperature inversion is dependent on the location of the PB. The following figures illustrate similar comparisons during the month of December.

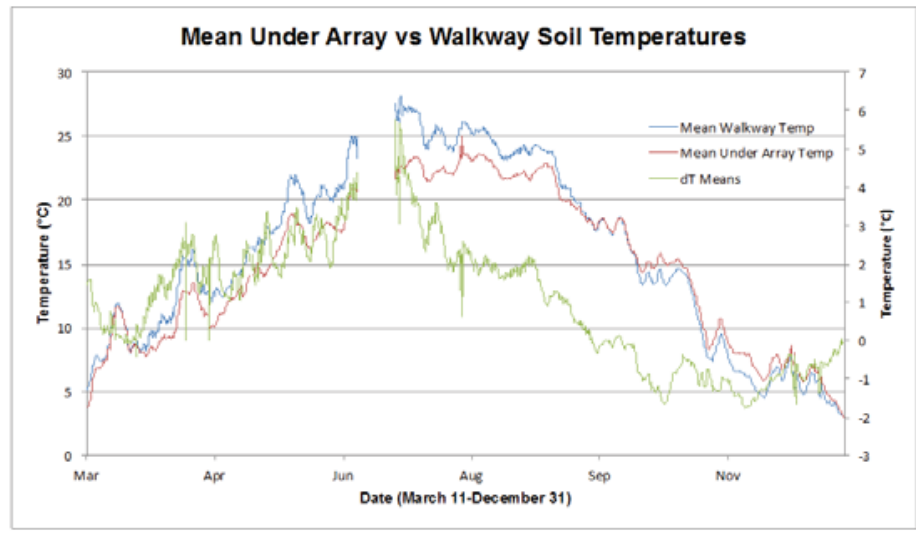

Figure 1: Mean Under Array Soil T vs. Walkway Soil T and dT 
From figures 3 and 4 we see that the temperature inversion does not always occur. Under array temperatures for PB-8 and PB-1 are higher than the walkway temperature but PB-10 is not inverted. To further understand how each PB behaves in the winter, a plot of the differences (walkway-under array) is provided in figure 5 .

\section{Discussion}

While it is not apparent, soil temperatures can be very influential on various basic environmental processes. Soil temperatures can also be influenced by the surroundings as well. One study utilized reed stalks at varying heights and density to see the impact it has on the temperature of the soil. It was found that as the density and height decreased, temperatures increased 3. On the LISF the vegetation varies at different PBs. Grass and plants are usually kept short to prevent casting shadows on solar panels. Under the panels, the vegetation can be overwhelming during the warmer months. Vegetation combined with the shading provided by the solar panels could further push temperatures lower than an open area such as the walkway. While it is obvious that temperatures

PB8 \& PB10 Under Array vs Walkway Soil Temperature

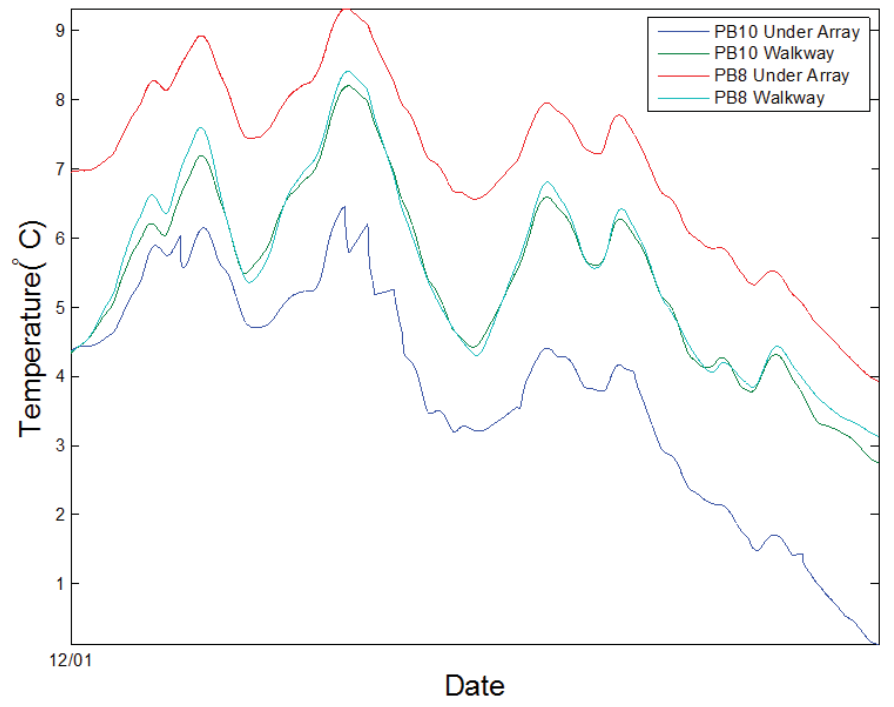

Figure 3: PB-1 vs PB-10 December Temperatures

PB1 vs PB10 Under Array vs Walkway Soil Temperature

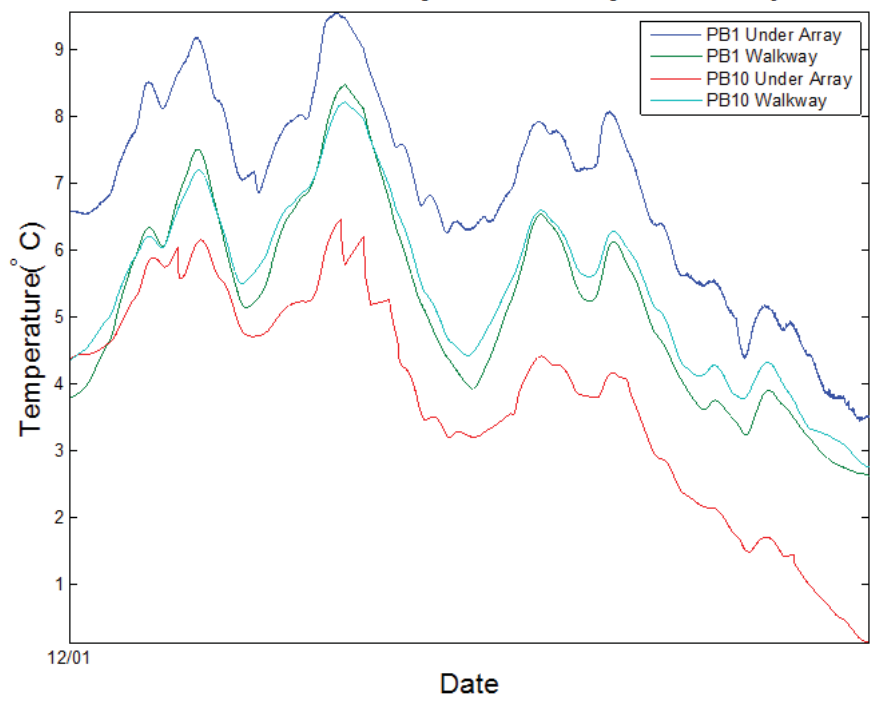

Figure 4: PB-1 vs PB-8 December Temperatures under the panels will be lower than the walkway, temperatures under the panels can climb above $50{ }^{\circ} \mathrm{C}$ on a given summer day.

During the month of December, we noticed something interesting; the soil temperature at powerblock $8(\mathrm{~PB}-8)$ and 10 (PB10) were inverted. At PB-10, under array reads lower than walkway and at PB-8, walkway reads lower than under array. This may be due to the height of the panel at PB-8, which is approximately 4-5 feet higher than PB-10. In the winter the sun approaches the lowest angle due to the earth's tilt, this results in increased sunlight under the arrays versus the walkways which may now be shaded by the panels.

The difference plot in figure 5 tells us that the geographic locations of PBs may influence the soil temperature. Figure 6 illustrates the difference in temperatures for May- December, as expected positive difference exists for the warmer months due to the walkway reading higher temperatures. In the colder months, there is a negative difference for all PBs excluding PB-9. This could be the result of increased sunlight as discussed before.

With this being the first year of data collection, we cannot say if there are dramatic changes in site ecology. Increased temperatures under the panels can possibly create a home for animals during winter or delay hibernation.

\section{Conclusions}

By analyzing the soil temperatures for both walkway and under arrays we saw that the under array soil temperatures were cooler for most part of the year as compared to the walkway. This trend was inverted during mid -September and continued that way through winter. Our explanation for this occurrence was increased

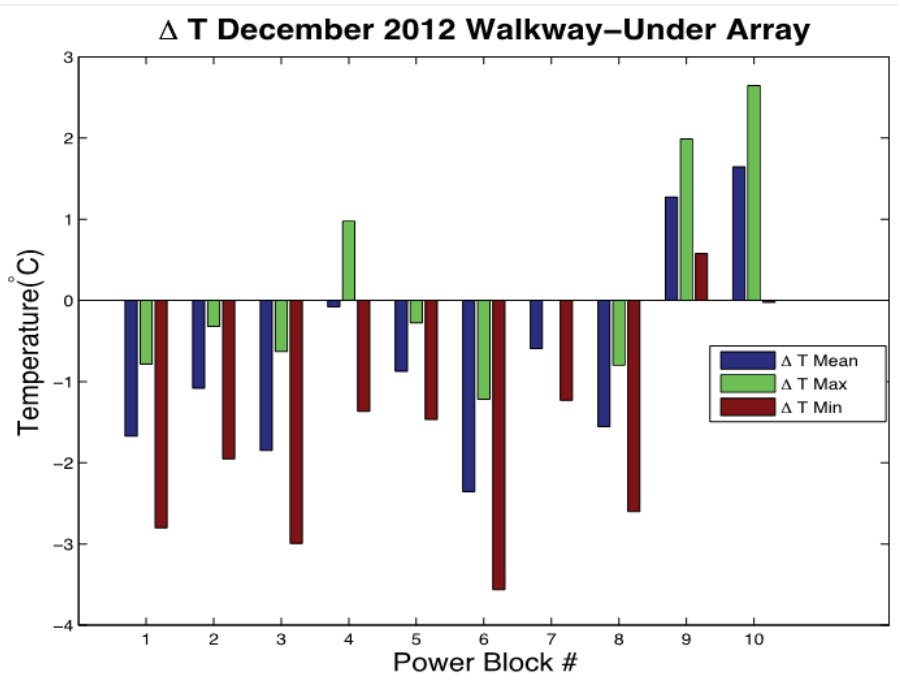

Figure 5: Walkway-Under Array Temperature Difference

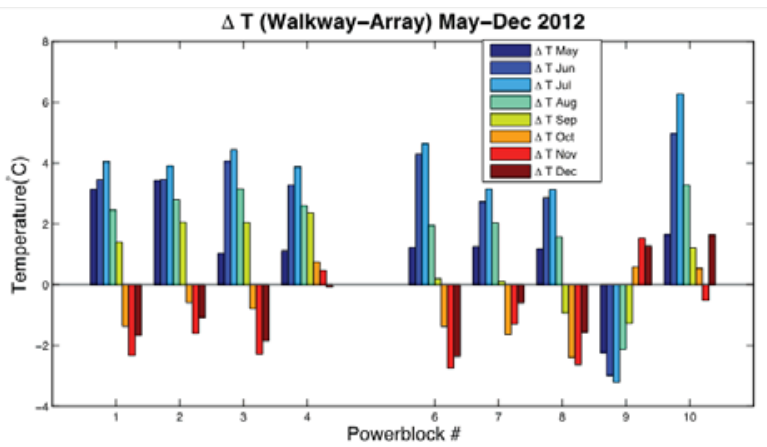

Figure 6: Mean temperature difference for walkway and under array May- December 
sunlight due to the winter solstice (the sun approaching its lowest angle of the year). Increased sunlight under arrays would heat the soil, due to this angle; light going to the walkways may be obstructed. As of now, it is not clear how much light gets under the arrays. One way to determine this is to install pyranometers under the panels to measure the solar irradiance. Using this approach we should be able to tell if the increased sunlight theory is valid and why there isn't a temperature inversion at PB-9 and PB-10.

\section{Acknowledgements}

The author would like to thank the following people and groups for their support during this project.

- John Heiser, Environmental Sciences

- Andrew McMahon, Environmental Sciences

- Scott Smith, Environmental Sciences

- Lauren Herrera, Stony Brook University

- Laurence Milian, Environmental Sciences
- Paul Kalb, Environmental Sciences

- BNL Environmental Sciences Staff

- Long Island Solar Farm Staff

- BNL Office of Educational Programs

- Timothy Green, BNL Environmental Protection

- Jennifer Higbie, BNL Environmental Protection

- Elizabeth Pooran

This project was supported in part by the U.S. Department of Energy, Office of Science, Office of Workforce Development for Teachers and Scientists (WDTS) under the Community College Internships Program (CCI).

\section{References}

${ }^{1}$ NASA GISS, (2013).

${ }^{2}$ Brookhaven National Laboratory, Environmental Assessment for BP Solar Array Project (Upton, 2009), p. 68.

${ }^{3}$ Y. Song, D. Zhou, H. Zhang, G. Li, Y. Jin, and Q. Li, Chinese Science Bulletin 58, 907 (2013). 


\title{
Studies in calorimetry using silicon photomultipliers, and tracking using gas electron multipliers for the super-PHENIX upgrade experiment
}

\author{
Marie Blatnik \\ Physics Department, Cleveland State University, Cleveland, OH 44115 \\ Martin Perschke, Bob Azmoun, Sean Stoll, John Haggerty, and Craig Woody \\ Physics Department, Brookhaven National Laboratory, Upton, NY 11973
}

\begin{abstract}
PHENIX, a multipurpose spectrometer surrounding a collision site at Brookhaven National Laboratory's Relativistic Heavy Ion Collider, studies the transition from normal nuclear matter to the quark gluon plasma, a state of matter so hot and dense that the baryons melt into a non-viscous liquid of quarks and gluons. Detector upgrades are being developed to better probe this transition state, which include an electromagnetic calorimeter that uses tungsten plates and scintillating fiber layers to sample the energy shower of penetrating particles. Small cavities, similar to integrating spheres, collect the light from the fibers and are read out with silicon photomultiplier sensors (SiPMs), were designed with optical simulations to maximize light collection efficiency and homogeneity. Additionally, the Zero Degree Calorimeter, used primarily to measure the energy of particles along the beam direction at RHIC, was also studied for calibration and radiation damage at the NASA Space Radiation Lab with an iron beam. Thirdly, a mini-drift GEM detector - a type of Time Projection Chamber with a small drift gap that uses gas electron multipliers (GEMs) to avalanche the signal which will be used to track particles in PHENIX - was used to image a leaf that had been given fluoridoxyglucose (FDG, a positron-emitting sugar), to demonstrate its particle tracking abilities.
\end{abstract}

\section{Background and Introduction}

PHENIX--the Proton High Energy Nuclear Interaction eXperiment-records energy and momentum tracking data from the heavy ion collisions of the Relativistic Heavy Ion Collider at Brookhaven National Lab. The current focus of the experiment is the characterization of the quark gluon plasma, especially around its transition conditions [1].

To create quark gluon plasma, heavy ions are accelerated to high energies, increasing the quark and gluon density of the nucleons by Lorentz contraction. Two beams of high-energy ions collide together in the heart of a detector, where the beam overlap creates an almond-shaped dollop of quark gluon plasma.

One would hypothesize that at these extreme energy regimes, the quark gluon plasma would act like an ideal gas of partons because the strong running coupling constant $\alpha$ s approaches zero:

$$
a s=\frac{g 2}{4 \pi}=\frac{2 \pi}{b \ln \left(2 \pi \frac{T}{\Lambda_{Q C D}}\right)}
$$

where $\mathrm{g}$ is the gauge coupling parameter, $\mathrm{b}$ is a constant, $\mathrm{T}$ is the temperature, and $\Lambda_{\mathrm{QCD}}$ is the QCD scale.

However, experimental observations show that at high tem- peratures, the new phase of matter behaves like a perfect fluid [2] and is therefore strongly coupled. All fluids, liquids and gasses, move in response to pressure gradients. If the quark gluon plasma behaved like a gas, the partons would not interact with each other and the pressure gradient would therefore be uniform on all sides. However, the soft (low energy) particles within the quark gluon plasma feel a larger pressure gradient on the shorter side of the "almond," and so more soft particles disperse from that shorter side [3]; this effect is known as the elliptic flow.

Jet suppression gives more evidence for the strong interactions within the quark gluon plasma. Jets tend to form in pairs, but the momentum of a jet can be absorbed within the quark gluon plasma and suppress the would-be jet from forming [4].

However, full azimuthal coverage is required to complete these jet studies and other important full-coverage analyses such as dilepton events. PHENIX does not have full azimuthal coverage.

Other questions that interest PHENIX include probing the bounds on the transition temperature, where the lower bound could be established with the ratios between the upsilon ground state to its excited states [1]. However, the current spectrometer cannot resolve the energy peaks between the upsilons' states;

PHENIX requires an upgrade of its close-range trackers and calorimeters. Super-PHENIX upgrades include a new full-azimuthal electromagnetic calorimeter and tracking detectors.

\section{Methods}

A. The mini-TPC

Time projection chambers utilize signal timing to reconstruct particle tracks; they are composed of a gaseous drift volume in a uniform electric field with an avalanching device and readout at the bottom of the field. When a particle passes through the gaseous drift volume of the detector, it ionizes the disturbed gas

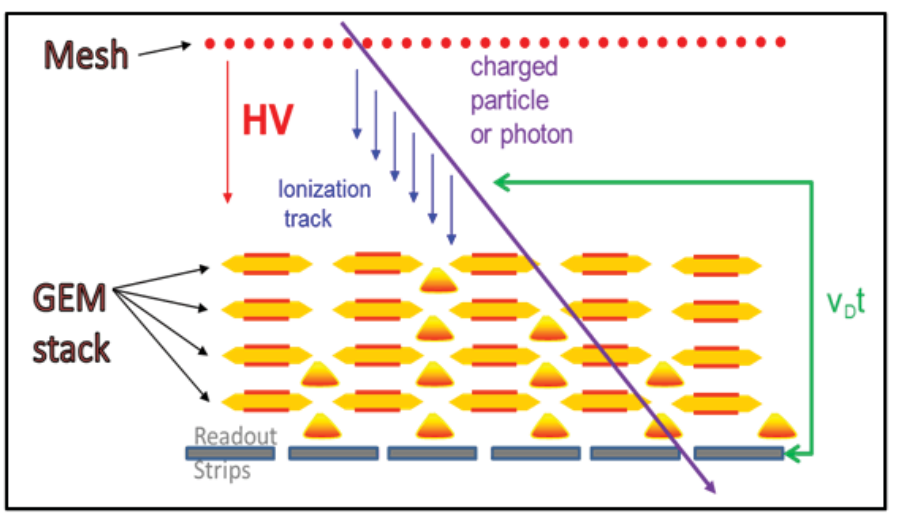

Diagram 1: The mini-TPC configuration, with its drift volume between the mesh and the GEM stack. 
molecules and leaves a trail of electrons in its wake. These electrons feel the electric field between the mesh and the avalanching device, and migrate to the avalanching device to produce a measurable signal (Diagram 1). The time it takes the signal to reach the readout system is proportional to its distance that it traveled along the field through the volume; its $\mathrm{z}$ coordinate can then be calculated from that time.

Typical time projection chambers have large drift volumes surrounded by an electric field cage to keep the electric field constant. This cage uses, for example, resistor chains and conductive strips to maintain the electric field in the large volume. However, these devices are massive, expensive, and inefficient (losing the electrons in the large volume). The mini-TPC has a drift gap of $16.31 \mathrm{~mm}$, orders of magnitude smaller than the meters of drift gap in the STAR TPC [5].

The avalanching device, which multiplies the electrons into a measurable signal, is a Gas Electron Multiplier (GEM) stack. Typical TPCs use wire readout planes, which requires a crosswork of parallel wires - at least a plane running along $\mathrm{x}$, a plane running along $\mathrm{y}$. These are messy and cumbersome for a number of reasons, the first of which being that wires break and short out a random number of other wires. Wires break easily because of their necessary design requirements - they must be thin and taut. The tension is to ensure a lack of sagging to maintain good position resolution, and the small diameter is the avalanching mechanism. As the electron approaches the wire, the energy it receives increases with an inverse distance relationship. Very close to the wire, it receives a large enough amount of energy to ionize more gas molecules. The resulting electrons ionize more molecules to create a cascade effect around the wire - an avalanche. This produces a readable signal in the sense wire, but also elongates the signal.

The second flaw in using wires for signal readout is the $\mathrm{E} x$ B effect; the field along the 'intended' wire can send an electron toward the next (wrong) wire, smudging/destroying position data. This is a grave difference of at least 150 microns, plus the extra time to produce a signal at a wrong wire will destroy the z-component and confuse one's tracking algorithms.

Gas electron multipliers, GEMs, are a brilliant answer to this struggle. Instead of using wires as avalanching devices, the answer lies in lithography. A GEM is composed of an insulating sheet coated in gold-coated copper with tiny holes $(50 \mathrm{~nm})$ etched through the sheet [6]. The idea is to stack these sheets (with a spacer between them) and apply a voltage across them. The elec-

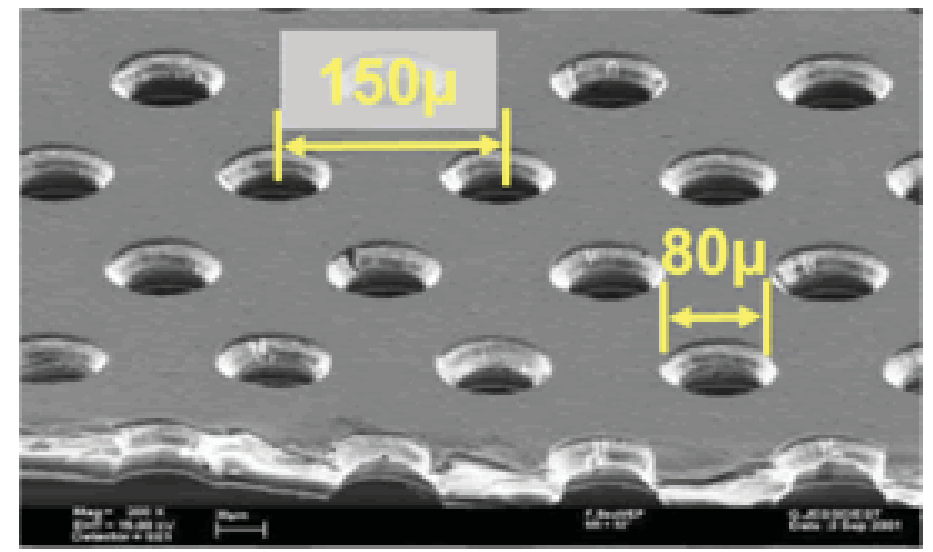

Diagram 2: SEM photograph of a GEM foil. tric field lines are then reminiscent of fringe-field capacitance; they squish in the holes with the strength to avalanche an electron that was driven into them. There are still some ExB effects, but the difference is on the order of $50 \mathrm{~nm}$ - and no wires and no headaches.

\section{B. The Electromagnetic Calorimeter}

Calorimeters measure the energy of an incident particle by absorbing the particle's energy in the form of a particle shower. Electromagnetic calorimeters sample the electromagnetic interactions as an energy signal as the particle shower creates bremsstrahlung and scintillation photons.

This calorimeter prototype is a sampling calorimeter, meaning that the light is collected in fibers sandwiched between dense metal plates. The plates are important to create the showers and stop the particle. The fibers are amassed into towers at the end of the calorimeter.

These towers prove to be an interesting engineering conundrum for a calorimeter with full azimuthal coverage. The optimal location of the towers would be behind the calorimeter, but the normal of the sandwiches cannot be perpendicular to the incident particle - some particles would pass through the scintillating fi-

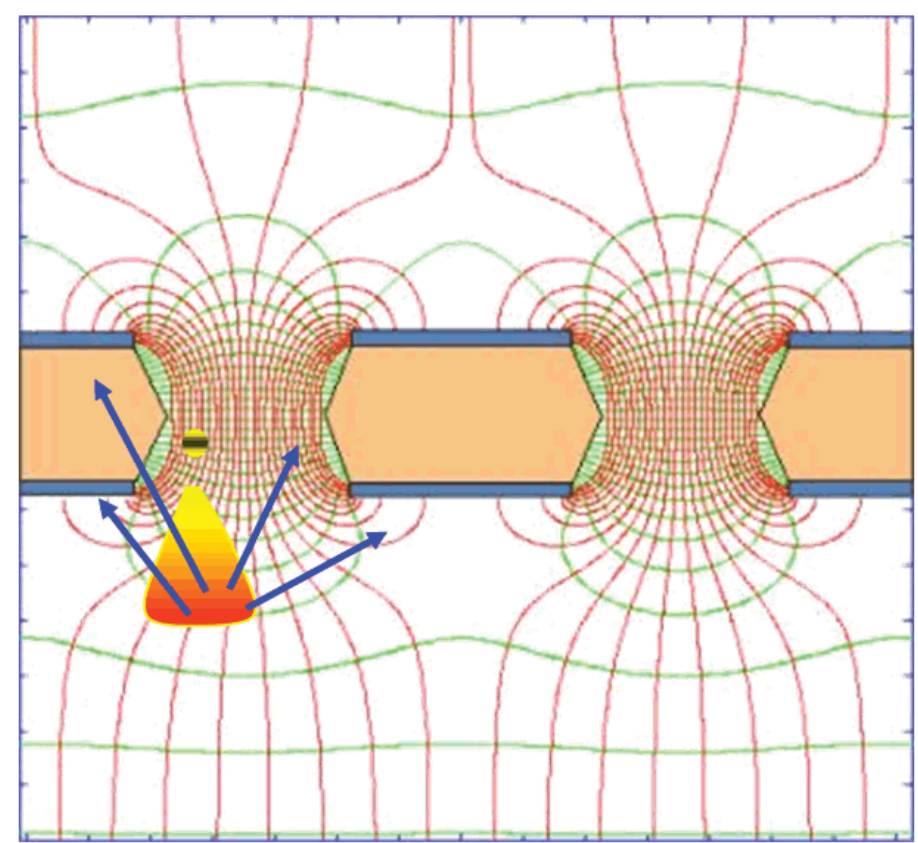

Diagram 3: Demonstrating the avalanching concept behind a GEM foil. The electric field is much stronger in the hole.

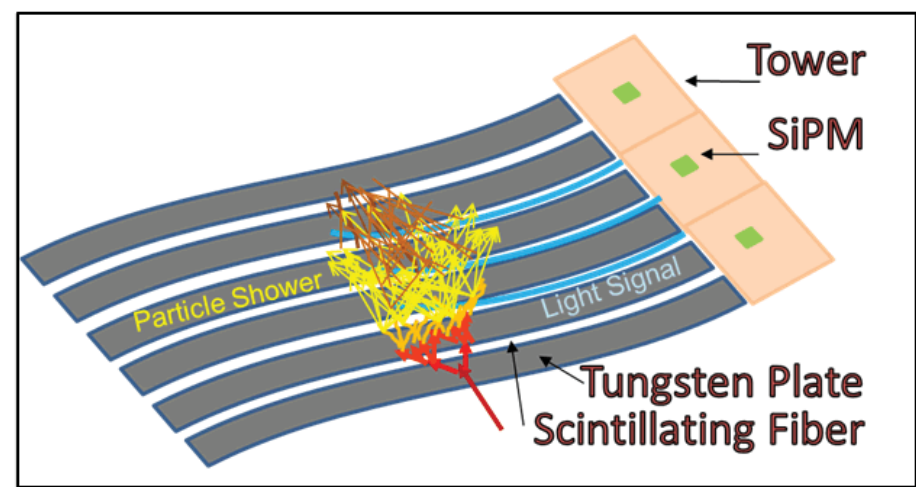

Diagram 4: A particle shower diagram. Ideally, the particle would arrive perpendicular to the tungsten plates, but such a configuration could not relegate the towers to the back. 
bers and not deposit their energy, while others would hit the tungsten and not be sampled.

An ideal situation would have the incident particles arriving normal to the tungsten plates. Such a configuration could not be read out without dead spots for the towers.

There are two solutions to this conundrum: the first is to angle the plates so that the particle would hit the tungsten and allow the shower to pass through layers of the fibers, and put the calorimeter towers in the back. The second solution is to bend the plates in a wave formation so that the particle would still pass through layers of sandwich. Both options are being explored.

The towers, too, ask an engineering question- how do they capture the light signal from the fibers? A silicon photomultiplier--an avalanching photo-sensitive diode array-converts light into an electric signal.

The towers work like an integration sphere-light enters the sphere through the fiber, than bounces around the very reflective surface until it hits the SiPM. However, special care must be taken in designing these cavities as to not allow the signal to be position-dependent. There cannot be any bright spots or shadows, depending on which fiber brought in the light.

\section{The Zero-Degree Calorimeter}

A second type of calorimeter is a hadronic calorimeter, which takes its measurements based on hadronic interactions and decays. The ZDC, or zero-degree calorimeter at PHENIX is this type of calorimeter.

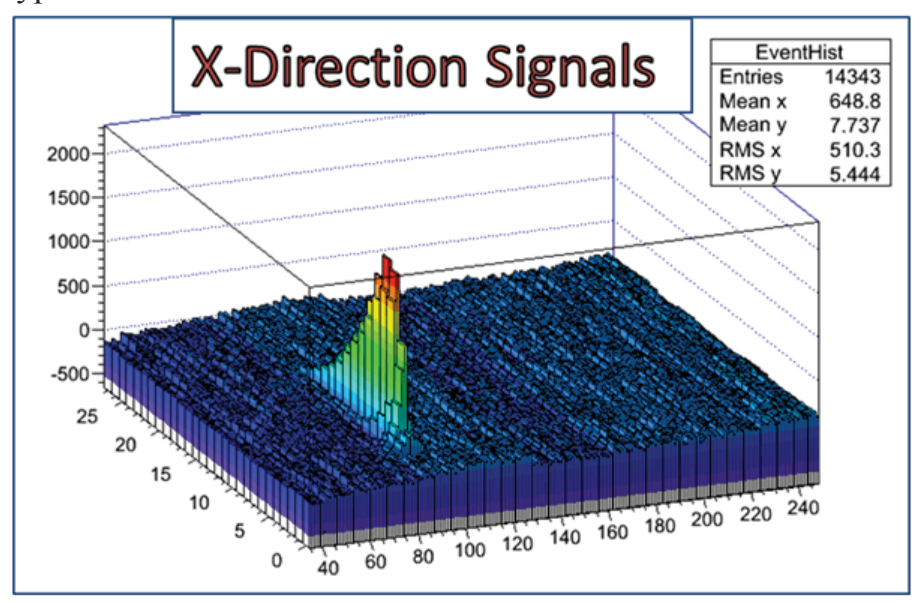

Diagram 5: A subset of all the data from the readout plane.

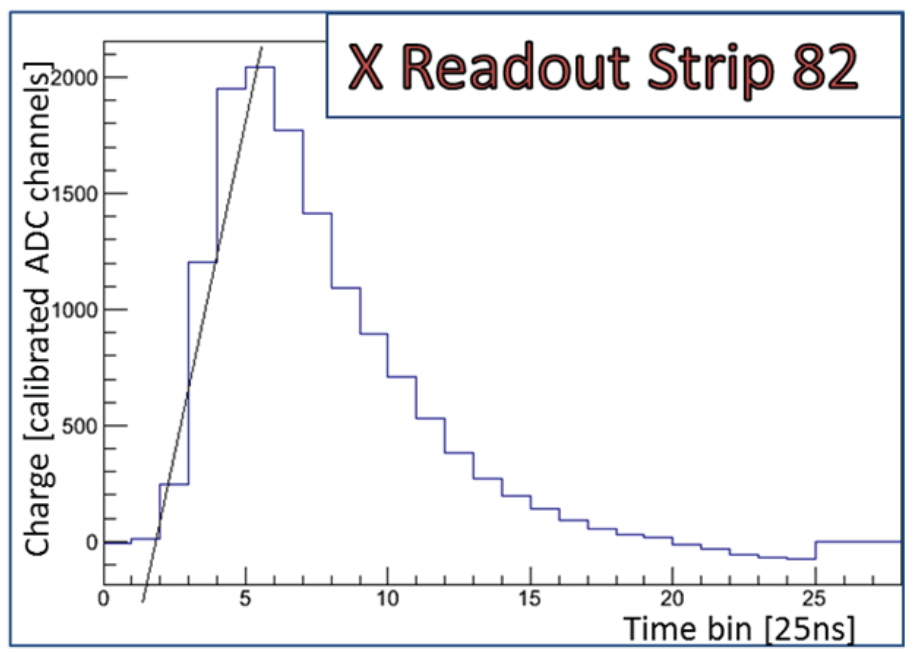

Diagram 6: A pulse on one readout strip, with its leading edge fit.
The specific purpose of the ZDC is to measure the energy from spectator neutrons - particles not produced by decays and undeflected by PHENIX's magnets.

This calorimeter is already tested, installed, and used in PHE-

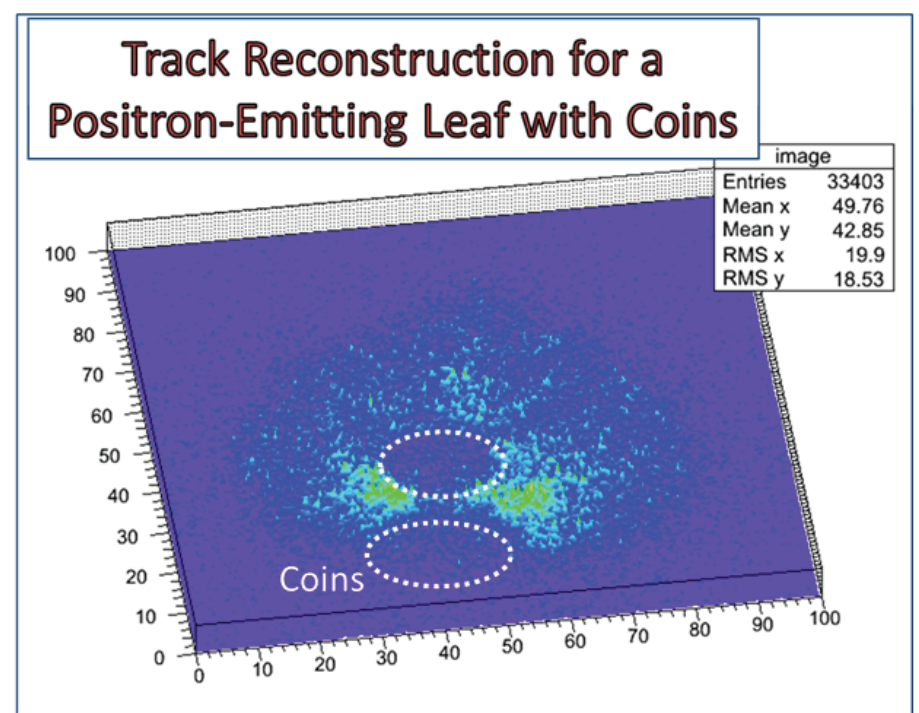

Diagram 7: Track reconstructions for a positron-emitting leaf.

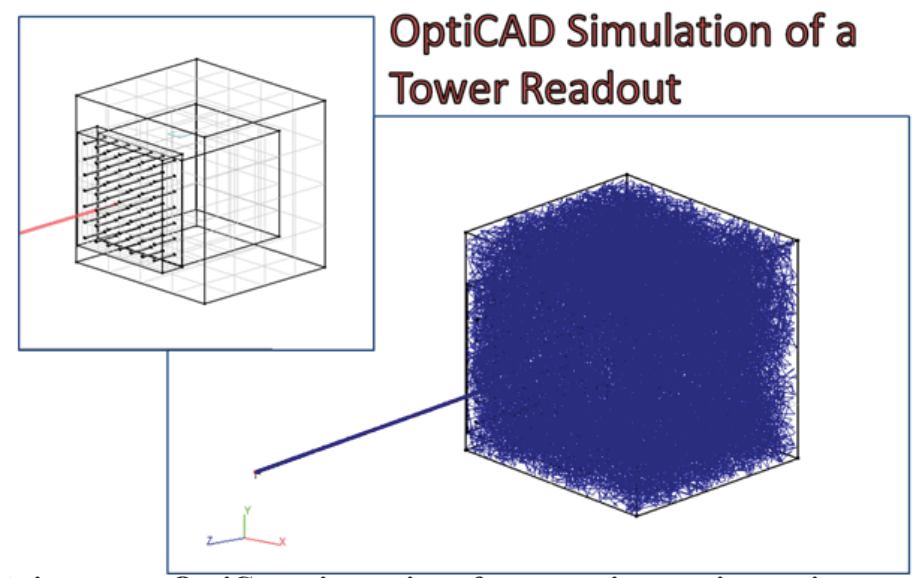

Diagram 8: OptiCAD simulation of a square integrating cavity.

\section{Measured Signal Efficiency vs Incident Light Position}

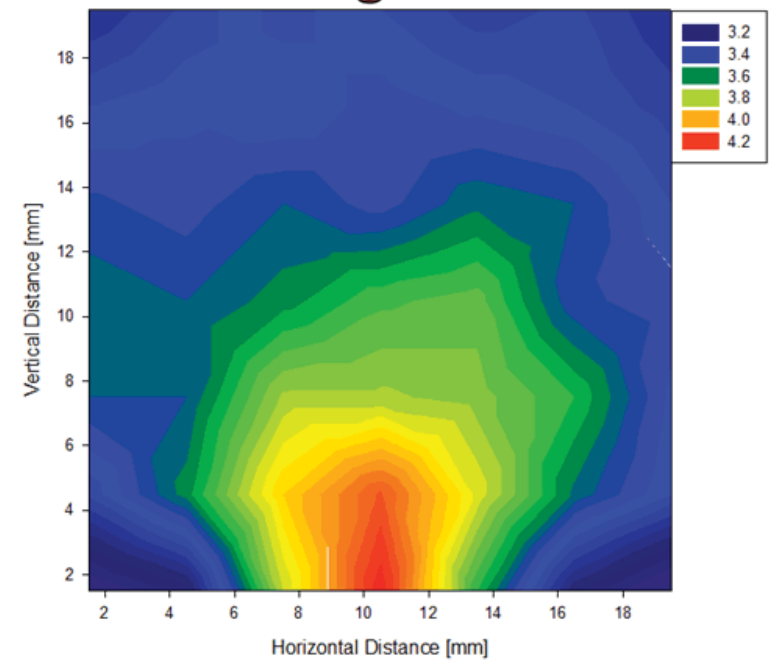




\section{9\% Reflective Surface}

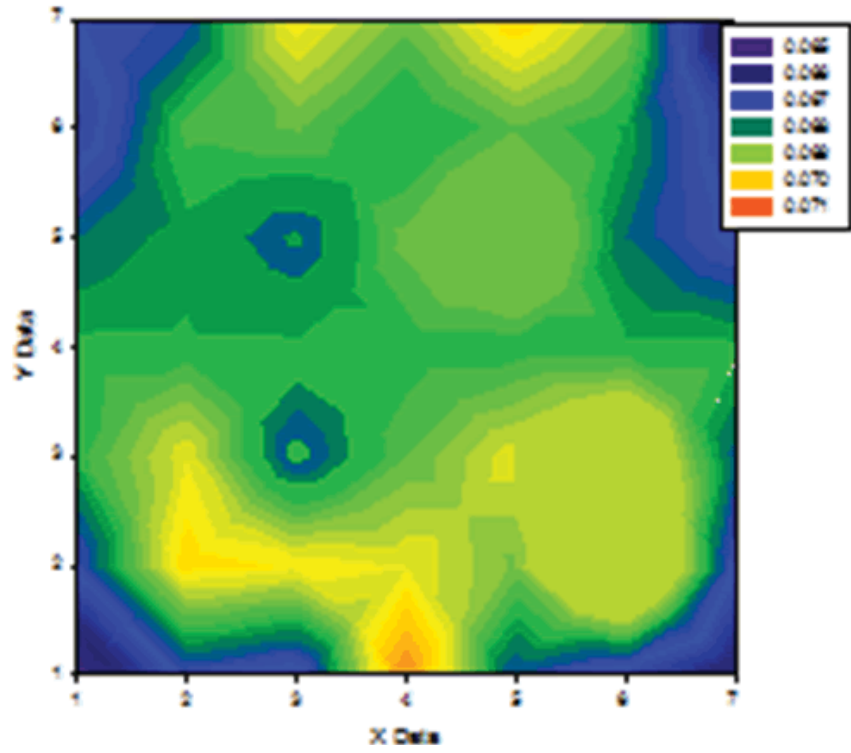

95\% Reflective Surface

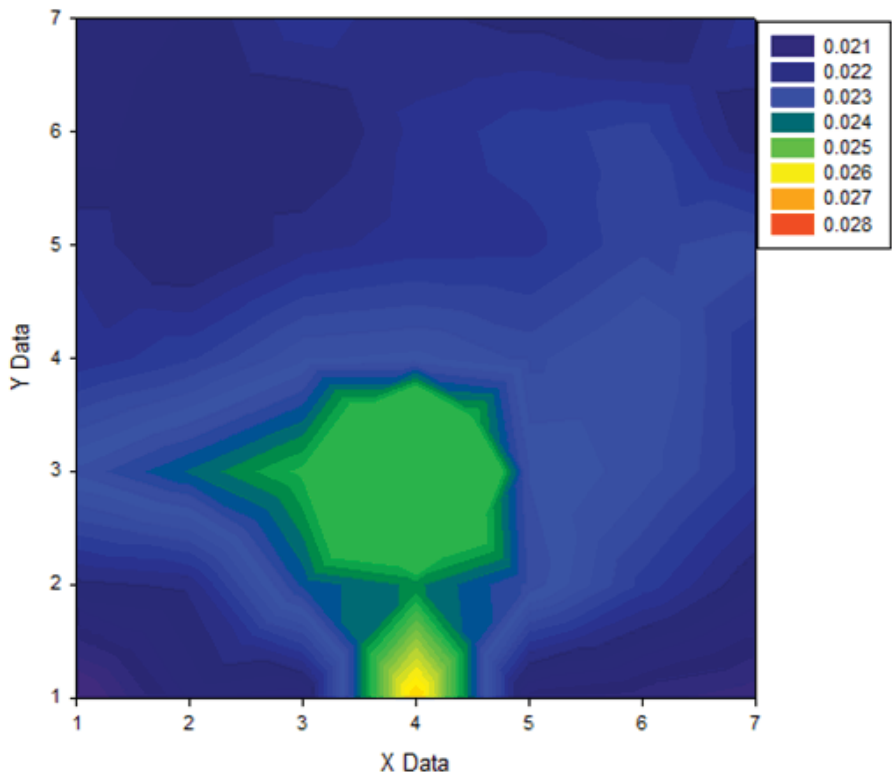

NIX runs. However, it had not been calibrated since 2000 [7]. A light-emitting diode was used as a check on the calibration for years. However, a measurement of the ZDC's response to an iron ion beam at the NASA Space Radiation Lab yields a fresh calibration.

\section{Results and Discussion}

\section{A. The mini-TPC}

The charge produced by the GEM stack is collected onto readout strips, which share the charge and are read out by an SRS or Scalable Readout System via four APV-25 cards, which are like 125 flash-ADCs (analog-to-digital converters). In other words, the SRS is like the readout electronics for 512 oscilloscopes, each connected to a strip.

The timing data is extracted by using the pulse arrival time. However, the pulse's arrival cannot be determined by the timing of the pulse's maximum or its passing of some threshold because of the "walk" effect--a more quickly-rising signal will pass a threshold at a different time than a slower signal. To minimize

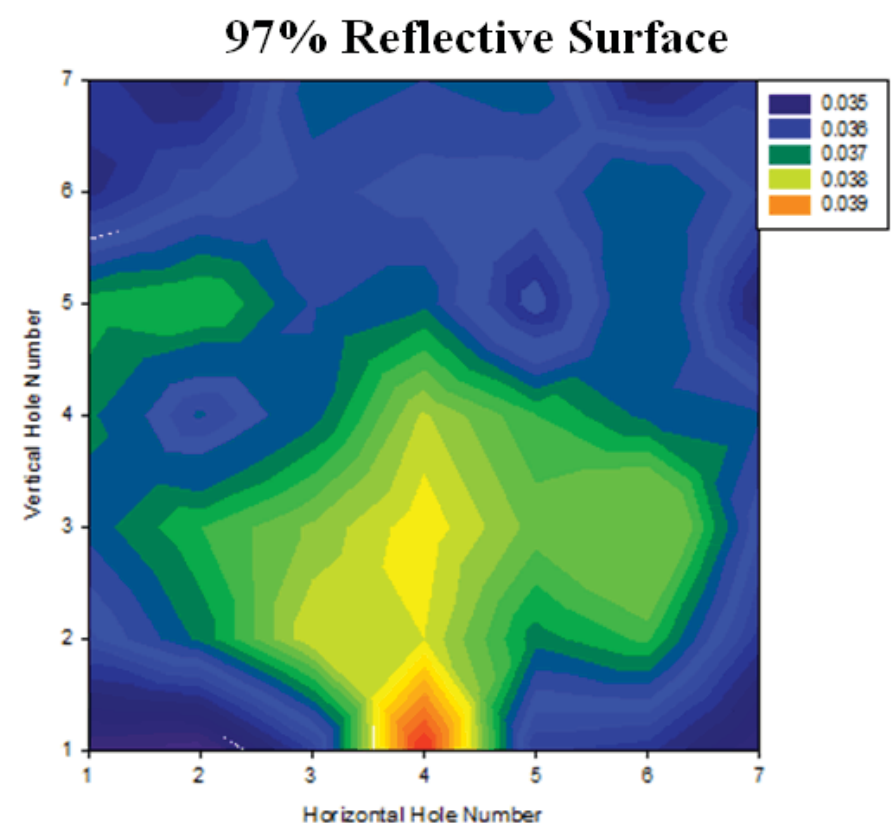

$\mathbf{9 0 \%}$ Reflective Surface
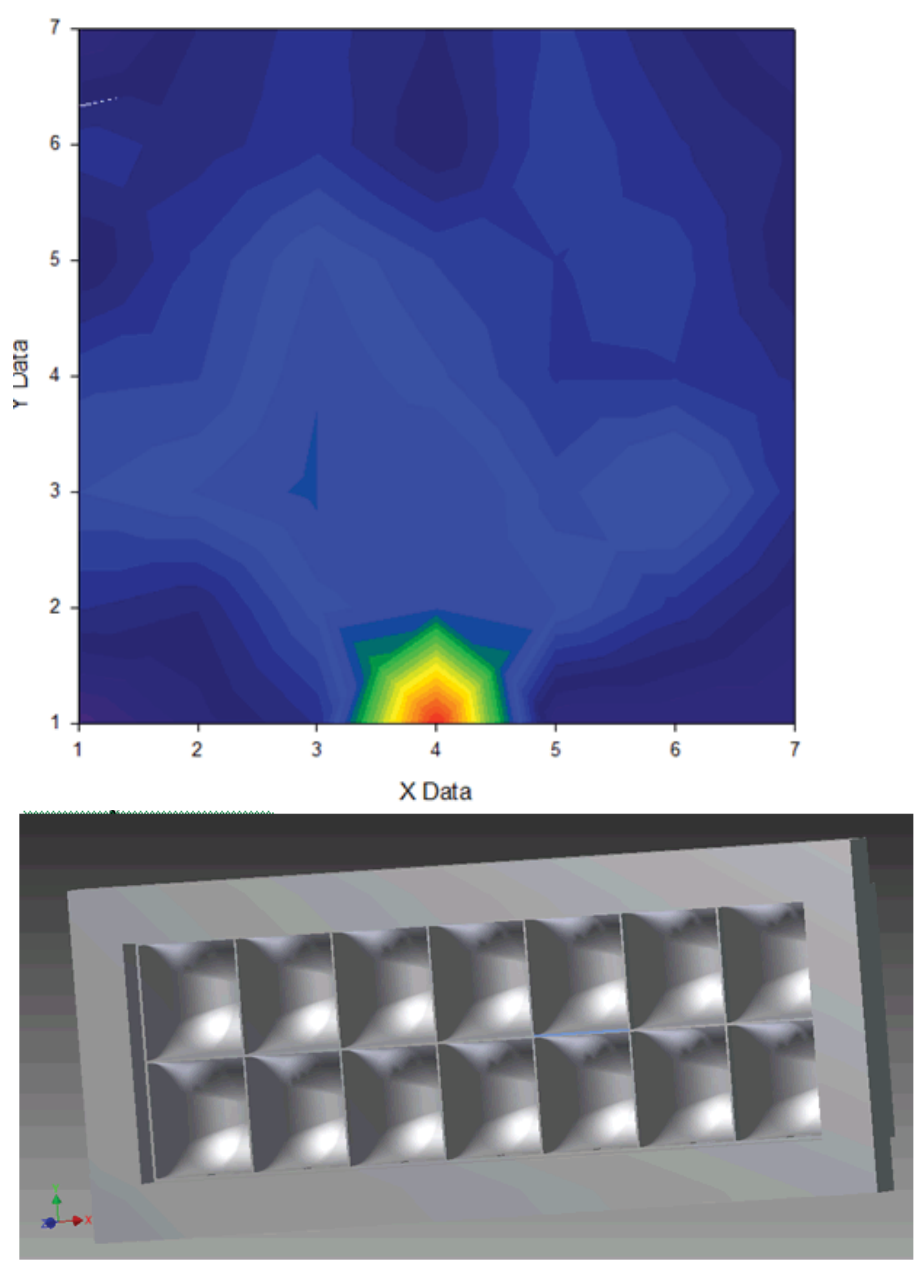

Diagram 8: A design for a potential integrating cavity cluster.

this error, we fit the leading edge of the pulse to a straight line, and its zero-intercept was used as the pulse timing.

These pulses were then used to reconstruct the tracks of a positron-emitting leaf.

In this leaf reconstruction, veins are slowly becoming more visible. To aid the data-acquisition trigger, the hot spots were covered with coins. 

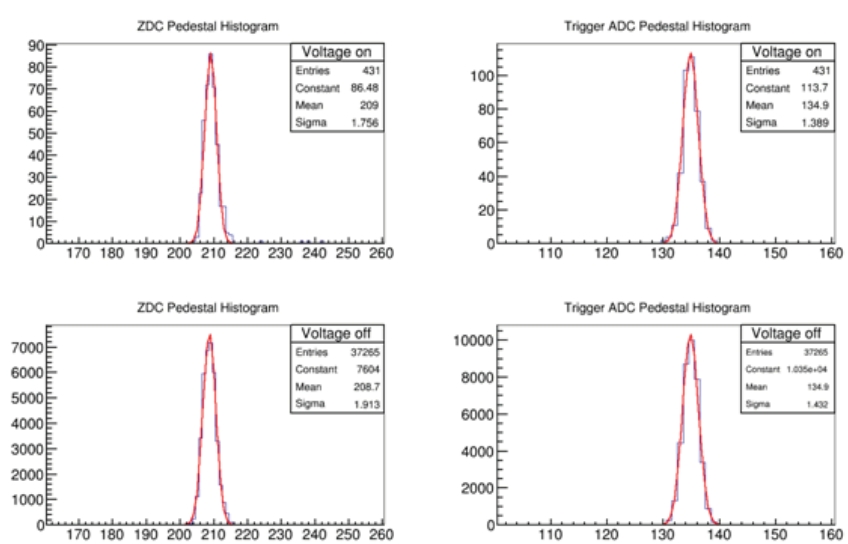

Diagram 9: The pedestals for the analog to digital converter for the trigger and the ZDC signal
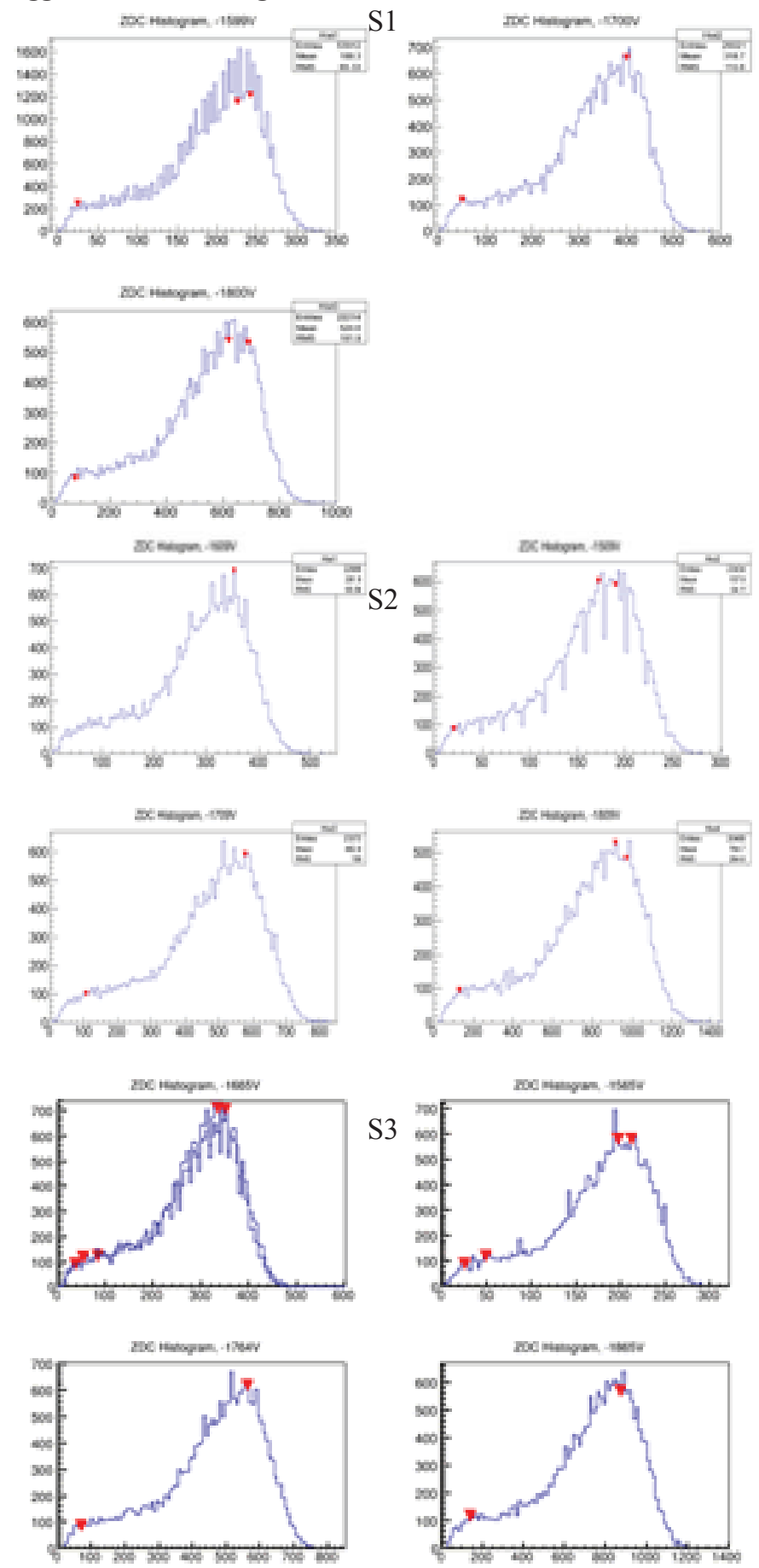

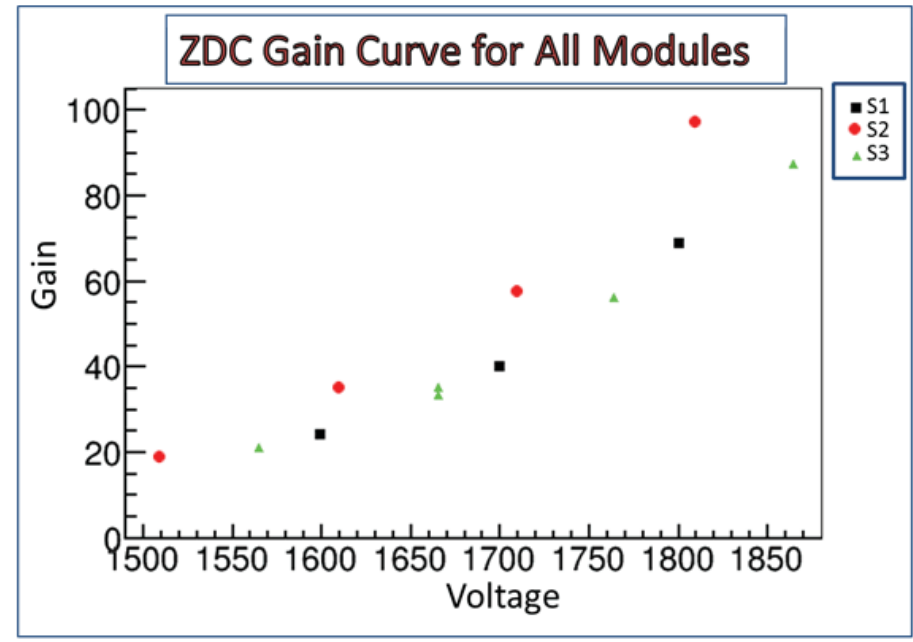

Diagram 11: The gain curve

\section{B. The Electromagnetic Calorimeter}

The integrating cavities must have a high efficiency, and no fiber-dependent bright spots or shadows. This can be modeled using a ray-tracing simulator called OptiCAD. OptiCAD bounces rays off of an imported CAD surface given specific characteristics.

The first cavity simulated was a square box. The square box was easiest to construct and measure, and so the simulation parameters - such as the reflectivity of the surface -- could be tuned to match the measured data.

The measured data from the integrating cavity demonstrated a bright spot for rays coming in near the SiPM and an efficiency from $3.2 \%$ to $4.2 \%$

The simulations show that this corresponds to a reflectivity between $95 \%$ and $97 \%$. Furthermore, the simulations show that as the reflectivity increases, the efficiency is more and more uniform.

Further tests will be completed elongating the square to show that as the SiPM moves further and further away, the bright spot will become less and less pronounced. Also, other shapes must also be tested, such as the egg shell in diagram 8:

\section{The Zero-Degree Calorimeter}

Pedestals are noise baselines upon which all signals sit and must be measured and subtracted off of signals. For the ZDC signal, the pedestals are in diagram 9:

The gains were calculated with the measured signal after smashing iron ions into each calorimeter module. The responses are as follows in in diagram 10.

The new gain curve is in diagram 11:

\section{Conclusions}

The mini-TPC has shown its ability to track positrons, and the tracking algorithms have been developed. Low statistics and poor track fits due to scattering of the low energy positrons are most likely to blame for the obscured leaf veins. While its efficiency and reliability will help with individual higher-energy particle tracking, other imaging techniques trump its ability to reconstruct biological PET images.

The OptiCAD program is powerful and will be a valuable tool to guide test prototypes for the integrating cavities. However,

Diagram 10: The responses of each ZDC module (S1, S2, and S3) 
the square cavity is not an ideal choice for the readout towers; it has a bright spot near the SiPM. More work must be done on the OptiCAD simulations for other configurations.

The new gain curves for the PMTs were measured and a new ZDC calibration was established. However, there is more work still to do with ZDC data, such as comparing this calibration to the LED signal.

In conclusion, I need two more weeks to finish these analyses.

\section{Acknowledgments}

I would like to thank all the coauthors of this paper, from my mentor, Craig Woody, to all the people I worked with this summer-Bob Azmoun and his brilliant work with the SRS and the mini-TPC, Sean Stoll and his calorimetric expertise and VME readout, John Haggerty and his PHENIX data acquisition help and beam run, and most especially to Martin Perschke for writing the data acquisition software and troubleshooting my own code.

I would also like to thank Brookhaven National Lab and the Office of Educational Programs for hosting this program. This project was supported in part by the U.S. Department of Energy, Office of Science, Office of Workforce Development for Teachers and Scientists (WDTS) under the Science Undergraduate Laboratory Internships Program (SULI), and DOE Contract No. DEAC02-98CH10886.

\section{Endnotes and References}

[1] sPHENIX: An Upgrade Concept by the PHENIX Collaboration, arXiv:12076378 (2012).

[2] K. Adcox et al. [PHENIX Collaboration] Formation of dense partonic matter in relativistic nucleus nucleus collisions at RHIC: Experimental evaluation by the PHENIX collaboration. Nucl. Phys.,A757:184-283, 2005. arXiv:nucl-ex/0410003 (2005).

[3] T. Renk. Jets in medium: What RHIC and LHC measurements of RAA and IAA can teach about the parton-medium interaction. arXiv:1111.0769 (2011).

[4] Y.-S. Lai. Direct jet reconstruction in $\mathrm{p}+\mathrm{p}$ and $\mathrm{Cu}+\mathrm{Cu}$ collisions at PHENIX, Nucl. Phys., A855:295-298, (2011).

[5] J. Thomas [STAR Collaboration], The STAR Detector at RHIC, http://instrumentationcolloquium.lbl.gov/The STAR_Detector_at_RHIC.pdf

[6] I. GEM: A New Concept for Electron Amplification in Gas Detectors, F.Sauli et.al., Nucl. Inst. Meth. A386 (1997) 531.

[7] C. Adler [RHIC Collaboration], The RHIC Zero Degree Calorimeter, arXiv:nucl-ex/0008005v1.pdf (2000). 


\title{
Analysis and fabrication of carbon micro-ribbon argets for proton polarimetry studies at RHIC
}

\author{
Kevin Bogaer \\ School of Materials Science and Engineering, Georgia Institute of Technology, Atlanta, GA 30332 \\ John Warren \\ Instrumentation Division, Brookhaven National Laboratory, Upton, NY 11973
}

\begin{abstract}
:
Spin-polarization studies at the Relativistic Heavy Ion Collider require high purity carbon micro-ribbon targets that are $25 \mathrm{~mm}$ long, $10 \mu \mathrm{m}$ wide, and $50 \mathrm{~nm}$ thick. Conductivity and transmission electron microscopy studies of previously tested targets showed the target microstructure to be amorphous. This amorphous structure results in low electrical conductivity and reduced lifetime when exposed to $250 \mathrm{GeV}$ protons at RHIC. Two approaches for material property improvement have been identified. First, the current generation of amorphous carbon micro-ribbons can be annealed to form conductive graphite. Graphite targets are over an order of magnitude more electrically conductive than amorphous carbon and should therefore have significantly longer lifetimes. Second, a new generation of tri-layer micro-ribbons can be made from copper, carbon, and nickel. Graphene is precipitated during an annealing and quenching process and the metal layers etched away to leave a graphene micro-ribbon. Graphene is known to be more electrically conductive and much stronger than graphite and should have a longer lifetime.
\end{abstract}

\section{INTRODUCTION}

The Relativistic Heavy Ion Collider (RHIC) at Brookhaven National Laboratory (BNL) collides two opposing beams of polarized protons to study the internal structure of the proton. Due to the presence of numerous depolarizing resonances and the necessity of having a highly $(>70 \%)$ polarized beam, the state of polarization of the proton beam must be continuously monitored by Coulomb-nuclear interference (CNI) polarimeters. ${ }^{1}$ The 250 $\mathrm{GeV}$ protons are elastically scattered from a carbon micro-ribbon (CMR) target during numerous months of continuous testing. Due to the length of the experiments, the ultra-high vacuum required for operation, and the expensive cryogenics needed for the beam's superconducting magnets, polarimeter target lifetime is of high importance. ${ }^{2}$ If a sufficient number of polarimeter targets fail while RHIC is in operation, testing must stop until new targets are loaded in the CNI polarimeter and ultra-high vacuum is again achieved.

For each experimental run, the CNI polarimeters require 48 amorphous carbon micro-ribbon targets with the dimensions of $2.5 \mathrm{~cm} \times 10 \mu \mathrm{m} \times 50 \mathrm{~nm}$. The targets are fabricated by the Collider-Accelerator Department at BNL following the procedure of Lozowski et al. ${ }^{2}$ Although CMR durability is critical to the polarized proton program at RHIC, the lifetime of these CMRs varies greatly. Many CMRs fail early during the experimental run, but a few targets survive numerous proton beam interactions without failure. We have studied the surviving CMR targets and compared them to as-fabricated CMRs to analyze the affect of the RHIC proton beam on the carbon microstructure. By determining the cause of survival of the "long-lived" targets, we propose to improve fabrication methods that will enable the routine production of CMR targets with a longer and more predictable lifetime.

It is thought that the low electrical and thermal conductivities of amorphous carbon are the sources of failure. ${ }^{3}$ When exposed to the $250 \mathrm{GeV}$ proton beam, inelastic scattering heats the target and ultimately causes mechanical failure. By replacing amorphous carbon with a more conductive crystalline allotrope of carbon such as graphite or graphene, the lifetime of the targets may be increased significantly with minimal change to the current fabrication procedure. The fabrication of large-scale patterned graphene films from amorphous carbon through a process of annealing and quenching has been well documented and this method is also compatible with the current CMR design parameters with only minor modifications. A $10 \mathrm{~nm}$ carbon layer will dissolve to form a solid solution within a $50 \mathrm{~nm}$ nickel layer at $700 \mathrm{oC}$ during annealing and then precipitate out as multi-layer graphene on quenching. ${ }^{4,5,6,7}$ The nickel can then be removed in a dilute nitric acid solution to leave a free-standing graphene CMR.

\section{EXPERIMENTAL}

Micro-ribbon targets were fabricated using electron beam deposition in a Lesker Lab 18 vacuum deposition system. Depositions were patterned to desired thicknesses through an anisotropically etched $<110>$ silicon wafer mask consisting of an array of slots $10 \mathrm{um}$ wide and $30 \mathrm{~mm}$ in length onto a frosted glass slide that was coated with a Tergitol- 8 thin film. ${ }^{8}$ The pressure during deposition was on the order of 10-4 Pa.

Following deposition, the micro-ribbon targets were lifted off from the glass substrate by dissolving the Tergitol-8 film in water. Each target was attached to two copper electrodes on a C-shaped insulating frame using silver paste. The frame/target assembly was then placed in a small vacuum chamber, flushed with argon, and pumped to $5 \mathrm{~Pa}$. Once under vacuum, the targets were resistance annealed by applying a potential of up to 300 volts to the copper electrodes of the frame. Resistivity and current readings for a given potential were collected during annealing using a Keithley 487 picoammeter/voltage source.

Structural characterization of targets and electron beam lithography of silicon wafer masks were performed in a JEOL JSM-6500F field emission scanning electron microscope. Electron beam lithography patterning of the mask used the Nanometer Pattern Generation System that was attached to the JSM-6500F. Energy-dispersive X-ray spectroscopy was performed using a Bruker XFlash 4030 silicon drift detector attached to the JSM$6500 \mathrm{~F}$. Diffraction patterns were generated at $200 \mathrm{keV}$ in a JEOL 2100 transmission electron microscope at the BNL Center for Functional Nanomaterials. Both as-prepared original amorphous targets and targets exposed to the RHIC proton beam were provided by the BNL Collider-Accelerator Department. All measurements were performed at room temperature unless otherwise stated. 


\section{RESULTS AND DISCUSSION}

\section{A. Existing targets}

Before developing new CMR targets, the first priority of this study was to analyze the successes and failures of the existing design. Transmission electron microscope diffraction patterns were acquired for several carbon samples shown in Fig. 1. In clockwise order, they range from an intentionally amorphous sample in Fig. 1(a) to a highly crystalline surviving RHIC target in Fig. 1(d). Interestingly, the as-prepared RHIC targets, shown in Fig. 1(b), strongly resemble the amorphous pattern of Fig. 1(a). This implies that the current method of CMR fabrication produces an amorphous carbon target with the characteristically low conductivity of amorphous carbon. While exposed to the high energy RHIC proton beam, annealing and crystallization occurred in sufficient quantity to produce the well defined diffraction pattern rings found in Fig. 1(d). These diffraction pattern rings strongly correlate with the calculated ring spacings of several interplanar spacings for the graphite crystal structure.

To improve the lifetime of the CMR targets, all targets should be fabricated at or as close to the level of crystallinity found in Fig. 1(d). This topic will be discussed in greater detail in Section III B but results so far have yielded the diffraction pattern present in Fig. 1(c). While the degree of crystallinity displayed by the CMR diffraction pattern in Fig. 1(c) is not as great as Fig. 1(d), it is still a significant improvement over the amorphous as-fabricated CMR that shown in Fig. 1(b).

a

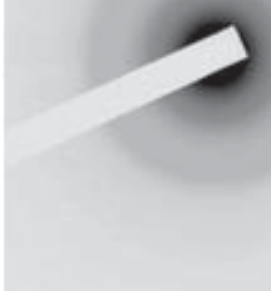

c

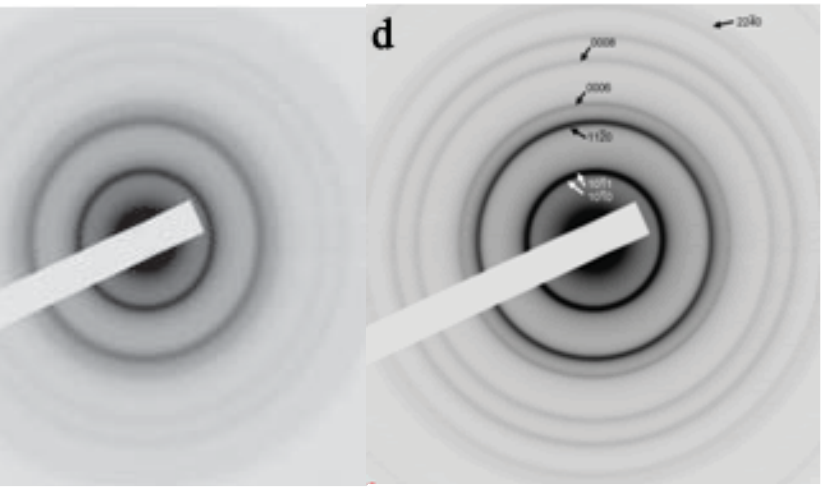

FIG. 1. Inverted contrast TEM diffraction patterns of (a) amorphous carbon (b) an as-fabricated CMR (c) an annealed CMR and (d) a surviving RHIC target labeled with the corresponding planes of graphite.

\section{B. Growing and crystallizing graphite}

To begin testing new CMR designs, a versatile method of target fabrication, compatible with the graphite and graphene design parameters was required. Given the nearly one-dimensional nature of the ribbons, it was crucial to have a consistent template that produces perfectly straight ribbons.

This was achieved by anisotropic etching, a method commonly used to fabricate high aspect ratio microstructures from silicon wafers. By exposing and developing a resist-coated $<110>$ silicon wafer with a rectangular design, the wafer can then be etched in potassium hydroxide at $60 \mathrm{oC}$ to create vertical grooves extending through the wafer. The close-packed (111) plane etches orders of magnitude slower than other low-index planes in silicon, enabling the creation of very sharp vertical walls. As shown in Fig. 2(a), a negative resist, SU8 was used initially to verify that a high aspect ratio could be achieved using anisotropic etching of silicon wafers. Following this, a positive resist, PMMA (poly-methyl methacrylate) was used to create silicon wafer masks. Each mask contained 10 grooves up to $100 \mu \mathrm{m}$ wide and $30 \mathrm{~mm}$ long. Fig. 2(c) demonstrates the perfectly parallel and vertical nature of the silicon wafer mask grooves. Fig. 2(d) shows the as-prepared CMR prior to annealing.

Early attempts at CMR liftoff from the deposition substrate involved using oxidized silicon wafers. Following deposition, the oxide would be dissolved in buffered hydrofluoric acid (BHF), allowing the CMRs to float to the surface. Due to a high failure rate, long dissolution period, preparation time required for re-oxidizing silicon wafers, and use of the toxic BHF solution, this approach was abandoned in favor of Lozowski's method of using Tergitol 8 and glass slides. 8 Each completed mask was clamped in close contact with a glass slide coated with a thin film of Tergitol- 8 that acts as a release agent and coated with $50 \mathrm{~nm}$ of carbon in the Lesker Lab 18 vacuum evaporator. This process improved the high failure rate, so that each deposition produced between two and five successful CMRs for mounting and annealing.

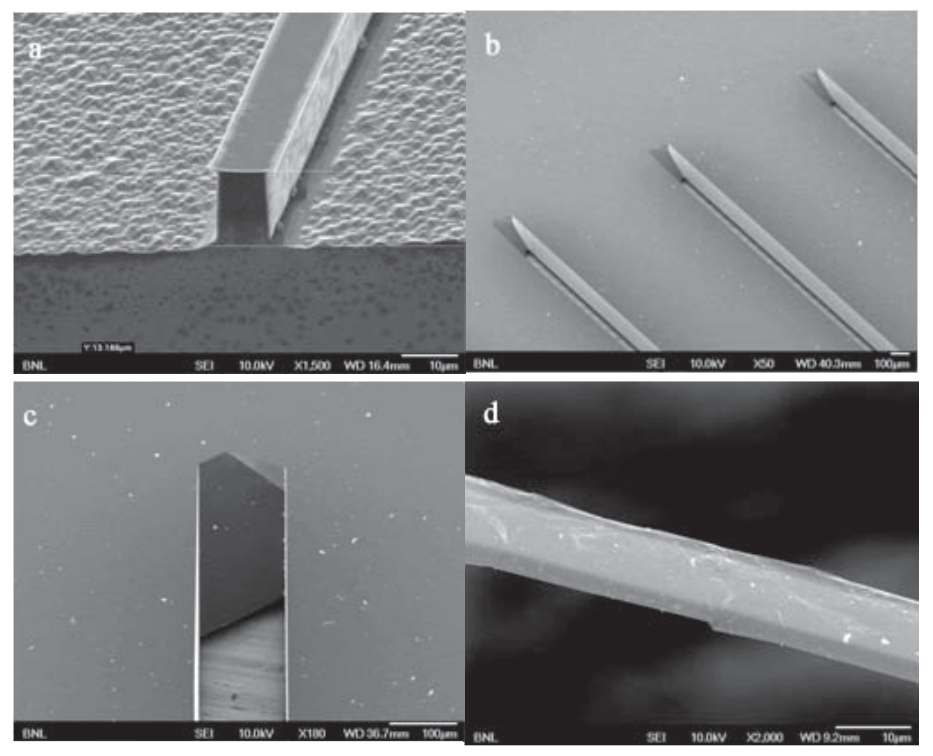

FIG. 2. (a) SEM of anisotropically etched of Si wafer coated with SU8 resist. (b) SEM of $<110>$ anisotropically etched Si wafer mask used for micro-ribbon deposition. (c) SEM overhead view of anisotropically etched Si wafer mask (d) SEM of amorphous carbon micro-ribbon as-prepared prior to annealing.

In an effort to close the crystallinity gap demonstrated by Figs. 1(b) and 1(d), vacuum annealing was explored as a means of crystallizing the amorphous targets and thus increasing the electrical conductivity. Using the Stefan-Boltzmann Law shown 
in Eqn. 1, targets were resistance-heated to increase conductivity. Fig. 3 shows the varying resistances and temperatures of a single CMR, measured at three points in time spanning approximately 30 minutes. As the resistance decreased by over an order of magnitude, the current passing through the CMR increased, resulting in a greater total power measured, and thus a higher temperature experienced by the CMR. Therefore, as the amorphous carbon crystallizes, it is capable of reaching greater temperatures and the process of crystallization accelerates.

$$
T=\left(\frac{V 1}{\varepsilon \sigma A}+T_{R}^{4}\right)^{1 / 4}
$$
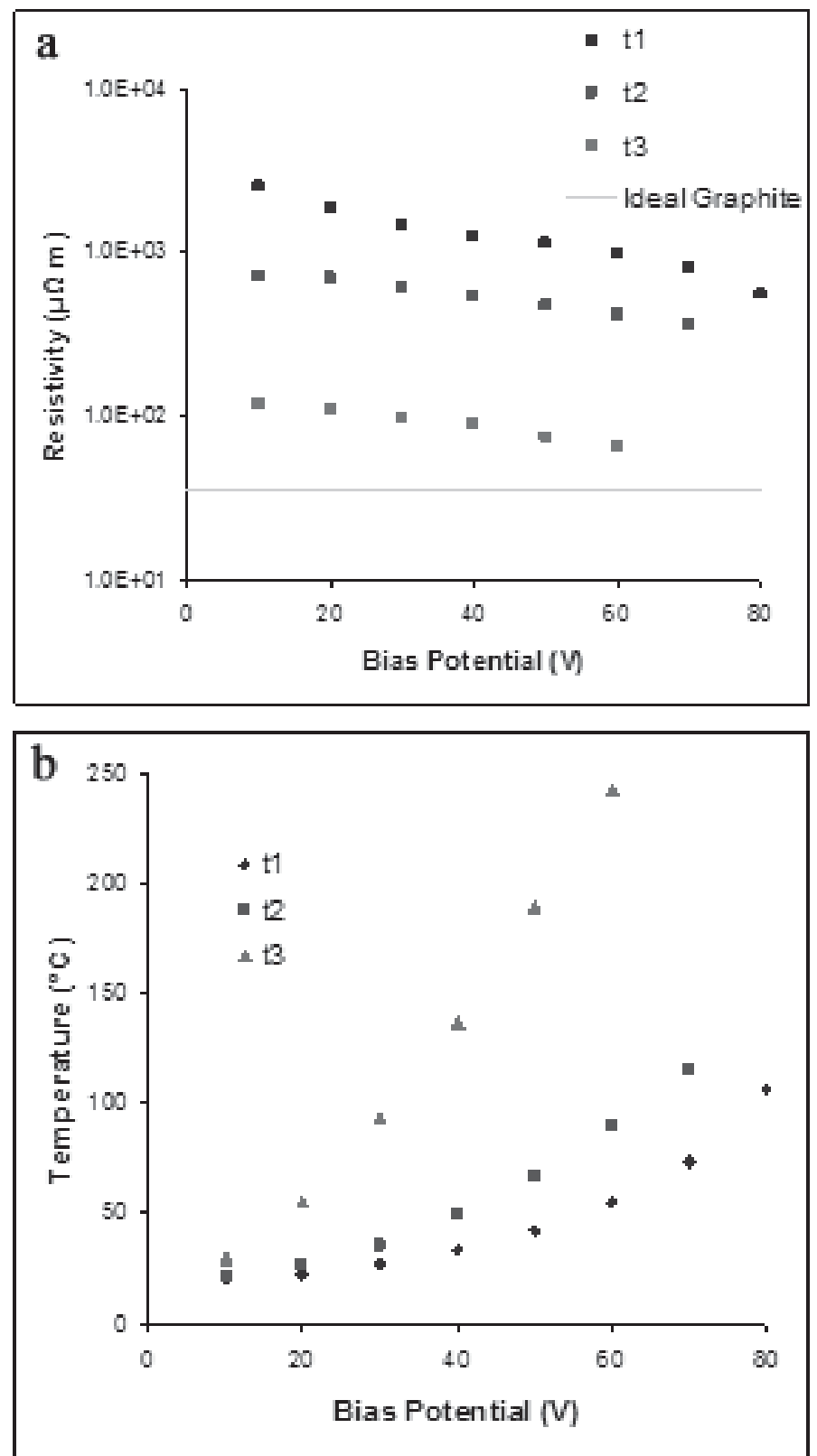

FIG. 3. (a) Micro-ribbon resistivity decreases and (b) temperature increases with time during annealing.

\section{Precipitating graphene}

While highly crystallized graphite is a significant improvement over the former amorphous CMR targets used, it is not necessarily the ideal solution. Using the same deposition method previously described for graphite fabrication, copper, carbon, and nickel layers were deposited through the anisotropically etched silicon wafer mask to create a tri-layer micro-ribbon. As shown in Figs. 4(a) and 4(b), the tri-layer micro-ribbon prefers to curl upon itself due to the differences in thermal expansion coefficients. These ribbons are deposited at elevated temperatures and contract on cooling. This phenomenon will be reduced in narrower $(10 \mu \mathrm{m})$ micro-ribbons as compared to the $100 \mu \mathrm{m}$ micro-ribbons pictured due to a reduction in torque.

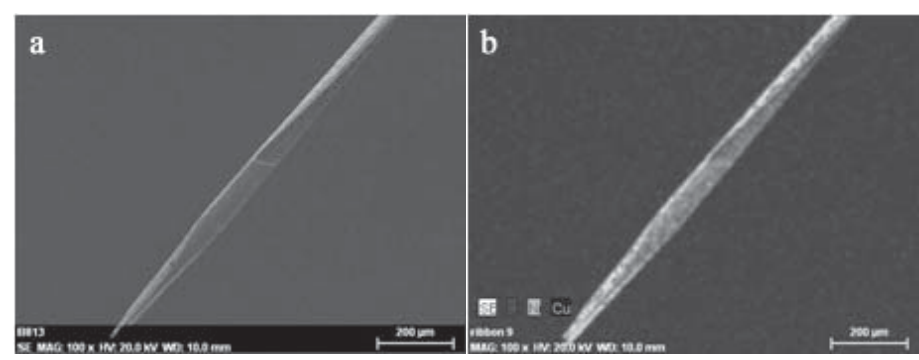

FIG. 4. (a) SEM and (b) EDX elemental map of tri-layer micro-ribbon. Part of the nickel layer peeled off, revealing the carbon middle layer.

\section{CONCLUSIONS}

The serendipitous survival of some RHIC CMR targets was originally thought to be the result of variations in electrical conductivity. Our TEM results indicate that the surviving targets were crystalline graphite, therefore possessing a higher conductivity than the as-fabricated amorphous carbon targets. While exposed to the high energy polarized proton beam at RHIC, these targets were annealed and crystallized into graphite.

Our research attempted to consistently duplicate these results by vacuum annealing fresh as-fabricated CMRs. Results indicate that although the crystallinity of the annealed CMRs shown by TEM diffraction patterns did not match that of the surviving RHIC targets, significant crystallization did occur. In terms of electrical conductivity, the annealed samples were over an order of magnitude better than their as-prepared CMR counterparts. Following approximately 30 to 45 minutes of annealing at temperatures below $500^{\circ} \mathrm{C}$, these CMRs were typically no more than twice as electrically resistive as the established values for graphite.

Thus far, only a proof of concept has been established that tri-layer micro-ribbons can be fabricated. Once graphite CMR research is mature and high-conductivity targets can be consistently and reliably mass-produced for implementation in RHIC, future work will focus on the fabrication of graphene micro-ribbons via the multi-layer deposition and annealing process described above.

Using the methods shown above for the fabrication and annealing of carbon micro-ribbons, we can expect significant improvements in RHIC target lifetimes. The order of magnitude of increase in electrical conductivity should prevent or at least inhibit the type of failure previously experienced by the CMR targets during the initial moments of beam exposure in previous RHIC experimental runs. This will allow RHIC researchers to collect data concerning beam polarization for longer durations of time with improved-functioning CMR targets, increasing scientific productivity and resource efficiency, while decreasing downtime and operational costs.

Beyond the scope of RHIC, these findings outline a method for the fabrication of thin-film, multi-layer ribbons from any ma- 
terial suitable for electron-beam deposition. Such samples are ideal for in-situ annealing experiments and can be immediately examined by electron microscopy to reveal many aspects of their microstructure and material properties.

\section{ACKNOWLEDGMENTS}

This project was supported in part by the U.S. Department of Energy, Office of Science, Office of Workforce Development for Teachers and Scientists (WDTS) under the Science Undergraduate Laboratory Internships Program (SULI).

\section{REFERENCES}

[1] I. Alekseev et al. Polarized proton collider at RHIC. Nucl Inst \& Meth. A499, 392-414 (2003).

[2] W.R. Lozowski et al. Carbon micro-ribbon and strip polarimeter targets for the AGS and RHIC. Nucl Inst \& Meth. A590, 157-163 (2008).

[3] A. Zelenski. RHIC polarimeter operation. Instrumentation Division Seminar, Brookhaven National Laboratory, Upton, NY, June 27, 2012.

[4] L. Baraton et al. On the mechanisms of precipitation of graphene on nickel thin films. Europhy. Lett. (96), 46003, (2011).

[5] K.K. Kim et al. Large-scale pattern growth of graphene films for stretchable transparent electrodes. Nat. Letters (457) 706-710, (2009).

[6] Q. Shao et al. High-temperature quenching of electrical resistance in graphene interconnects. Appl. Phys. Lett. (92) 202108, (2008).

[7] M. Zheng et al. Metal-catalyzed crystallization of amorphous carbon to graphene. Appl. Phy. Lett. (96), 063110, (2010).

[8] W.R. Lozowski and J.D. Hudson. Preparation of carbon micro-ribbon targets and open-edged stripper foils for the IUCF cooler ring. Nucl Inst \& Meth. A303, 34-42, (1991). 


\title{
Acoustic identification of Chiroptera species in eastern Suffolk County and Brookhaven National Laboratory using full spectrum call analysis.
}

\author{
Jessica Bristol \\ Eastern Connecticut State University, Willimantic, Connecticut, 06226 \\ Kathy Schwager \\ Brookhaven National Laboratory, Upton, NY 11973
}

\begin{abstract}
First discovered in Albany, New York in 2006, white-nose syndrome (WNS) has decimated bat populations throughout North America. WNS is caused by the cold-loving fungus Pseudogymnoascus destructans, which is native to Europe. Nine species of Chiroptera are known within New York State, 6 of which winter in caves. It is believed that P. destructans arouses these bats from torpor at inappropriate times, leading to a depletion of their fat-stores and causing death by starvation. Acoustic surveys were conducted in eastern Long Island in coordination with Brookhaven National Laboratory (BNL) and the New York State Department of Environmental Conservation (NYSDEC) in order to note trends among bat populations as a result of WNS. Ultrasonic bat calls were recorded along 4 different routes using a car-mounted microphone and the unique frequency of "search phase" calls used to identify the species of bat. Of the 6 species recorded, most prevalent were 2 species, the big brown bat (Eptesicus fuscus) and the eastern red bat (Lasiurus borealis), composing $83 \%$ of calls recorded. The data collected were compared with 2 previous years of collected data and indicated a substantial decrease in $E$. fuscus and increase in $L$. borealis. Acoustic surveys were also conducted within BNL in order to determine differences between the solar farm and a recently burned forest when compared to a control site. This research is helping to get a better idea of population trends and niche changes as WNS spreads across America.
\end{abstract}

\section{Introduction}

Chiroptera is the second most numerous order of mammals, composed of nearly 1,300 species commonly referred to as bats. Chiropterans are the only mammals capable of true flight and may travel distances of up to $50 \mathrm{~km}$ a night to forage, consuming nearly $50 \%$ of their body weight in insects per night. ${ }^{1}$ As one of the few nocturnal predators of flying insects, they provide pest-control services valued as high as 53 billion dollars that save crops and help to control the spread of insect-borne diseases. ${ }^{2}$

In 2006, a psychrophilic fungus commonly referred to as white-nose syndrome, recently reclassified from Geomyces destructans to Pseudogymnoascus destructans, was found within hibernacula in upstate New York. ${ }^{3,4}$ Since that time, WNS has spread throughout the United States and killed over 5.7 million bats. ${ }^{5}$ P. destructans is thought to be a novel pathogen from Europe and is only associated with the mortality of North American bats. Injections of both the American and European strains of $P$. destructans to Myotis lucifugus resulted in WNS infection, leading to hyper-arousal and eventually mortality. ${ }^{6}$ WNS infects the wing, ear, tail and muzzle tissue of hibernating cave bats, de- stroying tissue and increasing arousal from torpor to once every 10-20 days to as often as every 3-4 days. ${ }^{7}$ This quickly depletes the fat stores of these animals and can force them to forage during the winter months when the cold and low availability of insects become lethal. ${ }^{8}$

Forty-seven species of Microchiropterans are found in the United States, 9 of which are found within New York State. ${ }^{9}$ Of these, 3 species are tree bats which over-winter in hollow trees or migrate south. ${ }^{10}$ The tree bats, Lasiurus borealis (eastern red bat), Lasiurus cinereus (hoary bat) and Lasionycteris noctivagans (silver-haired bat) generally have more than one offspring per year and are less susceptible to WNS due to their roosting habits. ${ }^{11}$ These 3 species migrate south for the winter, with L. borealis roosting solitarily, using its furred tail membrane for insulation. $L$. cinereus may travel further south, often avoiding hibernation entirely and foraging within the tropics, while L. noctivagans hibernates in small clusters within the southern United States in rock crevices and trees. ${ }^{12}$

Cave bats are easily distinguished from tree bats due to the lack of fur on their tail membranes and their tendency to spend the winter hibernating in caves. ${ }^{13}$ Of the 6 species of cave bats found in New York, 5 have been drastically reduced in number due to WNS. Populations of Myotis lucifugus (little brown bat), Perimyotis subflavus (tri-colored bat) and Myotis septentrionalis (northern long-eared myotis) have all declined by over $90 \%$. Myotis sodalis (Indiana bat) numbers have decreased by $71 \%$ and Myotis leibii by $13 \% .{ }^{14}$ Eptesicus fuscus (big brown bat) is typically found in small numbers but is rarely sighted with WNS. ${ }^{15}$ Since these species typically only bear 1 offspring per year, these populations are in dire shape without immediate conservation initiatives. ${ }^{16}$

Of the 5 initially infected hibernacula, 3 have shown increases in M. lucifigus numbers but it is unclear if this growth is due to integration of new bats to the area or reproductive success of the colonies. $^{17}$

The purpose of this study was to continue ongoing population surveys within eastern Long Island in order to note any population trends among Chiropteran species as a result of WNS. We hypothesized that a continued decline in cave bat species would lead to an increase in tree species as more resources, such as habitat and obtainable food, became available. This study also provided a preliminary look at bat species present at BNL and examined habitat usage of these species. 


\section{Materials and Methods}

These procedures were adapted from: Bat Survey Training. (Herzog, Carl. 2010)

\section{A. Route Selection}

Four routes in eastern Suffolk County were plotted using DeLorme 2012@. Each route was approximately 15-25 miles in length. Routes were selected to avoid high speed areas and congestion when possible. Three of the routes pass primarily through the Pine Barrens Region while the Sound Ave. route is located further east to get an idea of bat populations near farmland. The North Street route travels along the Peconic River and Carman-Rocky Point route passes through the Carmans River watershed (Appendix 2).

Three mobile routes and 3 stationary locations were also plotted within BNL in order to cover areas of the Long Island Solar Farm and a recently burned forest to compare to a control (Appendix 3). One additional stationary survey was conducted in Manorville, NY at a private residence containing 3 bat houses and bordering the burned area from the 2012 Crescent Bow fire. ${ }^{18}$

\section{B. Survey Conditions and Setup}

Mobile surveys for the NYDEC were conducted from June 5th through July 1st and surveys on-site on BNL property were conducted July 17 th until July 23rd. Mobile surveys were conducted beginning 30 minutes after sunset and stationary surveys ran from 20:00-21:30 EST. Weather data was collected prior to recording and surveys were conducted if sustained winds were less than $15 \mathrm{mph}$ and temperature was greater than $55^{\circ} \mathrm{F}$ with no rain. Weather data was also collected immediately after recording was complete.

Mobile surveys within Suffolk County were conducted at a speed of 18-20 mph while surveys at BNL were reduced to speeds between 10-15 mph due to road conditions. Hazard lights were used to indicate the slow speed of the vehicle and we pulled off to the shoulder to let cars pass. The number of cars that passed in this manner were recorded to help determine whether or not the route should be repeated next season.

Calls were recorded using an acoustic monitoring device mounted by magnet to the vehicle's roof. The program Spect'R IIIC was used to record full-spectrum bat calls with a tuner that allowed for conversion of ultrasonic frequencies to audible levels and a sonographic analyzer used to visualize the call. ${ }^{19}$ The coordinates traveled and their associated times were logged using Delorme 2012@ in conjunction with a GPS unit attached to the roof of the vehicle. Each NYDEC route was run 4-5 times and BNL surveys were conducted once.

\section{Identification}

Calls were analyzed using the program Scan'R $(\mathcal{C}$ which visually the displayed frequency $(\mathrm{kHz})$, slope $(\mathrm{Sc})$ and shape of each pulse over time (ms). With the aid of a flowchart designed by Carl Herzog, calls were manually deciphered based on characteristic frequency $(\mathrm{Fc})$ and $\mathrm{Sc}$. In order to identify species using this method, the search phase call was used, defined by a minimum of 5 evenly-spaced pulses occurring approximately every $100 \mathrm{~ms}$. Calls inadequate in length or uneven in spacing were classified as unknown Chiropteran species. Due to the difficulty in differenti- ating E. fuscus and L. noctivagans by Fc, all calls fitting the criteria for these species were lumped into one category and referred to as " $E$. fuscus" in the results.

III. Results

A. Overall trends

A total of 500 positive Chiropteran calls were recorded over the course of this study. Of these calls, $12.02 \%$ were attributed to unidentified species (10.70\% at BNL, $12.82 \%$ Suffolk County).

\section{B. Changes in Suffolk County}

The mobile surveys for the NYDEC showed a decrease in E. fuscus calls (-19.76\%) and an increase in L. borealis (10.44\%) since $2012 .{ }^{20}$ E. fuscus was the most commonly encountered species representing $56.09 \%$ of positive calls. Also encountered were L. borealis (26.92\%), M.lucifugus (2.56\%), P. subflavus $(1.28 \%)$ and L. cinereus $(0.32 \%)$.

\section{BNL \& Manorville}

E. fuscus was also the most commonly encountered species within BNL (51.60\%) followed by L. borealis $(32.45 \%)$. Also recorded were P. subflavus (2.13\%), M. leibii (1.06\%), and L. cinereus $(2.13 \%)$. Unidentified bats composed $10.63 \%$ of the calls recorded.

\section{Discussion}

The results of this study indicate a decline in the number of $E$. fuscus calls recorded over the last two years (Figure 2). Correlating with this is the prevalence of $L$. borealis compared to previous years, having increased by nearly $10 \%$. It is important to note that the number of unidentified bat calls has also increased but not significantly enough to have had an impact on these trends. $M$. lucifigus was the only other Myotis species encountered during the NYDEC surveys, showing a $2.24 \%$ increase since $2011 .^{21}$ Continuing studies are needed to determine if populations are in fact growing and whether or not these changes are associated with migration or reproductive success in native bats. ${ }^{22}$

Bat surveys at BNL showed the most concentrated number of calls within and immediately adjacent to the burned forest. Of the 5 species recorded on-site, 4 were recorded in the wildfire area, including 2 individuals of $M$. leibii. This result suggests that burned forest may contain more usable habitat than unburned forest, potentially due to the low canopy density, increased sun exposure and high availability of roosts from standing dead trees..$^{23}$ The presence of $M$. leibii, an elusive species, suggests that this area may provide summer roosts to many species.

A stationary survey in close proximity to the Long Island Solar Farm (Meadow Marsh) yielded twice as many bat calls when compared to a control (Weaver Pond). This pond is technically outside of the fenced-off solar arrays, which are inaccessible at night. A comparison of these results to an internal solar farm survey site would be beneficial to identify bat usage of solar farm areas.

More acoustic surveying should be conducted on-site at BNL in order to provide an adequate comparison of these unique areas. Since acoustic surveys, particularly static surveys, introduce some bias regarding populations, mist-netting should also be employed in appropriate areas to estimate population density.

In future surveys measuring abundance, care should be taken to learn the map routes before conducting surveys. Surveying 
at BNL in particular was challenging due to the number of unmapped roads traveled. Surveys in Suffolk County often resulted in incorrect directions from Delorme 2012 C so a partner is needed to interpret directions unless the driver is completely comfortable with the area. Data points from false turns were often picked up and were included in the results despite the deviance from the route.

\section{Acknowledgements}

I would first and foremost like to thank my mentor, Kathy Schwager, for her guidance and insight with this project. I would also like to thank Tim Green and Jennifer Higbie for sharing their experience and lending support. Thanks to Carl Herzog and Kathleen O'Connor for resources and aid in identifying bat calls. Many thanks to my fellow interns who helped with data collection, especially Molly Foley. Finally, I would like to thank the Office of Educational Programs and the Department of Energy for making this experience possible.

This project was supported in part by the U.S. Department of Energy, Office of Science, Office of Workforce Development for Teachers and Scientists (WDTS) under the Science Undergraduate Laboratory Internships Program (SULI).

\section{Notes}

${ }^{1}$ Harvey, Michael J., J. Scott Altenbach, and Troy L. Best. Bats of the United States and Canada. Baltimore: Johns Hopkins UP, 2011. Print.

${ }^{2}$ Boyles, Justin G., et al. "Economic importance of bats in agriculture." Science 332.6025 (2011): 41-42.

${ }^{3}$ White-Nose Syndrome Confirmed in Illinois Bats | U.S. Fish and Wildlife Service Midwest Region. White-Nose Syndrome Confirmed in llinois Bats U.S. Fish and Wildlife Service Midwest Region. US Fish and Wildlife Service, 16 Apr. 2013. Web. 30 July 2013.

${ }^{4}$ Minnis, Andrew M., and Daniel L. Lindner. "Phylogenetic evaluation of Geomyces and allies reveals no close relatives of Pseudogymnoascus destructans, comb. nov., in bat hibernacula of eastern North America." Fungal Biology (2013).

${ }^{5}$ White-Nose Syndrome Confirmed in Illinois Bats | U.S. Fish and Wildlife Service Midwest Region. White-Nose Syndrome Confirmed in llinois Bats U.S. Fish and Wildlife Service Midwest Region. US Fish and Wildlife Service, 16 Apr. 2013. Web. 30 July 2013.

${ }^{6}$ Warnecke, Lisa, et al. "Inoculation of bats with European Geomyces destructans supports the novel pathogen hypothesis for the origin of white-nose syndrome." Proceedings of the National Academy of Sciences 109.18 (2012): 6999-7003.

${ }^{7}$ White-Nose Syndrome Confirmed in Illinois Bats | U.S. Fish and Wildlife Service Midwest Region. White-Nose Syndrome Confirmed in llinois Bats U.S. Fish and Wildlife Service Midwest Region. US Fish and Wildlife Service, 16 Apr. 2013. Web. 30 July 2013.

${ }^{8}$ Warnecke, Lisa, et al. "Inoculation of bats with European Geomyces destructans supports the novel pathogen hypothesis for the origin of white-nose syndrome." Proceedings of the National Academy of Sciences 109.18

\section{Appendix 1}

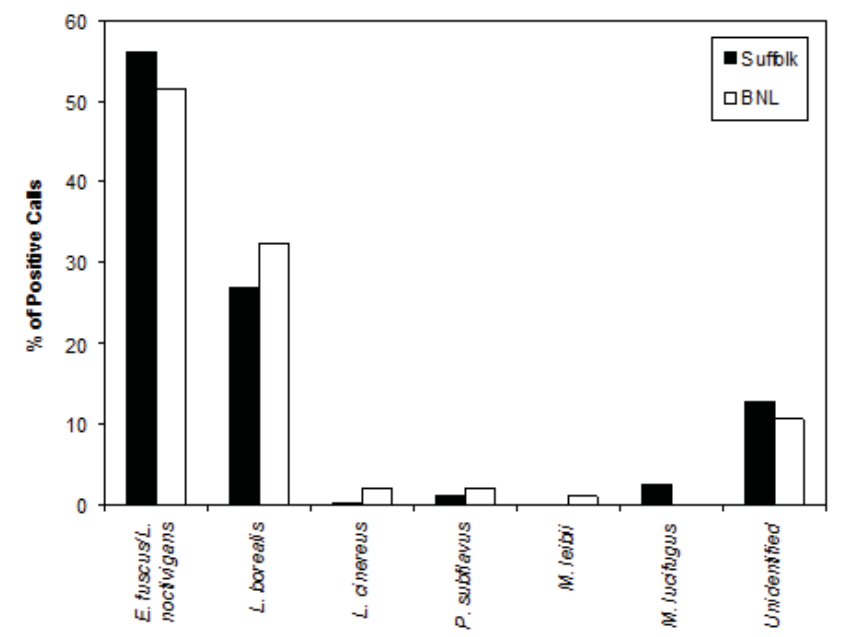

Figure 1. Chiropteran specieshipgtertifpedees in eastern Suffolk County and Brookhaven National Laboratory.

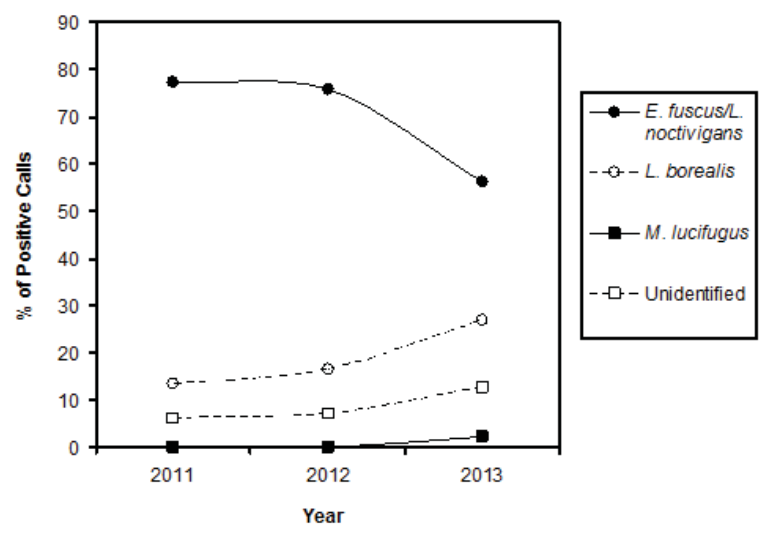

Figure 2. Population changes in prominent Chiropteran species within eastern Suffolk County.

(2012): 6999-7003.

${ }^{9}$ Harvey, Michael J., J. Scott Altenbach, and Troy L. Best. Bats of the United States and Canada. Baltimore: Johns Hopkins UP, 2011. Print.

${ }^{10}$ Stegemann E., and Hicks, A. "Bats of New York.” New York State Department of Environmental Conservation. 31 July 2012.

${ }^{11}$ Harvey, Michael J., J. Scott Altenbach, and Troy L. Best. Bats of the United States and Canada. Baltimore: Johns Hopkins UP, 2011. Print.

${ }^{12}$ Tuttle, Merlin D. "How North America's Bats Survive the Winter.” BATS Magazine 9.3 (1991). Web. 30 July 2013.

${ }^{13}$ Stegemann E., and Hicks, A. "Bats of New York." New York State Department of Environmental Conservation. 31 July 2012.

${ }^{142012}$ Winter Bat Survey Results.” NYS Dept. of Environmental Conservation. NYS Department of Environmental Conservation, 19 Apr. 2012. Web. 30 July 2013.

15“"White-nose Syndrome.” U.S. Fish \& Wildlife Service. U.S. Fish \& Wildlife Service, Apr. 2009. Web. 30 July 2013. 


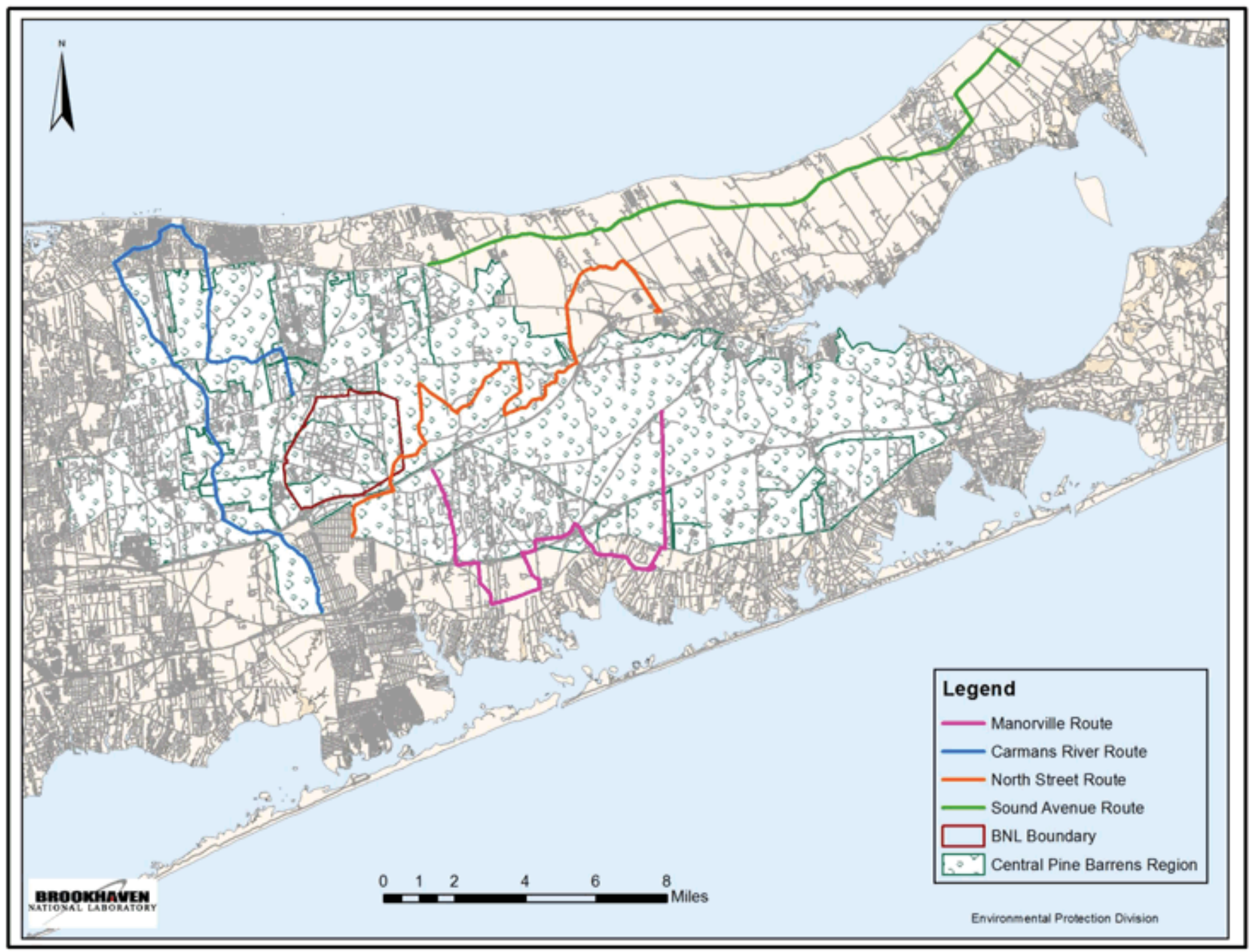

$<$ http://www.fws.gov/northeast/pdf/white-nosefaqs.pdf $>$.

${ }^{16}$ Harvey, Michael J., J. Scott Altenbach, and Troy L. Best.

Bats of the United States and Canada. Baltimore: Johns Hopkins UP, 2011. Print.

${ }^{17} 2012$ Winter Bat Survey Results.” NYS Dept. of Environmental Conservation. NYS Department of Environmental Conservation, 19 Apr. 2012. Web. 30 July 2013.

${ }^{18}$ Jacob, Andrew, and Bernadette LaManna. "On Patrol." NYDEC. New York State Conservationist, June 2012. Web. 30 Jul. 2013.

${ }^{19}$ Binary Acoustic Technology LLC. "Product details - binary acoustic technology." 13 June 2012. http://www. binaryacoustictech.com/batpages_files/spectr.htm

${ }^{20}$ Jackson, Nichelle and Kathy Schwager. "Identification of bat species in Suffolk County." July, 2012.

${ }^{21}$ Jackson, Nichelle and Kathy Schwager. "Identification of bat species in Suffolk County." July, 2012.

22012 Winter Bat Survey Results." NYS Dept. of Environmental Conservation. NYS Department of Environmental Conservation, 19 Apr. 2012. Web. 30 July 2013.
${ }^{23}$ Boyles, Justin G., and Doug P. Aubrey. "Managing forests with prescribed fire: implications for a cavity-dwelling bat species." Forest Ecology and Management 222.1 (2006): 108-115.

VII. Literature Cited

2012 Winter Bat Survey Results.” NYS Dept. of Environmental Conservation. NYS Department of Environmental Conservation, 19 Apr. 2012. Web. 30 July 2013.

Binary Acoustic Technology LLC. "Product details - binary acoustic technology." 13 June 2012.

Boyles, Justin G., and Doug P. Aubrey. "Managing forests with prescribed fire: implications for a cavity-dwelling bat species." Forest Ecology and Management 222.1 (2006): 108-115.

Boyles, Justin G., et al. "Economic importance of bats in agriculture." Science 332.6025 (2011): 41-42.

DEC Reports: 2012 Winter Bat Survey Results. NYS Dept. of Environmental Conservation. NYS Department of Environmental Conservation, 19 Apr. 2012. Web. 30 July 2013. 


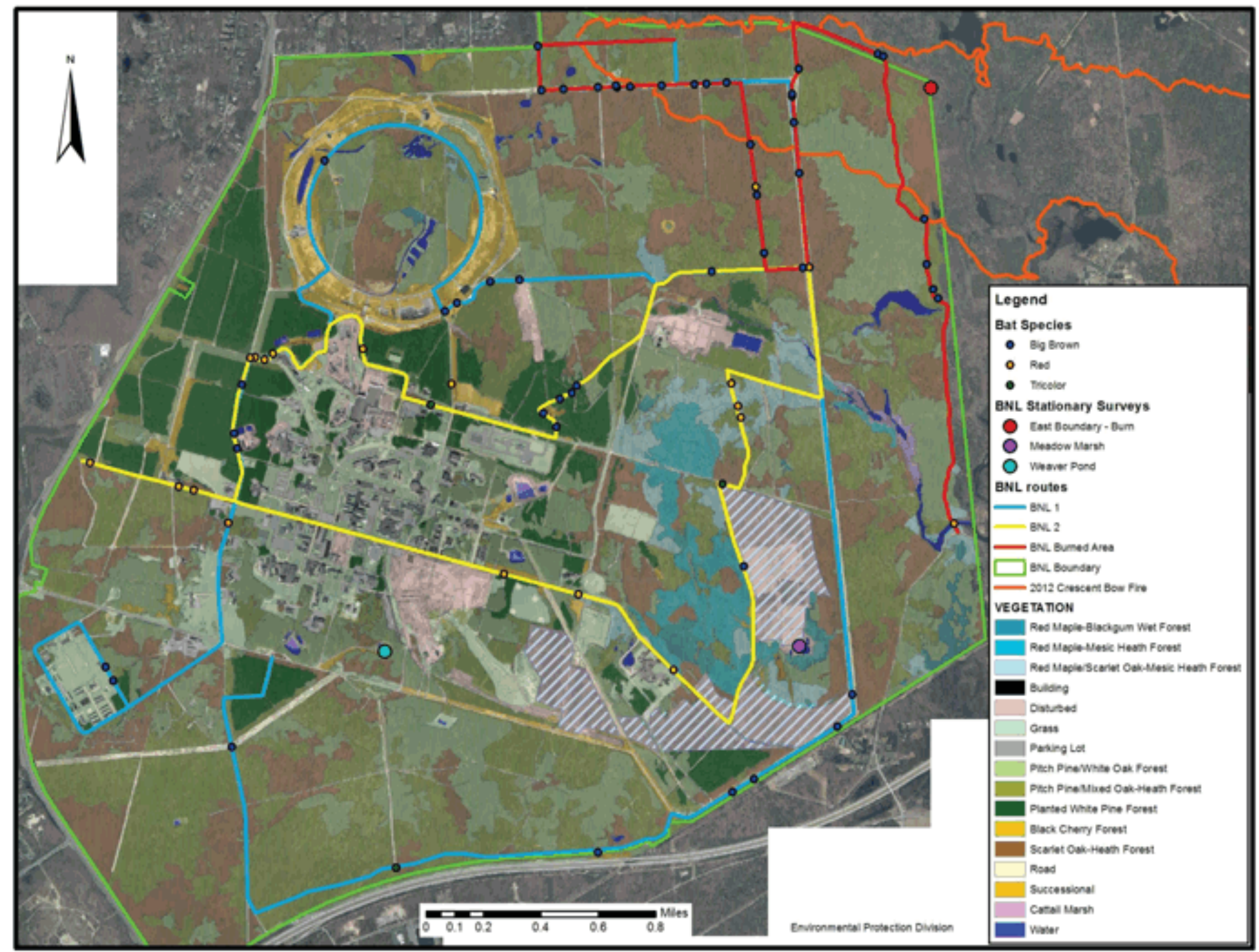

Harvey, Michael J., J. Scott Altenbach, and Troy L. Best. Bats of the United States and Canada. Baltimore: Johns Hopkins UP, 2011. Print.

Herzog, Carl. "Bat Acoustic Survey Protocols." New York State Department of Environmental Conservation. 22 April 2010.

Jackson, Nichelle and Kathy Schwager. "Identification of bat species in Suffolk County." July, 2012.

Jacob, Andrew, and Bernadette LaManna. "On Patrol." NYDEC. New York State Conservationist, June 2012. Web. 30 Jul. 2013.

Minnis, Andrew M., and Daniel L. Lindner. "Phylogenetic evaluation of Geomyces and allies reveals no close relatives of Pseudogymnoascus destructans, comb. nov., in bat hibernacula of eastern North America." Fungal Biology (2013).

Stegemann E., and Hicks, A. "Bats of New York." New York State Department of Environmental Conservation. 31 July 2012.
Warnecke, Lisa, et al. "Inoculation of bats with European Geomyces destructans supports the novel pathogen hypothesis for the origin of white-nose syndrome." Proceedings of the National Academy of Sciences 109.18 (2012): 6999-7003.

White-Nose Syndrome Confirmed in Illinois Bats | U.S. Fish and Wildlife Service Midwest Region. White-Nose Syndrome Confirmed in llinois Bats U.S. Fish and Wildlife Service Midwest Region. US Fish and Wildlife Service, 16 Apr. 2013. Web. 30 July 2013 


\title{
Characterization of Room-Temperature Radiation Detectors
}

\author{
Trevor Chan \\ College of Engineering, University of Alabama in Huntsville, Huntsville, AL 35758 \\ Giuseppe Camarda \\ Nonproliferation and National Security Department, Brookhaven National Laboratory, Upton NY 11973
}

\begin{abstract}
Throughout many years, one of the major goals of the Department of Energy (DOE) has been to insure the integrity and safety of the United State's nuclear materials, while advancing the concept, principals, and international acceptance of nuclear nonproliferation. Accordingly, one of the prime foci of the Nuclear Nonproliferation and National Security (NNS) Department at Brookhaven National Laboratory is to develop room-temperature radiation detectors to monitor and track nuclear materials. A main component of one of the more common types of radiation detectors is Cadmium Zinc Telluride (CZT), a good candidate for radiation monitors because it has the required material properties, viz., high resistivity, high density, a high atomic number, excellent photosensitivity, and a high absorbance of $\mathrm{\gamma}$-rays. A CZT crystal must be of extremely good quality since the presence of extended defects like Te inclusions, sub-grain boundaries, cracks, dislocations, and voids potentially can trap charges inside the material, resulting in devices that perform poorly. With help from industry, NNS acquires CZT samples grown by different methods, and then characterizes them in detail. First, optical- and infrared-microscopy is employed to inspect the sample for the presence of different types of defects. Then, using the different beam lines at the National Synchrotron Light Source, the performance of the different samples is detailed, and the effect of the defects on the performance of the detectors is measured. Particular attention is given to point defects that are investigated using Deep-level transient spectroscopy to identify electrically active defects. Using this spectrum of approaches might well enable the fabrication of detectors that will enhance our nuclear security.
\end{abstract}

\section{INTRODUCTION}

We received several samples from an outside crystal grower. We picked 2 bar shaped CZT crystals ( $6 \times 6 \times 15 \mathrm{~mm}^{3}$ ), which are all grown by using the High Pressure Bridgman method. We have characterized the material and determined the quality of the crystals and the performance of the detectors.
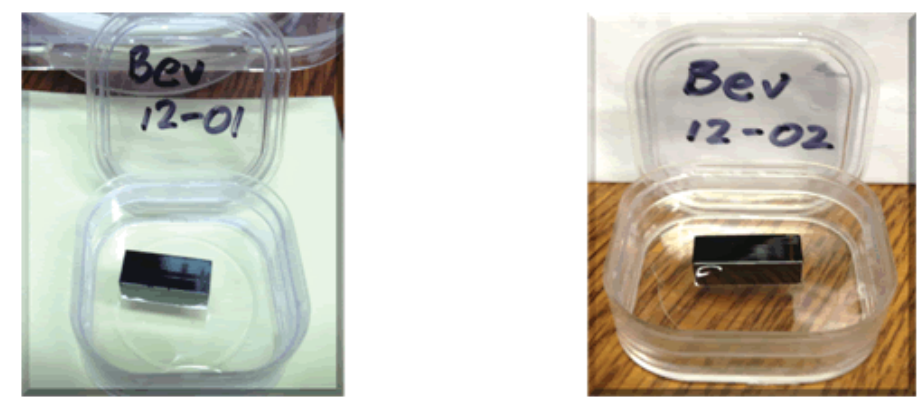

\section{CHARACTERIZATION TECHNIQUES}

A. IR TRANSMISSION MICROSCOPY

In IR Microscopy the CZT crystals appears transparent to the infrared light because the band gap of CZT is $\sim 1.45 \mathrm{eV}$, but the Te inclusions show up as dark masses (band gap of Te is $\sim 0.2$ $\mathrm{eV})$. Te inclusions are deleterious for the detector performance because they trap the charge created by ionization in the CZT by the incoming photons (X-rays, gamma-rays). Fluctuations in total collected charge cannot be corrected electronically, therefore the more Te inclusions there are in the crystal, the worse the performances of the detector fabricated from that crystal are. IR Microscopy method can count the number and size of Te inclusions in each volumetric region (or stack of images); usually we measure 5 volumetric regions and then average the concentration of Te inclusions in each of the volumetric regions to finally estimate the concentration of Te inclusions per $\mathrm{cm} 3$. As shown below IR Microscopy can project up to a 100 stack of images of on one plane to find the concentration of Te inclusions per $\mathrm{cm}^{3}$.
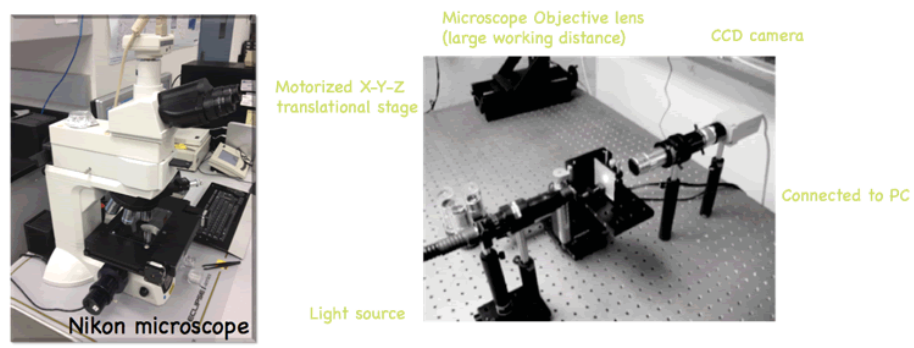

\section{B. WHITE BEAM X-RAY DIFFRACTION TOPOGRAPHY}

White Beam X-ray Diffraction Topography (WBXDT) uses a beam energies ranging from $5 \mathrm{KeV}$ to $54 \mathrm{KeV}$, and the actual beam size is varied depending on the crystal area to be measured. WBXDT is very efficient and take accurate diffraction images of detectors in only a few seconds. As shown in the figure below he WBXDT image provides information on the crystalline structure and orientation of a detector. The WXDT we can screen quickly CZT crystals to make sure they have only one domain and to see if there are extended defects in the crystal tested; we need high resolution X-ray films, to see some extended defects and strains fields. Unlike other methods of analyzing CZT detectors the WBXDT is very quick and nondestructive. 


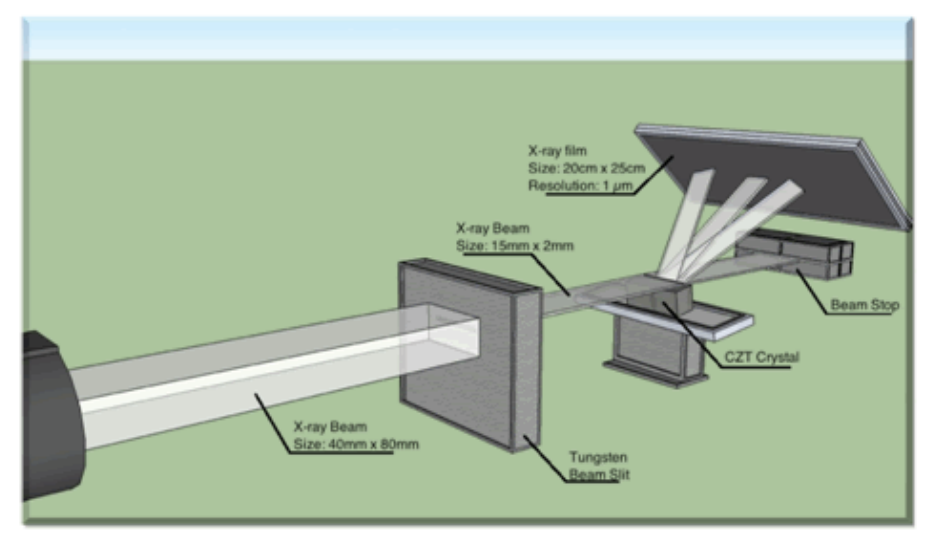

\section{MICRO-SCALE DETECTOR MAPPING}

The Micro-scale Detector Mapping (MDM) method uses a monoenergetic X-ray micro-beam to map the whole area of the sample. As shown in the figure below for each micro-area interrogated the energy spectrum is acquired, stored and subsequently used to plot an X-ray response map. The X-ray mapping technique is the main tool that provides high spatial resolution, limited by the diffusion only (low in the case of X-rays) and lets controls the interaction depths of $\mathrm{X}$ - rays (avoid surface and polarization effects). With this technique each pixel on a pixilated CZT detector is analyzed up to a depth of 10 microns and an X-ray response map of the pixel is created to show dislocations (imperfections), sub-grain boundaries, and the area where there is charge collection lost.

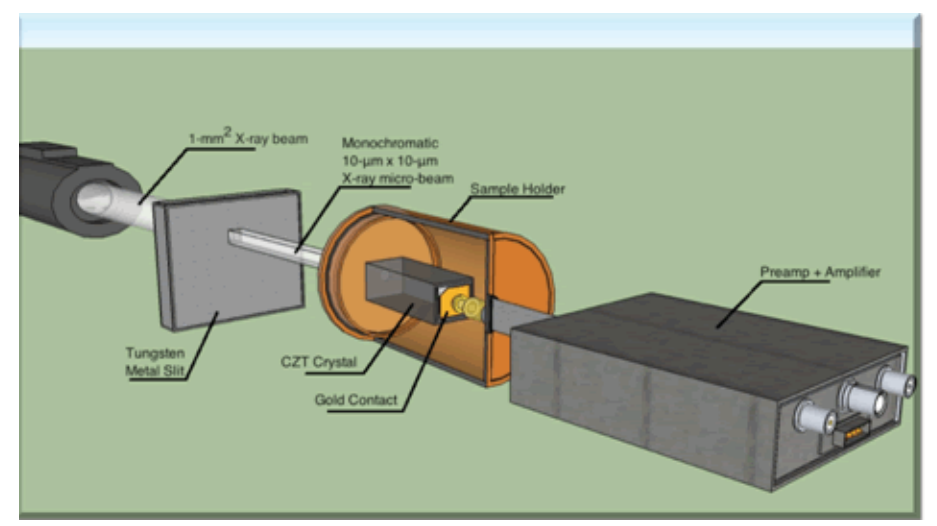

\section{Current-Voltage Measurement}

By applying a voltage (V) across the crystal, we can measure the current (I) and calculate the resistance of the crystal by using the equation:

$$
\mathrm{V}=\mathrm{IR}
$$

Then we can find the resistance $(\mathrm{R})$ we found earlier using the equation :

$$
\mathrm{R}=\mathrm{p}(\mathrm{L} / \mathrm{A})
$$

Where $\mathrm{L}$ is the length, $\mathrm{A}$ is the area and $\mathrm{p}$ is the electrical resistivity.

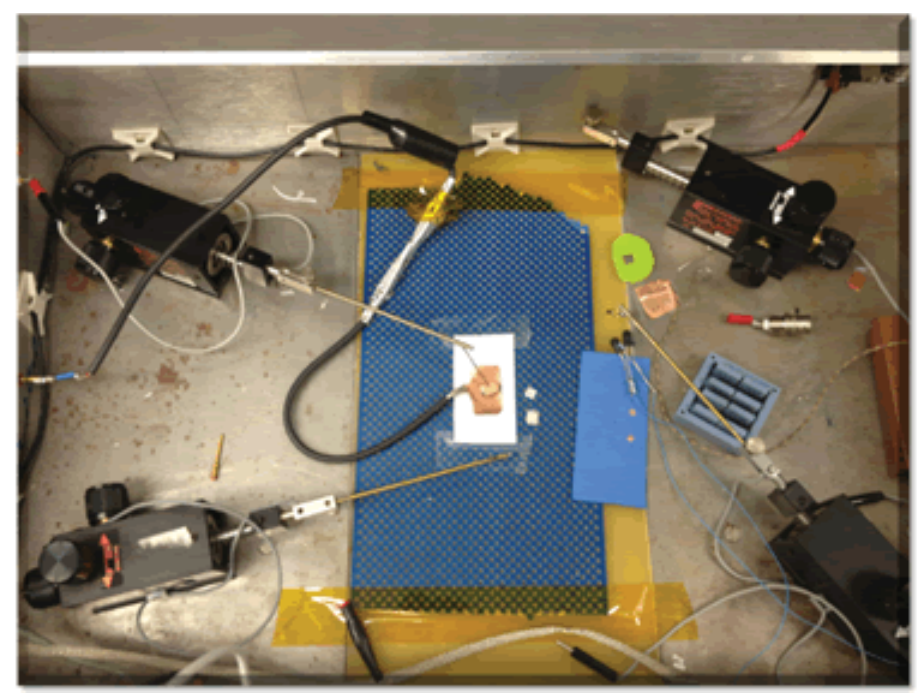

\section{RESULTS}

In this experiment and analysis, characterization of two $6 \mathrm{x}$ $6 \times 15 \mathrm{~mm} 3 \mathrm{CZT}$ radiation detectors. The detectors measured are used as imaging devices. Some crystals might be impaired with material defects which cause non-uniformity in the electric field (E-field), therefore aberrations of the image response can arise. This experiment characterized material defects and detector performance by using several techniques such as IR microscopy, White Beam X-ray Diffraction Topography (WBXDT), and Micro-scale Detector Mapping (MDM). After measuring the material defects by using IR and WBXDT, each device was then connected with the electronics inside a test box, and measured the performance of each device using the beam-line X27B at BNL's National Synchrotron Light Source (NSLS). The X-ray response map showed images of this pixilated detector, and the charge electron is severely disfigured because of numerous crystal defects (multiple sub-grain boundaries and star-punching dislocations (white spots)). The crystal defects affect the charge transport and distort the E-field and thus severely hinder detector performance. The detectors tested don't match up to the standards of an ideal pixilated detector.

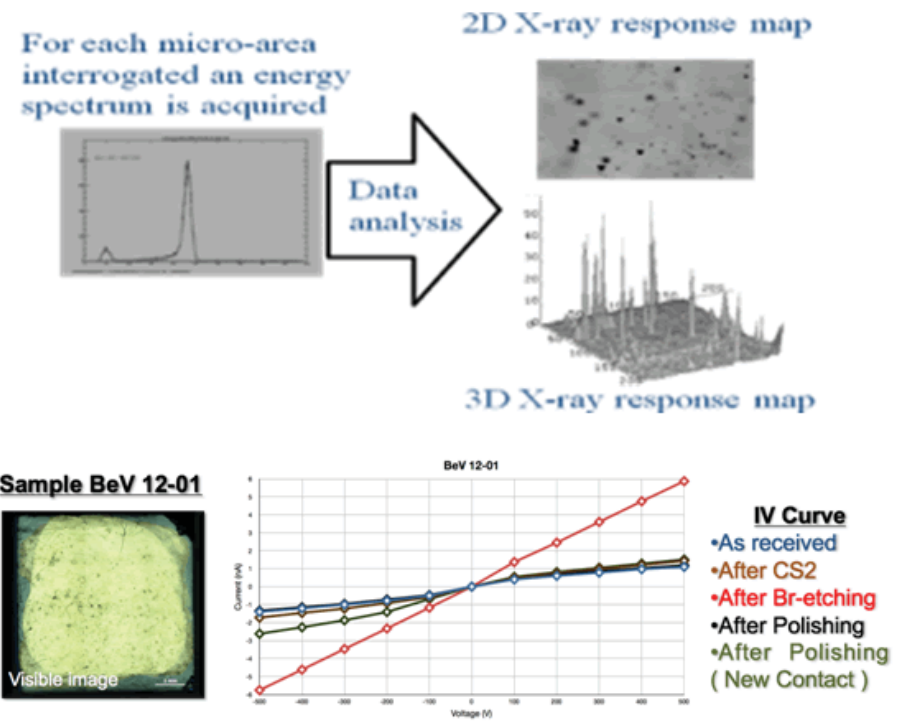



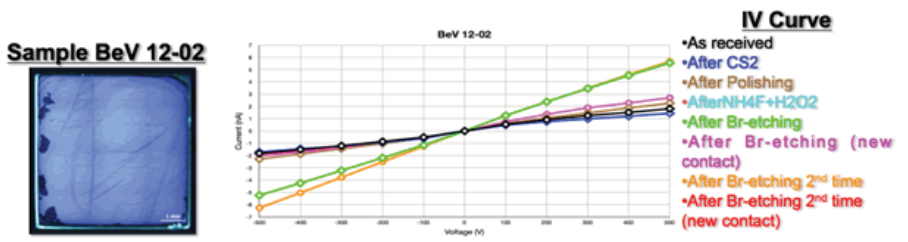

Right after we received the crystal from the outside grower, we use the MDM to generate a x-ray response map of the performance for both of the crystals.

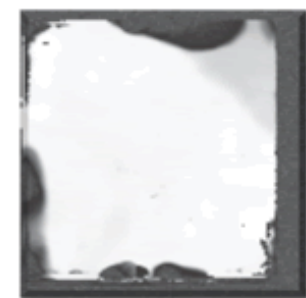

X-ray Response map As received

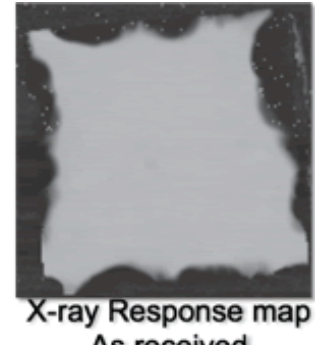

As received

Then we passivate each of the crystals with carbon disulfide. Then we will use the MDM again to generate another x-ray response map for both of the crystal.

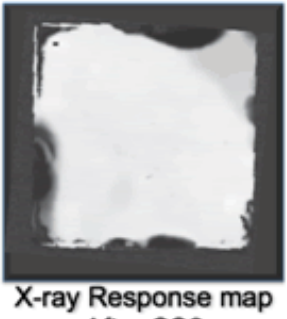

After CS2

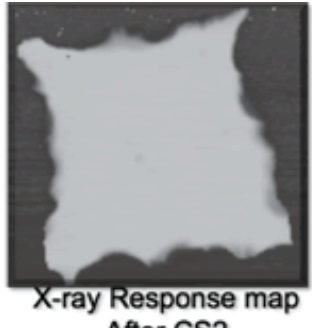

After CS2

Then we use another method to passivate the crystals call bromine-etching. By passivating the crystals in $1 \%$ bromine methanol for 1 minute, the use the MDM to generate another $\mathrm{x}$-rap map for both of the crystals.

\section{CONCLUSIONS}

In conclusion, we can observe that by passivating the crystals in the carbon disulfide solution. It had very minor effects on the performance of the crystals. But by using bromine-etching, we can observe that it is one of the most efficient way to improve performance of the crystals. 


\title{
Determination of thickness for laser ablation plasma generation on a sputtered target
}

\author{
David Cinquegrani \\ University of Michigan, Ann Arbor, MI 48109 \\ Masafumi Kumaki \\ Waseda University, Shinjuku, Tokyo, Japan \\ Masahiro Okamura \\ Collider-Accelerator Department, Brookhaven National Lab, Upton, NY 11973
}

\section{ABSTRACT}

The Laser Ion Source (LIS) group in Brookhaven National Lab's (BNL) Collider-Accelerator Department focuses mainly on the production and analysis of plasmas and ions created from matter interactions with lasers. A $1064 \mathrm{Nd}$ :YAG laser is used to irradiate a solid target and produce a plasma by laser mass ablation. The target that was created for this experiment is an aluminum plate with four different thicknesses of carbon that have been evaporated onto the surface. These thicknesses of carbon are used to investigate the depth of the crater created from the laser and to differentiate between the vaporized and plasma generated part of the crater. The plasma produced from this target contained ions of both carbon and aluminum, meaning the laser was able to irradiate past the layer of carbon and into the aluminum plate.

\section{INTRODUCTION}

When a laser with high power irradiates a target it produces plasma consisting of ions of the target species. These ions have many applications ranging from use in an accelerator, cancer therapy or use in experiments for NASA. The focus of ion production by the LIS is in high charge state ions. These are ions that have a higher charge than found naturally such as Carbon 6+ and Aluminum 12+ ion. The idea behind using a target that has thin foil of another element evaporated onto the surface is to use the thickness of the foil to adjust the plasma drift and expansion velocity, as well as to produce more high charge state ions. The target used for this experiment was an aluminum plate about 1.2 $\mathrm{cm}$ thick with four squares of carbon that were evaporated onto the surface. Each square is a different thickness of carbon: 500, $250,125,25 \mathrm{~nm}$. We expect to see an increase in the high charge state of the carbon in the resulting plasma as well as ions from the aluminum base plate. This was based on a similar experiment using a gold foil with no base plate that showed higher charge states were achieved using film thicknesses less than 1 micrometer1. We also expect there to be a greater amount of carbon ions compared to aluminum in the plasma generated from the target areas with thicker films of carbon.

This paper focuses on the results from the $500 \mathrm{~nm}$ carbon film and comparison of the results of an experiment using a pure aluminum target. Both experiments were performed under the same conditions in order to allow for direct comparison of the ion charge states of the respective plasmas. Our hypothesis was that the evaporated carbon target would exhibit a higher concentration of high charge state ions than the single species target. Our other goal was to produce a larger amount of carbon $6+$ ions than previously possible. The hope was that the temperature of the base metal and its thermal conductivity would be used to heat to a higher plasma temperature and produce more of these ions.

\section{EXPERIMENTAL SETUP}

The creation of the target for this experiment was made by a process called physical vapor deposition. The process involve heating an element to high enough temperatures that the sample sublimes and is turned into a vapor. This vapor is then deposited onto the target in a relatively even coating. For the deposition of carbon onto the aluminum plate we used an electron gun. The thickness of carbon we wanted to deposit was decided by estimating the depth of the crater that may be created by the laser. The four thicknesses decided on were 500, 250, 125, and $25 \mathrm{~nm}$. The size of the target used was $10 \times 10 \mathrm{~cm}$ and about $1.2 \mathrm{~cm}$ thick. This was placed in a target holder that was specifically made to hold this target. The plate was then attached to a two-axis controller. This controller was used to move the target each time it was shot with the laser. A fresh target surface was needed for each shot to be sure that there was a layer of carbon that would be shot. Another important aspect of the experiment was the determination of the distance between subsequent laser shots. If the laser shots were too close together the craters would overlap and there would not be carbon ions produced from the irradiation.

The target chamber is completely enclosed and at a pressure very close to vacuum. The laser used was a Nd:YAG laser with a short pulse of 170 picoseconds. A Faraday Cup (FC) was used to record the total current of the plasma produced by the laser. The next step was to start separating and collecting the ions from the plasma with the Electrostatic Ion Analyzer (EIA) and Secondary Electron Multiplier (SEM). The target was shot three times and resulting signal was recorded at many EIA voltages between 1 and $500 \mathrm{~V}$. At each voltage the ions of both carbon and aluminum were observed in the resulting plasma. Once all the plasma data was taken the FC signal was taken again for comparison. The target now had many craters on the surface that will be examined later for depth and ablation. Further analysis will be done using the Zygo 3D profilometer, which can create a 3D image of the surface of our target to determine the depth of the laser ablation.

\section{RESULTS AND DISCUSSION}

Irradiation of the pure aluminum target yielded results similar to those found in previous experiments. There was high production of low charge state ions and a significantly less amount of high charge state ions. There was an absence of the highest charge state that was possible for this experiment, $\mathrm{Al} \mathrm{12+.} \mathrm{These} \mathrm{results}$ gave us a control to compare the data that was obtained from our evaporated carbon target. The sum of the currents from each ion is equal to the Faraday Cup current taken after each experiment. The Faraday Cup was located about $2.8 \mathrm{~m}$ away from the target. The data shown in figures 1-4 is data adjusted to a Faraday Cup 
distance of $1 \mathrm{~m}$ and an aperture opening of $1 \mathrm{~cm} 2$. In the case of the pure aluminum the highest contribution to this sum comes from the $\mathrm{Al} \mathrm{9+}$ ion (Figure 2). This is because it has a relatively high amount of particle production (Figure 1) and a high charge to create a larger current than the $1+$ or $2+$ charge states of the ion.

These results are typical and to be expected from the irradiation of a single species. Our purpose was to see if we could improve on the production of the high charge state ion. The 500 $\mathrm{nm}$ thickness of carbon we tested proved to be the optimal thickness that was tested. The irradiation of the target generated a plasma that contained ions of both aluminum and carbon. The charge state distribution was drastically different than the pure aluminum target, not only because it included the carbon ions but also because in this experiment the $\mathrm{Al} 12+$ ion was generated. This ion alone helped to confirm our hypothesis for the generation of high charge state ions that were not present in the irradiation of a single species target (Figure 3).

Along with the high charge states of aluminum, the more exciting results were the generation of the $\mathrm{C} 6+$ ion. The generation of this ion was the goal of the entire experiment. The $\mathrm{C} 6+$ ion is important because it is the highest possible charge state of carbon. This ion will be easiest to accelerate because of the high charge and will also provide a higher current.

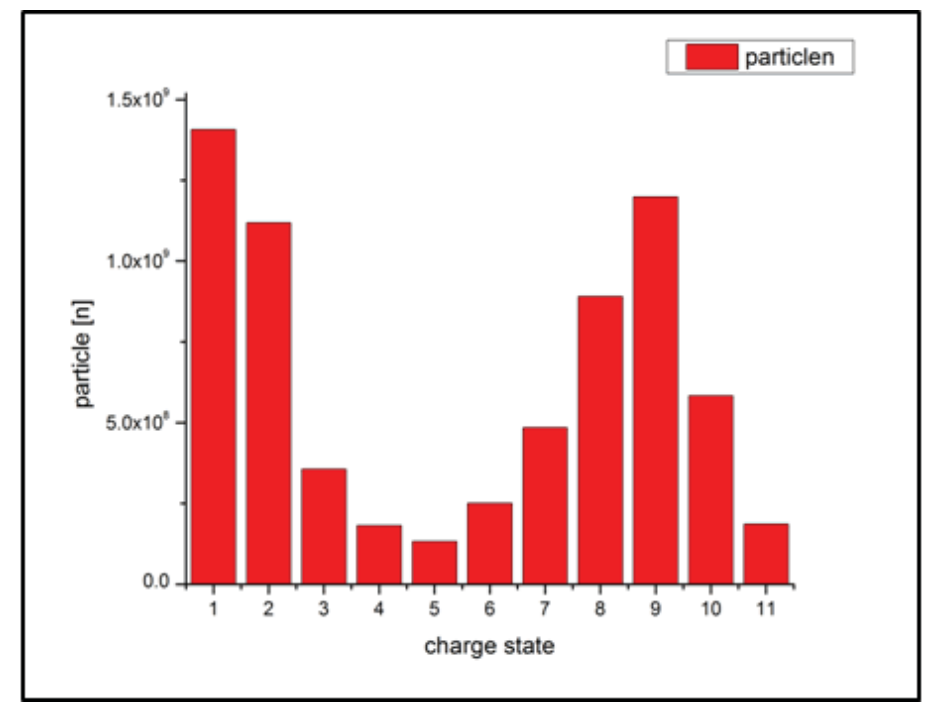

Figure 1: Distribution of Al Charge States by number of particles

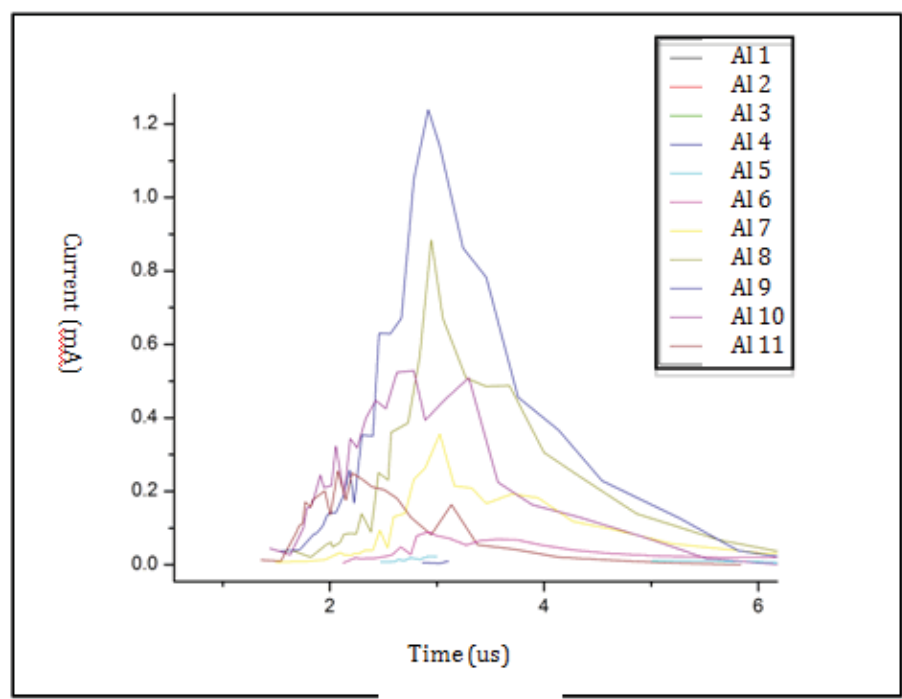

Figure 2: Current distribution for each ion charge state
The carbon $6+$ charge state had the most number of carbon particles in the resulting plasma (Figure 3). There was also a relatively high amount of carbon $5+$ ions generated, which is also good for this experiment. The current produced by the high

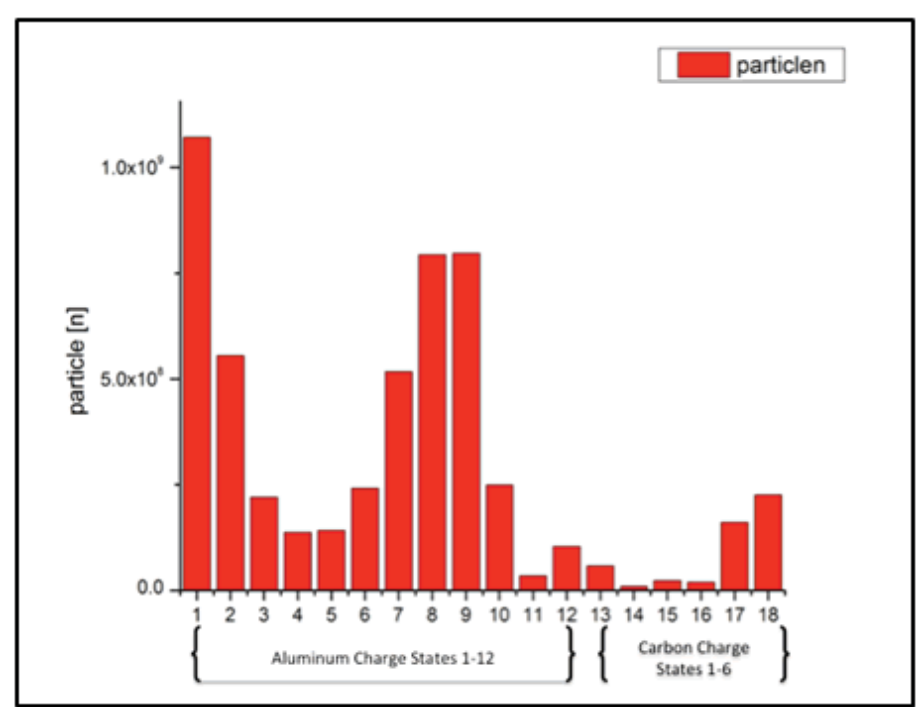

Figure 3: Ion charge states of both Aluminum and Carbon

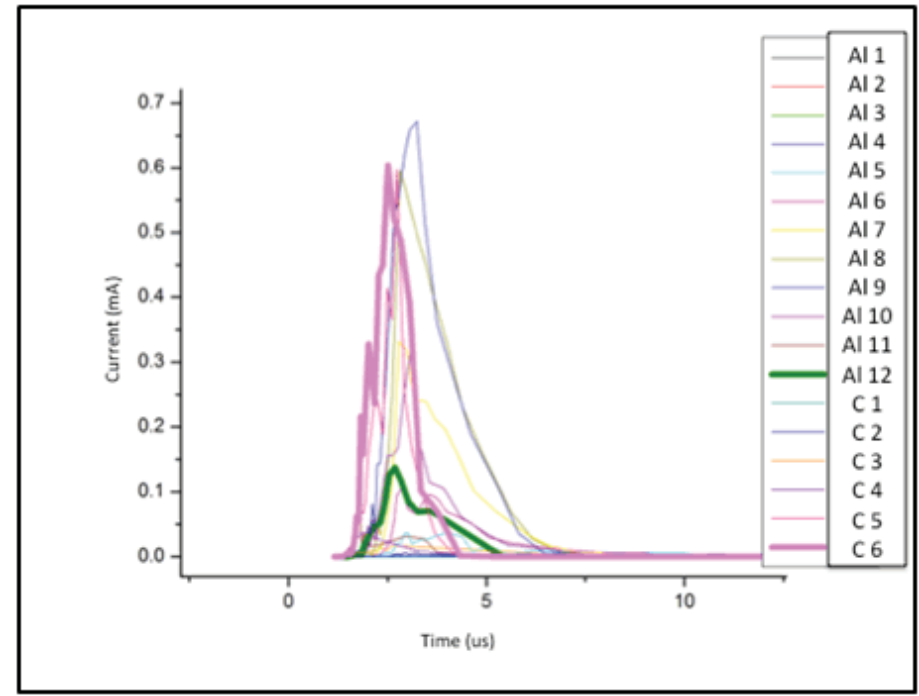

Figure 4: Current distribution of charge states with $\mathrm{C} 6+$ and Al 12+ ions highlighted.

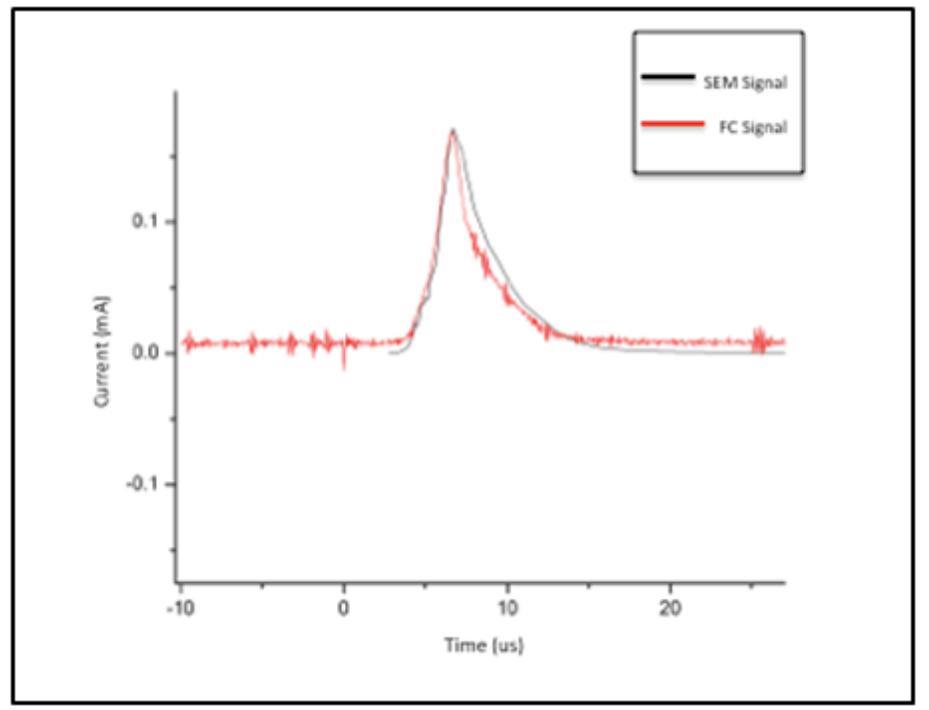

Figure 5: Comparison of Faraday Cup signal and sum of ion currents 
charge states of both carbon and aluminum is prominent in the plasma and very significant when compared to the pure aluminum plasma. The current attributed to the carbon $6+$ ion is the highest component of the plasma besides the aluminum $9+$ ion (Figure 4). This was the result we were looking for and proves that the high charge state was able to be obtained and have a large affect on the overall current of the plasma.

The current associated with each ion produced in the plasma is a division of the total current of the plasma. To validate our results the current of these ions was checked with the current taken from the Faraday Cup (Figure 5). This data was taken with the Faraday Cup about $2.8 \mathrm{~m}$ away from the target.

\section{CONCLUSION}

This experiment achieved exactly what we had hoped to achieve. The hypothesis we set of being able to generate a higher proportion of high charge state ions of the evaporated species was met by the higher production of the carbon $6+$ ion. This will be crucial for later studies of plasmas generated by the Laser Ion Source. This is the beginning of the production of ion beams for specific uses and shows more control of the field for future plasma applications.

\section{ACKNOWLEDGEMENTS}

This project was supported in part by the U.S. Department of Energy, Office of Science, Office of Workforce Development for Teachers and Scientists (WDTS) under the Science Undergraduate Laboratory Internships Program (SULI).

Along with my mentor Masahiro Okamura and graduate student advisor Masafumi Kumaki, I would like to thank Megumi Sekine, Takeshi Kanesue, Shunsuke Ikeda, Yasuhiro Fuwa, and Mark Romanelli for their work on the project. I would also like to acknowledge Dannie Steski for help in production of our target.

\section{REFERENCES}

${ }^{1}$ L. Torrisi, M. Cutroneo, L. Andò, and J. Ullschmied, "Thompson parabola spectrometry for gold lasergenerated plasma" American Institute of Physics, 2013 


\title{
Electrochemical and spectrophotometric studies of conjugated polymers
}

\author{
Hosts: Drs. John Miller and Steven Feldberg \\ Mentor: Alvin A. Holder \\ Interns: Ms. Altramiese Tippie and Ms. Tiera Corey
}

\begin{abstract}
Conjugated polymers are materials of interest for alternative energy applicators as active materials for solar cells because they are more easily processed and cheaper than the more commonly used silicon. In this study, we have collected and contrasted the cyclic voltammetry (CV) results for a conjugated polymer called poly(3-decylthiophene-2,5-diyl) (P3DT) a molecule of nearly similar reduction potential, fluoranthene (FLA), and a second polymer, poly[(9,9-di-n-octylfluorenyl-2,7-diyl)-alt-(benzo[2,1,3]thiadiazol-4,8-diyl) (F8BT). The $\mathrm{CV}$ for each species was acquired in tetrahydrofuran (THF) with tetrabutylammonium hexafluorophosphate (TBAPF ${ }_{6}$ ) $(0.10 \mathrm{M})$ as the supporting electrolyte in the presence of an $\mathrm{Ag} / \mathrm{Ag}^{+}$reference electrode and glassy carbon as the working electrode. The $E_{1 / 2}$ values for the FLA/FLA' and P3DT/P3DT redox couples were found to be $-\mathbf{2 . 5 0} \mathrm{V}$, while the value for the F8BT/F8BT redox couple was found to be $-2.14 \mathrm{~V}$. The diffusion coefficient for the $\mathrm{Fc}^{0 /+}$ redox couple in the presence of glassy carbon working electrode was determined as $2.1 \mathrm{x}$ $10^{-5} \mathrm{~cm}^{2} \mathrm{~s}^{-1}$ in the presence of the supporting electrolyte, 0.10 $\mathrm{M}$ tetrabutylammonium hexafluorophosphate and $\mathrm{a} \mathbf{A g} / \mathbf{A g}^{+}$ reference electrode. Spectrophotometric studies were carried out using the Laser Electron Accelerator Facility (LEAF). The $K_{\mathrm{eq}}$ value obtained for an electrolyte environment of $0.1 \mathrm{M}$ $\mathrm{TBAPF}_{6}$ was $34.7 \pm 5.5$, for which a $\Delta \mathrm{G}$ of $\mathbf{- 0 . 1} \mathrm{eV}$ was determined.
\end{abstract}

\section{Background}

In recent years, energy-related issues have received considerable attention concerning the rising costs of fossils fuels and the growing emission global greenhouse gases. ${ }^{1}$ There is an urgent need to develop clean and renewable energy technologies in order to circumvent these effects. The largest potential source of renewable energy is the solar energy incident on the Earth's surface. ${ }^{2}$ Solar cells are devices which convert solar energy directly into electricity, and the most common material used for solar cells is silicon, ${ }^{3}$ but today, the most widely studied organic solar cells use conjugated polymers due to their low cost and high processability. ${ }^{4-6}$

Polymer-based solar cells have attracted considerable attention as a promising long-term solution for clean, renewable energy due to advantages including simple and low cost fabrication, flexibility and lightweight. ${ }^{7}$ The utilization of the photovoltaic effect to generate electricity from solar energy has been hailed as an appealing solution to our growing need for clean, abundant, and renewable energy sources, and to our desire for protecting the environment. Over the last five years, organic photovoltaic devices have emerged as a new competitor to silicon-based solar cells.

For many years, chemists have been trying to understand the nature of charge distribution, delocalization, and charge carriers in conjugated polymers. 8 As such, the horizons for delocalization broadened with the advent of conjugated polymers, where in these molecules the concept of a bare charge carrier does not fully account for experimental observations. Instead, charges and excitons in conjugated molecules are thought to form polarons:8 quasiparticles composed of charges and their accompanying polarization field. Polarons are the essential charge carriers in solar cells, sensors, and electronic devices, and their natures have impact on charge separation as well as transport. ${ }^{8}$

Experiments $^{9-11}$ and theory ${ }^{10-13}$ on oligomers of definite lengths can yield rich information about what polarons are like for both charges and excitons. ${ }^{8}$ To understand how these species participate in electron-transfer reactions, (both charge separation and recombination) and how they move within and between polymer chains, there remains a need for a deeper understanding of the nature of polarons. Experiments and theory as by Miller and co-workers have sought to seek answers to questions such as: ${ }^{8}$ (1) What are the delocalization lengths of electrons and singlet excitons?, (2) When two electrons are introduced into an oligomer, how do they coexist in Fn chains of varied lengths?, (3) What are the natures of the visible, NIR, and "neutral" absorption bands? (4) What are the energies (redox potentials) to inject electrons?, and (5) How well do computational methods describe the behaviors of the polarons, bipolarons, and related species? A central goal of the research as by Miller and co-workers ${ }^{8}$ was to gain a better understanding, including a pictorial sense, of what polarons are like.

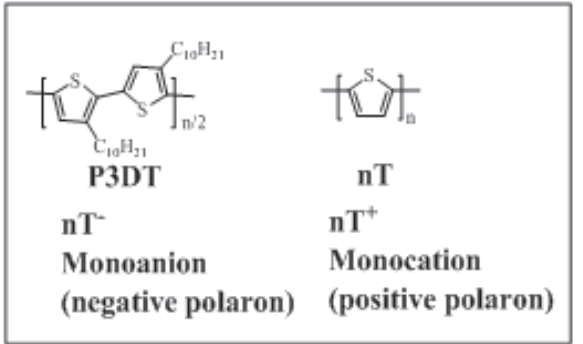

Recently, in a study as reported by Takeda and Miller, ${ }^{14}$ UV-visible absorption spectra of anions and cations of poly(3-decylthiophene) (P3DT) in solution were identified as single polarons. Pulse radiolysis of P3DT in THF determined the spatial extent of one negative polaron to be $\sim 11.5$ thiophene units by observing transient absorption of $\mathrm{P}^{2} \mathrm{DT}^{-}{ }^{-}$radical anions, which are principally free ions, at $850 \mathrm{~nm}$ with $\varepsilon=(7.25 \pm 0.47) \times 104 \mathrm{M}^{-1}$ $\mathrm{cm}^{-1}$ and bleaching of the neutral absorption band at $450 \mathrm{~nm}$. A $\mathrm{P}^{-1} \mathrm{DT}^{-}$radical anion was formed in a combination of diffusive reactions and fast "step" processes. Similarly, a positive polaron of P3DT was estimated to delocalize over $\sim 8.7$ thiophene units by pulse radiolysis in chloroform. Chemical reduction by sodium and oxidation by $\mathrm{FeCl}_{3}$ injected multiple charges into a single P3DT chain, while showing absorption spectra in the early stages of reaction resembling those observed by pulse radiolysis. Take- 
da and Miller ${ }^{14}$ concluded that the results indicated that multiple polarons exist in a single chain of P3DT before coalescing into bipolarons or transforming into other forms of polaron.

The main obstacles to practical applications of conductive polymers are the relatively poor environmental stability (mainly due to their oxidation by oxidation by oxygen and Joule heating) and their short lifetime when electrochemically cycled between oxidation states. Thus the development of new high-performance conducting polymers that possess high chemical, electrochemical, and thermal stability remains a challenge. Various methodologies have been proposed to overcome these problems, including the use of electrolytes (example, ionic liquids) ${ }^{15}$ nanoparticles, and the preparation of new high-performance conductive polymers by designing new monomers and/or controlling the effective $\pi$-conjugation of the polymer backbone. In this proposal, we will use our experience to use electrochemical and spectrophotometric studies on conjugated polymers in order to gather data so that we can design and synthesize more efficient and environmental stable conjugated polymers (with collaborators) that can exhibit the desired properties such as sunlight absorbers and light emitters.

The theory behind rotation disk electrochemistry (RDE):

Rotating disk electrochemistry (RDE) is a technique in which diffusional current is measured in a solution relative to an applied potential, using a rotating working electrode in contrast with a stationary working electrode as used in CV. The working electrode used for rotating disk electrochemistry contains a polished disc which is enclosed by an insulating sheath. The electrode is rotated along the axis perpendicular to the disc during measurement. This rotation provides a well-defined solution flow pattern in the form of a convection path which flows perpendicularly to the working electrode. ${ }^{16}$ This motion provides a steady flow of the detected species to the electrode surface and minimizes the effects of diffusion on transport measurements, making it a steady state measurement. ${ }^{17}$ The Levich current, $\mathrm{I}_{\mathrm{L}}$, being produced by the reduction or oxidation of the electroactive species can be described by the Levich equation:

$$
\mathrm{I}_{\mathrm{L}}=0.62 n \mathrm{~F} A \mathrm{CD}^{2 / 3} v^{-1 / 6} \omega^{1 / 2}
$$

where $\mathrm{n}$ is the number of electrons being transferred per molecule, F is Faraday's constant $\left(96,487 \mathrm{C} \mathrm{mol}^{-1}\right), \mathrm{A}$ is the electrode surface area $\left(\mathrm{cm}^{2}\right), \mathrm{C}$ is the analyte concentration $\left(\mathrm{mmol} \mathrm{cm}^{-3}\right), \mathrm{D}$ is the analyte diffusion coefficient $\left(\mathrm{cm}^{2} \mathrm{~s}^{-1}\right), \mathrm{v}$ is the kinematic viscosity of the solution $\left(\mathrm{cm}^{2} \mathrm{~s}^{-1}\right)$, and $\omega$ is the angular velocity of the rotating disk $\left(\mathrm{s}^{-1}\right)$.

\section{A. The LEAF}

LEAF is a facility equipped with an apparatus capable of performing picosecond and nanosecond-resolution pulse radiolysis. Radiolysis is a pump-probe technique whereby a sample (in our case a solution) is "pumped" with high energy radiation (in our case a $\sim 5 \mathrm{ps}, \sim 20 \mathrm{nC}$ electron pulse) and probed to observe the time-dependent quantitative change of some property of the system (in our case optical absorbance) corresponding with the radiolysis products and their reactions.

In LEAF, THF solutions of polythiophene (p3DT), fluoranthene (FLA), and binary solutions of the two were prepared in sets according to electrolyte content. The set of solutions of primary interest to this study involves the solutions containing $0.1 \mathrm{M}$ $\mathrm{TBAPF}_{6}$ as this was the supporting electrolyte for the CV exper- iments with which the LEAF data is meant to serve as a control. Whereas in the $\mathrm{CV}$ experiments, the number of electrons involved in the reduction process is not certain, in the LEAF experiments for this study, only one-electron reduction occurs. This is because radiolysis of the THF solution results in a byproduct of $\sim 1 \mu \mathrm{M}$ free electrons following ionization of THF molecules, which rapidly solvate and then react at diffusion-controlled rates with solutes (i.e. fluoranthene or polythiophene). In the case of the binary solutions, the concentration of p3DT was fixed at $20 \mu \mathrm{M}$ per chain while fluoranthene was varied in concentration in 5 concentrations ranging from $0.25 \mathrm{mM}$ to $10 \mathrm{mM}$, ensuring that fluoranthene molecules will capture virtually all of the electrons initially, preceding an equilibrium reaction modeled by scheme (1).

Fluoranthene (FLA) and p3DT have very similar reduction potentials, and therefore an equilibrium reaction of the form represented in Scheme 1 should be observable using LEAF and the equilibrium constant of the reaction, $\mathrm{K}_{\mathrm{eq}}$, can be calculated from equation

$$
K_{e q}=\frac{k_{f}}{k_{r}}=\frac{\left[F L A^{\cdot-}\right][p 3 D T]}{[F L A]\left[p 3 D T^{\cdot-}\right]}
$$

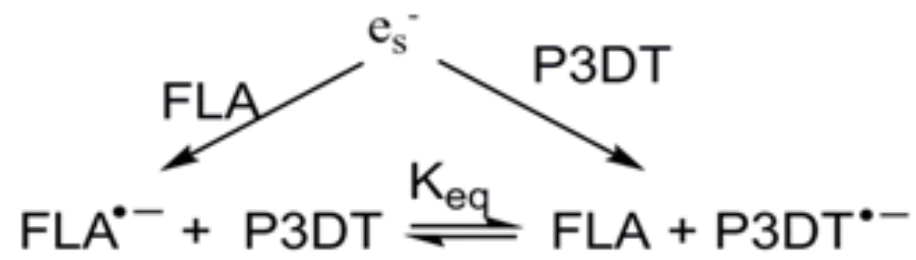

Scheme 1. The reaction scheme for LEAF experiments involving THF solutions containing fluoranthene (FLA) and polythiophene (P3DT), where [FLA] $>$ [P3DT]

In electrochemistry, the quantity in which we are most interested is E, the potential of the system. We can relate this idea of work done in electrochemistry to the thermodynamic concept of work, free energy, through the equation:

$$
\Delta \mathrm{G}=-\mathrm{q} E=-\mathrm{nFE}
$$

where "q" is the charge moved through some potential "E", $\mathrm{n}$ is the number of moles of electrons transferred in a cell reaction (a.k.a. half reaction), and " $F$ " is the Faraday constant - the number of coloumbs per mole of electrons $\left(9.648 \times 10^{4} \mathrm{C} \mathrm{mol}^{-1}\right)$. Also,

$$
\Delta \mathrm{G}=-\mathrm{RT} \ln \mathrm{K}_{\mathrm{eq}}
$$

where $\mathrm{R}$ is the universal gas constant $\left(8.314 \mathrm{~J} \mathrm{~K}^{-1} \mathrm{~mol}^{-1}\right)$, $\mathrm{T}$ is temperature, and $\mathrm{K}$ is the equilibrium constant.

The radiolysis data was used in conjunction with cyclic voltammetric data to try to learn about how many electrons are injected into (or removed from) a polymer chain during reduction (or oxidation) at the working electrode during the acquisition of a CV. In order to observe the reaction, we ideally chose a wavelength for measurement where (if possible) the ratio of extinction coefficients for the two species is $>>$ or $<<1$ (depending on which extinction coefficient is in the numerator).

If only one species absorbs at a given wavelength, its concentration can be easily determined by use of Beer Lambert's Law:

$$
\mathrm{A}=\varepsilon \mathrm{cl}
$$

where $\varepsilon=$ the molar extinction coefficient, $\mathrm{c}=$ the concentration, and $1=$ the path length of the light being absorbed.

The absorbances that we are collecting in LEAF are those of radiolysis products. In this project, for each solution, we wish to observe the effect of salt concentration on the equilibrium con- 
stant. The solutions were titrated with varying concentrations of $\mathrm{TBAPF}_{6}$ and the reaction in equation (1) will proceed with different rate constants, $\mathrm{k}_{\mathrm{f}}$ and $\mathrm{k}_{\mathrm{b}}$, and hence a shifting equilibrium constant.

\section{Hypotheses and research objectives and goals}

The main hypotheses are as follows: (i) a conjugated polymer will undergo multiple electron reduction during reduction in a $\mathrm{CV}$ scan, (ii) CVs of conjugated polymers are broad and featureless when acquired in non-aqueous media and (iii) the idea that the one-electron oxidation (or reduction) creates single polarons, and that the CVs for polymers and oligomers will comprise one- and multi-electron processes.

The main goal of the project is to gain an insight into multi-electron redox processes of conjugated polymers. No, with accurate one-electron potentials and well-characterized CVs in hand we hope to determine a quantitative relationship. We hope to create a mathematical model the CVs, and possibly DPVs, in order to obtain a series of potentials and to create a "map" giving potential versus number of holes (or electrons) injected into a single polymer or oligomers. The model will include least-squares fitting and digital modeling with DigiSIM electrochemical modeling programs.

We hope that the series of measurements we propose will also enable us to determine diffusion coefficients of the oligomers and polymers to be measured, and to delineate their effects on the currents in the CVs.

\section{Experimental:}

\section{A. Materials and methods}

Analytical or reagent grade chemicals were used throughout this study. Poly(3-decylthiophene-2,5-diyl) (P3DT) polymer was obtained from Rieke Metals, Inc. Poly[(9,9-di-n-octylfluorenyl-2,7-diyl)-alt-(benzo[2,1,3]thiadiazol-4,8-diyl) (F8BT) polymer, fluoranthene (FLA), tetrabutylammonium hexafluorophosphate, and tetrahydrofuran were obtained from Sigma Aldrich.

\section{B. Cyclic voltammetry:}

Cyclic voltammetry (CV) on P3DT, fluoranthene and ferrocene were carried out using a CHI Instruments Electrochemical Analyzer. Electrolyte solutions were prepared as 0.1 M TBAPF6 in THF in an argon-saturated glovebox, just prior to use. Analyte solutions of fluoranthene and ferrocene were prepared by directly adding the analytes to electrolyte solution in a Pyrex measurement cell after collecting $\mathrm{CV}$ measurements of the electrolyte solution. The P3DT solutions which were prepared by adding the polymer to electrolyte solution in vials were heated; then soni- cated. The solutions were prepared to achieve a volume of $10 \mathrm{ml}$. After cooling, molecular sieve was added to the vials to dry the solutions. Each CV measurement collected for a solution was preceded by purging with argon for 15 minutes. A glassy carbon working electrode $(3 \mathrm{~mm})$ was polished with $0.05 \mu \mathrm{m}$ alumina (Baikalox), rinsed with THF and carefully wiped and dried with kim wipes before use. A Pt wire and a $\mathrm{Ag} / \mathrm{Ag}^{+}(10 \mathrm{mM} \mathrm{AgNO}$ in $0.1 \mathrm{M} \mathrm{TBAPF}{ }_{6} / \mathrm{THF}$ ) were used as a auxiliary and a reference electrode, respectively. The instrumental resistance compensation was turned off. For calibration of uncompensated resistances, the CVs of ferrocene were collected at varied concentrations.

\section{Rotation disk electrochemistry}

Cyclic voltammagrams were acquired for P3DT using a rotating disk electrode (RDE) at various rotation rates using a Pine Instrument Company MSRX Speed Control rotator and a CHI Instruments Electrochemical Analyzer. A $2.5 \mathrm{mM}$ per repeat unit concentration of P3DT was prepared by adding the polymer to a $0.1 \mathrm{M}$ TBAPF6 solution in vials which were then heated and sonicated to fully dissolve the polymer. After cooling, $3 \AA$ molecular sieves were added to the vials to dry the solutions. The solution was prepared to achieve a total volume of $45 \mathrm{ml}$. CVs were acquired after purging the solution with argon for 40 minutes.

\section{The LEAF experiments}

Radiolysis data in LEAF were acquired with a $4 \mathrm{GHz}, 20$ GS s-1 Lecroy Waverunner 640Zi Transient Digitizer. Electrolyte solutions of TBAPF6 and TBABF4 at various concentrations containing P3DT and/or fluoranthene were prepared in $0.5 \mathrm{~cm}$ stopcock-sealed optical cells while under argon atmosphere in a glovebox. Data was collected on a $20 \mu$ s time scale and at a gain setting of 5 or $10 \mathrm{mV} /$ division.

\section{Results and discussion:}

A. The molecules used in this study:

The molecules used in this study are shown below: $\begin{array}{lll}\text { FLA FBBT } & \text { P3DT }\end{array}$

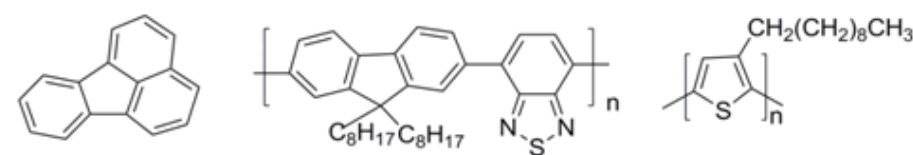

FLA $=$ fluoranthene, F8BT $=$ poly[(9,9-di-n-octylfluorenyl-2,7-diyl)-alt-(benzo[2,1,3]thiadiazol-4,8-diyl, and P3DT = poly(3-decylthiophene)

\begin{tabular}{|r|r|r|r|r|r|}
\hline [ferrocene] $/ \mathbf{m M}$ & \multicolumn{1}{|c|}{$\mathbf{i}_{\mathbf{p a}} / \boldsymbol{\mu} \mathbf{A}$} & $\mathbf{1 0 6} \mathbf{D} / \mathbf{c m}^{\mathbf{2}} \mathbf{s}^{\mathbf{- 1}}$ & $\mathbf{E}_{\mathbf{p a}} / \mathbf{V}$ & $\mathbf{E}_{\mathbf{p c}} / \mathbf{V}$ & $\mathbf{E}_{\mathrm{y} / 2} / \mathbf{V}$ \\
\hline 1.0 & -27.7 & 21 & -0.04 & -0.28 & -0.16 \\
\hline 2.0 & -46.9 & 15 & 0.02 & -0.32 & -0.15 \\
\hline 3.99 & -77.0 & 10 & 0.11 & -0.39 & -0.14 \\
\hline 5.99 & -98.0 & 7.2 & 0.20 & -0.43 & -0.12 \\
\hline 7.98 & -116.0 & 5.7 & 0.30 & -0.43 & -0.08 \\
\hline
\end{tabular}

Table 1. Tabulation of the calculations for the diffusion coefficient. Solvent $=$ THF, working electrode $=$ glassy carbon, auxiliary electrode $=$ Pt wire, reference electrode $=\mathrm{Ag} / \mathrm{Ag}^{+}$, Diameter of the working electrode $=3.0 \mathrm{~mm}$, area of the working electrode $=0.071 \mathrm{~cm}^{2}$, and supporting electrolyte $=0.10 \mathrm{M}$ tetrabutylammonium hexafluorophosphate. Scan rate $=100 \mathrm{mV} \mathrm{s}-1$. Temperature $=20.0^{\circ} \mathrm{C}=293.15 \mathrm{~K}$. 


\section{B. Cyclic voltammetry}

\section{Ferrocene:}

CVs were acquired for ferrocene in THF with 0.1 M TBAPF6 as a supporting electrolyte. Figure 1 shows the CVs for ferrocene at various concentrations. The ipa values were found to increase linearly with concentration of ferrocene, but with a constant of proportionality that is less than 1 . The values of $\mathrm{i}_{\mathrm{pa}}, \mathrm{E}_{\mathrm{pa}}, \mathrm{i}_{\mathrm{pc}}$, and $\mathrm{E}_{\mathrm{pc}}$ along with the calculated diffusion coefficients for each concentration of ferrocene are provided in Table 1.

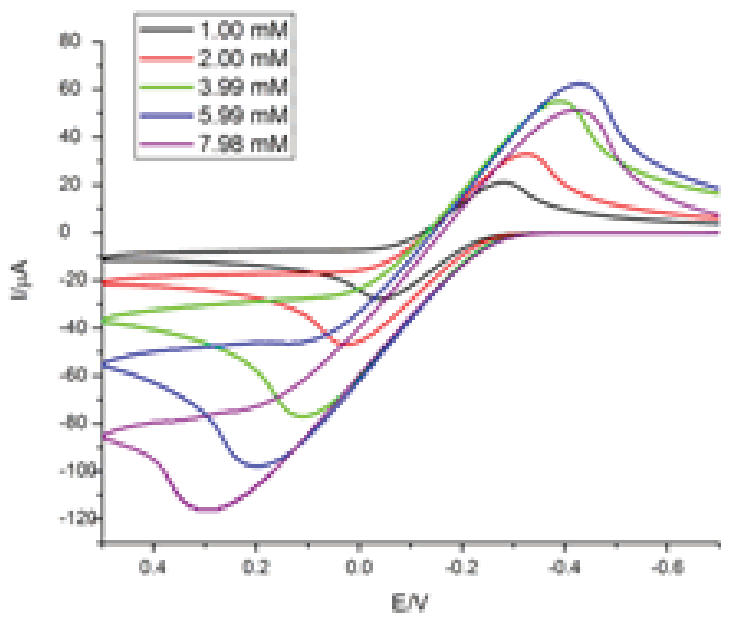

Figure 1. Cyclic voltammagrams of various concentrations of ferrocene in THF. Supporting electrolyte $=0.10 \mathrm{M}$ tetrabutylammonium hexafluorophosphate, working electrode $=$ glassy carbon, auxiliary electrode $=$ Pt wire, reference electrode $=\mathbf{A g} / \mathbf{A g}^{+}$, and scan rate $=$ $100 \mathrm{mV} \mathrm{s}^{-1}$

It has been proposed that the oxidation of ferrocene, $\left[\mathrm{Fe}\left(\mathrm{C}_{5} \mathrm{H}_{5}\right)_{2}\right]$ to the ferrocenium cation, $\left[\mathrm{Fe}\left(\mathrm{C}_{2} \mathrm{H}_{5}\right)_{2}\right]^{+}$is a standard one-electron transfer reversible process with an incredibly fast rate of electron transfer. For this reason, it has been useful as a standard reference with a well-known redox potential of $+0.64 \mathrm{~V}$ versus standard hydrogen electrode (SHE) 18 Therefore, it is expected that values of ipa should be linear with the concentration of ferrocene, which they are but with a proportionality constant that is less than one due to uncompensated resistance.

Ferrocene also serves as a good calibration standard from which uncompensated resistance can be determined since both uncompensated resistances and slow heterogeneous electron transfer are contributing factors to concentration-dependent separation between forward and reverse current peak potentials.

Diffusion coefficients (D) and $\mathrm{E}_{1 / 2}$ values of $2 \mathrm{mM}$ ferrocene as obtained by Tsierkezos 18 in various non-aqueous solvents were compared with the one calculated in this study using THF. It should be noted that the values obtained by Tsierkezos for D, increases according to solvent in the following order: DMSO $<$ NMK $<$ DMF $<$ PEN $<$ DCM $<$ CAN $<$ ACE (see Table 2). This order suggests inverse dependence on absolute viscosity with the $D$ values as predicted by the Stokes-Einstein equation:

$\mathrm{D}=\mathrm{kBT} / 6 \pi \mathrm{c} \mathrm{r}_{\mathrm{h}}$

where $\mathrm{k}$ is Boltzmann's constant, $\mathrm{T}$ is temperature in Kelvin, $r_{h}$ is the effective radius of the diffusing species, $\eta$ is absolute viscosity of the solvent medium, and $\mathrm{c}$ is a constant. According to the same equation, the value of D for ferrocene in THF is expected to be between that of dichloromethane and pentanone, and according to our calculation of D in THF, our result finds agreement with that expectation.

\section{P3DT and FLA \\ 1. P3DT}

CVs were acquired for P3DT in THF. Figure 2 shows a typical CV in THF with 0.1 M TBAPF6 for P3DT with a starting potential sufficiently positive to observe formation of $\mathrm{p} 3 \mathrm{DT}^{\circ+}$, while figure 3 shows the overlay of the CVs for $4.0 \mathrm{mM}$ fluoranthene and $4.0 \mathrm{mM}$ per repeat unit (PRU) of P3DT. A reversible redox reaction is observed, with an $\mathrm{E}_{1 / 2}$ of $-2.5 \mathrm{~V}$ for the redox couple. The fact that $\mathrm{E}_{\mathrm{pa}}$ is smaller in magnitude than Epc may indicate that the reduced P3DTn॰- (where ' $n$ ' is the unknown number of electrons added during reduction) is not fully stable.

According to the Randles-Sevcik equation

$i_{p}=0.4463 n \mathrm{FAC}(n \mathrm{FvD} / \mathrm{RT})^{1 / 2}$,

where $i_{p}=$ current maximum in amps

$n=$ number of electrons transferred in the redox event for a reversible process
$\mathrm{A}=$ electrode area in $\mathrm{cm} 2$
$\mathrm{F}=$ the Faraday Constant in $\mathrm{C}$ mol-1
$\mathrm{D}=$ diffusion coefficient in $\mathrm{cm} 2 \mathrm{~s}-1$
$\mathrm{C}=$ concentration in $\mathrm{mol} \mathrm{cm}-3$
$v=$ scan rate in $\mathrm{V} \mathrm{s}-1$

\begin{tabular}{|c|c|c|}
\hline Solvent & $105 \mathrm{D} / \mathrm{cm}^{2} \mathrm{~s}^{-1}$ & $\mathrm{E}_{1 / 2} / \mathrm{V}(\mathrm{vs} \mathrm{Ag} / \mathrm{AgCl})$ \\
\hline Acetonitrile & $2.24 \pm 0.06$ & $\mathrm{~N} / \mathrm{A}$ \\
\hline Acetone & $2.76 \pm 0.07$ & 1.020 \\
\hline N-methylformamide & $0.54 \pm 0.01$ & $\mathrm{~N} / \mathrm{A}$ \\
\hline $\mathrm{N}, \mathrm{N}$-dimethylformamide & $0.95 \pm 0.02$ & 0.951 \\
\hline N,N-dimethylacetamide & $0.76 \pm 0.02$ & $\mathrm{~N} / \mathrm{A}$ \\
\hline 3-pentanone & $1.41 \pm 0.04$ & $\mathrm{~N} / \mathrm{A}$ \\
\hline Dimethyl sulfoxide & $0.44 \pm 0.01$ & 0.869 \\
\hline Dichloromethane & $1.67 \pm 0.05$ & 1.125 \\
\hline THF & 2.1 & $-0.15^{\mathrm{a}}$ \\
\hline
\end{tabular}

Table 2. Diffusion coefficients and $\mathrm{E}_{1 / 2}$ values for $2 \mathrm{mM}$ ferrocene solutions in various solvents (collected for $2 \mathrm{mM}$ ferrocene solutions at 298.15 K).

${ }^{a}$ From our work with a $\mathrm{Ag} / \mathrm{Ag}+$ reference electrode and $2.0 \mathrm{mM}$ ferrocene at $20^{\circ} \mathrm{C}(298.15 \mathrm{~K})$. 
The $\mathrm{E}_{1 / 2}$ values for the redox couples of both FLA/FLA ${ }^{-}$and $\mathrm{P} 3 \mathrm{DT} / \mathrm{P} \mathrm{DT}^{-}$were determined to be $-2.50 \mathrm{~V}$ versus a $\mathrm{Ag} / \mathrm{Ag}^{+}$reference electrode, but the ip values of fluoranthene are significantly larger than those of P3DT at $4.0 \mathrm{mM}$ fluoranthene and $4.0 \mathrm{mM}$ (PRU) P3DT. The CVs for both were collected under identical experimental conditions, therefore the only variables affecting the ip were the diffusion coefficients and the number of electrons transferred during the redox event. Due to the fact that P3DT is so much larger than fluoranthene, the diffusion coefficient of P3DT is an order of magnitude smaller. Additionally, because an excess electron on P3DT occupies approximately 11 monomer units whereas each molecule of fluoranthene is capable of accepting an electron, $n_{\mathrm{P} 3 \mathrm{DT}} \leq(1 / 11) * n_{\mathrm{FLA}}$. Due to these two factors, the ipc of the $4.0 \mathrm{mM}$ P3DT is expected to be substantially smaller than the $i_{p c}$ of the equivalent molecular concentration of fluoranthene. Comparison of the $i_{p c}$ values of p3DT at $4.0 \mathrm{mM}$ with other concentrations of P3DT shows a linear relationship. A graph corresponding with the CVs of P3DT at various concentrations is shown in Figure 4.

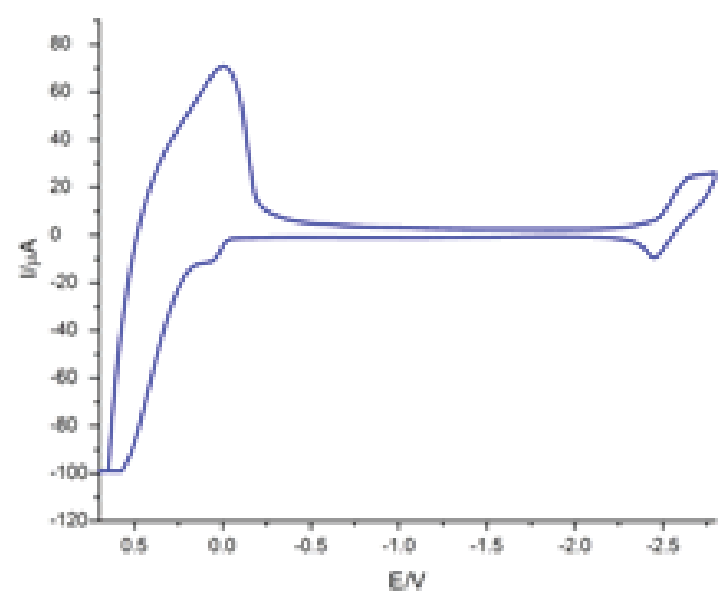

Figure 2. A CV of P3DT mM (PRU) in THF. Supporting electrolyte $=0.10 \mathrm{M}$ tetrabutylammonium hexafluorophosphate, working electrode $=$ glassy carbon, auxiliary electrode $=\mathrm{Pt}$ wire, reference electrode $=\mathbf{A g} / \mathbf{A g}^{+}$, and scan rate $=100 \mathrm{mV} \mathrm{s}{ }^{-1}$

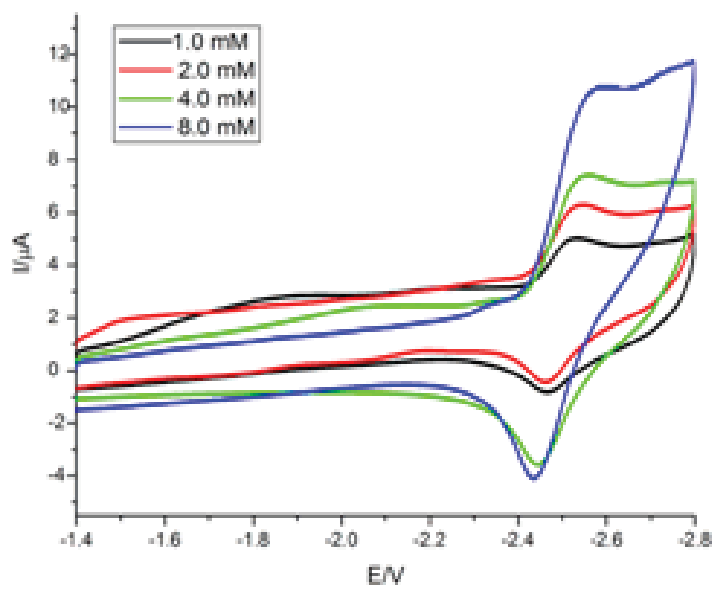

Figure 4. CVs of various concentrations of P3DT in THF are shown. Supporting electrolyte $=0.10 \mathrm{M}$ tetrabutylammonium hexafluorophosphate, working electrode $=$ glassy carbon, auxiliary electrode $=$ $\mathrm{Pt}$ wire, reference electrode $=\mathbf{A g} / \mathbf{A g}^{+}$, and scan rate $=100 \mathrm{mV} \mathrm{s}^{-1}$
The Randles-Sevcik equation was used to calculate theoretical values for DP3DT by assuming values of $n$ and assuming a concentration of $21.7 \mu \mathrm{M}$ (or $8 \mathrm{mM}$ PRU). The results are tabulated in Table 3. Work is in progress with modeling via Igor and DigiSim in order to determine the diffusion coefficient and the number of electrons for the P3DT/P3DT redox couple of $-2.50 \mathrm{~V}$.

\begin{tabular}{|l|l|l|l|}
\hline$[\mathrm{P} 3 \mathrm{DT}] / \mu \mathrm{M}$ & $\mathrm{n}$ & $\mathrm{D} / \mathrm{cm}^{2} \mathrm{~s}^{-1}$ & $i_{p c} / \mu \mathrm{A}$ \\
\hline 21.7 & 1 & $3.43 \times 10^{-3}$ & 7.17 \\
\hline 21.7 & 10 & $2.94 \times 10^{-6}$ & 7.17 \\
\hline 21.7 & 20 & $3.68 \times 10^{-7}$ & 7.17 \\
\hline 21.7 & 40 & $4.60 \times 10^{-8}$ & 7.17 \\
\hline
\end{tabular}

Table 3. Tabulated diffusion coefficient values calculated for assumed values of $n$. ip = peak current, $A ; a=0.071 \mathrm{~cm}^{2} ; F=9.65 \times 104 \mathrm{C}$ $\mathrm{mol}^{-1} ; \mathbf{c}=21.7 \times 10^{-9} \mathrm{~mol} \mathrm{~cm}^{-3} ; \mathbf{T}=298.15 \mathrm{~K} ; v=0.1 \mathrm{~V} \mathrm{~s}^{-1} ; \mathbf{R}=8.314 \mathrm{~J}$ $\mathrm{mol}^{-1} \mathrm{~K}^{-1}$; and $\mathrm{D}=$ diffusion coefficient, $\mathrm{cm} 2 \mathrm{~s}^{-1}$.

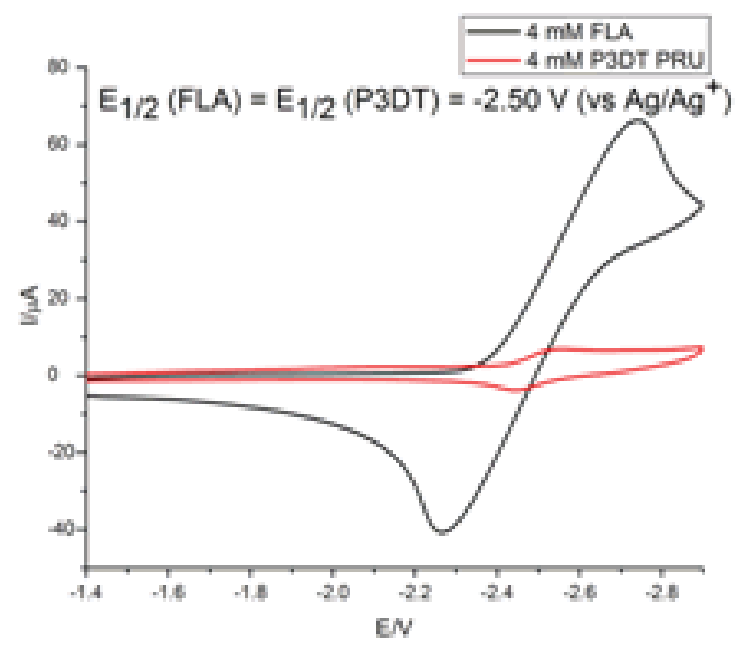

Figure 3. A CV of $4.0 \mathrm{mM}$ fluoranthene and $4.0 \mathrm{mM}$ P3DT PRU in THF. Supporting electrolyte $=0.10 \mathrm{M}$ tetrabutylammonium hexafluorophosphate, working electrode $=$ glassy carbon, auxiliary electrode $=\mathrm{Pt}$ wire, reference electrode $=\mathrm{Ag} / \mathrm{Ag}+$, and scan rate $=100 \mathrm{mV} \mathrm{s}^{-1}$

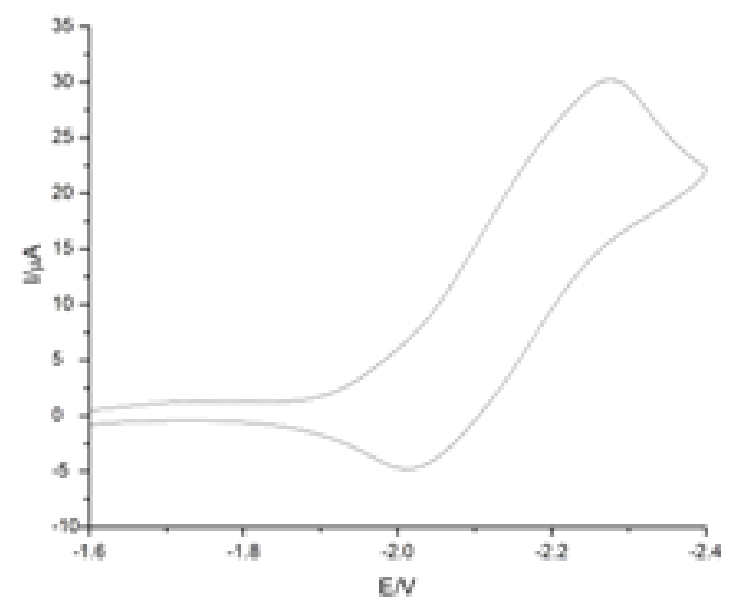

Figure 5. A CV of a 5.0 mM RPU F8BT solution in THF. Supporting electrolyte $=0.10 \mathrm{M}$ tetrabutylammonium hexafluorophosphate, working electrode $=$ glassy carbon, auxiliary electrode $=$ Pt wire, reference electrode $=\mathrm{Ag} / \mathrm{Ag}^{+}$, and scan rate $=100 \mathrm{mV} \mathrm{s}^{-1}$ 
A CV was acquired for $1.09 \mathrm{mM}$ fluoranthene in THF from which several parameters were calculated. The diffusion coefficient was calculated as $6.14 \times 10-6 \mathrm{~cm}^{2} \mathrm{~s}^{-1}$ for the reversible $\mathrm{CV}$ where the $\mathrm{E}_{1 / 2}\left(\mathrm{FLA} / \mathrm{FLA}^{-}\right)=-2.53 \mathrm{~V}$ versus $\mathrm{Ag} / \mathrm{Ag}^{+}$. Interestingly, with a $4.0 \mathrm{mM}$ FLA, the $\mathrm{E}_{1 / 2}$ value was calculated as $-2.50 \mathrm{~V}$ for the FLA/FLA- redox couple.

A literature reference for the reduction potential for fluoranthene has not yet been identified for comparison.

\section{E. F8BT}

The E $1 / 2$ value for the F8BT/F8BT- redox couple was determined as $-2.14 \mathrm{~V}$ (see Figure 5). The CV shows great reversibility and broad waves somewhat similar to those observed in CVs of P3DT as reported by Dong et al.19 Interesting enough, the authors did not report the type of working electrode used in this study, but they carried out the study with films on the working electrode in acetonitrile $/ \mathrm{CH}_{2} \mathrm{Cl}_{2}$.

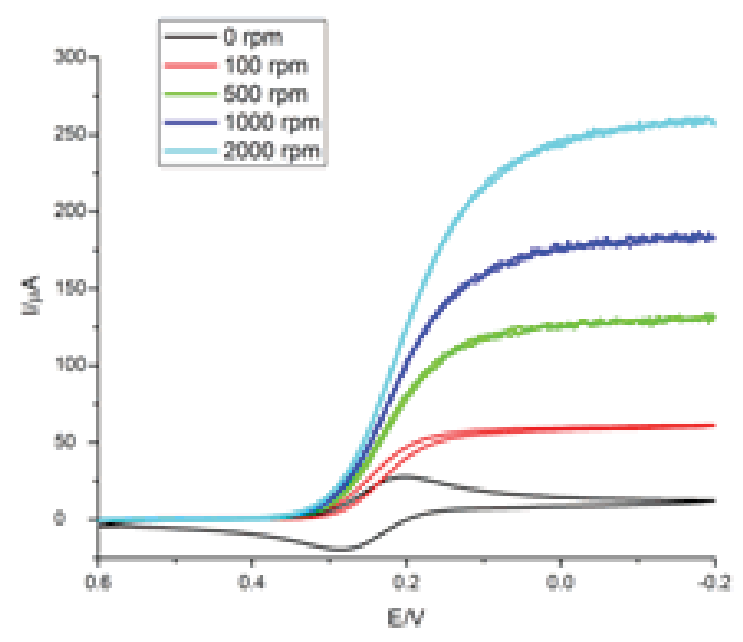

Figure 6. A CV of a $1.9 \mathrm{mM}\left[\mathrm{K}_{3} \mathrm{Fe}(\mathrm{CN})_{6}\right]$ in THF. Scan rate $=10 \mathrm{mV}$ $\mathbf{s}^{-1}$, working electrode $=$ glassy carbon, auxiliary electrode $=\mathbf{P t}$ wire, reference electrode $=\mathrm{Ag} / \mathrm{AgCl}$, and supporting electrolyte $=1.0 \mathrm{M}$ $\mathrm{KCl}$. Area of the working electrode $0.07065 \mathrm{~cm}^{2}$, and diameter $=$ $5 \mathrm{~mm}$.

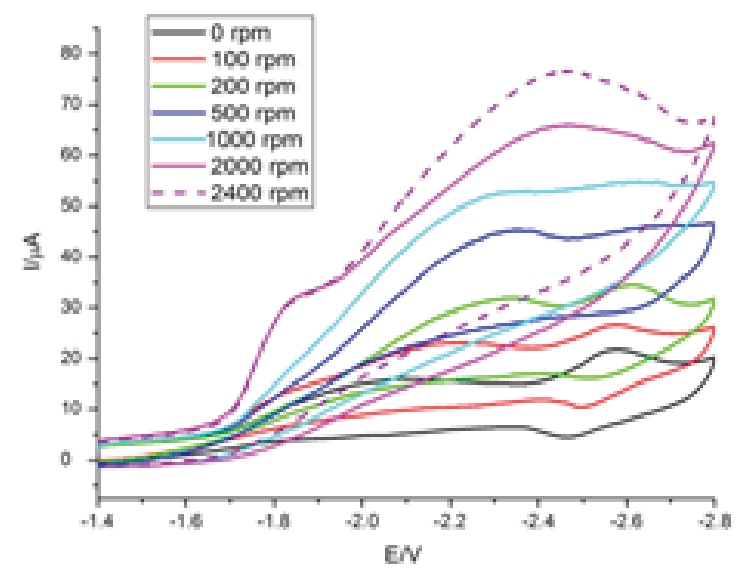

Figure 7: An overlay of rotating disk electrode voltammagrams for a $2.5 \mathrm{mM}$ (PRU) solution of P3DT in THF. Working electrode = glassy carbon, auxiliary electrode $=\mathbf{P t}$ wire, reference electrode $=\mathbf{A g} / \mathbf{A g}^{+}$, supporting electrolyte $=\mathbf{0 . 1 0} \mathrm{M}$ tetrabutylammonium hexafluorophosphate and scan rate $=50 \mathrm{mV} \mathrm{s}^{-1}$

\section{F. Cyclic voltammetry using an RDE:}

Potassium ferricyanide in an aqueous solution at $1.9 \mathrm{mM}$ served as a reference material for testing the voltage-dependent current response for the RDE measurement system. The supporting electrolyte used was $1.0 \mathrm{M} \mathrm{KCl}$. Figure 6 shows the CVs of potassium ferricyanide at several rotation rates. The resulting voltammagrams for potassium ferricyanide have negligible hysteresis as expected due to the measurement being steady state. The Levich currents, produced at the various rotation rates, allow for the diffusion coefficient of an analyte being accurately determined from the Levich equation due to the precision of the current measurements.

In contrast with the results of the potassium ferricyanide solution, the RDE for the P3DT solution at $2.5 \mathrm{mM}$ (PRU) shown in Figure 7, contains hysteresis that increases rather than decreases with electrode rotation rate. While this result is not yet understood, one possibility considered is that of a change in the distribution spread of the number of electrons per p3DT chain. Another important difference in the RDE voltammagrams of potassium fericyanide and P3DT is the observation of a current plateau in the former, and an increasing current "hill" which continues to increase with more negative potentials. This seems to suggest that P3DT is capable of being further reduced as the potential is pushed even more negative while potassium fericyanide levels after being reduced with one electron.

\section{G. The LEAF study}

The absorbance transients used for these calculations were measured at $1629 \mathrm{~nm}$ where only P3DT*- absorbs. This facilitated the calculation of $\left[\mathrm{P} 3 \mathrm{DT}^{*}\right] /\left[\mathrm{FLA}^{*}\right]$ at each concentration of fluoranthene via comparison of the absorbance at equilibrium with the absorbance when fluoranthene is not present. The Keq values were obtained for each fluoranthene concentration and $\Delta \mathrm{G}$ values were calculated from the average of the $\mathrm{K}_{\mathrm{eq}}$ values, with an accompanying standard deviation. For the $0.1 \mathrm{M}$ TBAPF6 electrolyte environment, the average $\mathrm{K}_{\mathrm{eq}}$ was determined as $34.7 \pm 5.5$, and $\Delta \mathrm{G}$ as $-0.1 \mathrm{eV}$.

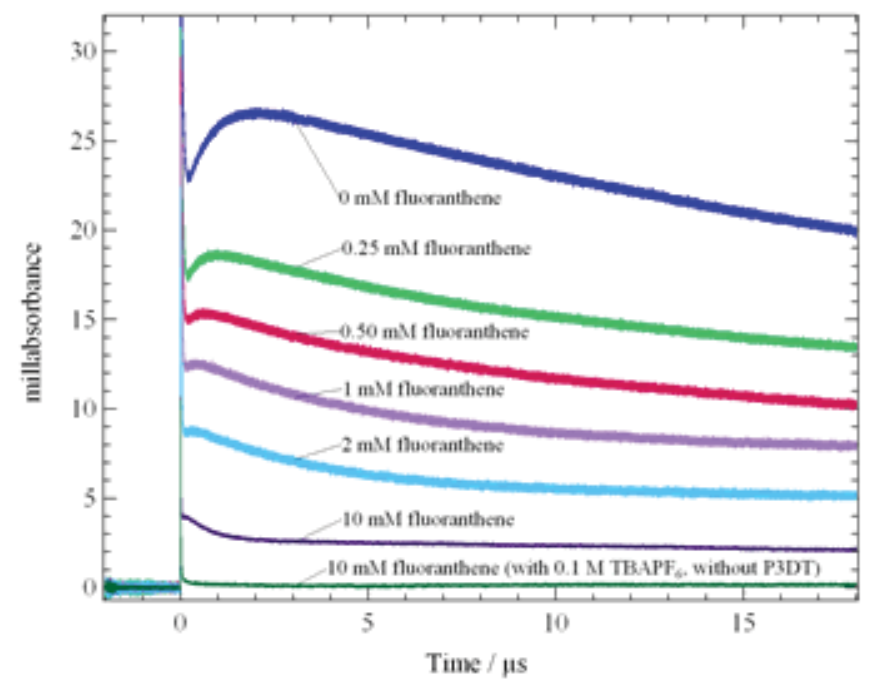

Figure 8. The radiolysis absorbance transients for a series of $20 \mu \mathrm{M}$ (per polymer) P3DT $(n=369)$ solutions containing varied concentrations of fluoranthene, collected at $1629 \mathrm{~nm}$ where only P3DT"- absorbs. 
A $\Delta \mathrm{G}$ of $-0.1 \mathrm{eV}$ corresponds to a potential difference of -0.1 $\mathrm{V}$ which would indicate that the $\mathrm{E}_{1 / 2}$ of the one electron reduced p3DT should be formed at $-2.60 \mathrm{~V}$ rather than the value of -2.50 $\mathrm{V}$ determined for $\mathrm{p}^{\mathrm{D}} \mathrm{T}^{\mathrm{n}-\boldsymbol{-}}$ from the $\mathrm{CV}$. This result along with the calculation of " $n$ " using a reasonable estimated value for the DP3DT in the Randles-Sevcik equation and known values for concentration and ipc of P3DT, suggest that " $n$ " is indeed more than one and perhaps close to 20 .

In addition to the radiolysis measurements performed on binary solutions of fluoranthene and P3DT in $0.1 \mathrm{M} \mathrm{TBAPF}_{6}$, measurements were collected for binary solutions in $0 \mathrm{M} \mathrm{TBAPF}_{6}$ and $0.6 \mathrm{mM} \mathrm{TBAPF}_{6}$. An interesting finding is that the value for the free energy is nearly the same in $0.6 \mathrm{mM}$ as in $0.1 \mathrm{M} \mathrm{TBAPF}_{6}$, while the value of the magnitude of the free energy has increased for the solution containing no electrolyte. This implies that at 0.6 $\mathrm{mM} \mathrm{TBAPF}{ }_{6}$ there is sufficient ion pairing for both FLA $^{-}$and P3DT"- to make the equilibrium conditions nearly the same. If most of the $\mathrm{FLA}^{-}$were ion paired with $\mathrm{TBA}^{+}$cations and most P3DT"- were not paired, then it is conceivable that the forward reaction rate would decrease substantially, leading to a $\Delta \mathrm{G}$ of even greater magnitude than observed for the case where neither anion is paired when no electrolyte is present. As the cation-paired FLA" $^{*}$ is expected to have a smaller dissociation constant than P3DT*-, there may exist a concentration of electrolyte less than 0.6 $\mathrm{mM}$, where ion pairing is dominant only for FLA" and a further study is underway to test this hypothesis.

\begin{tabular}{|l|r|r|}
\hline Polymer used & \multicolumn{1}{|c|}{$\left[\right.$ TBAPF $\left._{6}\right] / \mathbf{M}$} & \multicolumn{1}{|c|}{$\Delta \mathbf{G} / \mathbf{e V}$} \\
\hline P3DT $(n=170)$ & 0.10 & -0.09 \\
\hline P3DT $(n=170)$ & 0.00 & -0.15 \\
\hline P3DT $(n=170)$ & 0.0006 & -0.10 \\
\hline P3DT $(n=369)$ & 0.10 & -0.10 \\
\hline
\end{tabular}

Table 4. Calculated $\Delta G$ values for binary solutions of P3DT and fluoranthene under various electrolyte conditions.

In the future, further studies will be carried out in order to prove that P3DT conducts a multiple electron redox process which will make it a good replacement for silicon in solar cells. We would like to strive forward into achieving one of our challenging goals which is the acquisition of thermodynamic and one-electron potentials for electrons and holes in the kind of solid film environment in which the polymers would actually be used. Acquiring CVs via film in order to learn more about the polymer's holes and energies of the electrons seems to be of interest as well.

\section{References}

(1) Gratzel, M. Chem. Lett. 2005, 34, 8-13.

(2) Kamat, P. V. J. Phys. Chem. C 2007, 111, 2834-2860.

(3) Shah, A.; Torres, P.; Tscharner, R.; Wyrsch, N.; Keppner, H. Science 1999, 285, 692-698.

(4) Chen, J.-T.; Hsu, C.-S. Polym. Chem. 2011, 2, 2707-2722.

(5) Guenes, S.; Neugebauer, H.; Sariciftci, N. S. Chem. Rev. 2007, 107, 1324-1338.

(6) Thompson, B. C.; Frechet, J. M. J. Angew. Chem., Int. Ed. 2008, 47, 58-77.

(7) Boudreault, P.-L. T.; Najari, A.; Leclerc, M. Chem. Mater. 2011, 23, 456-469.

(8) Zaikowski, L.; Kaur, P.; Gelfond, C.; Selvaggio, E.; Asaoka, S.; Wu, Q.; Chen, H.-C.; Takeda, N.; Cook, A. R.; Yang, A.; Rosanelli, J.; Miller, J. R. J. Am. Chem. Soc. 2012, 134, 10852-10863.

(9) Hill, M. G.; Penneau, J. F.; Zinger, B.; Mann, K. R.; Miller, L. L. Chem. Mater. 1992, 4, 1106-13.

(10) Zade, S. S.; Zamoshchik, N.; Bendikov, M. Acc. Chem. Res. 2011, 44, 14-24.

(11) Fratiloiu, S.; Grozema, F. C.; Koizumi, Y.; Seki, S.; Saeki, A.; Tagawa, S.; Dudek, S. P.; Siebbeles, L. D. A. J. Phys. Chem. B 2006, 110, 5984-5993.

(12) Zade, S. S.; Bendikov, M. J. Phys. Chem. B 2006, 110, 15839-15846.

(13) Jansson, E.; Jha, P. C.; Aagren, H. Chem. Phys. 2007, 336, 91-98.

(14) Takeda, N.; Miller, J. R. J. Phys. Chem. B 2012, 116, 14715-14723.

(15) Lu, W.; Fadeev, A. G.; Qi, B.; Smela, E.; Mattes, B. R.; Ding, J.; Spinks, G. M.; Mazurkiewicz, J.; Zhou, D.; Wallace, G. G.; MacFarlane, D. R.; Forsyth, S. A.; Forsyth, M. Science 2002, 297, 983-987.

(16) Bard, A. J.; Faulkner, L. R. Electrochemical methods : fundamentals and applications; 2nd ed.; Wiley: New York, 2001.

(17) Burgess, K. S. D.; Kable, J. W.; Justice, J. B. Electroanalysis 1999, 11, 337-343.

(18) Tsierkezos, N. G. J. Solution Chem. 2007, 36, 289.

(19) Dong, W.; Xue, S.; Lu, P.; Deng, J.; Zhao, D.; Gu, C.; Ma, Y. J. Polymer Sci. Part A: Polymer Chemistry 2011, 49, 4549-. 


\title{
Detection of hydroxymethylcytosine in human cells exposed to ionizing radiation
}

\author{
Benjamin Daniels \\ Rensselaer Polytechnic Institute, Troy, NY, 12180 \\ Lucia Kolodiuk and Katherine Sanidad \\ Stony Brook University, Stony Brook NY, 11790 \\ Paula V. Bennett, Deborah J. Keszenman, Alicia Johnson, and Paul F. Wilson \\ Biosciences Department, Brookhaven National Laboratory, Upton, NY 11973-5000 3
}

\begin{abstract}
Gene regulation relies heavily on the level and order of the "5th" DNA base 5-methylcytosine (5-mC) which composes $\sim \mathbf{5 \%}$ of cytosine bases in mammalian DNA. Scientists are only beginning to understand how 5-hydroxymethylcytosine (5-hmC) affects gene expression. 5-hmC is formed by the addition of a hydroxyl group attached to the end of the methyl group in 5-mC. Ionizing radiation (IR) exposure has been shown to cause epigenetic changes in genomic DNA by oxidizing DNA bases and it can oxidize the methyl group on $5 \mathrm{mC}$ to 5-hmC. This project studies the levels of 5-hmC induction in human cells and detection with anti 5-hmC antibodies by developing an immuno-slot blot assay (ISB) for 5-hmC DNA base damage/modification assessment. The focus of this research is to determine if 5 -hmC levels increase in human fibroblast cells with increasing IR exposure and to determine if cellular 5-hmC levels change with time after gamma radiation exposure.
\end{abstract}

\section{Introduction}

Epigenetics is the study of heritable changes in an organism's observable traits or phenotype not directly encoded in its DNA sequence. It involves functional changes to an organism's DNA sequence and changes in gene expression influenced by environmental factors, without changing the sequence of DNA bases. In humans, epigenomic changes have been implicated in diseases such as cancer, cardiomyopathy, type II diabetes, autoimmune disorders, schizophrenia, and autism. The regulation of gene function in mammals is dependent on the methylation of the cytosine base in the DNA. This 5-methylcytosine base $(5-\mathrm{mC})$ can be oxidized into 5-hydroxymethylcytosine (5-hmC), affecting the expression of the genome. It is hypothesized that, ionizing radiation (IR) can oxidize $5-\mathrm{mC}$ to $5-\mathrm{hmC}$ radiochemically by creating highly reactive free radicals, which can bind to the methyl group. The ability to quantify increases or decreases in genomic 5-hmC levels would help to determine the types of epigenomic effects observed by modulation of 5-hmC in complex genomes.

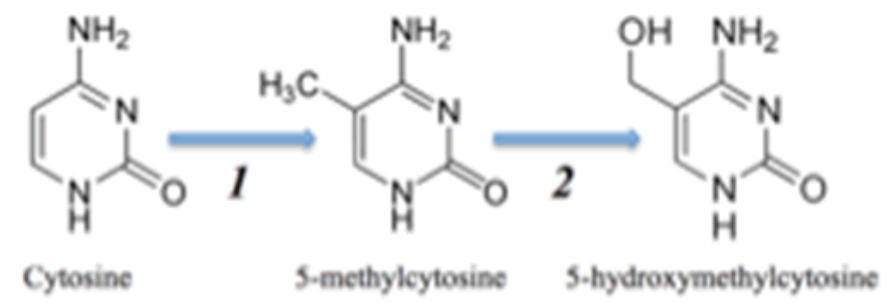

Figure 1: Pathway of 5-hydroxymethylcytosine creation

To determine if an immuno-slot blot (ISB) assay could be used to assay for DNA base changes, we developed an ISB assay to detect synthetic thymidine DNA base analog bromodeoxyuridine (BrdU), with an anti-BrdU antibody. BrdU is used to study the amount of DNA replication occurring in growing cells. When cells replicate their DNA in the presence of BrdU it is incorporated into their DNA as well as normal DNA base thymidine. The more BrdU incorporated the more the cells have replicated. For our study, osteosarcoma U2OS cells were grown in the presence of increasing concentrations of BrdU. The DNA was extracted and loaded onto nitrocellulose membranes, blocked and probed with BrdU antibodies. BrdU was detectable, so focus was switched to the detection of 5-hmC (a much rarer base modification in mammalian DNA) in 137Cs Gamma irradiated human fibroblasts cells. In this experiment an ISB assay using anti 5-hmC antibodies will be used to determine the extent of 5-hmC formation caused by IR. We hypothesize that ionizing radiation could increase 5-hmC levels in cellular DNA which could be observed by detection of 5-hmC in gamma irradiated DNA with antibodies against this base modification.

\section{Methods and Results}

A. Testing for the Incorporation BrdU

A culture of human osteosarcoma U2OS cells were grown in a humidified $37^{\circ} \mathrm{C} \mathrm{5 \%} \mathrm{CO} 2$ incubator. This culture was divided into four flasks and BrdU was added to $0,1,10$, and $100 \mu \mathrm{M}$ final concentrations in medium respectively. The cultures were then allowed to grow for 2 cell cycles to allow for the incorporation of the BrdU into the cellular DNA. The cells were harvested by trypsinization yielding $2.8 \times 106$ cells per flask. The cellular DNA was extracted and purified using the Qiagen $\AA$ DNeasy ${ }^{\circledR}$ Blood $\&$ Tissue Kit. The extracted DNA concentration was measured with a Thermo Scientific ND-1000 NanoDrop spectrophotometer as shown in Figure 2.

The quality of the DNA extracted from the cells was assayed using gel electrophoresis with neutral DNA as shown in Figure 3. DNA sizes of all the samples were approximately between 23 and 48 kilobase pairs in length indicating the DNA was of high quality for mammalian DNA extracted in solution.

In preparation for the ISB assay, the BrdU incorporated DNA had $10 \mathrm{mM}$ Potassium Phosphate (KP Buffer) added, along with $137 \mathrm{mM}$ NaCL, $2.7 \mathrm{mM} \mathrm{KCl}, 10.4 \mathrm{mM}$ Na2HPO4, $1.4 \mathrm{mM} \mathrm{KH}-$ 2PO4 (PBS solution). The samples were sonicated for 15 minutes, heat denatured $10 \mathrm{~min}$ at $95^{\circ} \mathrm{C}$ and, quick chilled on ice. Then, $2 \mathrm{M}$ Ammonium (NH4+) Acetate was added to neutralize the samples. Aliquots of 50, 100, 250, 500 and $1000 \mathrm{ng}$ were loaded onto BioRad nitrocellulose membrane inside a BioRad Bio-Dot SF apparatus. The samples were passed through the apparatus via gravity filtration in order to let the DNA have time to bind onto 
the membrane. After the samples were dry, they were baked at $80^{\circ} \mathrm{C}$ for $2 \mathrm{~h}$ and then placed in blocking solution of PBS-I $0.1 \%$ Tween-20 and 5\% dry milk (PBS-T-M) overnight.

Once prepared, the charged membrane was placed in a sealed container, and a 1:5000 dilution of the primary anti-BrdU antibody (Molecular Probes ${ }^{\circledR}$ anti-BrdU Mouse Monoclonal Antibody, (clone MuBU-1) Alexa Fluor ${ }^{\circledR} 488$ Conjugate) was added. The membrane was incubated on a rocker overnight at $4{ }^{\circ} \mathrm{C}$. At this point, direct fluorescent imaging $(480 / 520 \mathrm{~nm}$ wavelength excitation/emission) of the membrane was attempted unsuccessfully so we proceeded with chemiluminescent detection of the anti BrdU antibody. In brief, remaining anti BrdU antibody was removed and the membrane was washed 3 times with PBS-T. The secondary antibody - horseradish peroxidase (HRP anti-rabbit IgG, GE Healthcare)- was then added and the membrane incubated on the rocker for $1 \mathrm{~h}$. After the secondary antibody had been removed and the membrane washed, the membrane was treated with ECL in preparation for chemiluminescent electronic imaging. The imaging was used to detect for BrdU incorporation into the DNA. Images with exposure times of 10, 30, 60, 120, 300, and 600 seconds were taken with the imaging system. The presence of BrdU binding on the membrane can be seen in Figure 4.

\begin{tabular}{|r|r|r|r|}
\hline \multicolumn{1}{|c|}{ Sample \# } & \multicolumn{1}{|c|}{ Dose (Gy) } & $\begin{array}{c}\text { "Repair" } \\
\text { Time (hr) }\end{array}$ & $\begin{array}{c}\text { DNA Conc } \\
\text { ng/ } \boldsymbol{\mu L}\end{array}$ \\
\hline 1 & 0 & .083 & 141.8 \\
\hline 2 & 0 & 2 & 154.5 \\
\hline 3 & 0 & 6 & 160.0 \\
\hline 4 & 0 & 24 & 163.0 \\
\hline 5 & 5 & .083 & 152.3 \\
\hline 6 & 5 & 2 & 161.0 \\
\hline 7 & 5 & 6 & 158.0 \\
\hline 8 & 5 & 24 & 154.0 \\
\hline 9 & 10 & .083 & 146.6 \\
\hline 10 & 10 & 2 & 159.4 \\
\hline 11 & 10 & 6 & 166.4 \\
\hline 12 & 10 & 24 & 159.7 \\
\hline
\end{tabular}

Table 1: Dose, and exposure times for the irradiated human fibroblast cells

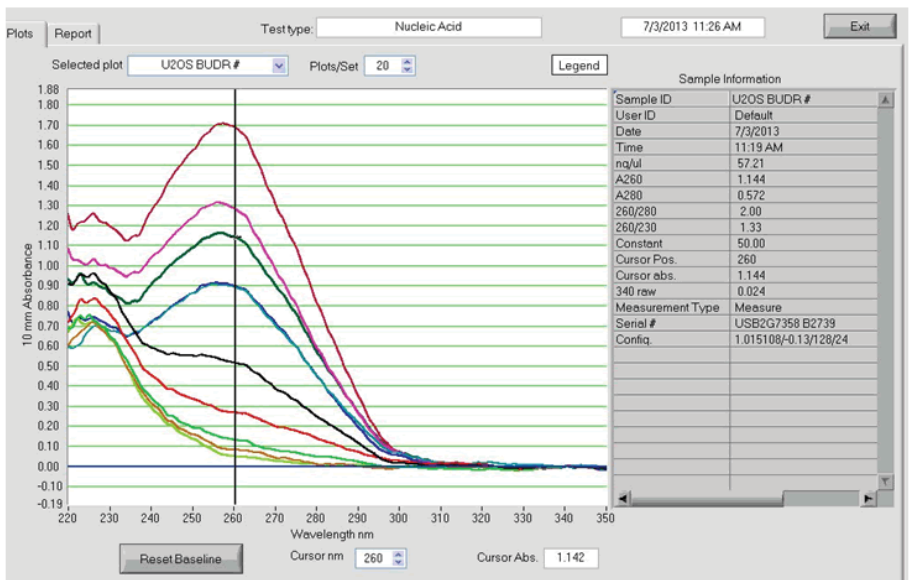

Figure 2: Spectrophotometer readout with concentrations and absorptions of BrdU incorporated DNA

\section{B. Testing for the Incorporation and Specificity of 5-hmC}

A culture of human fibroblast cells were grown in standard culture medium at $37^{\circ} \mathrm{C}$ with $5 \% \mathrm{CO}_{2}$. The culture was divided into 12 samples. They were placed inside the J.L. Shepherd Mark I Model 68A irradiator and irradiated with $662 \mathrm{keV}$ cesium-137 gamma rays at doses detailed in Table 1.

The DNA was extracted and purified from the cells, using the DNeasy ${ }^{\circledR}$ Blood and Tissue Kit, and the concentrations were again measured with the NanoDrop spectrophotometer, as shown in Table 1 . The quality of the DNA extracted was assayed by neutral gel electrophoresis. Anti 5-hmC antibody has the highest specificity for single stranded DNA on positively charged membranes. Concern for the chemical stability of the $-\mathrm{OH}$ group on 5 -hmC led us to experimentally determine the best possible DNA denaturation protocol, for anti 5-hmC antibody recognition. Three DNA heat denaturation protocols were designed, strong alkaline, moderately alkaline and weakly alkaline 4.0 M NaOH, $100 \mathrm{mM}$ Tris- $\mathrm{HCl} \mathrm{pH}$ 9, and $100 \mathrm{mM}$ Tris- $\mathrm{HCl} \mathrm{pH} 8$ respectively.

The irradiated fibroblast DNAs were prepared for the ISB assay by adding $10 \mathrm{mM}$ Tris- $\mathrm{HCl}, 0.5 \mathrm{mM}$ EDTA, pH 9.0 (AE buffer) to the DNA and 4.0 M NaOH, $100 \mathrm{mM}$ Tris- $\mathrm{HCl} \mathrm{pH} 9.0$ or $100 \mathrm{mM}$ Tris- $\mathrm{HCl} \mathrm{pH}$ 8.0. The DNA was heated for $10 \mathrm{~min}$ at $95{ }^{\circ} \mathrm{C}$, and quick chilled on ice. Denaturant treated DNA was then neutralized with $2 \mathrm{M} \mathrm{NH} 4+$ Acetate, bringing the final DNA concentration to $64.45 \mathrm{ng} / \mu \mathrm{L}$. Each of the 12 experimental DNA samples was split into 4 groups with increasing concentrations $100 \mathrm{ng}, 250 \mathrm{ng}, 500 \mathrm{ng}$ or $1000 \mathrm{ng}$, respectively. Sample volumes were equalized with the addition of loading buffer $(450 \mu \mathrm{L} \mathrm{AE}$ buffer, $50 \mu \mathrm{L}$ DNA denaturant, $500 \mu \mathrm{L} 2.0 \mathrm{M}$ NH4+ Acetate per $\mathrm{mL})$. Positively charged nylon membrane (Nitran ${ }^{\circledR}$ Scheicher and Schuell) was placed inside a Scheicher and Schuell MINIFOLD ${ }^{\circledR}$ dot blot apparatus. $50 \mu \mathrm{L}$ of each sample was loaded onto the dot

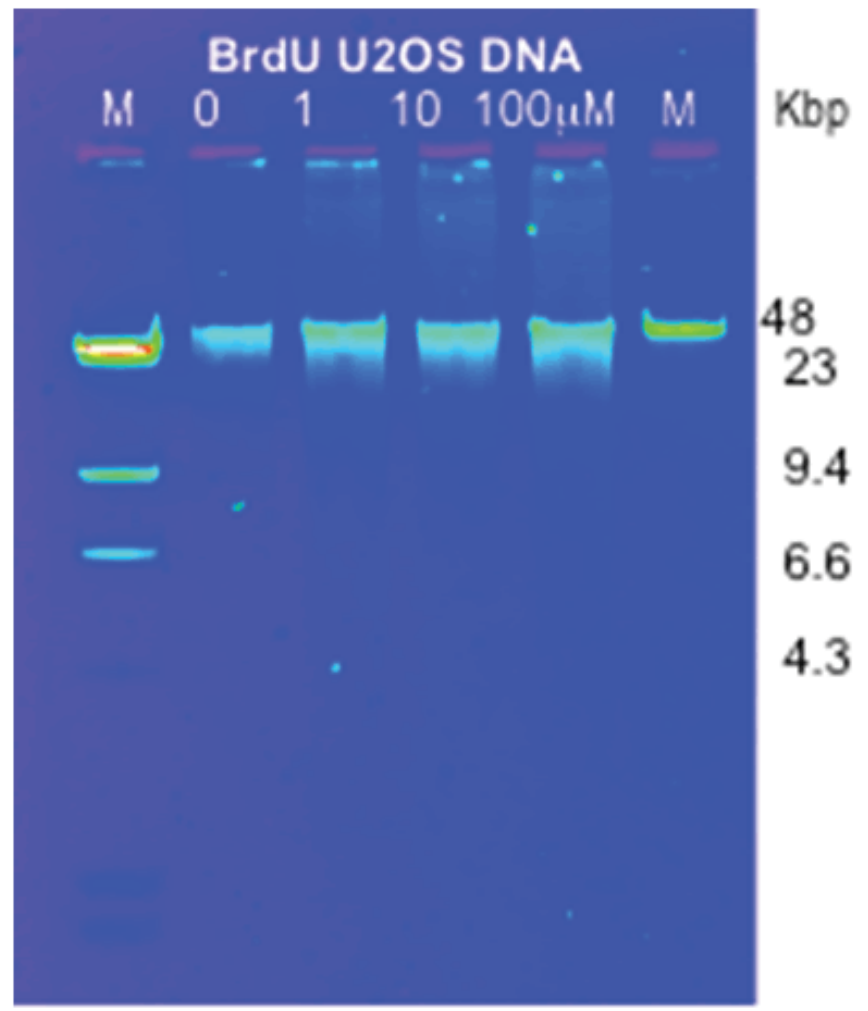

Figure 3: Image of a gel using the BrdU incorporated DNA 
blot apparatus. Three DNA controls were loaded onto the membrane to check for antibody specificity and nonspecificity, unmethylated and 5-mC negative control DNAs, and 5-hmC positive control DNA as shown in Figure 5. All samples were loaded onto the membrane by gravity filtration through the membrane. The membrane was washed once by gravity filtration with 6X SSC buffer (3.0 M sodium citrate 3.0 M sodium chloride $\mathrm{pH}$ 7.0).

Blots were air dried overnight at $37 \mathrm{oC}$. After the blots were dry, they were crosslinked with $30 \mathrm{~J} / \mathrm{m} 2254 \mathrm{~nm}$ ultraviolet radiation from a germicidal lamp in order to covalently bind the DNA to the membrane. The membranes were placed in blocking solution of $1 \mathrm{X}$ PBS-T-M (BioRad blotting grade) overnight at 4oC.

Once prepared, the nytran membrane was placed in a sealed container, and a 1:5000 dilution of primary anti-5-hmC antibody, (5-hmC antibody (pAb), Active Motif) was added. The membrane was incubated on a rocker overnight at $4 \mathrm{oC}$. The primary antibody was removed and the membrane was washed with PBS-T 3 times for $5 \mathrm{~min}$. A 1:1000 dilution of the Anti rabbit horseradish peroxidase conjugated secondary antibody, (HRP anti-rabbit IgG, GE Healthcare) was added. The membrane was incubated on a rotating platform for $1 \mathrm{~h}$ at $25 \mathrm{oC}$. After the secondary antibody was removed, the membrane was washed 3 times with PBS-T, blots were treated with ECL ${ }^{\circledR}$ (GE Healthcare) chemiluminescent detection reagents as per the manufacturer's instructions. Blots were electronically imaged immediately with a Star 138 CCD camera

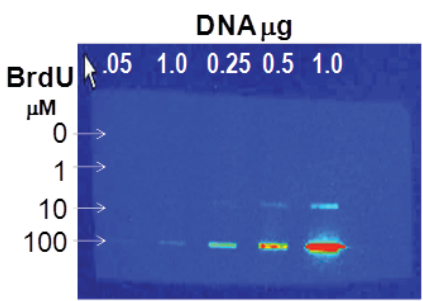

BrdU 120 sec

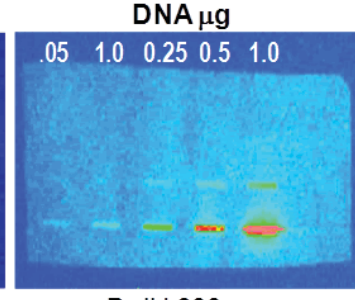

BrdU $600 \mathrm{sec}$
Figure 4: Image of charged membrane after ISB assay under chemiluminescent imaging

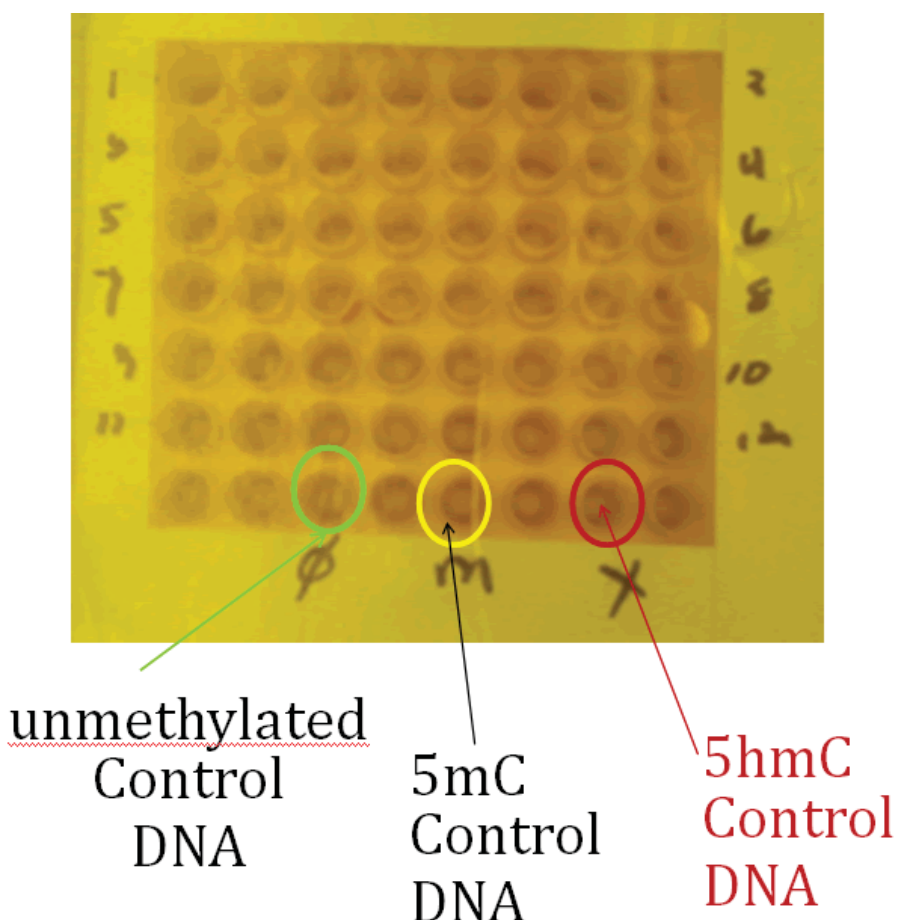

Figure 5: Layout of the samples for loading onto the nitrocellulose membrane
(Roper Scientific) to detect 5-hmC incorporation. Exposure times between 10 seconds and 900 seconds were taken and recorded with the imaging system. The presence of 5-hmC incorporation can be seen in Figure 6 and Figure 7

\section{Discussion}

In the ISB assay to detect BrdU incorporation into genomic DNA, the anti-BrdU antibody shows the highest affinity for binding with the cellular DNA from cells incubated with $100 \mu \mathrm{M}$ BrdU (Figure 3). This suggests that the anti-BrdU antibody that was used for the primary antibody is capable of detecting BrdU that has been incorporated into genomic DNA. No BrdU antibody reaction was detected in untreated cells. BrdU was not detectable in cells treated with $1 \mu \mathrm{M}$ BrdU until at least one $\mu \mathrm{g}$ of cellular DNA was loaded. BrdU positive bands were detected in cells treated with $10 \mu \mathrm{M}$ BrdU at the $0.25,0.5$ and $1.0 \mu \mathrm{g}$ DNA loading levels. BrdU positive bands were readily detected in all DNA loads when the cells were treated with $100 \mu \mathrm{M}$ BrdU concentrations in culture medium. Therefore, detection of BrdU incorporation into cellular DNA is possible with the ISB assay format. The reaction banding patterns are suggestive of a quantitative linear dose response with

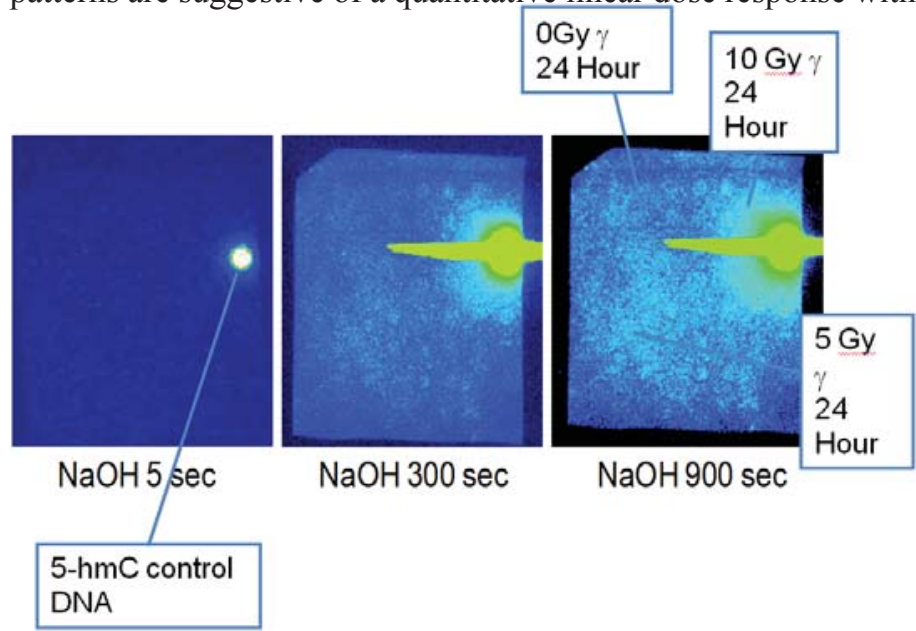

Figure 6: Chemiluminescent imaging of the 5-hmC binding with the NaOH denatured DNA

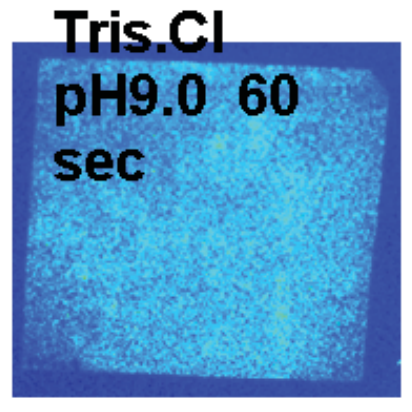

Tris.Cl
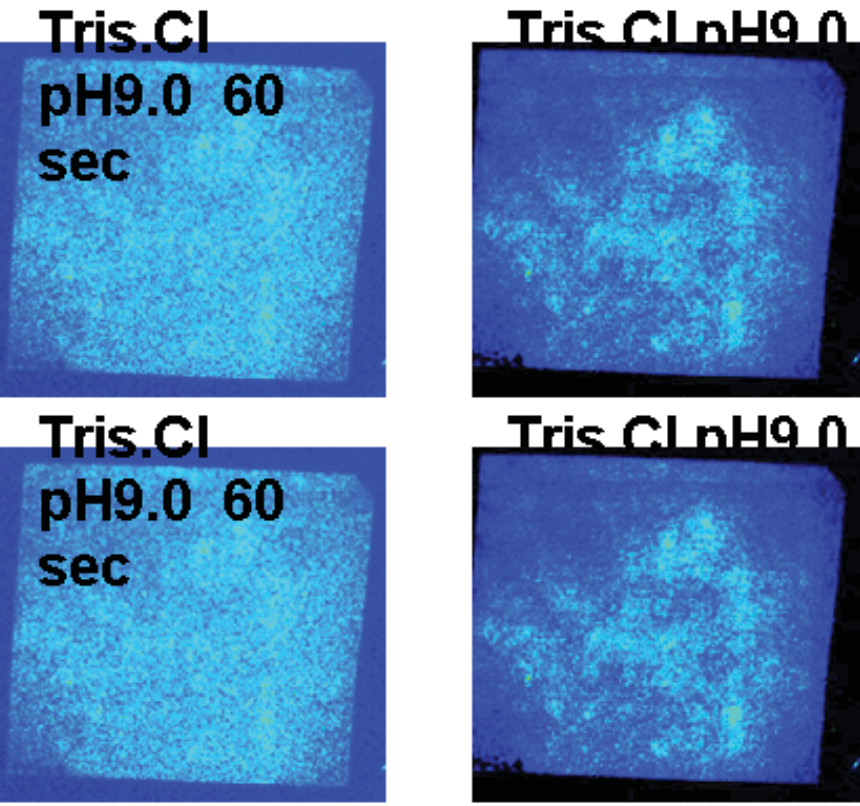

Figure 7: Chemiluminescent imaging of the 5-hmC binding with the Tris-HCl 9 denatured DNA 
increasing BrdU concentration and with amount of DNA loaded onto the membranes. We are currently analyzing the results to determine if the data is quantifiable.

In the ISB assay for detection of 5-hmC in gamma irradiated human genomic DNA with the anti-5-hmC antibody our results are promising but need further development. It is hypothesized that only $5 \%$ of $5-\mathrm{mC}$ bases in the human genome are oxidized to 5-hmC. Song et al. tested the human cell lines HeLa and HEK293FT and were able to detect 5-hmC in their genomic DNA by chemically enriching for 5 -hmC containing DNA. They estimate that $5-\mathrm{hmC}$ is present in those cell lines in very low abundance ( $\sim 0.01 \%$ of total nucleotides). That said, detecting hmC in unirradiated normal human fibroblast DNA might be difficult by ISB assay without enriching for 5-hmC containing DNA sequences. The anti-5-hmC antibody shows the highest affinity for binding with the positive control 5-hmC containing DNA standard (Figure 5). 5-hmC was stable to strong alkaline conditions because the control DNA was treated in exactly the same manner as the experimental DNAs. Our best detection results to date using un-enriched human genomic DNAs show that denaturing the DNA under strong alkaline conditions using $0.4 \mathrm{M} \mathrm{NaOH}$ as the denaturant is currently the most successful. We observe faint spots corresponding to cells irradiated with 5 or $10 \mathrm{~Gy}$ of gamma radiation. This suggests that the $\mathrm{NaOH}$ denatured samples and membrane were not optimized, as the signal to noise ratio was too low to be able to quantify the tested human fibroblast DNA samples. This assay also showed that anti-5-hmC antibody had almost no affinity for binding to the unmethylated negative control DNA standard or the 5-mC negative control DNA standard. Thus, the antibody binding occurs only in the presence $5-\mathrm{hmC}$ and is not cross-reactive with unmethylated cytosine residues or methyl cytosine base that are not oxidized.

In the ISB assay with the anti-5-hmC antibody with Tris- $\mathrm{HCl}$ $\mathrm{pH} 9$ as denaturant, the anti-5-hmC antibody showed the highest affinity for binding with the DNA samples gamma irradiated with 10 Gy. Faint spots were visible in this area of the blot but lower detection occured with the 5 Gy samples versus those done with $\mathrm{NaOH}$ denaturation.

Our results suggest, but do not prove that the DNA samples from cells irradiated with the highest dose of ${ }^{137} \mathrm{Cs} \gamma$-radiation, $10 \mathrm{~Gy}$, contained in the highest concentration of 5-hmC residues. Also, our results demonstrate that this correlation is detectable by the ISB assay however at this stage the blots are not quantifiable. $\mathrm{NaOH}$ was also a more effective DNA denaturant for the purposes of detecting the presence of 5-hmC by ISB assay, as the visible spots were detected with the lowest background effect on the images.

\section{Conclusions}

Ionizing radiation has been hypothesized to cause epigenomic changes in the DNA of an organism by modifying the bases of the DNA without modifying the base sequence. 5-hydroxymethylcytosine is a base modification whose effects are not fully known on gene expression. The detection for BrdU by performing an ISB assay showed that it is possible to determine the amount of incorporation of a base modification. Using a similar procedure for an ISB assay in irradiated human fibroblasts for detection of 5 -hmC in gamma irradiated cells suggests that an increase in radiation dose might be correlated with an increase in cellular 5-hmC levels.
The next step in this research would be to optimize blotting procedure and increase the signal to noise ratio to allow quantification of 5-hmC concentrations. $\mathrm{NaOH}$ was the most effective reagent tested for denaturing the sample DNA for the ISB assay. In all of our blots, the high relative background chemiluminescence made it difficult to determine how much incorporation of 5-hmC had occurred. Another step to improve the assay would be to test higher concentrations of blocking reagents and other chemiluminescent detection systems reported to be more sensitive than the GE Heathcare reagents used in this study. Success with detecting changes in genomic 5-hmC levels by ISB assay in genomic DNA that have not been enriched for 5 -hmC containing sequences would be a significant contribution to epigenetic studies.

Various DNA processing and repair mechanisms could be examined when there is an abnormally high concentration of 5-hmC in the genome from exposure to genotoxic agents. Changes to DNA repair mechanisms may demonstrate 5-hmC's epigenomic effects. Epigenomic changes can have serious consequences that determine which genes are expressed. The effect IR can have on the function of an organism's genome has been well known. However, not all of the effects of 5-hmC are as well known. Quantifying the relationship between ionizing radiation and the formation 5-hmC will help determine the full epigenomic effect of that particular base modification. This could be vital information concerning the treatment of diseases affected by epigenomic effects.

\section{Acknowledgements}

We thank John Trunk for assistance with the Star 138 chemiluminescent imaging system. We thank Dr. Stefan Tafrov for his generous advice with blotting and antibody detection methods.

This work was performed under the auspices of the U.S. Department of Energy by Brookhaven National Laboratory under contract DE-AC02-98CH10886 and supported by BNL LDRD 12-D12 to PFW

This project was supported in part by the U.S. Department of Energy, Office of Science, Office of Workforce Development for Teachers and Scientists (WDTS) under the Science Undergraduate Laboratory Internships Program (SULI).

\section{References}

Wu H, D’Alessio A.C, Ito S., Wang Z., Cui K., Zhao K., Sun Y.E., Zhang Y.Genome-wide analysis of 5-hydroxymethylcytosinedistribution reveals its dual function in transcriptional regulation in mouse embryonic stem cells. Genes \& Development 25:679-684, 2011.

Ito, $\mathrm{S}$. Role of Tet Proteins in $5 \mathrm{mC}$ to $5 \mathrm{hmC}$ conversion, ES-Cell Self-Renewal and Inner Cell Mass Specification. Nature, 2010 Aug 26;466(7310): 1129-33., Print

Song, C. et. Al. Selective Chemical labeling reveals the genomewide distribution of 5-hydroxymethylcytosine. Nature Biotechnology 29: 68-72.2011. 


\title{
Mapping Iron-based Fischer-Tropsch Synthesis Catalysts Using X-ray Computed Microtomography
}

\author{
Ryan Davella \\ Department of Chemical and Biomolecular Engineering, University of Delaware, Newark, Delaware 19716 \\ Devinder Mahajan \\ Department of Sustainable Energy Technologies, Brookhaven National Laboratory, Upton, New York 11973 \\ Keith Jones \\ Department of Sustainable Energy Technologies, Brookhaven National Laboratory, Upton, New York 11973
}

\begin{abstract}
Since the demand for crude oil has started to exceed its supply, finding alternative ways to power transportation vehicles is becoming a necessity. Fischer-Tropsch (F-T) synthesis is an effective pathway to produce fuels from most carbon-containing raw materials. The F-T process converts a mixture of carbon monoxide $(\mathrm{CO})$ and hydrogen $(\mathrm{H} \neg 2)$ into a mixture of liquid hydrocarbons, oxygenates, water, and carbon dioxide. At Brookhaven National Laboratory (BNL), previous data arising from a $120 \mathrm{~h}$ slurry phase $\mathrm{F}-\mathrm{T}$ synthesis reaction with Fe2O3-based BASF (20-80 nm), Mach-1 $(3 \mathrm{~nm})$, and UCI $(32.5 \mu \mathrm{m})$ catalyst showed that agglomeration was minimal in both nano-sized materials (BASF and Mach-1), while the micron-sized material (UCI) transformed into nano-sized (MPD $<10 \mathrm{~nm}$ ). These results provided some insight into the importance of a nano Fe catalyst in F-T synthesis. We are now characterizing these three materials using $x$-ray computed microtomography (CMT) at the National Synchrotron Light Source (NSLS) at BNL. The CMT measurements involve sample preparation, sample mounting in the beam and then recording the data. The data are then analyzed using software, such as IDL, ImageJ, and Drishti, to reconstruct images and determine how the iron catalyst behaves over extended periods of time. Results have shown that catalyst agglomeration does not occur, thus leading to a relatively even distribution throughout the sample. An even distribution is important for supported catalysts because it allows for improved efficiency through lesser amounts of material. These understandings will enable the utilization of a cost-effective catalyst, thus allowing for an option to produce fuels using F-T synthesis, lessening a dependence on crude oil. As a result of this experiment, I worked with high-pressure equipment and used research techniques, such as CMT at the NSLS, which is only available at select institutions. This valuable knowledge has helped build the skills needed to pursue a career in Chemical Engineering research.
\end{abstract}

\section{Introduction}

Since the 1950's, crude oil has emerged as one of the easiest ways to satisfy energy needs. Crude oil is widely used across the world because it can be easily refined into fuels for transportation vehicles, heating units, electrical power, and lubrication for machinery. Approximately $40 \%$ of the energy used in the United States comes from crude oil, and $99 \%$ of that is used for transportation purposes. ${ }^{1}$ According to the Energy Information Administration (EIA), the Middle East holds about $81 \%$ of the world's crude oil reserves, and approximately 109 years remain until their oil supply is fully depleted, basing their calculations off of the 2011 production trend. ${ }^{2}$ This has been an ongoing issue in the world for quite some time and the need for a solution is imminent.

One process that is independent on the use of crude oil is gas-to-liquid (GTL) conversion, which refines natural gas or other carbonaceous gases into gasoline, diesel fuel, syngas, and other useful products. ${ }^{3}$ Fischer-Tropsch (F-T) synthesis is a process that is part of GTL conversion and, if made cost-effective, can virtually eliminate the heavy use and dependence on crude oil. In 1925, Franz Fischer and Hans Tropsch developed this process that yields synthetic fuel (synfuel) from raw materials rich in carbon, such as coal, natural gas, biomass, or syngas. A conversion process utilizing F-T synthesis is shown in Figure 1.

This production method is highly favorable because the products contain lesser amounts of sulfur and aromatics which, when burned, are cleaner for the environment. Since the F-T pathway requires multiple chemical reactions, finding a catalyst to speed up these reactions is a crucial step in making the process cost-effective and efficient. The overall chemical process is shown in Figure 2.

Previous research has determined that transition metals, specifically nickel $(\mathrm{Ni})$, iron $(\mathrm{Fe})$, cobalt $(\mathrm{Co})$, and ruthenium $(\mathrm{Ru})$, are the most suitable for F-T synthesis. Although nickel tends to produce higher molecular weight hydrocarbons when used as a catalyst for F-T, it also produces methane, an undesirable product for the reaction. Due to the activity of Co and its ability to be designed for maximum wax selectivity, which is later used in a hydrocracking process, it is a favorable catalyst choice. ${ }^{4}$ The biggest downside to using $\mathrm{Co}$ is that it costs more and has less of a water-gas-shift activity in comparison to $\mathrm{Fe} .{ }^{5} \mathrm{Ru}$ can be said to be the overall best catalyst for the F-T synthesis. The ability to work at low temperatures while producing the highest molecular weight hydrocarbons and maintaining high activity are all favorable properties, ${ }^{4}$ but the cost of Ru exceeds the benefits it holds as a catalyst. ${ }^{5}$

Nano Fe has been tested and shown that when exposed to the F-T process at temperatures upwards of $513 \mathrm{~K}$, hydrocarbons can be produced while maintaining a good activity level. ${ }^{6} \mathrm{Fe}$ in general is an advantageous catalyst due to its high water-gas-shift activity and low cost. ${ }^{5}$ The water-gas-shift activity allows for Fe to utilize syngas rich in carbon monoxide as its feed, but it is unfavorable when using hydrogen-rich syngas. ${ }^{4}$

At Brookhaven National Lab (BNL), we are establishing the fate of the nano $\mathrm{Fe}$ catalysts during the F-T process. Examining the time resolved behavior of unsupported nano $\mathrm{Fe}$ and nano $\mathrm{Fe}$ catalyst supported on silicon dioxide $\left(\mathrm{SiO}_{2}\right)$ from a slurry phase F-T reactor was the focus of this experiment. Conducting x-ray 
computed microtomography (CMT) at the National Synchrotron Light Source (NSLS) provided insight into the catalyst behavior and what can be done to achieve maximum efficiency at the lowest possible cost.

\section{Experimental Methods}

The interest for this experiment was to determine the time resolved behavior of a nano Fe catalyst in a waxy sample from a slurry phase F-T synthesis reactor. These samples were prepared under a condition of $513 \mathrm{~K}$ and $2.77 \mathrm{MPa}$ in a reactor, which ran for a continuous 120 hours. By using $15.7 \mathrm{~g}$ of $\mathrm{Fe}_{2} \mathrm{O}_{3}, 300 \mathrm{ml}$ of ethyflopolyolefin-64, $\mathrm{CO}$ and $\mathrm{H}_{2}$ in a ratio of $1 / 2$, hydrocarbons were produced. The $356 \mathrm{~g}$ of solid yield contained catalyst particles, solvents, and other hydrocarbons. ${ }^{3}$ Using the CMT procedure at the X2B beam line at the NSLS enabled the visualization of catalytic behavior over an extended period of time. This research is crucial in determining how to make a more efficient and cost-effective catalyst for F-T synthesis.

At the NSLS, electrons are injected into a vacuum and soon reach the booster ring, where their energy significantly increases, usually by a factor of about 10 . Once these electrons have reached such high-speeds and high-energies, synchrotron light is produced. Using a monochromator, the synchrotron light is dispersed into smaller segments that can be focused onto the sample. At the X2B beam line, a bending magnet is used as well as a Sibased monochromator to analyze data. After the beam is focused on the sample, a scintillator absorbs the high energy and relays it to a charge-coupled device (CCD) camera where the information is then collected. CMT is a non-destructive technique used to take 2-D images of a sample rotated $180^{\circ}$.

To perform a CMT run, the sample must first be collected and then mounted between the $\mathrm{x}$-ray source and detector. The samples were taken from a slurry phase F-T reactor before this experiment, and held in a vile for storage. For this experiment, a large enough sample size was taken out of the vile and placed on a sample holder on the rotating stage. The rotating stage plays an important role because it allows for the sample to rotate 180 or 360 degrees, thus allowing a complete set of data consisting of 2-D CMT images. After the CCD has focused on the sample, manual entry of data, such as how long the sample will be exposed to the $\mathrm{x}$-ray and what energy is being used, is entered into a computer and a run can begin. ${ }^{7}$ Several CMT imaging scans were performed on each of the 3 samples at $10 \mathrm{keV}$ using a $5 \mathrm{X}, 10 \mathrm{X}$, or $20 \mathrm{X}$ magnification lens.

Once the 2-D data are collected, they are analyzed using Exelis' IDL Virtual Machine, which will reconstruct image slices into 3-D volumes and provide a spatial map of the x-ray attenuation coefficient values. This coefficient is unique for different materials at different $\mathrm{x}$-ray energies, so it can be used to distinguish between the different materials present in a sample. A medium with a higher $\mathrm{x}$-ray attenuation coefficient will absorb more of the $\mathrm{X}$-rays than a medium with a lower coefficient. After the 3-D volumes have been reconstructed, ImageJ is used to view a histogram of the attenuation values throughout each sample and confirms that two phases are present throughout. A final program, Drishti, renders the reconstructed data and provides a $3-\mathrm{D}$ viewing of the sample and allows for the removal of unwanted constituents, such as the wax. Using Drishti also shows how the catalyst is distributed throughout the sample without an occurrence of agglomeration.

\section{Data and Results}

Several CMT imaging runs were performed on each of the 3 samples and unreacted $\mathrm{Fe}_{2} \mathrm{O}_{3}$. The results from a few of these runs are listed in Table 1.

The BASF (20-80 nm) catalyst, shown in Figure 3, was imaged using a $20 \mathrm{X}$ lens. Figure 3 (a) is one 2-D image slice from a scan containing the BASF sample, and (b) shows a 3-D volume for the same run. As seen in Figure 3, the data collected displays areas of both darkness and brightness. The darker areas typically represent the less dense material with a lower attenuation coefficient. Air and the wax in which the catalyst is dispersed are the species that will exhibit a lower attenuation coefficient. Brighter areas represent the Fe catalyst because of its high density and high absorbance of x-rays. To more definitively differentiate where the Fe is located, the National Institute of Standards and Technology (NIST) has published theoretical $\mathrm{x}$-ray attenuation coefficient values for many materials that can be compared to the values obtained from these CMT images in ImageJ. Table 2 lists theoretical NIST values as well as experimental values from ImageJ for each material present. Since the iron is only $5 \%$ by weight, we scale the theoretical values from NIST by $5 \%$ in order to compare to our experimental values. It is clear that the brighter parts in Figure 3 (a) are the Fe catalyst because the attenuation range found in the picture for the brighter spots correspond to those listed on NIST. A range of $21.5 \mathrm{~cm}-1$ to $43.3 \mathrm{~cm}-1$ was observed in the image while the theoretical value for $\mathrm{Fe}_{2} \mathrm{O}_{3}$ is $31.75 \mathrm{~cm}-1$. This confirms that $\mathrm{Fe}_{2} \mathrm{O}_{3}$ is present in the sample after being reacted.

Similarly, CMT scans of the Mach-1 (3 nm) sample were taken using a $20 \mathrm{X}$ lens. Figure 4 (a) shows one 2-D image slice from this scan, and (b) shows a 3-D volume for the same run. As seen with the BASF catalyst, $\mathrm{x}$-ray attenuation coefficient values ranging from 28.6-44.83 cm-1 were found, which shows that $\mathrm{Fe}_{2} \mathrm{O}_{3}$ is present in the sample and remains stable after a period of time.

CMT scans of the UCI $(32.5 \mu \mathrm{m})$ sample were taken using a 20 X lens. Figure 5 (a) shows one 2-D image slice from this scan, and (b) shows a 3-D volume for the same run. Similar to the other catalysts, $x$-ray attenuation coefficient values ranging from 30.95$49.81 \mathrm{~cm}-1$ were found, thus showing that $\mathrm{Fe}_{2} \mathrm{O}_{3}$ is uniformly distributed throughout the sample.

The unreacted $\mathrm{Fe}_{2} \mathrm{O}_{3}$, shown in Figure 6, ran at energy of 10 $\mathrm{keV}$ and a magnification of $10 \mathrm{X}$. An attenuation value range of 27.37-36.90 cm-1 was observed in the sample after being tested. Since we know that the fresh sample of the UCI catalyst contained unreacted $\mathrm{Fe}_{2} \mathrm{O}_{3}$ on $\mathrm{SiO}_{2}$, we were able to compare its attenuation to the theoretical values from NIST and found that the theoretical value for $\mathrm{Fe}_{2} \mathrm{O}_{3}$ lies within the range, confirming the presence of $\mathrm{Fe}_{2} \mathrm{O}_{3}$ after testing.

After calculating attenuation coefficients at different points in each of these samples and observing the reconstructed image, a conclusion can be drawn that the nano Fe catalyst is uniformly distributed throughout the entire sample.

\section{Conclusions}

The United States Department of Energy (DOE) believes that creating an efficient energy system, by addressing the energy, environmental, and nuclear challenges of the United States, is the key to prosperity and security within the nation. This experiment holds a significant level of importance and provides valuable in- 
formation for future tests. After analyzing the data and looking at important factors, such as attenuation coefficient, we were able to determine that the supported nano Fe catalyst was evenly distributed throughout the sample. Due to the uniform distribution, less catalyst is required to perform the same operations in the slurry phase F-T reactor. Since lesser amounts of catalyst are required, the process can run more efficiently and more cost effective. ${ }^{8}$

Future research that can be done following this experiment includes varying the time the F-T synthesis samples were taken at, trying new metal catalysts, and varying the system pressure and temperature. Since the samples were created based off a slurry phase reactor that ran for $120 \mathrm{~h}$, seeing the catalysts as they would be in production settings was not feasible. Running a reactor for a longer period of time will provide information on the suitability of the catalyst for the F-T process. This leads into observations regarding new metal catalysts, such as cobalt, that may enhance the F-T synthesis and make for an even more efficient and cost-effective catalyst. Lastly, changing the systems pressure and temperature will allow for the viewing of the catalyst's behavior and conditions more suitable for production.

\section{Acknowledgements}

This project was supported in part by the U.S. Department of Energy, Office of Science, Office of Workforce Development for Teachers and Scientists (WDTS) under the Science Undergraduate Laboratory Internships Program (SULI).

\section{References}

1 “Oil," Commonwealth of Pennsylvania, 2013, <http://www. portal.state.pa.us/portal/server.pt/community/types_of_ energy/4568/oil/460041> (20 July 2013).

2 "Crude Oil," Organization of the Petroleum Exporting Countries (OPEC), 2013, <http://www.opec.org/opec_ web/en/press_room/180.htm> (22 July 2013).

${ }^{3}$ K. W. Jones, H. Feng, A. Lanzirotti and D. Mahajan, Nuclear Instruments and Methods in Physics Research Section B: Beam Interactions with Materials and Atoms 241 (1-4), 331-334 (2005).

${ }^{4}$ H. Schulz, Applied Catalysis A: General 186 (1-2), 3-12 (1999).

5 "Fischer-Tropsch (FT) Synthesis," The National Energy Technology Laboratory (NETL), 2013, <http://www.netl. doe.gov> (17 July 2013).

${ }^{6}$ D. Mahajan, P. Gütlich and U. Stumm, Catalysis Communications 4 (3), 101-107 (2003).

${ }^{7}$ Michael J Paulus, Shaun S Gleason, Stephen J Kennel, Patricia R Hunsicker, and Dabney K Johnson, Neoplasia 2 (2000 January), 62-70 (2000).

${ }^{8}$ U. NARKIEWICZ, presented at the CoNan Intensive Programme, Gdańsk University of Technology, Gdańsk, Poland, 2009 (unpublished).

${ }^{9}$ NETL, edited by ftsynthesis, Vol. 36.89 KB.

${ }^{10} \mathrm{~K}$. Willacy, edited by df2 (2004), Vol. 5.78 KB.

VII. List of Tables

\begin{tabular}{|l|l|l|l|l|l|}
\hline Sample & \multicolumn{1}{|c|}{ Run Name } & \multicolumn{1}{|c|}{ Magnification } & \multicolumn{1}{c|}{ Energy } & \multicolumn{1}{c|}{ Views } & \multicolumn{1}{c|}{ Attenuation Value Range $\left(\mathbf{c m}^{-1}\right)$} \\
\hline UCI & x2b-124-3 & $20 \mathrm{X}$ & $10 \mathrm{keV}$ & 1200 & $30.95-49.81$ \\
\hline Mach-1 & x2b-124-4 & $20 \mathrm{X}$ & $10 \mathrm{keV}$ & 1200 & $28.6-44.83$ \\
\hline BASF & x2b-125-3 & $20 \mathrm{X}$ & $10 \mathrm{keV}$ & 1200 & $21.5-43.39$ \\
\hline $\mathrm{Fe}_{2} \mathrm{O}_{3}$ & $\mathrm{x} 2 \mathrm{~b}-127-2$ & $10 \mathrm{X}$ & $10 \mathrm{keV}$ & 1200 & $27.37-36.90$ \\
\hline
\end{tabular}

Table 1: Specifications of the runs at the X2B Beamline at the NSLS for each of the catalysts.

\begin{tabular}{|l|l|l|l|l|}
\hline Compound & $\begin{array}{c}\text { NIST Attenuation } \\
\text { Coefficient }\left(\mathbf{c m}^{2} \mathbf{g}\right)\end{array}$ & Density $\mathbf{( g / \mathbf { c m } ^ { 3 } )}$ & $\begin{array}{c}\text { Theoretical Attenuation } \\
\text { Coefficient } \mathbf{( \mathbf { c m } ^ { - 1 } )}\end{array}$ & $\begin{array}{c}\mathbf{5 \%} \text { of Theoretical Attenuation } \\
\text { Coefficient }\left(\mathbf{c m}^{-1}\right)\end{array}$ \\
\hline $\mathrm{Fe}_{2} \mathrm{O}_{3}$ & $1.21 \mathrm{E}+02$ & 5.24 & $6.35 \mathrm{E}+02$ & 31.75 \\
\hline $\mathrm{FeO}$ & $1.34 \mathrm{E}+02$ & 5.74 & $7.69 \mathrm{E}+02$ & 38.45 \\
\hline $\mathrm{Fe}_{3} \mathrm{O}_{4}$ & $1.25 \mathrm{E}+02$ & 5.17 & $6.47 \mathrm{E}+02$ & 32.35 \\
\hline $\mathrm{Fe}$ & $1.71 \mathrm{E}+02$ & 7.874 & $1.34 \mathrm{E}+03$ & 67 \\
\hline
\end{tabular}

Table 2: Attenuation coefficient values obtained from calculations and the experiment. 


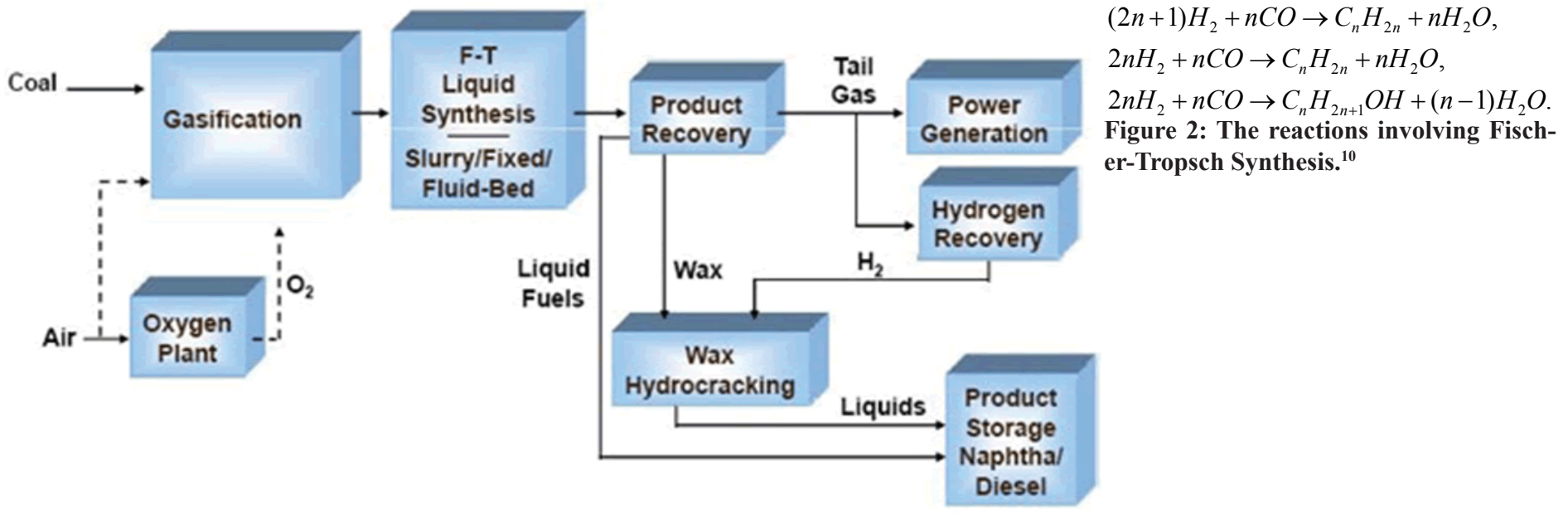

Figure 1: A gasification process involving the F-T synthesis reactions. ${ }^{9}$

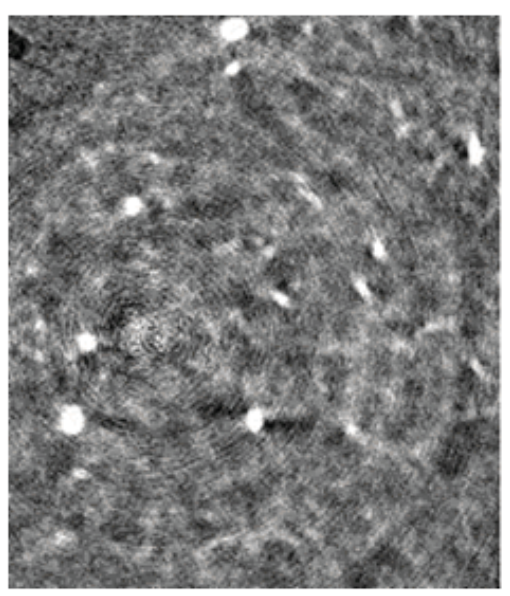

(a)

Figure 3: (a) A 2D Image Slice of BASF Catalyst (b) Reconstructed 3D BASF Catalyst
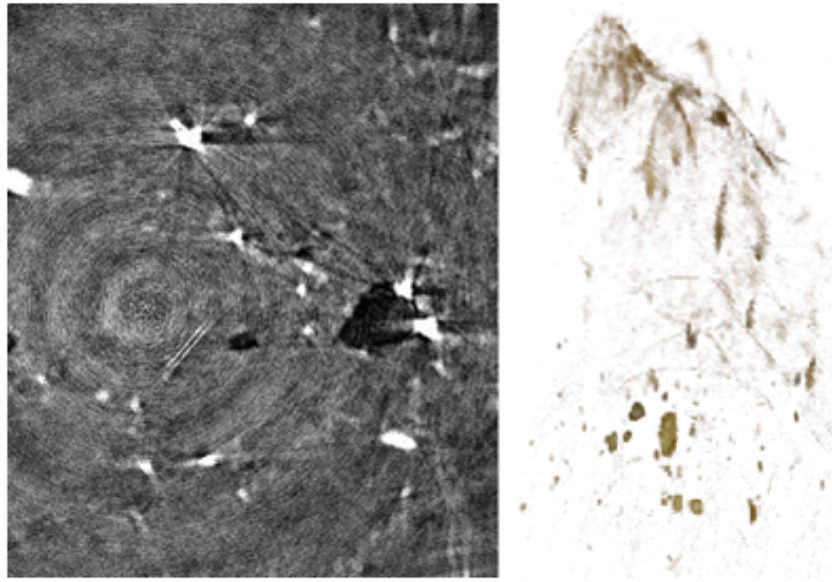

(a)

(b)

Figure 4: (a) A 2D Image Slice of Mach-1 Catalyst (b) Reconstructed 3D Mach-1 Catalyst

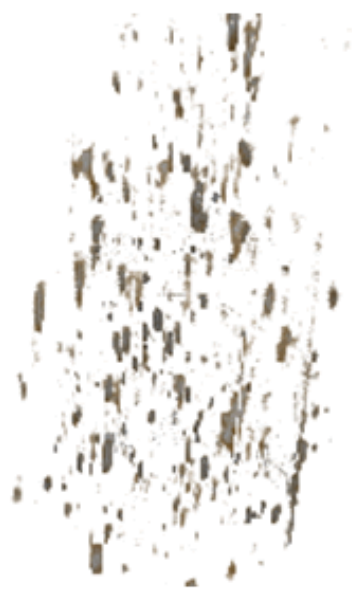

(b)
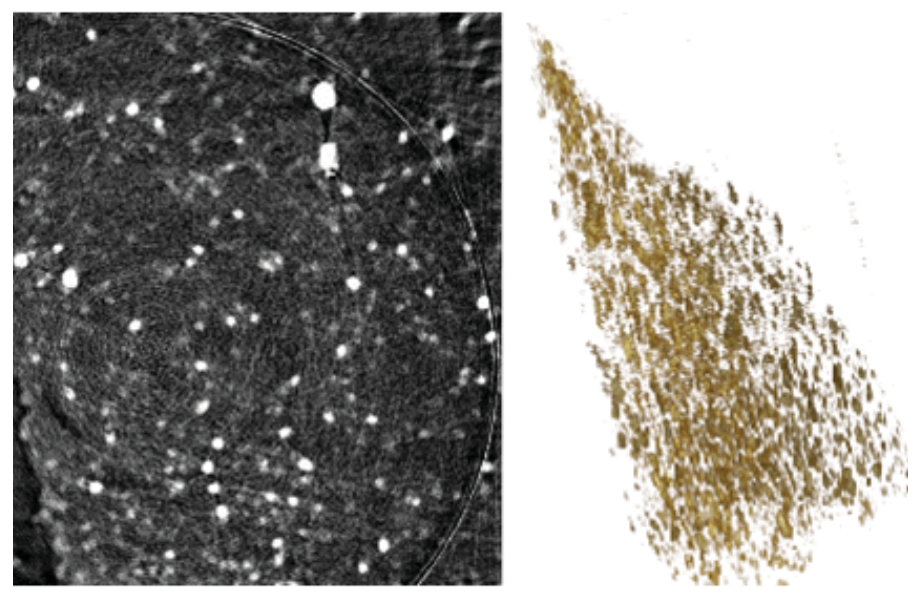

(a)

(b)

Figure 5: (a) A 2D Image Slice of UCI Catalyst (b) Reconstructed 3D UCI Catalyst
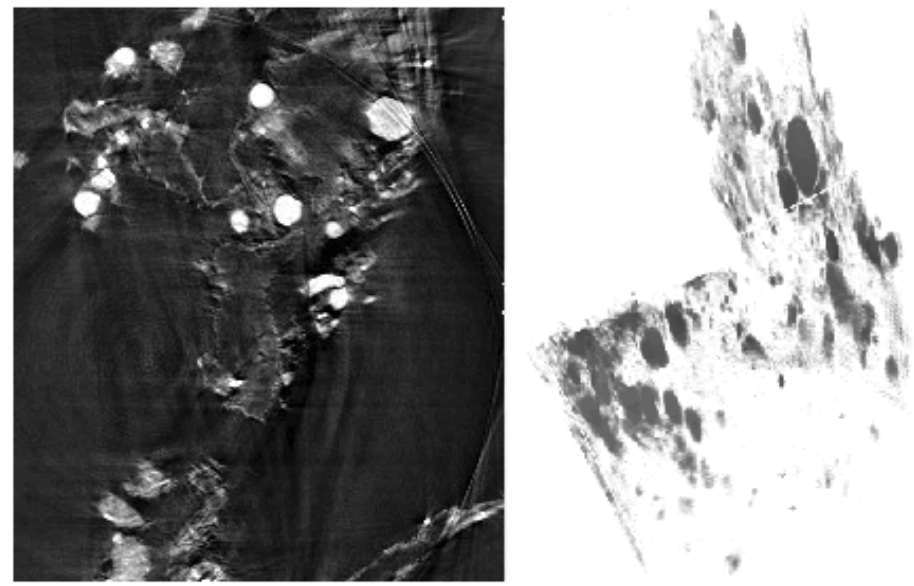

(a)

(b)

Figure 6: (a) A 2D Image Slice of unreacted Fe2O3 (b) Reconstructed 3D unreacted $\mathrm{Fe} 2 \mathrm{O3}$ 


\title{
Protein Crystallography Database programming, analysis, and modification
}

\author{
Kyle Davis \\ Photon Sciences Directorat, University of Delaware, Newark DE 19716 \\ Rick Buono \\ Photon Sciences Directorate, Brookhaven National Laboratory, Upton NY 11973
}

My work this summer as a Science Undergraduate Laboratory Intern (SULI) at Brookhaven National Laboratory (BNL) involved a wide range of computer programming and web development practice. I was stationed in an office at the National Synchrotron Light Source II (NSLS-II) building, under the guidance of my mentor, Rick Buono. Rick, also stationed at the NSLS-II, works primarily as a database administrator, computer software programmer, and systems analyst. His chief focus has been the development, evolution, modification, maintenance, and support of the Protein Crystallography Database, or PXDB. With his guidance, I was able to assist him with a large scale project involving this application. This project was supported in part by the U.S. Department of Energy, Office of Science, Office of Workforce Development for Teachers and Scientists (WDTS) under the Science Undergraduate Laboratory Internships Program (SULI). My primary objective concerning this project was to rewrite the PXDB application code using Python, along with selecting a web framework that will be utilized.

The Protein Crystallography Database -- BNL's PostgreSQL-based user-management and experiment tracking database system -- is a relational database system devised to provide experimenters with the ability to capture information related to macromolecular crystallography data collection at the National Synchrotron Light Source (NSLS). Utilized by all visiting scientists as well as the Protein Crystallography Research Resource scientific and administrative staff, PXDB is a web application that prepares, manages, tracks, records, and statistically analyzes every detail of the experimenters' NSLS beamline use. This may include, but is not limited to, scheduling, visits, projects, crystals, and x-ray data collections. The system architecture for this project currently utilizes the programming languages Perl, JavaScript, HTML, with embedded-SQL for the application code. There is a large-scale project currently underway to rewrite the application code for the PXDB, using the programming language Python for the underlying code. Using my previously acquired knowledge of Python, I was challenged to initiate this reconstruction, as well as develop and modify software to assist in this endeavor.

Having had only basic experience using the Python language, it was essential for me to first refresh my knowledge, as well as study more elaborate techniques that could aid in the ultimate completion of this project. Python is a very intuitive language with many advanced functions, and it was my goal to master these utilities. By reading a tutorial book entitled "Learning Python" I came as close as possible to completing this task. In order to initiate the reconstruction of the PXDB it was necessary to first commit to a web framework, the choices being Web2py and Django. Both frameworks are written, and programmable in Python. In addition they are both free and open source web frameworks, that follow the Model-View-Controller architectural pattern. This software pattern allows for the separation of concerns, provid- ing the ability to split the representation of information from the user's interaction with it. Similarities aside, there are many key differences between Django and Web2py that needed to be uncovered. It was my responsibility to delve into the workings of these two tools and compare their strengths and weaknesses, so the right framework could be chosen for the task ahead. By reading through the documentation of Web2py, via web2py.com, and Django, via djangobook.com and djangoproject.com, I was able to determine the pros and cons of each framework, as well as create a report stating this information. After a long process of studying, and experimenting with both of these frameworks, based off of its well written documentation, widespread community, and intuitive style, Django was our ultimate decision. Given that a language conversion is not an overnight task, the next step will continue to be an ongoing course of action for some time. Once a framework had been chosen and I had achieved a general understanding of the style of this utility, the process of translating the previously derived code into Django-Python code commenced. Beginning with a simple start-up screen, I was able to view this basic page's formerly written code, and recreate the screen using Django. While this process was underway I continued to explore this web development tool, uncovering more advanced techniques as I went. Progressing to more difficult pages, I soon became exceedingly accustomed to the process of implementing Django applications, producing pages identical to their predecessors in both style and function. In the time frame that I possessed I was able to recreate an estimated ten complete pages, ranging from straightforward screens such as a main menu and simple data display, to more intricate screens such as database entry and application forms. Unfortunately, being a very large-scale project, the complete recreation of the PXDB code will take an excessive amount of time, which because of my status as an intern, I did not have. As a result of my limited time it was decided that I was to construct a lesson-plan illustrating the basic processes of the Django framework. This lesson-plan will be used to optimize my colleagues' learning process, so they may understand and utilize the Django framework with ease and efficiency-- allowing for the further reconstruction of the PXDB.

My contribution to this project has paved the way for future integration, and improvement to the PXDB and other programs. By establishing a commitment to the framework that will be used towards the reconstruction of the application code, as well as initiating the modification process, I have not only saved time, but improved the quality of the outcome of this development. Python, as opposed to the previously implemented language Perl, is a current, more versatile programming language. It is used prominently by other departments within BNL, as well as being the primary programming language of major Fortune 500 Companies. Python will also adhere to standards currently discussed by National Laboratories to assimilate and regulate the software, hardware, and 
protocols utilized by the Labs. This conversion will establish a code base in the ever growing Python language, which will allow the PXDB to be continuously enhanced.

Having had essentially no experience with web development programming in the past, my time here at BNL has provided me with valuable tools for my future exploration in Engineering and Computer Science. I was able to learn a brand new programming style as well as significantly improve my understanding of web development, database programming, and the Python language in its entirety in comparison to the familiarity that I formerly possessed. I now recognize what the daily lifestyle at a laboratory institution is like: making difficult decisions, working with colleagues, and researching new topics I have never experienced before. As intricate and tiresome this process may have seemed, it has brought new light to my scientific career. 


\title{
Waveform digitizing electronics for time-of-flight detectors
}

\author{
Olivia Dickens \\ Department of Physics, Howard University, Washington, DC 20059 \\ Mickey Chiu \\ Department of Physics, Brookhaven National Laboratory, Upton, NY 11973
}

When cosmic rays enter the Earth's atmosphere they collide with molecules in the air and produce charged pions; which eventually decay into muons. The experiment that I assisted with at Brookhaven National Laboratory used the muons produced from cosmic ray interactions to test the timing resolution of a cost efficient time-of-flight detector. The two detectors used were a Multigap Resistive Plate Chamber (mRPC) and a Microchannel Plate Photo Multiplier Tube (MCP- PMT). I focused on the effect the electronics had on the timing resolution between the mRPC, MCP-PMT, and the data acquisition program for each. This was done by using a pulse generator to send signals through a cable to the wave form digitizer electronics. The timing difference between the signals was used to determine the amount of time the electronics added to the overall timing resolution of each detector. In order to get this number, I had to learn basic statistical analysis procedures and python programming. Based on the collected results, the root-mean-square (RMS) value for the timing resolution was 22.89 picoseconds, which is too slow for an efficient detector. By altering the amount of the time the electronics adds to the overall timing resolution of each detector, scientists working on the PHENIX project will be able to build better time-offlight detectors. They will also be able to run the Relativistic Heavy Ion Collider (RHIC) at higher energies; allowing for the identification of particles at higher momenta than currently obtainable.

The goal of this experiment was to determine whether or not it was possible for one to build a cost efficient time of flight detector with an overall timing resolution of ten picoseconds. In order to figure out the total timing resolution for the detector system, one would have to find the timing resolutions of both the detector and the electronics used with the detector separately. Assuming that the detector itself would take up a fraction of the time restraint, the electronics used would have to contribute less than eight picoseconds to the overall timing resolution. Whether or not this type of timing resolution is possible will depend on the type of electronics used and the signal to noise ratio for the electronics in question.
For the overall project, the two types of detectors used were multigap resistive plate chambers (mRPC) and microchannel plate photomultiplier tubes (MCP-PMT). In a MCP-PMT, a particle would travel through a fused quartz crystal and produce photons by Cherenkov radiation. These photons then traveled through a glass window with a photocathode on its backside; producing photoelectrons. The photoelectrons would then move through two glass plates lined with capillaries. As the photoelectrons passed through the capillaries, they would produce an avalanche of electrons. These electrons would accumulate on the cathode at the bottom MCP-PMT producing a signal.

For the mRPC a chamber, filled with a mixture of gases, would act as the holding cell for the detector. A particle would travel through the chamber, and the mRPC within the chamber, exciting the gas mixture inside. The gas mixture used for this project consisted of $95 \% \mathrm{r} 134 \mathrm{~A}$, which supplied the electrons needed for an avalanche, $4.5 \%$ isobutane, which acted as a quench gas to prevent too many electrons from building up, and $0.5 \%$ sulfur hexafluoride, which enhanced the effectiveness of the quench gas. The free electrons from the ionized gas would avalanche within the gaps of the mRPC producing various signals. The accumulation of the signals within the MRPC would be combined together in order to produce the main signal, which would be sent to the digitizing electronics. The electronics used to convert the signal for the MRPC were a PSI DRS4 Evaluation Board V3 and a customized preamplifier, while the electronics used for the MCPPMT was a CAEN DT5742 Desktop Digitizer.

This summer's portion of the project focused on the CAEN DT5742 Desktop Digitizer. The desktop digitizer is used to take analog signals from a detector and convert them into digital signals to be processed by a computer (Figure 1).

Signals taken from the detector are managed by digital pulse processing (DPP). DPP uses field programmable gate array (FPGA) firmware to filter the incoming data that will be sent to the computer for analyzing. This filtering allows the user to regulate the types of data that will be collected, and prevents the data acquisition program from taking in too many data points.

In order to get the signal to noise ratio for the electronics, the digitizer had to run continuously without any signal input. The

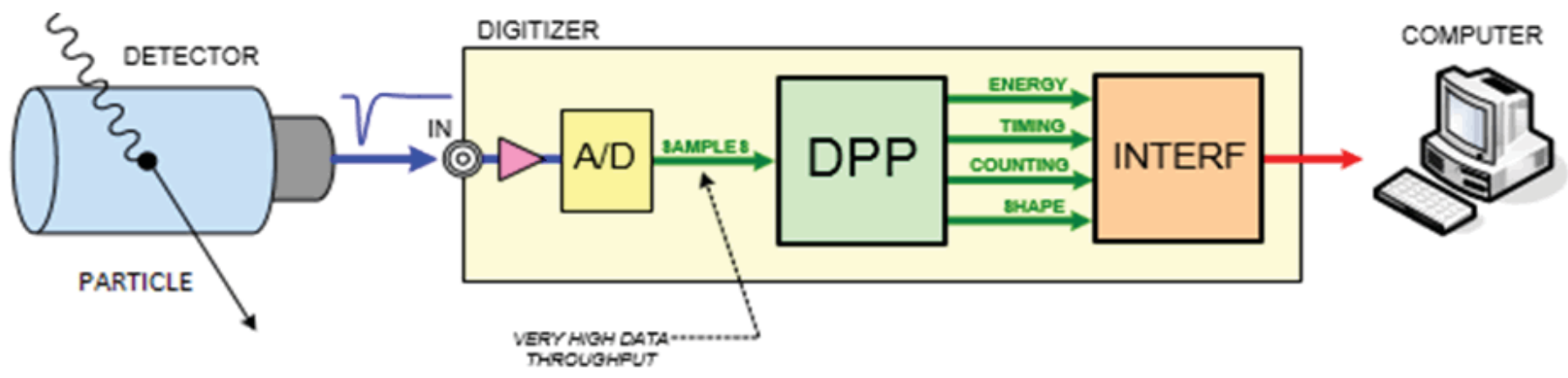

Figure 1 
FPGA firmware was programmed to recognize 233 events from the continuous input of data. Each event consisted of 1024 samples with 200ps between each event. This data was sent to the data acquisition software and statistically analyzed in order to extract the signal to noise ratio. If the FPGA firmware were not altered to only take in 233 event, the amount of data coming in from the digitizer would have overwhelmed the data acquisition software and possible shut down the computer.

For the timing resolution, an Agilent 33250A waveform generator was used to send a pulse through a cable connected to two channels of the digitizing electronics. The cable would split the pulse into two identical pulses and the difference between the amounts of time it took for the pulse to pass through each channel would be used to find the timing resolution of the digitizer. To prevent data overload, he FPGA firmware was modified to only take in data from the two specific channels and programmed to pay attention to the timing from the channel in picoseconds.

Analyzing the distribution for the signal to noise ratio (Figure 2A) gives a RMS value of 2.121, while the distribution for the timing resolution (Figure 3) gives a RMS value of 22.89. The re-
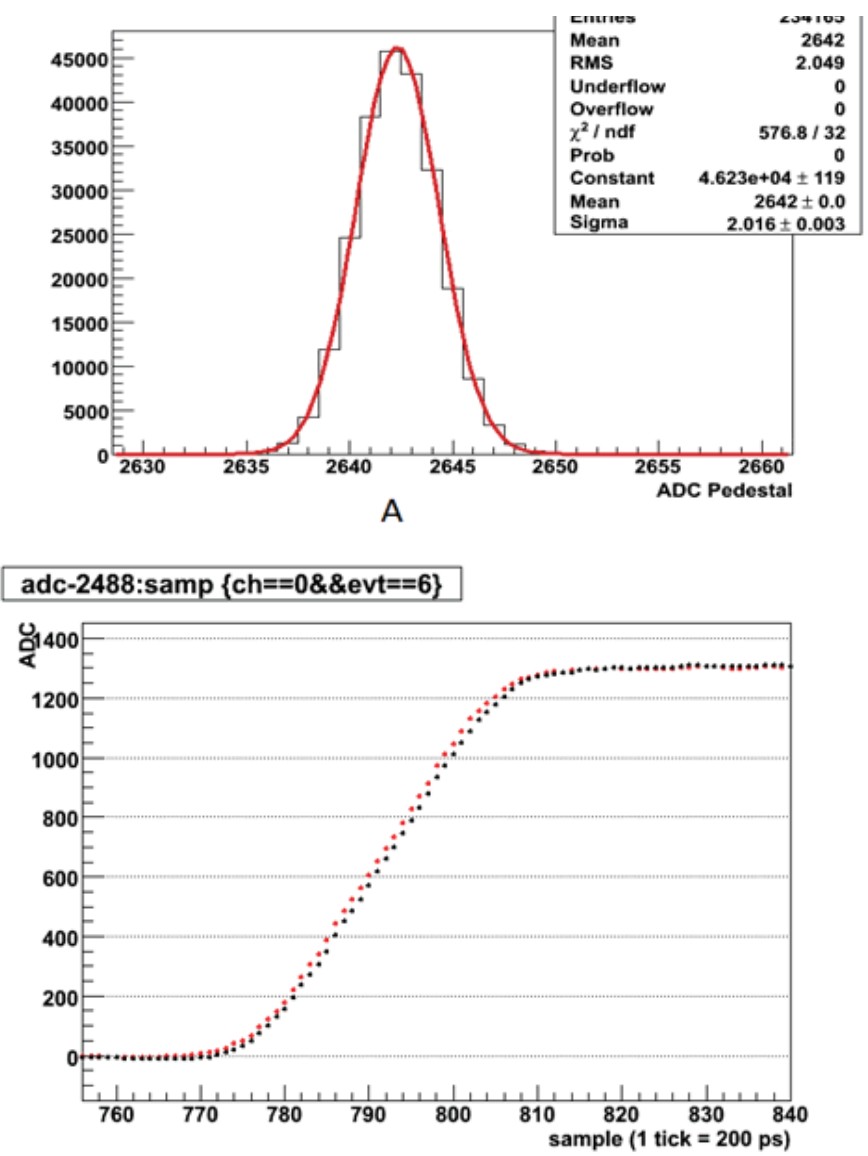

sults for both the timing resolution and the signal to noise ratio for the digitizer were significantly higher than expected. The RMS value for the timing resolution of the CAEN Desktop Digitizer was more than twice the RMS value for the timing resolution of similar, previously tested, digitizing electronic.

Our group concluded that this was due to the condition of the cables moving the signal and the quality of the power source to the digitizer. It was decided that we should change the power source from the traditional source that came with the digitizer, to a separate DC power supply which could provide more power. Sadly, this change had no effect on the timing resolution or signal to noise ratio for the electronics (Figure 2B).

Outside of altering the power supply and retiring some of the older wires, we were not able to significantly adjust the signal to noise ratio or the timing resolution collected for the electronics. Though the data collected from this summer's work was not within the range that we were expecting, more work will have to be done before we can determine whether or not the digitizer can be adjusted in order to contribute less noise and time to the overall detector system.
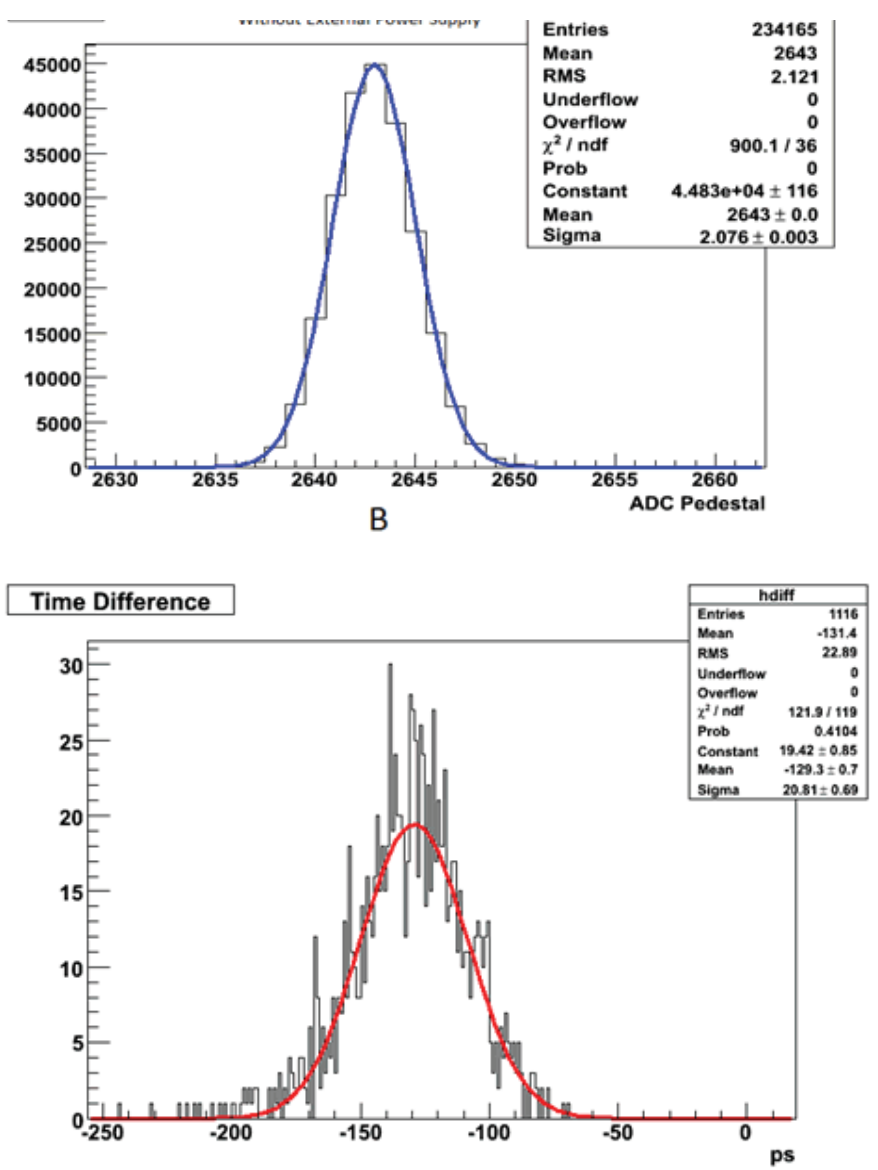

Figure 3 


\begin{tabular}{|l|l|l|l|}
\hline \multicolumn{1}{|c|}{ Participants } & \multicolumn{1}{|c|}{ Title } & \multicolumn{1}{c|}{ Institution } & \multicolumn{1}{c|}{ Role } \\
\hline Olivia Dickens & VFP participant & $\begin{array}{l}\text { Physics Department, Howard } \\
\text { University, Washington, DC } \\
20059\end{array}$ & $\begin{array}{l}\text { Finding and adjusting the timing } \\
\text { resolution and signal to noise ratio } \\
\text { for the digitizing electronics. }\end{array}$ \\
\hline Kahlil Dixon & SURP participant & $\begin{array}{l}\text { Physics Department, Howard } \\
\text { University, Washington, DC } \\
20059\end{array}$ & $\begin{array}{l}\text { Modifying the timing resolution } \\
\text { for the MCP-PMT. }\end{array}$ \\
\hline Nathaniel Lashley-Colthirst & SURP participant & $\begin{array}{l}\text { Physics Department, Howard } \\
\text { University, Washington, DC } \\
20059\end{array}$ & $\begin{array}{l}\text { Revising the timing resolution for } \\
\text { the mRPC and its components. }\end{array}$ \\
\hline Marcus Alfred & Faculty Mentor & $\begin{array}{l}\text { Physics Department, Howard } \\
\text { University, Washington, DC } \\
20059\end{array}$ & $\begin{array}{l}\text { Act as a mentor and aid in the } \\
\text { completion of the final project }\end{array}$ \\
\hline Mickey Chiu & Advisor & $\begin{array}{l}\text { Physics Department, Brookhaven } \\
\text { National Laboratory, Upton NY } \\
11973\end{array}$ & $\begin{array}{l}\text { Act as an advisor, explain the } \\
\text { parts of the project and facilitate } \\
\text { the completion of the project. }\end{array}$ \\
\hline
\end{tabular}

\section{Acknowledgements}

I would like to acknowledge each of the participants, Kahlil Dixon and Nathaniel Lashley-Colthirst, for helping with the completion of this project. I would also like to recognize my mentor, Mickey Chui, for explaining the project and the concepts behind it, and my faculty representative, Marcus Alfred, for aiding with the project and encouraging me to apply for this opportunity. This project was supported in part by both the Nuclear Regulatory Commission (NRC), and the U.S. Department of Energy, Office of Science, Office of Workforce Development for Teachers and Scientists (WDTS) under the Visiting Faculty Program (VFP).

\section{Scientific Facilities}

The facilities used to complete this project were the Physics Department in building 510, the Collider Accelerator Department in building 911, the PHENIX complex at building 1008 and the mRPC test lab in building 912. The mRPC test lab was where the entire project was pieced together and tested; the other buildings were used for meetings and simple data review.

\section{References}

[1] D. H. Perkins, "Introduction and Overview," in Introduction to High Energy Physics, 3rd ed., California, AddisonWesley Publishing Company Inc., 1987, pp. 1-25.

[2] D. H. Perkins, "Particle Detectors and Accelerators," in Introduction to High Energy Physics, 3rd ed., California, Addison-Wesley Publishing Company Inc., 1987, pp. 28-65.

[3] D. Halliday, R. Resnick, and J. Merrill, "Quarks, Leptons, and the Big Bang," in Fundamentals of Physics, Extended 3rd ed., vol. 2, New York, John Wiley \& Sons, 1988, pp. 1125-1137.

[4] G. F. Knox, "Photomultiplier Tubes and Photodiodes," in Radiation Detection and Measurement, 2nd ed., New York, John Wiley \& Sons, 1989, pp.251-260, 274-281.

[5] G. F. Knox, "Scintillation Detector Principles," in Radiation Detection and Measurement, 2nd ed., New York, John Wiley \& Sons, 1989, pp.239-247.

[6] CAEN Electronic Instrumentation, "Introduction to Digitizers", in Guide GD2080, CAEN S.p.A, 2011, PDF e-book. 


\title{
A performance study of the micro-channel plate photomultiplier tube
}

\author{
Kahlil Dixon \\ Department of Physics and Astronomy, Howard University, Washington, DC 20059 \\ Mickey Chiu \\ Department of Physics, Brookhaven National Laboratory, Upton NY 11973
}

\begin{abstract}
PHENIX, the Pioneering High Energy Nuclear Interaction Experiment at Brookhaven National Laboratory (BNL), is developing particle detectors of exceptional time resolution. These world-class detectors will serve as upgrade options for future modifications to the Phenix detector, a part of BNL's Relativistic Heavy-Ion Collider (RHIC). This summer, we strove to optimize the timing resolution of the prototypes to ten picoseconds. The most promising of the two being considered is a Photonis 85012xp micro-channel-plate photomultiplier tube (MCP-PMT). Our testing setup makes use of two scintillator paddles, to trigger on cosmic rays, and two MCPPMT prototypes, to determine the time resolution. After setup and testing we still have yet to reach our extraordinary goal of ten picoseconds; however, we are confident that this goal will be reached with more time. This project has been both intellectually challenging and enriching. I hope that I will continue to have the opportunity to work at BNL and participate in several more Phenix projects.
\end{abstract}

\section{Introduction}

In an effort to expand the capabilities of Brookhaven National Laboratory's (BNL) Relativistic Heavy-Ion Collider (RHIC) Phenix began exploring upgrade options for future installation. Consequently, our project is an exploration of the timing capabilities of the micro-channel-plate photomultiplier tube (MCP-PMT). In this project we set up a cosmic ray test and began the necessary modifications to achieve a time resolution of ten picoseconds. This technology, if used in the Phenix detector, could provide data pertaining to regions that are kinematically unobtainable with the currently installed technology. In addition, this could to RHIC becoming the world leader in time of flight detection technology.

\section{Progress}

To find the timing resolutions we setup a cosmic ray test. In the testing we used:

- Two Photonis 85012xp MCP-PMTs

- Two small scintillator panels with photomultiplier tubes

- DT5742 Desktop waveform digitizer with 16+1 channels

- Logic module and high voltage supply

- Test stand with clams and high voltage cables etc.

The two MCP-PMT detectors were arranged one over the other. This allowed us to be sure that the detected particles were muons form cosmic ray scattering. The separation and the width of each of the individual fused silica crystals gives a very small tolerance to the angle of the incoming particles; they must be close to perpendicular to the ground to be detected by both MCP-
PMTs. The scintillator panels where positioned around the top MCP-PMT; one above it on the stand and one below. These were connected to a logic module and set to fire on a coincidence between the two panels. This coincidence was what controlled our trigger for the waveform digitizer; this made it so that we only collected data when a cosmic ray passed though both our detectors. In our experiment incoming muons comes through the first scintillator panel and releases scintillator light that then strikes a photocathode. The photocathode will produce photo-electrons which travel across an electric potential amplifying the charge formed from the photo-electric effect about a million times; this is necessary to make the electrical pulse detectable. In the MCPPMT the muon creates an effect similar to that of a bullet breaking the sound barrier. The fused silica is used as a medium in which the speed of light is lower in the medium than in the air. The muon's initial downward velocity is near light speed. In a way, when this particle reaches the fused silica it could almost be thought as a breaking of the light barrier within that medium. Also, a cone of light is produced in this process. The photons formed from this effect, known as the Cherenkov Effect, interact with a photocathode. Then the electrons travel through the amplification micro-channel-plates also increasing the charge to a reasonable value. The digitizer will trigger and record the voltage changes due to the detected particles, assuming of course there is a coincidence in the scintillator panels.

More careful study must be completed before we can firmly say ten picosecond timing resolution has been achieved. The time resolutions that were achieved are still being reviewed and calculated; we have only had one run and, therefore, when the results are finalized they, most likely, will not be at our extraordinary goal. Noise was a small issue initially. However, this was not from our detector and will be resolved by switching from the DT5742 to our DRS4 evaluation board. We are carefully studying the correlations in hits between the top and bottom detectors. We found that some muons may come through one crystal on our top detector and pass through an unaligned crystal at the bottom MCP-PMT.

\section{Conclusion}

Overall, we are confident that this detector will be able to reach our ten picosecond goal. Only a few more adjustments need be made before we have the fully functioning prototype. Despite the extreme cost of the MCP-PMT detector, currently predicted to be approximately two million dollars per square meter, this technology will be a feasible consideration for future installation, specifically as a start time counter in Phenix. 


\section{Acknowledgements}

This project was supported in part by the U.S. Department of Energy, Office of Science, Office of Workforce Development for Teachers and Scientists (WDTS) under the Visiting Faculty Program (VFP).

Scientific Facilities: Building 912 at Brookhaven National Laboratory

\section{Appendix}

\begin{tabular}{|l|l|}
\hline Mickey Chiu & BNL Phenix Mentor \\
\hline Alfred Marcus & Howard University Visiting Faculty Program (VFP) professor \\
\hline Nathaniel Lashley & $\begin{array}{l}\text { Howard University VFP student intern. } \\
\text { Worked with the other high speed option; the multi-gap resistive plate chamber detector (mRPC) }\end{array}$ \\
\hline Olivia Dickens & $\begin{array}{l}\text { Howard University VFP student intern } \\
\text { Focused on the timing performance of the supplemental electronics such as the waveform digitizer, } \\
\text { High Voltage supply, logic modules, etc. (for both the mRPC and the MCP-PMT detectors) }\end{array}$ \\
\hline
\end{tabular}
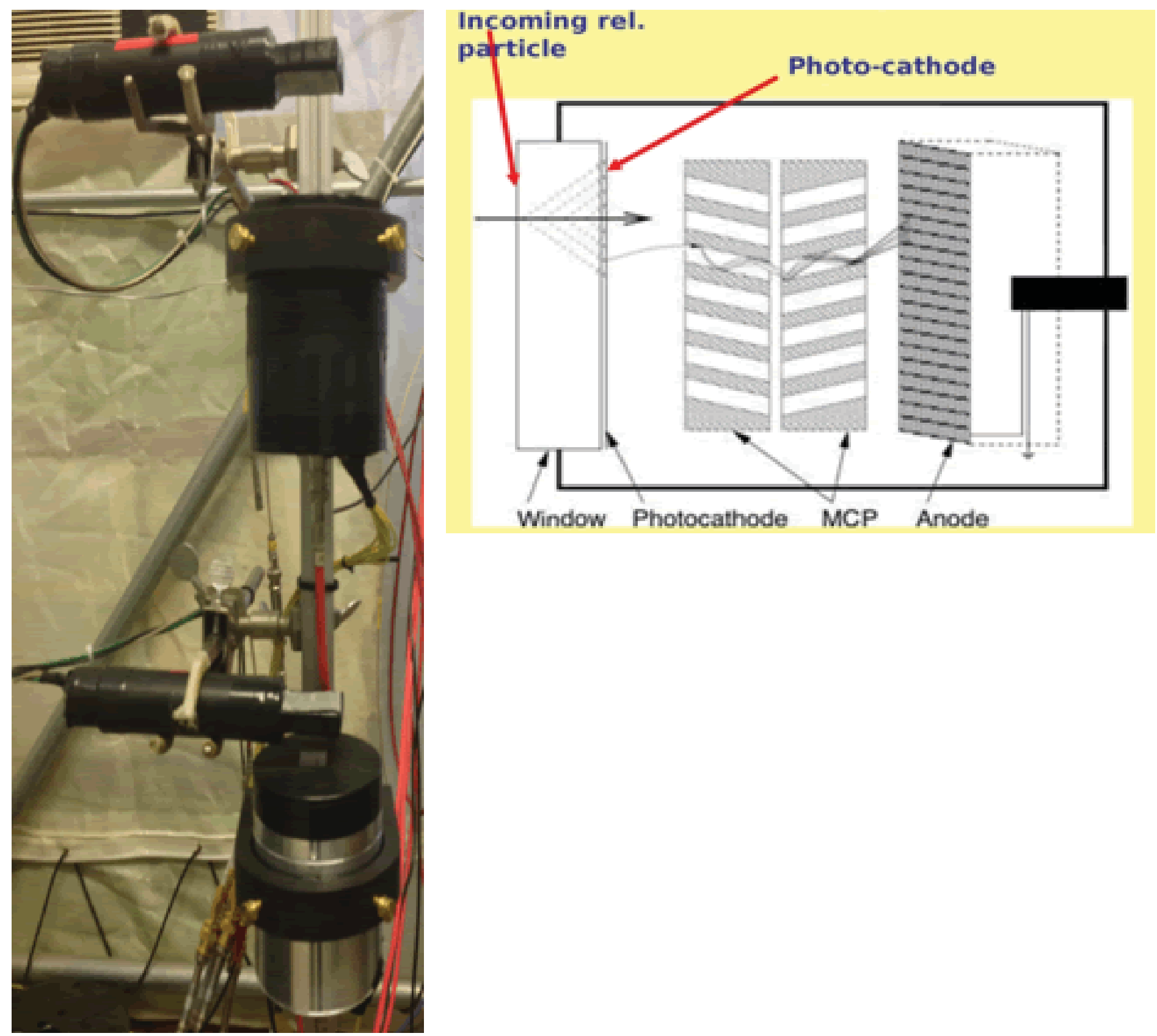


\title{
Analysis of cloud observations to determine its impact on solar panel output
}

\author{
Alison Egbon \\ Engineering, Stony Brook University, Stony Brook, 11973 \\ Michael Jensen and Mary Jane Bartholomew \\ Environmental Sciences, Brookhaven National Laboratory, Upton, NY 11973
}

\begin{abstract}
My research project consists of using remote sensing observations of cloud properties such as integrated liquid vapor (Lqint) from the microwave radiometer (MWR), and solar panel properties such as total power (TotP) from the broadband solar radiometer, to investigate the effects of clouds on solar power. Their comparisons showed that the output of the solar panel increases with total solar irradiance (CMPG) and decreases with Lqint. As a potential future environmental engineer, I explored the world an environmental engineer might venture into when trying to establish future green projects. I have improved my MATLAB skills and knowledge that will be useful in my engineering pursuit.
\end{abstract}

\section{Objective}

I predict that the total power will decrease as the integrated water vapor and global irradiance increases. If those properties increase, more light is reflected before the light reaches Earth's surface. As a result, there would be less total power output from the solar panel. In addition, I plan to at find at least one "bonus peak" from the data.

\section{Introduction}

Humans see the concept of "green energy" as one component towards the mitigation of global warming and the associated increase in surface temperature and sea level. Clouds play an important role in solar energy through the scattering of incoming solar radiation impacting the magnitude and vulnerability of solar radiation received at the surface. Solar panels are one "green energy" solution which converts solar radiation to electrical power. If we want to move from greenhouse gases to producing forms of clean energy, understanding the correlation between clouds and solar power is important. For this research project, I will use surface based observations of solar radiation and cloud properties to investigate the impacts of clouds on solar panel output.

Currently, the National Center for Atmospheric Research (NCAR) is working on a prototype that can foretell the amount of sunlight and resulting power every 15 minutes (Arm.gov). It will play a large hand in predicting clouds and diffusion particles that reduce incoming sunlight. Thus, solar farm managers will be able to supply the amount of energy when needed to its customers. I will be analyzing the properties that prevent sunlight from reaching the surface to understand when optimal power can be reached. This includes finding "bonus peaks" in which there is a spike in power output though it may be a cloudy day.

\section{Materials and Methods}

My three main devices were MATLAB, the MWR, and the broadband solar radiometer. MATLAB is a programming language I used to write, read, and run script files. The MWR measures the liquid water path in the clouds and the integrated water vapor in the atmosphere by detecting microwave emissions of these properties at two frequencies: $23.8 \mathrm{GHz}$ and $31.4 \mathrm{GHz}$. The broadband solar radiometer measures the amount of light that reaches the Earth's surface using a sensor with a rotating metal bar. The bar has three positions that represent three readings. In watts, the broadband solar radiometer takes readings norm to the surface, total diffusion when the metal bar covers the sensor and calibrates when the sensor is not covered.

For my procedures, I used MATLAB to compare the cloud and solar array properties. Firstly, I acquired the observations from the roof of $490 \mathrm{D}$ at BNL. I inserted the information on my account on cypher, a BNL server. These included the broadband radiometer observations of solar irradiance, MWR observations of integrated liquid water and output power from a single solar panel. Secondly, I acquired MATLAB from cypher to synchronize the observations in time. Lastly, I plotted the data and determined the correlations between the data.

\section{Discussion and Results}

My research showed a direct correlation between the data. I discovered that there is a linear relationship between the data of the cloud and solar array. For a given irradiance, power output decreases with greater Lqint. In addition, most of the power clustered around a low CMPG and Lqint.

Furthermore, my main difficulty was synching the data. I had to manipulate several script files to align the properties in the same time reference. But I had to also stop the data from overwriting for the month of May. For my first attempts, it only graphed the first day of May or the last day of May. I used a script file named match_times.m which contains a function that inputs data points and releases new variables. You input the time set you want to be sampled, dtin, the data points whose time you want to match, xin, and the desired output times. The function will output the time which was dtout and its data points. The output will be dtout and your new matched variable times xout. I also had to create a script file to save the new variables Lqintall, which symbolizes all the integrated water vapor points. After that, I saved all the sets of data to one script so I can easily graph the properties. I mainly used the plot function to analyze the data. I graphed using asterisks and other symbols because graphing a line would not give a good representation of the data.

I did not find any "bonus peaks" but a further study could identify them. I could pick a certain section of time for the TotP and compare the peaks over that course. For instance, if I look at TotP from eight in the morning to three in the afternoon, I could 
look at when the TotP would have large peaks. I would expect the peaks to have relatively low peaks before noon and high peaks after noon. Because the sun reaches its highest point around noon, the highest peaks should be around noon. However, if I see some high peaks around three in the afternoon, I would suspect that there are "bonus peaks." I would then look at the times of those peaks for CPMG and Lqint. I would contemplate what conditions must be set to get the "bonus peaks." What should be the angle of the sun? Does the CMPG and the Lqint have to be very low to give high peaks of power?

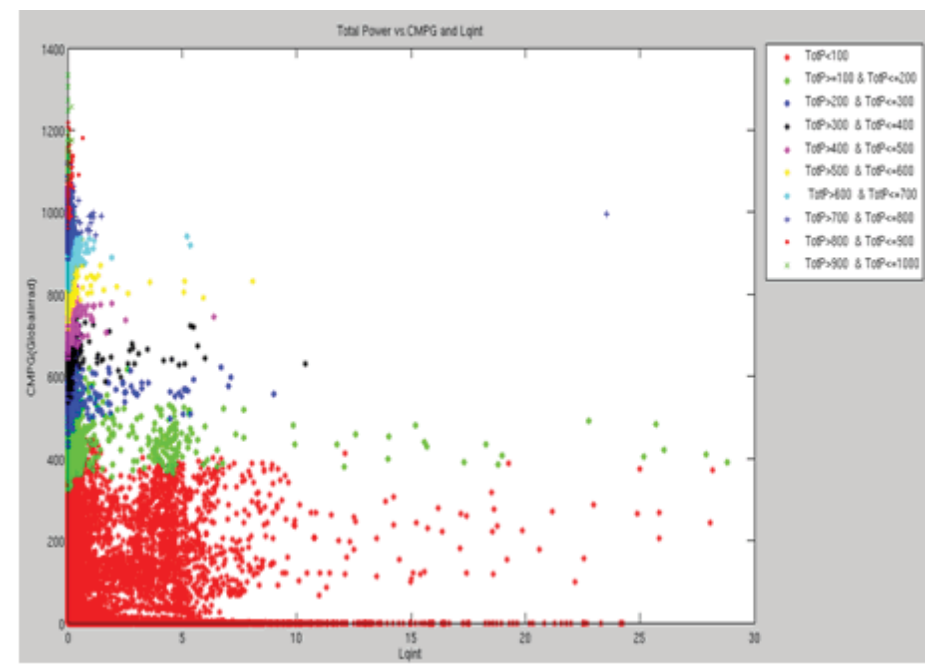

Figure 1

Figure $1 \mathrm{t}$ is a relationship between the TotP, Lqint, and the CPMG. Each color represents a range of power that varies from 0 to 1000 watts. There are 10 colors which signify that each color is a 100-watt power interval. Most of the points are clustered around a low CMPG of 200 and Lqint of 5. After the CMPG surpasses a value of 400, the TotP available decrease rapidly. The TotP becomes enclosed in an Lqint that is no greater than 3 in the graph. Because of the rapid change, the CMPG affects solar power output more than Lqint. If the CMPG is low, it is possible to yield high power output. In addition, on the graph is a dark blue spot at a Lqint of about 25. This point is separated from the rest which means there must have been an error in the reading. If there were more points clustered in that area, we could deduce that the area represents a "bonus peak."

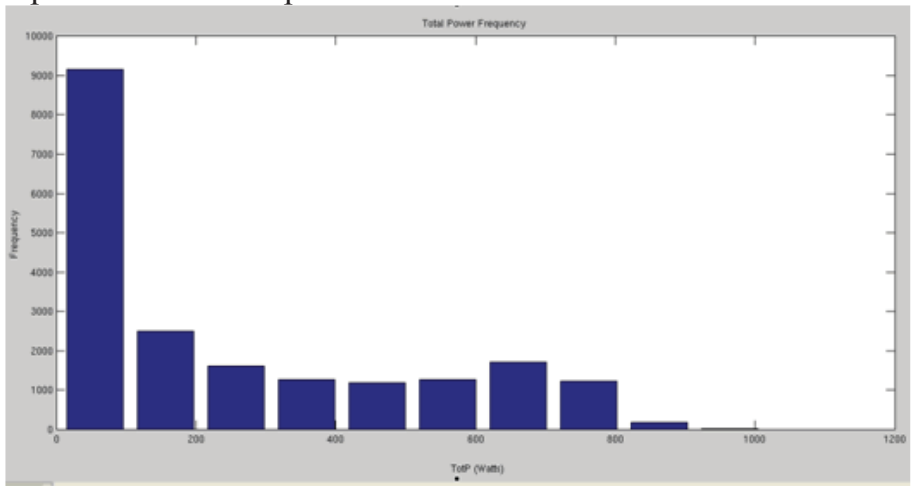

Figure 2
Figure 2 is a histogram of the TotP separated into 10 bins. As seen, most of the points are in the first bin and drastically decrease until there is a small increase for points between 600 watts and 800 watts. This might be the "bonus peak" the dark blue spot caused in the data. However, the data points whose power is lower than 5 watts were omitted. With the data included, the first bin is extremely large in which it was close to $3.5 \times 10^{\wedge} 4$ points. The other bins were really small compared to the first bin which made the data look a little unreal. Thus, I considered the values of 5 watts and lower as power that is close to zero.

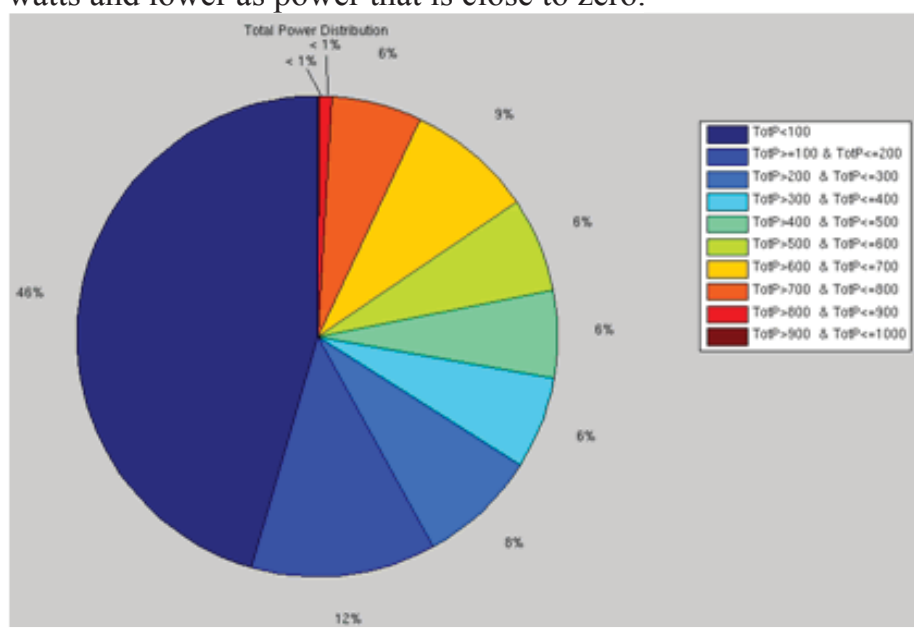

Figure 3

Similarly, the pie chart, Figure 3 have one large area of about $70 \%$ while the other nine parts were really small. Now, it seems more reasonable as the power below $100 \%$ only takes about $47 \%$ of the data. This pie chart is the best way to understand the amount of power you can acquire from a solar panel in a month in May. As seen, it would be difficult to acquire power more than 200 watts. The data below 200 watts account for about $60 \%$ of the data while the other slices each have less than $10 \%$ of the data.

\section{Further Study: Supplying power to Long Island Households}

A typical long island household uses 9,548 kilowatt hours (kwh) annually. This means about 795.6 kilowatts are consumed each month. From the pie chart, it can be seen that a typical solar panel generates most power in the range of 0 to 100 watts. If the solar panel is $119.7 \mathrm{~m}^{\wedge} 2$ and generates $3.2668 \times 10^{\wedge} 5$ watts monthly, a house will need about 3 solar panels to supply that power. You will need about $359.1 \mathrm{~m}^{\wedge} 2$ of land to place the solar panels. In addition, we must consider that the amount of land needed will only increase as we venture into the future.

According to Long Island Power Authority (LIPA), the current usage of power is a $268 \%$ increase from the annual usage of the mid-1950s. America has evolved from a Spartan-like society to a technology-ruled society. Power usage must be increased support the rise and use of technologies in the United States. These include air conditioners, fax machines, pc printers, electrical stoves, and televisions. Thus, we can expect the power usage rate to double to $536 \%$ in the next fifty to sixty years. The amount of land needed for one house would escalate to more than $500 \mathrm{~m}^{\wedge} 2$ of land. If we knew when the peaks of power could occur, we could garner power easily. 


\section{Acknowledgements}

This project was supported in part by the U.S. Department of Energy, Office of Science, Office of Workforce Development for Teachers and Scientists (WDTS) under the Science Undergraduate Laboratory Internships Program (SULI). I would also like to thank Brookhaven National Laboratory (BNL) and the Office of Educational Programs for this research opportunity.

\section{References}

Long Island Power Authority Summary:Recent Trends in Residential Electric Use, e U.S. Department of Energy (DOE)

Arm.gov 


\title{
In silico metabolic control analysis of Brassica napus seeds based on dynamic kinetic modeling
}

\author{
Michael Galazka \\ Housatonic Community College, Bridgeport Connecticut, 06604 \\ Jordan O. Hay \\ Biosciences Department, Brookhaven National Laboratory, Upton NY 11973
}

\begin{abstract}
Canola, a vital source of oil both for food and for fuel, is acquired from the developing seed embryos of Brassica napus. During the course of this summer, I used computer simulation software Computer Pathway Simulator (CoPaSi) and Python Simulator for Cellular Systems (PySCeS) to create a functional model of the metabolic pathway in $B$. napus responsible for the synthesis of lipids. The overall goal of this research is to eventually genetically engineer a new strain of $B$. napus that much more efficiently produces oils, and thus increases the availability, yield, and ease of harvesting canola oil. Using CoPaSi and PySCeS, I simulated large amounts of data relating to how various metabolites and reactions in the B. napus phosphoenolpyruvate branch point are sensitive to very minor changes in kinetic parameters (i.e., properties that effect the rate of enzymatic action). The end result of analyzing this data shows that of the two branches, the one beginning with Pyruvate Kinase contributes the greatest to lipid synthesis. The flow of matter through this branch, and therefore the overall amount of lipids synthesized, can be modified by changing variables of the system. Notably, modified enzyme activity in certain reactions such as Pyruvate Kinase or Phosphopyruvate Hydratase can drive up the amount of matter flowing through the oil producing branch of the system. To this end I learned a number of model simulation programs, as well as doing some programming for them. The overall experience I've gained from this summer at Brookhaven National Laboratory suggests that I need a stronger mathematics background, as I lack a deep understanding of the complicated calculus that makes up the backbone of all the data I've generated. I look forward to continuing my career in the sciences, aided by the things I've learned during my time here.
\end{abstract}

\section{Introduction and Background}

Canola oil is an important biofuel with applications for both food and industrial uses. Canola oil is harvested from the oil-rich seed embryos of the oilseed plant, Brassica napus. Although the metabolism that takes place inside $B$. napus is a very large network of over 572 reactions, ${ }^{1}$ for the purposes of our time here we focused on one small subset of this network, known as the phosphoenolpyruvate (PEP) branch point. In this small pathway, a series of 9 reactions and 11 metabolites operating in two separated branches work to synthesize proteins on one side, and lipids on the other. The goal of our research was to create a functional in silico dynamic kinetic model of the PEP branch point, and then perform a series of simulations aimed at discovering the best way to maximize the production of lipids in the developing embryos. These simulations were carried out via powerful metabolism modeling software, namely the programs CoPaSi (Complex Pathway Simulator) and PySCeS (Python Simulator for Cellular Systems). A dynamic kinetic model refers to a recreation of a metabolic pathway in which one can account for time, enzymatic flux properties, and subcellular compartments to the point of being able to create a functional, predictive simulation. A dynamic kinetic model will consist of some series of enzyme catalyzed reactions and their specific parameters (factors that can effect enzyme action). These include velocity (enzyme activity or its speed, which can be either forward or backward) and activation/inhibition constants (effects of substrates and modifiers on the reaction). ${ }^{2}$

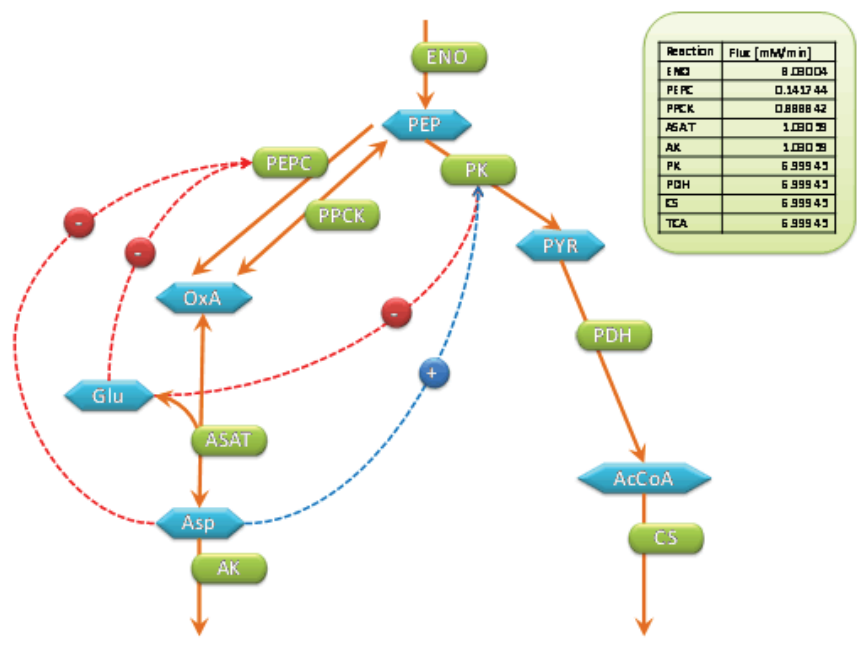

Figure 1. Structure of the PEP branch point dynamic kinetic model.

Starting from the top is PEP, which can be fed through either of the two branches. The PEP-carboxylase branch is responsible for directly creating amino acids. The Pyruvate-Kinase branch is more directly involved in the process of lipid biosynthesis. Both are allostericaly regulated and vital to the synthesis of oils in the developing seeds. Also present is a chart of flux rates at steady state, in $\mathrm{mM} / \mathrm{min}$.

\section{PEP Branch Point}

In the PEP branch point, one precursor reaction (Phosphopyruvate Hydratase, also known as Enolase or ENO) produces PEP, a vital metabolite involved in numerous pathways from glycolysis to seed oil synthesis. In this branch point, the PEP produced feeds into one of two branches, which will be abbreviated as the PEP Carboxylase / PEP Carboxykinase (PEPC/PPCK) branch which synthesizes proteins, and the Pyruvate Kinase (PK) branch which synthesizes lipids. The two branches, although occasionally sharing similar metabolites, do not interact with one another with the exception of two metabolites on the PEPC/PPCK branch that both influence the enzyme action of PK (aspartate and glutamate). Of particular note, the PEPC/PPCK branch contains a substrate cycle that operates almost outside of the influence of the rest of the pathway. PEPC functions as an irreversible reaction that turns PEP into oxaloacetate, with PPCK acting as a regulator that directs flux back towards PEP, allowing it to be redistributed to the opposite branch. PEPC and PPCK very strongly regulate 
each other; depending on the activity of PEPC, PPCK can act to move PEP back towards the PK branch or serve to feed PEP into aspartate transaminase (ASAT) should PEPC be attenuated. At the end of the PK branch is citrate synthase (CS), which acts as part of the Tricarboxylic Acid (TCA) cycle. Because both are accounted for in the dynamic model it is expected that they work to sort of cancel each other out. The data that can be gained from running simulations of this dynamic model includes reaction flux (the rate at which matter is moving through the specific reaction), concentrations of the different metabolites, control coefficients (a measure of the direct effect on flux or concentration that minute changes to enzyme activity cause), elasticity (a ratio of the rate of change of each reaction to changes in their constituent metabolites, in isolation) and sensitivities (a calculation of the direct changes to metabolite concentrations as a result of minute changes to the various kinetic parameters that make up each reaction). The goal of this data is to be able to analyze where matter flows through the PEP branch point pathway, and where making changes to reaction parameters or enzyme activity rates can affect this flow of matter and ultimately the buildup of finished metabolites and products, ultimately oil.

\section{A. CoPaSi}

CoPaSi (Complex Pathway Simulator) is a graphical user-interface-based program meant for running rigorous calculations and simulations on metabolic models. ${ }^{3,4}$ Although daunting at first, it is fairly straightforward given an understanding of enzyme kinetics. A model built in CoPaSi can either be built manually, inputting every reaction, metabolite, and rate law by hand, or it can be built automatically by the importation of a Systems Biology Markup Language (SBML) file. My colleague Mark Jensen was responsible for building a Python script which generates an SBML file; thus it is as simple as importing this file to have to latest revision of the dynamic model. Once the model has been input into $\mathrm{CoPaSi}$, the program is capable of running a series of simulations and calculations based on the model data. Of particular note, CoPaSi is able to calculate the concentrations and flux across the pathway once it reaches steady state (that is, once the system stabilizes and concentrations no longer change). The program can also run a metabolic control analysis, which provides vital data as to the system's control coefficients. A separate mathematical task can analyze the specific sensitivities of flux to miniscule changes in each of the kinetic parameters that make up the reactions. Perhaps the most useful function of CoPaSi to my work is the parameter scan, which runs a series of simulations and calculations based on specified changes in any specific factor of the pathway. In my work, I used the parameter scan to analyze effects on reaction flux that occur as a result of amplification and attenuation of enzyme activities.

\section{B. Automation \& PySCeS}

One of my tasks in kinetic modeling has been to attempt to create an automation script that would allow for importation of virtually any SBML file and automatically run the aforementioned simulations on the model. There exist two different versions of $\mathrm{CoPaSi}$, with the same functions but differing interface. CoPaSi$\mathrm{UI}$ is the graphical-interface variant, which I made extensive use of. CoPaSiSE is based entirely on the command line, and is meant for quick execution of simulations for very large, work intensive models. The command line version allows for some Python-based coding, but making a completely automated script would require significant time investment, coding experience, and use of other programming languages (such as $\mathrm{C}$ ). For the purposes of creating the script, I learned the use of another program, known as PySCeS (Python Simulator for Cellular Systems). ${ }^{5}$ PySCeS itself has no interface; it runs entirely as a plugin to the Python coding language. In essence, PySCeS uses a series of mathematical Python plugins to run the required calculations to complete the same sort of simulations that CoPaSi is capable of. As it is entirely Python based, it was much simpler to create an automation script that implements PySCeS; the resulting script that I wrote takes any SBML file and outputs all the relevant metabolic control analyses, sensitivity data, steady state calculations, and so on. The script itself has since been used and improved upon significantly by Dr. Hay, who simplified the code and added more functions. Unlike both versions of $\mathrm{CoPaSi}$, this script requires no direct input or participation from a user aside from pointing it towards the SBML file. The result is a script that will accept any dynamic model in SBML format and produce the same type of data each time. This is useful both for revisions of this or any other specific dynamic model, and also for the eventual analysis of the full B. napus metabolic network.

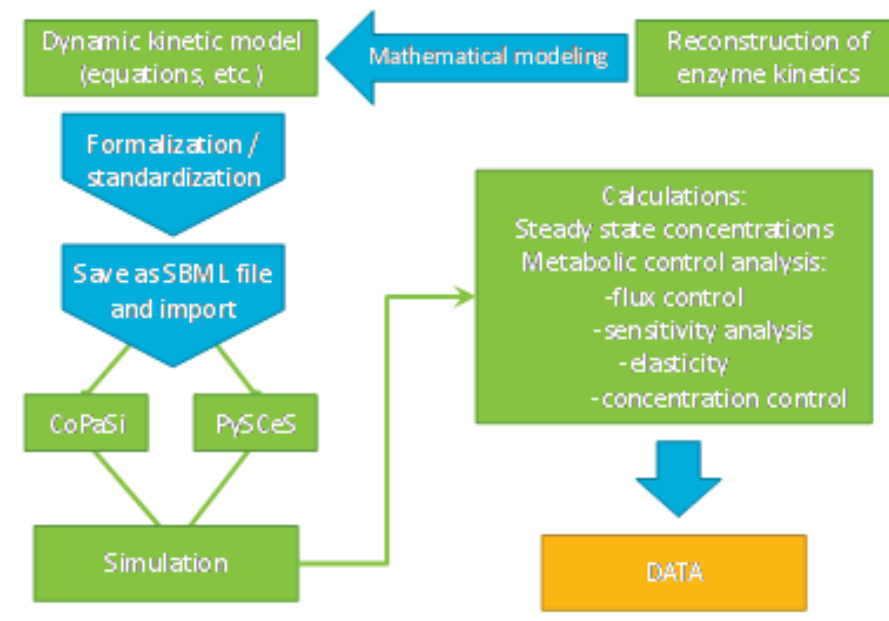

Figure 2. Description of workflow for in silico analysis of PEP branch point metabolism.

\section{Results}

The simulations provided large amounts of usable data describing the various aspects of the PEP branch point. The main portion of this data is raw data; much of it was summarized and compiled as a series of tables and charts. The majority of the data was based on the steady state, the point at which metabolite concentrations as well as reaction flux cease to change and instead stabilize. Table 1 demonstrates the concentrations and accumulation rates for the main metabolites at the steady state, while Table 2 shows the steady state flux of each reaction. These numbers represent the system at a state where the amount of material entering the system is equal to the amount leaving it. Assuming the parameters and rate laws that make up a system are correct, steady state is simulating the system as it would function in a homeostatic, in situ environment. In this case, the steady state calculations demonstrate the unperturbed function of the PEP branch point in $B$. napus. The remaining data represents parts of the system, and how they respond to changes. Table 3 shows the elasticities, a ratio of the rate of change of the system's reactions in isolation when concentrations of their metabolites change. Tables 4 and 5 
are all control coefficients, which show a scaled value representing how minimal perturbations enzyme activity effect the metabolite concentrations (Table 4) or the reaction fluxes (Table 5). Figure 3 contains graphs of parameter scans for each reaction. In each parameter scan, flux for all 9 reactions was calculated for enzyme activities between $0.01 \mathrm{x}$ to $100 \mathrm{x}$ the normal activity. These graphs demonstrate the effects of attenuation and amplification of enzyme activities and their effects on flux for each reaction.

\section{Data Analysis}

The majority of the connections I made in the analysis of the data of the simulated model verify assumptions that can be drawn from the structure of the model itself. CS and TCA function to cancel each other out for the most part. The flux through PK, Pyruvate Dehydrogenase (PDH), CS, and TCA (the PK branch) is the same for each individual reaction. Likewise, the flux through Aspartate Transaminase (ASAT) and AK (both of which are part of the PPCK/PEPC branch) are also the same; both of these branches are considered linear pathways and thus will usually share the same flux. Additionally, the flux through any part of both of the two branches will add up to the flux of ENO at steady state. This makes sense as the flux through the entire system should logically be equal to the flux entering the system. As ENO is the precursor reaction that governs all matter that ultimately flows through the pathway, increases to the enzyme activity of ENO will cause increases in the flux of both branches. Additionally, the data on steady state fluxes demonstrates that roughly seven times more matter flows through the PK branch than through the PEPC/ PPCK branch, or more specifically a ratio of $87 \%: 13 \% \mathrm{PK}: \mathrm{PEPC} /$ PPCK. Changes to ENO enzyme activity affect flux in the PK and PEPC/PPCK branches in the same ratio. Significant attenuation of ENO activity will lead to the system nearing fluxes of 0 across all reactions, as no matter entering the system will prevent flux throughout the system. ENO is also minimally affected by changes to reaction flux across all reactions except for itself and PK. PEPC and PPCK exist in a substrate cycle; changes in their activity affect one another as well as modifier metabolite concentrations (PEPC is inhibited by glutamate and aspartate), but otherwise do not affect flux or concentration significantly elsewhere in the pathway. Consistently across all the data I've simulated, PDH was shown to have no or minimal effect on flux, even when its activity is modified. This may be because PDH is an irreversible, one substrate to one product reaction with no allosteric modifiers. $\mathrm{PDH}$ is also preceded by a strictly irreversible reaction (PK) and is followed by CS/TCA, which together have no flux control over the system.

In regards to elasticities, the table of elasticity (Table 3) data shows a number of 0 values: these are metabolites that are absent in those specific reactions. As elasticities are a ratio of rate of change to metabolite concentrations in isolated reactions, metabolites that have no role in a reaction will have no effect on rate of change. A number of metabolite elasticities are almost equal and of the opposite sign- these represent reversible reactions, where the rate of change is dependent on the direction of the reaction. Aspartate transaminase (ASAT) presents a few interesting properties. ASAT enzyme activity alterations have an almost negligible effect on the flux of all reactions, as well as a minimal effect on metabolite concentrations (mostly affecting oxaloacetate and aspartate). However, the elasticities of oxaloacetate and aspartate compared to ASAT are abnormally large, at \pm 277 whereas all oth- er elasticities lie between 0 and \pm 10 . Based on the elasticities, aspartate has a positive effect on PK while negatively impacting PEPC: this is consistent with the branch point pathway as we know it in that aspartate is an activator of PK and an inhibitor of PEPC.

\section{Future Work}

The ultimate goal of this research into improving B. napusreaction flux is to increase the rate at which oils are synthesized in the developing embryos of oilseeds. This will eventually be accomplished by genetic engineering or selective breeding of the $B$. napus plant, to increase concentrations or enzyme activities of the parts of the pathway which feed into lipid synthesis. In time, having $B$. napus seeds with greater quantities of oil will serve to increase the yield of canola crops as well as the ease of harvesting larger quantities of canola oil. Over longer periods of time, this can lead to increased availability of canola as a biofuel in a world where renewable energy sources are becoming increasingly vital to our survival. Increased supply will also drive down the overall costs of canola oil, as well as potentially other types of fuel if used in mixtures, such as how ethanol and gasoline and mixed.

\section{Conclusions}

Although our work on the PEP branch point was thorough, it represents only a tiny fraction of the metabolism taking place in Brassica napus. The biggest impact of my work has been learning the various modeling software and being able to instruct my mentor in their use, along with creating a functional automation script for him. With the wealth of data I've managed to simulate I have demonstrated useful aspects of both CoPaSi and PySCeS, and their ability to potentially handle much larger metabolic systems. As for the actual data for the PEP branch point, the PK branch exhibits significant control over the rest of the system. Perturbations to PK enzyme activity can drive up the flux through the PK branch while taking flux away from the PEPC/PPCK branch. Although ENO activity has an effect on every reaction's flux, $\mathrm{PK}$ is the only enzyme to directly affect the flux of ENO itself. Interestingly, increased PEPC activity serves to drive up the concentration of aspartate in the PEPC/PPCK branch, which ultimately acts as an activator towards $\mathrm{PK}$ and drives up flux throughout the PK branch. PPCK, part of a substrate cycle with PEPC, can be amplified to bring up the flux of both the PEPC/PPCK cycle and ultimately the PK branch. All of these modifications can serve to increase PK branch flux, and theoretically drive up the production of oil in B. napus embryos.

\section{Acknowledgements}

I would like to thank my mentor Jordan O. Hay, and his colleagues Jörg Schwender, Inga Hebbelmann, Alistair Rogers, and Dhiraj Naik. Also thanks to Ann Emrick and Denise Monteleone for their assistance in providing our workstations.

Financial support to Jorg Schwender (PI) and Jordan Hay for quantitative analysis and modeling of central metabolism in plants is greatly acknowledged (Division of Chemical Sciences, Geosciences, and Biosciences, Office of Basic Energy Sciences, Field Work Proposal BO-133).

This project was supported in part by the U.S. Department of Energy, Office of Science, Office of Workforce Development for Teachers and Scientists (WDTS) under the Community College Internships Program (CCI). 


\begin{tabular}{|l|l|l|l|}
\hline \multicolumn{1}{|c|}{ Project Participants } & \multicolumn{1}{|c|}{ Departicipant } & \multicolumn{1}{|c|}{ Institution } & \multicolumn{1}{c|}{ Participant's Role } \\
\hline Jordan O. Hay & Bio Sciences Department & $\begin{array}{l}\text { Brookhaven National Laboratory, } \\
\text { Upton, NY 11973 }\end{array}$ & Project Mentor \\
\hline Michael C. Galazka & CCI Intern & $\begin{array}{l}\text { Housatonic Community College, } \\
\text { Bridgeport, CT 06604 }\end{array}$ & Kinetic Modeling \\
\hline Mark V. Jensen & CCI Intern & $\begin{array}{l}\text { Suffolk County Community College, } \\
\text { Selden, NY 11784 }\end{array}$ & $\begin{array}{l}\text { Python Scripting for SBML } \\
\text { Automation }\end{array}$ \\
\hline Bradley T. Salvato & SULI Intern & $\begin{array}{l}\text { College of DuPage, Glen Ellyn, IL } \\
60137\end{array}$ & Mathematical Modeling \\
\hline
\end{tabular}

\section{References}

[1] - Hay, J., \& Schwender, J. (2011). Metabolic network reconstruction and flux variability analysis of storage synthesis in developing oilseed rape (Brassica napus L.) embryos. The Plant Journal , 67(3), 526-41.

[2] - Rohwer, J. M. (2012). Kinetic modeling of plant metabolic pathways. Journal of Experimental Botany, 63(6), 2275-92.

[3] - Hoops, S., Sahle, S., Gauges, R., Lee, C., Pahle, J., Simus, N., Singhal, M., et al. (2006). COPASI--a COmplex

PAthway SImulator. Bioinformatics (Oxford, England),

22(24), 3067-74.

[4] - Mendes, P., Hoops, S., Sahle, S., Gauges, R., Dada, J., \& Kummer, U. (2009). Computational Modeling of Biochemical Networks Using COPASI. (I. V. Maly, Ed.) Systems Biology (XII, 460., Vol. 500). Totowa, NJ: Humana Press.

[5] - Olivier, B. G., Rohwer, J. M., \& Hofmeyr, J.-H. S. (2005). Modelling cellular systems with PySCeS. Bioinformatics (Oxford, England), 21(4), 560-1.

\section{Appendix}

Table 1. Steady state analysis of B. napus oilseed metabolites, in silico

Steady state is the point at which concentrations cease to change- the system has reached a plateau and no longer changes. The concentration at steady state represents the amount of each metabolite when the system stabilizes. Transition time represents the amount of time it takes each metabolite to reach steady state. Values calculated via CoPaSi software

\begin{tabular}{|l|r|r|r|}
\hline & Concentration $\mathbf{( m M )}$ & Accumulation Rate (mM/min) & Transition Time (min) \\
\hline AcCoA & 0.000700063 & $8.71 \mathrm{E}-16$ & 0.000100017 \\
\hline Asp & 14.7446 & $-4.31 \mathrm{E}-14$ & 14.307 \\
\hline OxA & 5.02035 & $4.44 \mathrm{E}-14$ & 0.625197 \\
\hline PEP & 1.22854 & $-1.74 \mathrm{E}-15$ & 0.152993 \\
\hline PYR & 0.0419944 & 0 & 0.00599967 \\
\hline
\end{tabular}

Table 2. Steady state fluxes of B. napus oilseed metabolites, in silico

Flux is the rate at which mass flows through the specific reactions, at the steady state point. It represents the rate at which metabolites move, and how they move, through the various reactions of the PEP branch point. Values calculated via CoPaSi software.

\begin{tabular}{|l|r|}
\hline \multicolumn{1}{|c|}{ Reaction } & Flux (mMol/min) \\
\hline ENO & 8.03004 \\
\hline PEPC & 0.141744 \\
\hline PPCK & 0.888842 \\
\hline ASAT & 1.03059 \\
\hline AK & 1.03059 \\
\hline PK & 6.99945 \\
\hline PDH & 6.99945 \\
\hline CS & 6.99945 \\
\hline
\end{tabular}


Table 3. Elasticities of metabolites in B. napus PEP branch point (scaled). Dimensionless.

Elasticity is a mathematical calculation of the ratio of the rate of change of the reactions compared to the value of a specific reaction parameter. Each elasticity is calculated only locally- it does not take into account the entire system of reactions, but only the reaction in which the metabolite being effected is present in. Calculated via CoPaSi software.

\begin{tabular}{|c|c|c|c|c|c|c|}
\hline \multicolumn{7}{|c|}{ Metabolite concentration being changed } \\
\hline & & AcCoA & Asp & OxA & PEP & PYR \\
\hline \multirow{5}{*}{ 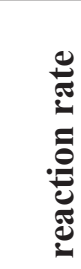 } & ENO & 0 & 0 & 0 & -0.2361 & 0 \\
\hline & PEPC & 0 & -1.14784 & 0 & 1.84818 & 0 \\
\hline & PPCK & 0 & 0 & -9.36831 & 9.38428 & 0 \\
\hline & ASAT & 0 & -277.757 & 277.763 & 0 & 0 \\
\hline & AK & 0 & 0.931294 & 0 & 0 & 0 \\
\hline \multirow{4}{*}{ 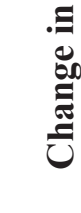 } & PK & 0 & 0.818849 & 0 & 0.847891 & 0 \\
\hline & PDH & 0 & 0 & 0 & 0 & 0.588268 \\
\hline & $\mathrm{CS}$ & 0.97328 & 0 & 0.003968 & 0 & 0 \\
\hline & TCA & 0.97328 & 0 & 0.003968 & 0 & 0 \\
\hline
\end{tabular}

Table 4. Concentration control coefficients in B. napus PEP branch point (scaled). Dimensionless.

Concentration control coefficients are a mathematical measure of the specific sensitivity that the major metabolite concentrations have to very miniscule changes in enzyme activity. The data below shows which enzyme activities have a greater, lesser, or negligible effect on the overall concentrations of the metabolites at steady state. As expected by the Summation Theory for concentration control coefficients, each row of coefficients adds up to 0. Calculated via CoPaSi software.

\begin{tabular}{|c|c|c|c|c|c|c|c|c|c|c|}
\hline \multicolumn{11}{|c|}{ Enzyme activity being changed } \\
\hline & & ENO & PEPC & PPCK & ASAT & AK & PK & PDH & CS & TCA \\
\hline \multirow{5}{*}{ 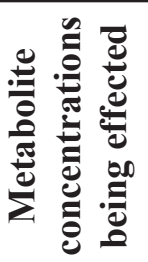 } & $\operatorname{AcCoA}$ & 0.9420 & 0.0008 & 0.0049 & 0.0002 & -0.1268 & 0.2063 & 0.0000 & -1.8872 & 0.8597 \\
\hline & Asp & 0.5242 & 0.0084 & 0.0528 & 0.0018 & -0.1303 & -0.4570 & 0.0000 & -0.8729 & 0.8729 \\
\hline & OxA & 0.5260 & 0.0085 & 0.0530 & -0.0018 & -0.1271 & -0.4585 & 0.0000 & -0.8758 & 0.8758 \\
\hline & PEP & 0.5775 & -0.0072 & -0.0451 & -0.0015 & -0.0203 & -0.5034 & 0.0000 & -0.1480 & 0.1480 \\
\hline & PYR & 1.5621 & 0.0014 & 0.0085 & 0.0003 & -0.2106 & 0.3383 & -1.6999 & -1.4283 & 1.4283 \\
\hline
\end{tabular}

Table 5. B. napus flux control coefficients (scaled). Dimensionless.

Flux control coefficients are a mathematical method of calculating the sensitivity of reaction flux to miniscule changes in enzyme activity. The below data demonstrates where changes to enzyme activity will cause positive or negative changes to the flux of each reaction. As expected by the Summation Theory for flux control coefficients, each row of coefficients adds up to 1 . Calculated via CoPaSi software.

\begin{tabular}{|c|c|c|c|c|c|c|c|c|c|c|}
\hline \multicolumn{11}{|c|}{ Enzyme activity being changed } \\
\hline & & ENO & PEPC & PPCK & ASAT & AK & PK & PDH & CS & TCA \\
\hline \multirow{9}{*}{ 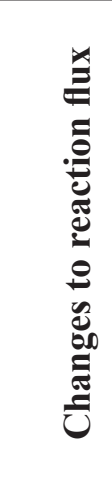 } & ENO & 0.8637 & 0.0017 & 0.0107 & 0.0004 & 0.0048 & 0.1188 & 0.0000 & 0.0349 & -0.0349 \\
\hline & PEPC & 0.4656 & 0.9770 & -0.1440 & -0.0049 & 0.1121 & -0.4058 & 0.0000 & 0.7285 & -0.7285 \\
\hline & PPCK & 0.4918 & -0.1467 & 0.0800 & 0.0027 & 1.0009 & -0.4287 & 0.0000 & 6.8161 & -6.8161 \\
\hline & ASAT & 0.4882 & 0.0078 & 0.0492 & 0.0017 & 0.8786 & -0.4256 & 0.0000 & -0.8129 & 0.8129 \\
\hline & AK & 0.4882 & 0.0078 & 0.0492 & 0.0017 & 0.8786 & -0.4256 & 0.0000 & -0.8129 & 0.8129 \\
\hline & PK & 0.9189 & 0.0008 & 0.0050 & 0.0002 & -0.1239 & 0.1990 & 0.0000 & -0.8402 & 0.8402 \\
\hline & PDH & 0.9189 & 0.0008 & 0.0050 & 0.0002 & -0.1239 & 0.1990 & 0.0000 & -0.8402 & 0.8402 \\
\hline & $\mathrm{CS}$ & 0.9189 & 0.0008 & 0.0050 & 0.0002 & -0.1239 & 0.1990 & 0.0000 & -0.8402 & 0.8402 \\
\hline & TCA & 0.9189 & 0.0008 & 0.0050 & 0.0002 & -0.1239 & 0.1990 & 0.0000 & -1.8402 & 1.8402 \\
\hline
\end{tabular}




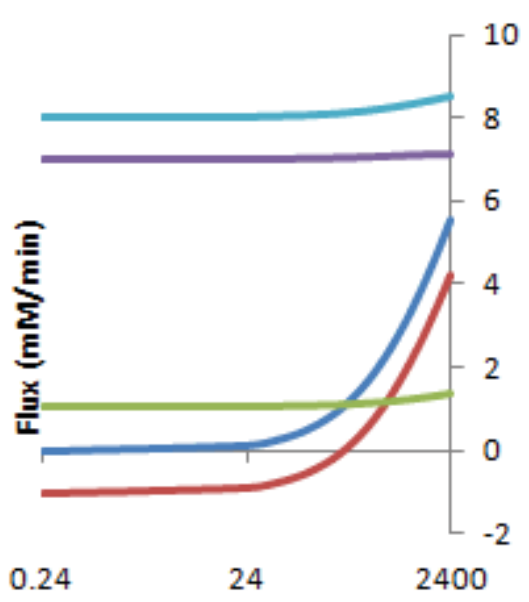

PEPC Enzyme Activity

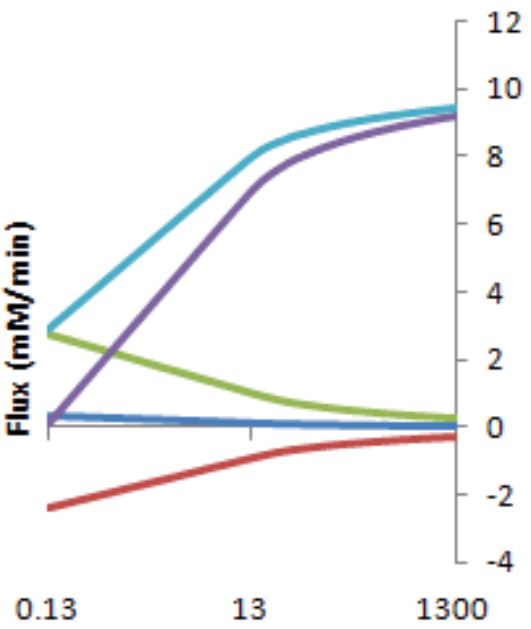

PKEnzyme Activity

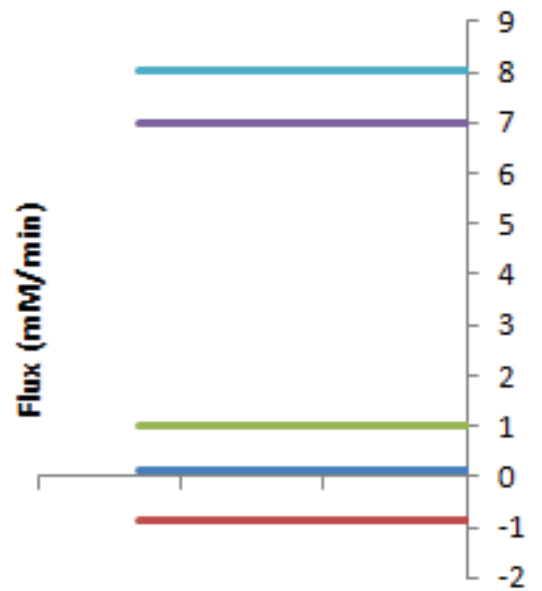

1.7
0.17

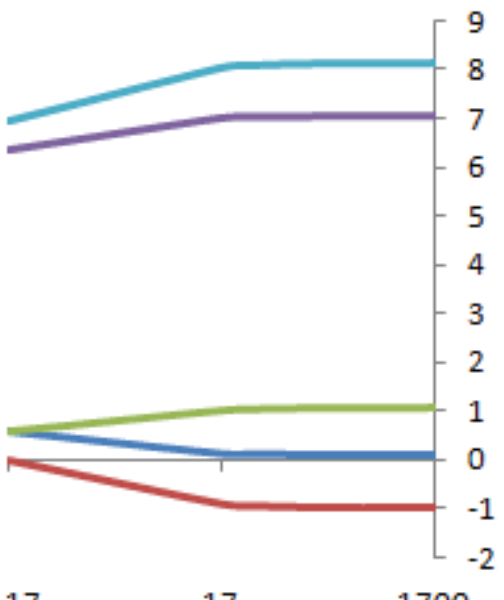

17

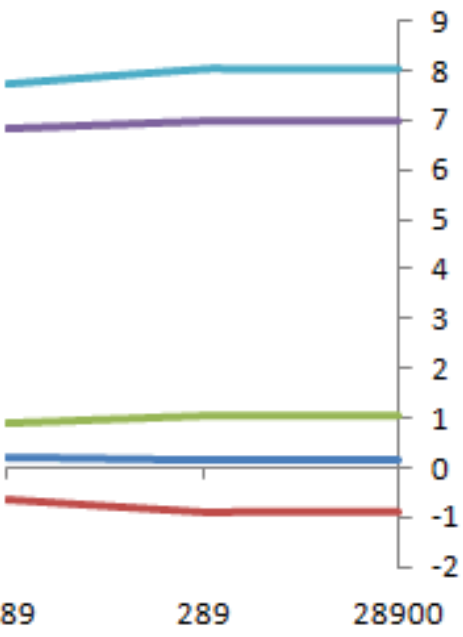

PPCK Enzyme Activity

ASAT Enzyme Activity
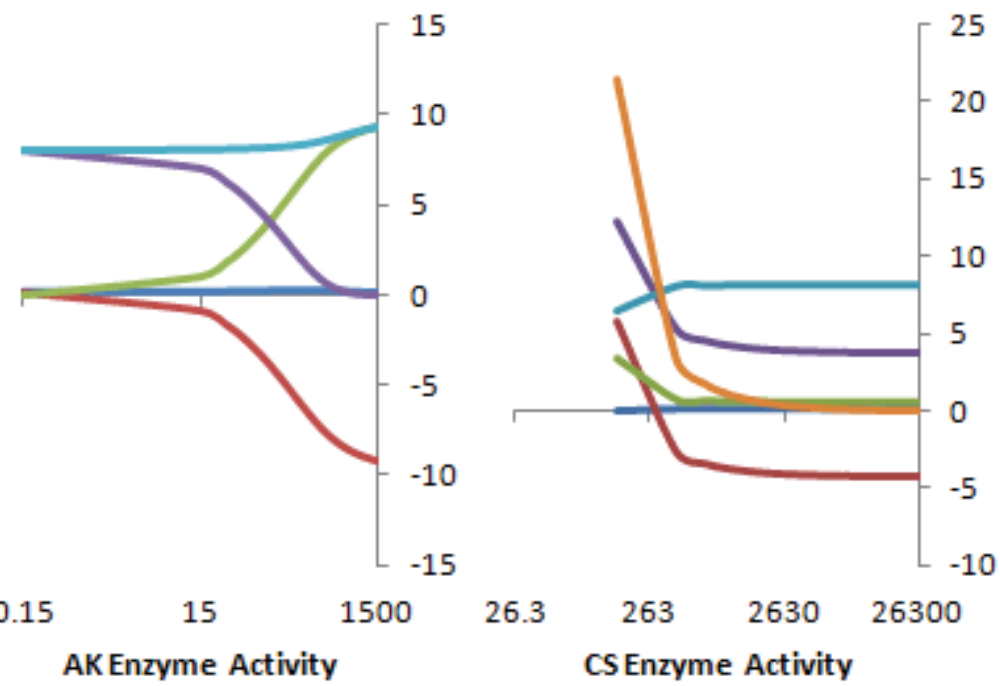

AK Enzyme Activity

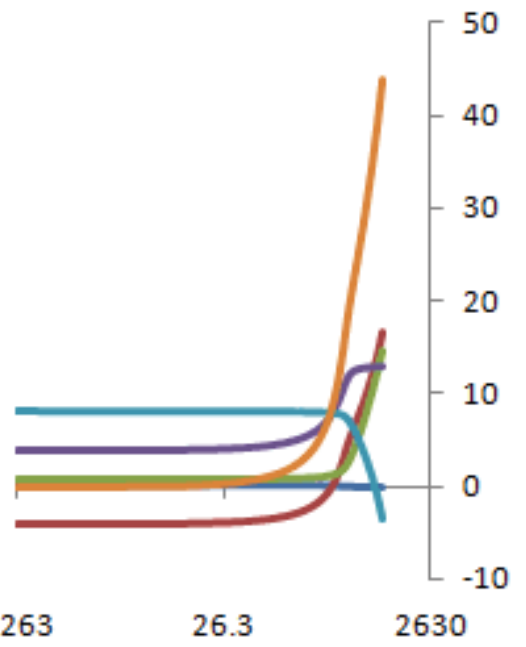

PDH Enzyme Activity

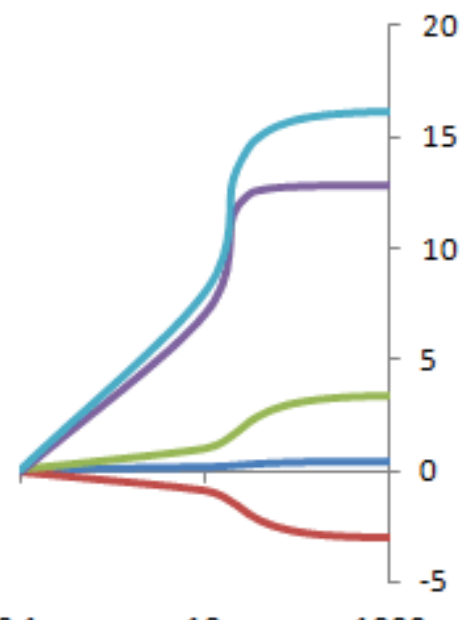

0.1

10

$1000 \quad 0.263$

TCA Enzyme Activity

25 


\title{
Reducing Hazardous Waste at Brookhaven National Laboratory
}

\author{
Kenneth Goodman \\ Chemistry Department, Long Island University, Brookville, NY 11548 \\ Joe Pavlak \\ Waste Management Department, Brookhaven National Laboratory, Upton NY 11973 \\ Peter Pohlot \\ Waste Management Department, Brookhaven National Laboratory, Upton NY 11973
}

\begin{abstract}
Excess chemical inventory across the Brookhaven National Laboratory (BNL) site has been an ongoing issue for many years. The issue of chemical accumulation has numerous problems associated with it such as aging chemicals that have gone past their expiration date, especially those in the peroxide forming category; chemicals stored in areas above the area's rated storage capacity such as flammable chemicals; and finally chemical hygiene and chemical compatibility during storage which could provoke very serious consequences including hazardous health situations. This internship has allowed me to work in various buildings and work with a countless number of scientists to determine how to effectively and economically reduce the BNL chemical footprint. Working with and assisting Joe Pavlak (BNL Waste Management Representative) has taught me methods of preventing and minimizing waste through speaking with researchers about the quantity of chemicals they order for their work and trying to reduce the amount they order as much as possible. A second method of avoiding waste used is to try and transfer chemicals that one person views as waste and give it to someone who will use it. Understanding methods for reducing waste consumption not only helps BNL scientists, but also the environment. Through this internship I have been put into a position to classify and test chemicals based on both Department of Transportation and Environmental Protection Agency standards which then gives the waste management technicians information about how to handle, pack, and transport these chemicals onsite. Working towards responsible chemical disposal will not only help reduce the chemical safety hazard of Brookhaven National Laboratory, but will also benefit the residents that reside around the lab that are at potential risk as well.
\end{abstract}

\section{INTRODUCTION}

Responsibly monitoring and disposing of chemical, radiological, and biological waste has become increasingly important to people as the negative effects of pollution can be observed in every corner of the globe. The weight of environmental stewardship has become a greater load for people to bear as fuel efficiency in their cars, organic foods, and their overall carbon footprint has become intertwined in mainstream thought. Here at Brookhaven National Laboratory, waste management has become crucial to the Department of Energy's mission to ensure America's security and prosperity by addressing its energy, environmental and nuclear challenges through transformative science and technology solutions. Preventing these environmental problems was the challenge proposed to me when beginning this internship. The challenge was attempting to reduce chemical inventory across Brookhav- en National Laboratory in every department on site. Working to resolve this issue has shown to be a difficult challenge for both my mentor and I. Using different methods of analyzing, reducing and disposing waste have been the most valuable knowledge learned from this internship. Implementing these methods into action have shown to be incredibly difficult due to vast amount of chemicals encountered and takes extensive knowledge and experience which I have only just begun to develop b. Determining how effectively these methods are working is crucial to rate how well the Department of Energy is meeting its mission of tackling tough environmental issues.

\section{DISCUSSION}

Throughout my time here at Brookhaven I have encountered various types and forms of hazardous chemicals. The etching solution used in nano-technology was one that was extremely bad. Piranha solution is the most common etching solution used at the lab and that consists of highly concentrated sulfuric acid an inorganic peroxide, usually hydrogen peroxide. The danger is that the solution has both a Department of Transportation (DOT) class nine substance (corrosive) and a DOT class 5.1 which is an inorganic peroxide, like hydrogen peroxide . Due to the peroxide presence in solution, it may cause many problems in handling, transporting, and treating. The reactive nature of peroxide causes this solution to possibly become explosive if left in the right conditions and could exothermically react with other waste chemicals in the immediate surroundings to form dangerous products. Due to the chemical activity of this solution, it was left to the Waste Management Department to write a treatment procedure to deactivate all the waste Piranha solution. My mentor and I came up with a plan to use ferrous sulfate which will cause the reduction of the hydrogen peroxide in solution by forming ferrous oxide, which is commonly known as rust. The production of non-toxic, non-hazardous rust creates a much safer environment for the technicians to handle. The complete reaction of the peroxide in solution allows for the waste to be handled as a single corrosive hazard once this done. Since the only hazardous material in solution now is the concentrated acid and it can be disposed of as such, rather than as an oxidative waste which takes many more steps and has many more hazards involved.

The proper treatment of chemical waste was the single largest job we performed throughout the summer. Since every department on site produces chemical waste whether it was used refrigeration oil at the maintenance shop or picric acid in the chemistry lab, we were required to be familiar with every type of waste produced $b$. This was a major undertaking to learn in just ten weeks, however I defiantly have a stronger understanding of how waste generated from research labs get handled once it leaves the hands of the re- 
searchers. Classifying chemicals according to both the DOT and EPA was essential to preserving the safety of the technicians who actually transport the waste. The DOT classifies chemicals into nine major categories ,a

If the waste being handled fits into any of these categories then it become regulated hazardous waste and must be appropriately disposed of by a licensed waste facility. The regulations for the packaging of these hazardous materials are very clear in what is required. The hazardous materials that are regulated by both the DOT and EPA required a standard red hazardous waste label with the chemical name clearly written on it with the date it will be put into the 90-day area. The 90 Day Hazardous Waste Storage Facility is an area at which hazardous chemical waste can be accumulated for a limited amount of time without a permit . All waste containers must have accumulation start date and be labeled as Hazardous Waste. Moreover, no waste container should remain in the storage beyond the 90 days including the first day waste was placed in the container. The satellite accumulation point, on the other hand, is an area at or near the point of generation where waste is initially accumulated and which is under the control of the operator of the process generating the waste; practically every research laboratory where chemical waste is generated. If the bottle is not large enough to place the label on it so it can be easily seen then it must be put into a plastic bag of appropriate size. Then the label can then be placed on the outside of the bag so it can be clearly seen from outside the 90 day area. Once the waste is properly labeled then the chemicals can placed into the 90-day area. The 90-day area requires secondary containment for the potential spills, which means that the waste must be placed into a bin or some type of container that will be able to hold the spill and will not react or corrode due to the hazardous waste. The most common type of containment is a bin usually made of high density polyethylene due to its stability against corrosives, oxidizers and solvents. After the waste is placed in the 90 day area the EPA representative designated to that area inspects it once a week for leaks and possible spills. The representative also inspects the labels for proper dates and chemicals names as well.

Transporting waste on site is another major aspect of the job of the Waste Management Department. Safely transporting waste requires a lot of knowledge about what chemicals are being transported and what sort of characteristics those chemicals have. Since the chemicals have to be safely packed so they cannot be spilt, a lot of the chemicals have to be packed together and knowing what can and cannot be placed together will help prevent any unwanted chemical reactions from occurring. Researching chemical properties such as flashpoint, reactivity in certain conditions and boiling point are all extremely important when moving chemicals in a warmer climate, such as the type encountered this time of year. Knowing everything about a chemical before it is sent out for shipment is the most important part of the whole process. Understanding the risks when shipping many of these chemicals falls into the hands of trained personnel at the Waste Management Department.

After the risks of each chemical being transported are understood the waste must be equipped with labels, at least four inches by four inches in size, are applied to the outside of hazardous materials shipping packages near the shipping name. These labels identify the primary and secondary hazard specific to the material being transported and give warning information about handling precautions in case of an emergency. If the diamond label will not fit on the package, shippers will put the label on a tag. For example, compressed gas cylinders that will not hold a label will have tags or decals . After the individual packages are labeled, the outside of the vehicle transporting the waste must be equipped with signs used to warn others of hazardous cargo and its DOT classification. A placarded vehicle must have at least 4 placards representing the applicable hazard. They are attached to each side and each end of the vehicle. Placards must be readable from all four directions. There are 22 DOT specification placards. They are 10 3/4 inches square, turned upright on a point, in a diamond shape. Cargo tanks and other bulk packaging show the ID number of their contents on placards, orange rectangular panels, or plain white square-on-point configurations having the same dimensions as placards .

Reducing chemical waste can be considered the single most important aspect of the waste management's mission of environmental stewardship. Many methods are put into place to reduce chemical waste, however they all stem from the idea of source reduction. This is defined as, any activity that reduces or eliminates the generation of chemical hazardous waste at the source. This can be accomplished by proper material management, substitution of less hazardous materials, and proactive laboratory procedures. Creating plans to reduce chemical consumption is the biggest portion of the efforts by waste management. Helping scientists order the correct amount of chemicals is another way to reduce the chemical inventory of the site. Creating a plan with the scientists that determine the amount of chemicals they should order is the easiest way to reduce chemical consumption. Many companies will give scientist a discount if the buy the chemicals in bulk, which would be fine if they actually used the amount they ordered. Since the scientists order the chemicals in bulk they are getting a better deal per unit, however if the chemicals sit around and then are disposed of later because of expiration the cost of disposal out weights the initial savings. Preventing this from happening is both environmentally and economically responsible. Another method of preventing a vast chemical inventory is a simple plan of recycling. Trying to use the waste material for another purpose, treated and reused in the same process, or reclaimed for another process will help ease the burden of hazardous waste accumulation. Many of the chemicals encountered by the waste management division are recycled with both environmental and economic issues in mind. During a short laboratory cleanout period the waste management division transferred about 40 bottles of various porphyrins form a laboratory that was being shut down to another laboratory that was doing research with these porphyrins. These bottles of porphyrins were recycled from a laboratory in the medical research department and given to another research laboratory in the chemistry department, ultimately preventing them from entering a chemical waste facility which is great for the environment. Moreover these porphyrins were valued at over ten thousand dollars which they now saved, could be spent on some other aspect of research in the chemistry department. Recycling these chemicals across departments that normally have limited communication is a great feat that would have not happened if it were not for the Waste Management professionals. The least preferable technique for waste minimization is chemical treatment. The most common treatment is the neutralization of corrosives like acids and bases. Chemical treatment of hazardous waste will help reduce the chance for chemical pollution since the waste has had its harmful characteristics removed. 


\section{CONCLUSION}

The need for a well operated and well trained Waste Management Division is a clear necessity for any large research facility. The fact is having a well trained and well operated Waste Management Division is somewhat a different story. A successful Waste Management Division will help drive the potential of any research facility by preventing federal fines, greatly limiting environmental pollution, and responsibly disposing of chemical, radiological, and biological waste. Luckily Brookhaven National Laboratory has a top tier Waste Management Division that pride them self on protecting the environment, researchers and residents of Suffolk County from further chemical, radiological and biological pollution. Working alongside these individuals has given many opportunities to understand why they work so hard to abide by and enforce the Department of Energy's mission of environmental protection through innovative solutions and technologies. Learning and implementing these methods procedures, and regulations at Brookhaven National Laboratory has been an incredible experience that has proven and will continue to prove in the future to be a valuable skill set that otherwise might not have been taught to me.

\section{AKNOWLEDGEMENTS}

I wish to thank my mentor, Peter Pohlot and Joe Pavlak, for their professionalism and generosity during the SULI program. Further, I would express my gratitude to the Office of Science Education. Finally, I wish to acknowledge the hospitality and kindness of Brookhaven National Laboratory and the Department of Energy. This project was supported in part by the U.S. Department of Energy, Office of Science, Office of Workforce Development for Teachers and Scientists (WDTS) under the Science Undergraduate Laboratory Internships Program (SULI).

\section{CITATIONS}

${ }^{1}$ The Hazardous Materials Regulation, 1 Code of Federal Regulations § 100-185 et seq. (2009). Print.

${ }^{2}$ The Hazardous Materials Regulation, 1 Code of Federal Regulations $\S 100-185$ et seq. (2009). Print.

${ }^{3}$ United States. Environmental Protection Agency. Hazardous Waste Generator Regulations. N.p., Aug. 2012. Web

${ }^{4}$ United States. Environmental Protection Agency. Hazardous Waste Generator Regulations. N.p., Aug. 2012. Web

${ }^{5}$ United States. Environmental Protection Agency. Hazardous Waste Generator Regulations. N.p., Aug. 2012. Web

61“"Frequent Questions | Waste Minimization." EPA. Environmental Protection Agency, 15 Nov. 2012. Web. 08 Aug. 2013.

${ }^{7}$ The Hazardous Materials Regulation, 1 Code of Federal Regulations $\S 100-185$ et seq. (2009). Print.

${ }^{8}$ The Hazardous Materials Regulation, 1 Code of Federal Regulations § 100-185 et seq. (2009). Print.

${ }^{9}$ The Hazardous Materials Regulation, 1 Code of Federal Regulations $\S 100-185$ et seq. (2009). Print.

${ }^{10}$ The Hazardous Materials Regulation, 1 Code of Federal Regulations § 100-185 et seq. (2009). Print.

11“"Hazardous Waste Reduction Techniques." Hazardous Waste Reduction Techniques. Idaho State Unniversity, n.d. Web. 08 Aug. 2013.

12“Hazardous Waste Reduction Techniques." Hazardous Waste Reduction Techniques. Idaho State Unniversity, n.d. Web.
08 Aug. 2013.

13“"Hazardous Waste Reduction Techniques." Hazardous Waste Reduction Techniques. Idaho State Unniversity, n.d. Web. 08 Aug. 2013.

\section{WORKS CONSULTED}

"Frequent Questions | Waste Minimization." EPA. Environmental Protection Agency, 15 Nov. 2012. Web. 08 Aug. 2013.

"Hazardous Waste Reduction Techniques.” Hazardous Waste Reduction Techniques. Idaho State Unniversity, n.d. Web. 08 Aug. 2013.

[49 FR 30903, Aug. 1, 1984, as amended at 73 FR 75595, Dec. 12, 2008]

The Hazardous Materials Regulation, 1 Code of Federal Regulations $\S 100-185$ et seq. (2009). Print.

United States. Environmental Protection Agency. Hazardous Waste Generator Regulations. N.p., Aug. 2012. Web. 


\title{
Comparison of measured and calculated light scattering by aerosol particles over the eastern North Pacific Ocean
}

\author{
Michelle Gostic \\ Environmental Engineering, Cornell University, Ithaca, NY 14850 \\ Ernie Lewis \\ Environmental Science, Brookhaven National Laboratory, Upton, NY 11973
}

\begin{abstract}
In order to understand how marine boundary layer clouds affect climate, the MAGIC field program deployed various instruments that measure cloud and aerosol properties on the Spirit, a cargo ship that travels from LA to Hawaii. Ultimately, knowledge gained from his data will be incorporated into global climate models and help prepare for future weather. This summer, I compared MAGIC data from two instruments measuring aerosol properties: the nephelometer, which measures light scattering by aerosols, and the ultra-high sensitive aerosol spectrometer (UHSAS), which measures the size and number of particles. Data from the UHSAS and the nephelometer provides information as to how radiation interacts with aerosols, which is important in determining the effect the particles have on climate. The data comparison has shown that within overlapping size ranges, the amount of light scattering measured by the nephelometer is generally in agreement with how much light scattering we would expect based on the size distribution of particles provided by the UHSAS.
\end{abstract}

About $70 \%$ of the earth is covered in water, yet nearly all in situ atmospheric measurements are made over land, which explains why climate models struggle to accurately describe the atmosphere. In order to understand how marine boundary layer clouds affect climate, the MAGIC field program deployed various instruments that measure atmospheric properties on the Spirit, a cargo ship that travels between LA and Hawaii. The effect that clouds and aerosols have on climate remains one of the biggest sources of error in climate modeling. The MAGIC project is part of the global Atmospheric Radiation Measurement (ARM) initiative, which aims to better understand how clouds affect the earth's radiation budget. The second ARM Mobile Facility (AMF2) is a collection of instruments deployed on the Spirit, which collects atmospheric data as the ship makes its way across the eastern North Pacific. The path the ship takes is close in proximity to the GPCI transect, an area of the Pacific Ocean that climate models inaccurately describe. Ultimately, MAGIC data will result in a better understanding of this region, specifically how marine boundary-layer clouds affect climate. This understanding will allow climate modelers to more accurately describe climate and better prepare for future weather.

Several instruments deployed on the Spirit measure the properties of aerosols, tiny particles suspended in the air. Aerosols impact the earth's climate in two ways: direct radiative forcing and indirect radiative forcing. Direct radiative forcing refers to the ability of aerosols to reflect incoming solar radiation, thereby cooling the atmosphere. Indirect radiative forcing pertains to how particles contribute to the formation of clouds, which absorb terrestrial radiation and warm the atmosphere. How light interacts with aerosols is affected by variations in particle size and composition, which is why achieving a thorough understanding of the properties of aerosols in our atmosphere is essential to determining the role that these tiny particles play in shaping climate.

This summer, my main goal was to provide closure between two instruments deployed by MAGIC. In the realm of atmospheric science, a closure experiment uses different techniques to take measurements that describe a given system or property. The data from both methods is compared, which can help to identify inaccuracies in one or both of the measurement techniques. I looked at data from two instruments measuring aerosol properties, the nephelometer and the ultra-high sensitive aerosol spectrometer (UHSAS). The nephelometer measures the amount of red light (700nm wavelength), green light (550 nm wavelength), and blue light (450 $\mathrm{nm}$ wavelength) scattered by particles, while the UHSAS categorizes particles by diameter into 99 size bins and counts how many particles are of each size, yielding a size distribution of particles. The UHSAS deployed on the ship measures particles ranging from $55 \mathrm{~nm}$ diameter to $985 \mathrm{~nm}$ in diameter. The size of particles that enter the nephelometer is regulated by an impactor, which alternates between $1 \mu \mathrm{m}$ and $10 \mu \mathrm{m}$ cuts. During a $1 \mu \mathrm{m}$ cut, the nephelometer measures scattering by particles smaller than $1 \mu \mathrm{m}$, and during a $10 \mu \mathrm{m}$ cut, the nephelometer measures scattering by particles smaller than $10 \mu \mathrm{m}$ in diameter. By using information about the size and concentration of aerosol particles from the UHSAS, we can calculate how much light scattering we expect to be measured by the nephelometer. Furthermore, if the calculated scattering agrees with the measured scattering, it is an indication that the instruments might be working properly and providing reliable data.

To calculate the expected scattering from UHSAS data, the equation below was used to determine how much light is scattered by particles in each size bin.

$$
\frac{d \sigma}{d \log (d)}=\frac{d N}{d \log (d)} \pi r^{2} Q_{s c a}
$$

The product of the number of particles in each size bin, the cross-sectional area, and the scattering efficiency (Qsca) yields the amount of light scattering from particles in a given size bin. The scattering efficiency was calculated using the Gamma Approximation, and the index of refraction $(\mathrm{m})$ for aerosol was assumed to be 1.4 .

$$
\begin{aligned}
& Q_{s c a}=Q_{\gamma}=0.85 z^{4} e^{-z} \\
& z=2\left(\frac{2 \pi r}{\lambda}\right)(m-1)
\end{aligned}
$$


Figure 1 shows how much particles from each size bin contribute to the scattering of blue, green, and red light. The black line shows a typical size distribution of particles. The graph shows that although most particles are between 100 and $250 \mathrm{~nm}$ in diameter, the particles that account for a bulk of the scattering are larger, between $200-600 \mathrm{~nm}$ in diameter. This implies that a few bigger particles play a greater role in light scattering than a bunch of tiny particles. This has large implications in terms of climate modeling in that larger sea-salt particles suspended over the Pacific Ocean will scatter light differently than the tiny anthropogenic pollution particles near LA.

Figure 1 also shows that for particles with diameters between $750 \mathrm{~nm}$ and $985 \mathrm{~nm}$, a phenomena known as anomalous scattering occurs. For smaller particles, blue light scatters the most and red light scatters the least. But particles with specific properties can cause anomalous scattering, where light scattering increases with increasing wavelength.

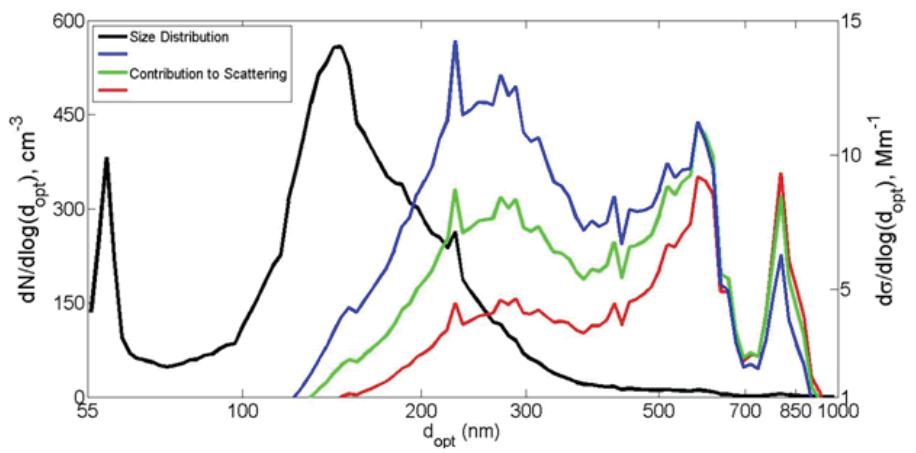

Figure 1. Typical size distribution of particles and contribution of various sized particles to light scattering

To determine whether the UHSAS and nephelometer measurements agree, the total calculated scattering was compared to the total measured scattering. The total calculated scattering was found by summing up the contribution to scattering by particles from all 99 size bins. The comparison of measured and calculated scattering is shown in Figure 2.

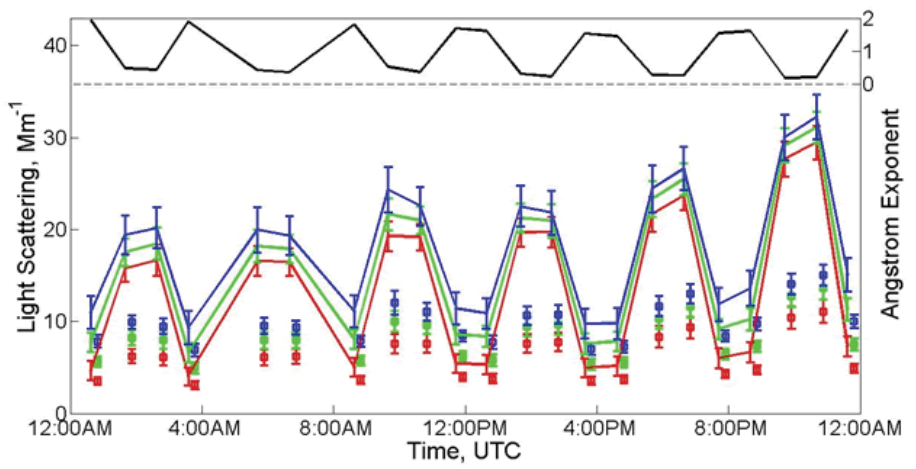

Figure 2. Correlation of the Ångström exponent with measured and calculated light scattering by aerosol.

The alternation between the agreement and disagreement of the measured and calculated light scattering is due to the switch between $1 \mu \mathrm{m}$ and $10 \mu \mathrm{m}$ cuts that affect the nephelometer, but not by the UHSAS. We expect the measured scattering to be higher than the calculated scattering during periods when the nephelometer measures scattering by particles smaller than $10 \mu \mathrm{m}$ diameter, because the UHSAS only sizes particles up to $985 \mathrm{~nm}(0.985 \mu \mathrm{m})$ diameter. As demonstrated by Figure 1, even a few large parti- cles cause a substantial amount of light scattering, so even if only a few particles with diameters greater than $985 \mathrm{~nm}$ were seen by the nephelometer (but not sized by the UHSAS) it would result in significant discrepancy between the measured and calculated values of light scattering. This means that the disagreement between the measured and calculated values is not because of instrument malfunction, but due to the disparity between the size ranges of particles being considered at these times. When the nephelometer is experiencing a $1 \mu \mathrm{m}$ cut, the upper diameter limit of particles measured by the UHSAS is only $15 \mu \mathrm{m}$ smaller than the upper diameter limit of particles measured by the nephelometer, thus the measured and calculated values are generally in agreement. Although the UHSAS only counts particles as small as $55 \mathrm{~nm}$, this would not cause disagreement between the measured and calculated values of light scattering because the contribution of very small particles to scattering is negligible, as shown by Figure 1 .

Figure 2 also displays the Ångström exponent, which is a measure of the dependence of light scattering on wavelength. For example, an Ångström exponent of zero means that scattering does not depend on wavelength, thus red, green, and blue light will all scatter equally.

$$
\text { Angström exponent }(\alpha)=-\left(\frac{\ln \left(\frac{N_{\text {Red }}}{N_{\text {Blue }}}\right)}{\ln \left(\frac{\lambda_{\text {Red }}}{\lambda_{\text {Blue }}}\right)}\right)
$$

In general, there is an inverse relationship between particle size and the Ångström exponent. Smaller particles generally scatter blue light the most and red light the least; however, light scattering by bigger particles is less dependent on wavelength. This idea is supported by the graph, which shows that the Ångström exponent is closer to zero when the nephelometer experiences a $10 \mu \mathrm{m}$ cut, due to the large contribution to scattering by larger particles.

Atmospheric particle concentrations are expected to gradually decrease due to the development of cleaner vehicles and the implementation of pollution controls. Some believe that the direct radiative effect of aerosol is so strong that lower particle concentrations will allow more solar radiation to penetrate the atmosphere, thus off-setting the Earth's energy budget and warming the atmosphere. In order to prepare for future weather, it is essential that scientists attain a fuller understanding of the aerosols in our atmosphere, and their effect on climate. If I had more time, I would investigate what causes fluctuations in aerosol concentrations and the amount of light-scattering in the middle of the ocean, where anthropogenic sources are hundreds of miles away. There is still much more work to be done in analyzing MAGIC data and in coming to conclusions about aerosols over the eastern North Pacific Ocean in order to better understand their impact on climate.

\section{Acknowledgements}

My sincerest thank you to Dr. Gunner Senum for his help throughout the summer, to my mentor Ernie Lewis for his continuous support and generosity, which made this summer memorable and informative, and to the Office of Educational Programs for this amazing opportunity. This project was supported in part by the U.S. Department of Energy, Office of Science, Office of Workforce Development for Teachers and Scientists (WDTS) under the Science Undergraduate Laboratory Internships Program (SULI). 


\title{
Expression, purification, and characterization of the Extender Module of PolyKetide Synthase (PKS)
}

\author{
Joshua Greenough \\ SUNY Oneonta, Oneonta, NY 13820 \\ Desigan Kumaran \\ Brookhaven National Laboratory, Upton NY 11973
}

\begin{abstract}
PolyKetide Synthase (PKS) is a multi-enzyme complex, found primarily in bacteria and fungus. Known best for its antibiotic activity, PKS can also be utilized to synthesize fatty acids. When reacted with short chain alcohols, fatty acids prove to be a viable source of clean energy. Due to the modular architecture of the PKS, fatty acid length is positively correlated with its size and hence the amount of energy that can be produced. Production of polyketide synthase, in E. coli is quite challenging due to the complexity and heterologous production of the protein and hence augmented risk of protein mis-folding. In this study we expressed, solubilized and purified the modules (with and without docking linker) corresponds to the extender units of De-oxy Erythronilide B Synthase (DEBS). These modules, molecular mass 210 and $218 \mathrm{kDa}$, oligomerized as a dimer in solution. Our current research shows that proteins with multi domains of moderate size $(\sim 200 \mathrm{kDa})$ are capable of soluble only in the detergents like as n-octyl- $\beta$-D-glucoside, N-lauroylsarcosinate, and n-Dodecyl $\beta$-Dmaltopyranoside with a chaotropic agent like Glycerol.
\end{abstract}

While society's across the world become ever more dependent on a steady supply of energy to maintain their technology based lifestyles, we are forced to face an unsettling reality. The reality is that man-kind's demand for fossil fuels has not only increased global temperatures and pollution, but it also far exceeds earth's supply. Keeping this reality in the forefront led my research team to analyze the Extender Module, a component of the PolyKetide Synthase (PKS) De-oxy Erythronolide B- Synthase (DEBS), which, if characterized could lead to the development of a fatty-acid based biofuel. One way we are trying to augment this naturally occurring biofuel is by manipulating the structure of the Extender Module, which controls fatty acid length; manipulation of this module could result in elongation of fatty acid chains which in turn would increase energy yield.

Characterization of the Extender Module is inherently difficult due to the complexity of it's protein domain organization. In an attempt to characterize the Extender Module, we created two different clones, ${ }^{1}$ S2A (218kDa), containing both the Extender Module and the Linker Region, and the second, S2B (210kDa), containing just the Extender Module. While expressing the protein in an E. coli host was not very challenging, solubilizing quantities of protein necessary for crystallization has presented an obstacle.

To address this issue we screened both samples for solubility with the aid of detergents, such as: n-octyl- $\beta$-D-glucoside, N-lauroylsarcosinate, and n-Dodecyl $\beta$-D- Maltopyranoside. Though the addition of detergents increased solubility, more research will be required before the Extender Module can be characterized.

Understanding the complexity of solubilizing and crystaliz- ing the Extender Module first requires an understanding of what PolyKetides are and what their function is. Found naturally within many bacteria, fungi, plants, and even some animal lineages,

PKS are most commonly known for their antibiotic characteristic. Other relevant features include use as: immune suppressants, anti-tumor agents, anti-cancer agents, and anti-cholesterol agents. The wide array of PKS products from their multi-enzyme complex structure; PKS structural diversity allows for the production of more complex products. More specifically, however, this project focused on the PKS DEBS. Though DEBS has been the subject of many studies, it's complete 3D structure is still a mystery. Currently we are aware of three components that comprise it's structure. Of which, two components have been identified, these are: the Loading Module and the Termination Module. The uncharacterized third module, the Extender Module, is our subject of study. If this component was to be characterized, we would be able to attain information as to how hydrocarbon chains are built by DEBS, thus gaining the insight as to how we could manipulate it's structure to produce unnaturally long fatty acid chains.

Initial procedures responsible for the production of our protein clones, S2A and S2B, included cloning and expressing pre-isolated Extender Module genes from DEBS. Genes corresponding to the Extender Module and also, in the case of S2A, the Docking Region were taken from Actinomycetes and ligated into an E. coli plasmid vector,

PAL-15 in BL21 (DE3) cells. After cloning was complete, we were free to express the samples. Expression of S2A and S2B began with inoculating clones in MDAG-135, non- inducing media, this facilitated culture growth to saturation over $\sim 12$ hours in an incubator set to $37^{\circ} \mathrm{C}, 200 \mathrm{RPM}$, without detectable induction of our target protein. Once mixed with glycerol and frozen, non-inducing media also allowed for the preservation of cell cultures for several weeks[2]. When MDAG-135 culture reached saturation it was then transferred into ZYM-5052, auto-inducing media for further growth, followed by induction of target protein. More specifically, auto-induction media allowed culture to metabolize glucose until saturation point was reached, at which time, lactose was metabolized and converted by $\beta$-galactosidase to the inducer, allolactose. Effectiveness of this auto-induction media is derived from the components listd in Table 1.

Based on turbidity of culture, expression of target protein was notably successful in all trials. Proceeding expression, the culture was harvested in the form of $500 \mathrm{~mL}$ cell pellets and ready for purification.

Once expressed, we were faced with the challenge of solubilizing our protein in a manor that was minimally-destructive to the protein structure. Due to the complexity and length of our clones, we had expected that they would not solubilize well without the use of a detergent. As hypothesized, SDS-page gel confirmed that target protein remained insoluble when lysed by BugBuster, with 
the aid of PMSF, Lysozyme, Protease Inhibitor, and DNAse (no detergents) at room temperature. In order to attain which detergents would be most affective in increasing protein solubility, while inflicting minimal damage, small samples of S2A and S2B were lysed with the above lysis buffer, but with the addition of the following screening variables (at $\mathrm{pH} 8.0$ ): 10\% Glycerol, $0.05 \%$ Triton X100, 2\% n-Octyl-ß-glucopyranoside (ßOG), 2\% Dodecyl Maltopyranoside (DDM), and 1\% Sodium-N-Lauroylsarcosinate (LSN). Though our samples still expressed low levels of solubility, enough protein had been dissolved to begin protein purification.

In order to separate our target protein from all non-specific protein, we executed two forms of purification, Metal Affinity (Histadine-Tagging (HT)) and Size Exclusion Chromatography (SEC). The first purification method, HT, was performed following host cell lysis. Total lysate was centrifuged at 16000 RPM, $4^{\circ} \mathrm{C}$, resulting supernatant was then binded to Nickel resin. A gradient of imidazole fractions, ranging from $10 \mathrm{mM}$ to $500 \mathrm{mM}$ were used to remove his tagged target protein for Nickel resin. tag. Nearly all HT trials released our target protein within the $100 \mathrm{mM}$ and $200 \mathrm{mM}$ Imidazole fractions. This was determined by running samples of each imidazole fraction, along with flowthrough, through SDS-page gel (you can give gel figure here, if you can). The fractions containing our purified protein $(100 \mathrm{mM}$ and $200 \mathrm{mM}$ ) were then concentrated via $100 \mathrm{k}$ Centricon filters to $\sim 5 \mathrm{~mL}$ total. Once concentrated, samples were injected into AKTA- FPLC, SEC, machine, in which the protein was passed through column and collected into glass tubes (fraction collector) in order of descending retention volume. The resulting chromatograph indicated which fraction contained our target protein, as determined by the degree of UV absorption. Peak fractions were collected and ran through gel to evaluate the purity of our target proteinFractions containing low levels of contaminants and high protein concentration were once again concentrated by $100 \mathrm{k}$ Centricon filters for further characterization.

When concentration and purification of our protein had been completed we were finally able to load our samples into crystallization screening plates for sitting drop vapor diffusion. The initial crystallization trials were carried out using automated
Art-Robbins Phenix crystallization robot, commercially available high-throughput (HT) crystallization reagent kits, A 96 well Crystal Screen HT and Index Screen HT. These crystallization plates were then examined under microscope on a frequent basis.

The target proteins, S2A and S2B, are expressed in E. Coli by auto induction procedure at $20^{\circ} \mathrm{C}$. These proteins were screened for solubility when lysed using bugbuster at room temperature with and without detergents at buffer $\mathrm{pH}$ 8.0. The screening variables are 10\% Glycerol, 0.05\% Triton X100, 2\% n-Octyl-ß-glucopyranoside (ßOG), 2\% Dodecyl Maltopyranoside (DDM), and 1\% Sodium-N- Lauroylsarcosinate (LSN). Protein lysed with glycerol and Triton X100 showed extremely low quantities of solubilized protein when ran through SDS-page gel. Other listed detergents proved to be slightly more effective in solubilizing our target protein. The solubilized proteins are purified by metal affinity (his-tag purification) followed by size exclusion chromatography. Even though S2A and S2B are expressing well in the auto induction media, final recovery of pure protein is very low due to the protein insolubility. Further research needs to be done to explore the possibility of recovering high yield of these proteins by denaturing and refolding the protein.

While characterization of the PKS DEBS could entail a great leap for the field of alternative energy, much more research must be conducted. Expressing DEBS genes responsible for the production of the Extender Module (and Docking region in the case of S2A) from within an E. coli host vector allowed us to research the effects of lysing the cells with various detergents. From these clones and several solubility tests, we were able to deduce that despite, the protein's structural complexity, S2A and S2B can be partially solubilized with the aid of detergents such as DDM, LSN, and BOG. We were also able to determine that based on one of our S2A-DDM chromatographs and the standard sizing chromatograph, a peak at 55 retention volume indicates that the oligomeric state of $\mathrm{S} 2 \mathrm{~A}$ is dimer (2 molecules). While this research provides a foot-hold for future Extender Module characterization research, many more trials must be conducted, especially in attempt at yielding greater rates of solubility. Only after protein solubility is increased will crystallization, followed by characterization of the Extender Module occur.

\section{References}

${ }^{1}$ F. W. Studier, Protein Expression and Purification 41: 207 (2005)

\begin{tabular}{|l|l|}
\hline \multicolumn{1}{|c|}{ Substance } & \multicolumn{1}{c|}{ Significance } \\
\hline ZY & Provides main energy source for E. coli to grow \\
\hline MgSO4 & $\begin{array}{l}\text { Fulfills nutritional requirements, allowing for increased saturation density (on average, 2mM } \\
\text { MgSO4 increased saturation density by 5-fold) }\end{array}$ \\
\hline 1000x Metals & Required to grow culture to high density \\
\hline $50 x 5052$ & Source of nutrients \\
\hline $50 x M$ & Source of nutrients \\
\hline Kanamycin & Prevents growth of non-specific bacterial growth \\
\hline
\end{tabular}

Table 1. 


\title{
Optimization of perfluorocarbon tracer data analysis using Visual Basic
}

\author{
Nathan Gubser and Terrance Sullivan \\ Department of Environmental Sciences, Brookhaven National Laboratory, Upton NY 11793
}

\begin{abstract}
Harmless, odorless, perfluorocarbon gasses can be deployed and captured to model environmental airflow using an application called perfluorocarbon gas tracing (PFT). An understanding of numerous airflow related issues within complicated physical environments can be gained using Brookhaven National Laboratory's advancing PFT process. A demand for more efficient ways to translate, sort, and present PFT data has developed as this process has evolved. My internship centered on the task of creating a program that would interact with information from any one of three gas chromatographers' (GC) comma separated value (.csv) format data files as well as .csv files output by machines used for capturing tracer gasses. This code was designed to coordinate information between these data sources, and then supply the user with a number of useful calculations and graphical display options. It allows for properties such as machine identity, GPS location, time series, and perfluorocarbon gas name to be selected in order to quickly and visually analyze data from thousands of samples in ways that have previously required time-consuming, manual efforts. This experience offered both a creative and analytical challenge that both exercised and strengthened my problem-solving capabilities. Furthermore, it allowed me the opportunity to provide a valuable contribution to my department while expanding my level of computer programming experience.
\end{abstract}

Deploying, capturing and measuring perfluorocarbon tracer (PFT) gasses is a powerful technique used in comprehensive analysis pertaining to a variety of airflow related inquiries. Brookhaven National Laboratory's (BNL) continually evolving, multi perfluorocarbon tracer (PFT) system, is the most advanced application of this practice. This paper will provide a general description of the PFT process and detail the approach taken in order to provide an automated method which has been developed by myself under the mentorship of Terry Sullivan, and supported in part by the Department of Energy under The Science Undergraduate Laboratory Internships program, in order to aid in the interpretation and presentation of accumulated PFT data.

In contrast to an arrangement of hypothesis, conclusion, methods, results, and a plentiful arrangement of clearly presented data, my research experience centered on the application of a solution which entailed the identification of a problem, working to understand this problem, developing an approach for dealing with it, and applying this approach as a solution. Both classical research internships as well as this sort of research experience are very valuable, yet I believe this difference worth mentioning, as I find it to be an underrepresented quality typical to many engineering oriented undergraduate research experiences. With this consideration, as I proceed to cover the components that comprise the problem scenarios, approaches, and results herein, I will do so more as a representation of a work based problem solving experience and less so of a classical research endeavor.

BNL's PFT system detects gas presence with a higher precision, is environmentally cleaner, and is also capable of studying more complicated scenarios in comparison to other tracer gas technologies,. This system employs a superior gas chromatography technology that allows measurements up to a level of resolution at parts per quadrillion versus the parts per trillion achieved through other methodology. This greater sensory ability results in the deployment of smaller quantities of PFT than other methods which deploy greater gaseous quantities that could be harmful to our atmosphere. Furthermore, BNL is able to deploy and analyze up to seven PFT's whereas most systems employ only one, and few are able to use as many as four. This creates the capability of studying a much more complex range of scenarios than other physical methods of verification.

Perfluorocarbon gasses are virtually non- existent in environmental air which make them ideal for using as tracing gasses. The term tracing refers to the action of following a released gas or gasses' air travel by testing for their presence in an air sample according to the known location of their release. The relative absence of atmospheric PFT allows for air samples to be collected with virtually no interference from pre-existing environmental levels. Brookhaven's PFT system consists of releasing up to seven known quantities of these gasses at known rates over predetermined periods of time. In conjunction with this, a strategic arrangement of air sampling units which draw air through suspended graphite capture spheres within capillary absorption tube systems are organized throughout the area of air flow inquiry. Upon the completion of tracer release and absorption, these tubes are collected and taken for quantitative analysis using any of three uniquely modified gas chromatographers which are designed to specifically handle these rare gasses.

After the chromatographic measurement of PFT's found within each sample tube, these quantities are ultimately interpreted through a series of calculations and described via graphical representation for use in reports and environmental analysis. This has always been accomplished manually. Recently, the NYPD and BNL executed the largest ever urban airflow study involving over 7000 samples from 200 machines throughout surface and subsurface locations across Manhattan furthering the demand for a more efficient way to process this data. The focus of my research experience consisted of approaching the problem of how to best optimize BNL's Department of Environmental Science's PFT data management process.It was decided that this optimization would occur in the form of Visual Basic based programming language.

In order to approach this task I began by developing a comprehensive understanding in regards to the sources of input data 
this program would operate in accordance with. PFT quantities would be interpreted from comma delineated files (.csv) output by gas chromatography (GC) machines. Three different machines are used for this process and consequently there are two separate formats of resulting .csv files to interpret. These files both link unique serial numbers to PFT sample quantities as well as additional measurements corresponding to tubes with known quantities and tubes that are empty for purposes associated to verifying and maintaining machine calibration. Each of these sample quantities is associated to a date and a time of measurement from a specific machine and a corresponding volume of air flow that was drawn through the sample's absorption tube. This data is output from each sample machine in another .csv file that typically contains data for twelve time periods / samples. As these samples are loaded into each machine another .csv is generated from a bar code scanner that contains each unique serial number with each machine number. Finally a separate identity file is created through user entered data that can contain a variety of information, such as physical addresses, serial numbers related to machine location and corresponding latitudes and longitudes.

With an understanding of where the input data originated from and what unique properties each file type contained that could be associated with one another, the next step was to hold discussions with the individuals that this program would be designed to serve. Through these verbal communication I ascertained what the needs of the users would be and was granted the freedom to develop this software in any way that would provide these features.

More specifically, these demands entailed reading from gas chromatography files and extracting measurements related to seven specific gasses as well as empty tubes and calibration samples. This extraction would first run calculations on the values associated to the calibration and blank samples in order to create three degrees of polynomial coefficient information in order to be applied to gc output values of measurement and be compared with the values that would be expected for viewing in terms of total error and percent error . Concurrently graphs would be generated using lines of best fit to give a visual frame of reference for evaluating data reliability. This allows the operator of the gas chromatography process to ensure that the machine is providing reliable readings for the study samples. If the readings are not satisfactory the samples quantities are inspected for spikes or anomalies in order to make manual determinations that will assure accurate coefficient values, wherein the process will repeat to update graphs and other numerically linked information. When these calibrated coefficients have been verified and approved, this program will then apply them to the air sample measurements for the seven specific gasses and calculate their value according to a third degree polynomial equation comprised of these new coefficients. Utilizing the unique associations between the aforementioned identiyy files, all of the identity information would then be incorporated into a sheet that contains the calculated air sample values. The associated total volumetric flow of related to each sample would then be used to adjust this quantity into a value of picoliters per liter (pL/L) completing the primary calculations. Finally this program would be designed to output graphic representations of gas type versus machine number and machine location versus gas quantity.

With these objectives laid out and knowing that there existed approximately seven weeks to complete this task there existed a number of options in how to deal with these demands. After con- sidering various designs I decided that despite a relatively short period of time it would be work undertaking the task of designing this program so that it could be used in the future for a variety of studies and not just pertain to this specific study. This means having the ability to choose any number of available pft's and establishing a protocol for multiple identity file formats to adhere to. It would mean that any number of machines, times, gasses, and locations could be requested for comparison with one another. Furthermore it would allow for multiple GC files to be analyzed with one another. With these functionalities to consider another important aspect taken into consideration was of the expansion of pft analysis capability and a potential desire for varying identity formats to be included. Regarding this steps were taken in order to allow for expansion to be easily retrofitted into the middle of processes so that this process might maintain its start and end procedures and proceed unaware of its own size.

This research experience extended an opportunity to me wherein I was able to gain practical knowledge about building code regulations, advanced gas tracing methodology, dynamic integration of programming application and the Visual Basic language.tionally, I was able to approach a problem and incorporate a coordinated series of steps through a programming implementation to increase productivity. This allows employees the ability to spend their limited time on other important aspects. Additionally the now automated processes will save costs associated to the handling, formatting and output of crucial information. 


\title{
Development and Application of "On-the- fly" Scanning for Fast XRF Imaging and Microspectroscopy
}

\author{
Chris Guerrero \\ Fordham University, Engineering Physics Department, Rose Hill Campus, Bronx, New York 10458 \\ Fabio B. Ono \\ Federal University of Lavras, Soil Science Department, 3037 Campus UFLA, Lavras, Minas Gerais, 37200-000, Brazil \\ Paul Northrup and Ryan Tappero \\ Photon Sciences Directorate, National Synchrotron Light Source, Brookhaven National Laboratory, Upton, NY 11973
}

This project creates a control system based on Python software (version 2.7) and channel access to obtain and analyze x-ray microprobe data. The purpose of this project is to apply new Python code to the sample stage control system at Beamline X15B in NSLS-I for "on-the-fly" scanning. This type of scanning rasters the sample with $\mathrm{x}$-rays in a four-cornered, polygon region typically through row by row increments. This row by row method is as efficient for people in visualization when reading or analyzing with the eyes. At the same time, we tested the current code at Beamline X27A and compared the codes at both beamlines to find improvement. The main tests at X27A include X-ray fluorescence (XRF) imaging and x-ray absorption spectroscopy (XAS) graphing, which fulfill the purpose of "on-the-fly" scanning. Aside from enabling "On-the-fly" scanning, the controls system takes struck values and assigns them into correct slots of arrays. As a result, the values are combined and normalized by different array manipulations.

In this project we mainly modify the Python code, however, other crucial components work along with this software. Delta Tau is the motion control gate that transfers and receives signals for all used channels. The Delta Tau has its own power supply as well. The Delta Tau power supply can be switched between 0 Volts, 24 Volts, and 48 Volts. The VME crate is the next component, which acquires data and allows the code to select data at designated times while the motors are moving. And the last major link between the Delta Tau and VME crate is the Linux computer that serves as the primary user interface. The computer operates a file of Python code that communicates with the Delta Tau and VME crate. The code is one hundred and thirty lines long, which mostly does input and output of values from the motors and detectors.

Calling the initial modules enable functions and commands to input and output values, data, or even other commands. The main modules used are sys, time, array, numpy, and epics. The code then initializes relevant (x, y, monochromator) motor and scaler (struck clock, struck i0, struck iF) objects. The code has a specific motor and device access to channels that were created using the PyEpics interface linked to the VME crate. Each individual motor or scaler device has a multiple channel access that reaches a capacity of 2048 channels. The user becomes more involved now that values must be entered for the start and stop positions of the fast and slow motors. In the code, these positions are named $\mathrm{x} \_$start, $\mathrm{x} \_$stop, $\mathrm{y} \_$start, and $\mathrm{y} \_$stop. After that, the user needs to input the pixel sizes in millimeters, which are named $x \_$pixel_size and y_pixel_size. The next step is to ask the user the dwell time, named dwell in the code. The dwell time computes how much time from the scan a motor can linger at a certain position. And the user finally needs to input a file name so that saved data can be accessed at a later time. Having a file created at the start is highly beneficial to the user because even if the rest of the experiment can not be performed, there will still be raw data available that the user can extract whenever needed.

The code computes the number of pixels for two motors and defines the row numbers. The scan row is initialized to zero and the array row number is initialized to one. The arrays are immediately created with empty slots large enough for the number of pixels to fit each dimension. For example, the code can have a one hundred by one hundred pixel size array and pull the first hundred values from the interval of the first row scan, which provides enough as 2048 values. So the code will be limited by the maximum size of arrays, but "on-the-fly" scanning is efficient enough to produce exceptional and reasonably sized arrays. In the code, three arrays are created for the struck values and additional temporary arrays are created so that the values can be passed on to the first three arrays correctly.

After the arrays are created and right before the scan loop begins in the code, the motors need to be set up at correct positons and other motor parameters need to be refined. The $\mathrm{x}$ and $\mathrm{y}$ motors are shifted by the move function at $\mathrm{x} \_$start and $\mathrm{y} \_$start. The code needs to pause until the motors reach their start positions, so a while loop for each motor follows after the move function so that the segment repeats until the motors stop moving. Now that the motors are near ready to move, the motor resolution is recorded by extracting the value and a prescale is calculated "on-the-fly" scanning motion is precise enough to stay on each pixel for a short time. The prescale is an integer of a half plus the ratio of $\mathrm{x} \_$pixel_size to the motor resolution to five. Then the code transfers that value onto the prescale memory and sets the number of $\mathrm{x}$ pixels to number used by all channels.

After the parameters have been set up, the code begins a scan loop which is a main while loop that repeats motor motion and data acquisition. The condition for the scan loop is: while the row number is less than the number of y pixels, repeat the scan. In the scan loop, the first step is to move the $\mathrm{x}$ motor by the number of $\mathrm{x}$ pixels plus one all multiplied by $\mathrm{x} \_$pixel_size. Then the $\mathrm{x}$ motor state value is extracted using PyEpics to simulate the checking loop the same way it was done for the first x_start motor motion. Then the code delays with a sleep function for a fourth of a second. After the short delay, the data values are extracted using the same PyEpics interface. The three multiple channel access extractions are on the initial energy data, the fluorescence data, and the clock data. The three sets of data are then copied on the 
three temporary arrays. The data are placed into the temporary arrays because the values are 2048 number units long. So there is a resize on the temporary arrays from 2048 values to number of $x$ pixels values. And these values are inserted into the three primary arrays. Next the arrays are saved onto the master file created in the start of the code and both the row number and the axis number is incremented by one. Finally the x motor returns to the start position of the current row and the y motor moves up one pixel. And the created master file is closed after the scan loop is completed.

Overall, the code is split to three primary operating branches: PyEpics, NumPy, and Larch. PyEpics provides modules and functions so that the computer can translate EPICS channel access. NumPy works with PyEpics to produce the data acquisition system of the code. And finally Larch is the data analysis and visualization tool for the data that was collected using PyEpics and NumPy. The Python code is tested with the following default scan parameters: a 1x1 $\mathrm{mm} 2$ sample area, of $0.01 \mathrm{~mm}$ pixel size, and 0.1 second dwell time per pixel. It takes approximately 9 minutes for full scan on 1x1 mm2 sample area. "On-the-fly" scanning effectively reduces overhead of detector initialization and motor motion. Further, scanning time for XRF improved tremendously from about 4.5 hours to about 9 minutes, which is thirty times faster.

In the application of "on-the-fly" scanning, our intention is to identify arsenic (As) species and oxidation sates in the mine tailings by bulk-XANES, micro-XANES, and micro-XRF analyses. Mine tailings are soil samples extracted beneath the top layer from an area in the largest gold mine in Brazil. The gold mine is a large scale open pit operation located near the city of Paracatu. The mine includes a processing plant and a tailings restricted area. It is the biggest gold producer in Brazil (circa 453,396 oz per year). Previous studies indicated high levels of arsenic in gold mine tailings (up to $2700 \mathrm{mg} \mathrm{kg}-1$ ) with low arsenic bioaccessibility $(<4.4 \%)$.

The gold mine tailings sample studied in this project was extracted from inside the mine area but the sample contains a variety and spread of arsenic species. Arsenic is present in the sample predominantly as the $\mathrm{As}(\mathrm{V})$ species, but interestingly enough there is a considerable amount of the As(II) species. There is a correlation between As and iron ( $\mathrm{Fe})$ as the main background in micro-XRF images. Appearing in the background of fluorescence images, Fe must have oxidized with the As to form a complex as the soil was extracted and exposed to air. Therefore, the minor phase was arsenopyrite (FeAsS) and its transformation products.

In conclusion, "on-the-fly" scanning permits investigation of heterogeneity in elemental abundance and speciation at millimeter to micron scales in reasonable amount of time. "On-the-fly" scanning for fast sample stage motors form the fluorescence images to locate spots on different maps and select a smaller region for more detailed fluorescence and spectroscopic analysis. In this project, maps zoom into collected full-spectrum images that reveal a shift from As(-I) to other As oxidation states (III, V) in tailings. Monochromator "on-the-fly" scanning allows As K-edge micro x-ray absorption near edge spectroscopy ( $\mu$ XANES) in about thirty seconds, which is impressively thirty times faster than the usual time. Arsenic mainly occurs as As (V) sorbed onto ferric oxyhydroxides. This supports the previous finding of low bioaccessible As and verifies the importance of Fe oxyhydroxides in immobilizing As in the terrestrial environment. 


\title{
The Role of $\mathrm{Cu} / \mathrm{CeO} 2$ Nanospheres in the Water- Gas Shift reaction: An X-ray Diffraction and Transmission Electron Microscopy study
}

\author{
Ilana Heckler \\ SUNY New Paltz, New Paltz, NY, 12561 \\ Sanjaya D. Senanayake, Wenqian Xu, Siyu Yao, Aaron Johnston-Peck, and Jose A. Rodriguez \\ Brookhaven National Laboratory, Upton NY 11973
}

\begin{abstract}
Catalysis aims to answer the demands for new sources of energy by converting sustainable chemicals into fuels. The Water-Gas Shift reaction (WGSR) is an important industrial catalytic process that helps to purify $\mathrm{H} 2$ from streams of $\mathrm{CO}$ upon reaction with water. Efficient processes such as the WGS are important to produce pure hydrogen so that it could be considered a practical, renewable fuel in what is termed 'the future hydrogen economy'. The aim of this work is to characterize a copper/ceria catalyst and its behavior in the WGSR, using several advanced characterization techniques. The $\mathrm{Cu}$ $\mathrm{CeO}_{2}$ catalysts are known for being highly active for the WGS because of the intrinsic redox behavior of ceria and its ability to disperse, support, and activate the overlying metal particle. Spherical copper/ceria nanoparticles are used in this study for optimum dispersion, and as a way to retain the smallest metallic particle size $(\mathrm{Cu})$ The unraveling of these structural properties was important in understanding the active components of the water-gas shift activity. We used X-Ray Diffraction (XRD), in conjunction with Transmission Electron Microscopy (TEM), to characterize the catalyst structure reaction in situ and ex situ. Our results showed that $\mathrm{Cu}$ was predominantly metallic during the WGS and there was a redox transformation of $\mathrm{CuO}(2+)$ to $\mathrm{Cu}(0)$ Ceria is reduced during this process $\left(\mathrm{Ce}+4 / \mathrm{Ce}^{+3}\right)$ as well. This knowledge is essential to the long-term goal of optimizing hydrogen production by engineering the next generation of catalysts.
\end{abstract}

\section{Introduction}

In an effort to systematically implement the 'hydrogen economy', the scientific community has recently taken a strong interest into the catalytic conversion, synthesis and production of fuels that can be used for processes in industry, small personal devices and automotive applications.

$\mathrm{CO}+\mathrm{H}_{2} \mathrm{O} \leftrightarrow \mathrm{CO}_{2}+\mathrm{H}_{2}$

The Water-Gas Shift reaction (WGSR) is an important industrial process used in the reformation of natural gases, such as methane or synthesis gas, that is processed to recover hydrogen. Conventionally, the reaction proceeds in two steps; a high temperature shift (HTS) using a Fe-Cr system, and a low temperature shift (LTS) with $\mathrm{Cu}$ on a mixed zinc/aluminum oxide support. The WGSR not only produces hydrogen, but in parallel also rids of carbon monoxide, a catalyst poison that is extremely harmful to humans. There are two commonly proposed mechanisms for the WGSR. The most commonly accepted reaction proceeds through the formation of a carboxyl (HOCO) intermediate followed by its decomposition, while formate $(\mathrm{HCOO})$ and carbonate $\left(\mathrm{CO}_{3}\right)$ intermediates are the other likely intermediates. Studies have shown that that the formation of formate on the $\mathrm{Cu}-\mathrm{CeO}_{2}$ catalysts does not correlate with activity results. [5] The most commonly accepted mechanism involves the associative reaction between $\mathrm{CO}$ and $\mathrm{OH}$ to result in the aforementioned intermediates (as tested on $\mathrm{Cu}$ (111)), and through decomposition and recombination leads to $\mathrm{CO} 2$ and $\mathrm{H}_{2}[3]$.

$$
\begin{aligned}
& \mathrm{CO} \rightarrow \mathrm{CO}(\mathrm{s}) \\
& \mathrm{H} 2 \mathrm{O} \rightarrow \mathrm{OH}(\mathrm{s})+\mathrm{H}(\mathrm{s}) \\
& \mathrm{OH}(\mathrm{s})+\mathrm{CO}(\mathrm{s}) \rightarrow \mathrm{HOCO}(\mathrm{s}) \\
& \mathrm{HOCO}(\mathrm{s}) \rightarrow \mathrm{CO}_{2}+\mathrm{H}(\mathrm{s}) \\
& \mathrm{H}(\mathrm{s})+\mathrm{H}(\mathrm{s}) \rightarrow \mathrm{H}_{2}
\end{aligned}
$$

The reaction, however, is limited by thermodynamic equilibrium and is not optimum over the commercially available catalysts $(\mathrm{Cu} / \mathrm{ZnO})$. The ability to dissociate water is the most difficult step. Typically $0.3-3.5 \%$ of CO remains in the converted gas [1]. Preferential oxidation (PROX) typically follows the WGSR to purify $\mathrm{H}_{2}(\mathrm{~g})$ further. Polymer Electrolyte Membrane Fuel Cells (PEMFC), for example, require a concentration of less than 20 ppm CO to ensure that the catalyst $(\mathrm{Pt})$ will not become poisoned. The WGSR is thermodynamically favored at low temperatures but higher temperatures are needed to reduce $\mathrm{CO}$ concentration to less than $5 \%$.

Commercially available catalysts such as $\mathrm{Cu} / \mathrm{ZnO}$ are pyrophoric in their reduced state, typically deactivate rapidly and therefore cannot be used in many applications such as automobiles or personal electronic devices. WGS catalysts must be altered to be stable during start up-shut down cycles. Research has thus been focused on noble metal catalysts $(\mathrm{Pt}, \mathrm{Ru})$ for their stability and activity [4].

Previous studies have identified various metal/oxide combinations as highly active WGS catalysts. Cerium oxide supports have been extensively studied due to the intrinsic redox chemistry of ceria $\left(\mathrm{Ce}^{4+} / \mathrm{Ce}^{3+}\right)$, great oxygen storage capacity (OSC) and ability to activate metals. In theory, $\mathrm{Ce} 3+$ is responsible for the dissociation of $\mathrm{H} 2 \mathrm{O}$ (rate determining step) while the metal nanoparticle absorbs $\mathrm{CO}$. The presence of the ceria support activates and supports the overlying metal particle. Oxygen vacancies in the bulk ceria result from the changing lattice structure. This expansion is an effect of the larger ionic radii of $\mathrm{Ce}^{3+} . \mathrm{Cu}-\mathrm{CeO}_{2}$ systems are also known to be good WGS catalysts so they have the potential to be used in many applications [2]

Previously, WGS experiments have been done on $\mathrm{Cu}-\mathrm{CeO}_{2}$ catalysts showing good activity for the WGSR attributed to greater distribution and smaller particle size of the copper although the exact reason is not yet known.

$\mathrm{X}$-ray diffraction (XRD) is a means of determining the crystalline structure and active phases of catalysts. In situ XRD measurements are crucial to be able to observe the catalyst un- 
der working conditions. This scattering tool can be used to study ceria-supported catalysts in the WGSR and show the changing of $\mathrm{CeO}_{2}$ lattice parameters with rietveld refinement due to the reduction and oxidation of cerium. The diffraction peaks will show the structural changes of the catalyst as $\mathrm{CuO}, \mathrm{Cu}_{2} \mathrm{O}$ and $\mathrm{Cu}$ evolve during the reaction. Transmission electron microscopy can be used to image the morphology of catalysts before and after the reaction. This combination of tools provides a local and global view of the catalyst structure and can help to correlate the structure to the activity of the reaction.

\section{Methodology}

XRD experiments were run at beamline X7b $(\Lambda=0.3196 \AA)$ of the National Synchrotron Light Source at Brookhaven National Laboratory. High resolution TEM/STEM imaging was conducted using a JEOL and Hitachi instrument $(200 \mathrm{kV})$ at the Center for Functional Nanomaterials (CFN). Catalyst samples were dried onto a grid after suspension in $\mathrm{H} 2 \mathrm{O}$.

For all XRD measurements, about $3 \mathrm{mg}$ of $5 \% \mathrm{Cu} / \mathrm{CeO} 2$ nanosphere powder sample was loaded into a quartz capillary tube secured in place by a small amount of quartz wool. A heating coil was fixed to heat the sample from below the tube. A Type K thermocouple was placed inside the capillary in the quartz wool next to the sample to monitor temperature. The capillary was loaded into the Klausen flow cell, which is then attached to the goniometer head. The sample was then aligned with beam. Heating wire is attached as well as gas lines $\left(1 \% \mathrm{CO}, 3 \% \mathrm{H}_{2} \mathrm{O}\right)$. The Initial inflow of $\mathrm{CO}$ was $+8.4,17.73$ PSI. Output gas flow was monitored with a mass spectrometer, with masses calibrated for $\mathrm{CO}_{2}(\mathrm{~m} / \mathrm{z} 44)$, $\mathrm{H} 2(\mathrm{~m} / \mathrm{z} 2)$ and $\mathrm{CO}(\mathrm{m} / \mathrm{z} 28)$ and $\mathrm{H} 2 \mathrm{O}(\mathrm{m} / \mathrm{z} 18)$. The temperature controller was set to $250^{\circ} \mathrm{C}$ and programmed to increase at a rate of $5^{\circ} \mathrm{C}$ per minute starting from room temperature. At $250^{\circ} \mathrm{C}$, once temperature had stabilized, and after 83 spectra, CO flow was stopped and helium carrier gas was flowed at +8.3 . After 204 spectra $\mathrm{CO}$ flow was resumed. Reduction ( $\mathrm{CO}$ flow) and oxidation (H2O flow) were performed with $\mathrm{He}$ as the carrier gas.

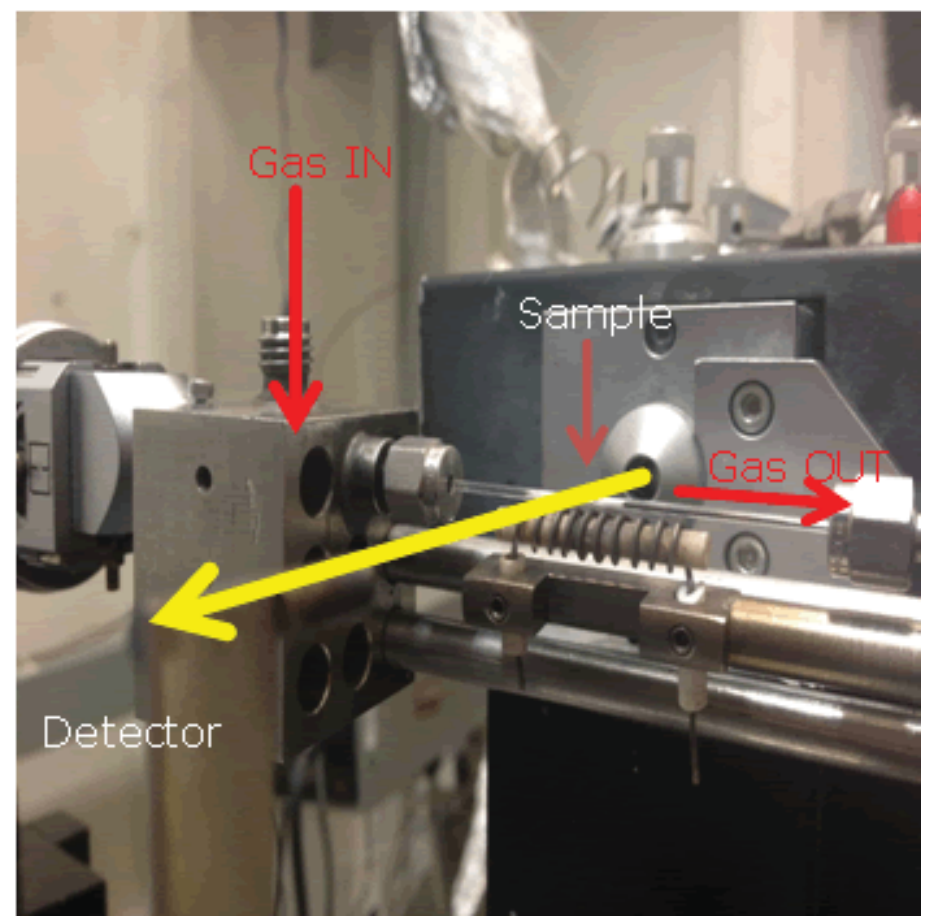

Figure 1: Flow cell set up for XRD measurments at X7b

\section{Results}

TEM data show spherical shape of the $\mathrm{CeO}_{2}$ nanoparticles. There is uneven distribution of the nanospheres and the mean particle diameter was found to be $9.142 \mathrm{~nm}$. Copper particles are not observed on TEM/STEM as dark spots. The $\mathrm{CuO}$ was approximately $5 \mathrm{wt} \%$ from ex situ XRD.

The first in situ XRD measurement was conducted on $\mathrm{CuO}$. Figure 4 shows the 3D pattern of XRD as a result of heating, $\mathrm{CuO}$ in $\mathrm{CO}$ gas and water vapor, to form $\mathrm{Cu} 2 \mathrm{O}$ through reduction, and formation of $\mathrm{CO}_{2}$. We identify $\mathrm{CuO}$ with peaks at $7.2(2 \theta)$ and the formation of $\mathrm{Cu}_{2} \mathrm{O}$ with peaks at $7.4(2 \theta)$. Further heating changes the $\mathrm{Cu}_{2} \mathrm{O}$ through reduction to metallic copper, that can be identified in XRD by a peak at 8.7 (20) At $200^{\circ} \mathrm{C}$ a very small amount of copper oxide remained. At $250^{\circ} \mathrm{C}$ metallic copper was the predominant copper species.

We obtained the information about the Ce through refinement, where the ceria lattice expansion is shown as being directly related to the reduction of copper oxide (Figure 9) As Ce is reduced oxygen vacancies are formed. These vacancies are highly active sites for WGS activity. [5] When CO flow is stopped, at $250^{\circ} \mathrm{C}$, metallic copper is oxidized by water to yield $\mathrm{Cu}_{2} \mathrm{O}$ (Figure $5)$. There is not enough oxygen in the system to reproduce $\mathrm{CuO}$. When $\mathrm{CO}$ flow is resumed copper is once again reduced (Figure 6). Two more cycles of $\mathrm{CO}$ flow being removed and resumed were performed (not shown) which confirmed the reproducibility of the experiment. RGA data shows, through $\mathrm{CO}_{2}$ production, that the activity of the catalyst remains constant with only a slight deactivation throughout the three cycles (Figure 8).

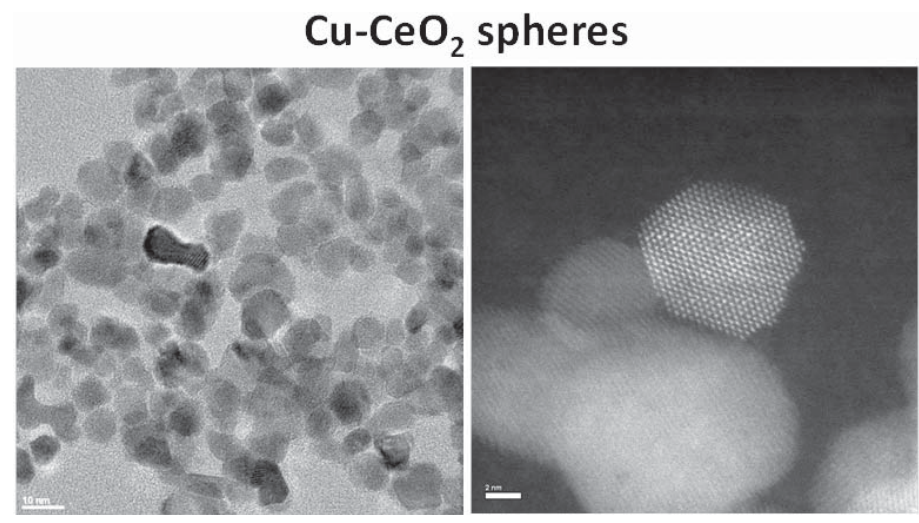

Figure 2: STEM/TEM images

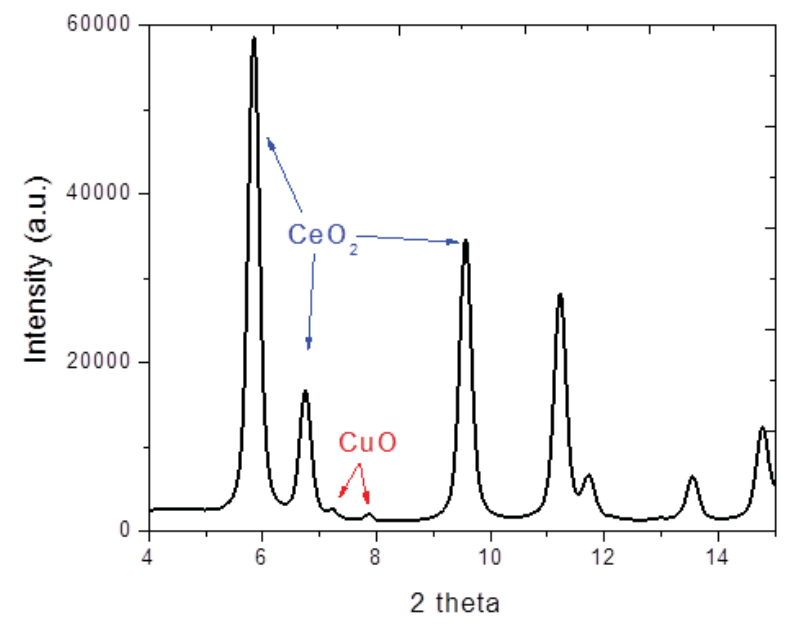

Figure 3: Ex situ XRD of the $\mathrm{Cu}-\mathrm{CeO}_{2}$ sample. 


\section{Conclusions}

$\mathrm{Cu}-\mathrm{CeOx}$ (nanospheres) are a good catalysts for the WGS and producing $\mathrm{CO} 2$ and $\mathrm{H}_{2}$. $\mathrm{CuO}$ is reduced to $\mathrm{Cu}$ in the WGS. Oxidation occurs with $\mathrm{H}_{2} \mathrm{O}$ to convert $\mathrm{Cu}$ to $\mathrm{Cu}_{2} \mathrm{O}$. Reduction with $\mathrm{CO}$ can then convert $\mathrm{Cu}_{2} \mathrm{O}$ to $\mathrm{Cu}$ metal. The expansion of the ceria lattice and $\mathrm{CeOx}$ reduction is shown as being directly related to the reduction of copper oxide. Expansion of the $\mathrm{CeOx}$ lattice leads to the formation of oxygen vacancies and conversion of $\mathrm{Ce}^{4+}$ to $\mathrm{Ce}^{3+}$. The structure of the catalyst is important to the activity of the WGS (known as the structure-activity relationship). In situ characterization is crucial to observe the catalyst under working conditions.

\section{References}

[1] Mierczynski, P., Maniukiewicz, W., \& Maniecki, T. (2013). Comparative studies of $\mathrm{pd}, \mathrm{ru}, \mathrm{ni}, \mathrm{cu} / \mathrm{znal} 2 \mathrm{o} 4$ catalysts for the water gas shift reaction. Central European Journal of Chemistry, 11(6), 912-919.

[2] Kušar, H., Hočevar, S., \& Levec, J. (2006). Kinetics of the water-gas shift reaction over nanostructured copper-ceria catalysts. Applied Catalysis B: Environmental, 63(3-4), 194-200.

[3] Gokhale, A. A., Dumesic, J. A., \& Mavrikakis, M. (2008). On the mechanism of low-temperature water gas shift reaction on copper. Journal of the American Chemical Society, 130(4), 1402-1414.

[4] Xu W., Si R., Senanayake S.D., Llorca J., Idriss H., Stacchiola D., Hanson J.C., Rodriguez J.A. In situ studies of $\mathrm{CeO}$ 2-supported Pt, Ru, and Pt-Ru alloy catalysts for the water-gas shift reaction: Active phases and reaction intermediates (2012) Journal of Catalysis, 291 , pp. 117126.

[5] Wang X, Rodriguez JA, Hanson JC, Gamarra D, MartínezArias A, Fernández-García M. J Phys Chem B. 2006;110(1):428

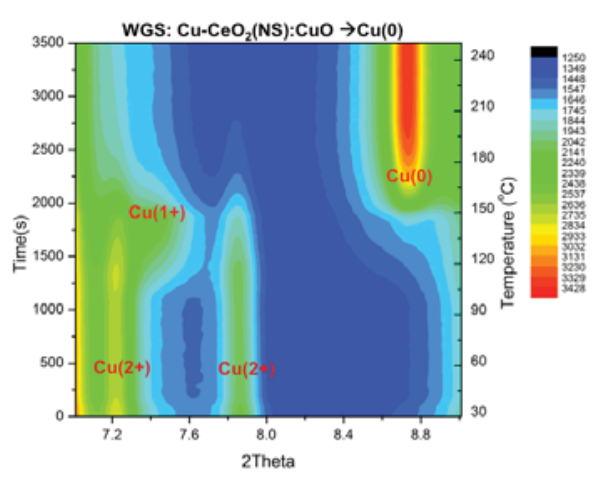

Figure 4: Heating $5^{\circ} / \mathrm{min}$ under WGS. $\mathrm{CuO}$ is first reduced to $\mathrm{Cu}_{2} \mathrm{O}$. At $200^{\circ} \mathrm{C} \mathrm{Cu}$ is predominant.

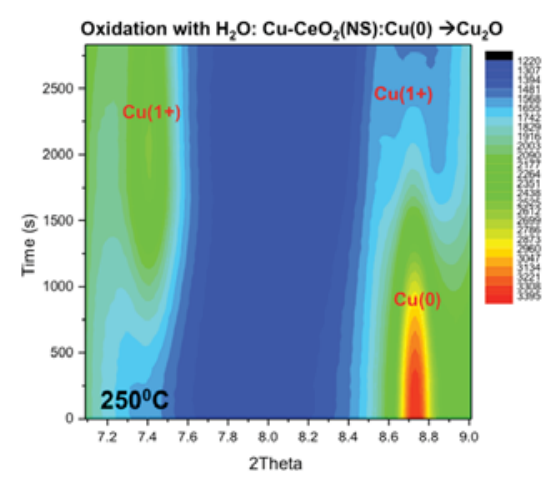

Figure 5: $\mathrm{CO}$ flow is stopped and metallic $\mathrm{Cu}$ is oxidized by water to yield $\mathrm{Cu}_{2} \mathrm{O}$ during oxidation

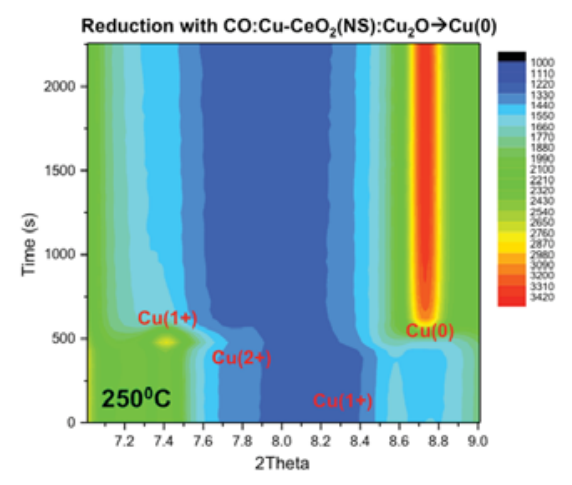

Figure 6: $\mathrm{CO}$ flow is started with $\mathrm{H}_{2} \mathrm{O}$ and $\mathrm{Cu}_{2} \mathrm{O}$ is reduced to metallic $\mathrm{Cu}$.

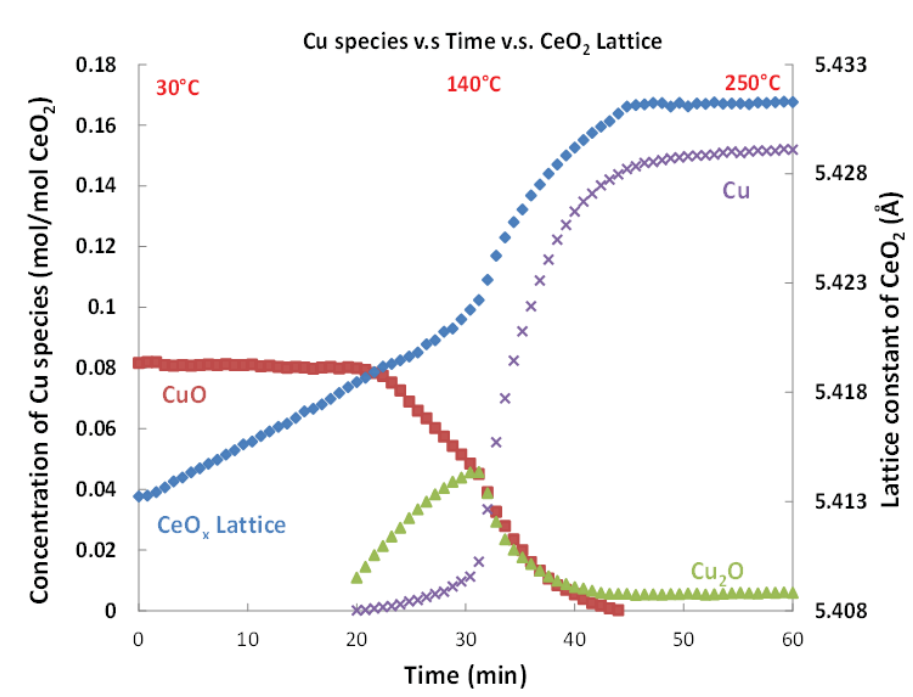

Figure 7: Refinement data showing expansion of ceria lattice, over time, as copper oxide is reduced to $\mathrm{Cu}$.

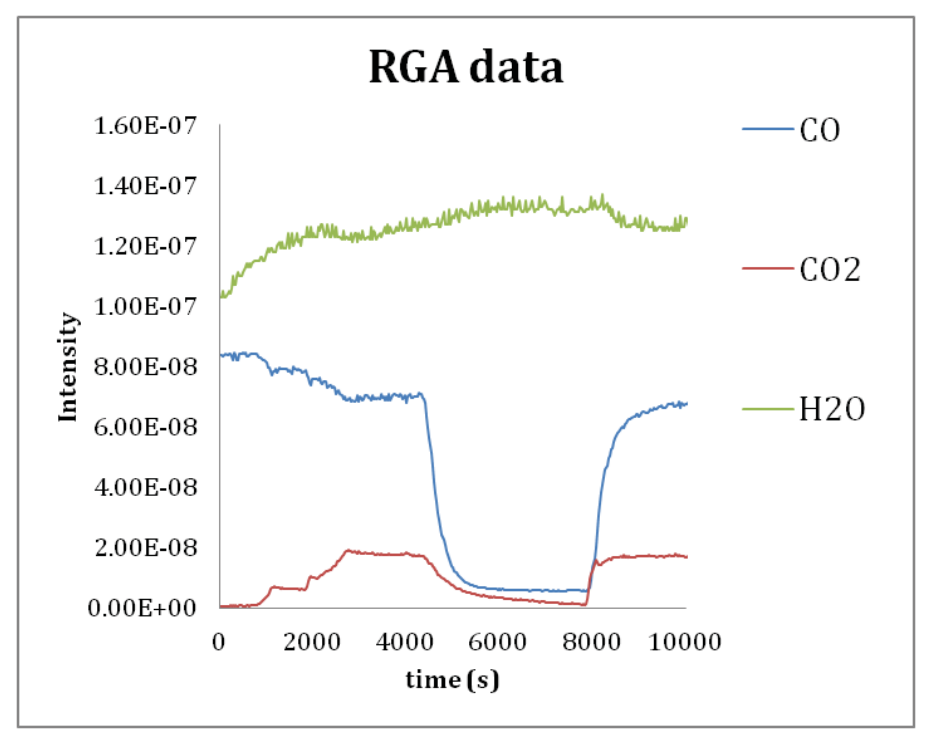

Figure 8: RGA data showing $\mathrm{CO}_{2}$ production during 3 cycles of stopping and starting $\mathrm{CO}$ flow. Activity of the catalyst does not significantly decrease. 


\title{
Investigation of Air Temperatures on the Long Island Solar Farm
}

\author{
Lauren Herrera \\ Stony Brook University, Stony Brook NY \\ John H. Heiser \\ Brookhaven National Laboratory, Upton, NY
}

\begin{abstract}
:
As the Department of Energy focuses its future on solar energy, Brookhaven National Laboratory has access to a two hundred acre solar farm that can power four thousand homes. Owned by BP Solar and MetLife, the Long Island Solar Farm (LISF) is the largest photovoltaic solar farm in the northeastern United States. The solar farm contains various sensors that monitor the meteorological conditions around the solar panels. This gives scientists a unique opportunity to collect valuable data that can be used to track and observe changes in the solar farm environment over the lifetime of the array. This paper focuses on the air temperature sensors throughout the solar farm and compares them to temperatures taken at the BNL meteorological (Met) field. The Met field is a large open grassy field that is maintained by mowing. The field is kept open so that the meteorological sensors, in particular, the wind sensors, are properly sited. The two-meter height (shaded) temperature sensor for the Met field is compared to the two meter under array temperature probes that are placed throughout the solar farm. A comparison of air temperature from the solar panel area to the meteorological field 2-meter tower can help determine if the solar panels are causing a heat island effect in the area. The temperature observations taken from the LISF and MET field can be compared to changes in Fauna and Flora in the area.
\end{abstract}

\section{Introduction}

As the county focuses their future on renewable energy, the department of energy is contributing their share of solar energy research. Brookhaven National Laboratory has access to a two hundred acre photovoltaic (PV) solar array (164,312 crystalline solar PV modules) that can generate up to $32 \mathrm{MW}$. Known as the Long Island Solar Farm (LISF), this solar array is unique when compared to large-scale solar farms around the world:

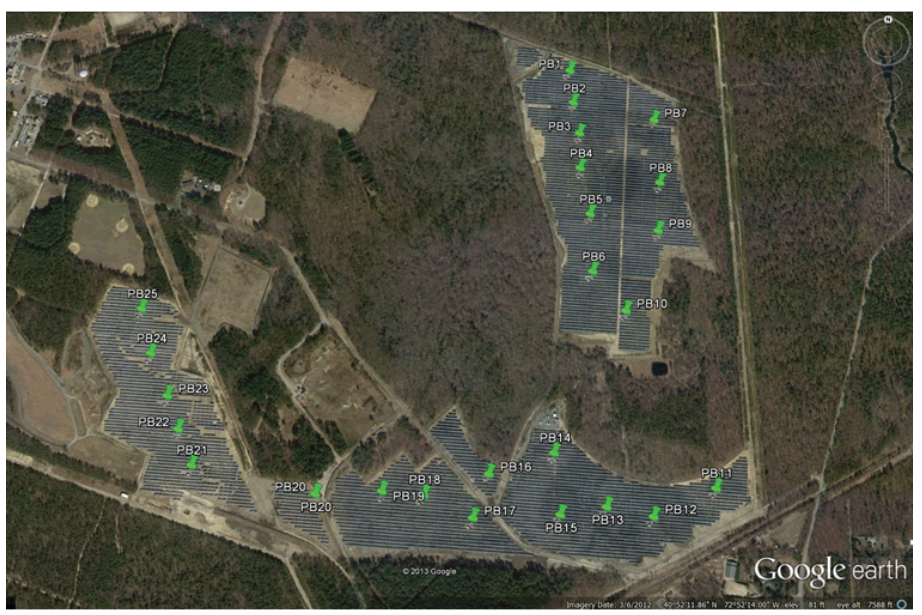

Long Island Solar Farm
Its numerous meteorological sensors, scattered throughout the solar array are collecting second data of current meteorological conditions on the Long Island Solar Farm. These observations will help scientist conclude any environmental changes occurring in the LISF area over the duration of the solar farm.

\section{A. Long Island Solar Farm}

The Long Island solar farm was built in collaboration with BP solar, MetLife, LIPA, and Brookhaven National Laboratory. This large-scale solar array consists of 25 inverter stations that convert the panels DC electrical current to AC.

\section{B. Meteorological Field}

The meteorological field is an open field that measures 140 meters by 240 meters, which is maintained by mowing. It is located approximately 2,000-3,000 meters from the Long Island Solar Farm. The field is kept open so that the meteorological sensors, in particular, the wind sensors, are properly sited.

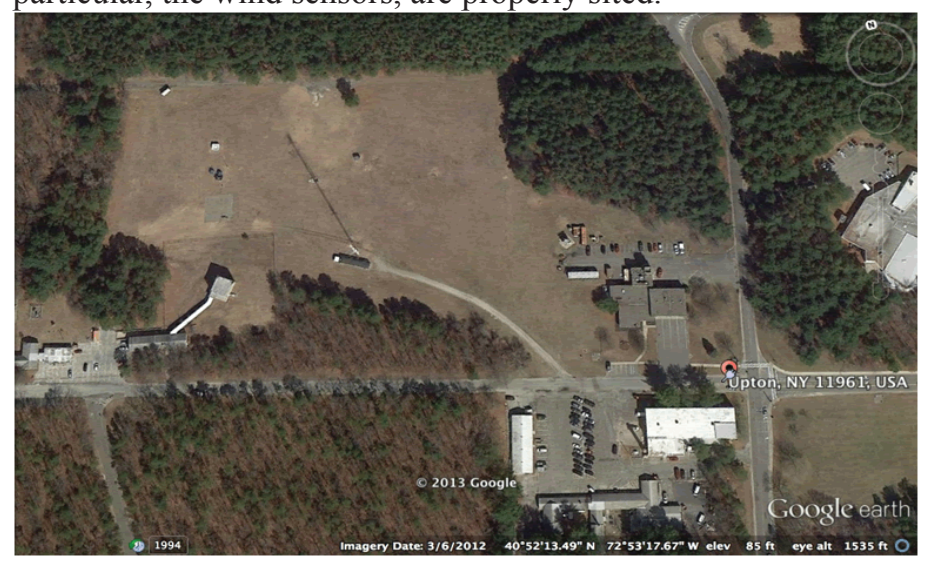

\section{Large Scale Solar Farms Consequences}

There are an increasing amount of large-scale solar farms around the world. As the world attempts to shift to solar energy, solar farms are becoming more popular and are being placed into different environments. The Ivanpah Project is a solar farm that is located in the Nevada Desert. There is a 1MW solar array on top of a canal in India. These solar arrays are being placed anywhere in the world and the consequences of placing these large scale solar farms are unknown. The solar panels can be causing changes in air temperature, which can affect local Flora and Fauna.

\section{Prediction}

Due to the large amounts of solar panels on the Long Island Solar Farm we expect to see a heat island effect in the area. This heat difference of $1-3^{\circ} \mathrm{C}$ is expected to effect local fauna and flora. 


\section{Materials and Methods}

A two-meter tower and a ten-meter tower are located in the center of the meteorological field. The two-meter height (shaded) temperature sensor is located in the Met field to ensure that the sun does not affect the air temperature readings. This tower includes a R.M. Young model $41372 \mathrm{VC}$ temperature sensor. The data collected is sent to Campbell CR1000 data loggers and stored in a main computer base.

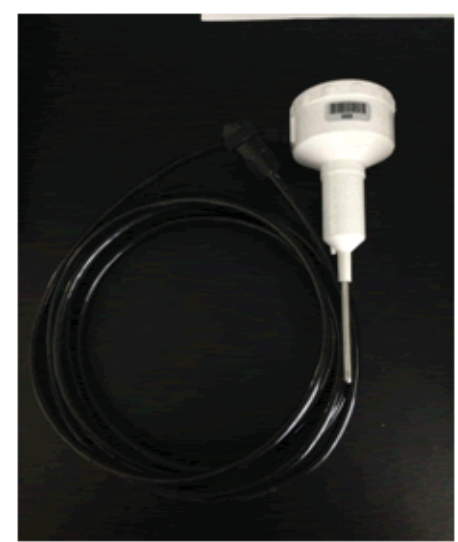

R.M Young Model 41342VC

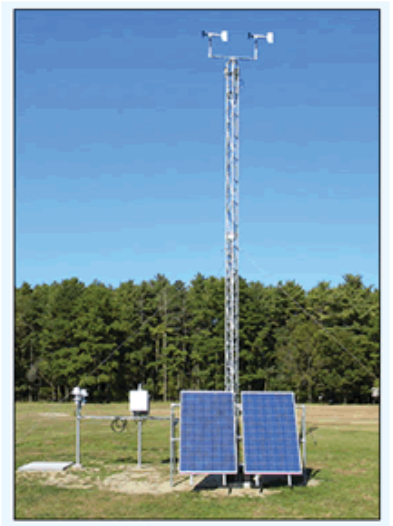
teorological field
$2 \& 10$ meter tower on the me-

\section{A. LISF Air Temperature Sensor}

The sensor that collects the air temperature data on the Long Island solar farm is a Omega model PRCU-10-2-100-1/4-6-e. This sensor is located underneath the solar panel to avoid the suns irradiance from affecting the air temperature readings.

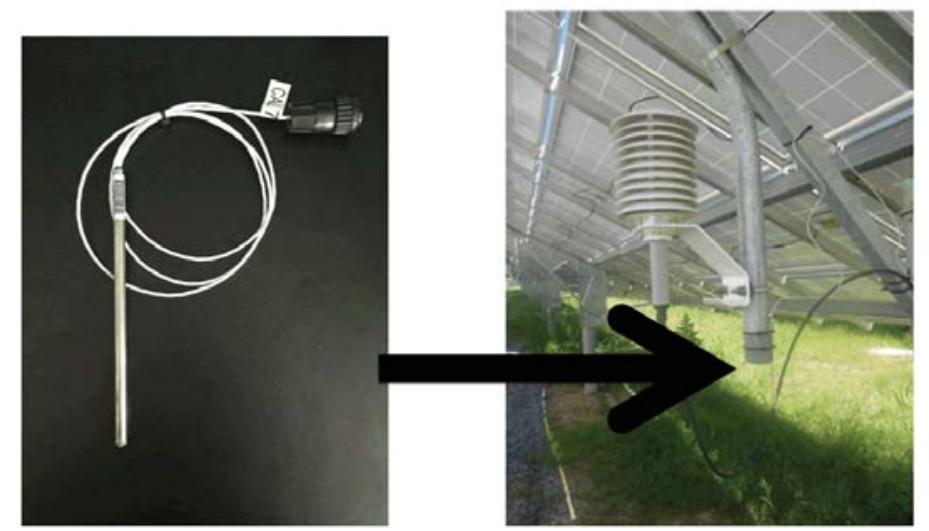

Omega model PRC-10-2-100-1/4-6-e sensor underneath solar panels

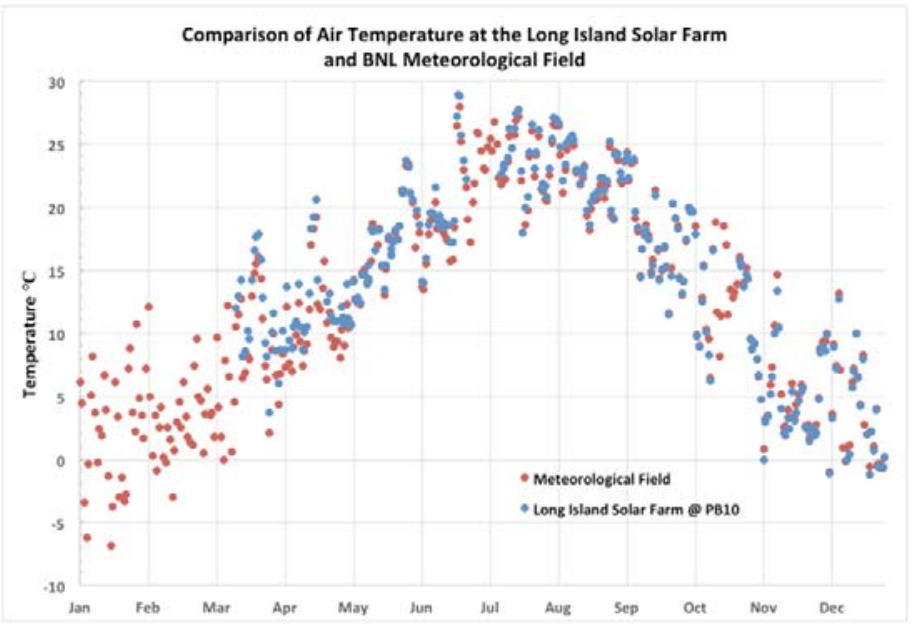

\section{Results and conclusions}

We plotted the daily air temperature means of the sensors located in the Met field and LISF (bottom, left). In plotting this means we were able to see that air temperatures for the year of 2012 were relatively the same. No significant air temperature difference was seen between the meteorological field and long island solar farm PB-10.

There was a slight difference of air temperature when comparing Area 6 August air temperatures to the rest of the 5 areas. For the month of August, Area 6 had higher air temperature reading than Area 1. We suspect that the higher elevation is causing a slight temperature difference in the extreme hot months. (Below,top)

There was a slight difference of air temperature when comparing Area 6 December air temperatures to the rest of the 5 areas. For the month of December, Area 6 had higher air temperature reading than Area 1. We suspect that the higher elevation in area 6 is causing a slight temperature difference in the extreme cold months.(Below, bottom)

\section{Future Work}

Daily air temperature collection will continue for the lifetime of the Long Island Solar Farm. The meteorological data will be complied and combined with studies from natural resources to see the consequences the Long Island Solar Farm has had to local Fauna and Flora.
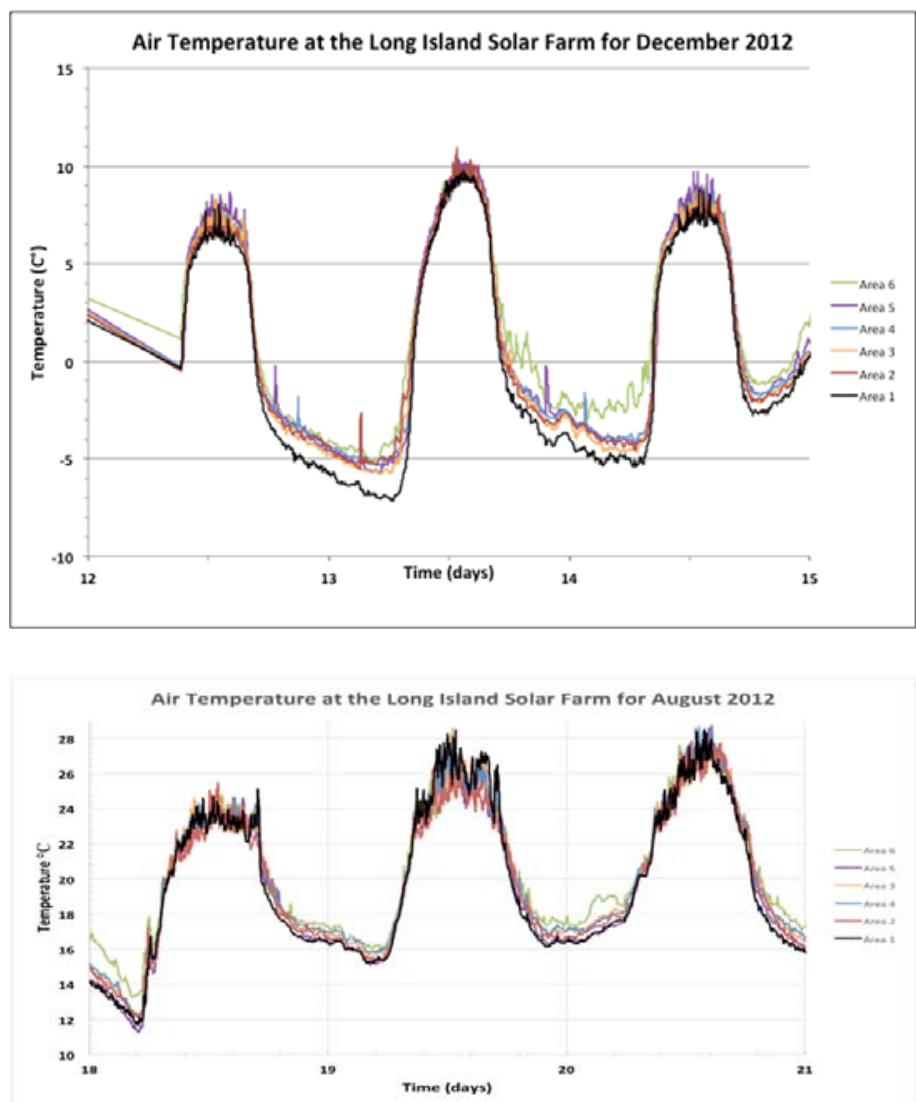


\section{Appendixes}

www.brightsourceenergy.com

www.thehindubusinessline.com

http://www.conergy.us/Utility-Solar-Case-Studies.aspx

http://www.bnl.gov/envsci/pubs/pdf/2013/BNL-99891-

2013-IR.pdf

http://www.bnl.gov/GARS/SET/LISF.php

http://www.bnl.gov/envsci/envtech/

http://www.bnl.gov/video/index.php?v=236

http://www.bnl.gov/today/story.asp?ITEM_NO=2719

http://libn.com/2011/11/18/lipa-flips-switch-on-long-island-

solar-farm/

\section{Acknowledgments}

- This project was supported in part by the U.S. Department of Energy, Office of Science, Office of Workforce Development for Teachers and Scientists (WDTS) under the Community College Internships Program (CCI).

- Environmental Science Department (BNL)

- Office of Educational Programs (BNL)

- John Heiser

- Scott Smith

- Lawrence Millian

- Andrew McMahon

- Paul Kalb

- Environmental Science Building Staff

- LISF Staff

- Jennifer Higbie

- Timothy Green 


\title{
Redesigning an events $\&$ issues database to allow for statistical analysis and accident prediction
}

\author{
Daniel Hirsh \\ Electrical Engineering Department, The City College of New York, New York, NY 10031 \\ Nancy Nair \\ Quality Management Office, Brookhaven National Laboratory, Upton, NY 11973
}

\begin{abstract}
An existing database used by the Quality Management Office containing safety data is currently unsuitable for statistical analysis. This project provides a preliminary redesign of the database structure which will allow for trend observation and accident prediction. Research into journal articles was conducted in order to explore prior studies regarding accident prevention. Limitations of the current system were explored in order to better evaluate the structure needed for the new system. Difficulties of the current procedures were examined and revised or new procedures were designed in order to address many of the limitations and challenges the department faces now. Care was taken to prevent the redesign from being unnecessarily complicated and to ensure the best compatibility with future requirements, which includes using existing standards where appropriate. A mock user interface was programmed in order to present the new design in a visual and interactive manner. Actual implementation and testing will not take place until the project moves forward and a commercial system is chosen and purchased.
\end{abstract}

\section{INTRODUCTION}

The Quality Management Office (QMO) offers many services to the laboratory. Among them include fact finding for events and issues, performing causal analyses, and providing corrective action plans. Many of the databases the QMO uses were initially designed only to log events, and not to provide a basis for statistical analysis. As a result, the data is incomplete and often inconsistent, making it insufficient for the goals of the laboratory, looking forward. Requests for proposals have been made of several vendors in order to find a new data management system to replace the old one. This allows for the opportunity to change the existing system and procedures in order to better handle the goals of the department, and ultimately, the laboratory in general.

The purpose of this project, therefore, is to provide a preliminary redesign of the structure of the Events and Issues database in order to allow for statistical analysis and accident prediction. The trends observed will then be able to guide the lab into proactive action in order to prevent accidents where they are most likely to occur. Research into journal articles was conducted in order to explore prior studies into accident prevention. The limitations of the current system were explored in order to better evaluate the structure needed for the new system. In addition, the current policies and procedures were examined in order to propose changes that will allow proper entry of logs and greater consistency of the data.

\section{METHODS AND DISCUSSION}

The Events and Issues database contains logs of problems and injuries that occur in the laboratory. If, for example, a non-conforming part is discovered in a contractor's materials, a chemical spill occurs, or a worker gets injured, all of these events get logged in this database.

In order to perform statistical analysis, the essential information must be separately notated in a granular fashion. To a computer, "2 people", "two people", and " 2 ppl" are all distinct items. In order to run a programmatic analysis, data must be entered in a manner that is uniform and leaves no room for interpretation. In addition, the information must be entered in a consistent manner. If fields are not being filled out, no meaningful analysis can be performed.

One of the most significant problems of the existing database is that the majority of relevant information is entered in the free-text description. This means that although all the required information may be present, it is in a form that renders it useless for programmatic analysis.

In order to address this, a new structure must be designed that overcomes this issue by separating the essential pieces of information and having them entered via fixed choices rather than a free text field. A final description field is provided that may still contain the information as it is entered now. However, the additional fields will possess the necessary data in the required form.

Below is a non-exhaustive listing of fields for the restructured database. Certain fields are non-relevant as they pertain to administrative requirements rather than statistical analysis, and have thus been omitted. In addition, the listed fields are considered primarily from the perspective of the user entering the data. The actual backend data structure is largely non-relevant to the purposes of this study and have also been omitted, with a few notable exceptions.

\section{A. User interface \\ - Event Date \\ - Event Time}

Note: The date and time should be separate in order to do analyses that look at time of day patterns.

- Title of Event/Issue

- Significance Category:

- $\mathrm{OE}$

- 1

- 2

- 3

- 4

- BNL

- NR

- Status

- Open

- Closed 
- Information on the person involved:

- If Employee or Guest:

- Life Number

- Lookup: All info listed below

Note: To protect Personally Identifiable Information (PII), the Life Number does not need to be stored in the database. It can be implemented to only pull non-PII data, such as type of employee, department, length of time at lab, etc...

- Person Involved:

- Employee

- Guest

- Visitor

- Contractor

- Student

- Unknown

- Department

- Length of time working at lab

- Age

- Employment Type:

- Administrative

- Professional

- Scientific

- Technical

- Union

- Other

- Job Title

- Multiple People Involved? (y/n)

- Participants

- General Location (i.e. buildings, streets, general grounds, etc...)

- Specific Location (free text field)

- Indoors/Outdoors (radio button)

Note:This is in regards to the location of the original incident, not where subsequent damage occurred.

- Equipment Responsible: (checkbox list)

- BNL

- Contractor

- Private

Note:This is in regards to whose equipment was responsible for the event, not whose equipment was subsequently damaged.

- Factors: (checkbox list)

- Weather

- Visibility

- Fatigue

- Event Classification:

- Property/Equipment/Service Issue (no injuries)

- Injury

- Inspection/Observation

- Branch - Property/Equipment/Service Issue (no injuries):

- Cause:

- Accident

- User error
- Faulty equipment

- Environmental

- Other

- Unknown/TBD

- Nature of Issue:

- Animal

- Blunt force damage

- Chemical

- Electrical

- Equipment Malfunction

- Fire

- Loss of service

- Non-Compliance/Violation

- Radiological

- Suspect Counterfeit

- Transportation

- Unwanted Release

- Unwanted Energy Release

- Waste Management

- Other

- Unknown/TBD

- Work Planning:

- Experimental Safety Review (ESR)

- Permit Planned Work

- Prescribed Work

- Worker Planned Work

- NA/None - no work involved

- Unknown/TBD

- General Activity (Combo box)

- Branch - Injury:

- Cause (Same as earlier)

- Nature of Issue (Same as earlier)

- Work Planning (Same as earlier)

- General Activity (Same as earlier)

- Nature of Injuries: (Note: Use the OI\&IC)

Note: There is no need to reinvent the wheel in regards to specifying the list of possible injuries. An existing reference already exists, the US Department of Labor Manual on Occupational Injury and Illness Classification. Therefore, this will be coded into the user interface, allowing standards-based classification of injuries.

- Injury Case \#

- Multiple people injured (y/n)

- Property/Equipment/Service Issue (y/n)

- Branch - Inspection/Observation:

- Nature of Issue: (Same as earlier)

- Work Planning: (Same as earlier)

- Property/Equipment/Service Issue (y/n)

- Event Description (free text narrative - exists for all branches) 
- Analysis:

- Relevant Facts

- Fact Analysis

- Corrective/Preventative Action

- Lessons Learned

\section{B. Mock user interface:}

A mock user interface (UI) was coded in order to provide a means to visually see and interact with an implementation of the suggested structure. The information has been split up into tabs in order for the user to not be overwhelmed with fields. In addition, the fields shown change based on user selections so that questions that are not applicable to the event being entered are hidden.

Figure 1 shows the first tab, which contains the basic information universal to all events.

Figure 2 shows one of the dropdown lists, which is used to avoid the problems that surface when typing the information in a free-text field.

Figure 3 shows the second tab, which is where most of the event-specific details are entered. This tab changes its appearance significantly depending on what type of event is selected. Shown is the Injury category. In addition, you can see how the Guidance section on the right provides detailed information to help the user make the correct choices.

\section{Analysis of safety data:}

The project parameters call for the purchase of a commercial system. As such, the software will come with various analysis routines built-in. However, for any advanced analysis that needs to be done, the data can be exported and the analyses run separately.

So long as the database structure provides the necessary granularity, the methods of analysis do not need to be determined in advance, as they can always and easily be changed after the fact. However, preliminary research was done via journal articles to explore possible techniques.

There are many methods that have been used to attempt to analyze accident statistics. Some methods use linear modeling, however, accidents and their causes often have complex, non-linear relationships. This causes such models to perform poorly on real-life data.

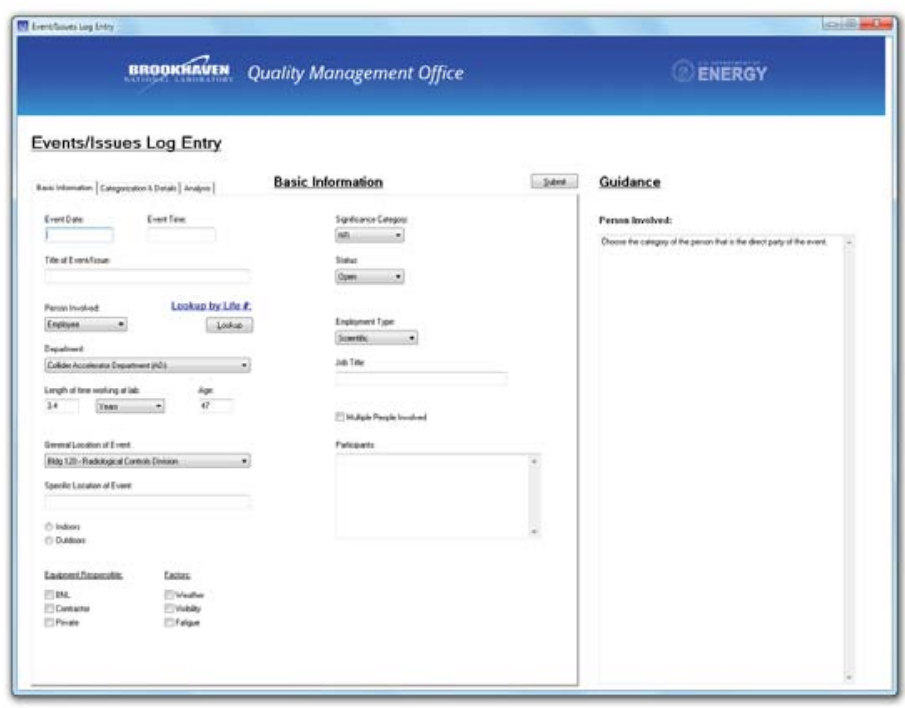

Figure 1
At the time of the research, the number of studies that compared different algorithmic techniques specifically for occupational accidents were few in number. In one of the more promising ones by Rivas, a study was done comparing several methodologies to test which data-mining techniques extract the most useful information on workplace accidents. They evaluated Bayesian networks, decision rules, classification trees, logistic regression and support vector machines. In this study, the Waikato Environment for Knowledge Analysis (WEKA) freeware developed by the University of Waikato was used to determine which variables were the most relevant. The study determined that the best results were obtained using a Bayesian network using the K2 algorithm, which had an $88.71 \%$ success rate in predicting future accidents. In addition, the Bayesian/K2 networks have the added advantage of allowing what-if analyses.

This offers a starting point for advanced analysis on the new system.

\section{Policies and procedures:}

No matter how well designed the database may be, if the policies and procedures that govern the entering, consistency, and integrity of the data are lacking, the resulting dataset is useless. Current policies allow many fields to remain blank, and do not

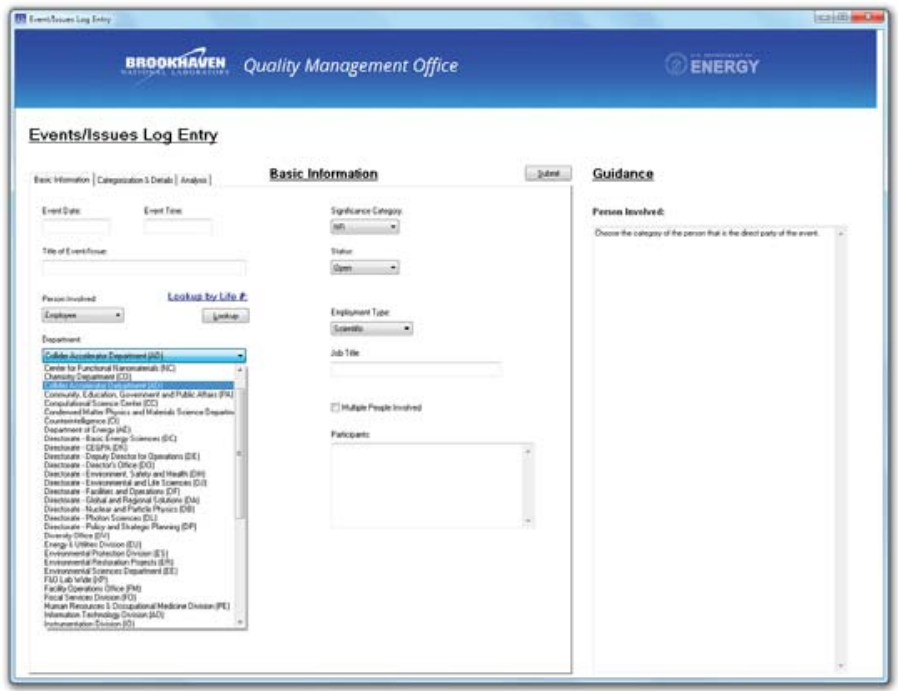

Figure 2

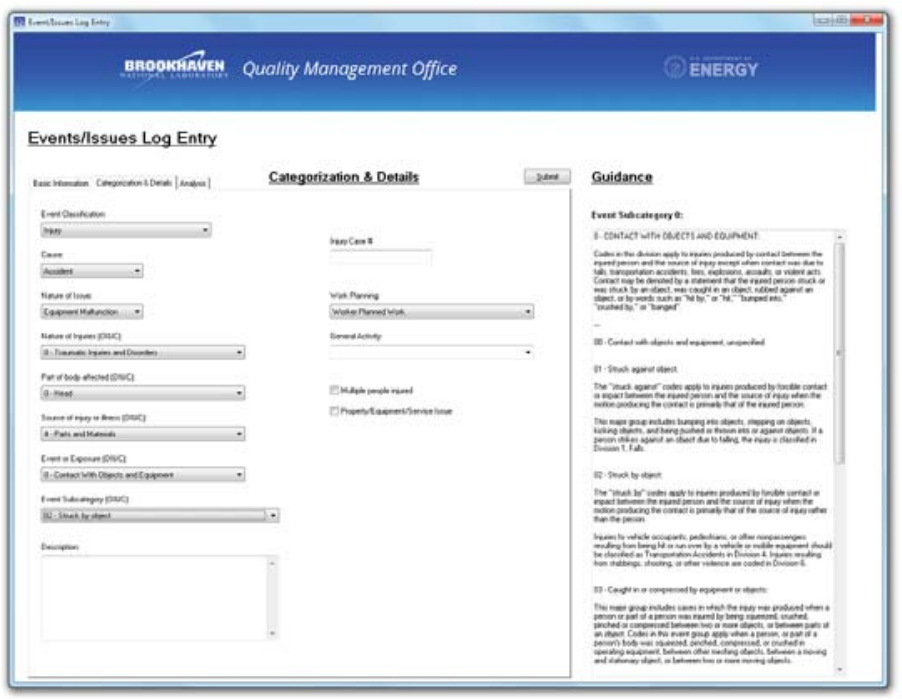

Figure 3 
require consistency in how logs are entered. As such, we design a set of goals and the procedures to implement them.

\section{Policy Goals:}

- Ensure that data is initially entered in a complete and accurate manner.

- $\quad$ Ensure that event logs are updated as new information and developments occur.

- Audit all event logs to confirm that the information is correct and complete. Follow up with log authors to correct any problems and to educate them on how to log events in the future.

\section{Implementation:}

- Ensure that data is initially entered in a complete and accurate manner.

This is accomplished with a two pronged approach through training for all users who have the authority to log events, and through careful design of the user interface of the logging application.

\section{User Interface Requirements:}

- $\quad$ Easy to understand and navigate.

- Clear and unambiguous choices and fields.

- An absolute minimum of free-text fields. Wherever possible, questions must have fixed selections of answers.

- Database lookups wherever possible and appropriate. (e.g. rather than risk misspellings, inconsistent names, and inaccurate data, if it is possible to enter a Life Number, the system should look up and autofill the employee details from the HR database)

- $\quad$ Appropriate fields must be made mandatory (i.e. the log cannot be saved or closed out unless all required fields are filled).

- Guidelines for fields where there might be confusion must be easily and clearly available.

- Ensure that event logs are updated as new information and developments occur.

Procedures must be set in place to update event logs as new information comes in. This involves multiple departments and is beyond the scope of this project.

- Audit all event $\operatorname{logs}$ to confirm that the information is correct and complete. Follow up with log authors to correct any problems and to educate them on how to log events in the future.

It is recommended that staff should be assigned whose responsibilities are to confirm all information is complete and thorough. Inconsistencies or overly terse and lacking descriptions would be followed up by the auditor with the author of the log, and if needs be, with the direct party.

An additional recommendation is that a full change log system should be in place to provide a complete record of all changes to data, the date/time they were changed, and the user who changed them.

\section{CONCLUSION}

The database structure was able to be redesigned so as to provide the necessary foundation for statistical analysis. Policies and procedures were suggested to allow for clear and consistent data entry, as well as to provide methods of insuring the integrity of the data. Other studies were researched for methods of advanced analysis, and among them, a Bayesian network using the K2 algorithm stood out as a very viable and accurate methodology. Future research can be made into other techniques such as neural networks. As the new structure gives the granularity needed for advanced analysis, future methods can be implemented with little or no change to the recommended design.

Actual implementation and testing will not take place until the project moves forward and either a commercial system is chosen and purchased, or changes are made to the current database and the user interface is rewritten.

\section{ACKNOWLEDGEMENTS}

This project was supported in part by the U.S. Department of Energy, Office of Science, Office of Workforce Development for Teachers and Scientists (WDTS) under the Science Undergraduate Laboratory Internships Program (SULI). I would like to thank my mentor, Nancy Nair, for her guidance on this project, as well as the Brookhaven National Laboratory, for their hospitality and kindness.

\section{REFERENCES}

${ }^{1}$ Vijia K. Karra, "Analysis of non-fatal and fatal injury rates for mine operator and contractor employees and the influence of work location," Journal of Safety Research 36(5), 413421 (2005).

${ }^{2}$ T. Rivas, M. Paz, J.E. Martín, J.M. Matías, J.F. García, J. Taboada, "Explaining and predicting workplace accidents using data-mining techniques," Reliability Engineering \& System Safety 96(7), 739-747 (2011). 


\title{
Using perfluorocarbon tracers to prevent chemical warfare
}

\author{
Richard Houanche \\ University at Albany, Albany, NY 12222 \\ John Heiser, andRichard Wilke \\ Brookhaven National Laboratory, Upton, NY 11973
}

\begin{abstract}
The Subway-Surface Air Flow Exchange (S-SAFE) study is to assist First Responders to determine the best course of action to minimize casualties and optimize the evacuation of residents who may be impacted by an accidental or intention chemical or biological release. Perfluorocarbon tracers (PFTs) are compounds that can be used for studying long range atmospheric transport and dispersion, as well as evaluating building ventilation dynamics and isolating underground utility cable leaks, etc. These tracers are nonreactive, biologically benign, and traceable to low atmospheric levels (10 parts per quadrillion). Gas Chromatography is used to analyze all of the data and to separate the different types of PFTs used in the study. We are using seven different PFTs, which allow us to study the air flow between many locations in the New York City area. In NYC the air can travel via a combination of natural wind patterns, vehicular traffic, solar convection, and the subway system. The S-SAFE experiment is focused primarily on gaining knowledge about the interaction between the surface air flows and the subway system.
\end{abstract}

\section{Introduction}

Tracer technology has many different uses: atmospheric transport dispersion, underground system integrity, air filtration systems, tower footprint models, characterization of heat exchange volumes, and more. To gain information on how the air travels in New York City Perflourocarbon tracers (PFTs) were released into the air. PFTs have been tools for studying long range atmospheric transport and dispersion since the early 1980s. Perflourocarbon tracer compounds are totally fluorinated cycloalkane compounds consisting of 4, 5, and 6 atom carbon rings (Thomas B. Watson). PFTs are extraordinary for many reasons, for this study the most important reasons are: nonreactive, biologically benign, traceable to low atmospheric levels. Non-reactive chemicals are those that do not readily combine with other common compounds, which allow scientists to accurately analyze them within the results. Biologically benign compounds are harmless to people, PFTs are sometimes used for medical procedures and put into a person's bloodstream. PFTs are traceable to low atmospheric levels as far as 10 parts per quadrillion (ppq), allowed us to only release them in small gram quantities. The high electronegativity of the PFTs is due to the fluorine that they contain, which in return makes them easier to located and very stable.

For the PFTs to be acquired from the air they are captured by capillary absorption tubes (CATS tubes). The CATS tubes contain an adsorbent called Ambersorb which can retain PFTs and can be cleaned out of PFTs. Another intern and I were responsible for cleaning nearly 10,000 capillary absorption tubes by heat to over $400^{\circ} \mathrm{C}$ and having nitrogen pass through the tubes. Nitrogen a "makeup gas" is used in this process because it is a gas that exhibits low excitation energy, making it easy to remove an electron from a nitrogen molecule. Before testing the data obtained in the study it was necessary for us to make standards for mock samples, which allowed us to create calibration curves. Calibration curves allow us to know better differentiate between PFTs and knowing how immense each PFT should appear at different concentrations. The Gas Chromatography is what we use to create all of the calibration curves and the method that analyses the results. A Gas Chromatograph (GC) has many components in which allow it to be very precise, for example the electron capture detector, and the mass spectrometer. An electron capture detector is used in a $\mathrm{GC}$ to detect trace amounts of chemical compounds in a sample, by distinguishing atoms and molecules in a gas throughout the attachments of electrons via electron ionization (LLC Books). A Mass Spectrometer is a device that gives access to conform the identity of compounds in the CATS tubes selectively and accurately.

\section{Procedure}

There were three days in the city that was throughout the month of July to perform this study. For this study to work it was necessary for there to be a vast amount of people working on it during each of those three days. There were about 20 teams created which had scientist and interns within them. Each team had about six people in it and we also had the help of NYPD. NYPD helped distribute all of the air samplers throughout the city to specific locations for the teams.

Each team had a team leader and an assistant team leader, for my team I was an assistant team leader. The teams on the surface would then make sure that the air samplers were placed onto the appropriate hooks, seven feet off the ground. The teams in the subways would attach the air samplers to benches at different stations and floors. From $8-2 \mathrm{pm}$ the air samplers were on and running, initially taking a background sample and then samples after the PFTs was released; there were 12 CATS tubes in each air sampler. The teams were regularly checking samples throughout the day to make sure they were still operational. From the time period they were on the samplers were programed to have a specific CATS tube running.

There were two different types of air samplers during the study, a BBS and a BATS II about 100 samplers of each were used each day the experiment was done. The BATS II actually had the wifi capabilities to be able to see which tube it was on and how was the flow rate at the moment, along with GPS that was turned on a 7:30 am. At the end of the day it was necessary to cape all of the tubes to prevent contamination. 


\section{Field Deployment}

Figure 1: Graph which shows that two PFTs reached the same air sampler during an Intensive Operational Period (IOP) on July 9th.

In this study we released seven different PFTs each in separate locations throughout Manhattan, Queens, and Brooklyn. The release of the PFTs was done in the subways and from there the travelled to locations all over the city, underground and above the surface. The data shown here in the graph above is as explicate as we are able to show to the public. It is a graph of what was found in one of the two hundred air sampler within this study, which was within the subway. From taking out the CATS tubes in this particular air sampler and placing them into the gas chromatograph we were able to notice that two different PFTs traveled to this air sampler. One of the PFTs had a much greater concentration of the PFTs that the other in this air sampler, this could be due to the distances the release points of both PFTs, or the traveling that was done by the subway trains during that time.

Both of the PFTs had concentrations that were increased, peaked, and decreased during the same time period which showed that the PFTs were traveling and dissipating throughout the city at the same time scale. An hour after the samples were released could be the time in which most of the air samplers have their peak concentrations; this is great to know for analyzing the results. By knowing where at what time each air sampler should be peaking tells us which CATS tubes are the most valuable for the areas where we may be expecting a very low concentration of PFTs. Also if the CATS tubes were somehow dropped and taken out of order, we could still decipher where each CATS tube should be. It is more than likely that air samplers in generally close proximities will have very similar results relating to the PFTs in which they contain and which of their CATS tubes have the peak concentration.

The CATS tubes in the study are very dependent on not only their displacement from the release points but for also if the subway trains make stops between stops quickly. For example if there was an unfortunate air release that happened at Penn Station, the data could show if and how fast will there have been a serious danger to Washington Square Park. Within ten minutes the air traveling between both stations on the A, C, or E trains could easily be transported. During the three days that we performed this study we tried to collect as much data as possible with having a very small amount of variables that could be different for the last study. On all of the same days of the study the wind was relatively, coming for the same direction with similar speeds. The only big differences between our first two days and our last day that we went in to the city was that the last day was very cold, fortunately the temperature of each day should not me a factor that changes any results.

\section{Discussion}

The S-SAFE Study was founded by NYPD department, there for the results are for official use only (confidential). No results will be given to the public in this study because of the fact that this is an anti-terrorism project. If the results are to be given to the public then they could be used against the people of New York City. The results will assist First Responders to determine the best course of action to minimize casualties and optimize the evacuation of residents who may be impacted by an accidental or intention chemical or biological release.

\section{Acknowledgements}

John Heiser and Richard Wilke

Environmental Science Department

Department of Education at Brookhaven National Laboratory bany

College Science and Entry Program at the University at Al-

College of Nanoscale Science and Engineering

National Science Foundation

New York State Police Department

\section{Bibliography}

LLC Books. Gas Chromatography: Katharometer, Gas-Liquid Chromatography, Inverse Gas Chromatography, Flame Ionization Detector, Electron Capture Detector. Books LLc, 2006.

Thomas B. Watson, Richard Wilke, RussellN. Dietz, John Heiser and Paul Kalb. "The Atmospheric Background of Perfluorocarbon Compounds Used as Tracers." American Chemical Society (2007): 4. 


\title{
Beam emittance measurements in the transfer line to RHIC at BNL
}

\author{
J. Huang \\ Duke University, Durham, North Carolina 27708 \\ D. Gassner, and M. Minty \\ Brookhaven National Laboratory, Upton, New York 11973, USA
}

\begin{abstract}
The Alternating Gradient Synchrotron (AGS)-to-Relativistic Heavy Ion Collider (RHIC) transfer line, abbreviated AtR, is an integral component for the transfer of proton and heavy ion bunches to RHIC. In this study using $23.8 \mathrm{GeV}$ proton beams, we focused on factors that may affect accuracy of emittance measurements used to determine the quality of beams in RHIC: background noise, calibration, resolution, and dispersive corrections. Ideal video Offset (black level, brightness) and Gain (contrast) settings were determined for consistent initial conditions in the Flag Profile Monitor (FPM) application. Using this information, we also updated spatial calibrations for FPM using corresponding fiducial markings and sketches. Resolution error was determined using the Modulation Transfer Function amplitude. To measure dispersive contribution, we conducted a scan of beam position and size at relevant Beam Position Monitors (BPMs) and Video Profile Monitors (VPMs, or "flags") by varying the extraction energy with a scan of the RF frequency in the AGS. The combined effects of these factors resulted in slight variations in emittance values, with further analysis suggesting potential discrepancies in the current model of the beam line's magnetic properties. In the process of testing various contributing factors, a system of checks has been established for future endeavors, providing an efficient, standardized, and reproducible procedure that might encourage greater reliance on the transfer line's emittance measurements.
\end{abstract}

\section{INTRODUCTION}

The 580m long AtR transfer line relies on a series of profile monitors equipped with mostly Charge-coupled device cameras (CCDs) [1] to help determine if acceptable beam parameters exist prior to injection into RHIC, thereby avoiding the use of corrupt bunches. This transfer line is used to make emittance measurements between the machines, which are of particular importance given that lower emittance allows for greater and more frequent collisions, in turn producing a desirably higher luminosity. By gathering accurate measurements, it becomes possible to control beam growth and therefore enhance performance of the transfer line. The beam emittances are derived from measurements of the horizontal and vertical beam sizes (called sigma, which refers to the RMS of the assumed Gaussian distribution of the beam profile) at three separate locations together with the known strengths of the magnets in the AtR [2]. By testing the sensitivity of emittance to contributing factors such as calibrations, resolution, dispersion, and noise under uniform conditions, a straightforward method of measurement transferable to future studies was established.

\section{OFFLINE EMITTANCE ANALYSIS}

A majority of our analysis was performed offline after the run cycle ended. In order to calculate and test sensitivity of emittance with logged data, we therefore used a script file that reads saved input files and outputs measurements. Given that emittance has a dependency on horizontal and vertical sigma values, we tested each potential factor by applying its impact - measured as a ratio or multiplier - to sigma. After editing a file of sigmas to adjust for the factor of interest, we ran the emittance script to determine the corrected measurements. A majority of this calculation was done in command prompt and file editing programs such as emacs and gedit, but some of the analysis also involved programs like matlab and excel. Acquisition of logged data required use of LogView, an internal program, and FPM. This latter program allowed us to manipulate and measure beam spots with an interface shown in figure 1 .

\section{INITIAL CONDITIONS}

The AtR transfer line relies on VPMs to test beam line conditions. They are plunged and removed from the beam pipe through the FPM controls application, an interface that allows users to alter configuration settings while viewing immediate results in one of four equally capable frame grabbers, or viewing windows. While inserted, the beam hits and illuminates the phosphor (Gd2O2S:Tb) screen. The twelve flags are spread out along the transfer line and separated into four sections, named the U, W, $\mathrm{X}$, and $\mathrm{Y}$ lines. Based on locations defined in figure 2, flags of interest for this study are UF3, UF4, UF5, WF1, WF2, and WF3 of the $\mathrm{U}$ and $\mathrm{W}$-lines.

The goal of our work was to test factors that affect emittance measurements as a means to produce an efficient method of acquiring accurate data during future run cycles. Before delving into these properties, however, ideal and uniform initial conditions were necessary. FPM offers a user-controlled environment for optimal beam shots and measurements by way of optional background subtraction, intensity adjustment, and Range of Interest (ROI) selection. Previous research has elaborated on the harmful effects of background subtraction and advantages of the ROI function [3]. Background subtraction was therefore switched off for all scans.

Intensity settings relate to Gain and Offset, or contrast and brightness, respectively. Using a python tape sequence, we acquired and plotted data sets to determine the effects of varying offset and gain settings on vertical and horizontal sigma and center. Figure 3a illustrates the ideal offset range of 242-252 for which sigma remains constant. To avoid saturation, values on the lower end of this range were considered ideal. In the second scan, Gain was confirmed as having little effect on sigma while remaining in the viable range of 80-160 (figure 3b). To confirm accurate beam parameters are not dependent on intensity variations, we performed a scan of intensity and employed neutral density filters to conclude that there was no problem between very low intensi- 
ties and 2E11 (see figure 4).

\section{CALIBRATION}

When discussing highly precise machinery and technology, it is reasonable to expect natural changes in positioning or accuracy of individual monitors and camera lenses. To counter this inevitable adjustment, we conducted a recalibration of Flags in the $U$ and $\mathrm{W}$-lines using screen fiducial drawings from the commissioning of the transfer line, as shown in figure 5. Marks on each drawing correspond to those on the phosphor screen of each flag, and the comparison of theoretical and measured distances on these screens provides a corrective ratio.

Precise measurements of distance on flag screen images require a saved image from FPM called a "bitmap". Given that these images are unix executable files, an additional image processing software is necessary. ImageJ, a freeware available online, not only recognizes this file type, but it also offers the capability of viewing coordinate pixels on any imported image. Using the line tool and pixel display in ImageJ, we calculated the $\mathrm{mm} /$ pixel relationship to match existing calibrations using appropriate conversion factors (note that the fiducial sketch must be rotated 90 degrees and turned over to match the perspective of the bitmap). By taking a ratio of the updated calibration over the existing value for each flag, we obtained corrective ratios, as shown in Table 1. Since the measurement of interest in this study was emittance, we multiplied these ratios by measured sigmas from the initial scan to derive input data that demonstrated calibration effects on emittance measurements. These new values are listed in Table 2.

TABLE 1. New to old corrective calibration ratios for FPM update

\begin{tabular}{|c|c|c|c|c|c|c|}
\hline & \multicolumn{1}{|c|}{ UF3 } & UF4 & UF4 & WF1 & WF2 & WF3 \\
\hline $\mathbf{X}$ & -0.987 & 0.994 & -0.996 & 1.255 & -0.978 & 1.088 \\
\hline $\mathbf{Y}$ & 0.986 & -1.045 & 0.997 & -0.983 & 0.980 & -1.122 \\
\hline
\end{tabular}

\section{RESOLUTION}

AtR imaging resolution is measured using a translation of the Modulation Transfer Function (MTF) for a bitmap under ideal intensity conditions with no suppression of the baseline. MTF is identified in equation 1 .

$$
M T F=\frac{(\text { Peak }- \text { Trough })}{(\text { Peak }+ \text { Trough })}
$$

Plot profiles of ruler sections on bitmaps in ImageJ provide average peak and trough values for each flag of interest. Resulting MTF amplitude values (contrast) ranged from 0.214 to 0.445 , with four flags falling short of the ideal threshold 0.33 that corresponds with a 200 micron resolution. To correct for these less than ideal values, we used the "PatternCalc" Excel program created by Peter Thieberger to generate a resolution coefficient, or Line Spread Function (LSF), from the contrast percentage. Using the adjusted input files, we corrected the emittance measurements based on the resolution of each flag.

\section{DISPERSION}

In the fall 1995 commissioning of the AtR transfer line, an early attempt to measure dispersion was made by adjusting magnet strengths to different extraction energies [4]. Unfortunately, too many influential variables were involved, thereby compromising the validity of results. During the 2013 run cycle, we per- formed a dispersion scan at BPMs and flags by changing the AGS extraction frequency in $1 \mathrm{~Hz}$ steps for a range of $-5 \mathrm{~Hz}$ to $+5 \mathrm{~Hz}$ (Fig. 6a). A correlation plot of this energy change $(\mathrm{G} \gamma)$ and BPM position (Fig. 6b) provides a slope equal to the dispersive contribution [eq. 2a-2d]

$$
\begin{aligned}
& \chi=a \cdot G \gamma+b \\
& \frac{\partial x}{\partial G \gamma}=a \\
& D x=\frac{\partial x}{\frac{\partial G \gamma}{G \gamma}} \\
& D x=a \cdot G \gamma \cdot 10^{-6}
\end{aligned}
$$

where $\mathrm{G} \gamma$ is a convenient energy scale, $\mathrm{G}$ is the anomalous part of the proton magnetic moment (1.7928), $\gamma=\mathrm{E} / \mathrm{m}$ with $\mathrm{E}$ as beam energy and $\mathrm{m}$ as proton mass. Equation $2 \mathrm{~d}$ represents horizontal dispersion in um.

Similarly, dispersion at each flag can be calculated with a correlation of $\mathrm{G} \gamma$ at each flag plotted against position (horizontal center in pixels). This method requires additional conversion factors to apply previously determined calibrations and convert units from pixels to meters. Dispersive contribution was measured using two methods at two locations for assurance, but this preferred method was applied to each flag location for additional corrective values. Interestingly, the introduction of dispersive corrections had negligible effects on emittance, suggesting future studies can plausibly ignore this correction (see Table 2).

\section{BACKGROUND NOISE}

The cameras used in FPM follow rules of the NTSC frame rate conversion. By measuring peaks and troughs in relation to the frame rate, specific frequencies can be determined. In the case of FPM, background noise visible in figure 7a is a high frequency synchronization error. The likely cause of this is coupling of transmission waves and an outside power source. If this is the case, expensive linear transmissions might be necessary as a means to avoid unreasonably high frequencies. Necessity of these expenses can be estimated by measuring emittance with and without noise. A basic solution is described here using Gaussian blur with a demonstrated $3-5 \%$ change. Total benefit, however, must be measured using experimental linear transmissions.

Figure 7a shows a high frequency noise pattern, or electromagnetic interference (EMI) drifting diagonally through the digitized image. This pattern is likely the result of upper harmonics in the switching power supply coupling to our analog video signal, but time has not yet been allocated to this study for confirmation. Background subtraction as a function in FPM was deemed overpowering in the 2011 study [3], so an alternative could be use of software filters like the Gaussian blur. This low pass filter blurs the EMI noise while maintaining integrity of the beam spot, in effect removing disruptive frequencies from raw data (Figure 7b).

In this study, EMI noise suppression is performed on the bitmap of each flag with the most noticeable noise for optimal corrections in emittance. We use ImageJ to obtain initial standard deviations as well as new values after applying a Gaussian blur with a tested sigma (radius) of 3.0. A ratio of initial to final standard deviation produces the corrective ratio, which is then multiplied by sigma values already corrected for the previous three factors. 
See Table 2 for noise-corrected emittance results.

\section{RESULTS/CONCLUSIONS}

Emittances were measured at two different intensities along both horizontal and vertical planes to test the effects of calibration, resolution, dispersion, and EMI Noise corrections. Both lower intensity and noise suppressed beams generally produced lower emittance measurements, whereas calibration and resolution corrections adjusted emittance on a case-by-case basis. Since our data collection method demonstrates no immediate problems, it is possible that the existing model holds some inaccuracies. Future studies might then benefit from closer examination of magnet performance between flags as well as the code used to evaluate emittance.

TABLE 2. Average horizontal and vertical emittance measurements (in $\mathrm{mm}$ mrad) for two intensities and varying factors at $U$ and W-lines. Dispersive corrections are not shown because dispersion is negligible.

\begin{tabular}{|l|r|r|}
\hline \multicolumn{1}{|c|}{ U-line X } & \multicolumn{1}{c|}{ 2.0E11 } & \multicolumn{1}{c|}{ 1.45E11 } \\
\hline Initial & $10.9+/-0.4$ & $11.0+/-0.5$ \\
\hline Spatial calibration & $11.7+/-0.3$ & $10.3+/-0.5$ \\
\hline Resolution correction & $10.6+/-0.4$ & $10.0+/-0.5$ \\
\hline EMI Noise suppression & $10.2+/-0.4$ & $9.7+/-0.5$ \\
\hline U-line Y & $23.5+/-0.3$ & $19.5+/-0.7$ \\
\hline Initial & $23.1+/-0.3$ & $19.4+/-0.6$ \\
\hline Spatial calibration & $23.2+/-0.3$ & $19.1+/-0.6$ \\
\hline Resolution correction & $22.6+/-0.3$ & $18.7+/-0.5$ \\
\hline EMI Noise suppression & $15.8+/-0.1$ & $13.2+/-0.1$ \\
\hline W-line X & $19.5+/-0.2$ & $16.3+/-0.1$ \\
\hline Initial & $18.6+/-0.2$ & $15.2+/-0.1$ \\
\hline Spatial calibration & $17.6+/-0.2$ & $14.4+/-0.1$ \\
\hline Resolution correction & $23.8+/-0.4$ & $21.4+/-0.7$ \\
\hline EMI Noise suppression & $26.8+/-0.4$ & $23.4+/-0.7$ \\
\hline W-line Y & $25.1+/-0.5$ & $21.9+/-0.7$ \\
\hline Initial & $23.4+/-0.5$ & $20.4+/-0.7$ \\
\hline Spatial calibration &
\end{tabular}

During my ten weeks working in the Collider/Accelerator Department of BNL, I discovered the intricacies of life as a science researcher. I gained a newfound appreciation for access to machinery during busy run cycles; I realized the difference cooperation among groups could make; I felt the excitement of drawing conclusions from data that I personally acquired and analyzed. I also developed practical skills in programs ranging from the internal FPM and LogView applications to the universally applicable programs such as Matlab and ImageJ. In updating the instrumentation wiki page, I not only documented my work for future exercises, I also practiced and learned how to operate this information source. My experience working with successful intellectuals in this field toward the submission of a Particle Accelerator Conference paper has been both educating and rewarding, and it is a summer I will always look back on as a period of rapid growth and achievement.

\section{ACKNOWLEDGEMENTS}

Thanks to Steve Tepikian, Peter Thieberger, Nick Tsoupas, and Vincent Schoefer for their time and dedication to this effort, as well as the incredible mentorship of David Gassner and Michiko Minty. Special thanks to Chris Zimmer for writing and running the tape sequence for camera setting optimization and Intensity scans during the busy end of the run. Thanks also to coordinators and operators in the main control room for their cooperation and help with data acquisition during the 2013 run cycle.

\section{$X$. REFERENCES}

[1] R. L. Witkover, "Design of the Beam Profile Monitor System for the RHIC Injection Line”, IEEE'96, p. 2589.

[2] M.C. Ross et al., "Automated Emittance Measurements in the SLC," PAC'87, p. 725.

[3] P. Thieberger et al., "Baseline suppression problems for high precision measurements using optical beam profile monitors," PAC'11, p. 486.

[4] T. Satogata et al., "Physics of the AGS-to-RHIC transfer line commissioning," EPAC'96.

\section{FIGURES}

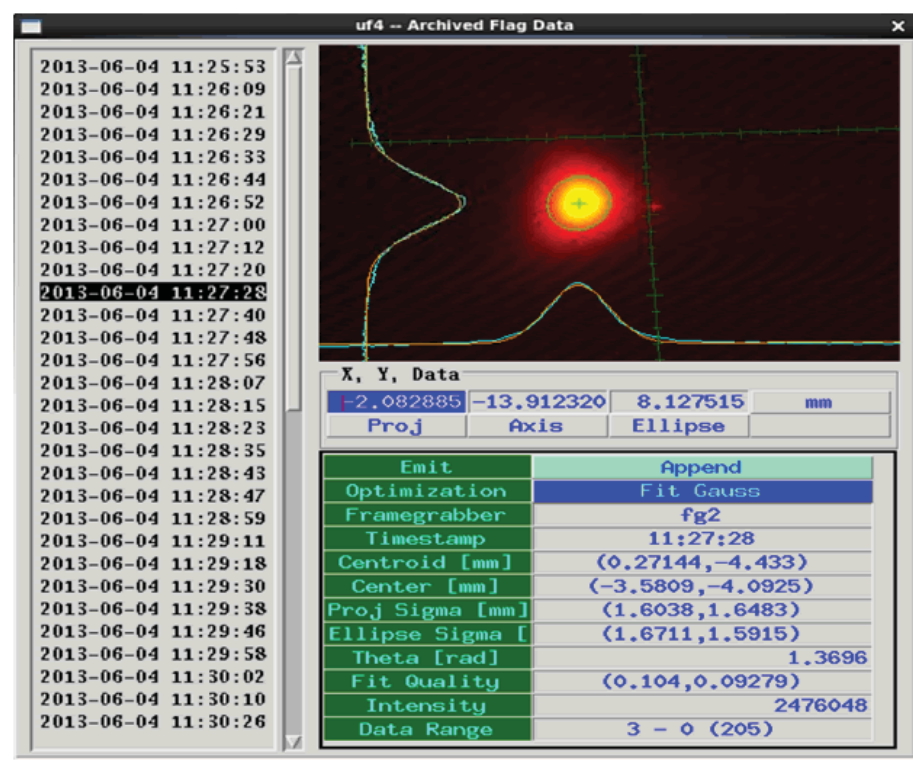

Figure 1: Example logged UF4 beam in FPM from June 4, 2013 


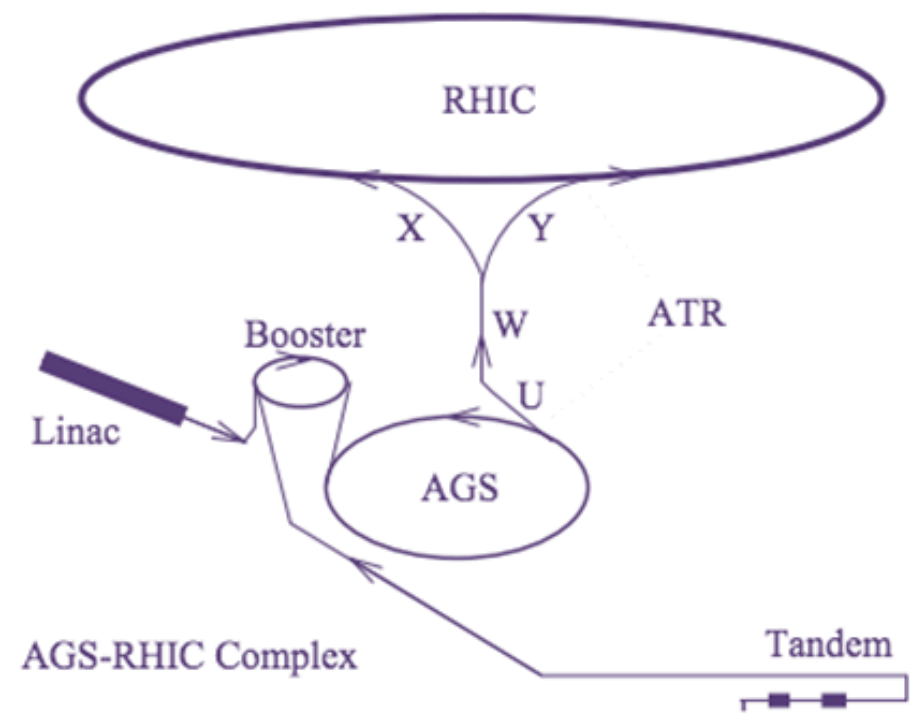

Figure 2: Diagram of RHIC beam progression including transfer lines
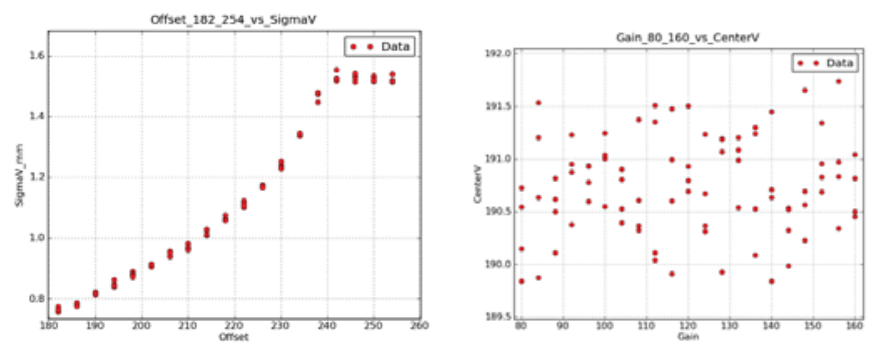

Figure 3a (left): UF4 1E11 intensity plot of offset vs. vertical sigma Figure 3b (right): UF4 1E11 intensity plot of gain vs. vertical center

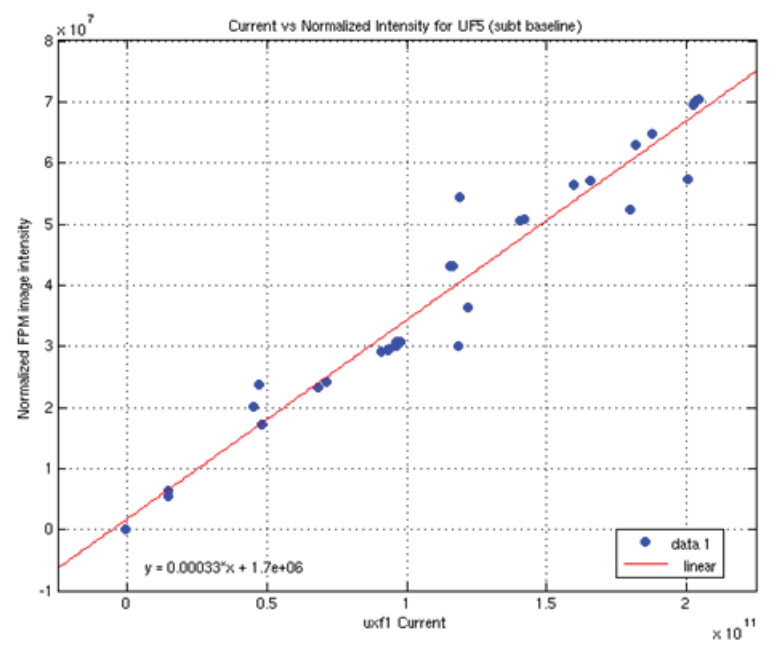

Figure 4: Linearity of current vs. normalized intensity; evidence that beams were not saturated.

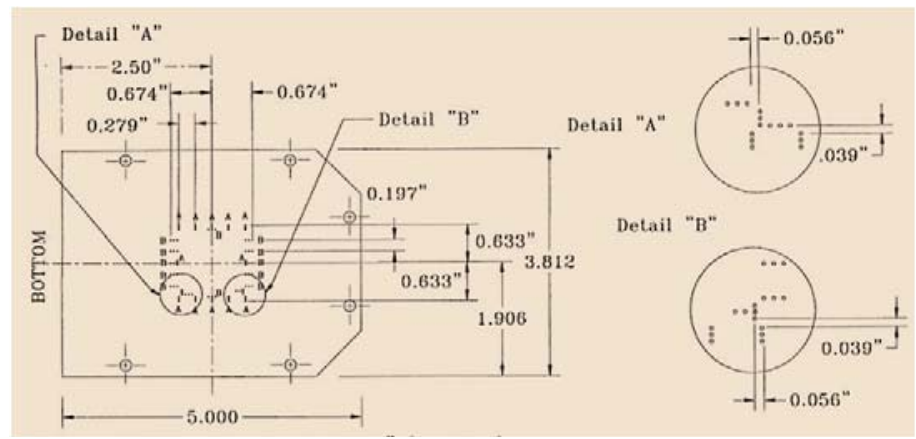

Figure 5: Screen fiducial diagram for UF4 and WF1

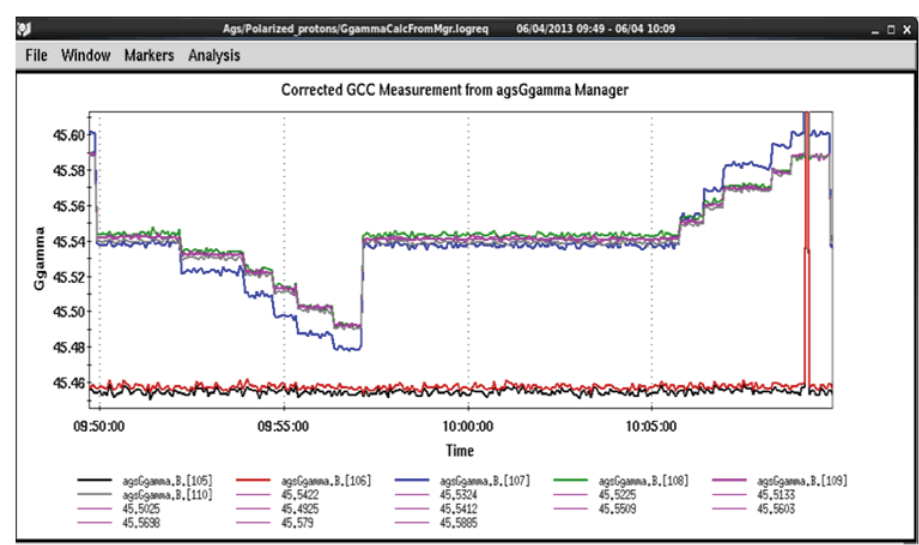

Figure 6a: Ggamma values for each time/frequency step

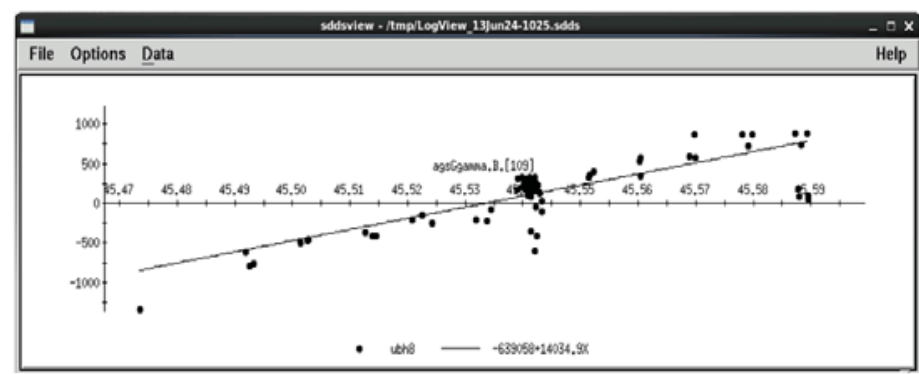

Figure 6b: Fitted correlation plot of Ggamma vs BPM position

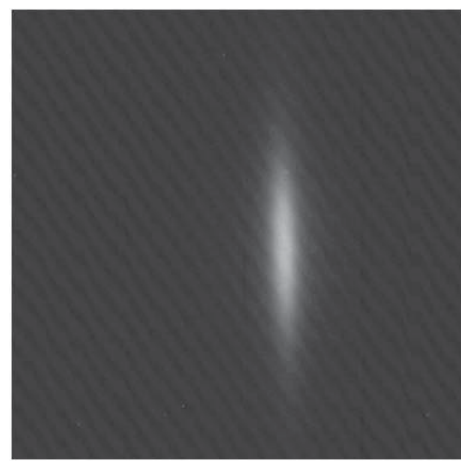

Figure 7a: original bitmap of UF3

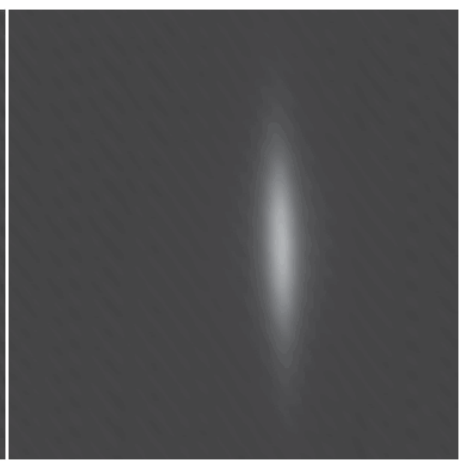

Figure 7b: UF3 bitmap with Gaussian blur 


\title{
Systems Biology Markup Language formulation of a dynamic kinetic model and its uses in quantitative and computational metabolic modeling
}

\author{
Mark V. Jensen \\ Computer Science, Suffolk County Community College, Selden, NY 11784 \\ Jordan O. Hay \\ Biosciences Department, Brookhaven National Laboratory, Upton, NY, 11973
}

\begin{abstract}
Brassica napus is currently the third largest source of vegetable oil in the world and, is used as food, feed, and to produce biodiesel fuel. Using a quantitative computational metabolic simulation model, the overall goal of this project is to identify which important metabolic pathways in Brassica napus can be increased or decreased in order to engineer a seed embryo yielding higher quantities of oil. With America being the largest biofuels producer in the world, accounting for 48 percent of global output, the Department of Energy's commitment to America's security and prosperity through transformative science and technology solutions has been pivotal in the project's progress. There are numerous techniques that can be used to represent the modeling and analysis of biological systems, but due to the growing prevalence, functionality, and versatility of computer technology, quantitative modeling of biological systems has become a necessity. Systems Biology Markup Language (SBML) was developed to create a standardized language from which the various computational metabolic models could import the necessary data. My goal for the project was, by inputting the data of one metabolic branch into a Python programming language, I set out to create the necessary corresponding code in order to automatically create an SBML file, and from there, have the SBML file imported and recognized by the various modeling software available for computation and simulation analysis. During my research at Brookhaven National Laboratory, I was able to develop such a Python code for SBML automation, and using free to use metabolic modeling packages such as COPASI, a graphical user interface modeler, and PYSCES, a command line modeler, I was able to import the automated SBML file and simulate the expected results successfully.
\end{abstract}

\section{Background and introduction}

Brassica napus is currently the third largest source of vegetable oil in the world and, is used as food, feed, and to produce biodiesel fuel. Using a quantitative computational metabolic simulation model, the overall goal of this project is to identify which important metabolic pathways in B. napus can be increased or decreased in order to convert a seed embryo containing low quantities of oil into a seed embryo containing higher quantities of oil. The ability to genetically modify B. napus in order to increase plant oil yield would have a tremendous impact on the biological oils industry, as well as the many industries that rely on the oils of which they produce.

There are numerous techniques that can be used to represent the modeling and analysis of biological systems, but due to the growing prevalence, functionality, and versatility of computer technology, quantitative modeling of biological systems has become a necessity. My goal for the project was straightforward, by inputting the data of the phosphoenolpyruvate metabolic branch point into a Python programming language, I set out to create the necessary corresponding code in order to automatically create an output file, and from there, have the output file imported and recognized by the various modeling software available for computational and simulation analysis.

\section{Data Format - SBML}

With so many different biological modeling software available for use, it was clear that rather than input the data into each modeling software individually, what was needed was a universally recognized file that could be easily imported into the various software with little to no modification, and produce the expected simulated results. This necessity is what originally gave rise to Systems Biology Markup Language (SBML). SBML is a machine readable format for representing biological models [1]. The development of SBML began in 2000 and continues today as an ongoing project, refining and improving the model format to meet the growing needs of the scientific community. The SBML file format is derived from the $\mathrm{xml}$ node tree structure, which proved to be ideal for node structure of biological models, and was adapted for SBML use. SBML does not represent an attempt to define a universal language for representing quantitative models. Since not all quantitative models are the same, such a goal would be impossible, however, the SBML creators sought to create a common intermediate format in which to represent the various models.

SBML has many benefits which promote the growing SBML community. Such benefits include the ability to use multiple modeling tools without the need to rewrite the biological model. For some smaller models, rewriting the model may not be that great a challenge, however, with larger models, entering the data into multiple software tools can become overwhelming. SBML allows the ability to exchange models with peers, allowing them to recreate the model using their own preferred software package. Having the ability to collaborate with one's peers regarding a biological model without the need for a mandatory software package is a great benefit. Not everyone will always have the same software available, therefore a simple data file import would be ideal. An SBML is also a way of archiving the model, ensuring it lasts well past the lifetime of the software used to represent it. No software is maintained forever, better products are released and developers move on, but the SBML file will always represent a machine readable record of the dynamic kinetic model.

\section{A. $\quad 2.1 \mathrm{SBML}$ format guidelines}

SBML files are designed to contain a specific order of el- 
ements which are to be represented in the model in a node tree structure. All well-formed SBML documents must begin with an XML declaration, which specifies both the version of XML assumed and the document character encoding. Following this XML declaration, the outermost portion of an SBML model consists of an object of class Sbml [2]. The Sbml class identifies the level and version of SBML you will be following in your document. The varying levels of the SBML correspond to the growing requirements as SBML versions have advanced over the years. With each new level of SBML that is released, more requirements, but also more details can be added to an SBML file. Currently, the latest version is level 3, version 1. Inside the Sbml class is where the model class is located. Every SBML file may only contain one model class, and this class will contain all the necessary container classes for the SBML. Inside the model node is where all of the list classes are placed. These lists are containers for their respective elements. Examples include: listOfUnitDefinitions, listOfCompartments, listOfSpecies, and listOfReactions. The list classes are not mandatory, but if one is created, it cannot be left empty. It is important to note that while all list classes are optional, some are dependent on the creation of others. In order to create a species, you must first create the compartment it will be assigned to, and in order to create a reaction, you must first create a species [2]. The created order of lists is important, and must adhere to the specified guidelines available on the www.SBML.org website.

\section{B. SBML element definitions}

Each of these list elements act as a container, and it is within these classes that the biological data is stored. The SBML file which our project is creating begins with the listOfUnitDefinitions class. This class contains all the descriptions of the units used in the model. They may be as general as seconds, or more complex, such as millimole per liter per second. After the units, the user defines the compartments, which specifies where the spe- cies are located. Next the user defines the various species in the listOfSpecies node. SBML allows users to define many conditions of the species, such as compartment location, initial concentrations, constant or state variability, and whether it is a boundary variable. Lastly, the user defines the reactions. The user begins by defining the reaction's id, name, and reversibility. Next, the user creates and defines the various reactants, products, and modifiers involved in the reaction. For any defined reactant, product, or modifier, there must be a corresponding species listed in the listOfSpecies section. Following the involved species would be the kineticLaw container, which holds the reaction's parsed equation in an SBML readable format. Using a MathML formatting style, the dynamic kinetic model's equation is separated into blocks grouped by parenthesis and mathematical symbols, and ordered appropriately. Along with the mathematics, kineticLaw also contains any additional factors not previously defined in the listOfSpecies, and lists them as parameters.

\section{SBML notes and annotations}

In addition to the many listOf classes, an SBML file can provide notes and annotations to assist in providing the user with additional information regarding the various elements, such as species or reactions. Notes are created for the purpose of being human readable text, while annotations are meant to be machine readable only. Currently, SBML supports two annotation schemes, Systems Biology Ontology (SBO), and Minimum Information Required in the Annotation of Models (MIRIAM). When applied, these schemes allow the SBML object to reference an online database which provides a more detail description of the object, as well as its biological composition, and activity.

$\mathrm{SBO}$ is a set of controlled, relational vocabularies of terms commonly used in Systems Biology, and in particular in computational modeling, and each SBML object may only be given one SBO term [3]. MIRIAM annotations have two parts, a reference

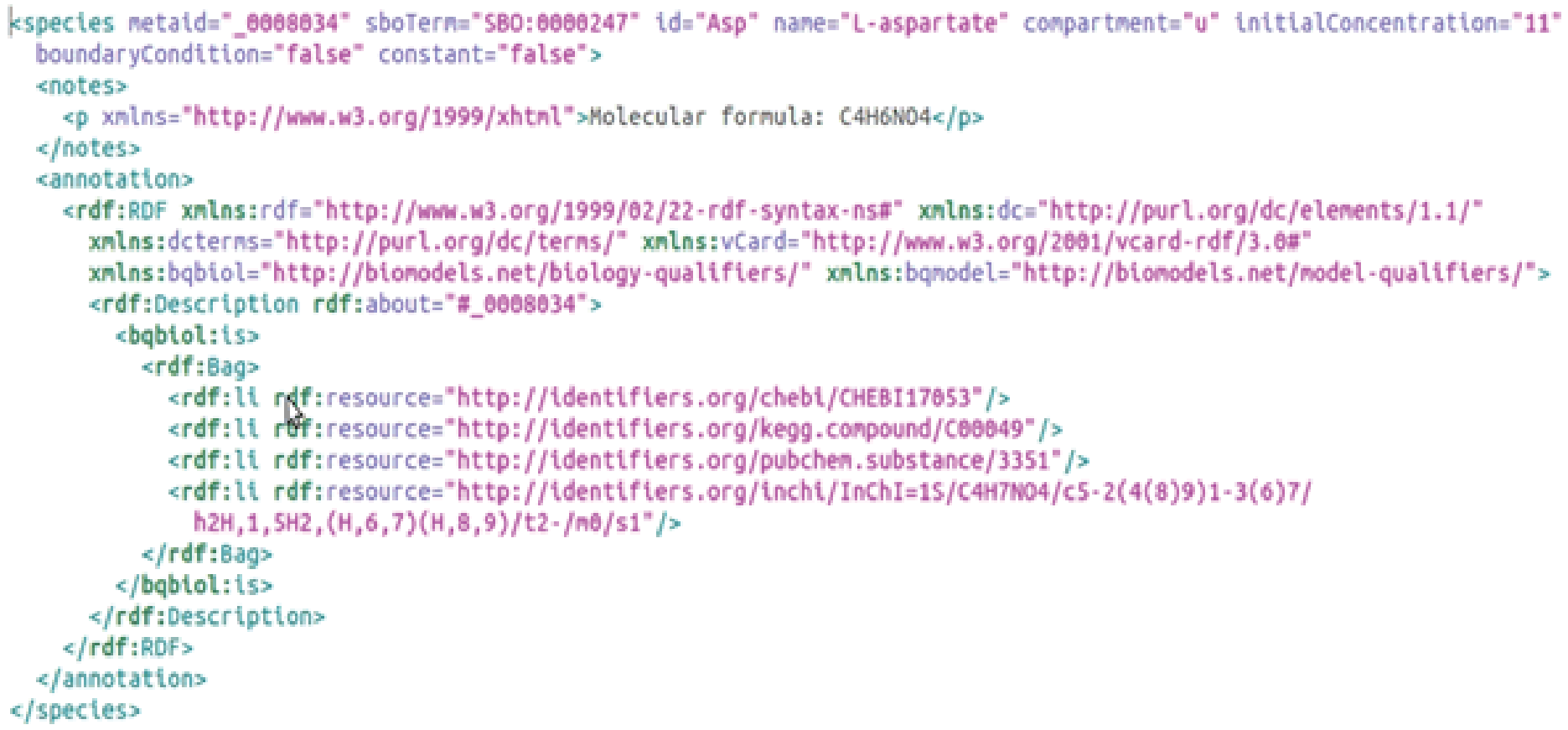


that matches each model to its description, which is often a journal publication, and a set of annotation schemes [4]. With MIRIAM identifiers, you can define the reference as being the actual object, being related to the object, being a part of, among other qualifiers. The MIRIAM identifier also links an SBML object to an online database which contains additional links providing detailed information regarding the SBML object, ranging from the object's molecular composition, to the object's citations in various articles. One SBML object can have multiple MIRIAM identifiers attached to it in order to provide a greater range of detailed information. It is possible that an SBML object may contain both SBO and MIRIAM identifiers. An annotation coding example for ATP which includes both SBO and MIRIAM identifiers can be seen in Figure 1.

\section{Python programming language}

The programming language in which I was to develop the automating script is Python, a free to use, open source programming language. The Python tool that we focused on was the use of IPython Notebook. IPython Notebook allowed us to code a Python script in pieces, allowing us to test out the individual pieces, or the combined code as a whole as we needed. Python is a flexible programming language for many reasons. With an easy to read and understand tab based format, Python script proved a very easy to learn programming language, considering having no previous experience with the language. The language is capable of handling small projects, as well as large ones. It is available for use on all the major operating systems, Windows, Mac OS X, and Linux. There are an abundance of third party packages and libraries available for use with Python, which add to its ever-growing functionality. One such library proved invaluable to the project I was given, libSBML.

\section{A. LibSBML}

LibSBML is an open-source library for writing and manipulating the Systems Biology Markup Language [5]. It gives the Python programming language access to an array of functions designed for the creation of and maintaining of SBML files. With the libSBML library, we are able to create SBML files, add models, compartments, species, reactions, and all the elements necessary for the creation of a usable and valid SBML document. Along with creation tools, libSBML offers a wide variety of functions which enable the editing and manipulation of previously created SBML files, as well as the ability to check SBML files for errors and validation.

\section{Progress}

The process by which I began my programming project started with research. I spent much of my time familiarizing myself with SBML. What SBML is used for, what is the standard layout, and what are the requirements, are some of the questions that needed answering, in addition to having been solely educated in Java programming language, I had never previously used the Python programming language. In the beginning, much of my time was spent using a trial and error technique. Using the SBML and libSBML websites as references, I began slowly by creating an empty SBML file in order to test the functionality of the libSBML library. The Python programming language proved to be extremely flexible and easy to learn. The functionality was easy to adapt to, with only minor semantic differences from what I currently was used to in Java.

\section{A. String parsing}

When my project had begun, my starting point was simply three lines of code, which were each a defined variable. The first, named model, was a string list of the model, containing all the metabolites in a single compartment, kinetic parameters, and equations, the second was the initial concentrations for each of the metabolites and kinetic parameters, and the third was an array of each metabolites stoichiometric matrix. From these three lines, the goal was to create a Python script that would formulate a valid, usable SBML file.

From the start, the project required a substantial amount of string parsing and string manipulation. The first object was to take the string variable named model and separate it into arguments and equations. By doing so, we were able to pair up the individual arguments with their respective initial concentrations and parameters, as well as have a separated list of all the equations. Due to the fact that Python's equation parsing function does not handle bracket symbols very well, I then passed the equation list through another parser which replaced all the brackets with parenthesis. This left me with the ability to separate all the metabolites from the argument list by counting the $\mathrm{x}$ number of arrays in the stoichiometric matrix and selecting the first $\mathrm{x}$ amount of arguments from the list, and a useable list of the reaction equations.

\section{B. SBML creation}

The process of SBML population was broken up into three different sections, the manual code entry, the automatically generated species list, and the automatically generated reaction list. The manual entry was used at the start of the SBML generating code, in order to code the items which are not represented in the string variable data that was given. In this section, I began with the libSBML functions that create the SBML file, including its level and version number, followed by the function used to create the model container. After the model, the list of units is created following the SBML requirement guidelines. And finally, the compartment is created. In our case, all the species given were in the same compartment, making this a feasible solution.

\section{SBML species creation}

Once the manual creation process is finished, I moved on to the automated processes of the SBML creation. To begin, the species were all given in the initial string variable allowing me to easily access the necessary metabolites. As previously stated, I had already parsed the arguments into an array, which allowed me to reproduce their names in order in the species list. I created an array which would contain all the species objects, and a loop which would populate the array with the specified entry from the argument list. Using the length of the stoichiometric matrix as a length for the iteration, I was able to define the number of items from the argument list which were to be used in the species list. These specific items also coincided with the correct initial condition parameters in the initial concentrations array, which allowed me to assign the species their correct values based on the index of the loop, which corresponded to the index of the array. Finally, I was able to determine whether the species was constant or not based on the stoichiometric matrix. If the particular row of the matrix contained no change for the reactions, the corresponding species would be constant. 


\section{SBML reaction creation}

Lastly, and most challenging, was coding the Python script which produced the automated reaction creation process. For the reactions I again used a loop for the automated process, however this time I used the length of the equation list array as my iteration counter. The reaction creation process begins with naming the created reaction. These particular equations all contained a forward velocity (Vf) which is named uniquely for each reaction such as Vf PEPC. In order to attain the correct name for the various equations, and planning ahead for further use, I created a couple different functions for the automated script to use. The first was yet another string parser, which would read in the given equation, and produce a list of arguments that are in the equation, eliminating any symbols and numbers. From this list of equation parameters, I was able to single out the parameter that began with Vf and identify the string that occurs after the underscore. This remainder string would correspond to the reaction's name. I then realized that two reactions could possibly contain the same name, so I created another function which will check for duplicate reaction names and add a number to the next reaction name accordingly, in order to differentiate.

The reaction should also contain reactants, products, and modifiers when applicable.

Using the matrix, I was able to two functions to identify reactants, denoted by a -1 , and products, denoted by a 1 , for any given reaction. Also, using the previously mentioned equation separating function, which created a list of parameters for each parameter, I was able to create a function which compared the reactants and products to the parameters and identifies any metabolites in the equation which are not in the reactant and product list. These extra metabolites will be placed in the modifiers section of the reaction.

Finally, we define the equation that the reaction uses in the reaction's kinetic law. Using a libSBML function named parseL3Formula, we were able to input a string into the function, and it will output an SBML machine readable formatted equation. After the equation was entered, we needed one last function in order to populate the kinetic law's parameter list. The function I created is named kineticParameters, and its function is to compare all the parameters in the equation to the modifiers and list them in the listOfParameters section of kinetic law without the modifiers. It then checks the index of the parameter in the original argument list and finds its corresponding initial concentration value. Once all the reactions are created, all that is left to do is write the file using a libSBML function named SBMLWriter.

\section{Project progression}

As the project progressed, I was able to add more detail to the SBML. My mentor suggested we look into SBO and MIRIAM identifiers, and I was able to input string variables for the various identifiers adding them as annotations to the various species, reactions and parameters throughout the code. The latest key point in the project was when I suggested we move from a string input method to a database import method. I understood that all the data was being copied into the script form somewhere else, so I thought it would be best to simply import the source and save even more time. I was successful in importing the Matlab structure (.mat file) into the project data for data manipulation and use. Not only was direct importing easier for data modification, but it proved to be more efficient in a coding aspect as well.

The data import reduced the majority of the necessary string manipulation code, and produced many benefits which previously caused hassles. The spreadsheet contained everything necessary for a detailed SBML file automation. It was able to add a greater level of detail to all aspects of the SBML, such as formal and informal names, elemental composition of species, and identifier listings to name a few. This allowed the user to share data files with colleagues, or modify any data necessary without the need to open the Python script.

\section{SBML simulation}

Having produced a fully functional SBML file, I needed to ensure that it was capable of being simulated using quantitative and computational metabolic modeling software. The first simulating tool I used was COPASI [6]. COPASI, a graphical user interface (GUI), offers a wide range of simulation tests to run, such as steady states, flux values, and sensitivity tests, to name a few. COPASI also verified the benefit and descriptive nature of the SBO and MIRIAM identifiers added to the SBML file. The identifiers were visible in the description section for each species, reaction, and parameter. The outputs for the tests are very descriptive and will even provide three dimensional charts and graphs where possible. The values for the steady states which it produced based upon the SBML import proved to be accurate, verifying that the generated SBML file is viable and valid. I also tested the same automatically generated SBML file using another GUI simulation software named SBMLSimulator [7]. SBMLSimulator is a simple simulator whose main function is testing the steady states of reactions. Again, the steady state results of our SBML file proved accurate, verifying the SBML file as a valid creation. The main benefit of SBMLSimulator would be the description page for each metabolite and reaction. SBMLSimulator contains a detailed and fully functional description page, not only offering descriptions, but fully functional hyperlinks, which lead the user to the correct publications for each identifier. The SBML file was also able to be run successfully in PYSCES, a command line simulation modeling software [8]. This proved that not only was the SBML valid, but it was also versatile in its possible uses.

\section{Conclusion}

During my research at Brookhaven National Laboratory, I was able to develop a Python script which enabled an automated SBML file generation for a given set of data. The script was able to not only create the required SBML contents, but also convert the ordinary differential equations, using $\mathrm{xml}$ based MathML, into SBML compliant code. The ability to include SBO identifiers and MIRIAM identifiers to the SBML, greatly adds to the description and understanding of the different elements and reactions involved. By means of free to use metabolic modeling packages such as COPASI, SBMLSimulator, and PYSCES, I was able to import the automated SBML file and simulate the expected results successfully.

This project showed that not only was SBML automation possible, but SBML automation proves to be a convenient method for simulating the expected metabolic results quickly and effectively using a given set of data, the created Python script, and a quantitative computational metabolic simulation modeler. The user has the ability to update the data of the dynamic kinetic model, or test alternate models, and rapidly produce a valid and functional 
SBML file that is easily shared with colleagues or imported into a modeling software of their choice, which is an optimal approach to quantitative and computational metabolic modeling.

\section{Acknowledgements}

I wish to thank my mentor, Jordan Hay, for his professionalism and generosity during my CCI program. His insights and guidance were pivotal in all that I accomplished while at Brookhaven National Laboratory. I would like to thank Jorg Schwender, Inga Hebbelmann, Alistair Rogers, and Dhiraj Naik for all their hard work and contributions to the overall goals of the project. I express my gratitude to Ann Emrick, and Denise Monteleone, for providing me with the exceptional workstation I needed to accomplish my goal. I wish to acknowledge the hospitality and kindness of Brookhaven National Laboratory. Financial support to Jorg Schwender (PI) and Jordan Hay for quantitative analysis and modeling of central metabolism in plants is greatly acknowledged (Division of Chemical Sciences, Geosciences, and Biosciences, Office of Basic Energy Sciences, Field Work Proposal BO133). This project was supported in part by the U.S. Department of Energy, Office of Science, Office of Workforce Development for Teachers and Scientists (WDTS) under the Community College Internships Program (CCI).

\section{References}

[1] Hucka, M., Finney, A., Sauro, H. M., Bolouri, H., Doyle, J. C., Kitano, H., Arkin, A. P., Bornstein, B. J., Bray, D., Cornish-Bowden, A. , Cuellar, A. A., Dronov, S., Gilles, E. D., Ginkel, M., Gor, V., Goryanin, I. I., Hedley, W. J., Hodgman, T. C., Hofmeyr, J.-H., Hunter, P. J., Juty, N. S., Kasberger, J. L., Kremling, A., Kummer, U., Le Novère, N., Loew, L. M., Lucio, D., Mendes, P., Minch, E., Mjolsness, E. D., Nakayama, Y., Nelson, M. R., Nielsen, P. F., Sakurada, T., Schaff, J. C., Shapiro, B. E., Shimizu, T. S., Spence, H. D., Stelling, J., Takahashi, K., Tomita, M., Wagner, J., Wang, J. (2003). "The Systems Biology Markup Language (SBML): A medium for representation and exchange of biochemical network models."

Bioinformatics, vol. 19, no. 4, pp. 524-531.
[2] Finney, A., and Hucka, M. (2003). “Systems Biology Markup Language: Level 2 and Beyond.” Biochemical Society Transactions, vol. 31, part 6.

[3] Le Novère N. (2006), "Model storage, exchange and integration.” BMC Neuroscience, 7(Suppl 1):S11.

[4] Juty N., Le Novère N. and Laibe C.,'Identifiers.org and MIRIAM Registry: community resources to provide persistent identification" Nucleic Acids Research (2012) 40 (D1): D580-D586.

[5] Bornstein, B. J., Keating, S. M., Jouraku, A., and Hucka M. (2008) "LibSBML: An API Library for SBML." Bioinformatics, 24(6):880-881, doi:10.1093/ bioinformatics/btn 051 .

[6] Hoops, S., Sahle, S., Gauges, R., Lee, C., Pahle, J., Simus, N., Singhal, M., Xu, L., Mendes, P., and Kummer, U. (2006). "COPASI - a COmplex PAthway SImulator." Bioinformatics 22, 3067-74, doi:10.1093/bioinformatics/ bt1485.

[7] Roland Keller, Alexander Dörr, Akito Tabira, Akira Funahashi, Michael J. Ziller, Richard Adams, Nicolas Rodriguez, Nicolas Le Novère, Noriko Hiroi, Hannes Planatscher, Andreas Zell, and Andreas Dräger. "The systems biology simulation core algorithm.” BMC Systems Biology, 7:55, July 2013.

[8] Olivier, B.G., Rohwer J.M. and Hofmeyr J.-H. S. (2005) "Modelling Cellular Systems with PySCeS" Bioinformatics, (21) pp 560-561

\section{Project Participants}

\begin{tabular}{|l|l|l|l|}
\hline \multicolumn{1}{|c|}{ Participant } & \multicolumn{1}{|c|}{ Department } & \multicolumn{1}{c|}{ Location } & \multicolumn{1}{c|}{ Participant's role } \\
\hline Jordan O. Hay & Bio Sciences Department & $\begin{array}{l}\text { Brookhaven National Laboratory, } \\
\text { Upton, NY 11973 }\end{array}$ & Project Mentor \\
\hline Mark V. Jensen & CCI Intern & $\begin{array}{l}\text { Suffolk County Community College, } \\
\text { Selden, NY 11784 }\end{array}$ & $\begin{array}{l}\text { Python script creation for auto- } \\
\text { matic SBML formulation }\end{array}$ \\
\hline Michael Galazka & CCI Intern & $\begin{array}{l}\text { Housatonic Community College, } \\
\text { Bridgeport, CT 06604 }\end{array}$ & Kinetic Modeling \\
\hline Bradley T. Salvato & SULI Intern & $\begin{array}{l}\text { College of DuPage, } \\
\text { Glen Ellyn, IL 60137 }\end{array}$ & Mathematical Modeling \\
\hline
\end{tabular}




\title{
Characterization of new Hamamatsu Multi-Pixel Photon Counters and the AdvanSiD 8x8 Silicon Photomultiplier array for use in positron emission tomography
}

\author{
Ankit Johari \\ Physics Department, Stony Brook University, Stony Brook, NY 11790 \\ C. Woody and S. Stoll \\ Physics Department, Brookhaven National Laboratory, Upton, NY 11973
}

\begin{abstract}
With the increased applications of positron emission tomography (PET) technologies in medical and non-medical imaging comes an increased demand for high precision PET imaging technologies. One such device used in these imaging systems is the Silicon Photomultiplier (SiPM) which is responsible for detection of photons generated by annihilation events. This research involves the characterization of the AdvanSiD 8x8 array SiPM as well as some new Hamamatsu SiPMs to assess their capabilities and limitations for use in PET systems. We examined noise, dark count, leakage current characteristics as well as gain, light saturation, and dynamic range. The measurements of the AdvanSiD 8x8 array SiPM demonstrated superior energy resolution, lower leakage current, and improved timing resolution to the AdvanSiD 4x4 array. Relative to the new Hamamatsu MPPC the AdvanSid 8x8 has a lower leakage current
\end{abstract}

\section{Materials and Methods}

A circuit board was developed to power the SiPM and its 64 pre amplifiers. Measurements were taken to ensure proper function and uniformity of all 64 channels of the device. Dark Current measurements were taken using the picoammeter. To Measure the timing resolution of the device a PMT with an LSO crystal array was placed equidistant to the Na22 source relative to the SiPM. The PMT was biased at -1320 Volts and the SiPM at 29.55 Volts. The $2 \times 2 \times 8 m m$ LYSO crystal was attached using optical coupling to a single pixel array on the SiPM. The energy resolution of the device was acquired by creating a vertical histogram at a defined threshold for a single channel on the SiPM. The coincidence timing measurement was taken by creating a horizontal histogram of the coincidence event using the signal from the SiPM and the CFD for the signal coming from the PMT.

\section{Results}

Figure 1 shows the new Hamamatsu MPPC leakage current properties with respect to operating voltage. Figure 2 shows the AdvanSiD 8x8's leakage current characteristics. The AdvanSiD 8x8 SiPM array has a lower dark current at its breakdown voltage relative to the new Hamamatsu MPPC. Figure 2 also shows that the $8 \times 8$ 's effective range, which falls between $28.55-30$ volts. The operating voltage of the AdvanSiD $8 \times 8$ array used in this experiment is 29.55 volts. This fell in the Geiger range of the device. Figure 3 Shows the timing resolution of the AdvanSiD 8x8 SiPM which was $1.07 \mathrm{~ns}$. This was calculated using the equation (sigma $x$ 2.36). Figure 4 shows the energy resolution of the AdvanSiD $8 \times 8$ SiPM, which is $38.6 \%$. This is calculated by dividing the Full width at half maximum by the mean. Figure 5 shows the change in gain of the $8 \times 8 \mathrm{SiPM}$ as a function of the operating voltage. Raising the operating voltage effectively increases the gain of the device. Comparing Figures 4 and Figure 6 shows the energy resolution of AdvanSiD 8x8 array SiPM compared to the $4 \times 4$ respectively. Figure 6 shows an energy resolution of $17.08 \%$ for the $4 \times 4$ array.

\section{Conclusion}

The AdvanSiD 8x8 SiPM tested has performed very well and is an excellent choice for use in PET imaging and High Energy Nuclear Physics. The device has a low operating voltage of only 29 volts. The device has low leakage current properties relative to the new Hamamatsu MPPC at breakdown voltage. The SiPM showed a good timing resolution of $1.07 \mathrm{~ns}$. The $8 \times 8$ array has an energy resolution of $38.6 \%$. The difference in energy resolution between the $4 \times 4$ and the $8 \times 8$ is $21.52 \%$. The AdvanSiD $4 \times 4$ and $8 \times 8$ have identical micropixel size of $50 \mu \mathrm{m}$ but the $8 \times 8$ has a smaller pixel size and pixel pitch. The new AdvanSiD monolithic $8 \times 8$ SiPM array has all the advantages of the new $4 \times 4$ array, and has greater timing and energy resolution.

\section{Acknowledgements}

I would like to thank both Dr. C. Woody and S. Stoll for their time and generosity. They have been great mentors to learn from and a pleasure to work with. I would like to thank Brookhaven National Laboratory and its employees for being such an inviting facility. Lastly, I would like to thank the Department Of Energy Office Of Sciences for allowing such research to take place

\section{Literature Cited}

[1] Yokoyama, M. et al. "Application of Hamamatsu MPPC to T2K Neutrino Detectors" Nucl.Instrum.Meth. A610 (2009) 128-130

[2] B. Kapoor, "Characterization of Silicon Photomultipliers for Positron Emission Tomography Imaging”.2013. p58 

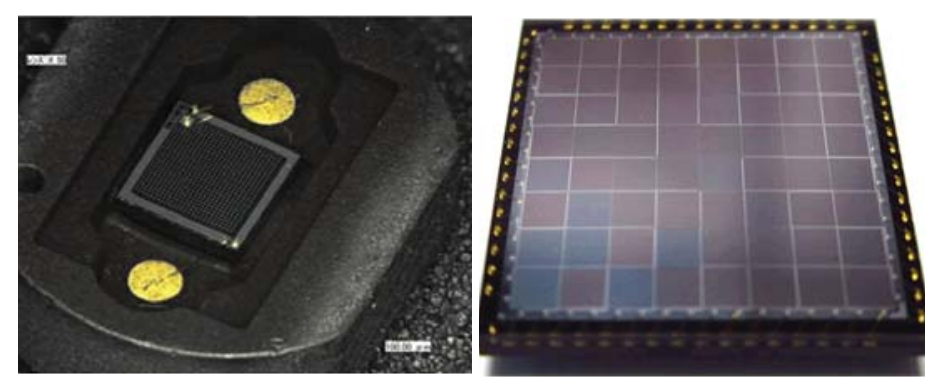

Image 1: Hamamatsu MPPC Image 2: AdvanSiD 8x8 SiPM image[1]

image

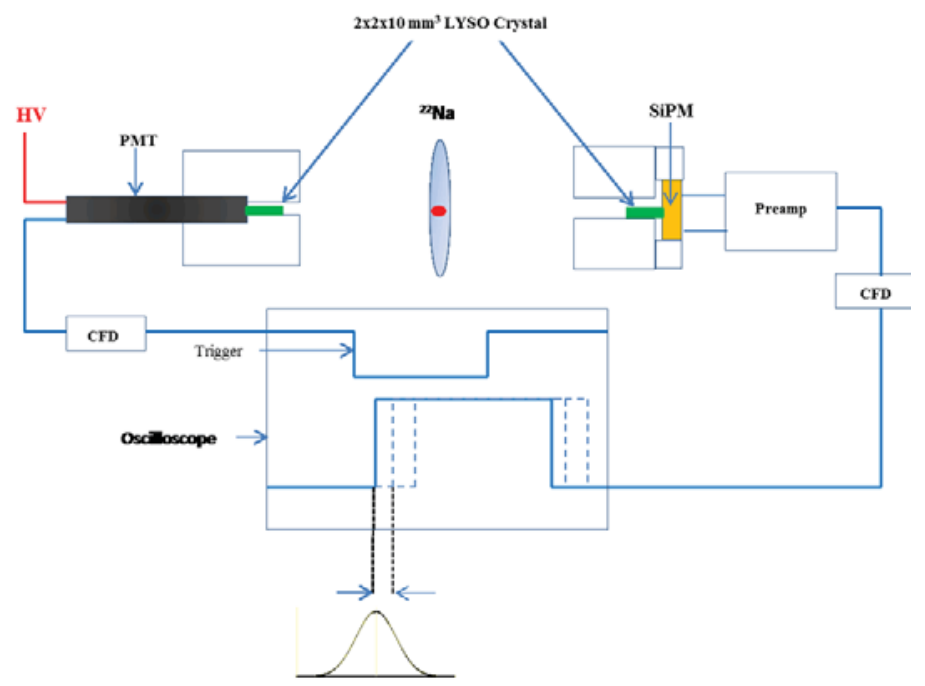

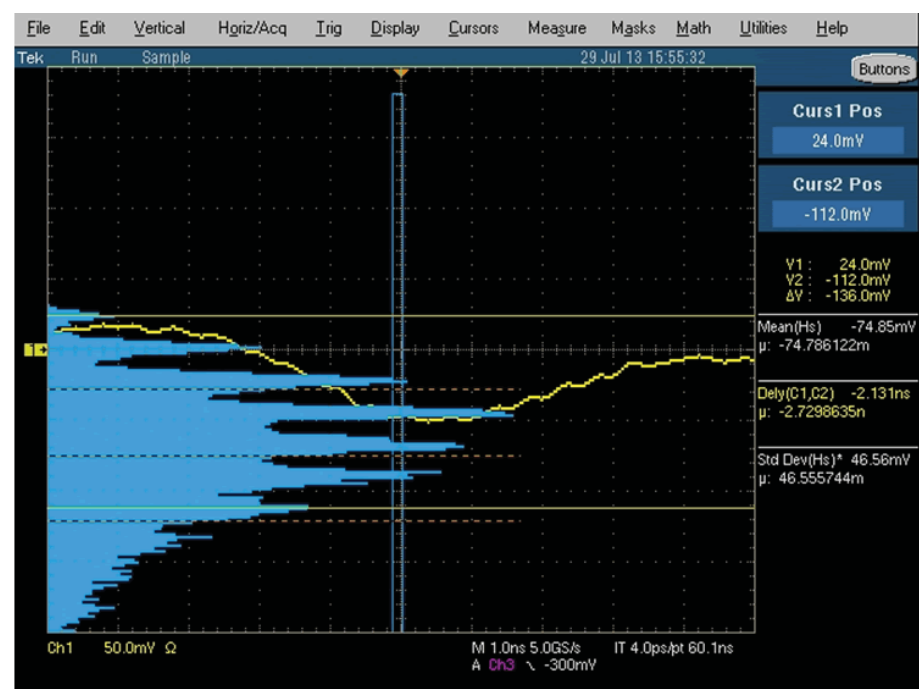

Image 4: This is an image demonstrating the ability to count the number of photopeaks at an operating voltage of 30 volts. Which was determined by figure 1 to be within Geiger mode.

Image 3: Diagram demonstrating the timing resolution setup[2]

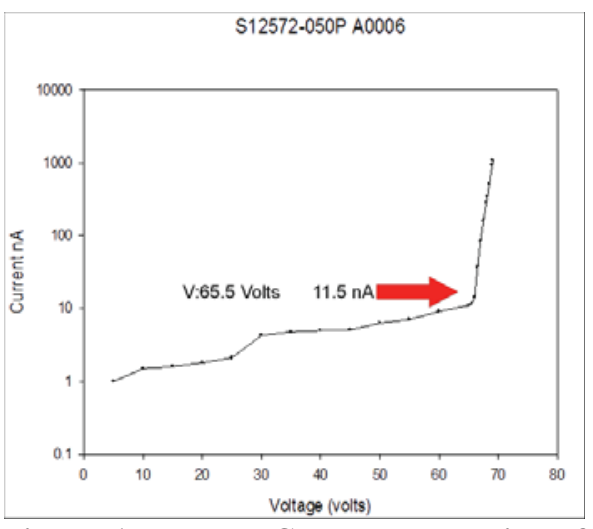

Figure 1:Leakage Current properties of Hamamatsu S12572-050P series A006 MPPC

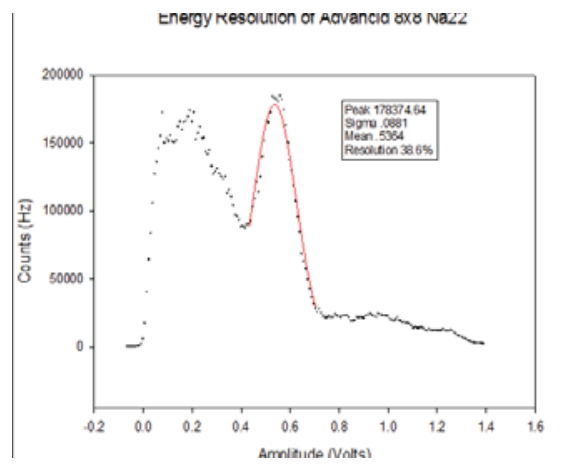

Figure 4: Energy Resolution of AdvanSiD 8x8 SiPM

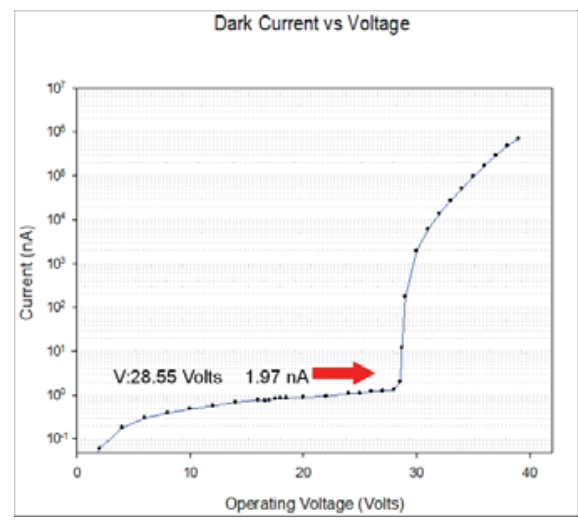

Figure 2:Leakage Current properties of AdvanSiD 8x8 SiPM

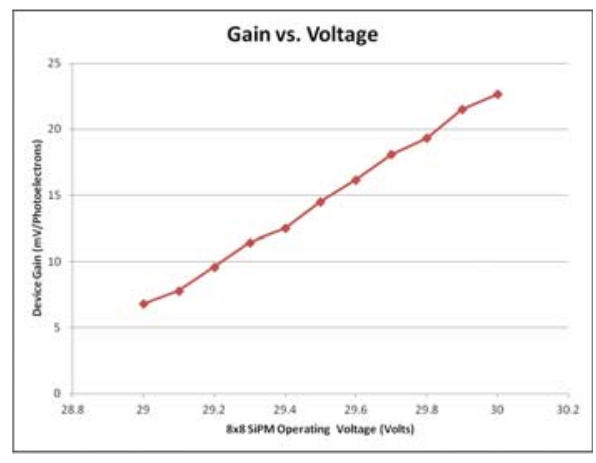

Figure 5: Gain of AdvanSiD 8x8 SiPM vs. SiPM operating voltage

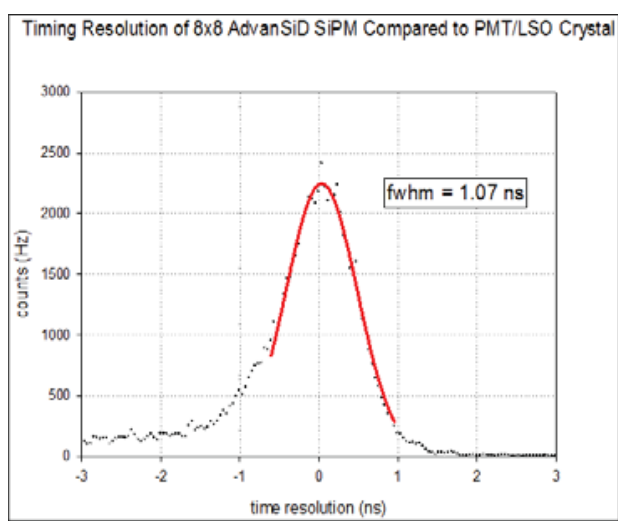

Figure 3: Timing resolution of AdvanSiD 8x8 $\mathrm{SiPM}$ is $1.07 \mathrm{~ns}$

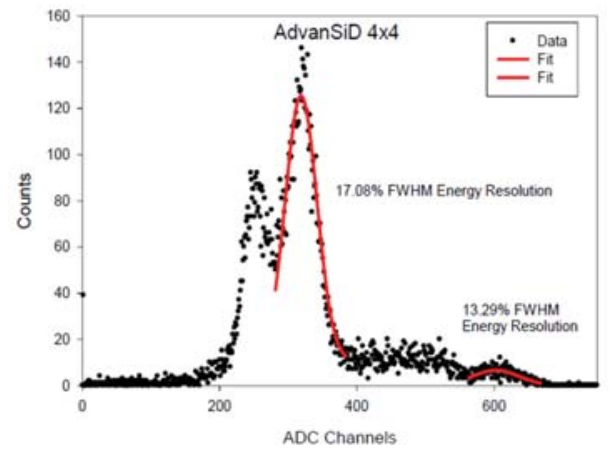

Figure 6: Energy resolution of AdvanSiD 4x4 array $\mathrm{SiPM}$, measured by fellow ratcap researcher[2] 


\title{
Monte-Carlo simulation of a slant-hole collimator in a trans-rectal gamma camera
}

\author{
Mark Kamuda \\ Nuclear Plasma and Radiological Engineering, University of Illinois, Urbana, Illinois 61820 \\ Yonggang Cui \\ Nuclear Proliferation and National Security, Brookhaven National Laboratory, Upton, NY 11973
}

\begin{abstract}
Having the ability to take an accurate 3D image of a tumor greatly helps doctors diagnose it and then create a treatment plan for a patient. One way to accomplish molecular imaging is to inject a radioactive tracer into a patient and then measure the gamma rays emitted from the high-uptake region, viz., the cancerous tissues. In large, expensive PET- or SPECT-imaging systems, the 3D imaging easily is accomplished by rotating the gamma-ray detectors and then employing software to reconstruct the 3D images from the multiple 2D projections at different angles of view. However, this method is impractical in a very compact imaging system due to anatomical considerations, e.g., the trans-rectal gamma camera under development at Brookhaven National Laboratory. The camera uses pixilated cadmium zinc telluride (CZT) detectors. Each pixel is exposed to a small parallel hole through which radiation enters, so creating a 2D projection of radiation intensity. This high- $Z$ high-density component, with its array of embedded parallel holes is called a collimator. Our research investigated the possibility of using a collimator with slanted holes to create a 3D picture of a radioactive source. The underlying concept is to take 2D projection images at different angles of view by rotating the collimator, then using the $2 \mathrm{D}$ images to reconstruct a 3D image. To do this, we first simulated the response of a pixilated CZT detector to radiation sources placed in its field of view. Then, we formulated an algorithm to use the simulation results as prior knowledge and estimate the distribution of a shaped source from its 2D projection images. From the results of the simulation, we measured the spatial resolution of the camera as $\sim 7-\mathrm{mm}$ at a depth of $13.85-\mathrm{mm}$ when using a detector with $2.46-\mathrm{mm}$ pixel pitch and a collimator with $60^{\circ}$ slanted angle.
\end{abstract}

\section{INTRODUCTION}

Prostate cancer is a very common disease in men. According to American Cancer Society, 1 in 6 men will develop the disease in his life, and 1 in 36 will die from it [1]. Although current nuclear-medical imaging techniques, such as magnetic resonance imaging (MRI), computed tomography (CT), single photon emission computed tomography (SPECT), and positron emission tomography (PET) are effective tools in diagnosing cancers, none are specific for prostate cancer because of their low sensitivity and low spatial resolution; this is especially problematic when the tumors are small, at their early stages, and are confined within the prostate gland. Currently, ultrasound-guided biopsy remains the gold standard in confirming the disease. However, ultrasound cannot pinpoint the location of the tumors. It only assists physicians in defining the boundaries of the glands; eventually, samples from the glands have to be taken randomly or evenly. Hence, small tumors very easily can be missed in this procedure. New imaging techniques are in high demand by patients and physicians. Ideally, such techniques should have high sensitivity and high spatial resolution in imaging prostate cancer, and in pinpointing tumors confined with the glands, both of which assist physicians during the biopsy and treatment of the disease. One promising way lies in devising a compact trans-rectal gamma camera that can acquire images of the prostate glands through the rectal wall. Such a short working distance can increase the sensitivity and spatial resolution greatly.

CZT radiation detectors, with their excellent material properties and performance, are attractive for applications in medical imaging. The detectors' modules, when fabricated in advanced pixilation- and hybridization-processes, can be very compact, and already are finding applications in endo-cavity measurements. However, in such usages there usually is very limited space for operation; the traditional detector and collimator design and arrangement are not suitable, especially for 3D imaging. To resolve this problem, we undertook a feasibility study of a slant-hole collimator that can be employed with planar pixilated detectors to generate 3D images. We modeled this collimator with the gamma camera using a Monte-Carlo simulation, and investigated the detectors' responses to various radioactive sources in their field of view. Then, we modified a Maximum Likelihood Estimation Method (ML-EM) and used the code to reconstruct 3D images of shaped radioactive sources.

\section{METHODS}

\section{A. Monte-Carlo Simulation}

We used a Monte-Carlo simulation package (Geant4) to simulate a slant-hole collimator and detector. We created a $6 \times 16$ array of 5-mm thick CZT detectors with a pixel pitch of $2.46 \mathrm{~mm}$. Their thickness was chosen specifically to ensure sufficient detection efficiency for $140.5-\mathrm{keV}$ gamma rays. The simulated collimator was a 6.4-mm-thick Tungsten plate with 1-mm-diameter holes at a $60^{\circ}$ slant-angle in line with the CZT detectors. To gather the data for the reconstruction, we simulated a $0.5-\mathrm{mm}$-diameter Tc-99m (140.5-keV gamma-rays) sphere source in each voxel in the detectors' field of view. The individual voxel size is $1.23-\mathrm{mm}$ (half a pixel) in the $\mathrm{x}-, 1.23-\mathrm{mm}$ in the $\mathrm{y}-$, and $\sim 1.07-\mathrm{mm}$ in the z-direction. In total, we simulated 2244 voxels. The simulation was run on a Linux cluster computer with 256 nodes.

Fig. 1 illustrates an example of a simulated point moving in the y-direction. When moving one pixel (from Fig. 1a to Fig. 1c, or from Fig.1b to Fig.1d) the image does not change, only its position. When moving one half-pixel (from Fig.1a to 1b), both the image and its position change. The results demonstrate the effective collimation of the collimator with simulated geometries. 


\section{B. Image Reconstruction}

The ML-EM algorithm developed for this collimator required two 2D images (sinograms) of the source to create a 3D one. It uses an iterative method to find the most likely distribution of the radioactive source(s) and the activity that would form the given sinogram. Both images were created using data simulated in one of the 2244 voxels used in the reconstruction algorithm. Fig.2 illustrates an example of the point source $(0,-1,3)$ being imaged. The first image was taken with the collimator in Position A (Fig. 3a). To obtain an image in Position B, we found the Position B point in the Position A space, then rotated the image $180^{\circ}$ about the axis of the field-of-view (Fig. 3b). In this way we cut by 50 percent the computer time needed for the Monte-Carlo simulation. The simulation results were converted to a $2 \mathrm{D}$ matrix that is the raw data the ML-EM algorithm uses to reconstruct a 3D image of the source(s). To reduce the noisy background caused by Compton scattered events and the electronics' noise, we set an energy window at 125 - to $155-\mathrm{keV}$, covering the photon peak of 140.5-keV gamma-rays. Fig. 4 shows an example of an image reconstructed using the ML-EM algorithm with the sinograms from Fig. 3.

\section{PERFORMANCE}

\section{A. Spatial resolution}

We ran two experiments to test the performance of the MLEM algorithm and the slant-hole collimator. The first was to find the y-axis resolution of the detector. For this, we simulated two separated point sources with the same radioactivity along a line on an arbitrary y-axis $13.85-\mathrm{mm}$ above the detector. From the simulated results shown in Fig. 5, only when the sources were dis-
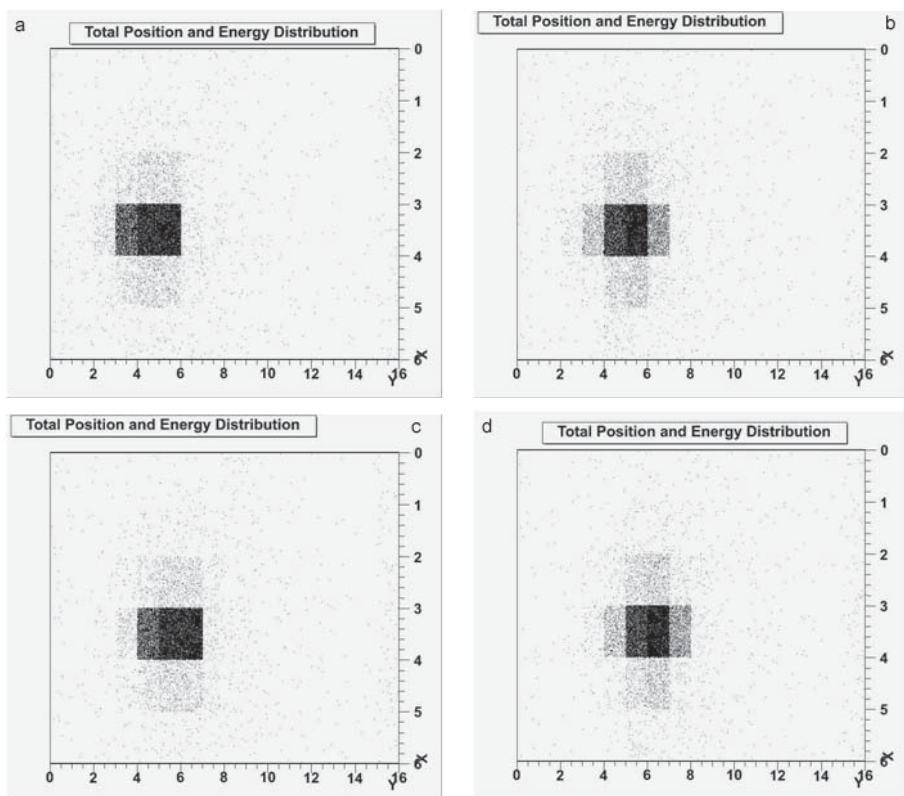

Fig. 1. A point translating along a line in half detector pixel increments in the y-direction

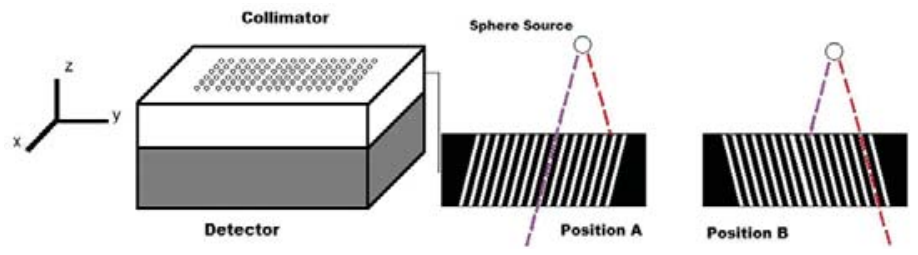

Fig. 2. Detailed sketch of the collimator in both viewing positions placed by 3 pixels could they be clearly resolved. Therefore, we estimated that the y-axis resolution was 3 detector pixels (7.38$\mathrm{mm})$ at $13.85-\mathrm{mm}$ above the detector. Appropriately addressing the $\mathrm{z}$-axis resolution requires further study. The $\mathrm{z}$-axis resolution depends on both the slant angle of the collimator and the relative position of the two separated sources on the y-axis.

\section{B. Phantom test}

For the second test, we simulated a phantom of 5 point sources of the same radioactivity. This test demonstrates the ability of the ML-EM and collimator to clearly reconstruct multiple radioactive hotspots sited near each other. All points were in line with a hole in the collimator and thus with a pixel of the detector. Fig. 6 shows the source orientation. All were on the same x-plane.

The 5 sources from the phantom were resolved clearly, but with some issues. The features of the phantom (distance between, position, and size of points) were well resolved (Fig. 7). The y-axis resolution of 3 pixels still is accurate with multiple sources.
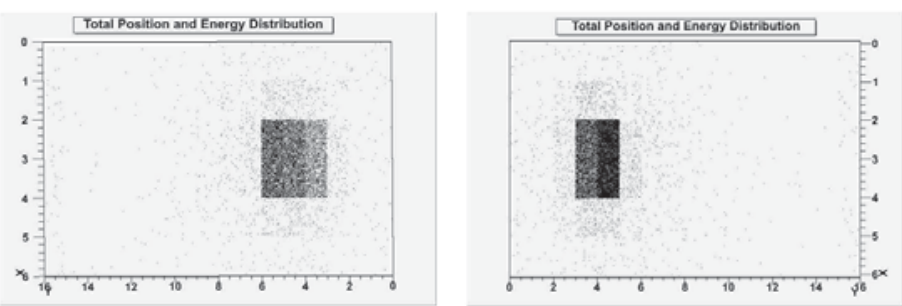

Fig. 3(a,b). Image of the same point source in Position A and Position B
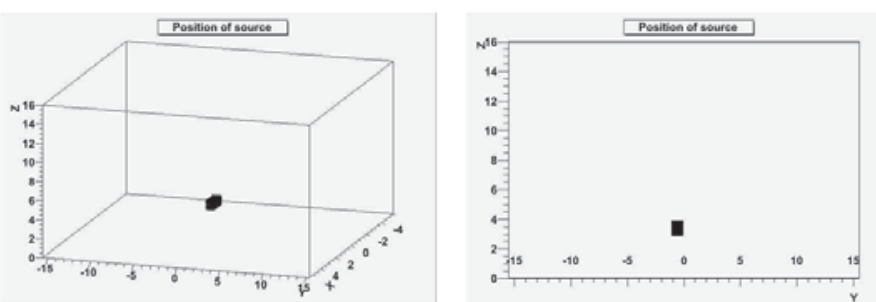

Fig. 4. Reconstructed 3D image of a single point source from two perspectives

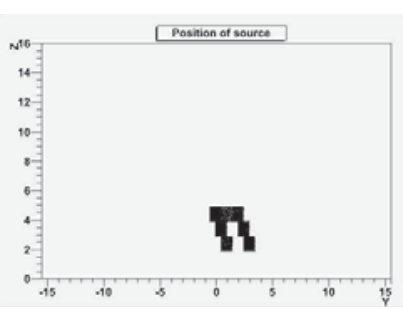

Source displacement: 1 pixel

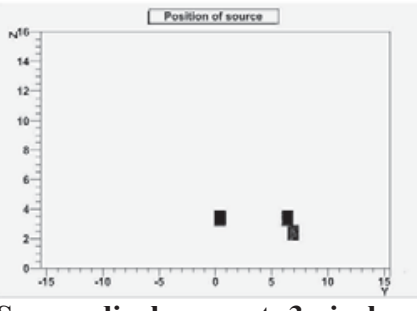

Source displacement: 3 pixel

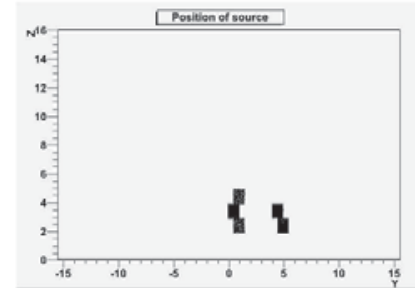

Source displacement: 2 pixel

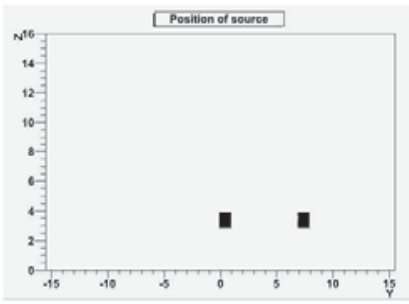

Source displacement: 3.5 pixel
Fig. 5. Two sources displaced on the y-axis 
The only problem with this test is the poorly resolved point. This inconsistency could be attributed to the thresholds used in feeding the sinogram matrices into the ML-EM algorithm, or to the number of ML-EM iterations. For comparison, Fig. 8 shows a reconstructed image of the same phantom but with different thresholds in the sinogram matrices used in the ML-EM algorithm. Here we see that the two original artifacts in Fig. 7 disappear and two new artifacts appear. It is promising for using this technique in medical applications that the images resolved well with different thresholds, but the presence of these artifacts suggests that we must optimize the reconstruction process.

\section{CONCLUSIONS}

We successfully simulated the trans-rectal gamma camera with slant-hole collimator, and modified the ML-EM algorithm for reconstructing a $3 \mathrm{D}$ image using this collimator. With the software code and the findings from the simulation, we studied the performance of the existing gamma camera coupled to a $60^{\circ}$ slant-hole collimator. This preliminary study demonstrated that the slant-hole collimator can be used in compact gamma camera, i.e., the trans-rectal probe, for 3D imaging. The results will guide us in our improving the design of the trans-rectal gamma camera.

The results of this work also suggest the needed further research. First, the results were based on computer simulation; the possible noise sources were not considered thoroughly. We plan to acquire real images in the laboratory using a slant-hole collimator with the same geometries as that simulated, and use the

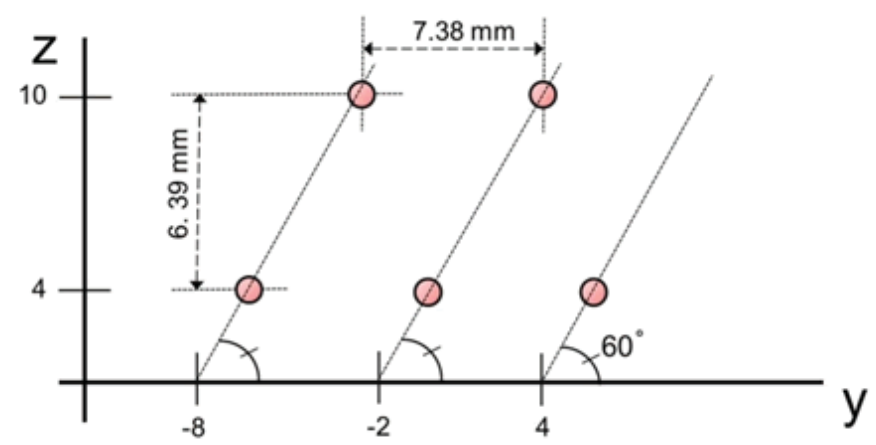

Fig. 6. Diagram of hot spot orientation for the phantom
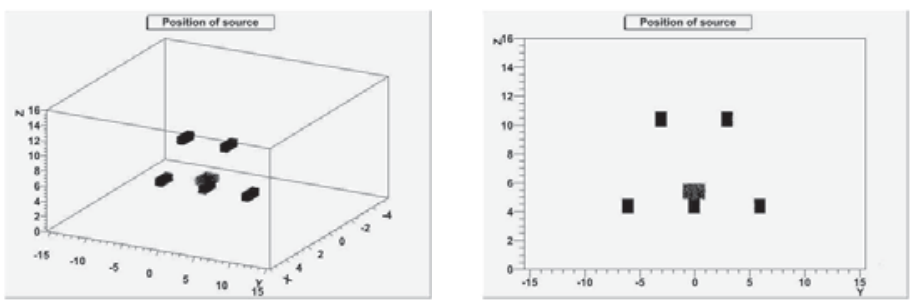

Fig. 7. Reconstructed 3D image of the phantom source from two perspectives
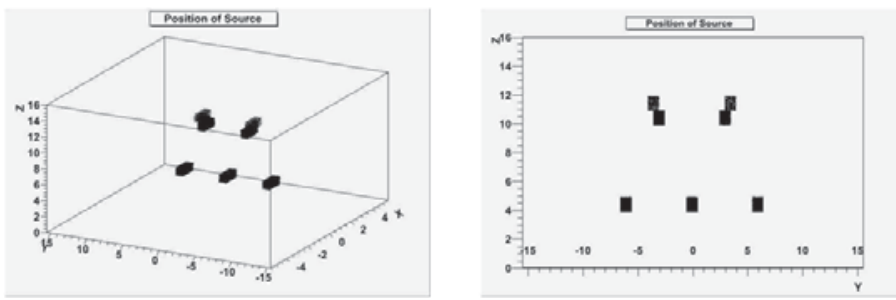

Fig. 8. Second reconstructed 3D image of the phantom source from two perspectives experimental data to test again the ML-EM algorithm. Second, the design of the collimator and angle of slant holes should also be explored further to optimize $y$ - and z-axis resolution so to meet the design specification of the 3D trans-rectal gamma camera.

\section{ACKNOWLEDGEMENT}

I would like to first thank my mentor, Dr. Yonggang Cui for his help, guidance, and time with this project. I learned valuable lessons and skills working with him that have prepared me to work on a larger range of programming projects. I also want to thank my colleges in the Radiation Detector and Nonproliferation R\&D Group for their friendship and lunchtime talks. I felt like part of a team while I was here.

This project was supported in part by the U.S. Department of Energy, Office of Science, Office of Workforce Development for Teachers and Scientists (WDTS) under the Science Undergraduate Laboratory Internships Program (SULI).

\section{REFERENCES}

[1] www.cancer.org 


\title{
Assessment of 5-hydroxymethylcytosine in the DNA of human skin fibroblasts exposed to ionizing radiation
}

\author{
Lucia Kolodiuk and Katherine Z. Sanidad \\ Biology, Stony Brook University, Stony Brook, NY 11790 \\ Benjamin Daniels \\ Biochemistry, Rensselaer Polytechnic Institute, Troy, NY 12180 \\ Deborah J. Keszenman, Paula V. Bennett, Alicia Johnson, Paul F. Wilson Jr. \\ Biosciences, Brookhaven National Laboratory, Upton, NY 11973
}

\begin{abstract}
Epigenetics refers to non-DNA sequence changes that are heritable and modulate gene expression, including DNA methylation and DNA-associated chromatin remodeling through histone post-translational modifications. Improper regulation of epigenetic states may contribute to different disease outcomes including carcinogenesis. Methylation of DNA bases, such as the conversion of cytosine to 5-methylcytosine (5-mC), is known to play a role in controlling transcriptional activities in developing neuronal tissues. Further chemical modification of 5-mC to 5-hydroxymethylcytosine (5-hmC) may occur by exposure to reactive oxygen species generated by ionizing radiation (IR). However, the biological function of 5-hmC in human cells and tissues is still largely unknown. We have recently shown that IR is capable of converting 5-mC to 5-hmC, and we have been developing assays to analyze 5-mC and 5 -hmC levels in human cells treated for $18 \mathrm{~h}$ with the histone deacetylase inhibitor SAHA (vorinostat; a drug that relaxes chromatin packing) and then exposed to gamma rays and protons at the NASA Space Radiation Laboratory (NSRL). DNA from human neonatal foreskin fibroblasts (NFF28) was isolated in agarose plugs and treated with the restriction endonuclease PvuRts1L, an enzyme that specifically cleaves DNA sequences containing 5-hmC. Relative levels of 5-hmC were then assessed by pulsed field gel-electrophoresis (PFGE) and gel image analysis. We determined that this enzyme can be used with DNA embedded in agarose plugs and tested the appropriate amounts of enzyme and buffer for optimal reaction conditions. Work is to be done to see a positive correlation between IR dose and 5-hmC occurrence. This project promoted my interest in science by helping develop a new assay to analyze epigenetic changes following different types of IR exposures, which can be utilized to better understand the impact of 5- hmC in irradiated human tissues.
\end{abstract}

\section{Introduction}

Epigenetics refers to non-DNA sequence changes that are heritable and modulate gene expression, including DNA methylation and DNA-associated chromatin remodeling through histone post-translational modifications. Open chromatin is related to active DNA transcription and it is usually characterized by unmethylated cytosines, acetylated histone tails and methylation of histones. Closed chromatin may happen when cytosines become methylated and acetyl groups are removed from histone tails by histone deacetylases (HDAC). ${ }^{1,2}$ Inappropriate regulation of epigenetic states may contribute to different disease outcomes in- cluding carcinogenesis.

Methylation of DNA bases, such as the conversion of cytosine to 5-methylcytosine $(5-\mathrm{mC})$, is known to play a role in controlling transcriptional activities in developing neuronal tissues. Further chemical modification of 5-mC to 5-hydroxymethylcytosine $(5-\mathrm{hmC})$ may occur by exposure to reactive oxygen species generated by ionizing radiation (IR). The figure below displays the pathway in which cytosine is modified into $5-\mathrm{mC}$ and further into 5-hmC.

$$
\mathrm{C}->5-\mathrm{mC}->5-\mathrm{hmC}
$$

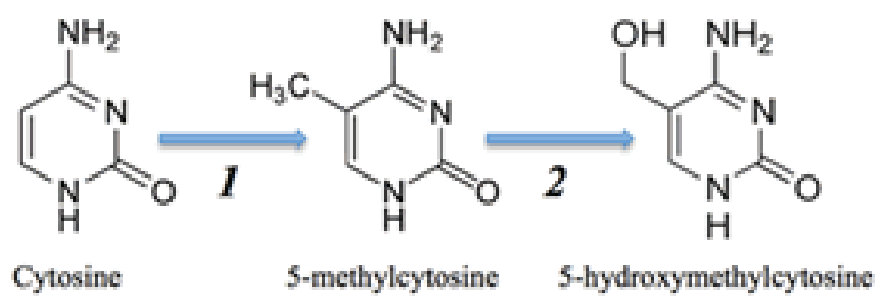

The creation of $5 \mathrm{hmC}$ can lead to demethylation of DNA, which may contribute to a change in the dynamics of DNA methylation. ${ }^{1,2}$ However, the biological function of $5-\mathrm{hmC}$ in human cells and tissues is still largely unknown.

There are many techniques to study DNA methylation and methylation patterns including PCR techniques. Nonetheless, most techniques do not distinguish between $5-\mathrm{mC}$ and 5-hmC. One way to differentiate $5-\mathrm{mC}$ from $5-\mathrm{hmC}$ is to use antibodies that recognize the two different modified bases. ${ }^{1}$ Another approach to determine the cytosine methylation patterns is based on the use of restriction enzymes sensitive to both methylated and hydroxymethylated cytosines cutting DNA if it is methylated. ${ }^{2}$ Recently, only the restriction endonuclease PvuRts1I has been identified to specifically recognize 5 -hmC and cleave DNA sequences containing this modified base. ${ }^{3}$

We have recently shown that IR is capable of converting $5-\mathrm{mC}$ to $5-\mathrm{hmC}$ and we hypothesize that the increase in $5-\mathrm{hmC}$ is IR dose dependent. To test this hypothesis we have been developing assays to analyze 5-mC and 5-hmC levels in human cells exposed to photon or charged particle radiation. Using human neonatal foreskin fibroblasts (NFF28) we proposed to study the levels of 5-hmC in cells exposed to increasing doses of IR alone or preceded by radiosensitization treatment. NFF28 cells were treated for $18 \mathrm{~h}$ with the HDAC inhibitor SAHA(Vorinostat) and then exposed to gamma rays or protons at the NASA Space Radiation Laboratory (NSRL). 


\section{Materials and Methods \\ A. DNA Substrate}

The unmethylated, 5-methylcytocine and 5-hydroxymethylcytosine double-strand DNA were created by the inclusion of either unmethylated duct, 5-methyl dCTP or 5-hydroxymethyl $\mathrm{dCTP}$ in the PCR reaction mix of a 338 base pair fragment from the APC (adenomatous polyposis coli) gene promoter (Active Motif, Catalog No. 55008 USA).

\section{B. Cell Culture and Irradiation}

Human neonatal foreskin fibroblasts (NFF28) were cultured in appropriate nutrient medium at $37^{\circ} \mathrm{C}$ in $5 \% \mathrm{CO}_{2}$ to confluency in $\mathrm{G} 0 / \mathrm{G} 1$ phase. Cells were trypsinized and resuspended in $0.5 \mathrm{ml}$ medium at a concentration of $2 \times 10^{6}$ cells $/ \mathrm{ml}$. Cell samples were exposed to gamma rays in doses ranging from 0 to $10 \mathrm{~Gy}$ at room temperature using a Shepherd Mark I Cesium ${ }^{137}$ Source (LET 0.80 $\mathrm{keV} / \mu \mathrm{m})$. Other samples were exposed at room temperature to $200 \mathrm{MeV}$ protons with doses from 0 to 4 Gy (LET $0.45 \mathrm{keV} / \mu \mathrm{m}$ ) at the NSRL. Proton irradiated samples were pretreated for $18 \mathrm{~h}$ with $0.2 \%$ DMSO (dimethylsulfoxide) or SAHA (Vorinostat). HDAC inhibitor SAHA was dissolved in $0.2 \%$ DMSO and used at a concentration of $10 \mathrm{mM}$. After irradiations, cells were immediately placed in dry ice and kept at $-80^{\circ} \mathrm{C}$ until further processing.

\section{DNA Agarose Plugs}

Cells or 338-bp DNA were subject embedded in 2\% InCert Agarose to form 1\% DNA agarose plugs. 338-bp DNA agarose plugs were kept in TE Buffer (10 mM Tris, 1mM EDTA, pH 8) at $4^{\circ} \mathrm{C}$.

The preparation of DNA agarose plugs from frozen cells is as follows. The cells were thawed rapidly in a $37^{\circ} \mathrm{C}$ water bath. Then, $50 \mu 10.5 \mathrm{M}$ EDTA pH 8.0 were added. Rapidly after, $600 \mu 1$ of $2 \%$ InCert Agarose (prepared in 50mM EDTA) were added and the mixture was placed in molds. The molds were set to solidify at $4^{\circ} \mathrm{C}$ for $10 \mathrm{~min}$. Next, the plugs were placed in tubes with Proteinase $\mathrm{K}$ Lysis solution $(1 \mathrm{mg} / \mathrm{ml}$ Proteinase K, 2\% N-Lauroyl- Sarcosyl in L-buffer: $100 \mathrm{mM}$ EDTA, $10 \mathrm{mM}$ Tris, $20 \mathrm{mM} \mathrm{NaCl} \mathrm{pH}$ 8.0) and chilled on ice for $15 \mathrm{~min}$ followed by incubation at $37^{\circ} \mathrm{C}$ overnight. On the next day, the Proteinase K Lysis buffer was changed. On ice, the old Lysis solution was removed and L-buffer was added. After $15 \mathrm{~min}$ on ice, the L- buffer was removed and new Proteinase K Lysis solution was added. Then, the plugs were incubated at $37^{\circ} \mathrm{C}$ overnight. For the next 2 days, the Proteinase $\mathrm{K}$ Lysis solution was changed using the process described above. On day 5, the plugs were washed with L-buffer and then treated with $0.2 \mathrm{mM}$ PMSF twice $30 \mathrm{~min}$ at room temperature. Then, the PMSF solution was removed and the plugs were rinsed with cold TE buffer several times until no traces of Sarcosyl detergent were left. The plugs were soaked in TE buffer overnight and stored at $4^{\circ} \mathrm{C}$ in the dark until they were to be digested with the enzyme.

The plugs from cells were treated with NotI restriction endonuclease to generate DNA fragments that can be analyzed by pulsed field gel electrophoresis (PFGE). The restriction digestion of the DNA in agarose plugs is as follows. The plugs were equilibrated on ice in 2-3ml restriction enzyme buffer (NEB3: $50 \mathrm{mM}$ Tris- $\mathrm{HCl}, 100 \mathrm{mM} \mathrm{NaCl}, 10 \mathrm{mM} \mathrm{MgCl}$, $\mathrm{pH}$ 7.9) without BSA (Bovine Serum Albumin) or DTT (dithiothreitol) added to the enzyme buffer. The samples were left to soak on ice overnight. The following day, the samples were equilibrated on ice in $2.0 \mathrm{ml}$ reaction mix containing $100 \mu \mathrm{g} / \mathrm{ml}$ acetylated BSA and $1 \mathrm{mM}$ Sigma molecular biology grade DTT and soaked for $2 \mathrm{~h}$. The reaction mix was replaced with $1.0 \mathrm{ml}$ reaction mix containing 2 to 3 units of restriction enzyme per microgram of DNA and incubated for at least $1 \mathrm{~h}$ on ice. The estimated concentration of DNA per plug was $1320 \mathrm{ng}$. Then, the samples were incubated at $37 \mathrm{oC}$ for $18 \mathrm{~h}$. The following day, the samples were moved samples to ice to stop reaction with a 1 hour soak in Argon treated TE. ${ }^{4}$

\section{DNA Digestion with PvuRts1I Restriction Enzyme}

PvuRts1I restriction enzyme specifically cleaves DNA sequences containing 5-hmC. The enzyme recognition site is hmCN11-12/N9-10G.

DNA in solution was digested at room temperature using 2 protocols. One protocol used was according to reaction conditions and reagents following the instructions provided by the vendor of the PvuRts1I enzyme (Active Motif, Catalog No. 55011, USA). The composition of the Reaction Buffer and the preferred reaction conditions were undisclosed. The other protocol used a reaction mixture containing 1X PvuRts Buffer $(150 \mathrm{mM} \mathrm{NaCl}, 20 \mathrm{mM}$ Tris $\mathrm{pH}$ 8.0, $5 \mathrm{mM} \mathrm{MgCl} 2), 1 \mathrm{mM}$ DTT, $50 \mu \mathrm{g} / \mathrm{ml} \mathrm{BSA}$, and DNA. The DNA in agarose plugs was digested using the second protocol described.

\section{E. Electrophoresis}

Static field gel electrophoresis was utilized to assay the activity of the enzyme PvuRts1I on the DNA in solution or in agarose plugs. Gels of $1.5 \%$ Sea Kem L.E. Agarose in $1 \mathrm{X}$ TAE buffer ( $40 \mathrm{mM}$ Tris, $20 \mathrm{mM}$ acetic acid, $1 \mathrm{mM}$ EDTA, $\mathrm{pH}$ 8.0) were run in 1X TAE Running buffer for 2 to $2.5 \mathrm{~h}$ or until the loading dye was visible half way down the gel at $30 \mathrm{~V}$ at room temperature. The gels were stained with ethidium bromide $(0.50 \mu \mathrm{g} / \mathrm{ml})$ and imaged using the Gel Image System $(4,5)$. Pulsed Field Gel Electrophoresis was used to detect DNA double- strand breaks (DSB) produced by IR (gamma or protons) and/or restriction digestion by PvuRts1I. DSB levels were determined using gel image analysis. ${ }^{4,5}$

\section{Results}

To test the hypothesis that 5 -hmC levels increase with IR dose, we have been developing assays to specifically determine 5 -hmC in irradiated DNA. To test the levels of 5-hmC we used the PvuRts1I enzyme that is commercially available. Since the composition of the commercial buffer supplied with the enzyme or the reaction mixture for optimal enzyme activity are not described by the vendor (Active Motif), we decided to test the PvuRts buffer described in the original article that reported this enzyme. ${ }^{3}$ Figure 1 shows the PvuRts1I enzyme activity assay.

Samples of unmethylated (UM), methylated (M), or hydroxymethylated (HM) DNA (100 ng/sample) in solution were treated at room temperature for $2 \mathrm{~h}$ using the commercial buffer with DTT (first 7 lanes after 100-bp ladder) or the non commercial buffer plus DTT and BSA (rightmost 6 lanes). In both treatments the UM and M DNA were treated with $1.0 \mathrm{U}$ of enzyme and increasing amounts of PvuRts1I enzyme (0.2, 0.4, 1.0, 1.6U) were used for HM DNA. As expected, in both cases we found that the enzyme did not digest the unmethylated or methylated DNA. Both restriction digestion buffers were effective in digesting hydroxymethylated DNA. Also, in both reaction mixtures the DNA was not fully digested at the highest amount of enzyme tested. From Figure 1, the range of optimal amount of enzyme per 100 
ng of DNA is from 0.4 to $1.0 \mathrm{U}$. In order to assess the kinetics of the digestion reaction we tested a shorter incubation period of $1 \mathrm{~h}$ in non commercial buffer using $0.5 \mathrm{U}$ of enzyme per sample of $\mathrm{U}, \mathrm{M}$, and HM DNA in solution (Figure 2, rightmost 3 wells). As seen in Figure 2, the hydroxymethylated DNA was not fully digested with a $1 \mathrm{~h}$ incubation.

Since we want to apply this enzyme in DNA isolated from mammalian cells in agarose plugs, we needed to test if the PvuRts1I enzyme was able to cleave HM DNA embedded in agarose plugs. Agarose plugs containing $125 \mathrm{ng}$ of the U, M, or HM DNA were prepared. The plugs were incubated in PvuRts buffer at $4^{\circ} \mathrm{C}$ overnight to equilibrate. The plugs were incubated in the reaction mixture containing non commercial buffer with $0.5 \mathrm{U}$ of enzyme for 2 or $4 \mathrm{~h}$ at room temperature. This is shown in Figure 2, in which the decrease of the band fluorescence displays that the reaction digestion indeed occurred when the HM DNA was embedded in agarose plugs. The action of the PvuRts1I endonuclease was more evident after $4 \mathrm{~h}$ of incubation. Also this figure further reinforces that the PvuRts1I enzyme cuts DNA containing 5-hmC and not methylated or unmethylated DNA.

To analyze the levels of 5-hmC induced by IR in human skin fibroblast NFF28 cells we propose to develop an assay based on the recognition and cleavage of 5-hmC sites in DNA by the PvuRts1I restriction endonuclease, leading to double-strand breaks (DSB). As a previous step, the levels of DSBs produced by exposure to increasing doses of IR must be determined. As previously described in the literature, ${ }^{4}$ we have employed the method of neutral PFGE and number average length analysis to study the levels of DSBs produced by exposure of NFF2 28 cells to $200 \mathrm{MeV}$ proton radiation. In addition, prior to radiation exposure, cells were treated for $18 \mathrm{~h}$ with DMSO or SAHA (an HDAC inhibitor). Figure $3 \mathrm{~A}$ shows the image of PFGE gel of proton irradiated samples pretreated with DMSO and figure $3 \mathrm{~B}$ shows the gel image for the proton irradiated NFF28 samples pretreated with SAHA. In both gels, a progressive fragmentation of DNA was observed with an increasing radiation dose as seen by the further migration in the gel away from the wells.

Figure 4 shows the frequencies of DNA DSBs obtained by the analysis of the gels shown in Figure 3. The graph represents the DNA DSBs dose response induced by increasing doses of 200 $\mathrm{MeV}$ proton radiation. For both DMSO and SAHA pretreatments, the DSB frequencies increased linearly with the radiation dose. In this experiment, the SAHA pretreated samples showed a higher frequency of DSBs as compared to the DMSO pretreated samples. However, more experiments are needed to determine the significance of the difference.

\section{Conclusions and Discussion}

Epigenetic changes such as 5-mC and the chemically modified 5-hmC are known to be important in controlling gene expression and under inappropriate regulation may contribute to cancer and other diseases $(1,2)$. Nonetheless, the role of $5-\mathrm{hmC}$ is still largely unknown. We have recently shown that IR is capable of converting $5-\mathrm{mC}$ to $5-\mathrm{hmC}$ and we hypothesize that the increase in $5-\mathrm{hmC}$ is IR dose dependent. To test this hypothesis we have been developing assays to analyze 5-hmC levels in human cells exposed to IR using the PvuRts1I restriction endonuclease commercially available.

Using DNA in solution we determined the reaction mixture for optimal PvuRts1I enzyme activity. As shown in Figure 1, the appropriate PvuRts1I enzyme reaction mix contained $1 \mathrm{X}$ PvuRts1I Buffer (150 mM NaCl, $20 \mathrm{mM}$ Tris $\mathrm{pH} 8.0,5 \mathrm{mM} \mathrm{MgCl}$ ), $1 \mathrm{mM}$ DTT, and $50 \mu \mathrm{g} / \mathrm{ml}$ BSA. Therefore, both the commercial and the non-commercial buffers may be used for the enzyme reaction mixture. In the same assay we studied the amount of PvuRts1I endonuclease to fully digest 5-hydroxymethylated DNA. The range was from 0.4 to $1.0 \mathrm{U}$ per $100 \mathrm{ng}$ of double- strand DNA.

Our results shown in Figure 2 evidenced that hydroxymethylated DNA embedded in agarose plugs was digested significantly by the PvuRts1I restriction enzyme under the tested reaction conditions. Thus, the PvuRts1I enzyme may be applied to the detection of 5-hmC present in DNA isolated in agarose plugs.

Since the PvuRts1I enzyme will recognize and cleave at DNA sites containing 5-hmC, and therefore producing DSBs, we assessed the frequencies of DSBs induced by exposure of NFF28 cells to IR alone or with radiosensitization pretreatment prior to testing the enzyme. As shown in Figure 3, the DNA fragmentation increased with the dose of $200 \mathrm{MeV}$ proton radiation. The analysis of the PFGE gels by number average length analysis demonstrates that the DSB frequencies increased linearly with the radiation dose for both DMSO and the HDAC inhibitor (SAHA) pretreatments (Figure 4).The higher frequencies of DSBs observed with SAHA pretreatment compared to DMSO is probably related to the open state of the chromatin. Work is to be done to see a positive correlation between IR dose and 5-hmC occurrence in DMSO or SAHA pretreated samples.

\section{References}

${ }^{1}$ Dahl C., Grønbæk K., Guldberg P. Advances in DNA methylation: 5- hydroxymethylcytosine Revisited. Clinica Chimica Acta 412: 831-835, 2011.

${ }^{2}$ Aypar U., Morgan W.F., Baulch J.E. Radiation-induced genomic instability: Are epigenetic mechanisms the missing link? Int. J. Radiat. Biol. 87: 179-191, 2011

${ }^{3}$ Szwagierczak, A., Brachmann A., Schmidt C.S., Bultmann S., Leonhardt H., Spada F. Characterization of PvuRts1I Endonuclease as a Tool to Investigate Genomic 5hydroxymethylcytosine. Nucleic Acids Research 39: 5149-5156, 2011.

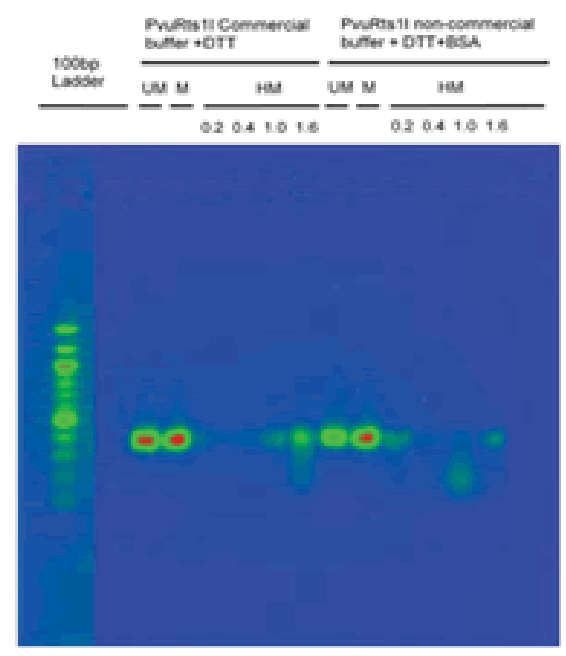

Figure 1.

PruRts1I enzyme activity assay. Samples of unmethylated (UM). methylated (M), or hydroxymethylated (HM) DNA (100 ng) in solution were treated at room temperature for $2 \mathrm{~h}$ using protocols a or b with increasing amounts of PvuRts1l enzyme $(0.2,0.4,1.0,1.6 \mathrm{U})$. The UM and M DNA were treated with $1.0 \mathrm{U}$ of enzyme. 


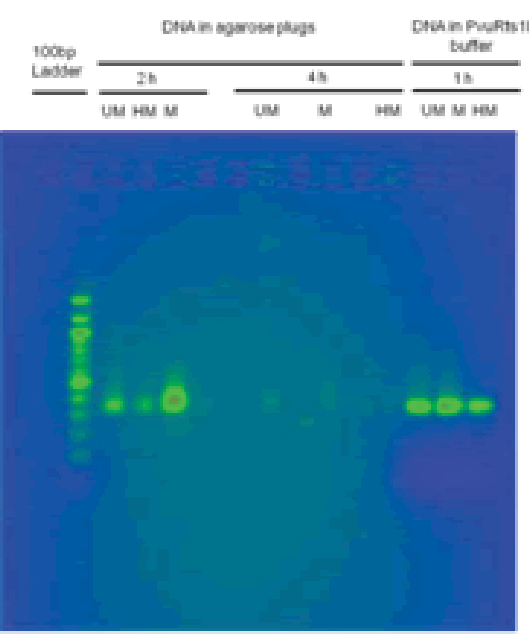

Figure 2.

PvuRts 11 enzyme DNA cleavage assayed in agarose plugs. Samples of $125 \mathrm{ng}$ of unmethylated (UM), methylated (M), or hydroxymethylated (HM) DNA embedded in agarose plugs were treated at room temperature for 2 or $4 \mathrm{~h}$ using protocol b with $0.5 \mathrm{U}$ of PvuRts 11 enzyme. $100 \mathrm{ng}$ of UM, M or HM DNA samples in solution were treated at room temperature for $1 \mathrm{~h}$ using protocol b with $0.4 \mathrm{U}$ of PruRts 11 enzyme.

\section{Figure 3.}

Images of neutral PFGE agarose gels for analysis of DS8s induced in NFF 28 cells exposed to $200 \mathrm{MeV}$ proton radiation. A. Prior to radiation exposure, cells were treated for $18 \mathrm{~h}$ with DMSO. tanes: Saccharomyces serevisiae $(1,13)$, Schirosaccharomyces pombe (14), Lambda Hind III (3), 0 Gy non-Notl digested (2), 0 Gy (4), $5 \mathrm{cGy}(5), 10 \mathrm{cGy}(6), 25 \mathrm{cGy}(7), 50 \mathrm{cGy}$ (8), 100 cGy (9), 200 cGy (10), 300 cGy (11). $400 \mathrm{cGy}$ (12).

B. Prior to radiation exposure, cells were treated for $18 \mathrm{~h}$ with SAHA. Lanes: Schinosaccharomyces pombe (1).

Saccharomyces cerevisiae (2), Lambda Hind III $(3,13), 0$ Gy (4), 5 cGy (5), 10 cGy (6), 25 cGy (7). 50 cGy (8), 100 cGy (9), 200 cGy (10), 300 $c G y(11), 400 \mathrm{cGy}$ (12), 0 Gy non-Noti digested (14).

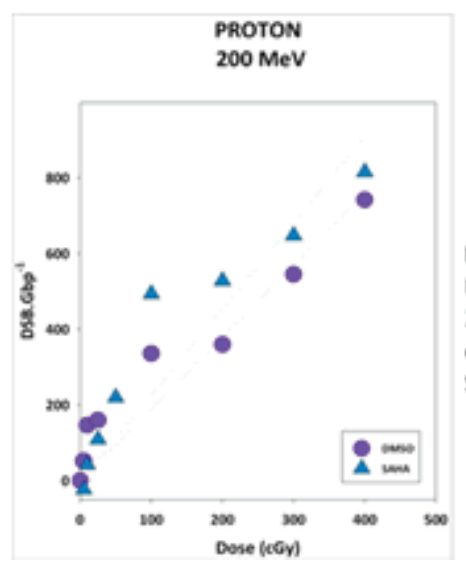

Figure 4.

DNA DSBs dose response induced by $200 \mathrm{MeV}$ proton radiation in NFF28 cells pretreated with DMSO (circles) or SAHA (triangles).
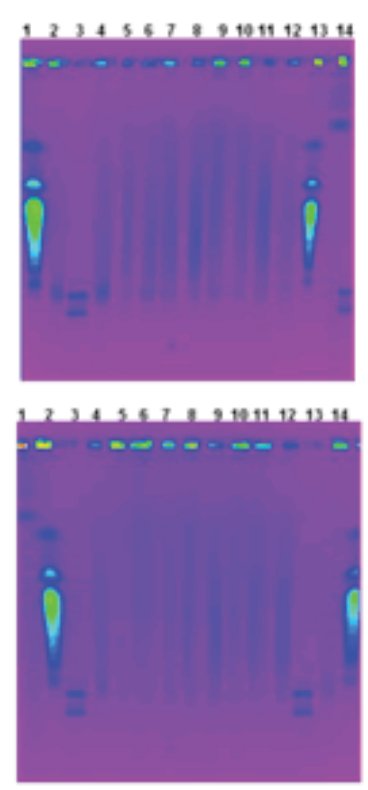

\section{B}

${ }^{4}$ Keszenman D.J., Sutherland B.M. Yields of clustered DNA damage induced by charged- particle radiations of similar kinetic energy per nucleon: LET dependence in different DNA microenvironments. Radiat Res.174:238-250, 2010. ${ }^{5}$ Sutherland B.M., Bennett P.V., Sutherland J.C., Laval J.

Clustered DNA damages induced by $\mathrm{x}$ rays in human cells. Radiat Res. 57:611-616, 2002.

\section{Acknowledgments}

I would like to thank my mentors Paula Bennett, Deborah Keszenman and Paul F. Wilson for all of their guidance and patience. Special thanks to Paul Wilson for instructing and supporting me in his lab. Thank you to Alicia Johnson for her assistance on this project. My appreciation goes to James Jardine for his laboratory support. Thank you to David Schlyer, Ann Emrick, John Trunk and Denise Monteleone for all of their help. Thank you to my fellow SULI coworkers Katherine Z. Sanidad and Benjamin Daniels for their assistance. This work was supported by the Department of Energy and the Office of Education Programs at Brookhaven National Laboratory. This work was performed under the auspices of the U.S. Department of Energy by Brookhaven National Laboratory and supported by BNL LDRD-12-012 grant to P.F. Wilson. 


\title{
Design and Installation of Oxygen Deficiency Sensors
}

\author{
Kevin Korol \\ Suny Maritime College, Throggs Neck, NY 10465 \\ Joseph Scaduto \\ Brookhaven National Laboratory, Upton NY 11973
}

\begin{abstract}
The Collider Accelerator Department's Water Systems Group deals primarily with the monitoring and maintenance of the water cooling systems, and plumbing for experimental projects. During the beginning of the internship, I was introduced to the daily operation of the Water Systems Group, including pump and valve maintenance, inline flow measurements and ultrasonic flow measurements. Recently I have been assigned a project in Building 1002: a request from the Access Control Group to install ODH (Oxygen Deficiency Hazard) sensors throughout the building. I met with Access Control at the job site and discussed the location of the sensor boxes and the materials that would be used. The first part of my job was to engineer the best way to run the piping and map it out using Google sketch-up and the program Q-Cad. Next I made a count of the amount of materials need and ordered them. I am currently installing the piping that I ordered. When this project is complete, it will make Building 1002 safer by alerting people if there is a helium leak from one of the compressors. Through this project, I believe that I have learned a great deal about the elements of a design project. I have worked on it from the beginning to the very end. It's a great feeling seeing what you have worked hard planning to actually come to life. This project especially taught me to take control of a situation and follow through.
\end{abstract}

\section{Introduction}

Safety is a key goal in making Brookhaven National Lab successful. The group that came up with ODH sensor project was Access Control. I worked directly with them through the project. The main object of this project was to make Building 1002 safe to operate in while the helium compressors are running. The Installation of these detectors is to ensure that people will be alerted if there is a helium leak in the building. The alerting system is comprised of a series of flashing light and sounding alarms. The project had to go through various groups and stages to be done successfully. My main objective was to come up with a design for the PVC piping and layout out the path it would take throughout the building. I used various programs to create this design which lead to the success of the project. By doing this project, it would create a safe and secure place for the tubing that went to the detector boxes, and monitoring the air.

\section{Materials and Methods}

Most of the project materials needed was comprised of PVC. The project called for 160 feet of $3 / 4$ inch schedule $80 \mathrm{PVC}$ piping. All of the fittings were $3 / 4$ inch schedule 80 PVC. Theses fittings included T's, 90 degree, 45 degree, couplings and sweeps. The metal electrical boxes that harbored the sensor in them were provided by Access control. The only other pieces I had to purchase were the materials to hang and secure the piping. My method for this project was to create a floor plan and map out which way I would install the piping. I used various programs to achieve the design aspect of the project. To design the basic layout of the piping I used the program Google sketch up. This program allowed me to design the building and add the components inside of it. This program was very easy to use and gave me a $3 \mathrm{D}$ view of the building without me being there. The next program that I used to create the actual blueprints for the project was Q-Cad. This program is slightly more complicated to use, but is very accurate. Through Q-Cad I was able to receive the exact measurements I would need for the piping. After I had a basic idea of the path through my drawings, I would start at one end and work off of that. I would make my rough cuts throughout the building and then come back once the piping is secured and make my final adjustments. After the piping was perfect, the sensor boxes had to be installed. For these sensor boxes I had to design a way for the PVC to go into the metal electrical boxes. I would also have to make a restraint for the PVC, so no one would be able to pull the PVC out of the box by accident. For this design, I used the program Google Sketch up again. The program made it easy to design the electric box, so I could figure out how to run the piping into box. I was able to create this transition from the PVC to the box by using a reducing coupling that feed into a one inch threaded female to male adaptor. For the restraint I used lock nuts on either side of the box. After this was completed I ran wax string through the piping. To run the string through the piping properly, I had to take a 25 foot wire pull and feed it through the piping in sections. I would then attach the string to the wire pull and pull the string through the piping. Each tube had an individual string so there was a total of six segments of string in the piping. The reason we ran the string instead of just running the wire pull was because of the 90 degree elbows and sweeps. The wire pull would become stuck in those pieces were as the string would coast through. This made the 6 capillary tubes very easy to pull through the PVC piping. These capillary tubes would go from the sensor to the main unit that detects the helium in the air. The main unit has a series of sensors and pumps in it to monitor the oxygen level. In the unfortunate event of a helium leak, the helium in the air would deplete the oxygen. The way the monitor works is by sucking the air from the room, and feeding it into the main monitoring unit through the capillary tubes. The sensors then detect how much oxygen is in the air and if the oxygen level is too low the alarm will sound. The materials and methods that I used on this project worked perfectly. Both Access Control and I were extremely pleased with the way the piping and sensor system turned out, and how efficiently the system operated. 


\section{Results and Conclusion}

The projects main goal was to make Building 1002 safe in the emergency of a helium leak. This goal was successfully achieved through the hard work of many people and proper planning. The final result of the project was the success of the detectors. After the completion of the detectors and tubing we were able to run test on the main unit. The unit was working perfectly monitoring the amount of oxygen present in the air. With this system in place we can safely monitor the oxygen level in the build and an alarm will sound if the oxygen level decreases. I did encounter minor problems throughout the project but none were really catastrophic. Working with Access Control on this project was a pleasure. We agreed on the same plan and they were always available for any questions that I had. I would definitely want to do another project with them. Safety is a key goal at Brookhaven National Lab and I am happy to be a part of this important project. This is also a personal achievement for me; this is the first actual engineering project that I have worked on. I was very fortunate to be able to take this project from the beginning to the end. I believe that this project has helped me become more proficient in the programs Q-Cad and Google Sketch Up, and has also taught me how to deal with various scenarios to complete the project. This project has truly helped me understand the hard work that goes into designing and installing a safety system.

\section{Acknowledgement}

This successful internship would not be possible without Mentor, Supervisor and Water Systems Crew. I would like to thank them for guiding me throughout this project and for always being there to offer their help or advice. My mentor, Joe Scaduto, has been a great role model and is someone that I aspire to be like in the work field. I would also like to thank my supervisor, John de Boer, for teaching me about the management aspect of Brookhaven National Lab. All of these people gave me lifelong lessons to further my career in mechanical engineering. I would also like to thank Brookhaven National Laboratory for giving me this opportunity to do an internship with them. I am extremely grateful to work for them this summer and experience all they offer. I believe that the internship with Brookhaven National Lab has set me on the right road for success. 


\title{
The use of mark-recapture techniques to study migration and population densities of Odonate species on a utility scale solar farm
}

\author{
Sarah Langan \\ Natural Sciences and Mathematics, Keystone College, La Plume, PA 18440 \\ Timothy Green \\ Environmental Protection Division, Brookhaven National Laboratory, Upton, NY 11973 \\ order Odonata, which consists of dragonflies and damselflies. Out \\ of over 5000 species worldwide, sixty-three are found at BNL. \\ Odonates are used as indicator species to evaluate aquatic habitat \\ because their larvae require clean water. These insects spend most \\ of their lives as completely aquatic nymphs, which transform into \\ air-breathing adults in what is known as emergence. The nymphs \\ are sensitive to many aspects of their environment, including wa- \\ ter temperature and acidity, vegetation, pesticide levels, and silt \\ content. ${ }^{2}$ To study the Odonate populations and potential migra- \\ tion patterns on the solar farm, visual surveys and mark-recapture \\ methods were used within two of the six solar farm Areas, at a \\ previously existing man-made pond near the solar farm, and at a \\ control pond at BNL.
}

\section{ABSTRACT}

The solar farm located on Department of Energy (DOE) property at Brookhaven National Laboratory (BNL) creates a unique opportunity to study the environmental impacts and habitat changes caused by the large scale use of solar energy. For example, the water accumulated underneath and around the solar panels creates aquatic habitats that did not exist prior to construction. The taxonomic order Odonata consists of dragonflies and damselflies, which are used as indicator species to assess environmental health. As part of a project to evaluate the overall environmental impact of the solar farm, I used mark-recapture techniques to study the population sizes and migration patterns of the various Odonate species found at five separate survey sites: two sections of Area One of the solar farm, known as Area 1 front (A1F) and Area 1 back (A1B); Area Two (A2) of the solar farm; the Meadow Marsh, which existed near the solar farm property prior to construction; and Weaver Road Pond (the control). The goal was to evaluate the existing Odonate populations on the solar farm and to see if Odonates migrate between the Meadow Marsh and the water in Areas One and Two. To track migration and population numbers, dragonflies were marked with Sharpie ${ }^{\circledR}$ markers, receiving a location symbol on the left hindwing and a number on the left forewing. Damselflies had their thoraxes dotted with paint, with the color identifying the original capture location. I have observed Odonate migration within Area One, but whether they are migrating between the different Areas and the Meadow Marsh remains undetermined. In addition, with the exception of $\mathrm{A1F}$, Odonate species present on the solar farm seem to be able to establish healthy populations during their flight seasons that experience growth and change.

\section{INTRODUCTION}

Few studies have been conducted on the environmental impacts of solar farms. The solar farm located at BNL on DOE property had been in operation since fall of 2011 and consists of 164,312 solar panels over approximately 80.94 hectares, making it the largest solar farm currently in operation in the Eastern United States. Because of its location relative to BNL, it creates a unique opportunity for the lab's Environmental Protection Division to study the effects of a large scale solar farm on the local wildlife.

Prior to construction, the land used for the solar farm was primarily forest and grassy fields. Currently, sections within the six Areas of the solar farm have poor drainage, and ponds were inadvertently created where there had not previously been standing water. To assess the quality of these new habitats, population studies were conducted on the aquatic insects of the taxonomic

\section{METHODS}

Five sites, shown in Figure 1, were chosen for study: A1F, $\mathrm{A} 1 \mathrm{~B}$, and $\mathrm{A} 2$ on the solar farm; the Meadow Marsh; and Weaver Road Pond. The sites on the solar farm were chosen based on the amount of water in them. The Meadow Marsh is a man-made pond that existed between Areas One and Two prior to solar farm construction. Weaver Road Pond is located on BNL property approximately 2,100 meters away from the solar farm and was used as a control. At least two sites were chosen to survey per week and each site was visited every other day. Field work was usually conducted from the morning until the afternoon.

Each day, the site(s) visited, time spent there, and general weather conditions were recorded. Visual surveys were conducted throughout the day to determine the species present and estimate the population levels. All species observed and captured were identified with a combination of three field guides: Dragonflies and Damselflies of the East, ${ }^{3}$ Damselflies of the Northeast, ${ }^{4}$ and Dragonflies Through Binoculars: A Field Guide to the Dragonflies of North America. ${ }^{5}$ When sites were revisited, binoculars were used to look for Odonates that had been marked in days prior. The Odonates were caught with a thirty-eight centimeter diameter net and removed by folding their four wings together and holding them between two fingers. Once removed, they were placed in wax paper specimen bags and stored in a cooler to slow down their metabolisms and prevent them from being crushed.

After thirty to sixty minutes in the cooler, the Odonates were processed. Dragonflies were held down by placing them sideways with their four wings together on top of the cooler. A thin plastic strip was placed over the wings near the body and taped to the cooler. A location symbol, detailed in Figure 2, was drawn on the left hindwing with Sharpie ${ }^{\circledR}$ marker. Then they received an individual number on the forewing. Damselflies were kept in the hand with four wings together and received a dot of acrylic paint on the back of the thorax, as suggested by Beirinckx, et al. ${ }^{6}$ The different 
colors, shown in Figure 2, indicated the original capture location. In the event of recapture, I would know where the damselfly was marked, but I unfortunately would not know which day it had been captured. The species, sex, and other notes, such as presence of parasitic water mites or damaged wings, were recorded. The Odonates were promptly released after being marked.

\section{RESULTS}

Detailed in Figure 3, a total of 247 individual Odonates from twenty-four species were marked in this study. Three were recaptured as shown in Figure 4. An additional five species were observed and recorded in visual surveys, but were not marked. Figure 5 details the population levels of all Odonate species seen at the survey sites. Out of the twenty-nine species, eight dragonfly and four damselfly species were found at all sites surveyed. Four dragonfly and two damselfly species were only found on the solar farm property, including within the three sites in the Areas and at the Meadow Marsh. Two dragonfly and four damselfly species were only found at the Meadow Marsh and Weaver Road Pond, and within that, one dragonfly and three damselfly species were only seen at Weaver Road Pond. In the following analysis, a small population is defined as one to five individuals, a medium population consists of six to twenty individuals, and a large population includes individuals numbering twenty-one and over.

A1F had the lowest species richness calculated at fourteen, with nine dragonfly and five damselfly species. Thirty individuals of eight species were marked here. Out of the species present, $7 \%$ had large populations, $7 \%$ had medium populations, and $86 \%$ had small populations. Pachydiplax longipennis was seen in small populations from mid-June to mid-July, and then the population grew to be significantly larger than any other species observed at the site. Plathemis lydia had consistent populations through the entire study, with males observed flying over standing water even when other Odonates were not present in significant numbers. Erythemis simplicicollis was not observed until mid-July when it became present in small to medium sized populations, which corresponds with the most productive time of emergence during the species' flight season. ${ }^{7}$ Two females of Erythrodiplax berenice, a species not previously documented at BNL, and one female Calopteryx maculata were seen in A1F on July 10th but were not seen at the site again. C. maculata was also not found at any other survey site.

A1B had the second highest species richness calculated at nineteen, with fourteen dragonfly and five damselfly species. Thirty individuals of eight species were marked at this site. Out of the species present, $21 \%$ had large populations, $11 \%$ had medium populations, and $68 \%$ had small populations. Included in the species with small populations were Epiaeschna heros, Celithemis martha, and Lestes disjunctus australis, which were only seen in late July and not found at any other survey site. L. disjunctus australis has also never been documented at BNL. P. lydia was consistently observed in medium to large populations, and $\mathrm{Li}$ bellula pulchella was observed in medium populations, until late July when the population declined. Ischnura verticalis and Ischnura hastata were consistently observed in large populations, with the latter being female dominated. As in A1F, P. longipennis was found in small populations until late July when the population became larger than any other dragonfly species previously observed at the site.

In A2, richness was calculated as eighteen, with thirteen drag- onfly and five damselfly species. Fifty-three individuals in eleven species were captured and marked. Out of the species present, $17 \%$ had large populations, 33\% had medium populations, and $50 \%$ had small populations. $P$. lydia was present in medium populations throughout the entire study, and E. simplicicollis was present in medium populations as of mid-July. One female $E$. berenice was present at the site in early July, but no others were seen during the rest of the survey. On July 17th, several species, including $L$. pulchella, P. longipennis, Libellula incesta, I. hastata, Enallagma aspersum, and Enallagma civile, experienced population growth and were present in larger numbers than had been seen previously within the site. However, the populations were smaller when the site was revisited on July 19th and 23rd. In addition, many adult and teneral damselflies were present in A2 on July 15th, 17th, and 19th, but when the site was revisited on July 23rd, very few damselflies were present.

Species richness at the Meadow Marsh was calculated at seventeen, with twelve dragonfly and five damselfly species. Ninety-six individuals of eleven species were marked here. Out of species present, $6 \%$ had large populations, 35\% had medium populations, and $59 \%$ had small populations. $P$. longipennis, $L$. incesta, E. simplicicollis, and $P$. lydia had consistently medium sized populations. E. civile and $I$. verticalis females were seen at the pond in early June, but during the rest of the study, E. aspersum males were present in a significantly larger population than at any other survey site, with only a few Lestes species and one I. hastata seen. Celithemis eponina populations were small when first seen in early July, but grew to medium sized by the end of the month, corresponding with the peak of emergence during their flight season. ${ }^{8}$ The reverse happened with the pond's Libellula cyanea population, a species with generally high populations in June that decrease in July. ${ }^{9}$ One female E. berenice and one male Lestes congener were seen on July 10th, but no other individuals were found at the pond. L. congener was also not found at any other survey site.

Weaver Road Pond had the highest calculated species richness at twenty-two, with fourteen dragonfly and eight damselfly species. Thirty-eight individuals of thirteen species were marked here. Out of the species present, 36\% had large populations, 28\% had medium populations, and $36 \%$ had small populations. Species such as Anax junius, Tramea lacerate, Tramea carolina, Libellula luctuosa, and Libellula semifasciata that had small populations on the solar farm thrived at Weaver Road Pond. T. lacerate, $P$. longipennis, L. incesta, and L. luctuosa experienced population growth between July 16th and July 29th. E. civile was always present in a large population, but significant population growth was observed when the pond was visited on July 29th. Four species that were observed at Weaver Road Pond, Lestes forcipatus, Lestes rectangularis, Enallagma durum, and Celithemis elisa, were not found at the solar farm survey sites.

On July 9th, L. pulchella \#2 was marked in A1B and was recaptured in A1F the next day, having travelled a distance of approximately 270 meters. Seven L. pulchella males were marked in A2 on July 17th, and at least one was seen at the same site through binoculars two days later. On July 29th, a previously-marked male L. forcipatus was captured at Weaver Road Pond. The marking was from Weaver Road Pond, but due to the damselfly markings only detailing the original location of capture, it is uncertain whether this individual was marked on July 17 th or 18 th. 


\section{A. Ectoparasites}

Odonates are subject to parasitism by various species of water mite larvae. After hatching, the larvae attach themselves to the underside of an Odonate nymph and are able to remain there after the nymph emerges as an adult. The mites feed on the adult Odonate's bodily fluids until they are ready to drop back into the water and become predatory nymphs themselves. Usually the Odonates are simply weakened to the point where reproductive efforts are diminished, but if many mites attach, they can suck out all of the host's fluids, leading to its death. ${ }^{10}$

Parasitic water mites were found on Odonates captured at A1F, A1B, A2, and the Meadow Marsh. The mites were unidentified, but black one and red ones were present in all locations. However, it is possible that both colors were the same species because many species of water mites can change color with age. ${ }^{11}$ Twenty of the marked Odonates, fifteen males and five females, had varying amounts of red and/or black water mites attached to the sides and underside of the thorax, underside of the abdomen, and/or the legs. Six different species, five dragonfly and one damselfly, were hosting the parasites: P. longipennis, $C$. eponina, $C$. martha, E. simplicicollis, P. lydia, and E. aspersum. Out of the twenty individuals, fifteen were found at the Meadow Marsh, including seven $P$. longipennis, two $C$. eponina, three E. simplicicollis, one P. lydia, and two E. aspersum. Both colors of water mite were seen at the Meadow Marsh, with most having only black or red water mites and some having a mix of both colors. In A1B, two $C$. martha, the only two marked in a species only seen in A1B, had black and red water mites, and in A1F, only one female E. simplicicollis had black water mites, but she had so many that her abdomen was swollen. Finally, in A2, two P. longipennis were found with both red and black water mites. Females and males of $P$. longipennis, E. simplicicollis, $C$. eponina, and $C$. martha had water mites, while only the males of $P$. lydia and $E$. aspersum had them.

\section{B. Solar Panel Interactions}

Studies have suggested that Odonates can be attracted to shiny black surfaces, such as black cars, polished black gravestones, and solar panels. ${ }^{12,13}$ Odonates find water by detecting the horizontally polarized light it gives off. When other objects give off similar light, the Odonates can mistake it for water. The object can then become an ecological trap if female Odonates lay their eggs on the surface. However, if the solar panels are divided with non-polarized white lines, Odonates become less attracted to them. ${ }^{13}$ Since the panels on the solar farm in this study are divided with white lines in a grid-like pattern, significant interactions with the Odonates were not anticipated. However, certain behaviors were observed.

Many species of male dragonflies seem to claim the panels as part of their territory, in addition to standing water, and will chase other males away from the panels. They fly over, under, and along the panels in the efforts to defend them. This behavior only occurs where there is also water underneath and around the panels. Female Odonates were not observed interacting with the panels in such a manner. Additionally, E. heros and L. pulchella individuals were seen perching on the backs of the solar panels. Finally, a wheeling pair of $P$. longipennis were observed to briefly land on a solar panel, but quickly flew away. Female Odonates were not seen ovipositing on the panels.

\section{DISCUSSION}

Out of all sites surveyed, Weaver Road Pond had the overall highest species richness and percent of large populations as well as the lowest percent of small populations. The solar farm Areas and the Meadow Marsh had a three to eight decrease in species richness and at least fifty percent small populations. Within the solar farm, A1B had the highest species richness and percent of large populations. However, A2 had more medium sized populations and fewer small populations than A1B. The Meadow Marsh had the second lowest species richness and consisted of primarily medium and small sized populations. A1F had the smallest species richness and, only one species, P. longipennis, experienced substantial population growth.

Of interest are the sightings of E. berenice females in early to mid-July since the species was not previously documented at BNL, and it was at three of the four survey sites on the solar farm. This species is primarily found in coastal regions and breeds in salt water and salt marshes. However, wandering individuals are frequently found inland, ${ }^{3}$ which accounts for the four females seen at the solar farm.

L. pulchella \#2 was the only recapture that migrated, traveling from A1B to A1F. However, seven species were found in A1B that were not found in A1F, indicating not all species migrate within the Area. Therefore, the reason for the migration is undetermined. However, based on the territorial and aggressive nature of male dragonflies, it is hypothesized that during the time between capture and release, the dragonfly's existing territory was taken over by another male. After release, if the dragonfly could not reestablish its territory, it was forced to move elsewhere. To test this hypothesis in future studies, the Odonates should be marked as quickly as possible after capture without storage in a cooler. Additionally, despite having marked 247 Odonates, only two were recaptured and one was seen through binoculars. A second hypothesis to the lack of recaptures is that the markings make the Odonates more visible to predators, such as birds, and they are eaten more readily than unmarked individuals.

Many teneral and adult damselflies were seen in A2 on July 15th, 17th, and 19th, but the damselfly populations were severely decreased when the site was revisited on July 23rd. A possible explanation for the drop in numbers is a high level of predation. At Weaver Road Pond, male and females of E. simplicicollis were seen consuming damselflies. This species was not seen at any survey site until mid-July, which is the peak time for emergence. A2 had a medium-sized population of E. simplicicollis at the time when many damselflies were emerging, which could have caused the dramatic decrease in the populations.

Parasitic water mites were seen on Odonates in all four of the survey sites on the solar farm property but were not seen at Weaver Road Pond. Since the mites can diminish the reproductive success of adult Odonates or lead to their deaths, they have the potential to decrease Odonate populations on the solar farm. To see how the parasites may affect the populations, a study should be conducted to see if water mites are present at other BNL ponds and how the Odonate populations compare to ponds without the parasites. Additionally, $75 \%$ of parasitized Odonates were male. Studies have shown that water mite larvae show sexual biases towards males, ${ }^{14}$ and while ectoparasite sexual preference was not the primary objective in this study, this data supports that argument. 
Based on the territorial behaviors observed, male Odonates seem to confuse the solar panels with water. However, since only males were seen defending them and females were not observed ovipositing on them, the solar panels are not harmful to the Odonate populations on the solar farm. However, if further studies are done, any additional interactions the Odonates have with the solar panels should be noted.

Weaver Road Pond has the healthiest populations out of the five sites surveyed, but, with the exception of A1F, Odonate species present on the solar farm seem to be able to establish healthy populations during their flight seasons that experience growth and change. One instance of migration of Odonates on the solar farm was observed within Area One, but it is inconclusive if the Odonates are moving between the Areas and the Meadow Marsh. More research on the solar farm Odonates is required if definitive results are to be found.

\section{ACKNOWLEDGEMENTS}

This project was supported in part by the U.S. Department of Energy, Office of Science, and Office of Workforce Development for Teachers and Scientists (WDTS) under the Science Undergraduate Laboratory Internships Program (SULI). I would also like to thank my mentors Dr. Timothy Green and Jennifer Higbie for providing me with this research opportunity; my helpers Jessica Bristol and Mika Lai; everyone who drove me to and from the solar farm; Gasper Giancontieri, Jim Doran, and the solar farm workers; and Bill Mauffray from the International Odonata Research Institute.

\section{NOTES}

1"Project Overview," Long Island Solar Farm, http://www.bnl. gov/GARS/SET/LISF.php (19 July 2013).

${ }^{2}$ Michelle Simon, "Dragonflies - Indicator Species of Environmental Health,” Earth Times, 11 Jun 2012, http:// www.earthtimes.org/nature/dragonflies-indicator-speciesenvironmental-health/2033/ (19 Jul 2013).

${ }^{3}$ Dennis Paulson, Dragonflies and Damselflies of the East, (Princeton University Press, Princeton and Oxford, 2011).

${ }^{4}$ Ed Lam, Damselflies of the Northeast, (Biodiversity Books, Forest Hills, 2004).

${ }^{5}$ Sidney W. Dunkle, Dragonflies Through Binoculars: A Field Guide to the Dragonflies of North America, (Oxford University Press, New York, 2000).

${ }^{6}$ Kirsten Beirinckx, Hans Van Gossum, Marc J. Lajeunesse, and Mark R. Forbes, "Sex biases in dispersal and philopatry: insights from a meta-analysis based on capture-markrecapture studies of damselflies," Oikos 113 (3), 539-547 (2006).

7 Jim Bangma, "Eastern Pondhawk Erythemis simplicicollis," Njodes, 2006, http://www.njodes.com/Speciesaccts/ skimmers/pond-east.asp, (06 Aug 2013).

8 Jim Bangma, "Halloween Pennant Celithemis eponina," Njodes, 2006, http://www.njodes.com/Speciesaccts/ skimmers/penn-hall.asp, (06 Aug 2013).

9 Jim Bangma, "Spangled Skimmer Libellula cyanea," Njodes, 2006, http://www.njodes.com/Speciesaccts/skimmers/ skim-span.asp, (06 Aug 2013).

${ }^{10}$ Cynthia Berger, Dragonflies, (Stackpole Books, Google eBook, 2004), 49-50.
${ }^{11}$ Rodger Mitchell, "The Use of Parasitic Mites to Age Dragonflies," American Midland Naturalist 82 (2), 359 (1969).

${ }^{12}$ Gábor Horváth, Péter Malik, György Kriska, and Hansruedi Wildermuth, "Ecological traps for dragonflies in a cemetery: the attraction of Sympetrum species (Odonata: Libellulidae) by horizontally polarizing black gravestones," Freshwater Biology 52, 1700-1709 (2007).

${ }^{13}$ Gábor Horváth, Miklos Blaho, Ádám Egri, György Kriska, István Seres, and Bruce Robertson, "Reducing the Maladaptive Attractiveness of Solar Panels to Polarotactic Insects," Conservation Biology, 1-9 (2010).

${ }^{14}$ Marc J. Lajeunesse, Mark R. Forbes, Bruce P. Smith, "Species and sex biases in ectoparasitism of dragonflies by mites," Oikos 106 (3), 501-508 (2004).

\section{FIGURES}

FIG. 1. Map of Odonate survey sites based on a Google Satellite image.

FIG. 2. Location marks for dragonflies and damselflies.

FIG. 3. Marked Odonate species where " $M$ " indicates male, " $F$ " indicates female, and an asterisk indicates the species was not marked and/or seen at the survey site.

FIG. 4. Recaptured Odonates.

FIG. 5. Odonate population size estimates based on visual surveys. Those listed in re were found in all locations, purple were only at the solar farm, and blue were only at the Meadow Marsh and Weaver Road Pond. Tan highlighting specifies marked species. The general population sizes are indicated as follows: " $S$ " for one to five individuals, "M" for six to twenty individuals, and " $L$ " for at least twenty-one individuals. Species that have multiple population estimates had a significant change in the population level during the course of the study. An asterisk indicates the species was not found at the survey site. 


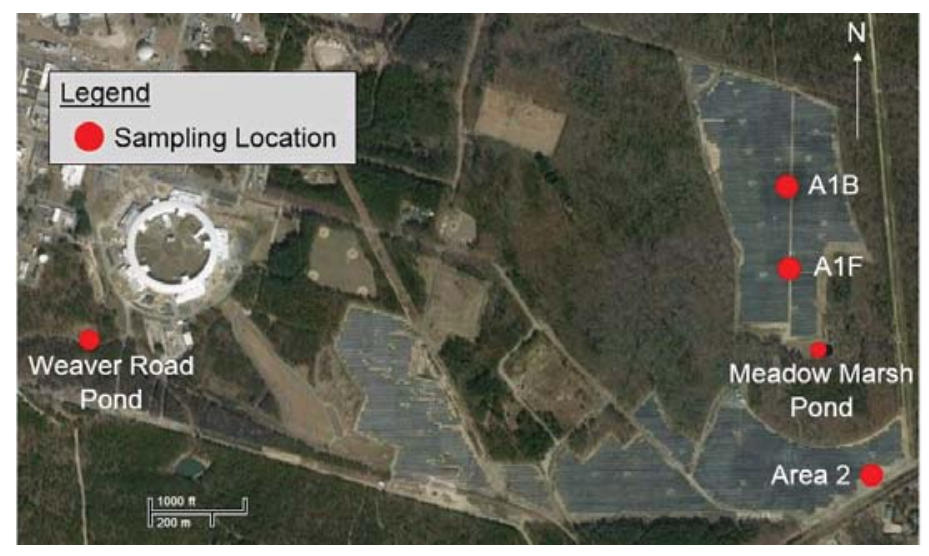

FIG. 1. Map of Odonate survey sites - Sarah Langan

\begin{tabular}{|c|c|c|}
\hline $\begin{array}{c}\text { Original Capture } \\
\text { Location }\end{array}$ & Dragonfly Symbol & Damselfly Color \\
\hline A1F & 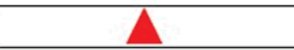 & O \\
\hline A1B & & 0 \\
\hline A2 & & (1) \\
\hline Meadow Marsh & D & 0 \\
\hline Weaver Pond & 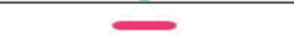 & 0 \\
\hline
\end{tabular}

FIG. 2. Dragonfly and damselfly location marks - Sarah Langan

\begin{tabular}{|l|ll|}
\hline Recaptures & Originally Marked & Recaptured \\
\hline Libellula pulchella \#2 & 7/9 A1B & 7/10 A1F \\
Libellula pulchella & $7 / 17 \mathrm{~A} 2$ & 7/19 A2 \\
Lestes forcipatus & $7 / 17$ or 7/18 Weaver & 7/29 Weaver \\
\hline
\end{tabular}

FIG. 3. Marked Odonates - Sarah Langan

\begin{tabular}{|c|c|c|c|c|c|c|}
\hline Dragonflies & A1F & $\mathrm{A} 1 \mathrm{~B}$ & $\mathrm{~A} 2$ & $\mathrm{MM}$ & Weaver & Total \\
\hline Anax junius & * & $1 \mathrm{M}$ & $1 \mathrm{M}$ & $2 \mathrm{M}$ & $7 \mathrm{M}$ & $11 \mathrm{M}+\mathrm{OF}=11$ \\
\hline Erythemis simplicicollis & $7 F$ & * & $2 \mathrm{M}$ & $4 \mathrm{M}, 1 \mathrm{~F}$ & $1 \mathrm{M}$ & $7 M+8 F=15$ \\
\hline Libellula pulchella & $2 \mathrm{M}$ & $3 \mathrm{M}$ & $9 M$ & $1 \mathrm{M}$ & $1 \mathrm{~F}$ & $15 M+1 F=16$ \\
\hline Tramea lacerata & * & $1 \mathrm{M}$ & * & * & * & $1 \mathrm{M}+O \mathrm{~F}=1$ \\
\hline Pachydiplax Iongipennis & $10 \mathrm{M}$ & $2 \mathrm{M}$ & $7 \mathrm{M}$ & $11 \mathrm{M}, 1 \mathrm{~F}$ & * & $30 M+1 F=31$ \\
\hline Plathemis Iydia & * & * & $3 \mathrm{M}$ & $2 \mathrm{M}, 1 \mathrm{~F}$ & $1 \mathrm{M}$ & $6 \mathrm{M}+1 \mathrm{~F}=7$ \\
\hline Libellula cyanea & * & * & * & $6 \mathrm{M}$ & * & $6 \mathrm{M}+\mathrm{OF}=6$ \\
\hline Perithemis tenera & * & $1 \mathrm{~F}$ & * & * & * & $O M+1 F=1$ \\
\hline Erythrodiplax berenice & $1 \mathrm{~F}$ & * & $1 \mathrm{~F}$ & $1 \mathrm{~F}$ & * & $O M+3 F=3$ \\
\hline Celithemis martha & * & $1 \mathrm{M}, 1 \mathrm{~F}$ & * & * & * & $1 M+1 F=2$ \\
\hline Celithemis elisa & * & * & * & * & $1 \mathrm{M}, 1 \mathrm{~F}$ & $1 \mathrm{M}+1 \mathrm{~F}=\mathbf{2}$ \\
\hline Libellula incesta & * & * & $6 \mathrm{M}$ & $9 M$ & & $15 \mathrm{M}+\mathrm{OF}=15$ \\
\hline Libellula Iuctuosa & * & * & * & * & $1 \mathrm{M}$ & $1 \mathrm{M}+O F=1$ \\
\hline Libellula semifasciata & * & * & * & * & $2 \mathrm{M}, 1 \mathrm{~F}$ & $2 M+1 F=3$ \\
\hline Celithemis eponina & $1 \mathrm{~F}$ & * & * & $4 \mathrm{~F}$ & & $O M+5 F=5$ \\
\hline Damselflies & A1F & $\mathrm{A} 1 \mathrm{~B}$ & $\mathrm{~A} 2$ & $\mathrm{MM}$ & Weaver & \\
\hline Enallagma aspersum & * & * & * & $52 \mathrm{M}$ & $2 \mathrm{M}$ & $54 \mathrm{M}+0 \mathrm{~F}=54$ \\
\hline Enallagma civile & * & * & $7 \mathrm{M}, 2 \mathrm{~F}$ & * & $2 M$ & $9 M+2 F=11$ \\
\hline Ischnura hastata & $2 \mathrm{~F}$ & $5 \mathrm{M}, 9 \mathrm{~F}$ & $2 \mathrm{M}, 1 \mathrm{~F}$ & * & $4 \mathrm{M}, 5 \mathrm{~F}$ & $11 M+17 F=28$ \\
\hline Ischnura verticalis & $3 \mathrm{M}, 3 \mathrm{~F}$ & $3 \mathrm{M}, 3 \mathrm{~F}$ & $4 \mathrm{M}, 7 \mathrm{~F}$ & * & $1 \mathrm{M}$ & $11 \mathrm{M}+13 \mathrm{~F}=\mathbf{2 4}$ \\
\hline Calopteryx maculata & $1 \mathrm{~F}$ & * & * & * & * & $O M+1 F=1$ \\
\hline Lestes congener & * & * & * & $1 \mathrm{M}$ & & $1 \mathrm{M}+0 \mathrm{~F}=1$ \\
\hline Lestes forcipatus & * & * & * & * & $5 \mathrm{M}, 2 \mathrm{~F}$ & $5 \mathrm{M}+2 \mathrm{~F}=7$ \\
\hline Lestes rectangularis & * & * & * & * & $1 \mathrm{~F}$ & $O M+1 F=1$ \\
\hline Ischnura posita & * & * & $1 \mathrm{M}$ & * & * & $1 M+O F=1$ \\
\hline Total individuals & & & & $\overline{96}$ & 38 & 247 \\
\hline Total species & & & & 11 & 13 & 24 \\
\hline
\end{tabular}

FIG. 4. Recaptured Odonates - Sarah Langan 


\begin{tabular}{|l|lllll|} 
Libellula pulchella & $\mathrm{S}$ & $\mathrm{M}-\mathrm{S}$ & $\mathrm{S}-\mathrm{L}$ & $\mathrm{S}$ & $\mathrm{L}$ \\
Tramea lacerata & $\mathrm{S}$ & $\mathrm{S}$ & $\mathrm{S}$ & $\mathrm{S}$ & $\mathrm{S}-\mathrm{L}$ \\
Pachydiplax longipennis & $\mathrm{S}-\mathrm{L}$ & $\mathrm{S}-\mathrm{L}$ & $\mathrm{S}-\mathrm{L}$ & $\mathrm{M}$ & $\mathrm{S}-\mathrm{L}$ \\
Tramea carolina & $\mathrm{S}$ & $\mathrm{S}$ & $\mathrm{S}$ & $\mathrm{S}$ & $\mathrm{M}$ \\
Plathemis lydia & $\mathrm{S}$ & $\mathrm{M}-\mathrm{L}$ & $\mathrm{M}$ & $\mathrm{M}$ & $\mathrm{L}$ \\
Celithemis eponina & $\mathrm{S}$ & $\mathrm{S}$ & $\mathrm{S}$ & $\mathrm{S}-\mathrm{M}$ & $\mathrm{S}$ \\
Epiaeschna heros & $*$ & $\mathrm{~S}$ & $*$ & $*$ & $*$ \\
Libellula cyanea & $*$ & $\mathrm{~S}$ & $*$ & $\mathrm{M}-\mathrm{S}$ & $*$ \\
Erythrodiplax berenice & $\mathrm{S}$ & $*$ & $\mathrm{~S}$ & $\mathrm{~S}$ & $*$ \\
Celithemis martha & $*$ & $\mathrm{~S}$ & $*$ & $*$ & $*$ \\
Anax longipes & $*$ & $*$ & $*$ & $\mathrm{~S}$ & $\mathrm{~S}$ \\
Celithemis elisa & $*$ & $*$ & $*$ & $*$ & $\mathrm{~S}$ \\
Libellula incesta & $*$ & $\mathrm{~S}$ & $\mathrm{~L}-\mathrm{M}$ & $\mathrm{M}$ & $\mathrm{S}-\mathrm{M}$ \\
Libellula luctuosa & $*$ & $*$ & $\mathrm{~S}$ & $*$ & $\mathrm{M}-\mathrm{L}$ \\
Libellula semifasciata & $*$ & $\mathrm{~S}$ & $\mathrm{~S}$ & $*$ & $\mathrm{M}$ \\
Perithemis tenera & $*$ & $\mathrm{~S}$ & $\mathrm{~S}$ & $*$ & $\mathrm{~S}$ \\
\hline Damselflies & $\mathrm{A} 1 \mathrm{~F}$ & $\mathrm{~A} 1 \mathrm{~B}$ & $\mathrm{~A} 2$ & $\mathrm{MM}$ & $\mathrm{Weaver}$ \\
\hline Enallagma aspersum & $\mathrm{S}$ & $\mathrm{S}$ & $\mathrm{M}-\mathrm{S}$ & $\mathrm{L}$ & $\mathrm{L}-\mathrm{M}$ \\
Enallagma civile & $\mathrm{S}$ & $\mathrm{M}$ & $\mathrm{S}-\mathrm{M}$ & $\mathrm{S}$ & $\mathrm{L}$ \\
\hline Ischnura hastata & $\mathrm{S}$ & $\mathrm{L}$ & $\mathrm{S}-\mathrm{M}$ & $\mathrm{S}$ & $\mathrm{L}-\mathrm{S}$ \\
Ischnura verticalis & $\mathrm{S}$ & $\mathrm{L}$ & $\mathrm{M}-\mathrm{S}$ & $\mathrm{S}$ & $\mathrm{M}-\mathrm{S}$ \\
Calopteryx maculata & $\mathrm{S}$ & $*$ & $*$ & $*$ & $*$ \\
Lestes disjunctus australis & $*$ & $\mathrm{~S}$ & $*$ & $*$ & $*$ \\
Lestes congener & $*$ & $*$ & $*$ & $\mathrm{~S}$ & $*$ \\
Lestes forcipatus & $*$ & $*$ & $*$ & $*$ & $\mathrm{M}$ \\
Lestes rectangularis & $*$ & $*$ & $*$ & $*$ & $\mathrm{~S}$ \\
Enallagma durum & $*$ & $*$ & $*$ & $*$ & $\mathrm{~S}$ \\
Ischnura posita & $*$ & $*$ & $\mathrm{~S}$ & $*$ & $\mathrm{~S}$ \\
\hline Species Richness & & 14 & 19 & 18 & 17 \\
\hline
\end{tabular}

FIG. 5. Odonate population size estimates based on visual surveys - Sarah Langan 


\title{
Multigap resistive plate chamber for fast timing
}

\author{
Nathaniel Lashley-Colthirst \\ Physics, Howard University, Washington, D.C, 20059 \\ Mickey Chiu \\ Physics, Brookhaven National Laboratory, Upton NY 11973
}

This summer I worked at Brookhaven National lab (BNL) with a group from Howard University under the guidance of Mickey Chiu. The goal of our research was to find the timing resolution of two detectors and the electronics involved. The detectors we tested were the multigap resistive plate chamber (mRPC) and the mutli-channel plate photomultiplier tube (MCP-PMT). The main electronic component we tested was an analog-to-digital converter (ADC) CAEN board. Along with finding the timing resolutions, we also wanted to modify the current design of the $\mathrm{mRPC}$ to attain a timing resolution of ten picoseconds down from a hundred, which is the current standard at the Pioneering High Energy Nuclear Interaction Experiment (PHENIX). With this timing resolution, particle identification will be possible at higher momenta at accelerators like the Relativistic Heavy Ion Collider (RHIC). To improve the mRPC we tested production modules from the PHENIX time of flight west (TOF.W) to find the current timing resolution using cosmic rays and to produce prototypes that will result in a better timing resolution.

The mRPC is currently constructed from copper sheets, layers of glass and fishing line that runs across the glass, and each layer is separated by a gap of two millimeters. The upgrades in consideration have an increase in the number of gaps and a decrease the gap size. In our tests, the mRPC was connected to a high voltage supply and sat in a gas filled box. The mRPC detects muons by the electrons that are freed when the muons pass though the detector and ionizes the gas within the gaps. The freed electrons then ionize more of the gas as they accelerate towards the anode, causing an avalanche of electrons which are picked up by the fishing line and induce a signal on the copper sheet. In our last setup we connected the output to a preamp, which was then connected to the analog-to-digital converter and then to the computer. To keep the data consistent we set up a trigger for the mRPC. Our trigger was setup with scintillator panels to keep the data form vertically passing muons.

The scintillators have panels that when a muon passes through it an electron is released. At the end of the panels are photomultiplier tubes which use the photoelectric effect over multiple dynodes. This process is able to take one electron and produce millions more for the signal. We used scintillators both above and below the mRPC to set up a coincidence. This will allow us to trigger on the mRPC when both the scintillators send a signal within a few nanoseconds of each other. So the data we got was of a muon that traveled close to vertically through the detectors. This reduced the possible paths a muon could travel making the path more consistent for our calculations.

Another detector we worked with was the MCP-PMT. This detector is less likely to be used on a large scale as it cost thousands of dollars per square meter for the fused silica. Fused silica is high grade quartz which is used to exploit the _effect to create photons which enter a photomultiplier tube and are read out. We also used scintillators panels to ensure that the data from MCP-PMT was from mouns passing vertically through the detector. Each of the detectors' setup were similar, expect that we used two MCP-PMTs allowing us to find data for the timing resolution relative to each other.

The last device we found the timing resolution of was the CAEN board. We found out that not only was the timing resolution lower even we wanted, the signal to noise ratio was higher than expect. To lower the noise we replace the power supply with a digital supply suggested by the manual. But the noise did not lower by much after the supply was switched. To test the timing resolution we used a signal generator and split the signal into two and sent both to the board. We used the difference in arrival times to find the timing resolution. In the end, we found the timing resolution to be around twenty picoseconds, which is above the ten picosecond resolution we were aiming for. This means to achieve our goal, we need to find other boards other than the CAEN if we want our setup to be around ten picoseconds.

The CAEN board was not the problem that occurred during the experiment. Another problem that occurred was that our gas box was not completely air tight. We think this was due to the metal warping when the screw holes were drilled in. To fix this we clamped the box as we did not have time to make a new box. The port in the box for the output did not match the cable being used inside, meaning there was a lose wire that was picking up signals and contributed to the noise. We fixed by simply changing the cable to one that matched the port. Another easy fix was when one of the scintillators became much noisier. To fix this we found the areas where the light was leaking in and patched them with black tape. We later found a problem with the signals we were getting from the mRPC. From the graphs, we could see a wave forming on the end of our signals. We thought this was due to an impedance miss-match from the fifty ohm impedance cables from the detector to the inside of the box and the hundred ohm impedance cables from the outside of the box to our other electronics. We later replaced the hundred ohm cables with fifty ohm cables, but we ran out of time before we could re-run the test to see if it was the cause. The main problem we had was that we only had access to one mRPC until the end of the summer when we got a second one, so we did not have the time to find the time resolution of the detectors relative to each other.

Even though we weren't able to find the timing resolution of the mRPC or the MCP-PMT, were able to get data from each detector and find the timing resolution of the CAEN board. We also were able to setup and troubleshoot the problems that came up. Considering the fact we started from nothing and had a relatively short time period to work, we were able to get lot done. The project doesn't stop with us and maybe the goal of ten picoseconds will be achieved and we will be able the accurately identify particles at higher levels. This summer was a welcomed learning 
experience. During my time here I have learned the programing code Python and Eagle, a circuit board creation program. I also have deepened my understanding of the related nuclear and particle physics.

\section{Acknowledgements}

I would like to thank the following people for their guidance.

- Mickey Chiu

- Marcus Alfred

\section{References}

Barlow, R.J. Statistics: A guide to the Use of Statistical Method in the Physical Sciences. Wiley, 1993.

Langtangen, Hans. A Primer on Scientific Programming with Python. Second Edition. Springer, 2009.

Veltman, Martinus. Facts and Mysteries in Elementary Particle Physics. First Edition. RIver Edge: World Scientific, 2003.

Tsoulfanidis, Nicholas. Measurement and Detection of Radiation. Hemisphere, 1983. 


\title{
Experimental gas configuration management
}

\author{
Raynold Lewis
}

The Pioneering High Energy Nuclear Interaction Experiment (PHENIX) is an ongoing research experiment designed to understand the smallest building blocks of the sub nuclear particles of the universe -- quarks and gluons. The internship at PHENIX provided the opportunity to understand a particular detector subsystem used by scientist to detect specific subatomic particles through certain gas mixtures

The internship at PHENIX experiment provided the opportunity to become more acquainted with the gas systems that run the detection process through assignments given by scientists and engineers.

The tasks assigned included updating 3D models of the gas pad area, mixing house, gas shed, and a new ethane tube trailer, updating 2D schematics for gas pad, mixing house, and gas shed with modifications provided by gas system expert and chief mechanical engineer, incorporating new schematics for the new ethane tube trailer into the gas pad schematics file, generating parts models and detailed drawings that were fabricated by a machinist for service in PHENIX MPC-Ex upgrade, and updating the excel documentation of the gas pad and mixing house gases as well as generating a new document for R134a.

The 2D schematics and 3D models were developed using the engineering software programs Autodesk Inventor and AutoCAD. The 2D schematic updates are an integral component of the PHENIX configuration control system. The 3D models are useful illustrations that will play an important role in planning and conceptualizing future upgrades. Becoming more skilled in detailed drawing of parts and models while using AutoCAD and Autodesk Inventor is inevitable, through the tasks assigned and techniques demonstrated by the PHENIX design engineer. In addition, the internship provides professional growth in developing skills in communication, writing, and comprehension. Lastly, participation in planning and status meetings provide interns a better understanding on how engineers communicate and collaborate efficiently, effectively, and accurately.

The PHENIX gas system consists of three connected gas subsystems essential to the experiments detection process. The subsystems include the gas pad area and the distribution gas for detector subsystems. The gas pad and gas shed stores important liquid and gases and dispatches them steadily through pipelines to control racks within the mixing house for effective gas distribution into the experimental area. Gas system documentation is important for reflecting added, removed, and switched components. The updating of this documentation require skills in $2 \mathrm{D}$ and $3 \mathrm{D}$ engineering design along with utilizing Autodesk and AutoCAD engineering software.

The student internship at PHENIX taught basic engineering techniques that will help improve any engineer's work quality. The designing of 3D models and parts include four processes/and or techniques that can be used by any designer engineer to obtain quality designs of component. The process began with creating a 2D and 3D sketch with dimensions incorporated on A2 blank paper. The importance of this process is to provide a rubric and criterion for the model or part being rendered. Second, sketch the front face of the 3D sketch on Autodesk Inventor to begin virtual design with specified dimensions. Third, use the extrude feature to develop the sketch from 2D to 3D. Lastly, use features in the engineering software to sculpt the part matching the 3D sketch.

The processes taught helped develop new parts for the PHENIX detector system including a light collector component. The light collector holds fiber optic cables, a light diffuser, and a transmitter. The particles are detected in the detector system through the computerized waveforms showing that certain particles are present through its energy. The detector system that calculates the energy of the particles consists of lead barriers that will absorb a particle's wavelength. When the wavelength is absorbed completely the strength of the particle can be easily calculated. The purpose of the light collector is to calibrate the detector system's waveforms that will calculate the energy of the subatomic particles. The detector system over time produces uncertainties through temperature, aging equipment, etc. The light collector's job is to keep the data collected accurate and consistent to the calibration (zero-out) waveform produced by the component's fiber optic cables.

The $3 \mathrm{~d}$ model of the gas pad assembly consists of many different parts and assemblies. The assembly's essential parts and components include gas cylinders, gas cylinder elements, dewars, and vaporizers. The same techniques used to create the light collector are the same used for these parts.

The essential features used in Autodesk Inventor in developing these parts were revolved, extrusion, fillet, and work planes. The feature revolve seems self-evident where a closed surface and an axis of rotation is used to turn the $2 \mathrm{D}$ image into a $3 \mathrm{D}$ solid structure through rotation around that specific axis (Figure 1). The fillet feature takes any square corner of a 3D model and makes a smooth round edge (Figure 2). Work planes are separate working areas for sketching new components to be added to an existing solid structure (Figure 3).

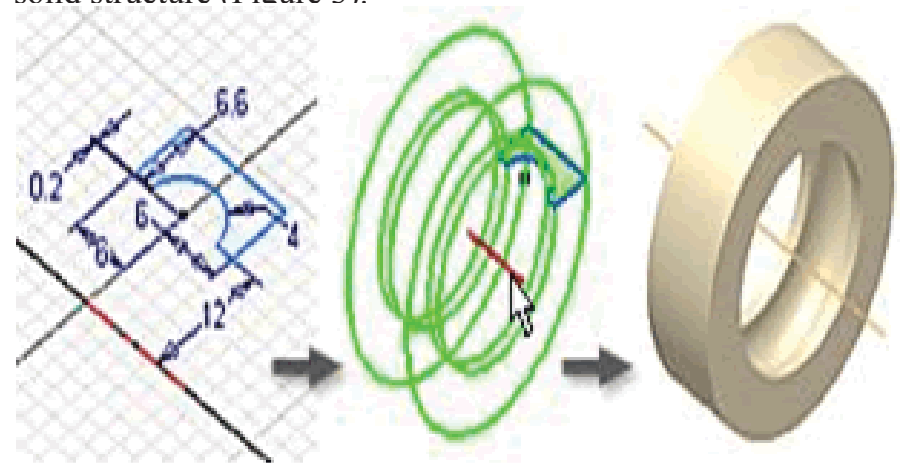

Figure 1: Revolve feature in Autodesk Inventor 
The gas subsystems of the PHENIX experiment include the gas mixing house, gas pad, and gas sheds that are connected the mixing house. The gas supply and distribution configuration management documentation are essential elements to the process of the experiment. The PHENIX experiment goes through many renovations and upgrades, which causes these essential documents to be updated. The updates for this documentation include a 3D model of the gas pad area, the 2D schematics of the gas pad, mixing house, and gas shed, and the gas subsystem's EXCEL documents. The purposes for these updates are to demonstrate how the gas subsystems are arranged presently, increase awareness of newly upgraded and/or missing components including valves, vaporizers, gas cylinders, pressure regulators etc., and identifying efficient space for future components to be added to the gas subsystems.

The 3D models of the gas pad and the mixing house with its connected sheds have been completed (Figure 4). The parts/components that were created for the assembly were gas cylinders, gas dewars, vaporizers, gas carts, a tube ethane trailer, the mixing house vinyl siding, roofs, control racks, pipelines, and gas ethane tube trailer. The many parts/components were created through using the same techniques specified above.

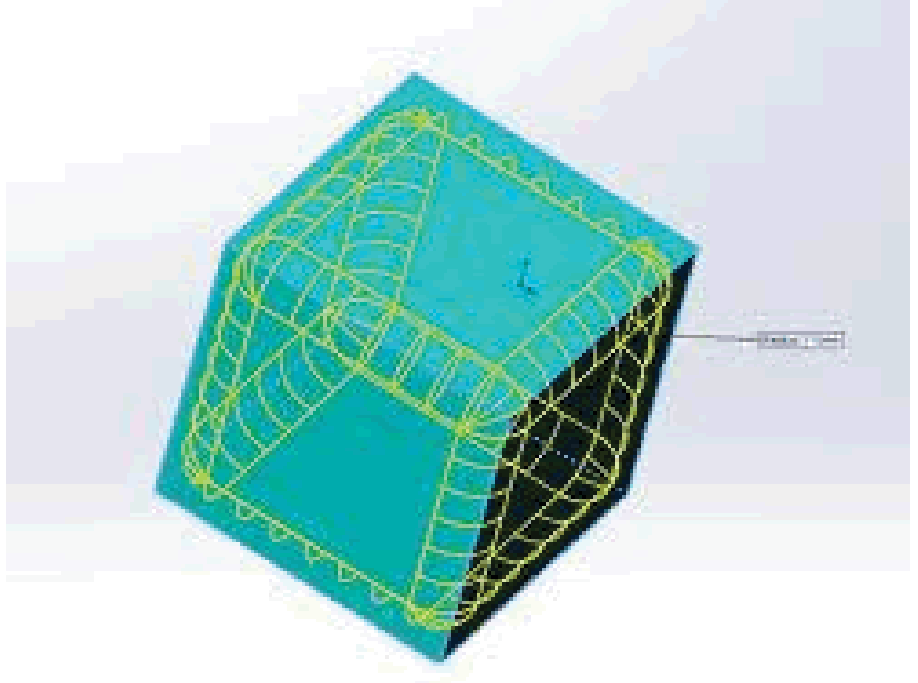

Figure 2: Simple illustration of fillet feature in Autodesk Inventor

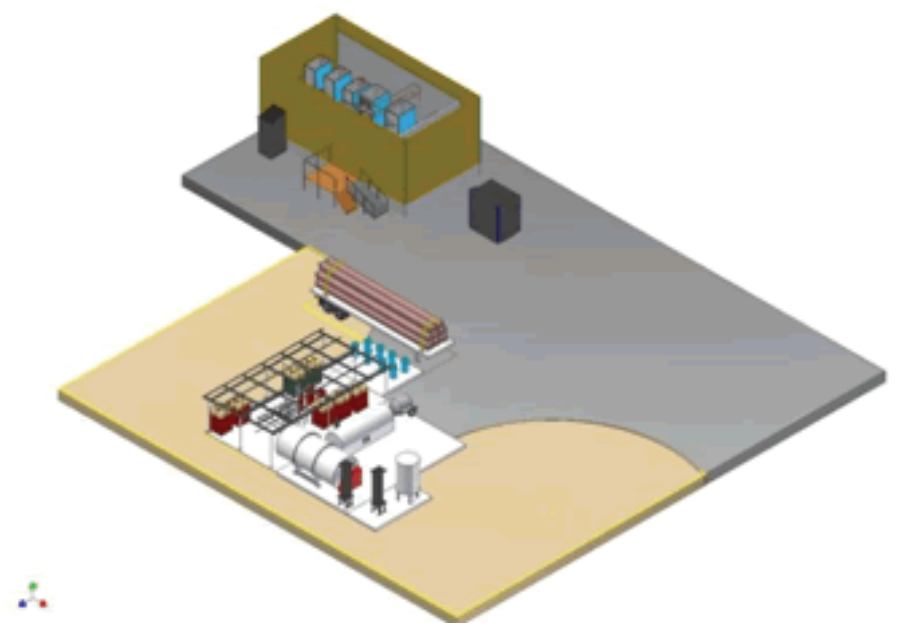

Figure 4: 3D model of the gas pad area including multiple components
The pipeline structures of the mixing house interior (Figure 5) should represent the $2 \mathrm{D}$ schematics accurately so that upgrades to the subsystems would be less difficult and more visually feasibly understandable to any scientist, technician, and engineer. The difficulty within creating the pipeline structures included having the right lengths measured and keeping the mixing house interior area scaled to the mixing house exterior design. Through carefully scaling the assemblies, placing these components into one another by simply dragging them was easily done.

The 2D pipeline schematics of the gas pad and mixing house with connecting sheds were completed field inspection with accompanied technical staff assistance. Updates to these schematics consisted of added and removed components. The components added to the gas pad schematics included an auxiliary gas dewar with vaporizer, ethane tube trailer, liquid argon dewar with vaporizer, and a new SF6 gas cylinder. In addition, the removed components included the CF4 standard line, a CF4 high purity tank and an $\mathrm{Ar} \% 5 \mathrm{H}$ gas cylinder. Updated schematics for the mixing house have new and removed components that have been modified within the document.

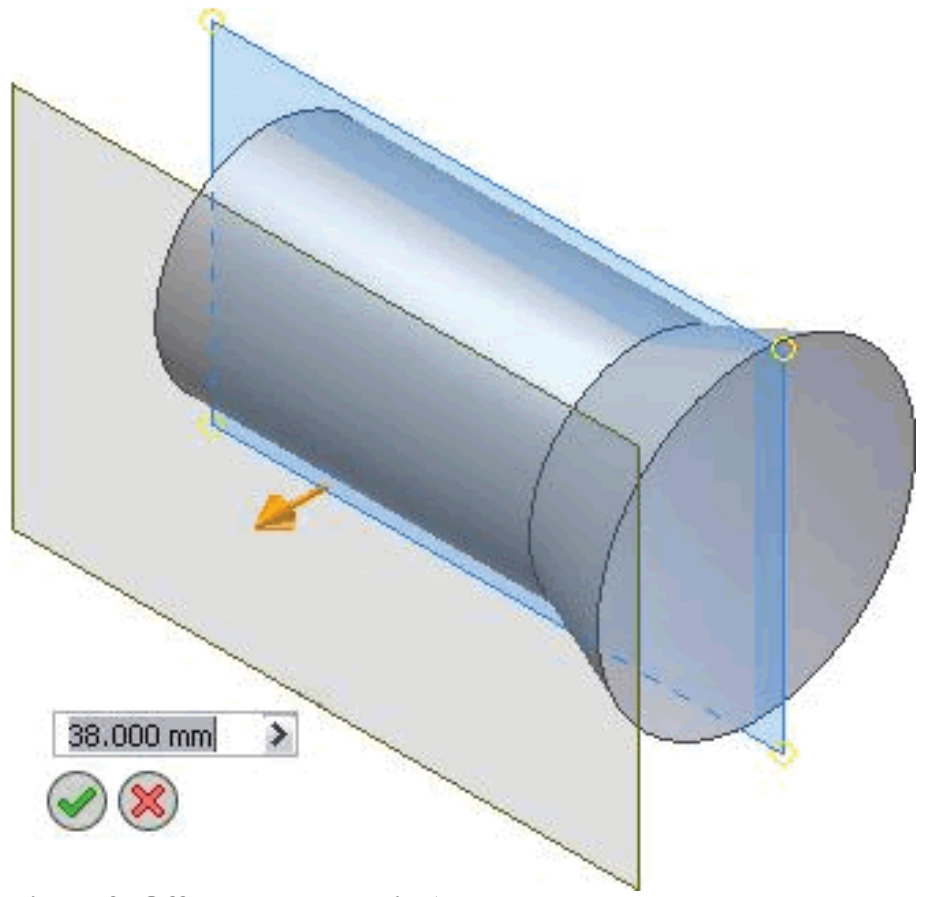

Figure 3: Offset Work plane in Autodesk Inventor

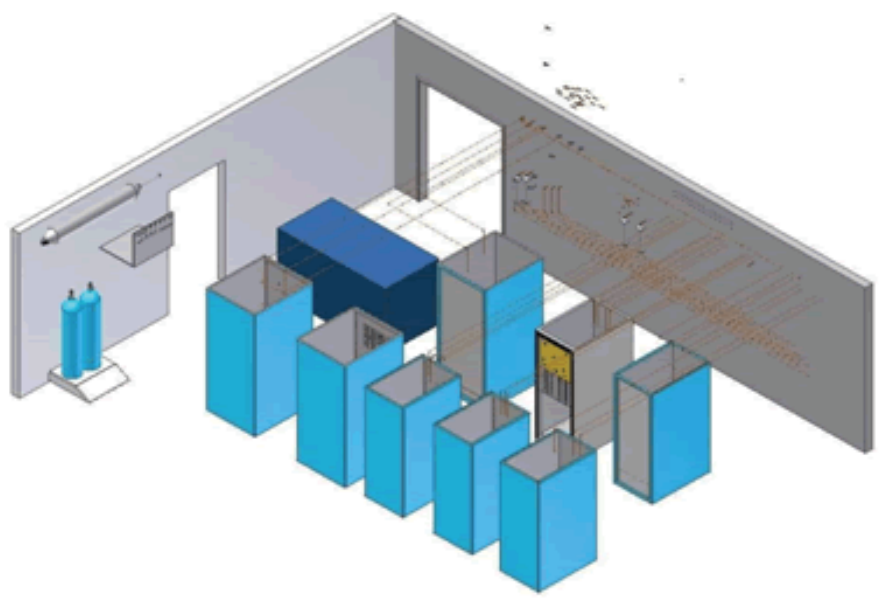

Figure 5: The 3D model of the interior of the gas mixing house 
The modifications include a new gas analyzer, pipelines running from the analyzer to the TEC and RPC control rack, TEC has been removed, and supply and return pipelines were switched between RPC and RICH control racks. A schematic needs to be signed off by the gas expert before becoming classified as an upto-date document. Successfully, all gas configuration documentation is up-to-date. The schematics will gain a drawing no. that will be used as references to other schematics for the gas subsystems (Figure 6a \& 6b).

The engineering programs used including Autodesk inventor, were easy to adapt to since it was similar many 3D Cad programs used. The only difference is the name set for certain features, which actually do the same exact action for a particular design. In addition, it is best to have a mentor who is able to provide tips and tricks that will improve the quality of designing. The SULI program provided me with three mentors that provided tips and tricks to improve my overall engineering skills. The three mentors were Don Lynch (Chief Engineer), Robert Pisani (Gas Expert), and Richard Ruggiero (Design Engineer).

Fortunately, I gained experience in the three aspects of engineering that are perspectives of mine. The three perspective careers in engineering include design, HVAC, and management. The opportunity to work with the SULI program, PHENIX team, and particular students was an exceptional experience. The orga-

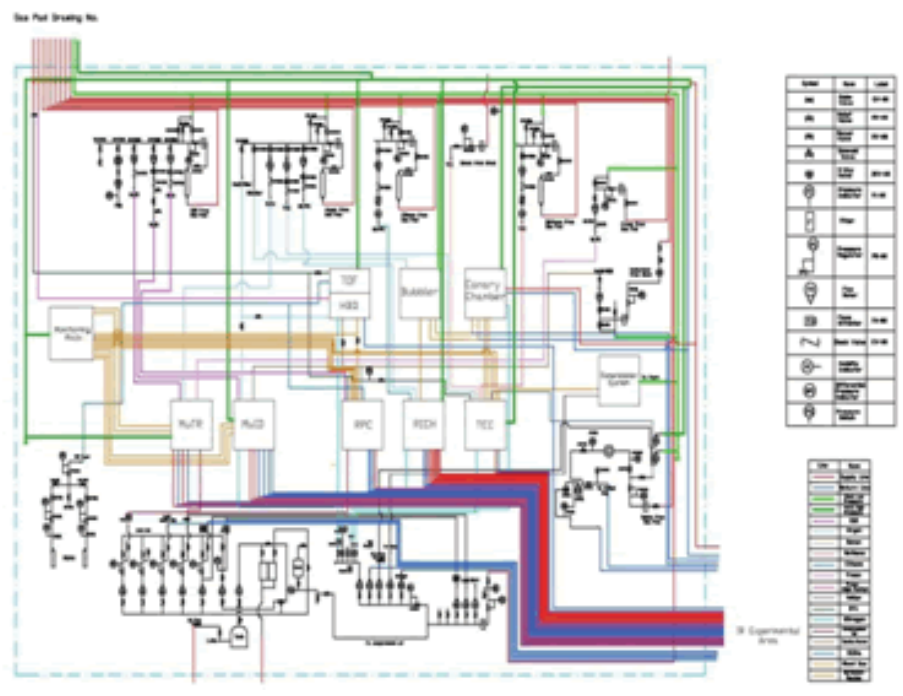

Figure 6a: The updated 2D schematic of the mixing house

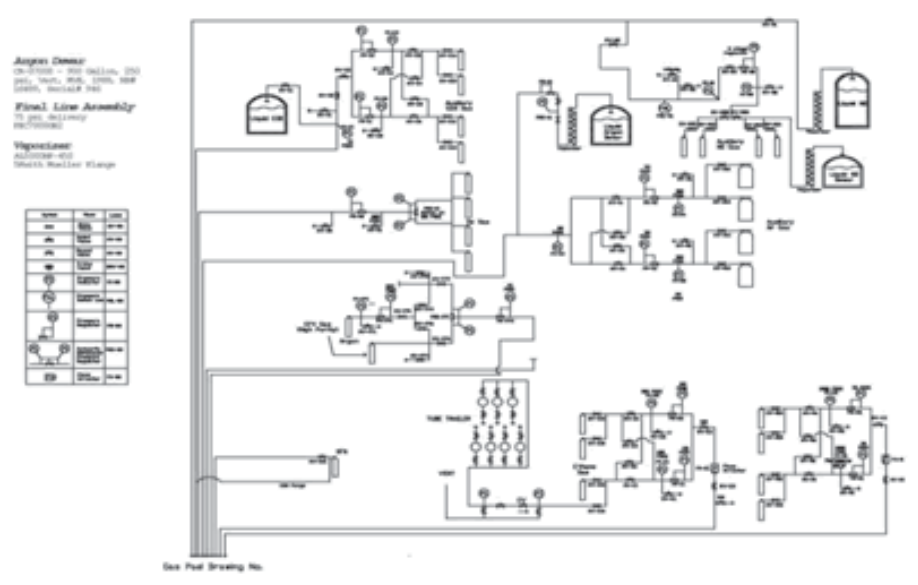

Figure 6b: 2D Schematics of the gas pad area recently updated nized activities and workshops from the SULI administration has provided techniques and tools that will help improve a student's overall performance in any endeavor in perspective.

\section{Bibliography}

https://d2t1xqejof9utc.cloudfront.net/pictures/files/12721/

large.png?1339159202

http://wikihelp.autodesk.com/Inventor/enu/2011/Help/

Tutorials/123AutodeskInventorTut/3593DirectManipula-

tion/3598CreateanOffsetParal

http://files.wikihelp.autodesk.com/Autodesk_Inventor/2012/ ENU/GUID-F44F14B1-F58B-4935-910D-78576CE20422-low. jpg 


\title{
Analysis and comparison of atmospheric aerosol concentrations measured using two instruments over the eastern North Pacific Ocean
}

\author{
Danielle Mallon \\ Department of Atmospheric and Environmental Sciences, University at Albany, SUNY, Albany, New York 12222 \\ Ernie Lewis \\ Environmental Science Department, Brookhaven National Lab, Upton, New York 11973
}

Aerosol particles play an important role in Earth's climate through the various processes in which they are involved. They affect Earth's energy balance by scattering and absorbing incoming solar radiation (i.e., light), the so-called "direct effect," and by acting as nuclei upon which cloud drops form, the "indirect effect." As the size and chemical composition of an aerosol particle determines the extent to which it scatters or absorbs light and to which it can form a cloud drop, knowledge of the size distribution and chemical composition of aerosol particles is important to understanding and quantifying aerosol affects on climate. My goal this summer was to compare the aerosol number concentrations measured over the Eastern North Pacific Ocean during a DOE-sponsored field campaign using two different instruments which operate over two different size ranges using different techniques. The Condensation Particle Counter (CPC) detects all particles with equivalent diameter greater than $10 \mathrm{~nm}$ using a mobility technique, which utilizes the ability of the particle to diffuse through air. The Ultra-high Sensitivity Aerosol Spectrometer (UHSAS) detects all particles with equivalent diameter greater than $55 \mathrm{~nm}$ using an optical technique, which is based on the ability of the particle to scatter light from a laser. Comparison of the concentrations determined by these two instruments provides information on the concentration of particles with diameters between 10 and $55 \mathrm{~nm}$. Knowledge of this quantity is important in understanding aerosol processes and inferring aerosol sources over the oceans, a topic of great current interest.

There are many feedbacks in the climate system and there are quite a few involving clouds and aerosols specifically. The main one is the aerosol direct effect, which basically says when there are clouds, incoming visible solar radiation hits the clouds and is scattered and reflected. This is also known as albedo. In general radiation being scattered and reflected causes a net cooling. Another is the aerosol indirect effect; which basically says, due to pollution being emitted, there are more particles in the air. This makes it so water vapor has more particles or cloud condensation nuclei (CCN) available for it to condense onto. This leads to an increased albedo because more light is being scattered from increased particles in the cloud. This also leads to a net cooling. There are more $\mathrm{CCN}$ and fewer cloud droplets that are large sized and more that are small sized, but the volume of water is the same. This causes the lifetime of the cloud to increase and reduced rates of drizzle and rain. A third effect is the black carbon effect; this involves the same mechanisms explained in the direct and indirect effects, except opposite. The particles absorb incoming solar radiation. This causes a positive radiative forcing and the atmosphere to warm, instead of a negative one due to reflection. Very small, carbon based particles, ranging in size from .1 to 1.0 micrometers are emitted as a result of incomplete combustion of fossil fuels, biofuels, and biomass. They are very effective at absorbing solar radiation, their atmospheric lifetime ranges from days to weeks and can have longer term effects if deposited on ice and snow, where it affects the earth's albedo. Black carbon is a short lived climate forcer and causes regional climate impacts. This is because its emissions are not the same everywhere and its lifetime is shorter, because it doesn't have time to mix throughout the atmosphere. The effects happen on a very small scale, much smaller than the grid point resolutions of climate models. Because of this, these effects have to be parameterized in climate models, the mathematical formulas to simplify and represent these mechanisms are input in the models. Because these mechanisms are not fully understood and happen on such a small scale, so they are not properly represented in climate models and their effect is not properly quantified. This leads to a source of error and inaccuracy in the model outputs.

There are many regions in climate models that are not accurately represented because in- situ data in certain areas are sparse. For example, places where there is very low population density like Polar Regions and over the oceans because it's very hard to have a stationary in-situ site over water. This is because the water isn't stationary and there are machine maintenance requirements. The direct and indirect effects and aerosols over the ocean, specifically the Pacific Ocean mostly come from sea salt and sea spray, these can act as CCN and contribute to cloud formation, later affecting a portion of the radiation budget. Chemical composition and size of these particles in clouds will affect the clouds albedo and absorption. Studying and trying to understand aerosols as

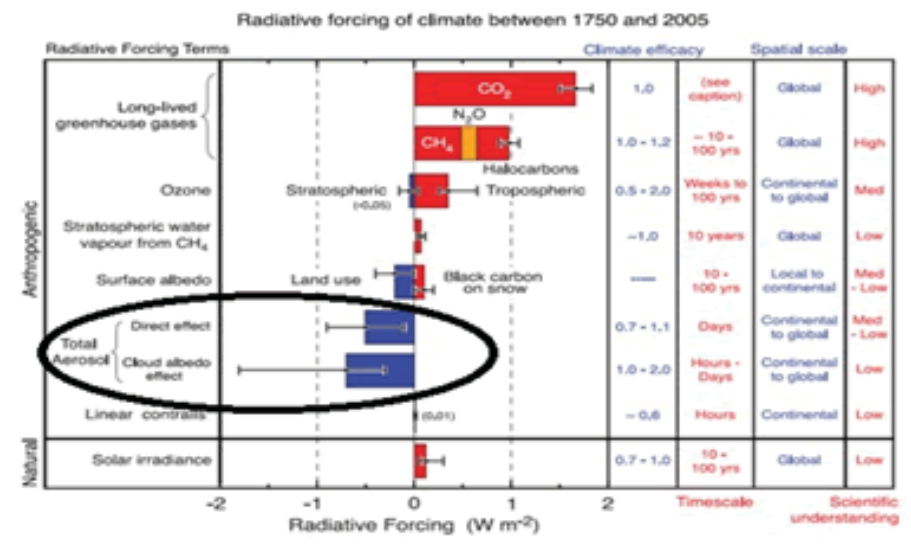

Figure and caption are from the IPCC 2007 synthesis report. The error bars are the largest for both of the aerosol effects compared to the other effects listed. 
well as other aspects of clouds, especially over this region, would be of great benefit to improving the accuracy of climate models. There are scientists and programs working to do just this.

The ARM (Atmospheric Radiation Measurement) Climate Research Facility is a DOE scientific user facility that provides the climate research community with strategically located in situ and remote sensing observatories designed to improve the understanding and representation, in climate and earth system models, of clouds and aerosols, as well as their interactions and coupling with the Earth's surface. Their goal is to provide a detailed and accurate description of the earth atmosphere in diverse climate regimes to resolve the uncertainties in climate and earth system models toward the development of sustainable solutions for the Nation's energy and environmental challenges (1). The second ARM mobile facility has been placed on a ship called Spirit, run by Horizon Lines, for a year from October 2012 through September 2013 to collect in-situ data for the first time over an ocean. The ship travels in the Pacific Ocean from Los Angeles to Hawaii. The goal of the MAGIC (Marine ARM GPCI Investigation of Clouds) initiative is to study low marine boundary layer clouds and the transition that happens between cumulus to strato-cumulus. The dynamics and aspects involved in this transition play an important role in climate and are poorly understood and therefore poorly represented in climate models because the lack of observed data. The primary objective of MAGIC is to improve this representation of the cloud transition in climate models. People working on MAGIC are using the data collected to document and understand the small- scale physical processes associated with aerosols, turbulence, convection, and radiation in a variety of marine cloud types. Because the Los Angeles/Honolulu route lies closely along the cross-section used for the GPCI (GCSS Pacific Cross-section Intercomparison), the data collected will provide constraint, validation, and support for the GPCI modeling activities and other associated international modeling efforts. (2)

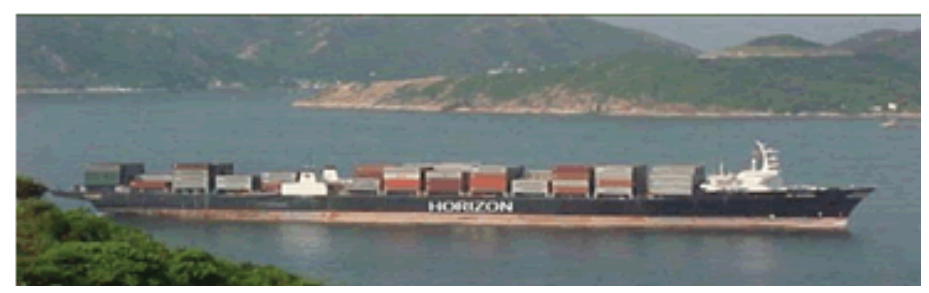

Horizon Spirit

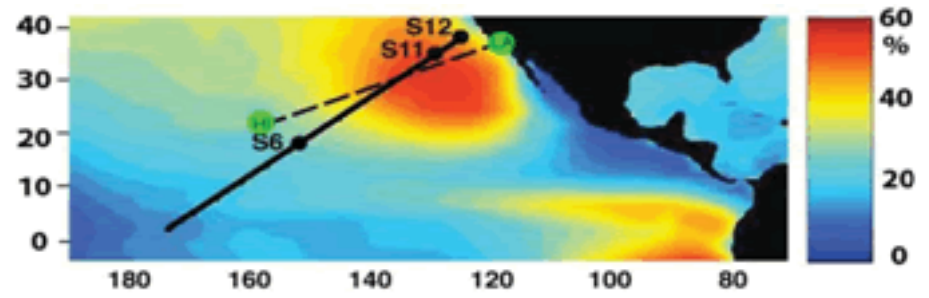

June-July-August average low level cloud cover from ISCCP, with MAGIC transect (dashed line) and GPCI transect (solid line), along which Points S6, S11, and S12 are used in the GCILS model intercomparison, adapted from Teixeira et al., J . Climate, v. 24, 2011

Two instruments aboard MAGIC are the Ultra-High Sensitivity Aerosol Spectrometer (UHSAS) and the Condensation Particle Counter (CPC). The UHSAS uses a relatively high- powered near infrared laser to illuminate the aerosol sample volume and collects and measures the scattered radiation over a large solid angle. It typically can resolve the size distribution between 55 nanometers and 3000 nanometers in 100 size-bins (3). The CPC measures sub- micron aerosol particle number concentration by growing the particles to a size that is easily detectable and countable by optical scattering. The condensating liquid is typically an alcohol (e.g., butanol) or water. It can cover a size range from 10 $\mathrm{nm}$ to $3000 \mathrm{~nm}$ (fine)(4).

This Summer I worked with data from the UHSAS and CPC instruments that were part of the suite of instruments aboard MAGIC. Each of these two instruments measures aerosol concentration (the number of aerosol particles in a given volume), but they have different size ranges. The difference between the two values gives the number concentration of aerosol particles in the intermediate size range, which is of great interest for aerosols over the ocean, as one outstanding question is whether the ocean produces many particles in this size range from breaking waves. I plotted the data from each instrument and the difference between them on a graph. I took the difference of the UHSAS concentration from the CPC concentration. Doing this subtracted out all of the particles that were above $55 \mathrm{~nm}$, leaving the particles in the intermediate size range between 10 -and $55 \mathrm{~nm}$. This was done from October to December 2012 on legs 3 to 7, because the UHSAS was only on the ship during this time period.

Particles in the small and intermediate size range are generally from anthropogenic sources. Graphing these variables was important because it was unclear if there were any particles in this size region over the ocean, which is far removed from anthropogenic sources. The UHSAS measures particles and separates their sizes into a range of size bins, but does not give a total number. The CPC only gives a total number and no information on the different sizes of particles it measures. There is currently no instrument that measures the total number of aerosols in the intermediate size range. My graphing the total number of both of these instruments and the difference was important because it was a way to quantitatively determine this.

A large majority of my summer was spent learning a computer language called Python. I did not have any prior programming knowledge. I quickly became aware that programming is a very useful and necessary skill in the research field for importing, displaying and manipulating data. This summer was very challenging for me because of this, but I learned so much and have gained a useful and transferrable skill that I can use in the future.

Although my end results may look straight forward, there were many steps that needed to be taken in Python to get there. For the UHSAS data, a code was written in MATLAB to process the data into a format Python could read in, this code also took the values the machine reported for each different size bin and added them to get a total concentration. For the CPC data, I created a script to take the data it recorded every second and average it so the points were every 10 seconds. This was done so the UHSAS and CPC points matched up. They didn't match up exactly, but the UHSAS was always within a few seconds of the 10 second interval. Within this script the data points were converted from hour, minute, second format into seconds after midnight format, so it graphed smoothly. Another script was created to do this with the UHSAS data because it was also in the same format. Next, a script was created to find where there were missing data points in both of the data types and insert a place holder. This was done so the arrays matched up exactly, if they didn't the program would 
crash and the shell would display an error message. Next, a script was created to plot each days worth of data from UHSAS, CPC and their difference on a graph. A portion of code in this script was created to output a file of all of these variables, including time into a text file in case it was needed in the future. After these programs were ran and new graphs and text files for each of the days from all of the legs were created, another script was created to take the minute average from each of the instruments and plot them and their difference on a graph and output another file with those values. This was run for all of the days in each of the legs. Lastly a script was created to take all of the 10 second data from each day in leg 7B and put it together and plot it on a graph. This was done to see if there was a general trend in a single trip. A better script that had a variable created to do this for all of the legs would have been created, but time ran out.

The graphs for the two dates were chosen because they clearly demonstrate some of the results found. The graphs on the left show the 10 second data averaged over minutes. This was done to reduce noise. The graphs on the right show the raw UHSAS data taken every 10 seconds and the CPC second data averaged over 10 seconds. Almost every day the machines were on, when graphed they show at least one period of time each day when UHSAS concentrations were greater than $\mathrm{CPC}$ concentrations. This ideally should not happen because the CPC covers the UHSAS' size range and below, so it includes more particles and should always be above. Both days show periods when the difference increased; for this to happen it means the particle number con- centration increased. Instead of happening proportionately, where each of the instruments measured the same rise in magnitude, it happened disproportionately and the CPC always measured an increase at least double of what the UHSAS measured, sometimes more.

Multiple conclusions could be made after studying and graphing the data from these machines. One of them is that a substantial amount of the total number concentration of particles had a diameter less than $55 \mathrm{~nm}$. Another is despite daily and weekly variability, over the ocean the particle concentrations were usually very low at around $100-200 \mathrm{~nm}$ total, indicating very clean air. As expected the UHSAS concentration (larger particles) were usually always lower than the CPC concentration (smaller particles). The most interesting finding was that when the particle concentrations were higher, the differences between the instrument measurements were larger. This means that the increase in small particles was disproportionately higher. Normally if there was an increase in background aerosols the increase in all of the plotted variables would be proportionate.

There are multiple things that could and still need to be done involving data from these instruments. First is to continue to write scripts in Python to manipulate and display the data in various ways in order to extract even more information. Second would be to cross reference and compare other instrument data to provide closure. Another would be to investigate discrepancies with expected relationship between CPC and UHSAS concentrations, and actual relationship. Another is to investigate the effect of ship
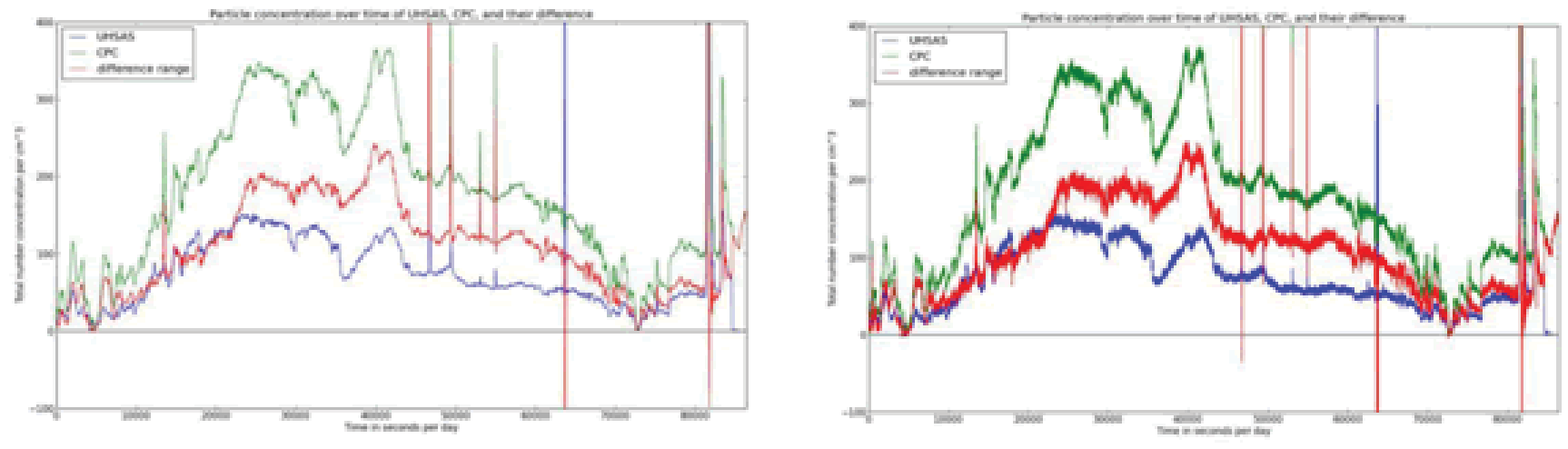

Leg $4 \mathrm{~b}$ of MAGIC, Hawaii to LA on 10/21/2012 This date illustrates two events where it likely rained due to a drop in concentration
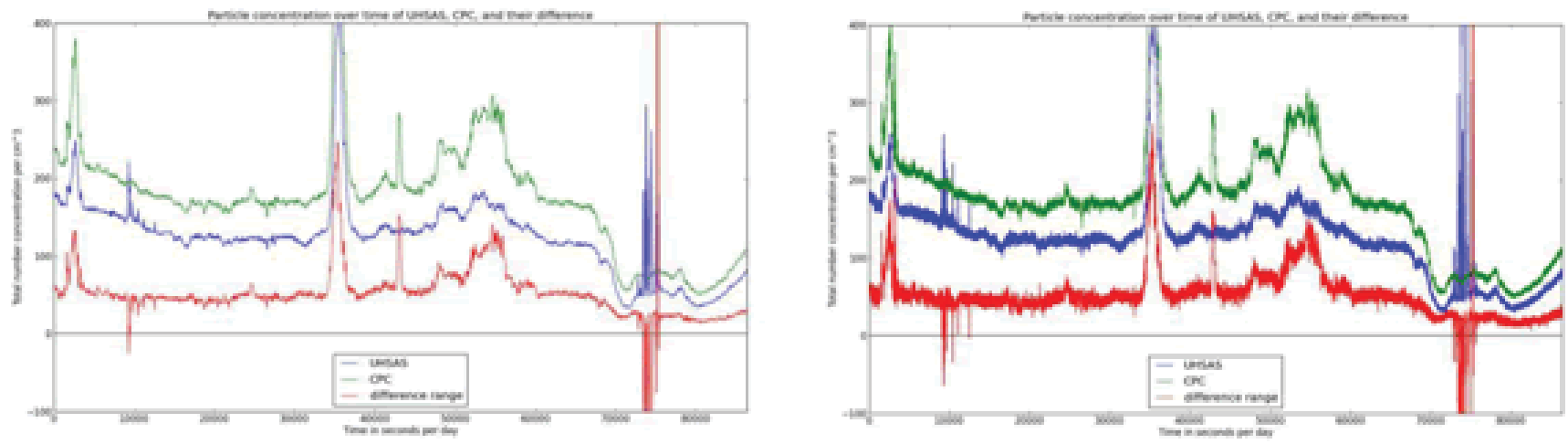

Leg 7a of MAGIC, LA to Hawaii on 12/03/2012

This date illustrates two large peaks in concentration which is likely due to an exhaust plume from the ship when the wind was blowing in the direction of the air flow inlet 
exhaust on the results, to see if ships played a role in the intermediate size of particles observed. One is to plot corresponding wind barbs on the $\mathrm{x}$-axis to see how the concentration, wind speed and direction correlate. The last thing that can be done in the future is to investigate the disproportionately large differences between small particles and larger ones when total number concentration increased and why when particle concentration didn't rise proportionately.

\section{References}

1 "Mission and Vision Statements." ARM.gov. US

Department Of Energy, n.d. Web. 30 July 2013.

2 “ARM - AMF Deployment, Los Angeles, California, to Honolulu, Hawaii.” ARM Climate Research Facility. US Department Of Energy, n.d. Web. 1 Aug. 2013.

$<$ http://www.arm.gov/sites/amf/mag/>.

$3 \quad$ "ARM - Instrument - uhsas." ARM Climate Research Facility. US Department Of Energy, n.d. Web. 1 Aug. 2013. <http://www.arm.gov/instruments/uhsas>.

$4 \quad$ “ARM - Instrument - cpc.” ARM Climate Research Facility. US Department Of Energy, n.d. Web. 1 Aug. 2013. <http://www.arm.gov/instruments/cpc>.

5. Aerosols, their Direct and Indirect Effects Content." Working Group 1: The Scientific Basis. Intergovernmental panel on Climate Change, n.d. Web. 7 Mar. 2013.

$<$ www.ipcc.ch/ipccreports/tar/wg1/160.htm>.

\section{Acknowledgements}

Thank you to the Office of Educational Programs at BNL, Ernie Lewis for being my attentive mentor this term, Chongai Kuang for giving me CPC data, Gunnar Senum for giving me UHSAS data and writing MATLAB code to put it in the correct format for me to read into Python, Karen Johnson for writing Python code to find and insert missing data points, and Mike Jensen for mentoring me when Ernie was not present and helping me edit my poster. This project was supported in part by the U.S. Department of Energy, Office of Science, Office of Workforce Development for Teachers and Scientists (WDTS) under the Science Undergraduate Laboratory Internships Program (SULI). 


\title{
Airflow in the big city
}

\author{
Mauro Mancilla \\ Department of Engineering, Tallahassee Community College, Tallahassee, Florida 32303 \\ John Heiser \\ Department of Environmental Science, Brookhaven National Laboratory, Upton, New York 11973
}

\begin{abstract}
The project that I worked at Brookhaven National Laboratory was called the Subway-Surface Air Flow Exchange (S-SAFE) study. The purpose of this project is to understand the pathway of gases or any other airborne contaminants above ground and below in the subways of New York City (NYC). Project S-SAFE, sponsored by the New York Police Department (NYPD), will help NYC to improve emergency response in case of any accidental or intentional release of danger contaminants. During the field study, a small quantity of harmless and colorless gas know as perfluorocarbon were released on three non-consecutives days on the subways and the streets of NYC and the dispersion of the gas was traced using about 200 air samplers installed around NYC. My role in this project was to help with the preparation of instrumentation and the tracking of the gas during the release days. Preparation involved assembling, organizing, and programing the samplers called black boxes (BBS) and Brookhaven Air Tracer Sampler II (BATSII). During each of the three release days my job was to place samplers on the assigned areas and check they were working properly during the six hours of sampling programmed in the samplers. Once the samplers were done with sampling the task was to remove the sampler tubing from inside the samplers where data was collected and turn it over to those collecting the samples. The data from this survey will be analyzed and given to the NYPD. No data will be provided to the public since this could be used against the people living in NYC. At the end of this internship the hope is that I learned new skills such as laboratory tool use, project planning and analysis of data while working with real scientists and professionals and also enriched my background as a student, person, and worker.
\end{abstract}

\section{Introduction}

This summer I was assigned to work in a very interested study called Subway-Surface Air Flow Exchange (S-SAFE). S-SAFE study, sponsored by New York Police Department (NYPD), is a study that was conducted and completed by Brookhaven National Laboratory (BNL). The study was scheduled for this summer in the principal boroughs of New York, especially Manhattan, and expected to cost $\$ 3.4$ million dollars. This study is known as the largest study ever on the understanding of airborne contaminants. The most important part for this study is to know how chemical, biological and radiological (CBR) weapons could be dispersed in the air since particles could disperse in the same way as the Perfluorocarbon gas (gas used for S-SAFE study) does. The reason for using Perfluorocarbon for this study is because Perfluorocarbon is a harmless, colorless, and odorless, gas and could be traceable with the technology used by scientists from BNL. The study was completed during three non-consecutives days during the month of July. These days were chosen according to favorable weather conditions such stable winds, clear days, and not rainy days. BNL worked in conjunction with meteorologist to determine the better days for the three different field studies done for S-SAFE. The S-SAFE members' organization was very professional and well planned, and the project coordinators were John Heiser and Paul Kalb. They managed the entire study checking that the work needed for this study got finished as wanted and predicted.

\section{Technical Approach}

The work that I performed during this study could be divided as the follow:

- Safety training for laboratory procedures and use of tools as follows:

- Back Safety Training: With this training I learned new techniques for proper ways to lift an object depending on its weight and size.

- Ladder Safety Training: With this training I learned the proper ways to use a ladder and how to distinguish a good from a bad ladder.

- Hazardous Waste Generator Training. With this training I learned to distinguish danger and special wastes that need special handling. Also to know the respective disposal for any of these wastes.

- Laboratory Standards Training: During this training I learned that the use of proper clothes and protective equipment are very important for any work in the lab.

- Preparation of the S-SAFE equipment including Black Box Samplers (BBS), Brookhaven Air Tracer Sampler II (BATS II), custom release units, etc. This preparation includes the following:

- Connect in a proper way all the wires used in the boxes, install all tubes called Capillary Adsorption Tube Sampler (CATS) in its respective place, check and connect all the hoses used for the air absorption to the air pump and tubes etc. Also tightening screws in the samplers and checking that all parts were in place and in good working conditions.

- Review that all numeration on the outer of box samplers and the inside content match with the respective number in the program for every single sampler.

- Charge all sampler batteries for BATS II and for BBS. To charge batteries in BATS II, the job was simply to connect them with their respective chargers to the electrical outlet. To charge BBS, the batteries had to be removed and plugged into a special charger box capable of charging up to 10 batteries at one time.

- Programming the samplers was another interesting labor. Since BATS II have WI-FI technology, we were able to install the program to 10 samplers at the same 
time and ran the program to test the proper working of the air pump and solenoids in the BATS II. For BBS the programming was different. We connected every single sampler to the main computer and installed and ran the program to test also the proper working of the air pump and solenoids in the BBS.

- Placed velcro on the outer side of the samplers in order to put the information signs as corresponding.

- Tested the schedule programs for the custom released units.

- Transported all the samplers and custom release units to the momentary operation area located on Hoyt St. in Brooklyn.

- The field study called Intensive Operation Period (IOP), which was done in three days called IOP 1, IOP 2, and IOP 3 respectively.

- One day before each IOP we had to get into the operation area to start loading tubes into the samplers. All the tubes were codified, so we installed and recorded in the proper numeration with the samplers. We also made sure that all the batteries for samplers were fully charged and ready to operate.

- On IOP days, the workday started at 5 am and started with the loading of samplers in the NYPD trucks. NYPD were in charge of distributing the samplers to the respective areas and also picking them back up after the IOP finished.

- Once loading was complete, we went to the respective dropping points assigned for our IOP routes where we were receiving a pre-determined number of samplers according to the IOP route.

- Once the samplers were received, we had to located each single samplers on their respective pole where the sampler was sampling.

- Our mission after the samplers were positioned was to monitor them for their proper working order. The BATS II were assigned for surface or above ground. These samplers were able to get check it by WI-FI connection with any device capable to connect via Wi-Fi. On the other hand, subways samplers, BBS, didn't have the WI-FI technology. The way that these samplers were checked was by looking at the indicators lights located on top of the samplers.

- The monitoring lasted 6 hours, from 8 am to $2 \mathrm{pm}$. Once samplers were done, we took them to the assigned dropping point where the NYPD took them back to the operation area.
- The next step was in the operation area where we collected all the tubes from the samplers and labeled them with their respective routes and packed them for future analysis.

- Finally we took all the equipment used in the operation area back to BNL.

\section{Results and accomplishments}

The project was successfully accomplished as predicted. The results will be obtained from the tubes collected from the samplers and analyzed by scientists at BNL. These Capillary Adsorption Tube Sampler (CATS) are capable of collecting PFT from the air on a charcoal filter, which is then analyzed using a gas chromatograph. The results obtained from this study will be given to the NYPD where they will kept confidential and not revealed to the public since they could be used against the population of NYC if the results fell in the wrong hands.

\section{Impact}

S-SAFE was a study that will help police to safeguard more than 8 million people who live in NYC and neighboring cities, not counting the thousands of visitors that the city welcomes every day. Also, this study will help the NYPD to understand other inhalation hazards, such as smoke from chemical spills. This study will help the scientists understand the world that surrounds us to find better ways to protect the people and environment from hazardous air pollutants.

At the end of this internship the hope is that I learned new skills such as laboratory tool use, project planning and organization and analysis of data. The experience working in BNL was amazing, working with real scientists and professionals is a big step that marked my life and enriched my background as a student, person, and worker.

\section{Acknowledgements}

This project was supported in part by the U.S. Department of Energy, Office of Science, and Office of Workforce Development for Teachers and Scientists (WDTS) under the Community College Internships Program (CCI).

I would like to thank the Departmental of Energy for the opportunity to participate with this great internship. Also thanks to my Mentor John Heiser for his support and professionalism and to all the other mentors that helped me in my journey with this experience at BNL. Thanks to the National Science Foundation for the support of future scientists. Finally thanks to all the personnel from the Office of Educational Programs who helped me with all the required documents to successfully complete this new step in my professional career. 


\section{Appendix}

\begin{tabular}{|l|l|l|l|}
\hline \multicolumn{1}{|c|}{ Name } & \multicolumn{1}{c|}{ Department } & \multicolumn{1}{c|}{ Institution } & \multicolumn{1}{c|}{ Role } \\
\hline Adedoyin Akingba & Department of Biology & State University of New York at Potsdam & Intern \\
\hline Gabe Vignato & Department of Environmental Sciences & Brookhaven National Laboratory & Mentor \\
\hline Ilia Vardishvili & Department of Electrical Engineering & Stony Brook University & Intern \\
\hline Jessica Saintibert & Department of Biology & Tallahassee Community College & Intern \\
\hline John Heiser & Department of Environmental Sciences & Brookhaven National Laboratory & Mentor \\
\hline Larry Millan & Department of Environmental Sciences & Brookhaven National Laboratory & Mentor \\
\hline Lezou Dali & Department of Electrical Engineering & Stony Brook University & Intern \\
\hline Marie Lamothe & Department of Engineering Sciences & Suffolk Community Colege & Intern \\
\hline Matthew B. Mayers & Department of Human Ecology & Cornell University & Intern \\
\hline Mauro Mancilla & Department of Engineering & Tallahassee Community College & Intern \\
\hline Paul Kalb & Department of Environmental Sciences & Brookhaven National Laboratory & Mentor \\
\hline Scott Smith & Department of Environmental Sciences & Brookhaven National Laboratory & Mentor \\
\hline
\end{tabular}




\title{
Oxigraf monitor installation
}

\author{
Aaron Mohammed \\ Texas A\&M University, College Station, TX 77843 \\ Vincent Castillo \\ Collider-Accelerator Department, Brookhaven National Laboratory, Upton, NY 11973
}

\begin{abstract}
The oxygen content of the atmosphere is required to be at some specific level, $\sim \mathbf{2 0 . 9} \%$, in order to sustain life. In industry the need for an alarm for when the oxygen concentration falls below $18.5 \%$ is critical. This project calls for the installation of an Oxigraf oxygen monitor in a service room of the Relativistic Heavy Ion Collider (RHIC) at the Brookhaven National Lab (BNL). This service room houses the Liquid Helium Facility (LHF) which is being used for the Coherent Electron Cooling (CEC) [1] project. The Oxigraf will take a reference sample of air from the outside atmosphere with known $\sim 20.9 \%$ Oxygen content and compare it to the sample of air from the LHF. If the LHF sample is $<\mathbf{1 8 . 5 \%}$ oxygen the Oxigraf unit will trigger an alarm which represents an Oxygen Deficiency Hazard (ODH). Through the use of Programmable Logic Controllers (PLCs), an operator at the Main Control Room (MCR) can monitor the alarm and take the required actions necessary to address the problem.
\end{abstract}

\section{INTRODUCTION}

The Relativistic Heavy Ion Collider (RHIC) at the Brookhaven National Laboratory (BNL) uses Superconducting Magnets (SCM) for the circulating and storing of the particle beams that are used for experiments. SCMs require liquid helium to reach the superconducting temperature of $4^{\circ}$ Kelvin.

The presence of Helium poses a threat of an Oxygen Deficiency Hazard $(\mathrm{ODH})$ which is addressed by a ring-wide $\mathrm{ODH}$ monitoring system. This system uses an electrochemical sensor with ancillary electronics developed by the Access Control Security (ACS) group of the Collider-Accelerator Department (C$\mathrm{AD})$, to respond to ODH alarms. The response to the alarm consists of turning on fans, opening vents, sounding audio alarms and turning on strobe lights wherever the alarm was triggered; fans and vents in adjacent areas will activate if the event propagates for $>10$ minutes.

There are many other ODH systems in C-AD not connected to the RHIC ring ODH system; one such system is the Building 912 ODH system that uses a PureAire [2] monitor that is specifically designed for detecting N2. There are also isolated standalone systems that use the RHIC ring sensors and electronics such as the Cold Snake [3] in the Alternating Gradient Synchrotron (AGS) ring.

The current Oxigraf project is another stand-alone industrial ODH system that will be monitoring the Liquid Helium Facility (LHF) for the Coherent Electron Cooling (CEC) machine. The purpose of the CEC project is to improve the coherence of RHIC beams by eliminating the parasitic halos that are generated by the like charges that constitute the particle beam.

\section{OXIGRAF UNIT DESCRIPTION}

For the CEC project, an Oxigraf, model O2iM unit, [4] will be installed to monitor the concentration of oxygen at the sample points in the LHF. The Oxigraf is an electro-mechanical device that uses laser spectroscopy to analyze the concentration of oxygen in a sample of gas.

The O2iM is composed of an oxygen sensor, a vacuum fluorescent alphanumeric display (VFD), a touch keypad, detection relays, 4-20 mA analog output, as well as its own horn and strobe light. Figure 1 shows the face view of the O2iM which has the touch keypad and the VFD.

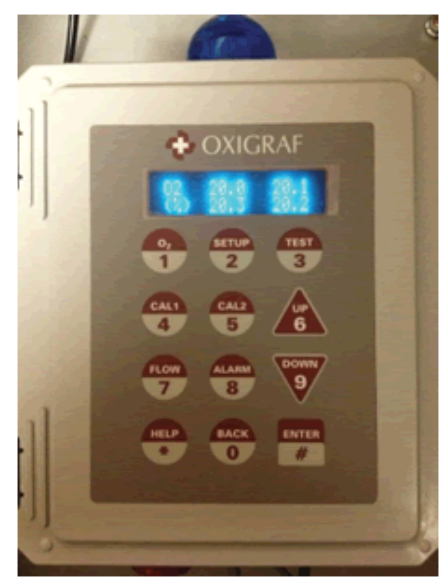

Figure 1. VFD, touch keypad and blue strobe light of Oxigraf

These components work together to measure and display the concentration of oxygen in a sample of gas that is drawn through the machine. The gas samples will flow through 4 sample input tubing channels into 4 input sample ports, then sequentially into the oxygen sample cell to be analyzed by laser spectroscopy after which it is released through the exhaust port. At the input of each sample tube is a hydrophobic gas filter, shown in Figure 2; its primary purpose is to prevent contamination of the sensors by only allowing gases to travel through the sample tubes and into the unit.

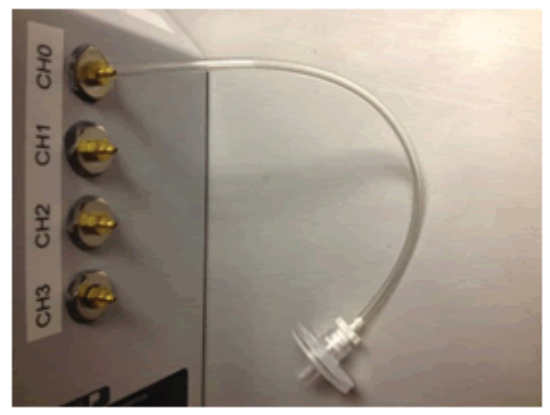

Figure 2. Hydrophobic gas filter at input end of sample tube

External components: strobes, horns, fans, and vents are activated by using the alarm signals from the Oxigraf in conjunction with the RHIC PLC ODH response system. 


\section{BENCH TEST RESULTS}

Many test bench simulations were performed to understand the complexity of the unit as well as understating what settings and features are necessary for the Oxigraf application at the CEC, see Appendix A: Settings that are specific to the CEC project. The purpose of these test bench simulations was to understand how the Oxigraf processes each gas sample from each respective input port and outputs the result to the numerical display on the unit.

Each sample tubing was $100 \mathrm{ft}$. long and was terminated with a hydrophobic gas filter at the input end. Styrofoam cups were attached to the filters to emulate the protective housing that would actually go around the filters when installed in the LHF, shown in Figure 3, and also to contain the sample shot of helium to the input being tested. Samples of helium were shot into each filter and then timed to see how long it would take for the sample to travel from the input of the line until an ODH alarm is registered by the unit. The internal pump was set to its maximum performance state which allows for the gas to travel through the line at $\sim 400 \mathrm{ml} / \mathrm{min}$.

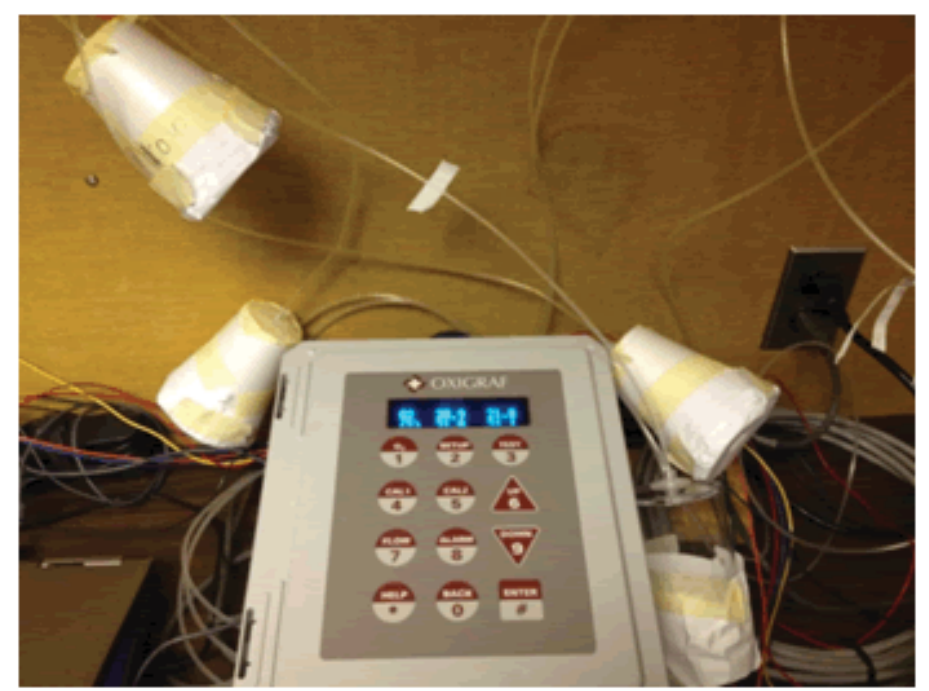

Figure 3. Styrofoam cups that represent the protective metal box used in test bench simulations

Three trials for each channel were performed at the workbench in our lab and the LHF to determine consistency in performance among the channels. The results can be shown in Table 1 and Table 2. Results in Table 1 are longer than those in Table 2 because the $100 \mathrm{ft}$. sample tubes were not stretched out thoroughly so that would have restricted the flow for trials in Table 1. It was found that the average time for the LHF tests was roughly 30 s, and the results from the workbench and the LHF show consistency in performance among the channels. Table 3 shows the time for the channels with the sample tubes installed at their required sam-

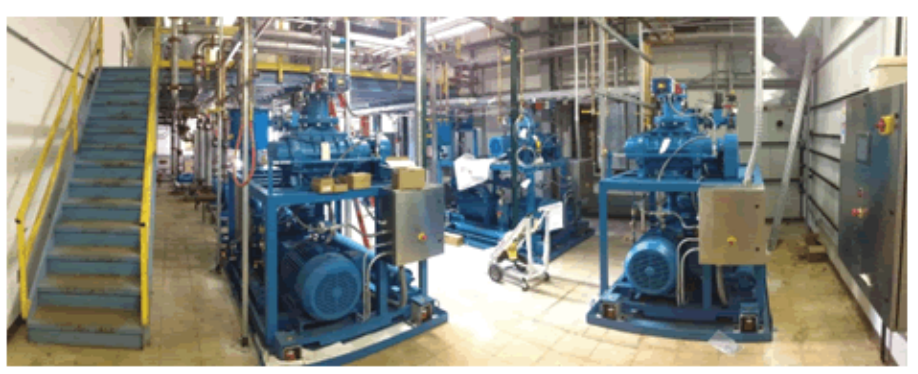

Figure 4. Liquid Helium Facility ple positions in the LHF. The final lengths for the sample tubes are as follows: Channel $0-88 \mathrm{ft}$. Channel $1-84 \mathrm{ft}$. Channel 2 and Channel $3-110 \mathrm{ft}$.

\begin{tabular}{|l|r|r|r|r|}
\hline & \multicolumn{4}{|c|}{ Channel } \\
\hline Helium & \multicolumn{1}{|c|}{$\mathbf{1}$} & \multicolumn{1}{c|}{$\mathbf{1}$} & \multicolumn{1}{c|}{$\mathbf{3}$} \\
\hline Trial 1 & $41 \mathrm{~s}$ & $41 \mathrm{~s}$ & $39 \mathrm{~s}$ & $24 \mathrm{~s}$ \\
\hline Trial 2 & $31 \mathrm{~s}$ & $39 \mathrm{~s}$ & $36 \mathrm{~s}$ & $24 \mathrm{~s}$ \\
\hline Trial 3 & $41 \mathrm{~s}$ & $24 \mathrm{~s}$ & $32 \mathrm{~s}$ & $38 \mathrm{~s}$ \\
\hline Avg. Time & $34.67 \mathrm{~s}$ & $28.67 \mathrm{~s}$ & $37.6 \mathrm{~s}$ & $35.67 \mathrm{~s}$ \\
\hline
\end{tabular}

Table 1. Results from the test bench simulations in our lab

\begin{tabular}{|l|r|r|r|r|}
\hline & \multicolumn{4}{|c|}{ Channel } \\
\hline Helium & \multicolumn{1}{|c|}{$\mathbf{0}$} & \multicolumn{1}{|c|}{$\mathbf{1}$} & \multicolumn{1}{c|}{$\mathbf{3}$} & \multicolumn{1}{|c|}{} \\
\hline Trial 1 & $24 \mathrm{~s}$ & $38 \mathrm{~s}$ & $26 \mathrm{~s}$ & $23 \mathrm{~s}$ \\
\hline Trial 2 & $34 \mathrm{~s}$ & $28 \mathrm{~s}$ & $39 \mathrm{~s}$ & $49 \mathrm{~s}$ \\
\hline Trial 3 & $38 \mathrm{~s}$ & $28 \mathrm{~s}$ & $31 \mathrm{~s}$ & $24 \mathrm{~s}$ \\
\hline Avg. Time & $31.33 \mathrm{~s}$ & $32 \mathrm{~s}$ & $32 \mathrm{~s}$ & $32 \mathrm{~s}$ \\
\hline
\end{tabular}

Table 2. Results from the field test simulations at the LHF

\begin{tabular}{|l|r|r|r|c|}
\hline & \multicolumn{4}{|c|}{ Channel } \\
\hline & \multicolumn{1}{|c|}{$\mathbf{1}$} & \multicolumn{1}{c|}{$\mathbf{2}$} & $\mathbf{3}$ \\
\hline Sample Time & $20 \mathrm{~s}$ & $24 \mathrm{~s}$ & $31 \mathrm{~s}$ & $25 \mathrm{~s}$ \\
\hline Sample Tube Length & $88 \mathrm{ft}$. & $84 \mathrm{ft}$. & $110 \mathrm{ft}$. & $110 \mathrm{ft}$. \\
\hline
\end{tabular}

Table 3. Sample times for the channels with the sample tubes installed at their required sample positions in the LHF

\section{LIQUID HELIUM FACILITY}

The LHF, shown in Figure 4, is located at the 2 O' clock service building of RHIC; the CEC machine is located in the 2 O' clock Experimental area of RHIC as shown in Figure 5. The LHF houses all the heavy machinery that will be used for cooling and compressing the helium gas into a liquid, hence we have to be prepared for a helium leak or spill in this area. This area is approximately 160 sq.-ft. The 2 O'clock Experimental area of RHIC is already monitored by the RHIC ODH System.

The Oxigraf will be located, $\sim 100 \mathrm{ft}$. away in another room of the 2 O' clock service building, adjacent to the PLC cabinet that controls the 2 O'clock RHIC sector. From here, the Oxigraf's hardware will be integrated into RHIC's PLC system. From the Oxigraf, the 4 sample tubes are run inside of PVC conduit to the LHF. There are 2 other tubes also in PVC conduit which are run to the outside of the $2 \mathrm{O}$ ' clock service building to be used for the sample-pump exhaust and the oxygen reference level ( $20.9 \%$ in atmosphere) respectively. Each tube terminates with a hydrophobic gas filter and is housed in a protective metal box. There will only be 3 sample points in the LHF so only 3 ports will be actively used while the fourth will be held as a spare. 


\section{PLC PROGRAMMING}

By using a PLC system, operators in the Main Control Room (MCR) are able to monitor and respond to the Oxigraf in the event of an ODH incident. A PLC is a digital processor that is used for the automation of electromechanical systems. The current operating system in RHIC uses a PLC to which the hardware of the Oxigraf is configured to be compatible. Only 3 ports from the Oxigraf will be used in monitoring the LHF, which means $3 \mathrm{ODH}$ signals have to be monitored.

The PLC is programmed such that when the unit detects an ODH in any port, a blue strobe, audio alarms, vents and fans will all become activated to contain the ODH event and to alert workers in the LHF as well as the MCR that an ODH event has occurred. The operators in the MCR use a Human Machine Interface (HMI) display to monitor the alarms. This HMI display will show exactly which port or ports registered the ODH event and the safety devices that are responding as a result. Figure 6 shows a sample ODH display from the MCR ODH operations screen.

For the PLC to work with the HMI, the following programs were used: RSLogix 500, RSLinx Classic Lite and FactoryTalk View Studio. With RSLogix 500, Ladder Logic is the language that is used to control bits which represent inputs and outputs. For instance, the inputs all correspond to each individual channel on the Oxigraf while the outputs represent the strobe lights, audio alarms, vents and fans. See Appendix B: Tutorial on PLC programming for how specific instructions on this PLC were formed.

The use of timers and delays within the code are also pertinent because there is an order in which events must occur. For

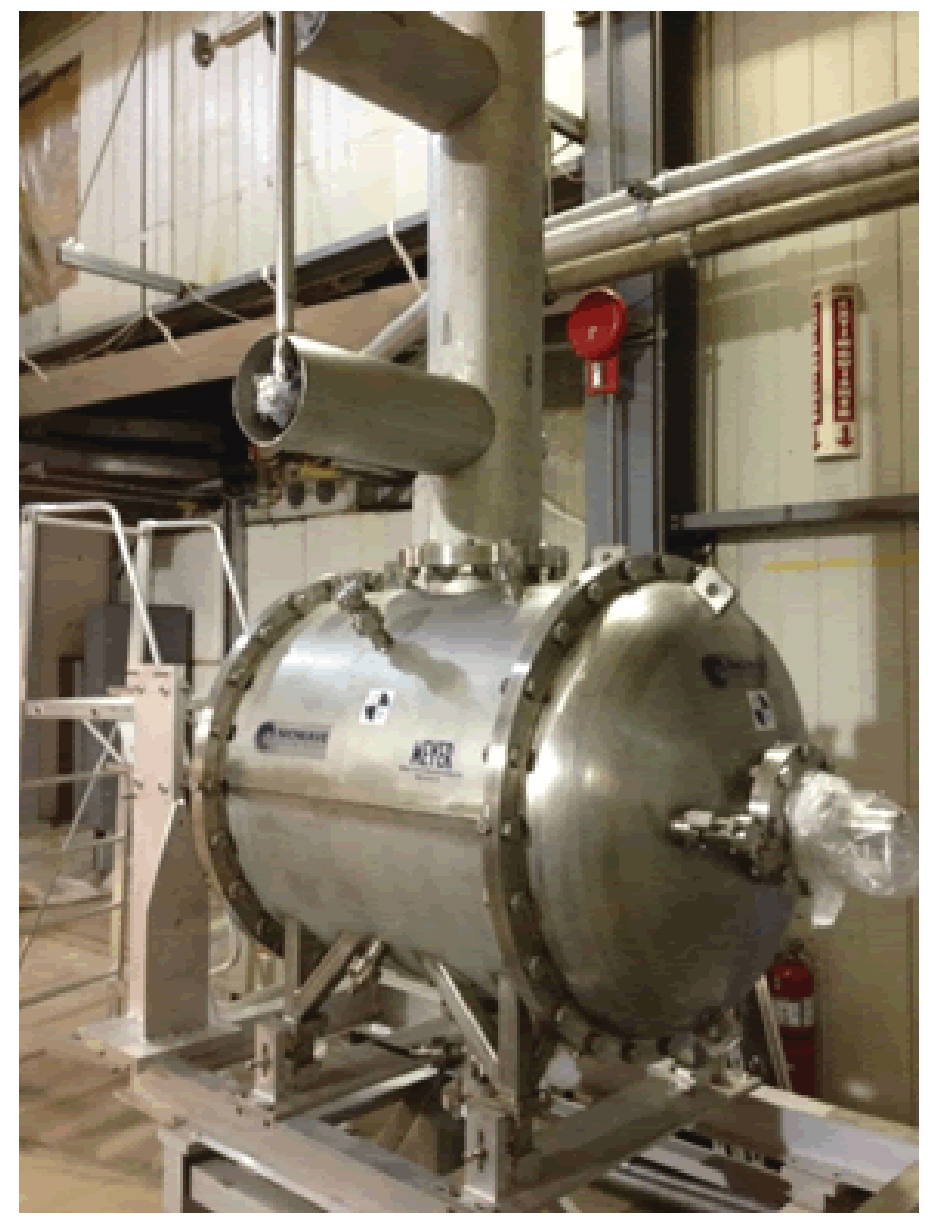

Figure 5. CEC equipment in the 2 o'clock Experimental area of RHIC example, the readings from the Oxigraf will be outputted onto the HMI display. If the scan time is set to $5 \mathrm{sec} /$ channel that means for every 5 seconds a channel reading will be updated on the display. There must be a timer that does the same thing in the code so that the values being displayed on the HMI are in sync with what the Oxigraf is displaying.

\section{CONCLUSIONS}

The Oxigraf monitor installation is expected to be completed by September 2013. At the time of writing, the engineers in the ACS group are waiting for the electricians to prepare the installation site for the Oxigraf unit in building 1002a. All sample tubes have been set up for the sample points in the LHF and tested for proper operation. Next the Oxigraf hardware will be integrated into RHIC's PLC system along with the necessary PLC code to complete the integration. In addition, an HMI screen will be made for the MCR so that the operators can monitor ODH events and respond accordingly. Finally this Oxigraf ODH system will be tested and certified by the ACS certification program.

\section{ACKNOWLEGEMENTS}

I wish to thank everyone in the ACS group for all of their support and help throughout the summer: Group Leader Jonathan Reich, Heather Hartman, Darren Hillis, Joel Vasquez, Susheng Xin, Rich Conte, Walter Lamar, Dan Carroll, Frank Scheifele and my mentor Vincent Castillo. This project was supported in part by the U.S. Department of Energy, Office of Science, and Office of Workforce Development for Teachers and Scientists (WDTS) under the Science Undergraduate Laboratory Internships Program (SULI).

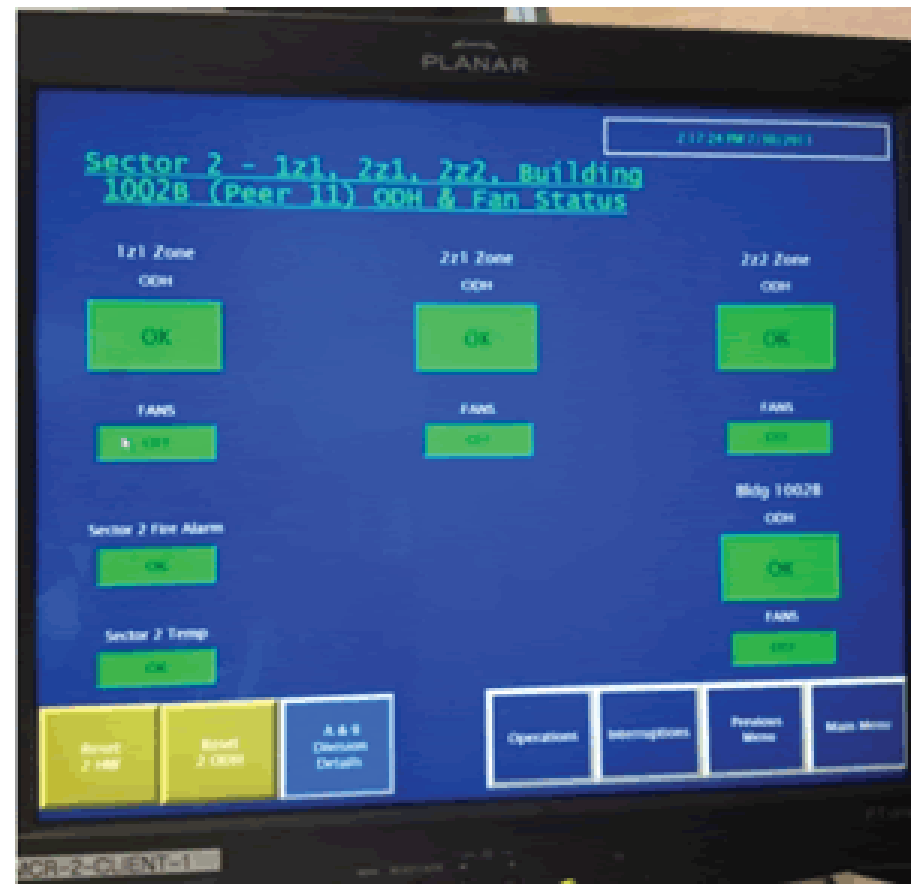

Figure 6. ODH HMI from RHIC MCR operations screen 


\section{REFERENCES}

1"The Past 6 Weeks for Nuclear, Particle Physics at BNL." RHIC News. N.p., n.d. Web. 07 Aug. 2013. <http://www. bnl.gov/rhic/news/070108/story1.asp>.

2"SRF Systems for CeC PoP Experiment in RHIC." Lecture. Collider-Accelerator Dept. Brookhaven National Lab. Web. 7 Aug. 2013. <http://www.c-ad.bnl.gov/ardd/ ASSRC $\% 20 \mathrm{CeC} \% 20 \mathrm{PoP} /$ Presentations/SCRF\%20 Presentation.pdf>.

3“Air Check O2 Oxygen Deficiency Monitor for CO2, N2 Storage Areas." Air Check O2 Oxygen Deficiency Monitor for CO2, N2 Storage Areas. N.p., n.d. Web. 01 Aug. 2013. <http://www.pureairemonitoring.com/allcategoriesgas-monitorsair-check-o2-oxygen-deficiencymonitor-for-co2-n2-storage-areas/>.

${ }^{4}$ Luccio, A.U, R. Gupta, W.W MacKay, and T. Roser. "Cold AGS Snake Optimization by Modeling." Diss. Brookhaven National Laboratory, n.d. (n.d.): n. page. Dec. 2003. Web.

5“O2iM Manual.” 2012. MS. Mountain View. Oxigraf, 9 Jan. 2012. Web. 1 July 2013

\section{Appendix A: Settings that are specific to the CEC project.}

a. Configuring Parameters

The Oxigraf can be programmed to various settings which can all be accessed from the Startup menu. These setting can be modified to specific parameters, all of which depend on the specific use of the unit. For the CEC project, only the functions listed below will be used (the number in front of the setting name is where that setting can be referenced in the Oxigraf O2iM Manual); although the unit can be programmed to do much more, they are extraneous for this project. In order to scroll through the settings, press the SETUP key and the parameter that needs to be configured. From there use either the UP/DOWN (U/D) key to set the parameter.

\subsection{Multipoint Scan Mask}

The scan mask allows for any channel to be active when selected; when active, the gas sample from each channel is scanned by the multiplexer valves. Use the U/D key to select which channel is active. For the CEC project, only 3 channels (0-2) will be active.

\subsection{Scan Sample Time}

The sample time is the time the channel sample is analyzed and displayed. Each sample time is measured in seconds. Use the U/D key to increase or decrease the time. If set to zero the sample time is disabled and channels must be displayed manually by pressing the $\mathrm{O} 2 \mathrm{key}$. The sample time is set to $5 \mathrm{sec} / \mathrm{channel}$

\subsection{Display All Channels}

This parameter can be turned On/Off by using the U/D key. If selected On, then the $\mathrm{O} 2$ level for all channels will be displayed at once. If selected Off, then each channel will be displayed with respect to order of scan along with its flow rate. This setting needs to be set to ON.

\subsection{Flow Setpoint}

This parameter sets the target flow rate for the sample flow controller. If set to zero, the sample pump will permanently be set to zero. Set this parameter to $400 \mathrm{ml} / \mathrm{min}$

\subsection{Pump State}

The pump state has three different parameters for the flow controller. Use the U/D key to select either Off, On or Max. Since the channels will be measuring samples that are up to a $100 \mathrm{ft}$. away, the pump state needs to be on Max.

\subsection{O2 Alarm Thresholds}

The threshold parameter consists of a pair of high and low alarm limits. Use the SETUP key to highlight the Low O2 Limit A and set the limit by either using the U/D keys or manually entering a numerical value for the lower limit. Do likewise for Limit B. Both limits need to be set to $18.50 \%$

\subsection{Low Flow Alarm}

This is a useful function to be active because it sets a low limit to the flow of the gas in the line. If this alarm becomes active that means the flow in the line is low which probably means that the hydrophobic gas filter has gone bad. Set this function to alarm at the [accepted flow rate -50$] \mathrm{ml} / \mathrm{min}$.

\subsection{Relay Failsafe}

The purpose of the failsafe is to have the channels deactivate the alarm relay on the alarm/warning condition. The Oxigraf unit needs the relay failsafe option to be turned "On". Use the U/D key to enable this feature.

\subsection{Relay Allocation}

For our specific project the Oxigraf needs to be programmed to channel relay mode. Use the U/D key to select this option. Channel relay mode allocates each channel to a relay whereas the default relay system assigns alarm functions: System OK, Warning, Limit A and Limit B to the 0-3 relays respectively.

The channel relay mode allocates the channels as follows: Channel 0 - System OK relay Channel 1 - Warning relay Channel 2 - Limit A relay Channel 3 - Limit B relay

The following settings in the manual will not be used: 5.4, 5.5, 5.6, 5.7, 5.8, 5.9, 5.10, 5.11, 5.12, 5.13, 5.14, 5.15, 5.19, 5.21, $5.22,5.23,5.24,5.26,5.28,5.29,5.30,5.31,5.32,5.33,5.34$ and 5.35 


\section{Appendix B: Tutorial on PLC programming Processor, I/O Modules}

A PLC system consists of a PLC processor and input and output (I/O) modules in a PLC rack. I/O modules may also be in remote racks that communicate with the processor by a data buss or Ethernet. Modules come with various channel availabilities: 8 or 16 channels.

\section{a. RS Logix 500}

RS Logix 500 is a graphical based coding program developed by Allen Bradley for PLC programming. The code is written using Ladder Logic; there are rungs with commands that control bits which perform specific tasks. To write the program:

1. 1. Connect your I/Os into the processor and use a RS232 cable to connect the processor to the computer.

1. 2. Open the program and create a new file and select the processor type.

1. 3. Then configure the $\mathrm{I} / \mathrm{O}$ modules by selecting the proper size rack and the location of each $\mathrm{I} / \mathrm{O}$ module in the rack.

1. 4. Now take the system online where you can begin writing PLC code.

1. 5. Assign an input bit for each individual channel of the Oxigraf.

1. 6. Assign outputs to be activated when an input bit, representing an ODH alarm, becomes energized. For example, the $24 \mathrm{~V}$ blue strobe light, a small fan and a sonalert are all assigned bits on the output module.

1. 7. Write the PLC code that calls for the proper interaction of the inputs and outputs.

1. 8. Once done, move onto Factory Talk View to set up the HMI display.

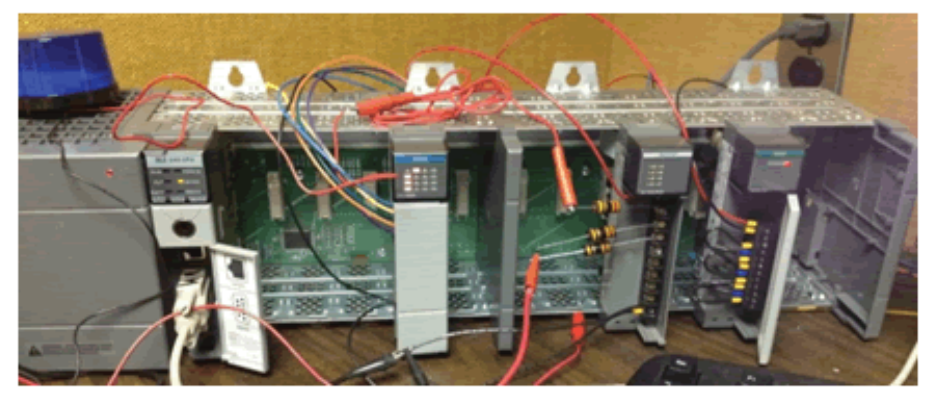

Figure 1. PLC processor with I/O modules
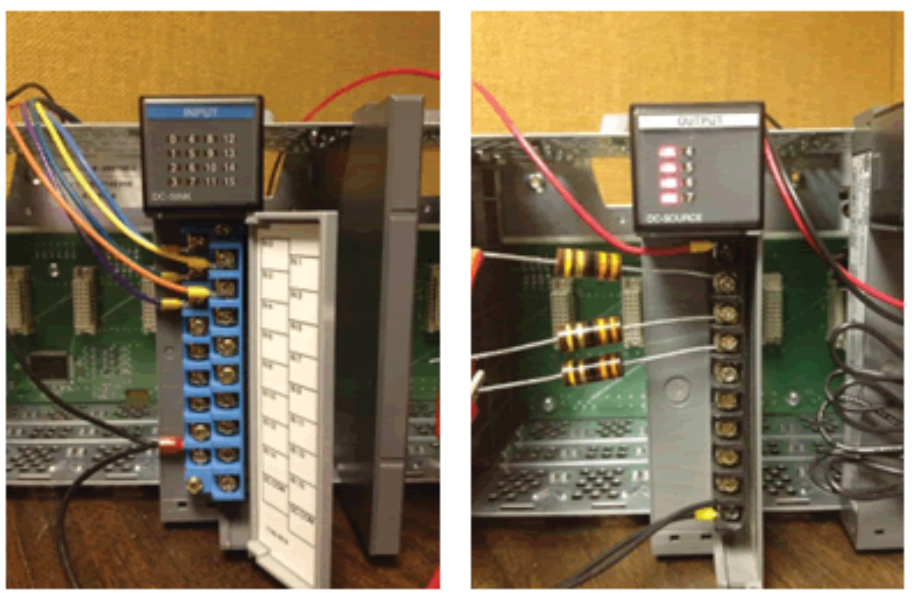

Figure 2. 16 channel Input mod- Figure 3. 8 channel Output module

\section{a. Factory Talk View}

Factory Talk View is another program developed by Allen Bradley to configure HMI displays.

1. Open RS Linx Classic Lite, which is a program that connects the HMI display to the processor. Configure the drivers and the processors so that Factory Talk View can recognize the processor.

1. Using Factory Talk View, create a new file and map it using RS Linx Classic Lite. The processor stores the bits in memory. The tags are assigned to buttons and indicators in the HMI. RS Linx Classic Lite will map the tags to the bits which will allow the button and indicators to act in conjunction with the status of the bit assigned to it.

1. Create a new display.

1. Create 4 indicators which have to be tagged to the input bits of the 4 channels.

1. Create 4 separate tags for each indicator and assign them to the indicators.

1. Using the indicator properties, assign different states for the indicators. State 0 could be used for a normal state, meaning no ODH alarm, which appears green. State 1 could be used for an ODH alarm which appears as light blue.

1. Repeat the same process for the indicator and the tags that control the vent and the fan.

1. Then create momentary push buttons following the same procedure above assigning bits and tags as described. Create button that can acknowledge the alarm and reset the display manually.

1. Then organize the buttons and indicators on the screen so it is both user-friendly and looks professional. 


\title{
Supporting the Standards-Based Management System staff on key initiatives
}

\author{
Brittany Morgan \\ English Department, Binghamton University, Binghamton, NY 13902 \\ Roy Lebel \& Joe Canestro \\ Quality Management Office, Brookhaven National Laboratory, Upton, NY 11973
}

I am supporting the Standards-Based Management System (SBMS) staff within the Quality Management Office by helping to edit and verify institutional procedures. I am assisting in making these web based procedures more user-friendly by doing a variety of activities that include providing a fresh perspective on content, creating and revising flowcharts, using HTML and other web tools in order to build subject areas on the SBMS website, and by editing existing subject areas and newly created ones. One of my larger, more focused projects was to create and update a spreadsheet full of metrics related to the document development process. This spreadsheet lists all documents available on their website and the details of the revision process, such as when the notification of a needed change or revision was received by the staff, the type of revision needed (be it major or minor), as well as the dates of when various parts of the process were completed. This spreadsheet assists the SBMS Manager by informing him how long it takes to complete the process and where they may improve the efficiency of the complete document development process.

SBMS is used to deliver policies and procedures to Laboratory staff, guests and visitors that are derived from diverse laws and directives (DOE Directives, federal, state, or local laws, etc.) that we have to abide by. Due to the fact that each month, an average of ten changes and updates needs to be incorporated into the way BNL operates, the SBMS staff must efficiently support the entire lifecycle of the documents housed on the SBMS website. Working with the Subject Matter Experts (SMEs) on the evaluation of the many existing and new requirements to assessing whether or not the information in documents is effective, the staff, with the help of the Information Technology Division (ITD), continually maintains and improves the system and its tools in order to deliver these documents to serve the needs of all staff, facility users, guests, visitors, contractors, and students. As a part of this process, I revised and edited documents and webpages, used Microsoft Office to create a variety of documents that include, but are not limited to process maps, interactive PowerPoint presentations, and data sheets, and used online tools to create interactive features on various documents.

As a part of the revisions I assisted in, I creating a case study practice in the form of a Microsoft Word document from a PDF. Although Adobe Reader can, in fact, create such documents from PDFs for you, at times, the conversion process skews the document. Things such as spacing issues, word placement, and picture transfer were just a few of the issues I encountered when converting the document. I also converted a PowerPoint presentation into a participant workbook for a Yellow Belt Course the Competitiveness Improvement Project (CIP) Team titled Process Improvement which will be used at a later date. It will be given to those participating in the course for them to write in and keep as a reference. It contains various definitions, instructions, examples, diagrams, etc. that will provide instruction on how to improve processes. First, I created a piece of SmartArt that displayed the course of the Process Improvement procedure. From there, I created a contents page before creating a variety of pages that will be edited and rearrange until needed.

One of the other documents I reviewed is a part of the SBMS Improvement Plan for the fiscal year of 2013. The document lists their software requirements as of July 2013. Its intention is to list requirements for software developments for particular projects which include Dynamic Subject Areas, the 2013 improvements, and Dynamic Management System Descriptions. The features needed to be implemented are listed in order of priority. My role was to go over the document as a new user and input suggestions for possible features or questions about what was listed since I had been working within the Subject Areas and the backend on the SBMS website.

This website houses the institutional policies and procedures for Brookhaven National Laboratory. The SBMS staff manages this website through data management tools which can only be accessed by those with special permission. There, the coding behind the page is formatted, subpages can be added to the main pages, and process maps can be added to display various subsections of a process in an area known as the backend. There, any and all formatting changes or textual revisions are made. A new format has been introduced into SBMS that displays the procedures in an innovative way using icons for important related information. This includes Requirements, Lessons Learned, Training, etc. for the content and active flowcharts used for navigation between sections. I navigated this format in my assistance with various Subject Area (SA) revisions.

The Alcohol and Substance Abuse (ASA) SA was one of the larger projects I assisted the staff with during my time at Brookhaven National Laboratory. It is located on the SBMS website and is in the drafts section to be edited for publication. First, the body of the future pages for the SA was formatted into a Microsoft Word document. In that form, I assisted in reviewing and revising the various topics that would be included in the ASA SA. From there, the text was put into the backend before I began to code the pages using HTML. I input links, headers, created lists, and formatted text amongst other things. Each page was created using the backend and inputting all of the coding by hand. Once the text [including comments to the Subject Matter Expert (SME)] and coding had been inputted into the backend, the next step was to take and insert the images of the process map on each page.

Process maps, also known as workflows, use the Business Process Modeling Standard to depict a process that Laboratory staff is going to use. The most recent standards were drafted at the end of June of this fiscal year. Within the standards document itself contains an explanation of what process maps are, how they 
are used, as well as the standard shapes to be integrated throughout the Laboratory. Process maps are used in any process that involves multiple management systems or functions. Although I did not create the ASA SA process maps used, one of the process maps I did work on was the Facility Use and Agreement (FUA) Revision Process. Within this particular process map are step-bystep directions of what is supposed to occur during the process, whom is to be following these steps, and when certain actions need to be taken.

Process maps are used within SAs because they are interactive and include the option of clicking on individual steps in order to view a more detailed description, outlines of activities, or additional resources and contacts pertaining to a process. The Online Image Map Editor Tool makes it possible to click on individual steps and be taken to another corresponding page within the SA. In order to create the interactive process map, I first took the links from each section of the SA. Next, I created a Microsoft Word document and placed all of the links within the document for future use by the SBMS staff. Then, I mapped the coordinates of each process on the image and linked them to the appropriate pages with the image map tool. Finally, I inserted the code created by the tool into the backend. Other areas of the backend include a section for definitions, requirements, training, as well as forms/ exhibits which are all displayed on each page of a Subject Area.

I also used the Online Image Map Editor Tool to adjust the Lockout/Tagout (LOTO) for Installation, Demolition, or Service and Maintenance SA which has been created and will be published and effective at the end of September of this year. Here, I assisted in updating the page for the final installment. The images of the flowchart on the website were too large. Once they were resized, I was given the images in order to place into the Online Image Map Editor Tool. The process for creating the code through mapping coordinates before putting it into the backend was the same as with the ASA SA. These interactive processes allow the staff to make following procedures more user-friendly for the Laboratory.

Another interactive feature I helped to create involved using Microsoft PowerPoint. I created draft slides for an upcoming causal analysis training seminar using the Causal Analysis handbook. The subjects that will be focused on include fact finding, conducting interviews for the remainder of the Process Improvement $\mathrm{Cy}$ cle (PIC), and reporting conclusions. The presentation itself will be interactive and will include scenarios and other features added to the definitions and steps already within the document. One of the other PowerPoints I worked on included creating a Management System Distribution example. Its contents contain various areas of Management Systems such as people, facilities, research, etc. and which Management System Descriptions (MSDs) located on the SBMS website fell under said categories. MSDs were also involved in the larger process data project I completed.

There is a spreadsheet with data on the entire document revision process for all documents found on the SBMS website. I created the spreadsheet for the data found in the third quarter (Q3) of this fiscal year, i.e., April to June. The spreadsheet lists the document type (be it a Subject Area (SA), Program Description (PD), Management System Description (MSD), or BNL Manual), the revision number for the document, and the type of revision (major, minor, or new) to start. It is then a list of dates that correspond with specific actions in regards to the document. For example, a Notice of Intent (NOI) must be filled out when making major revisions to any document. A major revision is when a change in said document affects the way a staff member performs their work. The dates of when the NOI was submitted and received executive approval are included in the document. From there, information taken from various databases is inputted into the spreadsheet. The key dates that are calculated are when the initial draft for a change is received from the Subject Matter Expert (SME) by the SBMS staff, when it is returned and has been approved by the SME after needed revisions have been made and when it is published. These dates show where in the process that improvements can be focused, evaluated, and explored i.e., whether or not it takes more time for the SBMS staff to respond to an initial draft change or if it takes longer for the SME to approve the changes.

One of the larger projects I had to complete included creating a spreadsheet with the assorted policies the Laboratory has in effect. Policies put into effect by either the Department of Energy of Brookhaven National Laboratory are extremely important and are required to be followed by any and all persons on Laboratory property. I first searched for policies using the Laboratory search engine located on the Laboratory website's main page. Although highly regarded in level of importance here at the Lab, the term "policy" is strewn throughout various departmental websites giving over 13,000 results on the main page. After combing through these results, it was found that often the word was used as a keyword despite the document or link not linking to a policy, e.g., policy council homepages or links to newsroom articles. The SBMS homepage's search engine produced similar results; nearly 6,000 results containing the term policy were found, but not every result linked to a policy. Using these search results, I identified fifty-four Laboratory-wide policies in effect. The locations of these policies varied from the SBMS website to the Human Resources (HR) website as well. Because policies are effective lab-wide, some of the policies discovered are not considered policies, rather department protocols. A variety of information was discovered about certain policies such as the fact that some policies links are not listed when searched on the Laboratory's main page or other links take you to SAs rather than policies. I then created a spreadsheet which contains the policy name, the location of the policy, the link to where the policy can be found, comments about the policy, and the owners of the policies i.e., the Management System Stewards (MSS).

The ultimate goal of the new interface being incorporated by the SBMS staff is to make their website and lab-wide procedures more user-friendly. Whether it is for a new staff member looking to purchase inventory or for a SME looking to revise a Subject Area, clarity and simplicity is important. Mistakes can be made if certain steps of a process are overlooked or misunderstood and the SBMS staff assists the SME with trying to prevent this by continually updating and revising using their new interface. My assistance in making work processes more efficient for the staff through the improvement of the quality of documented Laboratory-wide procedures could help to reduce the time and effort needed for future documents. By assisting the staff with these and other projects this summer, I am learning about how safety and the quality of a workplace environment play a key role in day-to-day work situations. I am learning to manage my time between various assignments and meetings. I have improved my skills in areas involving Microsoft Excel, HTML coding, as well as Microsoft Visio, among other things. My assistance also plays a key role at the Lab by helping to improve the quality of documented Laboratory procedures which impacts the Laboratory's work environ- 
ment. Through this work at Brookhaven National Laboratory, I am helping to fulfill part of the Department of Energy's mission statement of management and operational excellence.

I would first like to thank the Manager of the Quality Management Office, Roy Lebel for his generosity in accepting me into his department and for being one of the best representations of professionalism and courtesy I have ever encountered. Next, I would like to thank my mentor, Joe Canestro, SBMS Manager, for his guidance and for being a supportive role model during my time in the SULI program. I am grateful to all SBMS staff as well as the staff of the Quality Management Office for their professionalism and the hospitality with which they shared their knowledge with me. I would also like to thank the Office of Educational Programs, and Brookhaven National Laboratory for allowing me to have this opportunity. This project was supported in part by the U.S. Department of Energy, Office of Science, Office of Workforce Development for Teachers and Scientists (WDTS) under the Summer Undergraduate Research Program (SURP). 


\title{
Vibrational analysis at Brookhaven National Lab ground sites and on existing $x-y$ stages
}

\author{
Scott Oldham, Mechanical Engineering, Michigan State University, East Lansing, MI 48824 \\ Sushil Sharma, Photon Sciences, Brookhaven National Laboratory, Upton NY 11973
}

\begin{abstract}
The new facility at Brookhaven National Lab known as the National Synchrotron Light Source II is designed to produce very high brightness of $x$-rays also known as synchrotron radiation which will allow beam scientists to conduct research. To maintain the high quality brightness it is necessary for optical components, such as slits, photon shutters and mirrors to remain free of the vibrations which come from the ground or utilities throughout BNL. The $x-y$ motion stages on which the optical components are mounted were designed so that the vibration remains at a recommended level and that the load of the optical components can be supported. The use of vibrational analysis employing accelerometers, a Labview program, and an Excel spreadsheet all assisted in arriving at a function that defined the transfer of vibrations between the floor and the $x-y$ stage as a value of magnification close to 1 . After assisting in the assembly of the new prototype $x-y$ stage, engineers needed to make modifications in certain areas which made it unable for vibration testing until 7/30/2013. The old $x-y$ stage was tested for vibration and its magnitude on average was around 1-1.8 in all directions. An optimized old $x-y$ stage was done through the adjustment of assembly parameters such as torqueing bolts up to $150 \mathrm{ft}-\mathrm{lbs}$ ( $50 \mathrm{ft}$-lbs increment) and tightening screws then taking vibrational measurements to see the magnitude it displaced. It was seen that the frequency of the $x-y$ stage remained at $50-60 \mathrm{~Hz}$, yet when the Glidcop slits were attached, the frequency dropped to around $35-40 \mathrm{~Hz}$ due to the increased mass. The new $x$ - stage was then tested for vibration and became evident that the magnification between the bottom invar stand and the top of the Glidcop slit was almost equal. Testing these $x-y$ stages has allowed me to gain knowledge of how vibration affects these components. I have learned new skills such as vibrational analysis and calibration of seismometers that will allow me to move further in my field.
\end{abstract}

\section{Introduction}

It is clear that vibration has an effect on everyday life, whether riding the train or flying in an airplane, vibration exists everywhere. Since the NSLS II requires a certain specification from the beam, vibration can alter that position drastically with only a few nanometers of magnitude. The goal is to find the source of vibration, take measurements on the ground and on optical components to innovate a plausible solution. Ground vibration data was taken at the beginning of the NSLS II project at a frequency range above $4 \mathrm{~Hz}$, which outputted a magnitude that was in range of specification. Yet the magnitude of vibration increased when the range of frequency was lowered to $2 \mathrm{~Hz}$, implying that the ground vibration had a higher magnitude than originally thought. Ground vibration was tested in several locations around the BNL site, as well as in 1 of the 5 service buildings within the NSLS II.
The service buildings consist of many machines such as pump skids and air handlers which could differentiate vibration levels if they were turned on or off. It was noted that the increased vibration could be caused from the Long Island Expressway traffic, not necessarily the cars but the semi-wheelers since there weight is 20 times larger, causing higher magnitude of vibrations as they drive up and down the road. It was essential to conclude this point, so we started by taking seismometer measurements in five different locations around the BNL site. Gradually increasing the distance from the L.I.E to see if vibration decreased as we got further, this graph can be seen in figure 2. It was also important to figure out the amount of trucks that pass by during a 24 hour period and correlate the vibrational data to the number of trucks. After I was comfortable using the vibrational equipment I started taking measurements on the old $x-y$ stage. Once the invar stand was grouted to the floor, I took separate measurements, one with just the stand, one with the stand and x-y stage and then finally adding the Glidcop slit. Each displayed a common frequency range and corresponding PSD but the transfer function on average got closer to 1 , which indicated that heavier mass will alleviate vibration magnification from the floor to the top of the stage. Knowing the vibrational levels I am able to determine specifications for the $x-y$ stage that will optimize its performance (Torqueing bolts etc.). Since there were some complications with the prototype assembly, unreleased modification drawings were created and used to fix the problems. One of which was to add clearance holes to access the bearing way mounting screws to allow proper fit. The analysis on the $x-y$ stage assemblies is discussed further in the results section of this paper.

\section{Methods}

There are two different types of devices that will measure vibration, one being an accelerometer and the other being a seismometer. An accelerometer is a device that measures proper acceleration using a piezoelectric effect, which contain microscopic

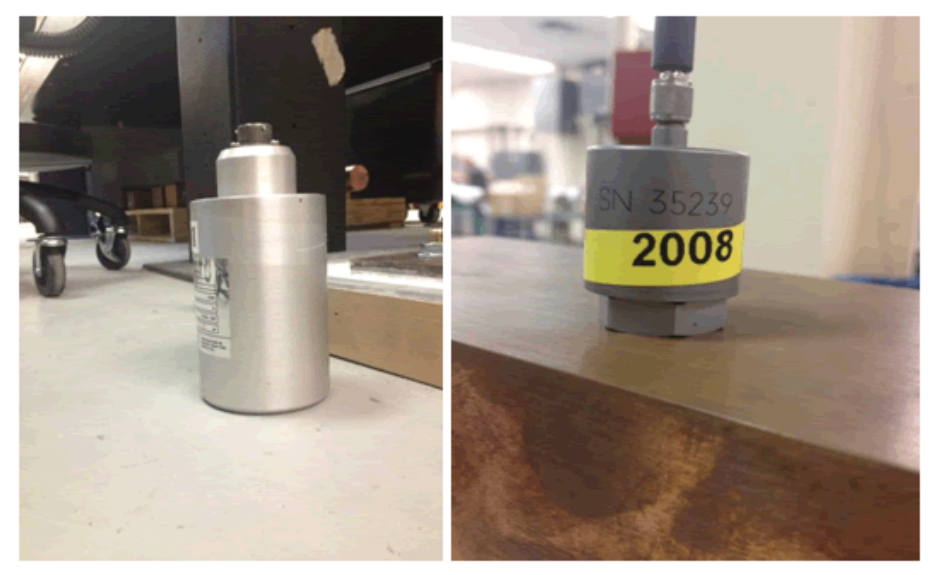

Figure 1: Seismometer (left) and Accelerometer (right) 
crystal structures that get stressed by accelerative forces and causes a voltage to be generated [1]. A seismometer measures velocity and uses a spring mass system to pick up vibration. Each device has a very low natural frequency in comparison to the frequency of the vibrations to be measured so that the ratio becomes very large. Figure 1 below depicts each device.

The equipment for the seismometer vibrational measurements used out on the five BNL test sites is listed in table 1 below.

Table 1: Equipment used for APS vibration measurements (Seismometers)

\begin{tabular}{|l|}
\hline 2 Sercel Seismometers (\#101 and \#103) \\
\hline NI 6259 USB 16 Bit Analog to Digital Convertor \\
\hline 2 amplifiers (Gain=100-500) \\
\hline Cables with Compensation Box \\
\hline Laptop with Labview vibration acquisition software \\
\hline
\end{tabular}

The equipment used for the accelerometers on the $x-y$ stage is slightly different and is located in table 2 .

Table 2: Equipment used for APS vibration measurements (Accelerometer)

\begin{tabular}{|l|}
\hline 6 Accelerometers \\
\hline NI PXI-1042Q Analog to Digital Convertor \\
\hline Laptop with Labview vibration acquisition software \\
\hline
\end{tabular}

I used the six accelerometers to establish five different data sets, each located somewhere on the x-y stage setup. Whether it was on the floor, grouted steel plate, bottom of the stand, top of the stand or on the x-y stage I would use three accelerometers for each location, one for each direction $\mathrm{x}, \mathrm{y}$ and $\mathrm{z}$. There were five channels used on the analog to digital convertor, the corresponding accelerometers and direction are located in table 3.

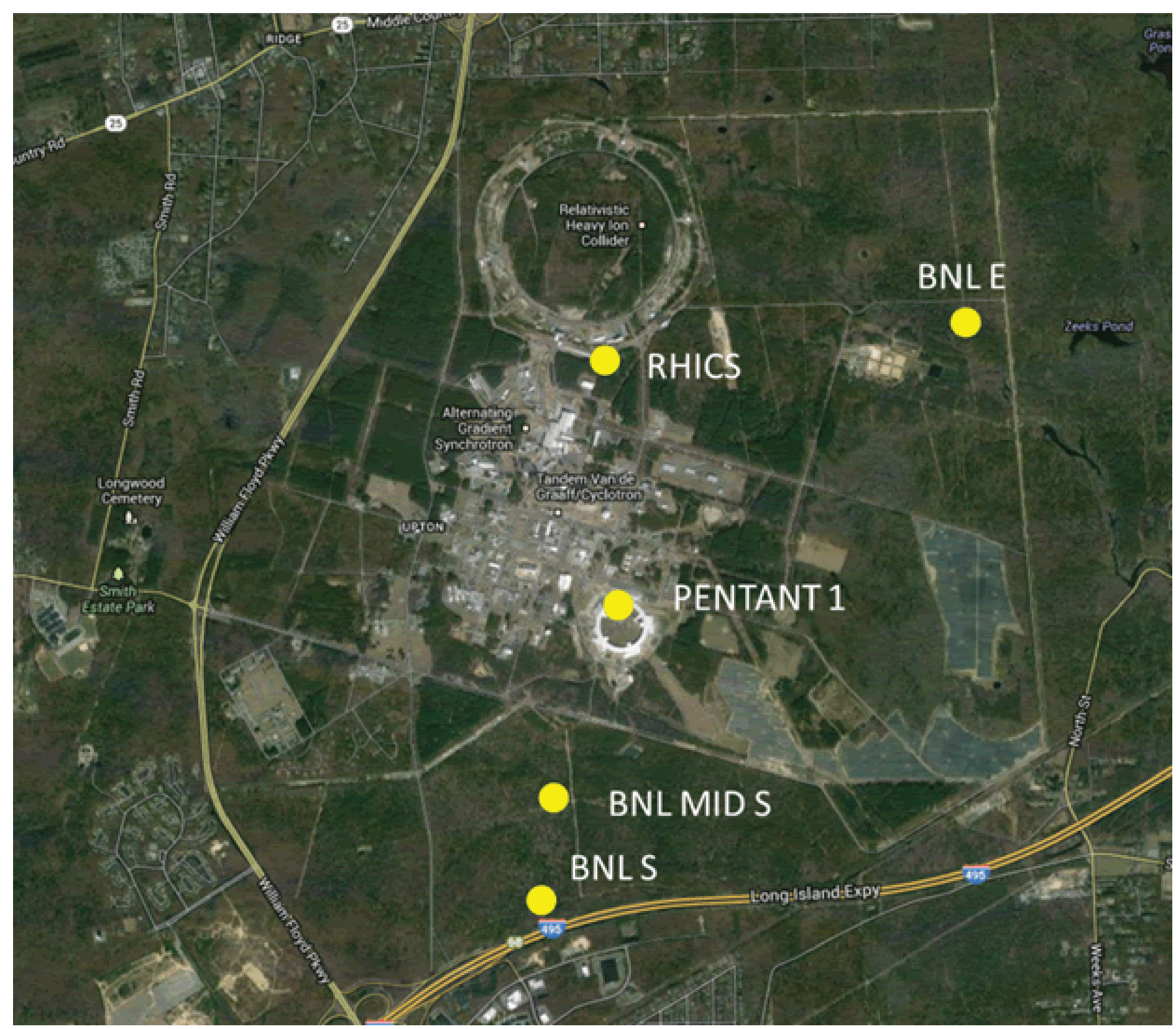

Figure 2: BNL Vibrational Test Site Map 
Table 3: Accelerometer setup

\begin{tabular}{|c|c|c|}
\hline Channel & $\begin{array}{c}\text { Serial Number } \\
\text { (Accelerometer) }\end{array}$ & Direction \\
\hline 0 & 2010 & $\mathrm{x}$ \\
\hline 1 & 2008 & $\mathrm{y}$ \\
\hline 2 & 2005 & $\mathrm{z}$ \\
\hline 3 & 2002 & $\mathrm{x}$ \\
\hline 4 & 2000 & $\mathrm{y}$ \\
\hline 5 & 2007 & $\mathrm{z}$ \\
\hline
\end{tabular}

I started by putting three on the grouted steel plate in which the invar stand sits on and three accelerometers on the bottom stand, and then progressed further up the stand. I ran each test with the vibration acquisition software at a rate of $1.6 \mathrm{kHz}$ for a total of 16000 samples, which is 10 seconds of data. Once I collected the data it was imported into an excel file that calculated the RMS displacement at $2 \mathrm{~Hz}$, these numbers were then divided top over bottom and the output was the magnification between two locations. When the seismometers were used in the vibrational measurements, it was necessary to test the correlation and accuracy of the seismometers prior to taking measurements. First the seismometers would be placed right next to each other and tested to see if they correlated the same signal. Secondly the seismometers are placed horizontally and tested to see if the vibrational measurement is close to zero. Once these tests are finished, the seismometers were ready to take accurate vibration data. For each BNL test site (Figure 2), data was taken to measure the correlation between two seismometers and the ground from zero feet to eighteen feet in nine foot increments. Then the two seismometers were placed right next to each other and were tested to take data every five minutes for one hour.

\section{Results/Conclusions}

After taking vibration data at the five locations located in figure 2 with seismometers, a graph was made to show how plausible it is that the Long Island Expressway is the cause for high levels of vibration. Figure 3 displays the BNL site green field RMS motion vs. Time.

It is noted from the above graph that as the vibration measurements moved farther away from the L.I.E there is a significant drop in RMS motion. This depicts that the traffic on the L.I.E is directly related to the relative ground motion throughout the BNL site. At BNL S which is the closest location from the L.I.E mea-

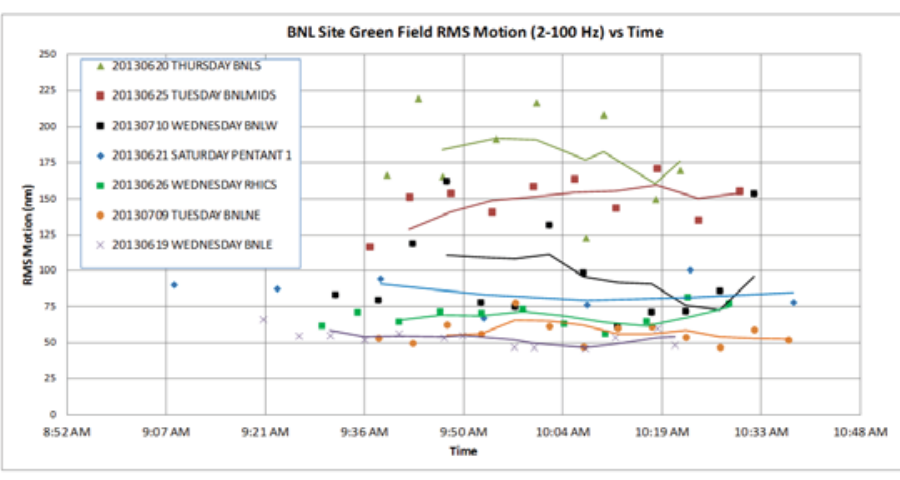

Figure 3: BNL Site Green Field RMS Motion vs. Time surements peak around $223 \mathrm{~nm}$ of RMS motion, yet at the farthest point BNL E, the RMS motion is around $50 \mathrm{~nm}$.

The $x-y$ stage assembly consists of a grouted invar stand, $x-y$ stage and a Glidcop slit. When measurements were being taken, it was best to test the natural frequency in all directions $(\mathrm{x}, \mathrm{y}, \mathrm{z})$ by placing each component of the assembly in order to see the difference. Using Labview sound and vibration assistant, the first test consisted of using six accelerometers, three on the bottom of the invar stand and three on the top of the invar stand. The second test was to put the $x-y$ stage on the invar stand and place three accelerometers on the $\mathrm{x}-\mathrm{y}$ stage in $\mathrm{x}, \mathrm{y}$ and $\mathrm{z}$ directions and three accelerometers on the base of the invar stand. The third test involved placing the Glidcop slit on top of the $x-y$ stage and putting three accelerometers each in $\mathrm{x}, \mathrm{y}$ and $\mathrm{z}$ directions on the slit and three accelerometers on the base of the invar stand. A sample rate of $16 \mathrm{k}$ and a rate of $1.6 \mathrm{k}$ were used with an iteration of 8 ; the results were taken as a screenshot. Figure 4, 5 and 6 depict each of the frequencies displaced from test 1 , test 2 and test 3 respectively.

From the above figures it is clear that the natural frequency seems to significantly drop as the mass increased. Test 1 outputted a frequency value around $70-75 \mathrm{~Hz}$, test 2 resulted in a frequency around $50-60 \mathrm{~Hz}$ and test $3 \mathrm{had}$ a frequency range of around 30-35 $\mathrm{Hz}$. The next step was to test the transfer function with the Glidcop on each $x-y$ stage old and new. Initially I torqued the bolts that hold the stage in place to $50 \mathrm{ft}-\mathrm{lbs}$ then increased to $150 \mathrm{ft}-\mathrm{lbs}$ in $50 \mathrm{ft}-\mathrm{lb}$ increments. Table 4 displays the magnification values

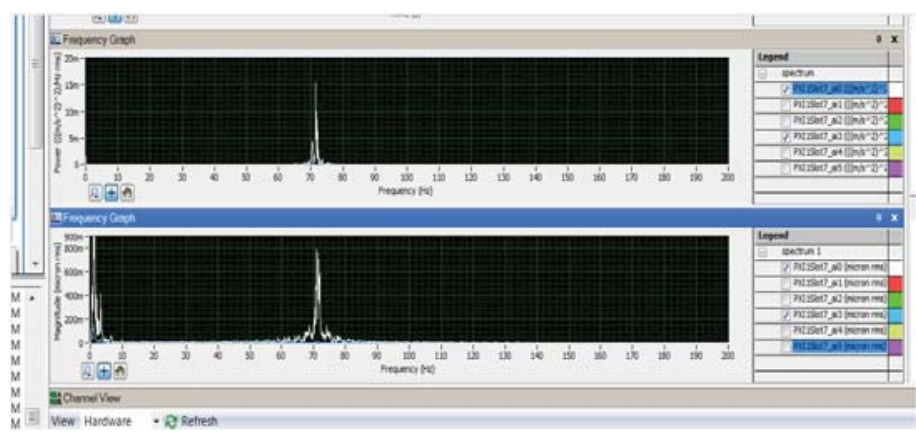

Figure 4: Natuaral Freq, PSD and displacement. Invar Stand

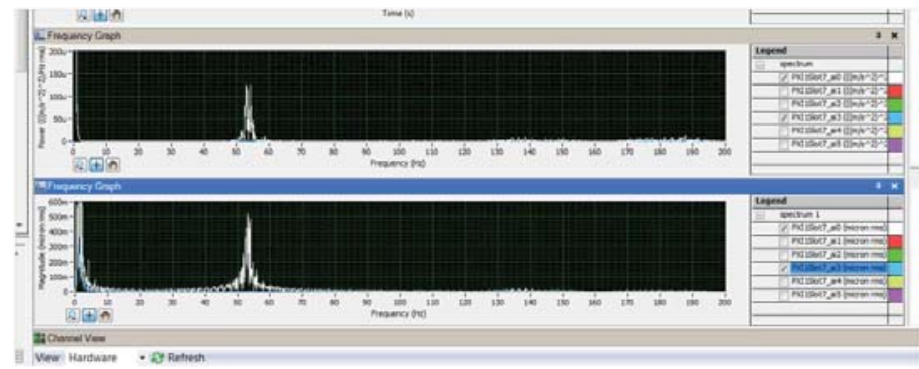

Figure 5: Natuaral Freq, PSD and displacement. Invar stand w/ x-y stage mounted.

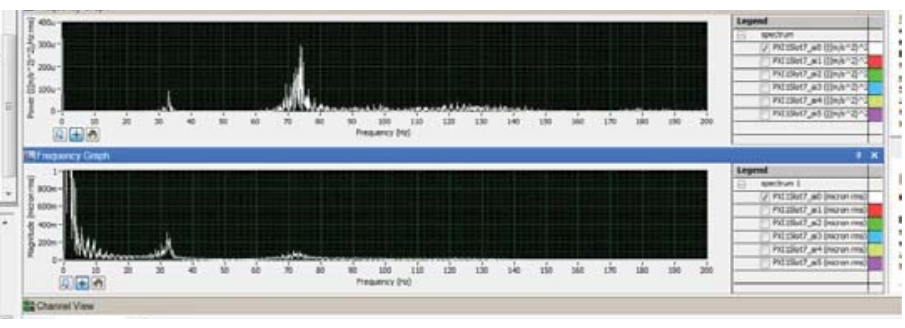

Figure 6: Natuaral Freq, PSD and displacement. Invar stand w/ x-y stage and Glidcop slit 
from the top of the stage to the bottom of the invar stand. Table 4: x-y stage w/ Glidcop Transfer Function @ 50 ft-lbs

\begin{tabular}{|c|c|l|l|}
\hline Channel & Direction & \multicolumn{1}{|c|}{ Location } & Magnification \\
\hline Ch 0 & X & $\begin{array}{l}\text { x-y stage } \\
\text { Bottom of } \\
\text { Invar Stand }\end{array}$ & 1.43 \\
\hline Ch 1 & Y & $\begin{array}{l}\text { Top of Glid- } \\
\text { cop slit } \\
\text { Bottom of } \\
\text { Invar Stand }\end{array}$ & Approx. 1 \\
Ch 4 & Y & $\begin{array}{l}\text { x-y stage } \\
\text { Bottom of } \\
\text { Invar Stand }\end{array}$ & 1.71 \\
Ch 2 5 & Z &
\end{tabular}

The goal to reach for the transfer function is to get a value that is close to 1 , the values above are relatively close at $50 \mathrm{ft}-\mathrm{lbs}$. Table 5 illustrates the magnification values for a torque specification of $100 \mathrm{ft}-1 \mathrm{bs}$.

Table 5: x-y stage w/ Glidcop Transfer Function @ 100 ft-lbs

\begin{tabular}{|c|c|l|l|}
\hline Channel & Direction & \multicolumn{1}{|c|}{ Location } & Magnification \\
\hline Ch 0 & X & $\begin{array}{l}\text { x-y stage } \\
\text { Bottom of } \\
\text { Invar Stand }\end{array}$ & 1.13 \\
\hline Ch 4 & Y & $\begin{array}{l}\text { Bottom of } \\
\text { Invar Stand } \\
\text { Top of Glid- } \\
\text { Cop slit }\end{array}$ & Approx. 1 \\
\hline Ch 2 & Z & $\begin{array}{l}\text { X-y stage } \\
\text { Bottom of } \\
\text { Ch 5 }\end{array}$ & 1.45 \\
\hline
\end{tabular}

It is noted from above that the magnification value decreased significantly in the $\mathrm{x}$ and $\mathrm{z}$ direction, this defined the hypothesis that an increase in stability will decrease magnification from the ground to the top of the stage. Table 6 is the last torque specification of $150 \mathrm{ft}-\mathrm{lbs}$ and is displayed below.

Table 6: x-y stage w/ Glidcop Transfer Function @ 150 ft-lbs

\begin{tabular}{|c|c|l|l|}
\hline Channel & Direction & \multicolumn{1}{|c|}{ Location } & Magnification \\
\hline Ch 0 & X & $\begin{array}{l}\text { x-y stage } \\
\text { Bottom of } \\
\text { Invar Stand }\end{array}$ & Approx. 1 \\
\hline Ch 4 & Y & $\begin{array}{l}\text { Bottom of } \\
\text { Invar Stand } \\
\text { Top of Glid- } \\
\text { cop slit }\end{array}$ & 1.16 \\
\hline Ch 2 & Z & $\begin{array}{l}\text { x-y stage } \\
\text { Bottom of } \\
\text { Invar Stand }\end{array}$ & 1.03 \\
\hline
\end{tabular}

It was evident after three separate trials at $150 \mathrm{ft}-\mathrm{lbs}$ torque that the magnification dropped significantly close to one. This is recognized as a stable assembly since vibration magnification from the ground to the top of the $x-y$ stage is limited. Once the old stage was finished with its testing, I was able to place the new $x-y$ stage up on the invar stand and begin the same transfer function testing. The $50 \mathrm{ft}-\mathrm{lbs}$ torque magnification specifications for the new $\mathrm{x}-\mathrm{y}$ stage with Glidcop are located in table 7.

Table 7: new x-y stage w/ Glidcop Transfer Function @ 50 ft-lbs

\begin{tabular}{|l|c|l|}
\hline Channel & Direction & Magnification \\
\hline $\mathrm{Ch} 0 \& 3$ & $\mathrm{x}$ & 1.7 \\
\hline $\mathrm{Ch} 1 \& 4$ & $\mathrm{y}$ & Approx 1 \\
\hline $\mathrm{Ch} 2 \& 5$ & $\mathrm{z}$ & 1.17 \\
\hline
\end{tabular}

Looking back at table 4, the comparison in table 7 between directions is similar, only being off by a fraction of 1 . This implication states that the vibration magnification of the old vs. new stage is resonating at almost the same rate. Tables 8 and 9 will display the magnification rates for $100 \mathrm{ft}-\mathrm{lbs}$ and $150 \mathrm{ft}-\mathrm{lbs}$ torques respectively.

Table 8: new x-y stage w/ Glidcop Transfer Function @ 100 ft-lbs

\begin{tabular}{|c|c|l|}
\hline Channel & Direction & \multicolumn{1}{|c|}{ Magnification } \\
\hline Ch $0 \& 3$ & $\mathrm{x}$ & 1.65 \\
\hline Ch $1 \& 4$ & $\mathrm{y}$ & Approx 1 \\
\hline Ch $2 \& 5$ & $\mathrm{z}$ & 1.28 \\
\hline
\end{tabular}

Table 9: new x-y stage w/ Glidcop Transfer Function @ 150 ft-lbs

\begin{tabular}{|l|c|l|}
\hline Channel & Direction & Magnification \\
\hline $\mathrm{Ch} 0 \& 3$ & $\mathrm{x}$ & 1.6 \\
\hline $\mathrm{Ch} 1 \& 4$ & $\mathrm{y}$ & Approx 1 \\
\hline $\mathrm{Ch} 2 \& 5$ & $\mathrm{z}$ & 1.24 \\
\hline
\end{tabular}

Now that the transfer function between the bottom stand and top of the $\mathrm{x}-\mathrm{y}$ stage are clear for each torque setting, this data can be used to optimize certain parameters of the $x-y$ stage so that the beam can be efficient and stable.

\section{Acknowledgements}

This project was supported in part by the U.S. Department of Energy, Office of Science, Office of Workforce Development for Teachers and Scientists (WDTS) under the Science Undergraduate Laboratory Internships Program (SULI). I would also like to thank my mentor Sushil Sharma as well as Charles Spataro and everyone in building 820 for their support and knowledge in assisting me through this internship.

\section{References}

NSLS-II CDR Conceptual Design Report, Brookhaven National Laboratory, 2006

http://www.bnl.gov/nsls2/project/CDR/

Present Status of the Mechanical Systems of NSLS-II,

Sushil Sharma (BNL), presented at The 2012 Medsi

Conferecnce, Shanghai, China 


\title{
The study of the carbon monoxide oxidation reaction using copper-titania catalysts
}

\author{
Naa Adokaley Pappoe \\ The City College of New York, New York, NY, 10031 \\ Jose Rodriguez, Sanjaya D. Senanayanke, Wengian Xu, Zongyuan Liu, Kumudu Mudiyanselage and Siyu Yao \\ Chemistry Department, Brookhaven National Laboratory, Upton, NY 11973 \\ Aaron Johnston-Peck \\ Center for Functional Nanomaterials, Brookhaven National Laboratory, Upton, NY 11973
}

\begin{abstract}
Carbon monoxide (CO) oxidation is a catalytic reaction that uses metal based catalysts $(\mathrm{Metal}=\mathrm{Cu}, \mathrm{Pt}, \mathrm{Au})$ in the conversion of $\mathrm{CO}$ to carbon dioxide $\left(\mathrm{CO}_{2}\right)$ upon reaction with oxygen $\left(\mathrm{O}_{2}\right)$. This is an important reaction used in industry to clean gas streams of $\mathrm{CO}$. We aimed to understand this reaction using a novel catalyst composed of both copper and titania. Our experiments were focused on two powder catalysts; $\mathrm{CuTiO}_{\mathrm{x}}$ and pure $\mathrm{CuO}$ to be studied with $\mathrm{CO}$ oxidation with the aid of several characterization techniques including Transmission Electron Microscopy (TEM), X-ray Diffraction(XRD) and Diffuse Reflectance Infrared Fourier Transform Spectroscopy (DRIFTS) to provide information pertaining to the catalyst morphology, structure and adsorbed surface species, respectively. Our goal was to better understand and interpret the activity of these catalysts in the carbon monoxide oxidation reaction, and to correlate it to the structure and identify likely active components. Our experiments focused particularly on the reaction mechanism, showing changes in the catalyst surface during the $\mathrm{CO}$ oxidation reaction. Comparisons between the two catalysts were performed under the same reaction conditions. Our results identified that $\mathrm{CuTiO}_{2}$ was more active than bulk $\mathrm{CuO}$ in the $\mathrm{CO}$ oxidation reaction due to its lower onset temperature point and better stability at higher temperatures. It also identified that the surface $\mathrm{Cu}^{1+}$ species visible in the $\mathrm{Cu}-\mathrm{TiO}_{2}$ is likely to be a key player in the $\mathrm{CO}$ oxidation mechanism.
\end{abstract}

\section{INTRODUCTION}

Carbon monoxide (CO) is a highly toxic poison, produced in large quantities from numerous industrial processes and automotive exhausts. Research focused on limiting or remediating the emission of this pollutant is very important to protect the environment and the health of the general population. The removal of $\mathrm{CO}$ can be achieved by a catalytic process that involves oxidation with $\mathrm{O} 2$ and conversion into carbon dioxide $\left(\mathrm{CO}_{2}\right)$.

$$
2 \mathrm{CO}+\mathrm{O}_{2} \rightarrow 2 \mathrm{CO}_{2}
$$

This process is commonly used in industry at low temperatures1, to clean carbon monoxide streams, typically following other reactions such as the Water-Gas Shift Reaction (WGSR). The $\mathrm{CO}$ even at low concentration can affect the use of $\mathrm{H} 2$ as a viable fuel, and $\mathrm{CO}_{2}$ is a gas that is less toxic to the environment as opposed to carbon monoxide, but is nonetheless a major contributor to greenhouse gases. In automotives, catalytic converters are utilized to 'convert' the carbon monoxide emitted from the engine by reaction against $\mathrm{O}_{2}$ gas and nitric/nitrous oxide $\left(\mathrm{NO}_{\mathrm{x}}\right)$ to make carbon dioxide (and nitrogen $\left(\mathrm{N}_{2}\right)$. In both cases, catalysts are used for these conversion processes. Typically, the types of catalyst used for this reaction involve expensive precious metals such as $\mathrm{Au}, \mathrm{Pt}, \mathrm{Rh}, \mathrm{Pd}$ and oxides such as $\mathrm{CeO}_{\mathrm{x}}, \mathrm{FeO}_{\mathrm{x}}$ and $\mathrm{TiO}_{\mathrm{x}}$.

Numerous fundamental investigations and studies are devoted to this reaction to understand: how these catalysts work, what the active components are, and what the predominant reaction mechanisms are? Some of these reaction mechanisms include Langmuir -Henshlewood(L-H) which is based on the theory that both reactants are adsorbed on the catalysts surface in order to form a bimolecular reaction and the Ely-Rideal (E-R) mechanism which is based on the theory of only one reactant adsorbed firmly on the catalysts while the other strikes the adsorbates from the gas phase in order to form a bond without adsorbing. ${ }^{2}$ Between these two mechanisms, the commonly favored towards the co oxidation reaction is that of the Langmuir-Henshelwood (L-H), reason being that the $\mathrm{CO}$ oxidation reaction is a surface reaction occurring at the solid-gas interface.

Our approach includes experiments with Transmission Electron Microscopy (TEM), X-ray diffraction (XRD) and Diffuse Reflectance Infrared Fourier Transform Spectroscopy (DRIFTS). Transmission Electron Microscopy is a technique in which a beam of electrons, travel through an ultra-thin specimen, and interacts with it forming a well focused image on a fluorescent screen. ${ }^{3}$ The images formed are at high atomic resolution and contain information on elemental composition and electronic structure at the ultimate sensitivity of a single atom. ${ }^{3}$ X-ray diffraction (XRD) is the technique used to determine the crystalline structure of a sample on the basis of scattering or diffraction of the x-ray beams. Diffuse Reflectance Infrared Fourier Transform Spectroscopy (DRIFTS) is a method of collecting and analyzing scattered infrared rays on powdered samples to determine the adsorbed surface species during the reaction. Data from most of these techniques are collected in situ. In situ allows one to take measurements and observe changes occurring in the sample during the catalytic reaction process.

The aim of this project is to study a powder catalyst composed of $\mathrm{Cu}-\mathrm{TiO}_{2}$, a cheaper alternative, which is also active for the $\mathrm{CO}$ oxidation reaction. Oxide based catalysts were used due to their selectivity towards particular gases as well as their lower costs as compared to that of noble metals such as gold, platinum, silver and palladium. ${ }^{4}$ We compare the results with that of a base catalyst, $\mathrm{CuO}_{2}$ to elucidate the role of the $\mathrm{Cu}$-Ti using several advanced characterization tools and techniques. The key questions we hope to answer about the $\mathrm{Cu}-\mathrm{TiO}_{2}$ catalyst include: what is the $\mathrm{Cu}$ species $\left(\mathrm{Cu}(0), \mathrm{Cu}_{2} \mathrm{O}(1+), \mathrm{CuO}(2+)\right)$ and $\mathrm{Ti}$ species (Ti4+, $\mathrm{Ti} 3+)$ that prevails during the reaction, which components are the most important (interface, $\mathrm{Cu}, \mathrm{Ti}$ ), and what is the reaction mechanism? This study can lead to a better understanding of how these catalysts behaves in the presence of carbon monoxide and how 
this molecule is converted into carbon dioxide.

\section{EXPERIMENTAL}

\section{A. Catalyst preparation}

Samples of anatase $\mathrm{TiO}_{2}$ was calcined to $500^{\circ} \mathrm{C}$ in air flow prior to incorporating a cupric nitrate precursor $\left(\mathrm{Cu}\left(\mathrm{NO}_{3}\right)_{2}\right)$. The $5 \mathrm{wt} \% \mathrm{Cu} / \mathrm{TiO}_{2}$ catalysts was prepared by a deposition-precipitation method (DP) using $\mathrm{Na}_{2} \mathrm{CO}_{3}$ as the precipitating agent, which was added to the $\mathrm{TiO} 2$ and $\mathrm{Cu}$ precursor in order to keep a neutral $\mathrm{pH}$ of 7 at $70^{\circ} \mathrm{C}$. In these conditions, the copper precipitated as $\mathrm{Cu}(\mathrm{OH})_{2}$. The $\mathrm{Cu} / \mathrm{TiO}_{2}$ sample was calcined to $500^{\circ} \mathrm{C}$ in air flow following the deposition-precipitation process. The reduction experiments of the $\mathrm{Cu} / \mathrm{TiO}_{2}$ in $\mathrm{CO}$ and the catalytic oxidation of $\mathrm{CO}$ were conducted at temperatures less than $300^{\circ} \mathrm{C}$, much lower than that of the calcination process at $500^{\circ} \mathrm{C}$.

\section{B. STEM}

The high resolution TEM images of the $\mathrm{Cu} / \mathrm{TiO}_{2}$ sample was taken using a High Angular Annular Dark Field Scanning Transmission Microscopy (HAADF STEM) instrument. The powder sample was dispersed as a suspension in deionized water, sonicated for 60 seconds then introduced in drops onto a Holey-C grid and allowed to dry before imaging. Electron energy loss spectroscopy (EELS) and energy disperse X-ray spectroscopy (EDS) chemical maps were acquired on a Hitachi $2700^{\circ} \mathrm{C}$ operated at $200 \mathrm{kV}$ using convergence semi-angles of 23 and $28 \mathrm{mrad}$, respectively.

\section{XRD}

This study was carried out on beamline X7B $(\lambda=0.3196 \AA)$ of the National Synchrotron Light Source (NSLS) at Brookhaven National Laboratory (BNL). The powder samples of 2-3 mg were loaded into a silica capillary ( $0.9 \mathrm{~mm}$ ID, $1.0 \mathrm{~mm}$ OD) mounted in the flow cell system. A Perkin Elmer Amorphous Silicon Detector was used to collect two-dimensional transmission diffraction data, which were subsequently processed with the program Fit2D to obtain XRD profiles( Intensity versus $2 \theta$ ). Lattice parameters and phase quantities were analyzed by the Rietveld Refinement using the program GSAS.

\section{DRIFTS}

DRIFTS data were collected on $\mathrm{CuTiOx}$ and pure $\mathrm{CuO}$ under carbon monoxide oxidation conditions with the help of a Bruker Equinox 55 FTIR spectrometer equipped with a modified Harrick Praying Mantis DRIFT cell that was connected to a gas flow system and a mass spectrometer. Carbon monoxide, oxygen and helium with concentrations of $5 \%, 20 \%, 30 \%$ respectively, were allowed to flow through the sample at ratio of $8: 1: 7$. The gas flow rate used in this study was $10 \mathrm{cc} / \mathrm{min}$. Stepwise heating and cooling with an increment of $50^{\circ} \mathrm{C}$, starting from room temperature up to $250^{\circ} \mathrm{C}$ was applied. The temperature was held for $20-30$ mins at each step were the temperature reading stabilized. The composition of the outflow gas was analyzed with the mass spectrometer.

\section{RESULTS}

\section{A. STEM}

The STEM images illustrate the orientation of this catalyst composed of tiny copper cluster sample on the bulk $\mathrm{TiO}_{\mathrm{x}}$. It is arranged in this manner because $\mathrm{TiO}_{\mathrm{X}}$ is the supporting material; also with this orientation copper has the chance of interacting with its support during the reaction. In a catalysts the support carries majority of the weight, thus leaving a little portion of the active phase, which in this case is copper. The sample contains spherical particles of both $\mathrm{CuO}$ and $\mathrm{CuTiO}_{2}$ with sizes of 2-3 nm and 20-50 nm respectively.
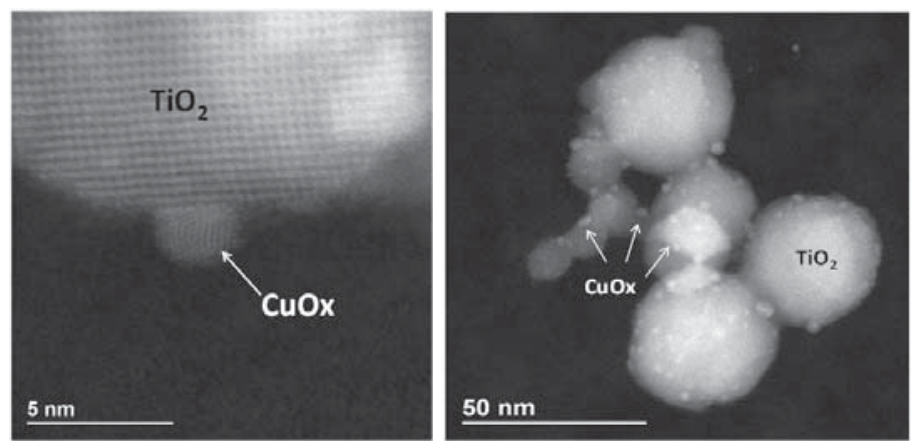

Figure 1. STEM images of CuTiOx catalysts

\section{B. XRD}

$\mathrm{TiO}_{2}$ exists in various crystalline forms, the most common forms being anatase and rutile $\#$. The powder diffraction pattern indicates the presence of these two crystalline structures alongside that of $\mathrm{CuO}$. The peak intensity variations in this graph are higher for that of the anatase phase and lower for that of the rutile phase. This indicates that the $\mathrm{TiO}_{2}$ present in this sample is predominantly anatase. The compositions of the various crystalline structures are $85 \%, 13 \%$ and $2 \%$ respectively.. The $\mathrm{CuO}$ appears assymetrical to the rutile peak at around 7.20.

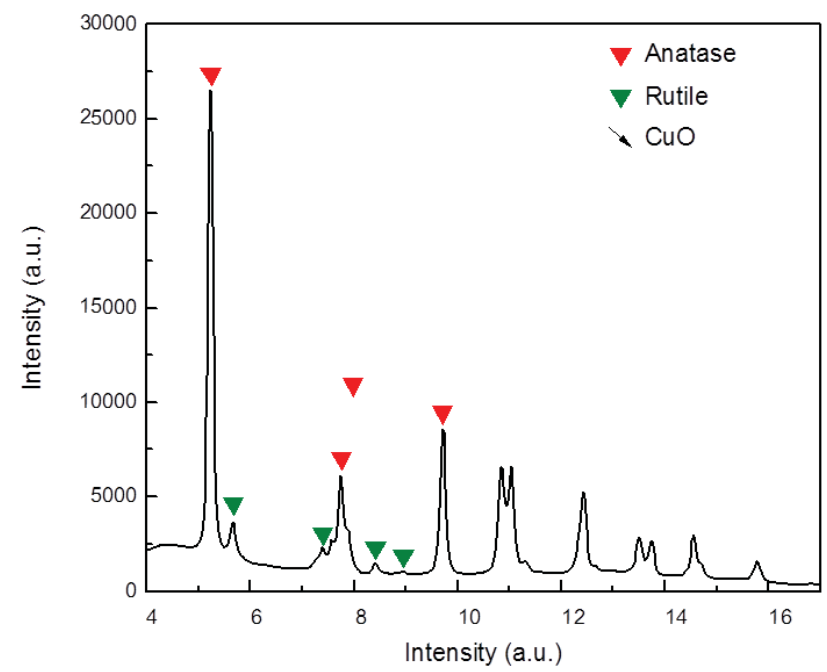

Figure 2. XRD of CuTiOx catalysts

\section{DRIFTS}

Figure 3 shows infrared spectra recorded while $\mathrm{CuTiO}_{2}$ is heated to different temperatures. At $25^{\circ} \mathrm{C}$ room temperature, $\mathrm{CO}$ adsorbed on $\mathrm{Cu}^{1+}$ is formed having a peak centered at $2120 \mathrm{~cm}^{-1}$. There is the formation of a peak at $2697 \mathrm{~cm}^{-1}$ indicating the presence of hydrocarbons due to contamination. The $\mathrm{CO}$ adsorbed on $\mathrm{Cu} 1+$ peak gradually decreases as the temperature increases. At a $100^{\circ} \mathrm{C}$ a new peak formation begins. This peak is the $\mathrm{CO}_{2}$ gas phase and it is identified by it characteristic CO band 2349 $\mathrm{cm}^{-1}$ indicating the start of the co oxidation reaction. The $\mathrm{CO}_{2}$ gas phase peak intensifies as the temperature increases to $250^{\circ} \mathrm{C}$, where as the $\mathrm{CO}$ on $\mathrm{Cu}^{1+}$ decreases. There is an increase in the 
production of $\mathrm{CO}_{2}$ gas with increasing temperature and depletion in the $\mathrm{CO}$ adsorbed on $\mathrm{Cu}^{1+}$ content.

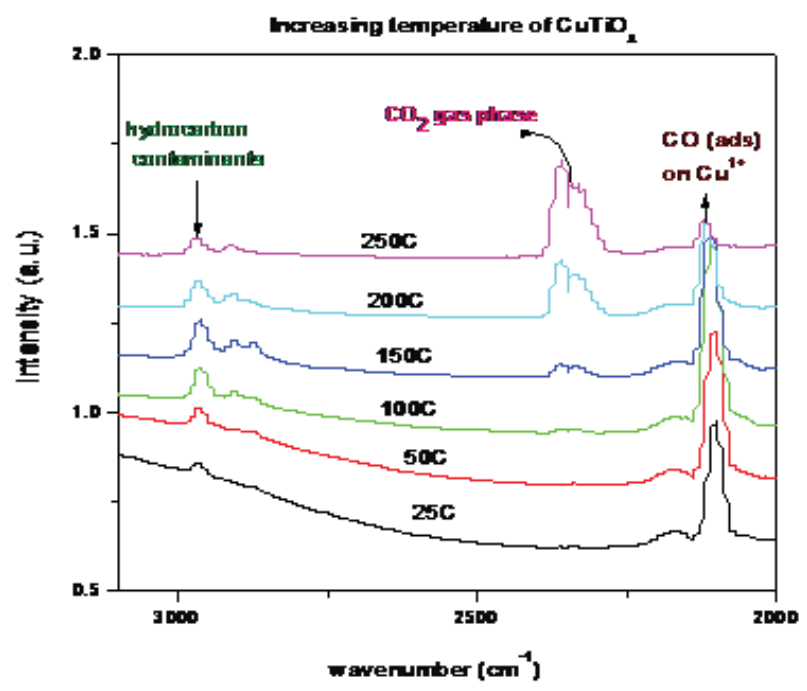

Figure 3. DRIFTS of CuTiOx at increasing temperatures of 25, 50, $100,150,200$ and $250^{\circ} \mathrm{C}$ during $\mathrm{CO}$ oxidation reaction.

Figure 4 shows the graph at decreasing temperatures for $\mathrm{CuTiO}_{2}$. At 250C two peaks appear at $2349 \mathrm{~cm}^{-1}$ and $2141 \mathrm{~cm}^{-1}$ each indicating the $\mathrm{CO}_{2}$ gas phase and $\mathrm{CO}$ adsorbed on $\mathrm{Cu} 1+$ respectively. The $\mathrm{CO}_{2}$ gas phase peak gradually decreases where as the $\mathrm{CO}$ adsorbed on $\mathrm{Cu}^{1+}$ increases. At $25^{\circ} \mathrm{C}$ the peak centered at $2349 \mathrm{~cm}^{-1}$ diminishes completely but the peak centered at 2141 $\mathrm{cm}^{-1}$ intensifies greatly. This point indicates where $\mathrm{CO}$ oxidation process ceases. The graph represents the inverse relationship to that of $\mathrm{CuTiO}_{2}$ at increasing temperatures.

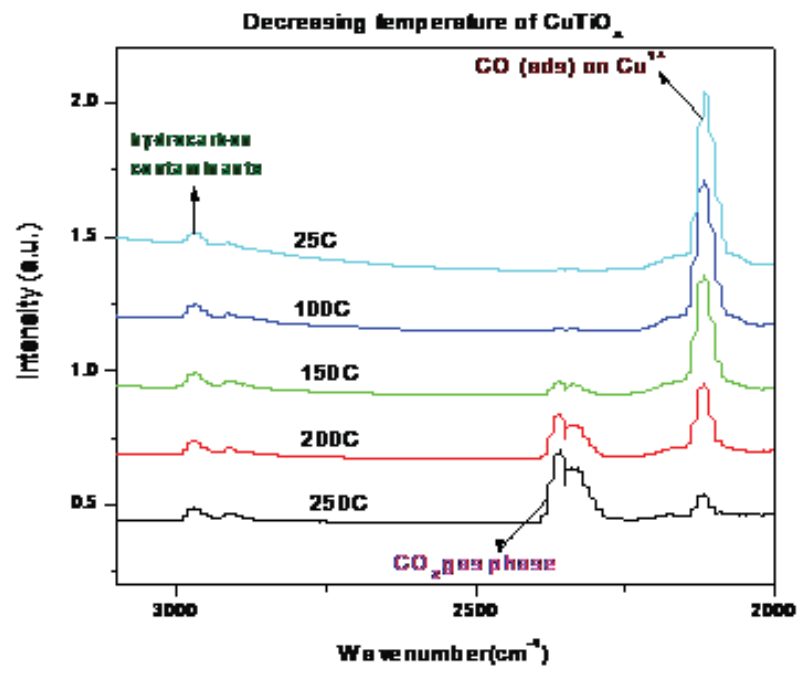

Figure 4. DRIFTS of CuTiOx at increasing temperatures of 25, 50, $100,150,200$ and $250^{\circ} \mathrm{C}$ during $\mathrm{CO}$ oxidation reaction.

In figure 5 at $25^{\circ} \mathrm{C}$, room temperature there is a hump-like feature formation with center at $2141 \mathrm{~cm}^{-1}$. This peak indicates the production of $\mathrm{CO}$ gas phase in $\mathrm{CuO}$. However as the temperature is increased, the hump gradually reduces till it flattens out at tem- peratures of $250^{\circ} \mathrm{C}$. With the temperature increasing so does the formation of a new peak emerge at $150^{\circ} \mathrm{C}$. The peak constantly intensifies from temperatures $150^{\circ} \mathrm{C}$ to $250^{\circ} \mathrm{C}$ with peak centered at $2349 \mathrm{~cm}^{-1}$. There is absence of absorbed $\mathrm{CO}$ on $\mathrm{Cu}^{1+}$ which is often seen with a peak centered at $2120 \mathrm{~cm}^{-1}$. There are no peaks within $2697 \mathrm{~cm}^{-1}$ region of this graph thus indicating the absence of hydrocarbon contaminants.

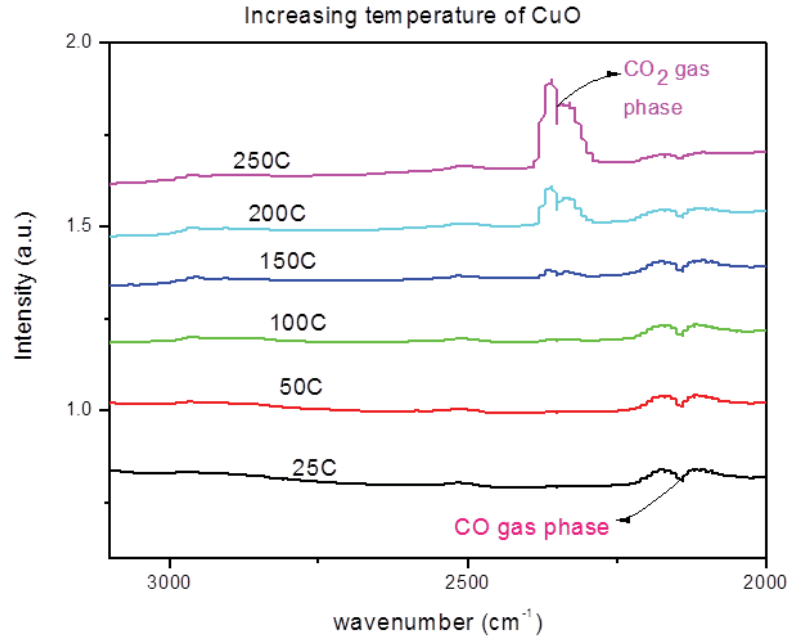

Figure 5. DRIFTS of $\mathrm{CuO}$ at increasing temperatures of 25, 50, 100, 150, 200 and $250^{\circ} \mathrm{C}$ during $\mathrm{CO}$ oxidation reaction.

Figure 6 represents the comparison of both $\mathrm{CuTiO} 2$ and $\mathrm{CuO}$ at different temperatures of $100 \mathrm{C}$ and $150 \mathrm{C}$. Analyzing the $100 \mathrm{C}$ graph, it was observed, there was a production of more $\mathrm{CO} 2$ gas content on the $\mathrm{CuTiO} 2$ than $\mathrm{CuO}$. There is an appearance of an intensified peak feature, which centers at $2120 \mathrm{~cm}-1$ symbolizing $\mathrm{CO}$ adsorbed on $\mathrm{Cu} 1+$. However on the $\mathrm{CuO}$ there is only the production of $\mathrm{CO}$ gas phase with no indication of $\mathrm{Cu} 1+$ species. This is represented by the hump-like feature with centers at 2141 $\mathrm{cm}^{-1}$. With the graph showing the comparison of both samples at $150^{\circ} \mathrm{C}$, there is a higher production of the $\mathrm{CO}_{2}$ gas phase on $\mathrm{Cu}-$ $\mathrm{TiO}_{2}$ with a peak centered at $2349 \mathrm{~cm}^{-1}$. On the $\mathrm{CuO}$ the production of the $\mathrm{CO}_{2}$ gas phase is relatively small compared to that of the $\mathrm{CuTiO}_{2}$. On the same graph there is an intensified peak feature representing the $\mathrm{CO}$ adsorbed on $\mathrm{Cu}^{1+}$ on the $\mathrm{CuTiO}_{2}$, whereas on the $\mathrm{CuO}$ there is the formation of a hump-like peak feature with very less intensity centered at $2141 \mathrm{~cm}^{-1}$, representing the $\mathrm{CO}$ gas phase. The absence of the sharp peak representative of $\mathrm{CO}$ adsorbed on $\mathrm{Cu}^{1+}$ species is shown on that of the $\mathrm{CuO}$ sample. Placing these figures side by side it is evident that at $150^{\circ} \mathrm{C}$ the activity of $\mathrm{CO}$ oxidation is faster than at lower temperatures of $100^{\circ} \mathrm{C}$. The peak in the $150^{\circ} \mathrm{C}$ graph is lower compared to $100^{\circ} \mathrm{C}$ graph, meaning at higher temperatures $\mathrm{CO}$ oxidation is efficient in the conversion of $\mathrm{CO}$ to $\mathrm{CO}_{2}$. 

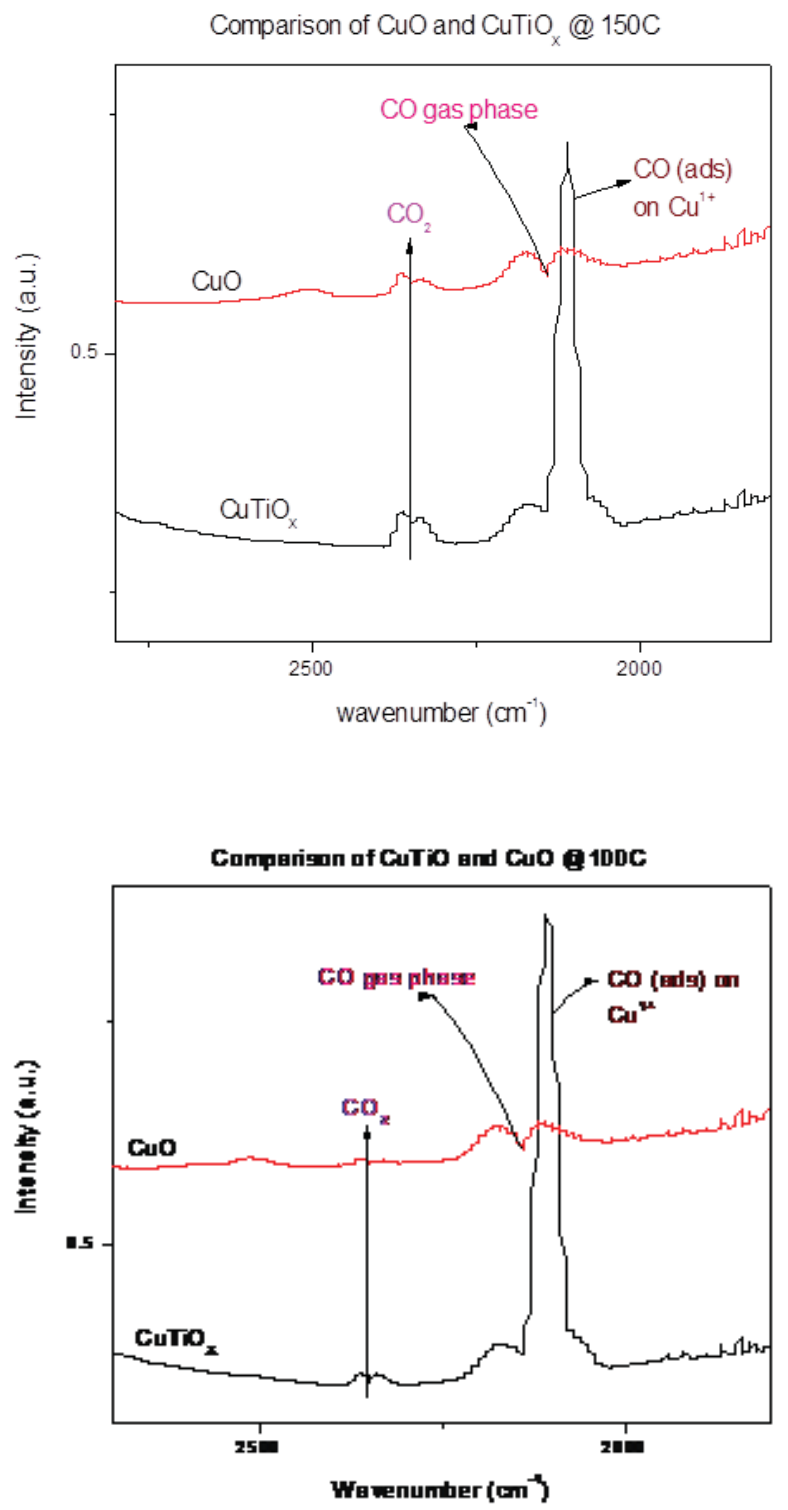

Figure 6. DRIFTS of CuTiOx and $\mathrm{CuO}$ temperatures of 100 and $150^{\circ} \mathrm{C}$ during $\mathrm{CO}$ oxidation reaction.

In figure 7 , there is a relationship between $\mathrm{CO}$ and $\mathrm{O}_{2}$ being that at $8000 \mathrm{~s}$ there is a gradual decline in intensities with increase in temperature. At $250^{\circ} \mathrm{C}$, it forms a slanted plateau and then it increases at $13000 \mathrm{~s}$ while the temperature is being decreased. The $\mathrm{CO}_{2}$ increases at $50^{\circ} \mathrm{C}$ within a time difference of $750 \mathrm{sec}$. It shows the formation of $\mathrm{CO}_{2}$ at $12500 \mathrm{sec}$ forming a slanted plateau like feature and decreases at around $14000 \mathrm{sec}$. These individual changes of $\mathrm{CO}$ and $\mathrm{O}_{2}$ occur simultaneous with the production of $\mathrm{CO}_{2}$.

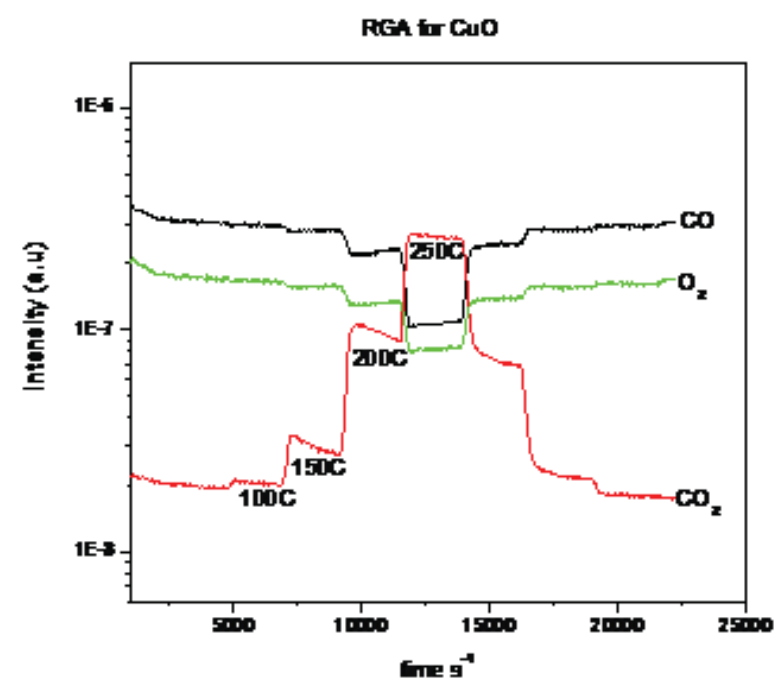

Figure 7. RGA data of $\mathrm{CO}$ oxidation over $\mathrm{CuO}$, showing $\mathrm{CO}(\mathrm{m} / \mathrm{z}$ 28), $\mathrm{O} 2(\mathrm{~m} / \mathrm{z32})$ and $\mathrm{CO} 2(\mathrm{~m} / \mathrm{z} \mathrm{44)}$.

In figure 8 , there is resemblance in pattern consumption of both the $\mathrm{CO}$ and $\mathrm{O}_{2}$ being that at $89250 \mathrm{sec}$ there is a gradual drop in intensities with increase in temperature. At $250^{\circ} \mathrm{C}$, it forms a steady plateau and then it increases at $96250 \mathrm{sec}$ while the temperature decreases. The $\mathrm{CO}_{2}$ increases gradually starting at 87500 $\mathrm{sec}$ at a temperature of $100^{\circ} \mathrm{C}$. It shows the formation of $\mathrm{CO}_{2}$ gas at $94500 \mathrm{sec}$ forming a steady plateau like feature and decreases at around $98000 \mathrm{sec}$. The $\mathrm{CO}_{2}$ gas formation occurs simultaneously with the consumption of $\mathrm{CO}$ and $\mathrm{O}_{2}$.

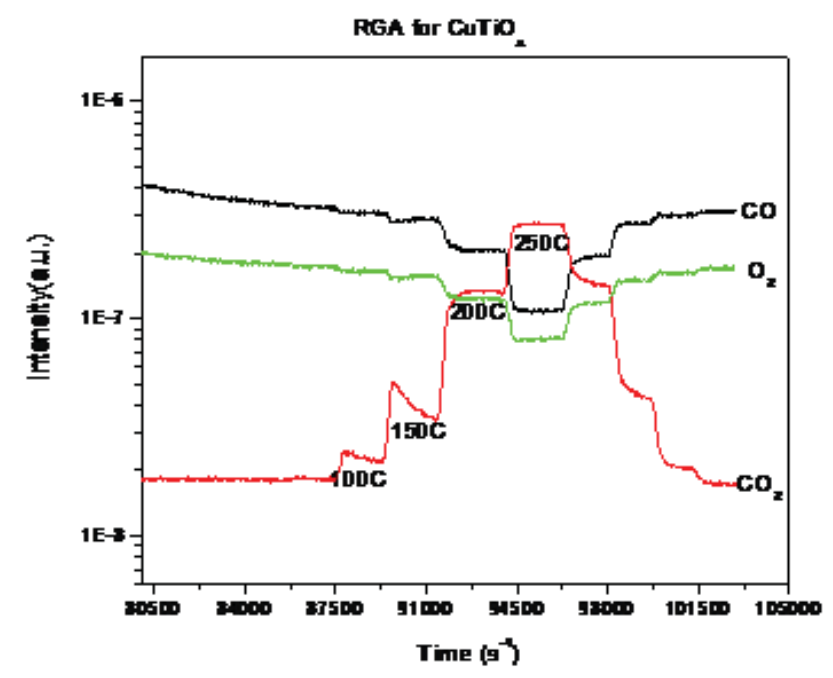

Figure 8. RGA data of $\mathrm{CO}$ oxidation over $\mathrm{CuTiOx}$, showing $\mathrm{CO}(\mathrm{m} / \mathrm{z}$ 28), $\mathrm{O} 2(\mathrm{~m} / \mathrm{z32})$ and $\mathrm{CO} 2(\mathrm{~m} / \mathrm{z} 44)$.

\section{DISCUSSION}

Both $\mathrm{CuTiO}_{2}$ and $\mathrm{CuO}$ showed activity for the $\mathrm{CO}$ oxidation reaction, but $\mathrm{CuTiO}_{x}$ showed an increase of $\mathrm{CO}_{2}$ at lower temperature with a unique $\mathrm{CO}$ adsorbed on $\mathrm{Cu}^{1+}$. This could be an important result for the $\mathrm{CO}$ oxidation reaction, implicating the presence of the $\mathrm{Cu}^{1+}$ aiding in the oxidation of the $\mathrm{CO}$. It may also be likely that at high temperatures like $250^{\circ} \mathrm{C}, \mathrm{CO}$ adsorbed on to $\mathrm{Cu}^{1+}$ is stable on $\mathrm{CuTiO}_{2}$ surface thus leading to the greater 
production of $\mathrm{CO}_{2}$. In addition, the intensified production of the hydrocarbon contaminant peaks could be as a result of $\mathrm{TiO}_{\mathrm{x}}$ poisoning with some $\mathrm{C}-\mathrm{H}$ residue from the reactant stream. The hydrocarbon poison is only obvious on $\mathrm{CuTiO}_{2}$ and not on $\mathrm{CuO}$, at $25^{\circ} \mathrm{C}$ shows a small peak but then intensifies gradually till $250^{\circ} \mathrm{C}$ where the peak reduces due to decomposition at a high temperature.

Furthermore, the RGA graphs of both, $\mathrm{CuTiO}_{\mathrm{x}}$ and $\mathrm{CuO}$ show the consumption of $\mathrm{CO}$ and $\mathrm{O}_{2}$ and production of $\mathrm{CO}_{2}$, but however at different concentrations of gases. The $\mathrm{CuTiO}_{\mathrm{x}}$, has the production of $\mathrm{CO}_{2}$ at a pressure of 2.725 torr whereas $\mathrm{CuO}$ has its production at 2.639 torr. The higher the pressure, the more the production of the $\mathrm{CO}_{2}$ gas. Also in the $\mathrm{CuTiO}_{2} \mathrm{RGA}$, at $250^{\circ} \mathrm{C}$, there is a more enhanced and stable production of $\mathrm{CO} 2$, even though a much smaller quantity of $\mathrm{Cu}$ species was used, however it is well dispersed on the $\mathrm{TiO}_{2}$ and the role of the interface between the $\mathrm{Cu}-\mathrm{Ti}$ is now important.

\section{CONCLUSIONS}

I have studied an important industrial reaction for the remediation of $\mathrm{CO}$ to $\mathrm{CO}_{2}$ using a novel metal oxide catalyst composed of $\mathrm{Cu}$ and $\mathrm{TiO}_{2}$. This catalyst is more active for this reaction than either $\mathrm{CuO}$ or $\mathrm{TiO}_{2}$ on its own. With the use of XRD, TEM and DRIFTS I was able to identify the morphology, structure and surface states that prevailed when the $\mathrm{CO}$ and $\mathrm{O}_{2}$ reacted to produce $\mathrm{CO}_{2}$. We discovered that the $\mathrm{CuTiO}_{2}$ has a unique structure that helped to lower the $\mathrm{CO}$ oxidation reaction temperature, and that the interface between the $\mathrm{Cu}-\mathrm{TiO}_{2}$ provide for the stabilization of $\mathrm{CO}-\mathrm{Cu}^{1+}$ that is likely to be an important part of this reaction.

\section{REFERENCES}

[1] Spendelow, J.S.; Good paster J.D.; Kenis P.J.A.; Wieckowski A. Mechanism of CO oxidation on $\mathrm{Pt}(111)$ in alkaline Media.2006, 110, 9545.

[2] Johnson, R.S.; DeLaRiva, A.; Ashbacher, V.; Halevi B.; Villanueva, C.J.; Smith G.K.; Lin S.; Datye, A.K.; Guo, $\mathrm{H}$. The $\mathrm{CO}$ oxidation mechanism and reactivity on PdZn alloys. 2013, 15, 7768.

[3] Savage, N. O.; Akbar, S.A.; Dutta, P.K. Titanium dioxide based high temperature carbon

monoxide selective sensor. 2001, 239-248.

[4] Xanthopoulou, G.; Vekinis, G. Investigation of catalytic oxidation of carbon monoxide over a $\mathrm{Cu}$-Cr-oxide catalysts made by self-propagating high-temperature synthesis. 1998, 37-44.

[5] Xu, W.; Liu, Z.; Johnston-Peck, A.C.; Senanayake, S.D.; Zhou, G.; Stacchiola, D.; Stach, E.A.; Rodriguez, J.A. Steam reforming of ethanol on $\mathrm{Ni} / \mathrm{CeO} 2$ : Reaction Pathway and Interaction between $\mathrm{Ni}$ and the $\mathrm{CeO} 2$ Support. 2013. 


\title{
Design proposals for the Linac central malfunction user interface
}

\author{
Divas Persaud \\ Department of Electrical Engineering, City College of New York, New York City, NY 10031 \\ Omar Gould \\ Collider Accelerator Department, Brookhaven National Laboratory, Upton, NY 11973
}

\begin{abstract}
Currently, the $200 \mathrm{MeV}$ Linear Accelerator (Linac) Control Room of the Collider Accelerator Department employs a malfunction summation panel that uses SN 7400 TTL logic circuits, miniature light bulbs, and push-button light switches that light up to indicate faults. While this design has been in operation for more than four decades, the setup's cumbersome layout make any malfunction assessment considerably time consuming. Through a new proposed hardware and software system, the upgraded malfunction panel will allow for simpler navigation, diagnostic menus, malfunction logs, and the addition of new devices to better isolate the source of malfunction. Based on the researched conducted on the existing signal characteristics, four designs: Programmable Logic Controllers (PLC), Field-Programmable Gate Array (FPGA), Synoptic, and a hardware-only design: Complementary Metal-Oxide-Semiconductor (CMOS) were researched to determine which one was best capable of handling the existing system while bringing forth the most reliable and ease of use interface.
\end{abstract}

\section{Introduction}

In the current malfunction panel for the Linac, signals are summed to indicate malfunctions from a specific set of locations and individual malfunction conditions of a specified system. For example the security indicator is defined as: The Linac, HEBT or BLIP Tunnels are on Restricted or Controlled Access, or an Access Controls Failure. This one signal can represent a total of 9 malfunctions, such as Access Controls Failure in the BLIP Tunnels and not Controlled Access in the Linac. Other signals require the assessment of multiple panels, while in this situation the malfunction is further isolated, it is still necessary to obtain information from a distinct panel in the Linac Control Room. An example of this is the inline valve indicator, this is defined as: A vacuum isolation valve is closed between any Linac cavities. Once this icon is activated, there is another panel that directs the user to what valve was actually closed.

The primary concept behind this new malfunction panel is to allow the user to seamlessly navigate their way through the interface to locate the source of failure. As the user navigates through the system, the initial breakdown will be done by location, further along, by equipment and/or components. On the main screen, the division of the Linac (LEBT, MEBT, LINAC, HEBT, AGS, Booster and BLIP) will be viewable to immediately inform the user the location of the malfunction. BLIP, Booster and AGS depend on Linac for beam operations; hence certain signals from the current panel contain specific malfunction signals from BLIP, Booster and AGS. A malfunction from those locations not receiving beam might result from a fault in the Linac. If a problem were to arise in LEBT, an indicator will alert the user to LEBT, further along the devices will be indicated, such as the Cryo Pumps and Steering Magnets, which are currently not a part of the existing panel. To continue, the user would navigate through the interface until they view a display that has solid indicator over the display isolating where exactly the source of error is located. The new system will isolate and identify the issue as best possible to decrease downtime of the Linac beam.

\section{Methods and Materials}

Gathering the schematics to show the layout of the Linac was the first step in the research. The Linac is broken down as follows: LEBT (which comprises of the H-minus, OPPIS, and the remaining LEBT region), MEBT, LINAC, and HEBT. For each region, all the devices were taken into account which would be used for the malfunction breakdown. This would also further help in determining how many signals would need to be accounted for, and how much hardware would be needed to input all these signals. Further research and resources accounted for 325 analog signals and approximately 325 digital signals.

Next, were determining the characteristics of the existing signals used in the Linac. This was needed to determine what specifications the new proposals should be capable of handling. Research showed a mixture of analog and digital, DC and Pulsed, with ranges from $0 \mathrm{~V}-12 \mathrm{~V}$ or $0 \mathrm{~V}-15 \mathrm{~V}$, and a minimum pulse width of $35 \mathrm{~ms}$. Therefore, the upgraded malfunction system should be capable of reading pulse width of at least $25 \mathrm{~ms}$. Further research revealed that certain devices contain malfunction cards; this would be one of the simplest and quickest way to detect any malfunction. For example, each power supply has a malfunction card. If a power supply were to go bad in a Linac cavity, determining which cavity and which power supply would occur instantly.

Feedback back from the staff was a crucial criterion for the design, as they will be interfacing with the new panel. A wish list was proposed in the beginning of the project to acknowledge what the staff in Linac would want to see monitored. Given the specifications of the navigation process, a formal breakdown was created. This breakdown included all the items from the existing panel, what was gathered through the schematics, and speaking to the staff. The wish list could now be implemented after the design breakdown, making it easier to integrate the items on the wish list, and avoid any repetitions of the same ideas. Receiving feedback for the malfunction breakdown concept was also taken into account.

Along with acquiring feedback from the staff on functionality of the design, their assessment of the current panel and how it could be improved were taken into consideration. Terminology is an issue; the same device has a different name in some of the newer Allen Bradley monitors compared to what is on the malfunction panels. In this case, there should no discrepancy when referring to a device. Avoiding abbreviations and using the full 
device name will limit discrepancy, allowing staff to simply know which device is being referenced.

A written malfunction $\log$ is also kept inside the control room. The two most recent malfunctions were analyzed. For the first malfunction, HEBT, Booster, and Inline Valve lit up. When HEBT and Booster lit up, it was not an actual malfunction but a result of not receiving beam. Evaluation of Inline Valve revealed that the valve between tank 1 and tank 2 were closed. The source of malfunction was not determined, after a reset, it continued operating properly. For the second malfunction, HEBT 2/3 Vac Closed, High Energy Beamstop Closed, and Inline Valve were lit up. After technicians resolved the Inline Valve issue, the other two indicators were still lit. Later through consultation, it was determined that these two indications were not malfunctions, but remain closed, and are open during testing.

From speaking with technicians and other staff, grasping what the existing malfunction panel offers and doesn't offer, and acknowledging what needs to be addressed, a more intuitive design is needed. Any discrepancy in the design delays staff from fixing a problem. Another addition to the system would be a malfunction log, which would document every malfunction that happens as a reference. This reference will not just be used to document the errors, but isolate what equipment causes numerous malfunctions which might need upgrading or replacement. The malfunction log will be stored in a separate hard drive, where it would be accessible through the display panel. Another reason for the malfunction log being stored in a hard drive is if the malfunction panel were to fail and require maintenance or rebooting, the hard drive would still be accessible with a laptop any other type of device with viewing capabilities. The need for this setup is to decrease downtime in unforeseeable circumstances.

\section{Results}

A hardware-only option was considered due to the longevity and robustness of the existing malfunction panel. As mentioned, the existing malfunction panel is all hardware and has been operating over four decades. CMOS technology using LEDs was chosen because it has the same properties. One of the major criteria that CMOS accounts for is a design without an overhead. An overhead is liable to crashes and malware, so if the network were to ever have issues, this design would still be functioning. LED technology is also reliable, and to account for the LEDs going bad, a test signal would be implemented to make sure all the LEDs are working properly. While a full graphical layout of the Linac can be used to make this design more intuitive, it will not be feasible to account for the number of devices that will need to be monitored. Expandability is another concern, adding new devices to be monitored would require an extended time period compared to the other designs, both on the microchips as well as the display.

Originally, Synoptic was the first design to be considered. Synoptic is a pre-set GUI that originated in Fermilab. It allows for real-time monitoring, fault alarms, and has a visually easy to understand graphical representation. While it does require an overhead, in this case Linux, research showed that Linux is an open source software, allowing users to find security flaws and assist with improving the platform. Linux also offers great server security, along with the fact that it will be behind the Collider Accelerator Department (CAD) firewall, should improve its security. Synoptic has the capability to write and read from hardware through an ADO (Accelerator Device Object). An ADO is a high level language code implemented in an FEC (Front End Computer) that communicates with lower level languages written to speak with the hardware. A driver is used to communicate with a VME Bus and SNAP for FPGAs. The issue is that not all devices carry an ADO. If this design was implemented, it would not immediately be useful, as there are some ADOs and hardware interfaces that would require troubleshooting. The three other proposals would be completely new and schematics would be kept on the layout for future resources, whereas this design would require significant documenting of the existing field configurations.

National Instruments (NI) has a cRIO (Compact Reconfigurable I/O) package, which is an all-in-one chassis that consists of I/O modules, a controller, and FPGAs. A PCI bus transfers data between the controller and FPGA's. The FPGA's reside in the backplane on the chassis where the I/O module will be inputted. The configuration is done through a network connection from NI LabVIEW, where it will communicate with the chassis. In LabVIEW, the block diagrams are created for your design configuration, and the front panel is where the user sees the graphical representation. FPGA hardware far exceeds the design specifications of this project, and it would come at an increased cost. The display however, doesn't fit the criteria of the design, it is time consuming to navigate and is not intuitive. This design would require educating the staff on NI LabVIEW and how to interchange back and forth between the block diagrams and front panel to isolate a malfunction.

Designing a system using PLC would not only allow for $\mathrm{OK} /$ Fail status, but real-time monitoring of analog signals, and it would all be on a visually pleasing display. Its display also allows for navigation with malfunction and alarm logs, and a recommended course of action. This design allows for a versatile user interface that is based on very reliable hardware. However, this comes at a cost since the display would be running on an operating system, making it vulnerable to crashes and malware. The design is space saving, since all the malfunction racks can be replaced by a single monitor. The remote I/O module itself, would save a lot of wiring, it only requires a communication wire to the processor. Currently there is about 325 analog signals and roughly 325 digital signals at the Linac. Using I/O modules with 16 inputs could decrease the amount of long distance wires used to the processor by a factor of 15 . If new devices are added later on, they can be tied into I/O modules with empty slots, and would only have to be configured in the processor through programming. If the decision is made to monitor oscilloscope readings that may be monitoring faster signals, for example in the $\mu$ s range, it will be doable at an increased expense. Network access would also allow for the staff to have access from remote locations. This is a downside since server security is always a concern.

\section{Conclusion}

This system upgrade will immediately determine the source of an error, making troubleshooting faster and easier since the designs are isolating the malfunction, allowing for less downtime, ensuring that the nuclear energy physics experiments receive proton beam in an optimal manner. The existing panel relies heavily on empirical knowledge of the current staff. Through the four new designs, individuals with less expertise on the Linac will be aided in expediting in troubleshooting a malfunction, such as when the majority of the Linac staff leave for the day, and the C-AD Main Control Room takes over. 


\section{Acknowledgments}

Thank you to my mentor, Mr. Omar Gould for his tutelage and guidance during my time at BNL and growing once I enter my career. I would like to thank Pablo Rosas and Yurii Bezpalko for their help and feedback in my research and designs. Also, Brian Briscoe, Frank Carlino, Thomas Flanagan, Vincent LoDestro, Deepak Raparia and the remaining Linac and CAD staff for their assistance in my research. 


\title{
Charge-Coupled Device Calibration Methods: The Photon Transfer Curve
}

\author{
Kevin Powell \\ Electrical Engineering, Florida A\&M University, Tallahassee, FL 32307 \\ Ivan Kotov \\ Instrumentation Division, Brookhaven National Laboratory, Upton, NY 11973
}

\begin{abstract}
Calibration procedures are performed for all scientific CCDs before they go into operation. A valuable technique for CCD camera calibration is the photon transfer curve. This method is one of the first steps to determine the overall health of the CCD and it reveals such performance parameters as gain, linearity, full well capacity, pixel non-uniformity and read noise. The Charge Coupled Device (CCD) used in a camera system, is a photon detecting sensor. Its vast sensitivity and huge wavelength range response make it great for imaging giant regions of the sky. Because of its elevated sophistication, a CCD sensor is vulnerable to a number of subtle performance problems and/or issues. The Photon Transfer Curve (PTC) technique is a tool that can detect and diagnose many of those issues. Satisfactory PTC parameters imply that the CCD is ready for producing accurate data.
\end{abstract}

\section{Introduction}

As our optical technologies become more sophisticated, we are capable of probing further into the vast skies. The Large Synoptic Survey Telescope (LSST) will be the world's largest camera system. It will possess a 3.2 giga-pixel camera, composed of about 200 CCDs. A CCD's primary function is to detect photons, both visible \& non-visible (IR part of the spectra), and convert to electrical signals which then can be readout by a computer to form an image. The $189 \mathrm{CCD}$ will be used to form the entire camera array, and 12 will be used to guide and focus the camera [4]. Each CCD will need to be tested and calibrated for optimal performance. By implementing the PTC method we were able to reveal important characteristics from our CCD. The PTC calibration algorithms have been implemented as $\mathrm{C} / \mathrm{C}++$ code written for ROOT framework. Three methods of the PTC technique have been explored. The PTC_mono.cpp program implements the first two methods(standard \& paired). The PTC_grad.cpp program implements the third method (gradient).

\section{Methods}

Test data from the CCD are stored in Flexible Image Transport System (FITS) files. Each FITS file contains header data units (HDU), one unit per each CCD channel. In some cases, there is also tabular data within the FITS files. To read this data, special FITS routines are utilized within the regular read programs. Table layout properties are first gathered from the header and are used to prepare the program for table read-out. The data is read into array buffers and then displayed. If there is no tabular data in the file then the regular image read functions are used. When performing the image analysis, the program first runs the bias files analysis in order to establish the base line for later correction. The base line can be calculated for each pixel simply by averaging over all available bias frames [3]. FITS routines are called to read in the value (or amplitude) of each pixel on the images in each file. Amidst this process, mean, variance, and gain calculations of the pixel amplitudes are being performed. The equation used for the pixel amplitude is

$$
A m p_{i}=N_{i}^{e} \times g,
$$

where $N_{i}^{e}$ is the number of electrons registered by pixel i, and $\mathrm{g}$ is the gain, or inverse of the electronics sensitivity in of a.d.u./e [2]. To obtain the mean, and variance of the amplitudes,

$$
\begin{gathered}
\overline{A m p}=\frac{1}{N} \sum_{i}^{N} A m p_{i}=A v g\left(N_{i}^{e}\right) \times g, \\
\operatorname{Var}=\frac{1}{N-1} \sum_{i}^{N}\left(A m p_{i}-\overline{A m p}\right)^{2}=\operatorname{Var}\left(N_{i}^{e}\right) \times g^{2},
\end{gathered}
$$

respectively are used [2]. Following the Poisson's distribution property, the mean equals the variance with the number of electrons registered (i. e., $\operatorname{Avg}\left(N_{i}^{e}\right)=\operatorname{Var}\left(N_{i}^{e}\right)$ ) [2]. Then the variance and mean values can be used to estimate the gain, $g=$ $\operatorname{Var} / \operatorname{Avg}(\mathrm{amp})$. After mean, variance, and gain values are computed for a pad of pixels, ROOT's histogram tools are used to store and later plot points on both one and two dimensional graphs, of which we used to yield the linearity, read noise, and other important parameters mentioned above.

\section{Photon Transfer Curve}

We implement Photon Transfer Curve methods, which have been proven to be the most valuable for CCD calibrating, characterizing and optimizing performance.[1] The photon transfer curve is a response from a CCD that is uniformly illuminated at different levels of light [1]. As part of the PTC analysis process, three different methods have been explored. The first method or standard method involves reading each pixel amplitude and performing the mean, variance and gain calculations across the image for each CCD segment file by file. This method is vulnerable in the fixed pattern noise presence (i.e. pixel non-uniformity, pixel dark current). The second method, known as the paired method alleviates the noise problems, because the difference of two identical exposures removes fixed pattern features. The paired method involves pairing the like exposure files into a multi-map container by using the newly added Exposure Mapping class within the code. Then, pixel amplitudes are read in for both files in a pair, and are summed and differenced before, the mean, variance, and gain calculations are performed. This method requires more memory for computing. The last method explored called the gradient method is quick and computationally economical. For the gradient method only single FITS file is used. In this method, the row over-scan region is averaged and subtracted from each pixel in the row before mean, variance, and gain calculations are made. This is done on a row by row basis of the image. The gradient 
method only uses one file so its statistics are limited. All three of these PTC methods implemented have different approaches and different plots, but disclose the same facts.

\section{Results}

From the three different PTC approaches, there are three different sets of curves produced. The standard and paired method plots are produced from the same set of data. Within the set, there are five important plots. The first and second plots depict one-dimensional histograms of the amplitude and variance occurrences, respectfully. The third plot is generated by plotting the Variance versus the Average Amplitude. The fourth plots average amplitude as a function of the exposure time. The fifth and most important plot depicts the Gain versus the Average Amplitude.

For the standard and paired methods, which employ relatively large exposure times, the data points fall out of order after about 30,000 a.d.u. This amplitude level is reached with exposure time of more than 30 seconds. This amount of light causes pixels to become saturated. This level of pixel amplitude represents the full well capacity of the CCD. The Amplitude vs. Exposure time also shows the CCD linearity range. The observed straight sloped line for all three methods indicate good linearity up to full well saturation. The Variance versus Average Amplitude histogram in the standard method demonstrates non-linear behavior expected for illumination and pixel non-uniformities. In this same plot there is also a "shadow-like" scatter trail of points above the main curve of points. In the standard method Gain versus Average Amplitude histogram there is a similar "shadow-like" trail that tries to split the data. These effects are mentioned above and are corrected for as part of the paired method. Observed in the Variance versus Average Amplitude plot for the paired method, the curve is more linear and there is no "shadow-like" trail. The gain should ideally be independent of the amplitude but the fixed pattern noise is completely removed only from the Variance of the difference and is still present in Sum. This explains the slight negative slope visible in the Gain versus Average Amplitude plot of the paired
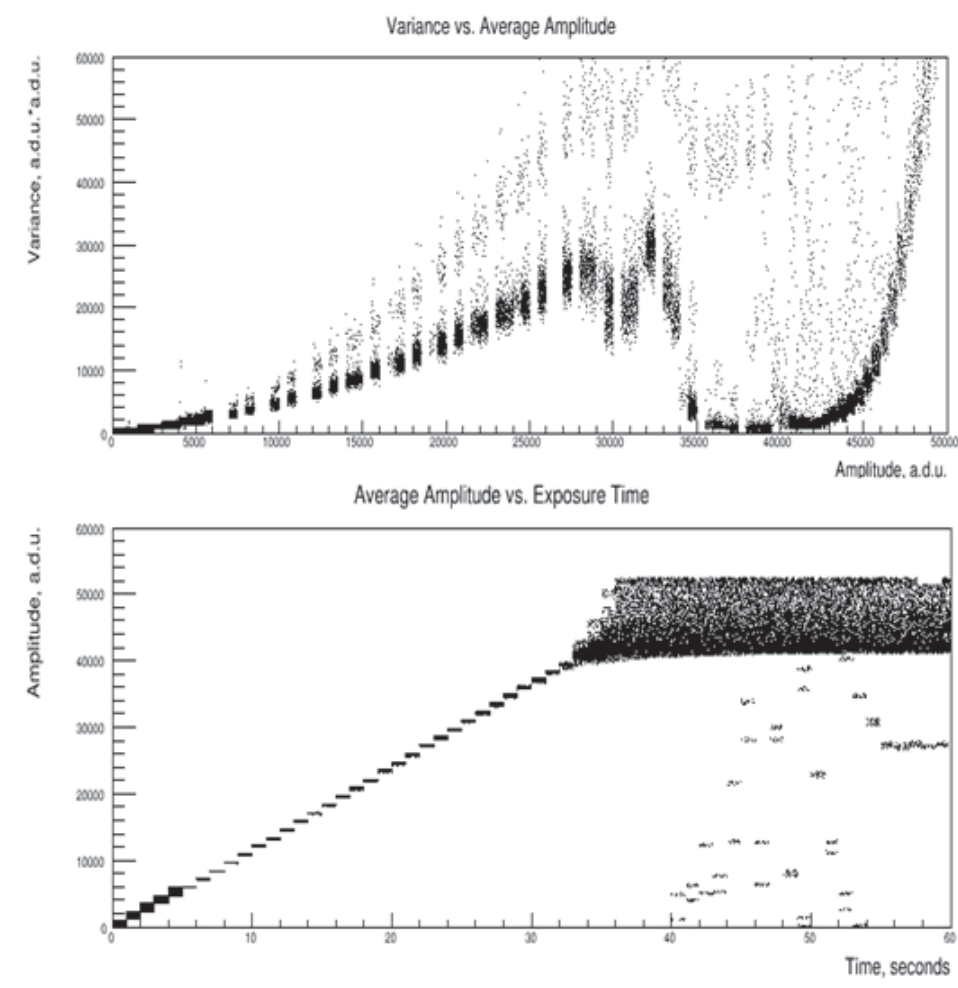

method.

The data for the gradient method has been obtained with different CCD bias voltages. Under these conditions we expect a different gain to be measured. In the gradient's method Gain versus Average Amplitude plot, the curve is getting flat when shot noise starts to dominate over readout noise. These results helped confirm that the CCD is appropriate for employment.

\section{Conclusion}

Three algorithms of the PTC method have been implemented and compared. It was found that gradient \& paired methods are more robust. The paired method results in more reliable gain measurements, though it requires more memory. Clearly, it is most convenient and rewarding to use the gradient method. In conclusion, the photon transfer technique utilized in several different methods have demonstrated and confirmed value and handiness in assistance to the calibration, characterization, and performance of CCDs. My involvement was writing, editing, and testing the $\mathrm{C}++$ code needed to implement this PTC technique under several algorithms and also writing code for the table read function. Before writing any official program code, I had to familiarize myself with the ROOT environment and learn basic commands in order to productively carry out my tasks. I also had to study several CFITSIO routines, used to access the FITS files, in order to know the flexibility and accessibility I have with these files. With my $\mathrm{C}++$ programming abilities and combined familiarity with CFISIO library and ROOT environment I was able to carry out my all tasks. By taking part in this research I was able to grow both skillfully and professionally while working within a large and diverse community. I got the opportunity to significantly improve my programming skills, improve my communication abilities, learn several techniques for data analysis, and lastly I was able to familiarize myself in a genuine research environment, somewhere I potentially see myself in few years.

\section{Gain vs. Average Amplitude}

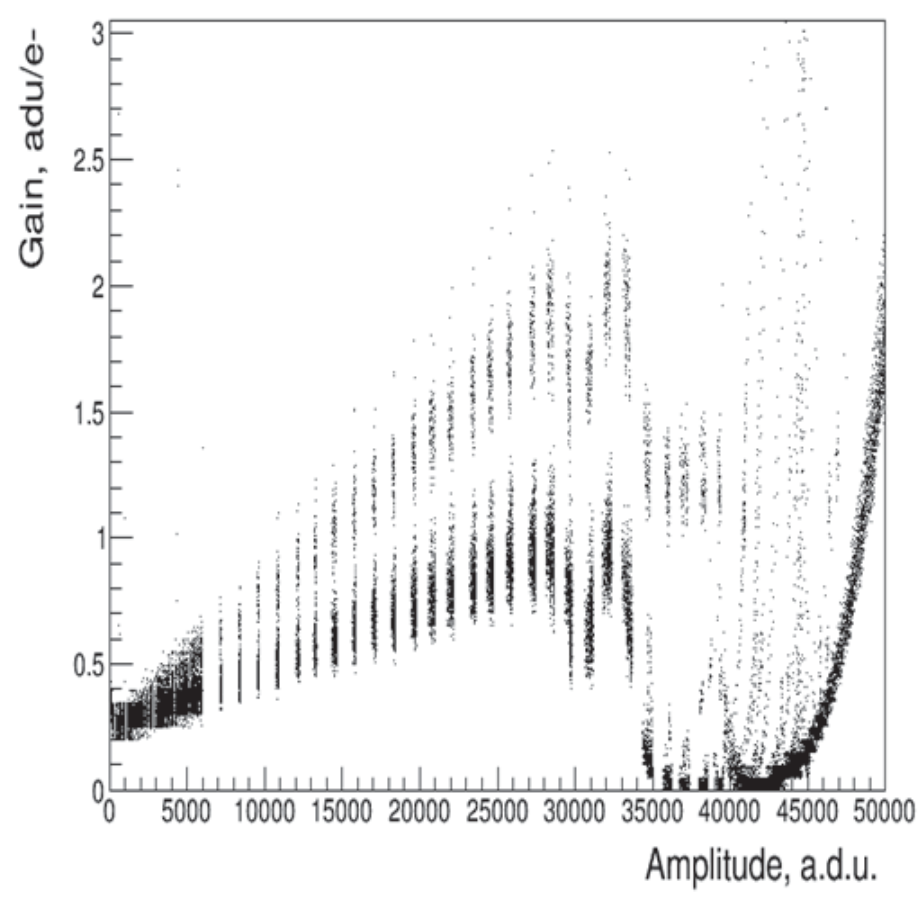




\section{References}

[1] James R. Janiseck, Scientific Charge-Coupled Devices, SPIE Press., Bellingham, Washington, (2001)

[2] I.Kotov, "CCD gain determination using properties of Poisson distribution", 2009

[3] I.V. Kotov, A.I. Kotov, J.Frank, P. O'Connor, V. Perevoztchikov, P. Takacs, "CCD Base Line

Subtraction Algorithms," IEEE Trans. Nucl.Science 57, (2010)

[4] BNL Large Synoptic Survey Telescope, Digital Sensor Array, http://www.bnl.gov/LSST/
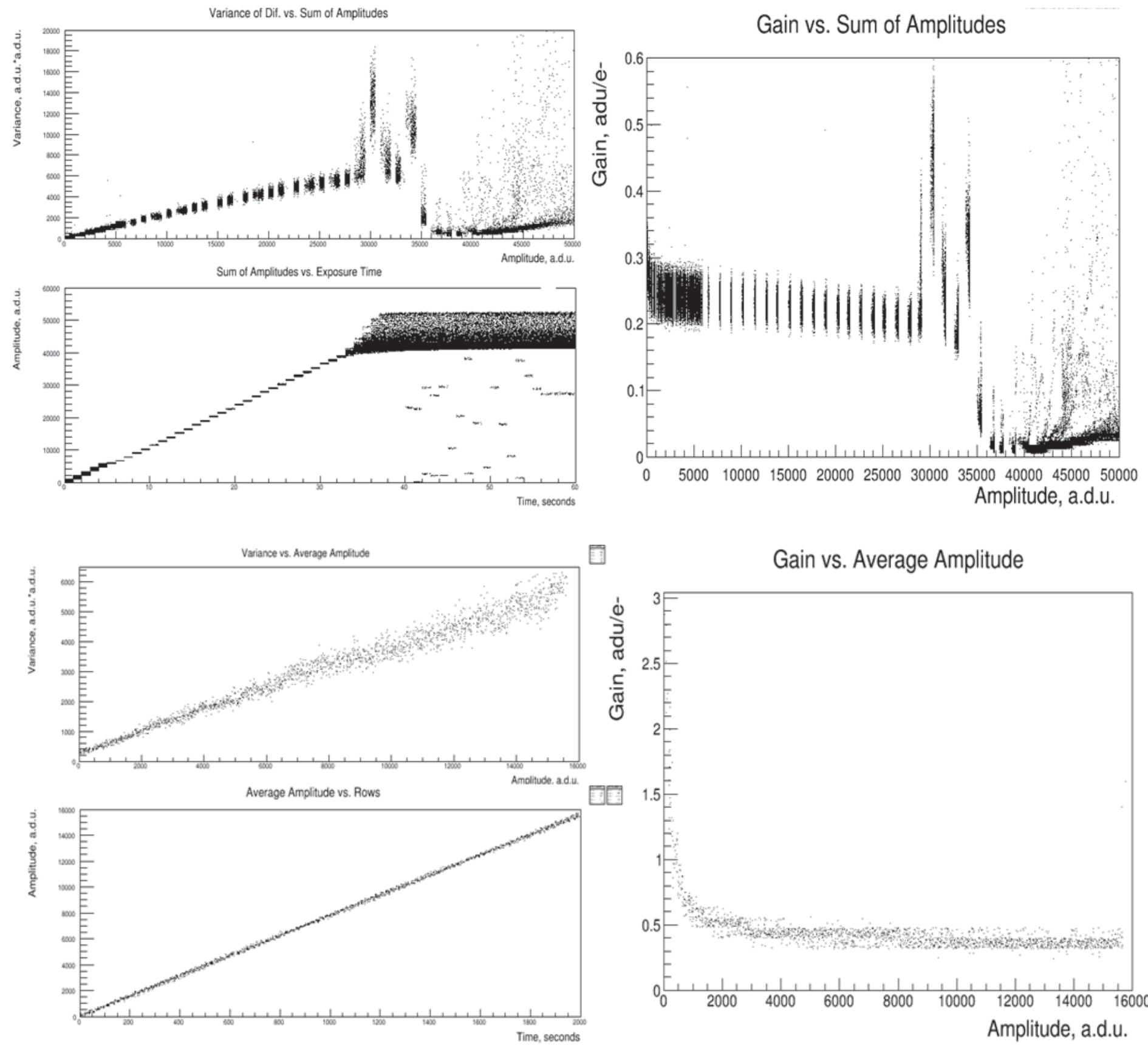


\title{
Tick population analysis and use of 4-poster devices by white-tailed deer (Odocoileus virginianus)
}

\author{
Lucas Price \\ Wildlife and Fisheries Resources, West Virginia University, Morgantown, WV 26508 \\ Timothy Green \\ Environmental Protection Division, Brookhaven National Laboratory, Upton, NY 11973
}

\begin{abstract}
Tick populations on Long Island are a problem for its residents because they carry various diseases, such as Lyme (Borrelia burgdorferi). Fire Island and Shelter Island have both used 4-poster systems to decrease tick populations in these isolated communities. The 4-poster device feeds deer corn and uses this attractant to apply pesticide to a deer's head, neck, and ears via rollers. Fourteen 4-poster devices have been setup at Brookhaven National Laboratory, on Long Island, New York, during spring 2013. This study looks at the following questions: What is the current population of ticks at Brookhaven National Laboratory? Do these ticks prefer forested areas or grass fields? How often are white-tailed deer feeding at 4-poster systems? The 4-poster systems were monitored using wildlife cameras and by monitoring how much corn and permethrin (pesticide) was added. Tick surveys were also conducted at each 4-poster location and two control sites. Deer use was calculated based on photos taken by wildlife cameras and corn use. Forested areas did not contain significantly more ticks then grass areas. On average, twenty-one whitetailed deer visited a 4-poster per day in July 2013 based on corn use, which appears to be the more accurate calculation. This is up from the fourteen deer per day calculated for April. These results show that deer are using the 4-poster systems. This report is the initial data that will be used for comparisons with data collected later in this three year longitudinal study.
\end{abstract}

\section{INTRODUCTION}

Brookhaven National Laboratory (BNL) is located on Long Island in New York, and consists of 5,265 acres. BNL is composed of mainly forested areas and open fields. It also has an abundance of white-tailed deer present on site. The forest areas have been fragmented due to building and recreational area development, along with the construction of a solar farm.

Tick populations on Long Island have caused problems for residents for years. These ticks carry various diseases including Lyme (Borrelia burgdorferi), Babesiosis (Babesia microti), Erhlichiosis, and others. These diseases also are hard to diagnose, leading to serious problems to people going without treatment1. These diseases are picked up by ticks from animals such as whitetailed deer and white-footed mice, both of which are abundant on the island2. The diseases have higher incidences on the island due to the high deer populations, which influence the high tick populations.

Ticks have a multi stage life cycle. They go through a larval stage, nymph stage, and adult stage over a two year period. The tick has to feed twice to progress to the adult stage. The feeding has to occur once in the larval stage and once in the nymph stage3. Ticks feed on mammals and birds extensively4.

Historically ticks were managed using a variety of methods.
To control areas that had high tick populations, pesticides are sprayed on the area. Education also helps to protect the public from ticks. They are informed what vegetation types to avoid and how to protect themselves. Personal protective equipment to prevent tick bites include closed shoes, long pants, permethrin treatment of clothing, and long sleeves.

The "4-poster" program that took place at Shelter and Fire Island, which are in the same area as Long Island, were the first studies of "4-posters" in the area. These locations saw the success of the "4-poster" in decreasing the tick population. They monitored deer use of "4-posters" by camera and corn consumption. These areas were carefully monitored for three years and the NYDEC now allows the use of the 4-Poster on Long Island5.

In an attempt to decrease the population of ticks, BNL has set up fourteen "4-poster" devices around the constructed portion of the site during spring 2013. The 4-poster works by attracting deer to it with a bait source of corn. This corn is stored in the vertical bin and comes out in a feeding tray on either side of the device. A restrictor plate allows the corn to come out in moderation. The feeding tray is accessed by white-tailed deer by sticking their head down along a slanted wall. This slant requires the deer to tilt its neck, head, and ears against the rollers on each end. These rollers contain a pesticide, permethrin. This permethrin will stay on the deer's neck, head, and ears killing present ticks and ones that come in contact with the deer in the near future. White-tailed deer revisit these devices for the food source and get reapplied with pesticide6.

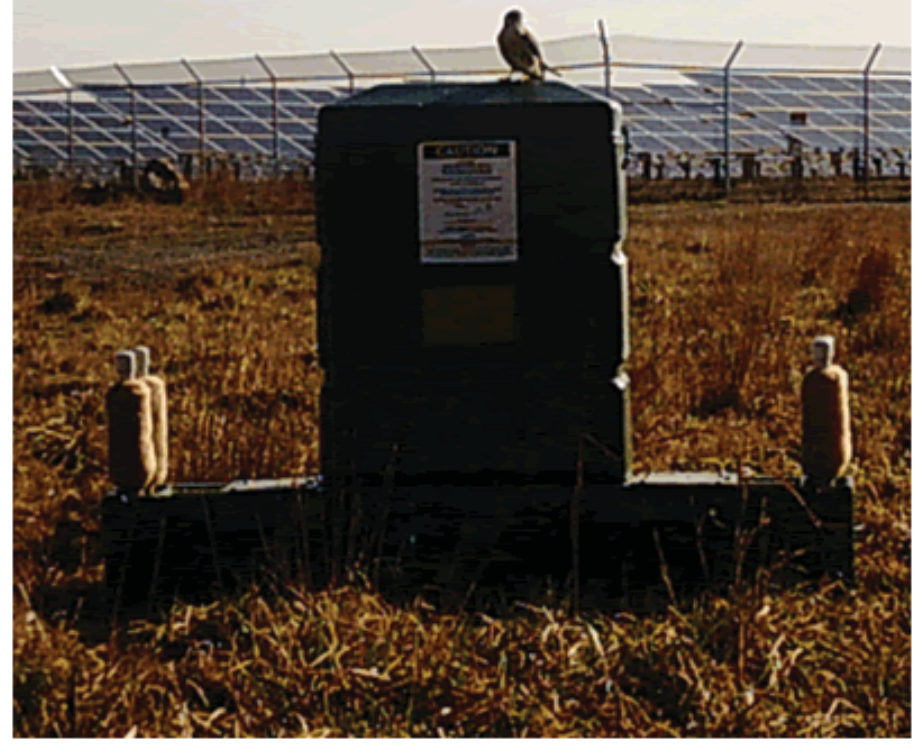


A permit from the NYDEC is required to operate the "4-poster" system. To keep this permit, permethrin and corn have to be monitored twice a week, and each 4-poster is required to be monitored for species using the device. Ticks also have to be monitored through tick population assessments to show the tick populations over time.

This study looks at the effectiveness of the first summer's deployment of the "4-poster" devices. The number of deer using the device is calculated, and the first year tick surveys are completed for later comparison. The tick population will also be compared between open grass and forested areas. The two different methods for calculating deer use are compared.

\section{METHODS}

Fourteen 4-poster devices were set up across BNL according to NYDEC regulations. 4-poster devices are maintained with corn and permethrin twice weekly. The amount of corn used, corn added, permethrin added, time, and date were recorded. Each device is monitored by a Wildgame Innovations Model W5EGC wildlife camera.

The cameras were initially set to recycle and collect pictures every minute when there is motion, and on a medium sensitivity for motion. These settings were changed to recycle every five minutes when there is motion and low sensitivity for motion beginning in June 2013. All pictures are on a medium picture quality setting. Memory cards containing pictures were collected every two weeks until June, after which they were collected approximately every week. The cameras were not checked if rain was falling or vegetation was excessively wet.

Pictures were organized based on content. Categories for sorting included white-tailed deer, wild turkey, raccoon, other animal, or no animal. A picture with a deer always went into the deer folder no matter what other animal was present. Pictures with raccoons may also have contained wild turkey or other animals. Pictures with turkey may also contain other animals. Humans went into the no animals' category.

Pictures with deer were used to calculate deer use. A camera's deer pictures were observed for 24 hours to calculate the average number of deer in each picture. Then a week's worth of deer pictures was evaluated to establish the average number of deer pictures per day. The average deer pictures per day was multiplied by the average number of deer in each picture. The number calculated was the estimated deer per day using a device. This followed the method used on Shelter and Fire Island5, and was calculated each month. For April and May, the number of deer pictures was divided by five to account for the difference in how often pictures were taken.

Corn consumption was also used to calculate usage of "4-posters" by deer. A week's worth of corn usage was averaged into corn consumed per 24 hours. This was divided by 1.5 to give the deer usage per day. 1.5 takes into account usage by other animals that occurs and was first used for the Shelter and Fire Island study5.

Tick surveys were completed at the fourteen 4-poster systems and two control sites. The surveys were completed in July. Each tick survey was conducted using a white flag. The flag was dragged through vegetation for thirty seconds and each site had this completed thirty times. The ticks were counted and identified in the field. Larval ticks had numbers estimated instead of fully counted. Vegetation type was identified for each thirty second sweep. End time and start time were recorded along with direction traveled from the 4-poster.

\section{RESULTS}

Pictures from all cameras contained both mammals and birds. Mammals include white-tailed deer, opossums, raccoons, and squirrels. Birds included wild turkeys, blue jays, northern cardinals, American kestrels, and various other species.

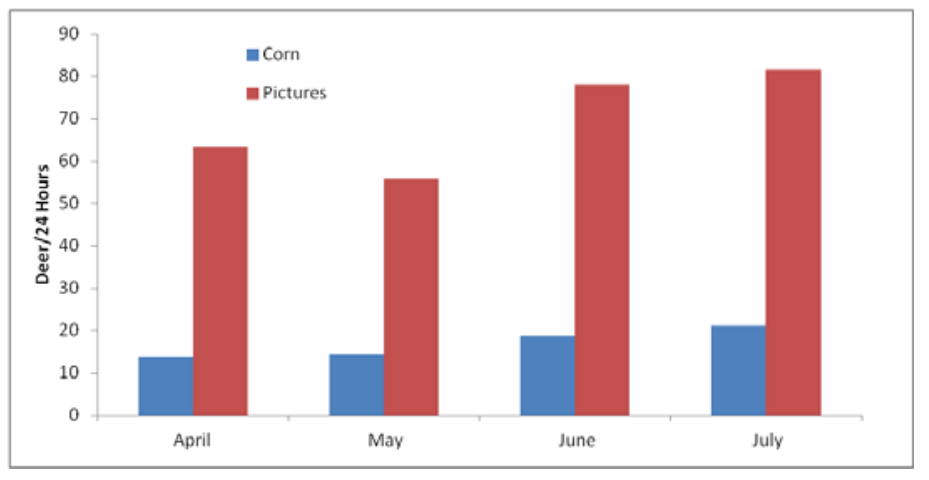

Fig. 1. Bar graph displaying deer use per day analysis by corn use versus by picture analysis. Both pictures and corn analysis are averages across all fourteen "4-poster devices".

The picture deer use analysis is statistically different from the corn deer usage analysis (Table 1). The picture deer use analysis is consistently higher. The corn deer use analysis consistently increases while the picture analysis decreases then increases.

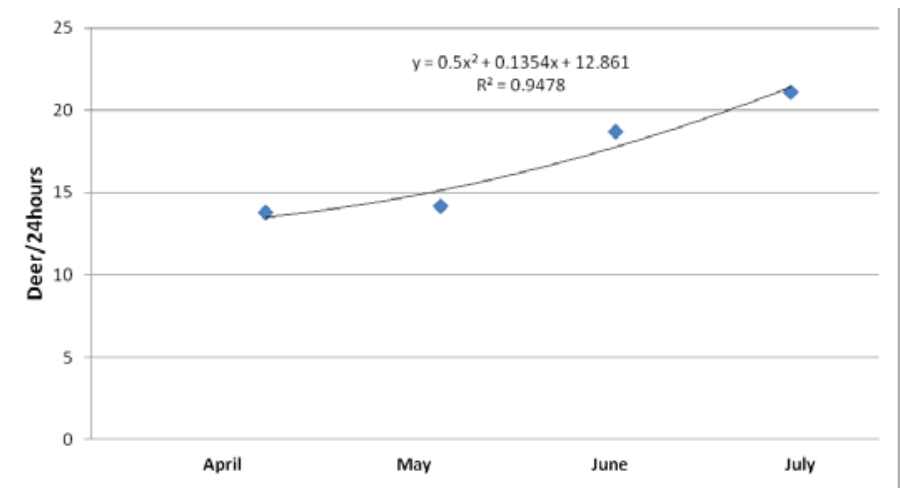

Fig. 2. Line graph displaying the increasing use of "4-posters" by deer based on corn consumption averaged across all "4-poster" devices for four months.

In July "4-poster" 11 and 12 had the greatest deer use (Table 2). "4-Poster" 2 had the greatest increase in deer use from April to July. "4-Poster" 9 had the lowest deer use in July, as it was too low to be measured. "4-Poster" 1 had the second lowest use by deer in July. The average use by deer increased each month.

The difference between tick numbers in grass versus forest is not statistically significant. Two-way ANOVA was run giving a p-value of 0.39 for rows and 0.47 for columns. The data collected shows higher tick numbers switching between forest and grass (Table 3). Larval ticks seemed to prefer grass, but more testing is required for conclusive data.

The current tick surveys show a variety of ticks and stages present at Brookhaven National Laboratory (Table 4). For dog ticks only adult males were found on site. Only one adult blacklegged tick was found, a female. Nymphs and larval black-legged ticks were found in higher abundance. All stages of lone star ticks 
were found.

\section{DISCUSSION}

\section{A. Picture vs. corn analysis}

The two methods of calculating daily deer use of a "4-poster" device yield significantly different results. The picture analysis calculates at the minimum 20 more deer per day on average then the corn deer use analysis. Both have flaws in the calculation. The corn use analysis is not precise because it is impossible to tell how much corn is consumed precisely in each "4-poster." Each "4-poster" has lines telling how much corn is present inside the bin. These lines are not exact. In some feeders corn will be eaten slowly enough that it does not noticeably lower the level. This is what occurred for the July "4-poster" unit 9 estimation. To help compensate, an average can be taken over a period of at least a week instead of using only one maintenance report, but this is still not perfect.

Picture analysis leads to a high estimate of deer use. This is due to the multiple images that are captured of a single or same group of deer at a device. The deer do not always leave after 5 minutes, and get recorded again on the camera. This is not accounted for in the calculations, and is hard to account for since each deer stays for a different amount of time. Deer will also stand by the device even if there is no corn in it. With no corn, they will probably not use the device, but still get included in the picture calculation.

The corn analysis seems to be the more reliable method for recording deer use of the "4-poster" device. It is also the easier method to calculate. Pictures took a few hours to calculate all the months, after the time spent sorting pictures. Corn use was easily completed within an hour of work.

\section{B. Deer use}

As time has gone on with the "4-posters", more deer use them each month on average. This trend shows that deer at BNL are using the device, and more deer on site are finding the devices over time. Supplemental feeding can bring deer closer together then during normal grazing7. It is discouraged and even banned in some states due to the possibility of transmitting diseases around the population of deer8. Feeding deer is illegal in New York. Therefore a permit is required for the use of the 4-Poster device in Suffolk and Nassau counties allowing feeding of deer in association with the use of the device. This possibility means disease is an issue to be aware of going forward in this project. Careful observation of the deer should mitigate the issue, though. The disease of main concern is chronic wasting disease. Chronic wasting disease is a prion disease that causes weight loss and change of behavior before death9.

\section{Forest vs. grass ticks}

No significant difference were found between forested and grass field tick surveys. While numbers change between forest and grass in a couple sites, they were not close to significant changes. One sight had close to identical numbers for grass and forest, though. A study done in Argentina found more ticks in forested areas then open areas. This could be the result of a preference for forested areas10. It is also possible that higher temperatures in open areas lead to dehydration of the ticks, decreasing the life span11. It is possible more data will reveal different results at BNL.

\section{Tick survey}

Tick surveys were completed as the baseline in a three year longitudinal study. They will be used for comparison in later years. Only one adult black-legged tick was found this year, and three adult dog ticks. No nymph or larval dog ticks were found this year. These results were not expected. It is possible they will be found in higher abundance in later surveys.

\section{E. White-tailed deer and white-footed mice}

White-tailed deer are believed to be the main cause of the tick problem. A study done at parks on Long Island shows nymphal ticks were 93\% less abundant when deer were absent. Larval ticks were also only at $2 \%$ of the levels at where deer were present 12 . If the deer population at BNL could be lowered it would likely help with the tick problem.

BNL is also a site consisting of many fragmented sections of forest. These sections are fragmented by roads and buildings, along with the open fields created. White-footed mice reach higher densities in fragmented forest habitat, and they are one of the animals that carry Lyme (Borrelia burgdorferi). Larger patches of forest carry a lower risk of disease transmission, as nymphal ticks are also more abundant in smaller patches of forest. Habitat fragmentations can influence people's health13. This problem is hard to see a solution to.

\section{ACKNOWLEDGEMENTS}

I would like to thank Timothy Green for being my mentor and always being there when help was needed. I would also like to thank Jennifer Higbie for assisting me when needed and exposing me to new experiences. I would like to thank the other environmental interns for helping with my project and sharing space with me. I would like to thank the Office of Educational Programs and Brookhaven National Laboratory for their generosity. This project was supported in part by the U.S. Department of Energy, Office of Science, Office of Workforce Development for Teachers and Scientists (WDTS) under the Science Undergraduate Laboratory Internships Program (SULI).

\section{NOTES}

1. Walker, DH. "Tick-Transmitted Infectious Diseases in the United States." Annual Review Public Health. 19 (1998): 237-269.

2. Bosler, Edward, Brian Ormiston, James Coleman, John Hanrahan, and Jorge Benach. "Prevalence of the Lyme Disease Spirochete in Populations of White-Tailed Deer and White-Footed Mice." The Yale Journal of Biology and Medicine. 57 (1984): 651-659.

3. Qiu, Wei-Gang, Edward Bosler, Jason Campbell, Gregory Ugine, Ing-Nang Wang, Benjamin Luft, and Daniel Dykhujzen. "A population genetic study of Burrelia brrrgdorferi sensu stricto from eastern Long Island, New York, suggested frequency-dependent selection, gene flow and host adaptation."Hereditas. 127 (1997): 203216.

4. Anderson, JF. "Mammalian and avian reservoirs for Borrelia burgdorferi.” Ann. NY Acad. Sci. 539 (1988): 180-191.

5. Curtis, Paul, Daniel Gilrein, and Susan Walker. "Shelter Island and Fire Island 4-Poster Deer and Tick Study." (2011): 1-113. 
6. Pound, JM, JA Miller, John George, and Craig Lemeilleur. “The '4-Poster' Passive Topical Treatment Device to Apply Acaricide for Controlling Ticks (Acari. Ixodidae) Feeding on White-Tailed Deer." Journal of Medical Entomology. 37.4 (2000): 588-594.

7. Thompson, Abbey, Michael Samuel, and Timothy Van Deelen. "Alternative Feeding Strategies and Potential Disease Transmission in Wisconsin White-Tailed Deer." Journal of Wildlife Management. 72.2 (2008): 416-421.

8. Bartelt, G.L., J. Pardee, and K. Thiede. "Environmental impact statement of rules to eradicate chronic wasting disease in Wisconsin's free-ranging white-tailed deer herd." Wisconsin Department of Natural Resources PUBSS-980. (2003): n. page.

9. Williams, E.S. "Chronic Wasting Disease." Veterinary

Pathology. 42.5 (2005): 530-549.
10. Guglielmone, A.A., A.J. Mangold, D.H. Aguirre, and A.B. Gaido. "Ecological Aspects of Four Species of Ticks Found on Cattle in Salta, Northwest Argentina." Veterinary Parasitology. 35. (1990): 93-101.

11. Lees, A.D. "The water balance in Ixodes ricinus and certain other species of ticks.” Parasitology. 37. (1946): 1-20.

12. Duffy, David, Scott Campbell, Dara Clark, Chris DiMotta, and Susan Gurney. "Zxodes scapularis (Acari: Ixodidae) Deer Tick Mesoscale Populations in Natural Areas: Effects of Deer, Area, and Location." Journal of Medical Entomology. 31.1 (1994): 152-158.

13. Allan, Brian, Felicia Keesing, and Richard Ostfeld. "Effect of Forest Fragmentation on Lyme Disease Risk." Conservation Biology. 17.1 (2003): 267-272.

\section{TABLES}

Table 1. Paired two sample t-test analyzing corn deer use analysis versus picture deer use analysis.

\begin{tabular}{|l|r|r|}
\hline & Variable 1 & Variable 2 \\
\hline Mean & 16.94898 & 69.67566 \\
\hline Variance & 83.20889 & 2667.414 \\
\hline Observations & 56 & 56 \\
\hline Pearson Correlation & 0.804018 & \\
\hline Hypothesized Mean Difference & 0 & \\
\hline df & 55 & \\
\hline $\mathrm{t}$ Stat & -8.83824 & \\
\hline $\mathrm{P}(\mathrm{T}<=\mathrm{t})$ one-tail & $1.92 \mathrm{E}-12$ & \\
\hline $\mathrm{t}$ Critical one-tail & 1.673034 & \\
\hline $\mathrm{P}(\mathrm{T}<=\mathrm{t})$ two-tail & $3.84 \mathrm{E}-12$ & \\
\hline $\mathrm{t}$ Critical two-tail & 2.004045 & \\
\hline
\end{tabular}

Table 2. Deer use of each "4-poster" device across four months based on corn consumption.

\begin{tabular}{|l|r|r|r|r|}
\hline Device \# & April & May & June & \multicolumn{1}{c|}{ July } \\
\hline $\mathbf{1}$ & 3.809524 & 4.666667 & 4.761905 & 4.761905 \\
\hline $\mathbf{2}$ & 3.809524 & 10 & 14.28571 & 23.80952 \\
\hline $\mathbf{3}$ & 23.80952 & 20 & 28.57143 & 23.80952 \\
\hline $\mathbf{4}$ & 19.04762 & 23.33333 & 23.80952 & 23.80952 \\
\hline $\mathbf{5}$ & 14.28571 & 13.33333 & 23.80952 & 19.04762 \\
\hline $\mathbf{6}$ & 14.28571 & 16.66667 & 23.80952 & 23.80952 \\
\hline $\mathbf{7}$ & 14.28571 & 13.33333 & 4.761905 & 14.28571 \\
\hline $\mathbf{8}$ & 9.52381 & 10 & 14.28571 & 14.28571 \\
\hline $\mathbf{9}$ & 4.761905 & 9.666667 & 19.04761 & 0 \\
\hline $\mathbf{1 0}$ & 9.52381 & 17.33333 & 33.33333 & 23.80952 \\
\hline $\mathbf{1 1}$ & 38.09524 & 26.66667 & 19.04762 & 38.09524 \\
\hline $\mathbf{1 2}$ & 19.04762 & 16.66667 & 23.80952 & 38.09524 \\
\hline $\mathbf{1 3}$ & 9.52381 & 13.33333 & 19.04762 & 28.57143 \\
\hline $\mathbf{1 4}$ & 9.52381 & 6.666667 & 18.70748 & 19.04762 \\
\hline Average & 13.80952 & 14.19048 & & 21.08844 \\
\hline
\end{tabular}




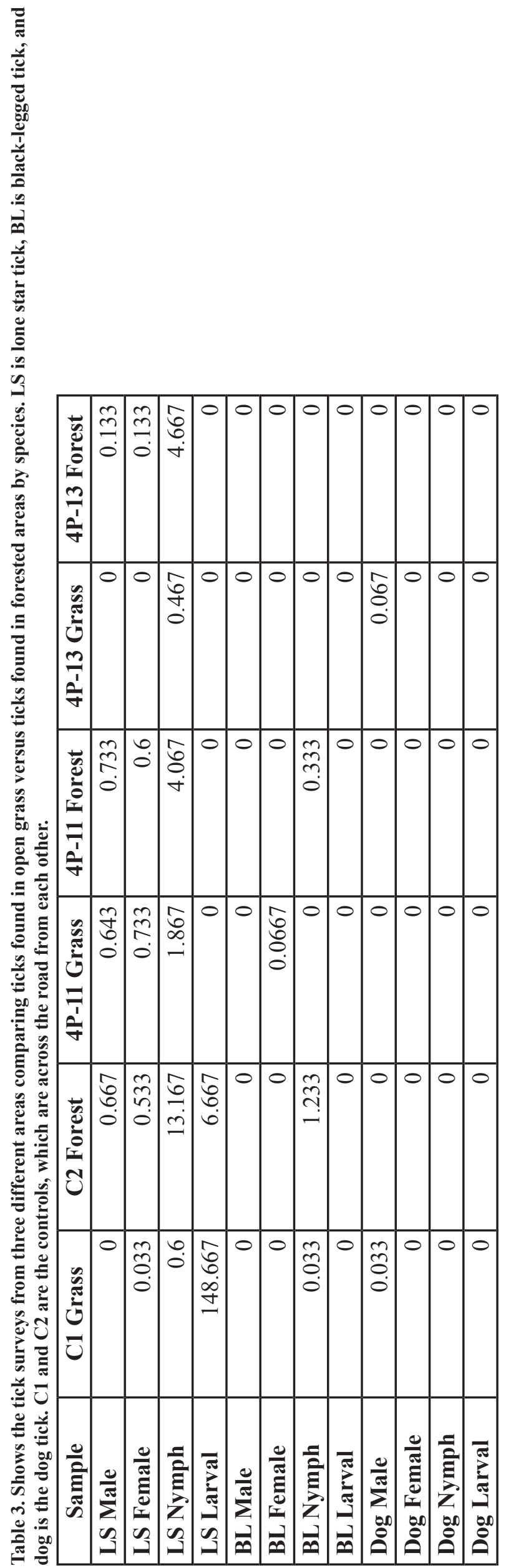

\begin{tabular}{|c|c|c|c|c|c|c|c|c|c|c|c|c|c|c|}
\hline Ư & & $\begin{array}{l}\hat{8} \\
\stackrel{0}{0} \\
0\end{array}$ & $\begin{array}{c}m \\
\tilde{n} \\
0 \\
0\end{array}$ & $\begin{array}{l}\hat{\sigma} \\
\dot{v}\end{array}$ & \begin{tabular}{|l|}
$\mid \begin{array}{l}0 \\
0 \\
0\end{array}$ \\
\end{tabular} & & 0 & 0 & רֶ & 0 & 0 & 0 & 0 & 0 \\
\hline 'َ' & & 0 & $\begin{array}{c}m \\
\hat{o} \\
\dot{0}\end{array}$ & $\begin{array}{l}0 \\
0 \\
\end{array}$ & 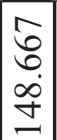 & & 0 & 0 & $\begin{array}{l}n \\
\tilde{0} \\
0\end{array}$ & 0 & $\begin{array}{l}m \\
\tilde{2} \\
\dot{0}\end{array}$ & 0 & 0 & 0 \\
\hline$\frac{\nabla}{\frac{J}{\sigma}}$ & & 0 & $\begin{array}{c}m \\
\hat{0} \\
0 \\
0\end{array}$ & $\begin{array}{l}\hat{\sigma} \\
\tilde{r} \\
\dot{\gamma}\end{array}$ & 0 & & 0 & 0 & $\begin{array}{l}n \\
\tilde{n} \\
0\end{array}$ & 0 & 0 & 0 & 0 & 0 \\
\hline$\frac{a}{\frac{1}{\sigma}}$ & & $\begin{array}{l}\hat{8} \\
\circ \\
0\end{array}$ & $\begin{array}{l}1 \\
0 \\
0 \\
0\end{array}$ & $\begin{array}{l}\hat{\sigma} \\
\sim \\
\sim\end{array}$ & 0 & & 10 & 0 & 10 & 0 & $\begin{array}{l}m \\
\tilde{0} \\
0 \\
0\end{array}$ & 0 & 0 & 0 \\
\hline$\frac{\mathfrak{Z}}{\mathfrak{z}}$ & & 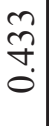 & $\begin{array}{l}\hat{\sigma} \\
+ \\
\dot{0}\end{array}$ & 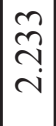 & $\begin{array}{l}\hat{0} \\
\dot{0} \\
\dot{0} \\
\sim\end{array}$ & & 0 & 0 & 10 & 0 & 0 & 0 & 0 & 0 \\
\hline$\frac{\bar{\gamma}}{\bar{\gamma}}$ & & $\begin{array}{l}\mathfrak{b} \\
0 \\
0\end{array}$ & $\begin{array}{l}1 \\
0 \\
0 \\
\dot{0}\end{array}$ & $\begin{array}{l}\hat{\sigma} \\
\check{2} \\
\text { in }\end{array}$ & 0 & & 0 & $\begin{array}{l}m \\
\stackrel{m}{0} \\
\stackrel{0}{0}\end{array}$ & $\frac{1}{0}$ & 0 & 10 & 0 & 0 & 10 \\
\hline$\frac{\Theta}{e^{\prime}}$ & & กิ & $\begin{array}{c}\hat{\sigma} \\
\dot{0}\end{array}$ & $\stackrel{⿱}{r}$ & 0 & & 0 & 0 & $\begin{array}{l}1 \\
0 \\
0 \\
0\end{array}$ & 0 & 0 & 0 & 0 & 0 \\
\hline$\frac{\hat{i}}{\vec{f}}$ & & $\begin{array}{l}\hat{b} \\
\stackrel{0}{0}\end{array}$ & $\begin{array}{l}\hat{\sigma} \\
\overrightarrow{0}\end{array}$ & $\begin{array}{l}0 \\
0 \\
0\end{array}$ & $\begin{array}{c}m \\
\tilde{n} \\
\tilde{c}\end{array}$ & & 0 & 0 & 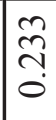 & 0 & 0 & 0 & 0 & 0 \\
\hline $\begin{array}{l}\infty \\
i \\
\dot{f}\end{array}$ & & $\begin{array}{l}\hat{\sigma} \\
0 \\
0\end{array}$ & $\begin{array}{c}m \\
\hat{\sigma} \\
0 \\
0\end{array}$ & $\begin{array}{c}m \\
\stackrel{m}{\sigma} \\
\dot{0}\end{array}$ & 0 & & 0 & 0 & $\begin{array}{l}n \\
\tilde{o} \\
0\end{array}$ & 0 & $\begin{array}{l}0 \\
\tilde{\delta} \\
0 \\
0\end{array}$ & 0 & 0 & 0 \\
\hline$\hat{f}$ & & $\begin{array}{l}\hat{\sigma} \\
\circ \\
0 \\
0\end{array}$ & $\begin{array}{c}m \\
\stackrel{1}{\hat{n}} \\
0\end{array}$ & $\begin{array}{c}m \\
\tilde{n} \\
-n\end{array}$ & 0 & & 0 & 0 & $\frac{m}{2}$ & 0 & 0 & 0 & 0 & 10 \\
\hline $\begin{array}{l}0 \\
\stackrel{i}{f} \\
\hat{f}^{\prime}\end{array}$ & & $\begin{array}{l}\hat{\sigma} \\
\text { Na} \\
0\end{array}$ & $\stackrel{1}{0}$ & $\underset{\dot{\infty}}{+}$ & 0 & & 0 & 0 & $\stackrel{1}{0}$ & 0 & 0 & 0 & 0 & 0 \\
\hline $\begin{array}{l}n \\
\dot{1} \\
\dot{f}\end{array}$ & & $\frac{m}{m}$ & $\begin{array}{l}\hat{6} \\
0 \\
0 \\
\end{array}$ & $\begin{array}{l}\hat{\sigma} \\
6 \\
0\end{array}$ & 0 & & 0 & 0 & $\begin{array}{l}10 \\
0 \\
0\end{array}$ & 0 & 0 & 0 & 0 & 0 \\
\hline $\begin{array}{l}\dot{I} \\
\qquad \\
\end{array}$ & & 0 & $\begin{array}{c}m \\
\hat{0} \\
\dot{0}\end{array}$ & $\begin{array}{l}\hat{\sigma} \\
\tilde{0} \\
0\end{array}$ & 0 & & 0 & 0 & 0 & 0 & 0 & 0 & 0 & 0 \\
\hline $\begin{array}{l}\tilde{f} \\
\hat{F}\end{array}$ & & $\begin{array}{l}\hat{\sigma} \\
\circ \\
0 \\
0\end{array}$ & $\begin{array}{c}m \\
\hat{n} \\
\vdots\end{array}$ & $\sim$ & 0 & & 0 & 0 & $\frac{\hat{\sigma}}{0}$ & $\nabla$ & 0 & 0 & 0 & 0 \\
\hline$\hat{I}^{\mathcal{I}}$ & & $\frac{m}{m}$ & $\begin{array}{c}\hat{\sigma} \\
-0 \\
0\end{array}$ & $\begin{array}{l}0 \\
\\
\end{array}$ & 0 & & 0 & 0 & $\begin{array}{l}n \\
\hat{\sigma} \\
0\end{array}$ & 0 & 0 & 0 & 0 & 0 \\
\hline$\vec{\prime}$ & & $\overrightarrow{0}$ & $\begin{array}{l}1 \\
0 \\
0 \\
0\end{array}$ & $\begin{array}{l}\hat{\sigma} \\
- \\
-\end{array}$ & 0 & & 0 & 0 & $\begin{array}{l}\hat{\sigma} \\
+ \\
+ \\
0\end{array}$ & 0 & 0 & 0 & 0 & 0 \\
\hline 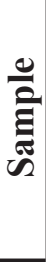 & ב & $\sum^{\frac{0}{\pi}}$ & 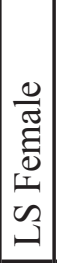 & 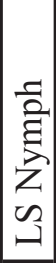 & 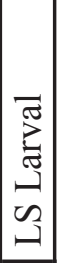 & $\vec{n}$ & $\frac{0}{\sum}$ & 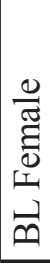 & 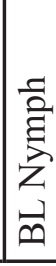 & 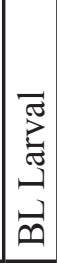 & $\begin{array}{l}\frac{0}{\pi} \\
\sum^{\pi} \\
00 \\
0 \\
0 \\
0\end{array}$ & $\begin{array}{l}\frac{0}{\pi} \\
\tilde{\Xi} \\
0 \\
0 \\
00 \\
0 \\
0 \\
0\end{array}$ & 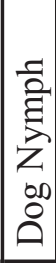 & 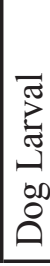 \\
\hline
\end{tabular}




\title{
Hot-pressing and physical properties
}

\section{characterizations of polycrystalline specimens of dysprosium titanate and dysprosium hafnate for elasticity studies at extreme conditions}

\author{
Brandon Rhymer \\ Department of Science and Mathematics, University of the Virgin Islands, Charlotte Amalie, VI 00802-9990 \\ Gabriel D. Gwanmesia \\ Department of Physics \& Pre-Engineering, Delaware State University, Dover, DE 19901 \\ Mineral Physics Institute, State University of New York at Stony Brook, Stony Brook, NY 11794 \\ Lars Ehm \\ Mineral Physics Institute, State University of New York at Stony Brook, Stony Brook, NY 11794 \\ Photon Sciences Directorate, Brookhaven National Laboratory, Upton, NY 11973
}

Polycrystalline specimens of dysprosium titanate (Dy2TiO5) and dysprosium hafnate (Dy2HfO5) have been fabricated using hot-pressing techniques developed for a Kawai-type 2000- ton uni-axial split sphere apparatus (USSA-2000). The starting materials for the hot-pressing experiments were finegrained dysprosium titanate and dysprosium hafnate previously synthesized by solid state synthesis techniques using dysprosium oxide $\left(\mathrm{Dy}_{2} \mathrm{O}_{3}\right)$, titanium oxide $\left(\mathrm{TiO}_{2}\right)$ and hafnium oxide $\left(\mathrm{HfO}_{2}\right)$ powders. Polycrystalline specimens of $\mathrm{Dy}_{2} \mathrm{TiO}_{5}$ and $\mathrm{Dy}_{2} \mathrm{HfO}_{5}$ were hot-pressed at $10 \mathrm{GPa}$ and $1275 \mathrm{~K}$, for duration of 2 hours. The bulk densities of the polycrystalline specimens have been determined by Archimedes' method yielding $8.99 \pm 0.09 \mathrm{~g} / \mathrm{cm}^{3}$ for dysprosium hafnate, and 7.36 $\pm 0.03 \mathrm{~g} / \mathrm{cm}^{3}$ for dysprosium titanate, both within $1 \%$ of their respective theoretical densities. The bulk densities of the second set of specimens hot- pressed at $5 \mathrm{GPa}$ and $1275 \mathrm{~K}$ for a duration of 2 hours are $9.07 \pm 0.03 \mathrm{~g} / \mathrm{cm}^{3}$ for dysprosium hafnate, and $7.84 \pm 0.01 \mathrm{~g} / \mathrm{cm}^{3}$ for dysprosium titanate.

\section{INTRODUCTION}

High melting point, high corrosion resistance and compatibility with jacket materials (platinum, rhenium, steel, zirconium, etc.) are important properties that some oxygen compounds of elements have, which makes them valuable for nuclear applications. Examinations show that irradiated samples volumetric size can increased depending on the temperature conditions and neutron fluence. This increase in unit-cell volume can cause materials to swell, crack and deteriorate over time. However, a specimen that is denser and contains a more symmetric structure has a better radiation-resistance. An increase in the degree of lattice symmetry helps to explain this increase in resistance against radiation. ${ }^{1}$ Therefore, materials with these properties are crucial for replacing inferior nuclear control rod materials like boron carbide.

Boron carbide $\left(\mathrm{B}_{4} \mathrm{C}\right)$ is generally one of the nuclear control rod materials used as a relatively high-thermal neutron absorption material. However, it undergoes radiation induced damage due to $\mathrm{B}(\mathrm{n}, \alpha) \mathrm{Li}$ reactions on the $\mathrm{B}^{10}$ isotope, which causes the formation of helium bubbles leading to nucleate swelling and fractures, and thereby diminishing the lifetime of control rods. ${ }^{2,3}$ Consequently, there are studies to explore alternative materials that are safe, reliable, and effective and do not deteriorate over an extended peri- od of usage. Current research has focused on examining absorber materials based on dysprosium (Dy), europium (Eu), samarium (Sm), hafnium (Hf) and cadmium (Cd) alloys to substitute boron carbide and other $(n, \alpha)$-absorbers in nuclear reactors. Studies conducted on post-irradiated lanthanoid oxides with the cubic fluorite structure have been proven to be the most resistant to radiation damage. ${ }^{3,4}$ Dysprosium titanate and dysprosium hafnate are among the materials under investigation, and they are generally fabricated by hot-isostatic pressing method. Even though fabrication methods do yield fully dense and well-structured pellets, a better understanding of non-metallic materials under extreme conditions can aid in finding more proficient and less expensive fabrication techniques that can contribute to the future of nuclear engineering material designs.

The main advantages of dysprosium titanate and dysprosium hafnate are their high- thermal neutron capture cross-sections, good thermophysical properties, relatively high density and exceptional radiation resistance. The synthesis reactions are Dy2O3 $+\mathrm{TiO} 2 \rightarrow$ Dy2TiO5 and Dy2O3 + HfO2 $\rightarrow$ Dy2O3.HfO2. In comparison with dysprosium titanate control rods, $\mathrm{B}_{4} \mathrm{C}$ rods start out being more efficient. Conversely, since the efficiency of Dy${ }_{2} \mathrm{TiO}_{5}$ decreases at a slower rate, the efficiency of dysprosium titanate surpasses boron carbide over an extended period of time. ${ }^{4}$ However, unlike boron carbide where boron is the only neutron absorber and dysprosium titanate, which only has dysprosium as the neutron absorber, dysprosium hafnate has both Dy and Hf as neutron absorbers. Therefore, its physical efficiency in nuclear control rods is greater than that of boron carbide and dysprosium titanate. 3 In regards to nuclear relevance, property characteristics and elasticity studies aid in better understanding the swelling and cracking of pellets that occurs within nuclear reactors. They also assist in the study of expanding the lifetime limit and reducing the porosity of pellets. Measurements of the elastic properties of pellets as a function of pressure and temperature are also important for engineering design applications. The objective of this project is to utilize hot-pressing techniques that have been developed for the 2000 ton uni-axial split sphere apparatus (USSA-2000) to synthesize fully dense polycrystalline dysprosium specimens of high-acoustic quality for nuclear control rod applications. The aim of this project also involves completely characterizing the physical properties of the samples by X-ray diffraction, optical 
and electron microscopy and by density measurements, and further investigating the elasticity of the materials by acoustic interferometry techniques at high pressure and temperature.

\section{EXPERIMENTAL PROCEDURES}

Four hot-pressing experiments were carried out in a Kawaitype multi-anvil high pressure device, the USSA 2000 also known as the Sumitomo Press ${ }^{5}$ installed in the High Pressure Laboratory at Stony Brook University. The press consists of a two-stage anvil system driven by a ram force and can generate pressures up to 30 $\mathrm{GPa}\left(300000\right.$ atmospheres) and sustain temperatures to $2500^{\circ} \mathrm{C}$ $\left(4532^{\circ} \mathrm{F}\right)$. Specimen sizes can range from $3 \mathrm{~mm}^{3}$ at $30 \mathrm{GPa}$ to $30 \mathrm{~mm} 3$ at $10 \mathrm{GPa}$. The first stage of these high-pressure systems consist of six pieces of tool steel cut from a sphere that are well situated into lower and upper guide blocks. The second pressure stage consists of 8 tungsten carbide (WC) cubes of high strength whose corners are truncated to triangular faces to form an octahedral cavity to host an octahedral $\mathrm{MgO}$ pressure medium that houses the sample under investigation. Six phenolic sheets are used to insulate the second stage from the first and hold the eight WC cubes together with the semi-sintered $\mathrm{MgO}$ ceramic pressure medium well seated inside the octahedral cavity created by the cubes. The eight cubes are insulated from one another by pyrophyllite gaskets and balsa wood spaces that also provide structural support when pressurizing. Two of the phenolic sheets each carry a small copper sheet through which current is transmitted from the external circuit to a graphite resistance furnace to heat the sample.

The $\mathrm{MgO}$ octahedron pressure medium has an edge length of $14 \mathrm{~mm}$ and is used in combination with WC carbide cubes whose corners are truncated to an $8 \mathrm{~mm}$ triangular face, generally called the 14-8 cell assembly. This magnesia pressure medium contains a cylindrical graphite resistance furnace that makes contact with two opposing cubes faces via molybdenum (TZM) rings. The furnace is surrounded by a zirconia sleeve that serves to minimize heat loose from the sample's environment. The $\mathrm{Dy}_{2} \mathrm{TiO}_{5}$ and Dy${ }_{2} \mathrm{HfO}_{5}$ powders were loaded into a platinum capsule and insulated from the graphite furnace by a $\mathrm{NaCl}$ sleeve in order to provide a pseudo- hydrostatic environment around the sample.

\section{RESULTS AND DISCUSSION}

Using the Sumitomo Press and 14-8 cell assembly, four 2.5 to $3 \mathrm{~mm}$ long cylindrical specimens were fabricated at a pressure range of 5 to $10 \mathrm{GPa}$ and temperature condition of $1275 \mathrm{~K}$. These pellets were examined under a microscope and appeared to be well-sintered and without any noticeable defects. The polycrystalline specimens of $\mathrm{Dy}_{2} \mathrm{TiO}_{5}$ and $\mathrm{Dy}_{2} \mathrm{HfO}_{5}$ that were hot-pressed at $10 \mathrm{GPa}$ and $1275 \mathrm{~K}$, for duration of 2 hours had bulk densities determined by Archimedes' method yielding $8.99 \pm 0.09 \mathrm{~g} / \mathrm{cm}^{3}$ for dysprosium hafnate, and $7.36 \pm 0.03 \mathrm{~g} / \mathrm{cm}^{3}$ for dysprosium titanate, both within $1 \%$ of their respective theoretical densities. The bulk densities of the second set of specimens hot-pressed at $5 \mathrm{GPa}$ and $1275 \mathrm{~K}$ for 2 hours were $9.07 \pm 0.03 \mathrm{~g} / \mathrm{cm}^{3}$ for dysprosium hafnate, and $7.84 \pm 0.01 \mathrm{~g} / \mathrm{cm}^{3}$ for dysprosium titanate. The relatively high increase in density of the second dysprosium titanate specimen may be due to some type of phase transformation. However, the density measurements show that dense polycrystalline $\mathrm{Dy}_{2} \mathrm{TiO}_{5}$ and $\mathrm{Dy}_{2} \mathrm{HfO}_{5}$ can be fabricated at moderate pressure and temperature conditions and are feasible for mechanical, thermal and industrial applications.

\section{CONCLUSION}

Although work has been carried out on dysprosium titanate and dysprosium hafnate more research is required in order to improve their general applications and usage. After a comparative analysis on the temperature versus input power to the graphite heater for four successive hot- pressing experiments in the Kawaitype multi-anvil apparatus, reproducibility of the cell temperature is feasible at high pressures. The samples will be further characterized by X-ray diffraction, optical and electron microscopy. Elasticity studies by acoustic interferometry techniques will also be carried out at high pressures and temperatures for engineering design applications.

\section{ACKNOWLEDGEMENTS}

We thank the Science Undergraduate Laboratory Internships Program (SULI) for the summer internship privilege, the Mineral Physics Institute at Stony Brook University (SBU) for use of their High Pressure facilities and x-ray equipment and the National Synchrotron Light Source 2 (NSLS II) for use of their office area. We also extend much gratitude to the Office of Educational Programs (OEP) at Brookhaven National Laboratory (BNL). This project was supported in part by the U.S. Department of Energy, Office of Science, Office of Workforce Development for Teachers and Scientists (WDTS) under the Science Undergraduate Laboratory Internships Program. We are grateful to William Woerner and Richard Triplett for assisting with the x-ray diffraction analysis and for useful discussions. We are also thankful for the support of Professor Robert C. Liebermann and Noel Blackburn, Educational Programs Administrator for the Office of Educational Programs at Brookhaven.

\section{REFERENCES}

${ }^{1}$ V. M. Kosenkov and S. A. Vorob'ev. "Crystal-Chemical Aspect of the Radiation Resistance of Nonmetallic materials," Atomic Energy, 84, 23-27. 1998.

${ }^{2}$ G. Panneerselvam et al., "Thermophysical measurements on dysprosium and gadolinium titanates," Journal of Nuclear Materials, 327, 220-225 (2004).

${ }^{3}$ G. Panneerselvam, R.V. Krishman, K. Nagarajan and M.P. Antony. "Thermal expansion and heat capacity of dysprosium hafnate," Journal of Thermal Analysis and Calorimetry, 101, 169-173. 2010.

${ }^{4}$ V.D. Risovany, E.E. Varlashova, and D.N. Suslov. "Dysprosium titanate as an absorber material for control rods," Journal of Nuclear Materials, 281, 84-89 (2000).

${ }^{5}$ G. D. Gwanmesia and R.C Liebermann. "Polycrysals of High-Pressure Phases of Mantle Minerals: Hot-Pressing and Characterization of Physical Properties," Am. Geophysical Union 117-135 (1992). 


\title{
An Analysis of the mPower Small Modular Reactor Design
}

\author{
Jonathan Rolland
}

\begin{abstract}
As part of the Nuclear Regulatory Commission's (NRC) initiative to usher in a new era of nuclear power reactors the Small Modular Reactor (SMR) Program was started. Since SMR designs are unique compared to typical reactors of the past modeling and analyzing these reactors may not be appropriate using the same methods as previous generations. Although there are more SMR designs being submitted, only the mPower design by the company Babcock \& Wilcox (B\&W) was analyzed by our group. The mPower design is a pressurized water reactor (PWR) design that has been scaled and integrated into a single pressure vessel to fill the requirements of the SMR proposal. The analysis of this reactor was done using the data provided by B\&W with the NRC's neutronics packages: SCALE 6.1, and PARCS 3.2. These have been used to analyze the steady state operation of the mPower design at a beginning of life state with no depletion. This work involved modeling many individual 2D sections of a fuel assembly with the TRITON module in the SCALE package which uses deterministic codes to calculate the macroscopic cross sections for each configuration. When this was completed the results were then converted for use in the core neutronics package PARCS using the GenPMAXS code. PARCS was used to model the core including the control rods and reflectors. This model was then used to find the range of k-effective values from no control rods to full insertion. Another important factor was analyzing the reactor leakage which was expected to be higher than previous generations of reactors due to the smaller geometry intrinsic to SMR design. The findings of this analysis resulted in a higher than expected excess reactivity with an uneven power distribution both radially and axially, an issue which could be caused by a variety of factors including the unavailability of information and the assumptions that were used to fill in such gaps. In the future this model will have to be refined as more information from $B \& W$ is obtained but stands as a successful backbone of a full 3D core model and as a good proof-of-concept for modeling SMR designs
\end{abstract}

\section{Introduction}

As part of the Department of Energy Science Undergraduate Laboratory Internships (SULI) program I was assigned to work at Brookhaven National Laboratory (BNL) as part of the Nuclear Analysis Group to model the reactor core of the mPower Small Modular Reactor. The Small Modular Reactor (SMR) program was started in order to usher in a new era of nuclear power reactors that could fill a different role than traditional large scale reactors. These new reactors are to be lower in electric energy output per reactor, below $500 \mathrm{MWe}$, but have the advantage of being modular from construction through to installation and operation. These reactors are to be assembled in a factory and be small in size so they may be moved to the plant site by rail or freight, then assembled on location upon delivery, as opposed to being built entirely on site. A major advantage of this design includes saving on overhead costs of construction as well as being integral to the modular nature of the reactor, each reactor would be assembled in this manner and delivered to the site and each site may operate multiple reactors in banks of up to 12 . This modularity enables scalable power production to meet the needs of the location over time and allows for limited periods of complete shutdown as each reactor can be refueled individually, taking only a marginal amount of power away from the grid instead of shutting down the entire plant as in a traditional reactor. With these requirements and subsequent advantages in mind different vendors began to submit designs for SMRs to the Nuclear Regulatory Commision (NRC) which are currently under review for approval. This analysis by our group pertained to a single SMR design by Babcock \& Wilcox $(\mathrm{B} \& \mathrm{~W})$, the mPower reactor.

The purpose of this analysis was to take the documents provided by the NRC on the mPower reactor and develop a 3D nodal core model to analyze the neutronics of the reactor core at steady state beginning of life operation. This procedure was possible by using the neutronics packages: the Standardized Computer Analyses for Licensing, or SCALE 6.1 developed at Oak Ridge National Lab, and the Purdue Advanced Reactor Core Simulator (PARCS 3.2), currently under development at the University of Michigan. The SCALE 6.1 package is capable of solving the neutron transport equations using the TRITON sequence and delivers data on cross sections, leakage, and reactivity based on the calculations. PARCS was used with the results of the SCALE model to take these $2 \mathrm{D}$ results and combine them into a single $3 \mathrm{D}$ core. The end result would model the entire length of the core including reflectors and deliver data on reactivity (k-effective), power distribution, temperature, and leakage based again on solutions to the transport equations. These results from PARCS are ultimately needed to evaluate the core as a whole as part of the review of the reactor design and to prove that this methodology for analysis is valid for the review of SMRs.

\section{Cross Section Generation}

To begin this analysis the documents received from the $\mathrm{NRC}$ were evaluated and critical information for the mPower reactor was collected. Using this design information the SCALE 6.1 package was used through the GEEWIZ graphic interface to begin creating a 2D model of an mPower assembly. The mPower core contains five assembly types in the core and each assembly has an axially zoned fuel, this required a separate TRITON model to be generated for each of these zones. The assemblies have quaternary symmetry, so only a quarter assembly was modeled with reflective boundary conditions to minimize computing time. The mPower design documents gave a single enrichment for each zone and a layout for a single assembly type. The design documents received by our group also lacked full information of the core design that led to assumptions during this analysis. Three of 
the five assembly types had a different number of burnable poison pins but the design documents did not show the layout of these pins, symmetry was assumed based on other standard PWR assemblies. The composition and dimensions of the control rods and their guide tubes was also unavailable, they were assumed based on standard PWR data. A final important missing component was the operating temperature of the fuel, moderator and clad, this was again assumed by PWR standards. With these assumptions in place the quarter assembly was configured by inputting all of the compositions and geometries for pins and placing them into an array in the GEEWIZ interface. Branches were established for the presence of control rods, and for different moderator densities. For our calculations the transport equations were also collapsed to two groups, fast and thermal, based on cutoffs used in other PWR simulations. The model did not include any depletion and only modeled a steady state beginning of life assembly. The TRITON sequence was then run on the BNL linux cluster and the outputs generated, this was repeated for over 20 permutations of the assemblies to cover the five assembly types and the axial zoning in each of them, all of these had the same branches and differed only slightly in composition and layout of the fuel pins. A geometry plot was created to check the parameters of the inputs, and example of such is seen in Figure 1.

\section{Figure 1: TRITON Quarter Assembly}

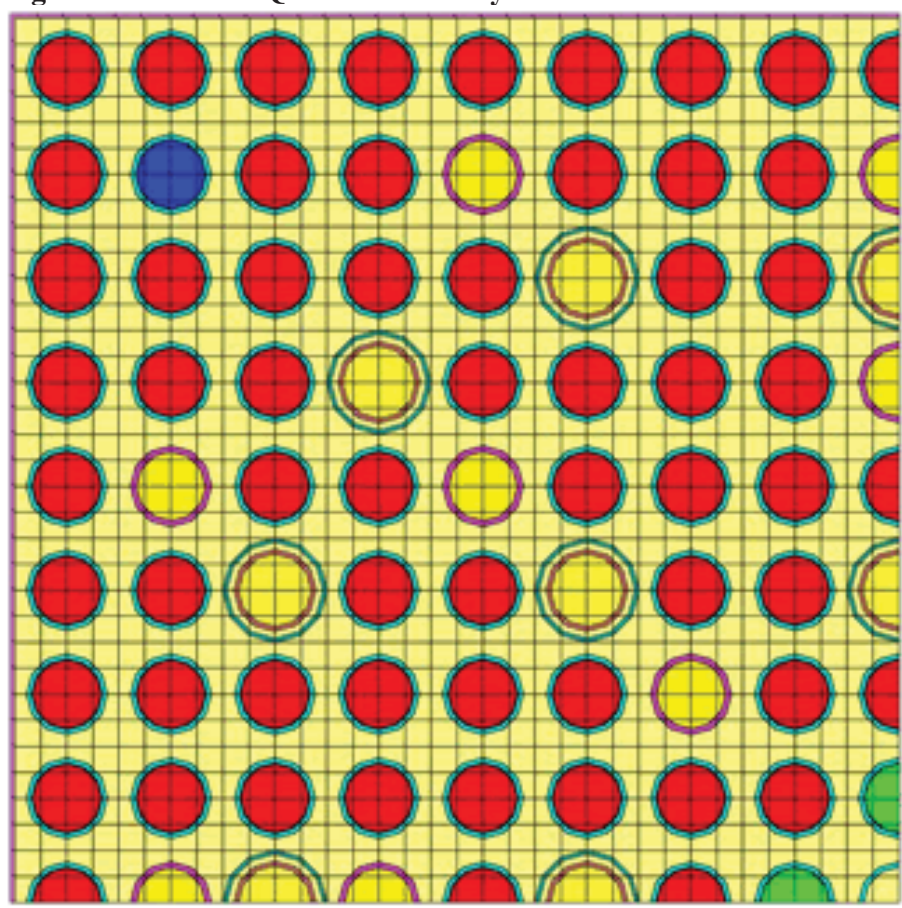

Fig. 1: A TRITON geometry plot of a quarter assembly, fuel pins, control rods and guide tubes can all be seen

Once the data was collected from the SCALE model it was converted via the Generation of the Purdue Macroscopic Cross Section Set program, or GenPMAXS which prepares the binary output files of TRITON into cross sections files readable by PARCS. This program was again used on the Linux cluster to convert each of the TRITON calculation's results into a corresponding PMAX file. When all of these were created and reviewed to make sure the information was converted correctly the PARCS model could begin to be assembled.

\section{PARCS Core Model}

The PARCS input is generated in through a simple ASCII file and inputs the conditions and geometries of a full reactor core. Since single assemblies were modeled by SCALE a radial map was generated of the five assembly types and radial reflectors. Since the reflectors were not specified in composition and geometry in the design documents, the cross sections used were generic PWR reflector cross sections with standard geometries. Since the assemblies are also axially zoned and PARCS operates in 3D by creating axial nodes of the $2 \mathrm{D}$ radial maps 20 nodes were created and the individual assembles were assigned different PMAX files by axial layers in order to recreate the design described. Once the assembly map was completed the next important parameter was the banking and insertion of the control rods. The design information indicated that every assembly had control rods but gave no information on the banking of these rods, for simplicity the banks were assumed based on quarter core symmetry for this model. With the control rod banking completed the other parameters were finalized and PARCS was executed for two cases for initial evaluation: zero control rod insertion and full control rod insertion. These two cases would be used to begin analysis of the mPower core.

\section{Results}

PARCS delivers a detailed output file of power distributions and thermal hydraulic calculations based on the solutions found to the transport equation but most importantly the simulation delivers a measure of reactivity- $\mathrm{k}$-effective. K-effective is the eigenvalue of the reactor as a result of the transport equations and is ideally 1 during operation, lower and the neutron chain reaction won't self propagate and higher and a runaway power situation can occur. The results from PARCS determined with complete control rod insertion the reactivity was computed to be 0.858 , and with no control rod insertion was computed as 1.11. Although these values seem reasonable, reaching criticality and being possible to control with full insertion, the amount of control for k-effective to reach unity was very high. By inserting all the control rods together stepwise into the core through the PARCS model, as seen in Table 1, it was found that the rods needed to be inserted over $85 \%$ for k-effective to reach unity. Although this model considers a steady state beginning of life 'fresh' core with no burnup and will therefore only have its reactivity decrease over its operation, the reactivity is still higher than expected and too much control rod insertion is required to reach criticality. Possible causes of this are the incomplete nature of the design and the assumptions made to fill these gaps. The assumptions made such as the fuel enrichment, assembly layout, control rod specifications, and the reflector cross sections could all have impacted this calculation. 
Table 1: K-effective vs \% control rod insertion

\begin{tabular}{|r|r|}
\hline \% Insertion & k-eff \\
\hline 0 & 1.110117 \\
\hline 5 & 1.090275 \\
\hline 10 & 1.08746 \\
\hline 15 & 1.086334 \\
\hline 20 & 1.085518 \\
\hline 25 & 1.084654 \\
\hline 30 & 1.083684 \\
\hline 35 & 1.082569 \\
\hline 40 & 1.081261 \\
\hline 45 & 1.079686 \\
\hline 50 & 1.077716 \\
\hline 55 & 1.075201 \\
\hline 60 & 1.07193 \\
\hline 65 & 1.067467 \\
\hline 70 & 1.061274 \\
\hline 75 & 1.052237 \\
\hline 80 & 1.038305 \\
\hline 85 & 1.015345 \\
\hline 90 & 0.974575 \\
\hline 95 & 0.897098 \\
\hline 100 & 0.858899 \\
\hline & \\
\hline
\end{tabular}

Looking past k-effective to the other parts of the PARCS output calculations it was seen that the density range of the moderator was covered by the branches in the TRITON data input, but that the distribution of power both axially and radially was skewed. In standard PWR the axial power is normally expected to have a cosine shape over the height of the core, peaked in the axial center of the core, where as the results of this reactor showed the power shape as seen in Figure 2.

Figure 2: PARCS Axial Power Map

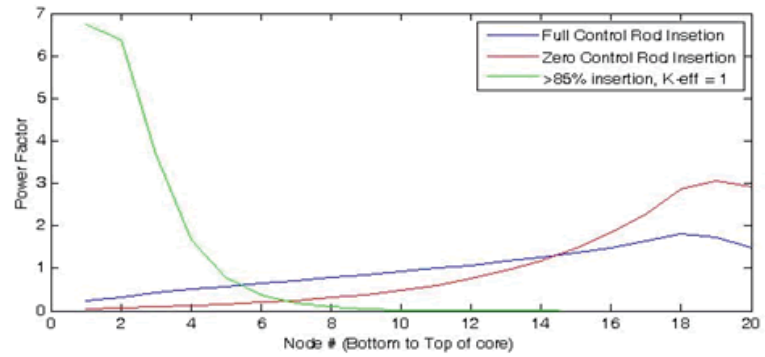

Fig. 2: Axial power map- plotted as the node \# of the PARCS calculation in the axial direction from the bottom to top of the core versus the power multiplier of the average power.

This result is easily attributed to the lack of clear data on the control rod layout and insertion which could change the power shape to a shape that is more expected. This lack of control rod data is also the likely cause of the problem that was also of concern; the radial power distribution of the reactor had extreme highs and lows. An ideal core has a fairly uniform power distribution in the central part of the core, with lower power at the periphery due to leakage. This core did have its lowest power on the periphery as the small core did have more leakage than its larger counterparts but also had large power spikes in different assemblies, especially the central one. This can be seen in Figure 3 where the quarter symmetric axial power map has been plotted. An attempt was made to understand the inconsistencies in the power distribution seen by inserting different groups of control rods but it was not successful in search of normalizing all of the fuel assemblies. As single control rod groups were inserted in areas of higher power the power was simply shifted and PARCS failed to converge to a k-effective under some conditions. The variables of k-effective, radial power shape, and axial power shape became too unruly to balance without any design information of the control rod banking or control rod insertion during reactor startup. These problems coupled with the existing high reactivity made finding a reasonable design for reactor startup untenable during the time allotted for this analysis.

Figure 3: PARCS 1/4 Radial Power Factor Map

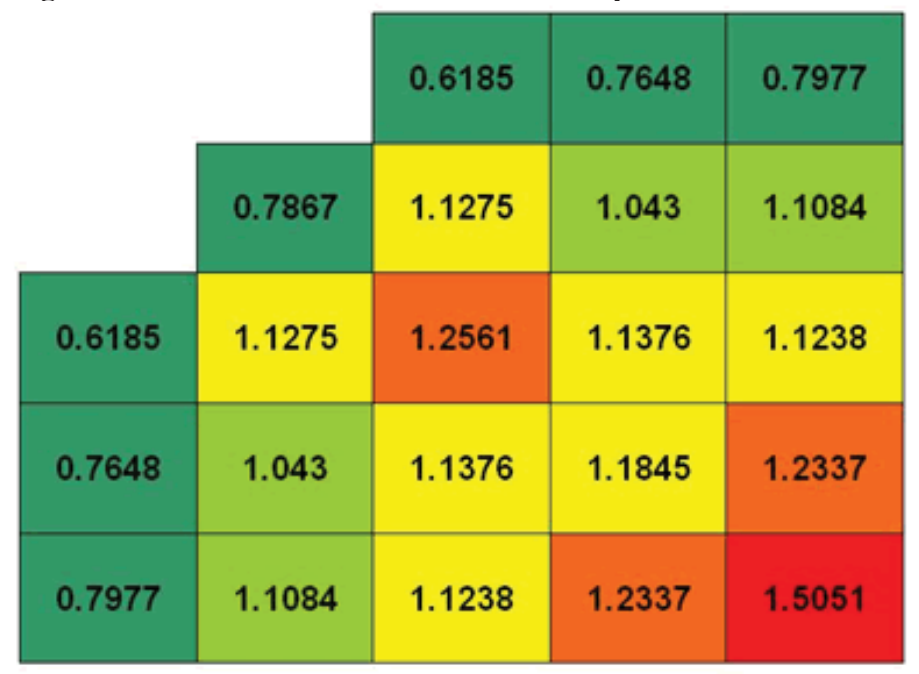

Fig. 3: Radial power map, numbers representing the power multiplier in each assembly based on the average power expected for a single assembly.

As part of the analysis to balance the power distributions in this reactor, a MATLAB script was generated that can take a PARCS output file and plot the axial power of each individual assembly in the core. This code was useful in visualizing the axial power shape and the radial differences on a single plot. These plots were similar to that of figure 2, with each assembly being plotted individually instead of the radial average. This code was generalized to be used with any PARCS output and can be used in the future for visualization of other reactors.

\section{Conclusions and Future Work}

This analysis achieved its goal of successfully building a 3D core model of the mPower small modular reactor by coupling the 2D code TRITON, part of the SCALE 6.1 package, with the 3D nodal code PARCS. Problems with the power distributions and the higher than expected k-effective were reasoned to be caused by the nature of being a freshly loaded core and the assumptions made in the design. As more information becomes available the model can be refined to be more accurate and the assumptions made in this process can be eliminated, making it possible to eliminate variables from the troubleshooting. Eventually with 
the appropriate data this model may be expanded and used as the backbone to model transients and further conditions of the full core as well as on a per assembly basis. Alongside this the MATLAB script used to graph the axial power of the reactor has been generalized to be useful to visualize any PARCS output.

The final part of this analysis was to create quarter core SCALE model. Modeling a quarter core in SCALE would simplify the reactor to two dimensions but provide useful data on the leakage of the core. This model was developed but due to the number of elements present in a quarter core the model could not generate a geometry plot within a reasonable time frame and by extension not solve for the leakage data needed during this ten week analysis. This may be due to a weakness in the SCALE software's handling of multiple arrays or simply be too large to compute. This result would be useful moving forward to see the results of leakage that come from a TRITON result in comparison to that of PARCS. This model and issue can be evaluated in further analysis.

The data resulting from this analysis will be helpful to have a working model at BNL to help understand SMR designs, although it will be necessary to refine the model for an accurate result. Despite this the models generated are a successful proof-of-concept and starting point for future modeling of SMRs as a reference case.

\section{Acknowledgements}

I would like to thank the Department of Energy, Brookhaven National Lab, and the Nuclear Analysis Group for hosting me for this summer term and affording me this opportunity to apply the concepts I have learned at the University of Illinois to a real world scenario. I would like in particular to thank my mentor Arantxa Cuadra Gascon for her patience and guidance as well as Nick Brown for his help in understanding PARCS and finally Arnie Aronson in his support and advice for this project every step of the way.

This project was supported in part by the U.S. Department of Energy, Office of Science, Office of Workforce Development for Teachers and Scientists (WDTS) under the Science Undergraduate Laboratory Internships Program (SULI). 


\title{
Magnetic shaping of laser ablation plasma
}

\author{
Mark Romanelli \\ College of Engineering, Cornell University, Ithaca, NY, 11787 \\ Shunsuke Ikeda, Megumi Sekine \\ Tokyo Institute of Technology, Yokohama, Kanagawa, Japan and Riken, Wako, Saitama, Japan \\ Masahiro Okamura \\ Collider-Accelerator Department, Brookhaven National Laboratory, Upton, NY, 11973
}

\begin{abstract}
Laser Ion Sources (LISs) have become an important area of research at Brookhaven National Laboratory. Since beams extracted from plasma are accelerated and collided in the Relativistic Heavy Ion Collider (RHIC), the production of plasma is of major importance for RHIC's continued operation. Ion beams are also used by the NASA Space Radiation Laboratory (NSRL) in their study of cosmic rays. Laser ablation may be a better way of generating the plasma from which these beams are formed. Before it can be utilized by RHIC or NSRL, we must have the ability to control and shape the plasma pulses created with laser ablation. As a part of this study, we used a variety of magnetic pulses and static fields to alter the shape of plasma created by laser ablation. We have demonstrated that it is possible to alter the usual shape of the plasma, which is typically a Maxwell-Boltzmann distribution. By inducing a magnetic field $2 \mu$ s before the most of the plasma has passed through the solenoid, we could dramatically increase the peak current and the total charge of a pulse. Also, by inducing large magnetic field $2 \mu$ s after the peak plasma flux has exited the solenoid, we could increase the time of flight to the maximum current, which can lead to the production of an ion beam with a steady current. With this knowledge, future scientists will be able to create plasma pulses in the exact shape needed for specific experiments.
\end{abstract}

\section{Introduction}

Ion beams are currently being used in several areas of research at Brookhaven National Laboratory. The NSRL is currently using beams composed of heavy ions to simulate cosmic rays in their biology experiments. In addition, RHIC uses an ion beam in its collider experiments.

The current means of producing these beams requires the use of a Hollow Cathode Ion Source (HCIS). The HCIS sends an electrical current through a vacuum chamber to create a beam of positive ions. This beam can then be sent to RHIC or the NSRL for use in their experiments.

LISs are a new way of producing ion beams. While a LIS is able to produce beams with much higher current than HCIS can, it also presents a major weakness when compared to HCIS. This weakness is that beams formed by a LIS typically form a Maxwell-Boltzmann Distribution in their current output. This means that the output is peaked and does not give constant current over the pulse's length. Because of this, experiments requiring a more steady supply of current cannot use LISs as their ion source.

The ability to shape the current output of a LIS would increase its versatility as a source of ions. In our experiments, we used magnetic fields, both pulsed and constant, in an effort to alter the shape of the current output and change the Maxwell- Boltzmann distribution which is typically produced.

\section{Methods}

In our experiments, we assembled the test set-up seen in Figure 1. All equipment listed was enclosed in a vacuum chamber to prevent the plasma or laser from interacting with gases and causing experimental error. We used a $1064 \mathrm{~nm}$ wavelength $\mathrm{Nd}$ :YAG laser with a Q-switch delay of $250 \mu$ s and a pulse energy of 600 $\mathrm{mJ}$. We chose an iron target for our experiments because iron ions are one of the major components of cosmic rays. Laser pulses were fired into the target chamber at an iron target. The target then ablated into a plasma consisting of +1 iron ions and electrons. The plasma moved past $20 \mathrm{~cm}$ of empty space into a $12 \mathrm{~cm}$ long solenoid, the pulsed solenoid. The plasma then passed through a $3 \mathrm{~m}$ long solenoid, the constant solenoid, and into a Faraday cup. The Faraday cup removed the electrons from the plasma, forming an ion beam, and recorded the current density of the beam over time.

The pulsed solenoid was attached to the Laser Source Solenoid Pulser (LSSP) which took in a constant voltage signal and sent a pulse with a $10 \mu$ s rising time. We have included a graph of a typical pulse as Figure 5 in the Appendix. All pulses that we created were similar in appearance, but we did change the time that the pulse was fired relative to the laser's firing time, and the amplitude of the pulse, which determined the magnetic field created by the pulsed solenoid. Because of the high voltage running through it, the pulsed solenoid had to be shielded with grounded aluminum foil so that the electrical fields from the wires did not interact with the plasma. We determined the magnetic field at the center of the solenoid by the following equation:

$$
B=\frac{\mu_{0} I N}{2 \sqrt{R^{2}+\left(\frac{L}{2}\right)^{2}}}
$$

Where B is the magnetic field at a specific point in the solenoid, $\mu 0$ is the permeability of free space, $I$ is the current running through the solenoid, $\mathrm{N}$ is the number of turns in the solenoid, $\mathrm{R}$ is the radius of the solenoid, and $\mathrm{L}$ is the length of the solenoid.

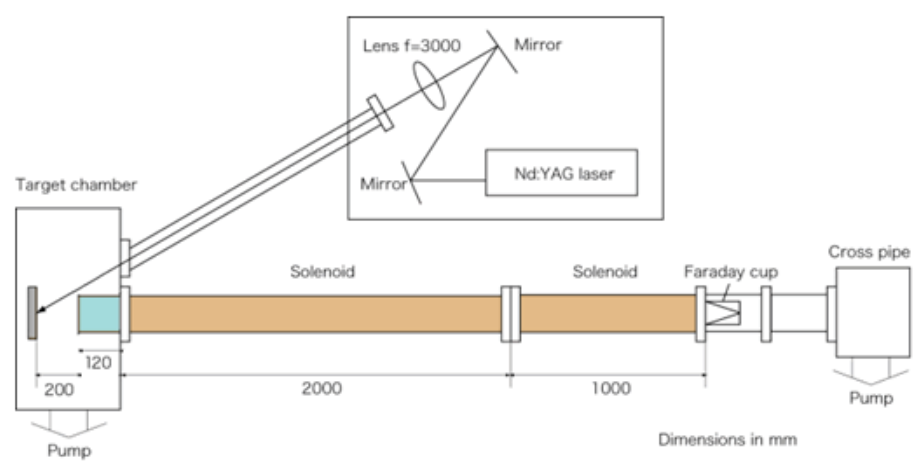

Figure 1: Set up used in our experiments. 
For this specific solenoid, the number of turns was 50, the length was $12 \mathrm{~cm}$, and the radius was $44 \mathrm{~mm}$. It can be seen that the magnetic field produced by this solenoid was linearly proportional to the current supplied to it which was in turn proportional to the voltage of the pulse from the LSSP. We verified this relationship with a Hall sensor.

Our first test was to ablate the iron without magnetic fields from either solenoid. This gave us the time it took each part of the plasma to reach the Faraday cup $3.32 \mathrm{~m}$ away from the target. Working backwards, we could determine the velocity of each section of the plasma. We could further use this information to determine when each section of the plasma was inside the pulsed solenoid. We determined that the peak of the plasma reached the opening of the pulsed solenoid at $4 \mu$ s and exited the pulsed solenoid at $7 \mu \mathrm{s}$.

After determining the plasma's velocity in the first test, we proceeded to run trials with different variables altered. The pulsed solenoid was given several voltages from $273 \mathrm{~V}$ to $2570 \mathrm{~V}$, which correspond to magnetic fields from 26.9 gauss to 510.6 gauss. We altered the time that the pulsed solenoid was fired from $1 \mu \mathrm{s}$ after the laser was fired to $20 \mu \mathrm{s}$ after the laser was fired and several times in between. The constant solenoid was given static magnetic fields of 0 gauss, 4 gauss, 15 gauss, and 50 gauss.

\section{Results}

Once all the data was recorded, we analyzed the data in terms of total charge created, peak current, time of flight to the peak, and half-width. After compiling this data, several interesting patterns began to emerge.

The use of the long solenoid in the 4 gauss range appears to have had a guiding effect on the plasma, compared to tests without the long solenoid's magnetic field. However, the use of a 15 gauss magnetic field had a disruptive effect on the experiments

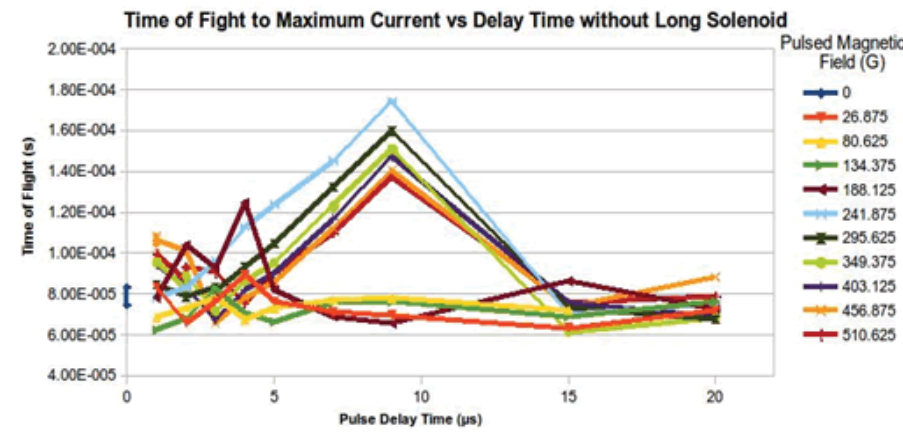

Figure 2: Time of flight reaches a maximum at $9 \mu$ delays.

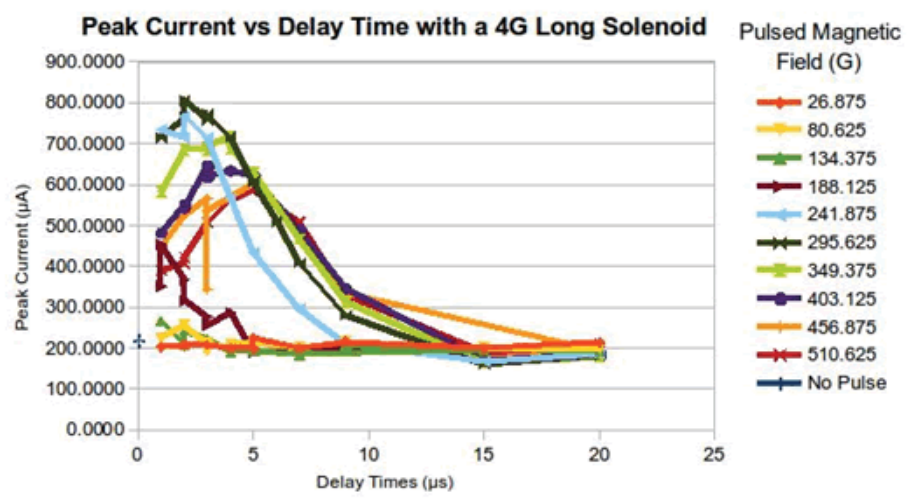

Figure 3: Maximum current for different delays and magnetic fields and tests in this range did not have reproducible results. For constant magnetic fields of 50 gauss, there was a great increase (almost twenty-fold) in peak current and in total charge created compared to tests without the constant solenoid. However for tests in this range, the pulsed magnetic field had little effect compared to the effect of the long solenoid's magnetic field. We believe that for both the 15 gauss and 50 gauss long solenoid trials the pulsed solenoid did not produce large enough of a magnetic fields to overpower the constant magnetic fields of the long solenoid. The lack of variation in trials with the 50 gauss long solenoid suggests that there was almost no plasma in the test chamber that was unaffected by the long solenoid. At that point we had recorded all of the plasma that it was possible to record with our set-up and no action of the pulsed solenoid could alter that.

This is not to say that the pulsed solenoid never had an effect on the plasma. On tests in the 0 and 4 gauss long solenoid range, pulses with a magnetic flux density of 241 gauss and greater, timed up to $9 \mu$ s after the laser fired were observed to greatly increase time of flight to the point of maximum current. Figure 2 below illustrates the effect that we observed. In these cases, this was most likely because the pulsed solenoid was able to guide plasma, which ordinarily would not have left the target chamber, towards the long solenoid and from there into Faraday cup. Thus, the current maximum recorded was not composed of particles which would have made up the "natural maximum" which would normally occur but rather one made up of particles that were only recorded because of the pulsed solenoid.

This hypothesis is supported by two other related trends observed in our tests. Firstly, in all trials that this behavior occurred, the maximum current was greater than the maximum current for tests without the pulsed solenoid. Secondly, in trials that the time of flight was made to increase, the half width of the plasma pulse was also seen to increase. In essence, this means that both the long and the pulsed solenoid were able to alter the shape of the plasma recorded and with the correct timing and field strength, could increase the time that the plasma exhibits its maximum current. This effect was noted in both tests with magnetic fields of 0 gauss and 4 gauss in the long solenoid.

Additionally, on the theme of shaping the plasma, we noticed that we could use the same factor that was delaying maximum current to greatly increase the maximum current instead. We found that by firing the solenoid anywhere from $2 \mu \mathrm{s}$ before to $1 \mu \mathrm{s}$ after the peak amount of plasma had entered the solenoid, the magnetic pulses were able to gather extra plasma from the target chamber. The extra plasma at this timing served to build on the current in the "natural maximum' rather than to create a new maximum as

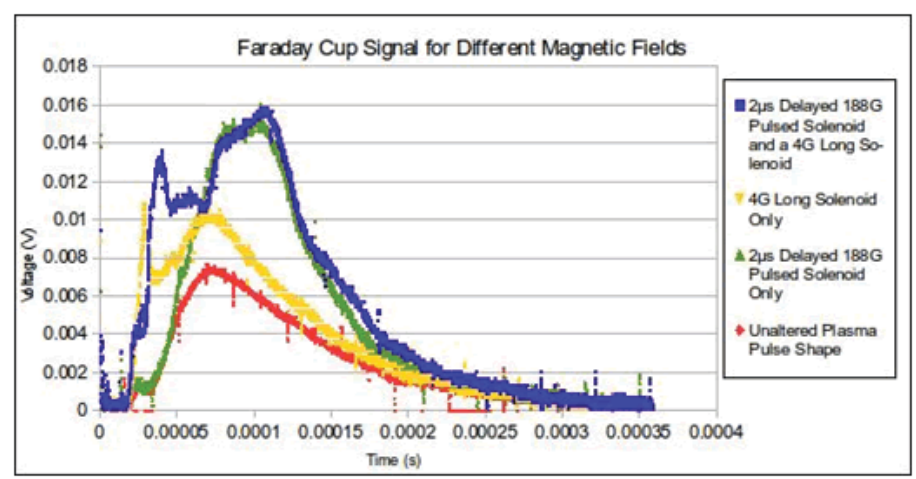

Figure 4: Current distributions produced by different magnetic fields 
it did in the $9 \mu$ s delay case. A magnetic pulse of magnitude 295 gauss at a delay of $2 \mu \mathrm{s}$, with a 4 gauss long solenoid, created a maximum current of $805 \mu \mathrm{A}$, almost 4 times as much as the 215 $\mu \mathrm{A}$ current created with only the 4 gauss long solenoid.

It is further interesting to note the relationships between the different plasma properties. We noticed a very clear relationship between the peak amount current and the total amount of charge created. Cases where high current was observed also tended to have a high total amount of charge, and similarly for low current.

We did discover some of the limits of this new technique as well. It appears that the usefulness of our pulsed solenoid was maximized in ranges from 250 gauss to 300 gauss. As we increased the maximum magnetic field in the pulsed solenoid, the pulsed field was able to gather more and more plasma from the test chamber. However above 300 gauss, the total charge collected and the maximum current observed began to decrease. We believe this may have been due to the curvature of the magnetic field lines in the pulsed solenoid. In all cases, the plasma followed the curvature of the pulsed solenoid's magnetic field lines into the pulsed solenoid. But at magnetic fields higher than 300 gauss, we believe that the plasma may have continued following the pulsed solenoid's magnetic field lines into walls of the long solenoid. Plasma that flew into the long solenoid's walls did not reach the Faraday cup and was therefore not recorded. At pulsed magnetic fields above 300 gauss, the field from the long solenoid may not have been strong enough to prevent this from occurring.

There were cases where our magnetic pulses failed to have an effect. Magnetic pulses with delays in the $15 \mu$ s and $20 \mu$ range yielded results that looked almost identical to the results obtained without a pulsed solenoid. Likewise, magnetic pulses with magnitudes lower than 188 gauss also had almost no effect on the shape of the plasma that we recorded.

While these results did show us some of the limits of our technique, the other trials show that our technique can be very effective when used correctly. Figure 3 gives an example of the different shapes of current output that we were able to achieve in our experiments. The differences between the unaltered pulse in red and the pulse in blue are very clear.

\section{Discussion}

The ability to lengthen the peak current of plasma output with a pulsed solenoid has allowed us to overcome one of the greatest weaknesses of LISs, namely their inability to supply continuous current. With more precise timing adjustments and input currents, one can make an ion beam with relatively constant current output, or any other output required.

In our trials, we were unable to alter the pulse width, but we believe that changing that variable will also have important effects on the ion beam. We would recommend this as an area of future study.

The only consideration preventing us from putting forward that this technique be put into practice immediately is that of emittance. At the writing of this paper, the emittance of the ion beams produced with this method has not been analyzed. Once the emittance is better studied, we would recommend that our magnetic shaping of plasma be put into practice. Our technique should be suitable for both the needs of RHIC and NSRL.

\section{Acknowledgements}

We would like to thank the following people for their contributions to this experiment: David, Chinquegrani, Masafumi Kumaki, Yasuhiro Fuwa, and Takeshi Kanesue.

This project was supported in part by the U.S. Department of Energy, Office of Science, Office of Workforce Development for Teachers and Scientists (WDTS) under the Science Under- graduate Laboratory Internships Program (SULI).

\section{References}

"Experiment 7: Magnetic Fields." The University of Tennessee At Chattanooga. N.p., n.d. Web. 22 July 2013. <http:// www.utc.edu/Faculty/ Tatiana-Allen/magfield.html>. Takahashi, Kazumasa, Masahiro Okamura, Megumi Sekine, Eric Cushing, and Peter Jandovitz. Effect of Solenoid Magnetic Field on Drifting Plasma. BNL.. 29 July 2013. Poster Presentation.

\section{Appendix}

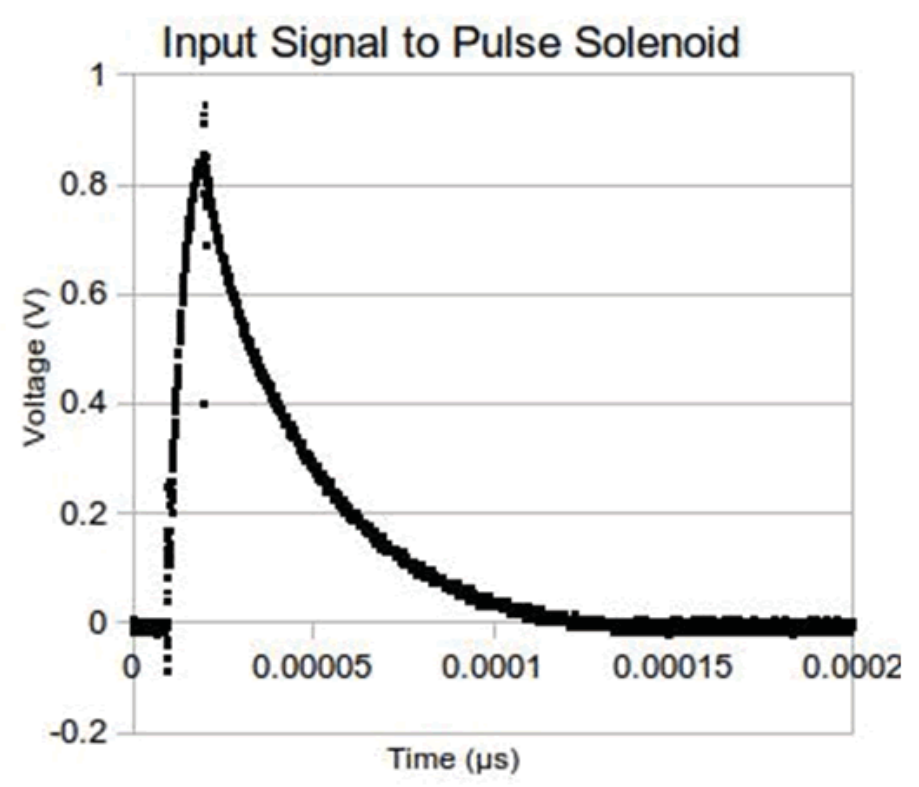

Figure 5: Typical input voltage to pulse solenoid

Peak Current vs Static Magnetic Field without the Pulsed Solenoid

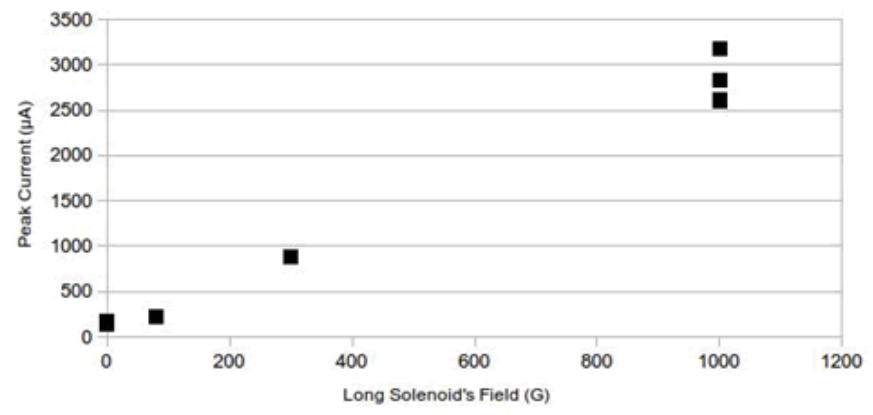

Figure 6: Effect of the long solenoid on the peak current recorded 


\title{
Control of the beam orbit in the NSLS-II storage ring injection straight section
}

\author{
Evgeniya Rubin \\ Chemical Engineering Department, City College of New York, New York, NY, 10031 \\ Timur Shaftan \\ Photon Science Department, Brookhaven National Laboratory, Upton, NY, 11973
}

\begin{abstract}
The National Synchrotron Light Source II (NSLS-II) project is an advanced, highly optimized, third-generation medium energy storage ring that will provide sophisticated new tools for discovery-class science in clean energy technologies. The objective of this work is to model and calculate the trajectory of electron bunches through a series of magnets located in the injection straight section of the NSLS-II storage ring. In this section the incoming beam from the booster ring merges with the beam circulating along the storage ring. Various focusing and bending magnets are used to enable the efficient injection of the particles into the storage ring. Consequently, varying the strength of a magnetic field through the injection magnets controls the injected and stored beam trajectories. As a result of my project I developed a python program with a graphic user interface (GUI) that will allow NSLS-II machine operators to adjust the beam orbit in the vicinity of the injection straight section.
\end{abstract}

\section{Introduction}

Particle accelerators come in many forms applying a variety of technical principles. All are based on interaction of the electric charge with static and dynamic electromagnetic fields and it is the technical realization of this interaction that leads to the different types of particle accelerators. The National Synchrotron Light Source II is a 331,385-square-foot facility contains an electron liner accelerator(linac), a booster ring, an energy storage ring, beam lines, experimental stations, and science research offices. A $100 \mathrm{kV}$ electron gun with thermionic cathode produces electrons that are injected into a booster, $158 \mathrm{~m}$ synchrotron, where they gain energy of $3 \mathrm{GeV}$. Then electrons are extracted into a storage ring where they circulate emitting a synchrotron radiation due to transverse acceleration during deflection in the bending magnets. This radiation is highly collimated in the forward direction, of high brightness and therefore of great interest for basic research, technology, and medicine.

Extraction from the booster is an important procedure during which injected and stored bunches have to be brought close to each other in order to be kicked into the storage ring. At NSLS II the extraction is performed with a four bump scheme. A kicker imparts a $5 \mathrm{mrad}$ angle, which is then followed by thin pulsed septum, and a large DC septum into the booster to storage ring line. In order to calculate and control the injection process a special monitoring routine is developed as a goal of this project. This routine generates a golden trajectory of the beam base on the input parameters of the magmatic elements and compares it with the real trajectory of stored and injected beams.

II. Theory

\section{A. Girder Magnets}

Two typical girders (Fig.1) are located on both sides of the injection straight section (Fig.2) contain various focusing and directing magnets and monitoring elements: quadruples, dipoles, sextopoles, correctors, beam position monitors (BPM), and drifts. In order to calculate the vector of the particle optical coordinates $\mathrm{Z}$ going through the elements we implement a map matrix, $\mathrm{M}$, that describes the physical changes in the beam coordinates

$$
\begin{aligned}
& Z_{2}=M \cdot Z_{1} \\
& Z=\left(\begin{array}{c}
x \\
y \\
x^{\prime} \\
y^{\prime} \\
t \\
\delta
\end{array}\right)=\left(\begin{array}{c}
x \\
y \\
x^{\prime}=p_{x} / p_{0} \\
y^{\prime}=p_{y} / p_{0} \\
t=k\left(t-t_{0}\right) \\
\delta=\left(E-E_{0}\right) / E_{0}
\end{array}\right)
\end{aligned}
$$

where $x, y$ are the beam position coordinates, $x$ ', $y^{\prime}$ are particle relative momentums, $\mathrm{p}_{\mathrm{x}}, \mathrm{p}_{\mathrm{y}}$ are particle momentum, $\mathrm{p}_{0}$ is reference particle momentum, $\mathrm{t}$ - time of flight of a particle, $\mathrm{t}_{0}$ is the time of flight of the reference particle, $\mathrm{k}$ is a constant of dimension velocity, $\delta$ - relative energy, $\mathrm{E}$ - particle energy, $\mathrm{E}_{0}$ - energy of the reference particle.

It is easier to understand the concept of the traveling map using a simple drift example. Drift is the most common element of the girder. Traveling through a drift electros change their relative positions $\mathrm{x}$ and $\mathrm{y}$ keeping a constant momentum $\mathrm{x}$ ' and $\mathrm{y}$ '. We can express these changes just for an $\mathrm{x}$ coordinate as:

$$
\begin{aligned}
& x_{2}=x_{1}+m_{1} \cdot l \\
& x_{2}^{\prime}=x_{1}^{\prime}
\end{aligned}
$$

This can obviously be written in the matrix form:

$$
\left(\begin{array}{l}
x_{2} \\
x_{2}^{\prime}
\end{array}\right)=\left(\begin{array}{ll}
1 & l \\
0 & 1
\end{array}\right)\left(\begin{array}{l}
x_{1} \\
x_{1}^{\prime}
\end{array}\right)
$$

It is important to note that matrix $\left(\begin{array}{ll}1 & l \\ 0 & 1\end{array}\right)$ depends only on the characteristic properties of the element which here is the length 1 . On the other hand vector $\left(\begin{array}{l}x_{1} \\ x_{1}^{\prime}\end{array}\right)$ depends only on the
parameters of the beam.

Another interesting element of the girder is a Quadrupole Magnet (Fig.3). It is used for particle focusing and consists of groups of four magnets laid out so that in the planar multipole 
expansion of the field the dipole terms cancel and where the lowest significant terms in the field equations are quadrupole. Quadrupole magnets are useful as they create a magnetic field whose magnitude grows rapidly with the radial distance from its longitudinal axis. The derivation of a quadrupole magnet map is shown below:

The equation of motion is $x^{\prime \prime}+k x=0$. Assuming the solution $x(s)=A \cos \sqrt{k} s+B \sin \sqrt{k} s$ and taking initial conditions as $x=x_{0}$ and $x^{\prime}=x_{0}$ at $s=0$ we obtain

$$
\left(\begin{array}{c}
x(l) \\
x^{\prime}(l)
\end{array}\right)=\left(\begin{array}{cc}
\cos \sqrt{k} l & \frac{\sin \sqrt{k} l}{\sqrt{k}} \\
-\sqrt{k} \sin \sqrt{k} l & \cos \sqrt{k} l
\end{array}\right)\left(\begin{array}{c}
x_{0} \\
x_{0}{ }^{\prime}
\end{array}\right)
$$

where $x$ is a position of the beam in the vacuum chamber with respect to the center of the vacuum chamber, $x_{0}$ is an initial position of the beam entering the magnet with respect to the center of the vacuum chamber, and $\mathrm{s}$ is a location of the magnet on the beamline with respect to a reference point.

Dipole magnet is another electromagnet of the girder used to create a homogeneous magnetic field over some distance. Particle motion in that field will be circular in a plane perpendicular to the field and collinear to the direction of particle motion and free in the direction orthogonal to it. Thus, a particle injected into a dipole magnet will travel on a circular trajectory. There are two types of dipole magnets in the injection straight section of NSLS II, closed bump correctors and kicker magnets. The physics behind these elements is described later in this section.

Sextupole magnet consists of groups of six magnets set out in an arrangement of alternating north and south magnetic poles arranged around an axis. They are used to control momentum distribution of an electron bunches, in other words, particles spread in the direction of motion. These magnets are not taken into consideration in this project due to their complex non-liner effect on a beam trajectory.

Beam Position Monitors (BPMs) are used for the beam diagnostics. The readings from the monitors give us information about a real beam trajectory that theoretically has to follow closely the golden or calculated beam trajectory. On the other hand, the real trajectory is being distorted by various small defects in the vacuum chamber. Using the four-bumps correction method, we can adjust the orbit displacement and the orbit angle to facilitate ease of injection and extraction, to avoid unwanted collisions, and to avoid the limiting-aperture in the accelerator. In the injection straight section of the storage ring we use a four-bump method facilitated by four thin dipoles (correctors) with kick angles $\theta_{i}(i=1$, $2,3,4)$. From equation for a particle coordinate

$$
y_{c o}(s)=\frac{\sqrt{\beta(s)}}{2 \sin \pi v} \sum_{i=1}^{4} \sqrt{\beta} \theta_{i} \cos \left(\pi v-\left|\psi-\psi_{i}\right|\right)
$$

where $\theta_{i}=(\Delta B \Delta s) / B \rho$ and $(\Delta B \Delta s)$ are the kick-angle angle and integrated dipole field strength of the i-th kicker. The conditions that the closed orbit is zero outside of these four dipoles are $y_{c o}$ $\left(s_{4}\right)=0, y_{c o}^{\prime}\left(s_{4}\right)=0$, or

$\sqrt{\beta_{1}} \theta_{1} \cos \left[\pi v-\psi_{41}\right]+\sqrt{\beta_{2}} \theta_{2} \cos \left[\pi v-\psi_{42}\right]+\sqrt{\beta_{3}} \theta_{3} \cos \left[\pi v-\psi_{43}\right]+\sqrt{\beta_{4}} \theta_{4} \cos \pi v=0$ $\sqrt{\beta_{1}} \theta_{1} \sin \left[\pi v-\psi_{41}\right]+\sqrt{\beta_{2}} \theta_{2} \sin \left[\pi v-\psi_{42}\right]+\sqrt{\beta_{3}} \theta_{3} \sin \left[\pi v-\psi_{43}\right]+\sqrt{\beta_{4}} \theta_{4} \sin \pi v=0$ where $\psi_{j i}=\psi_{j}-\psi_{i}$ is the phase advance from $\mathrm{s}_{i}$ to $\mathrm{s}_{j}$. Expressing $\theta_{1}$ and $\theta_{2}$ we obtain

$$
\left\{\begin{array}{l}
\sqrt{\beta_{3}} \theta_{3}=-\left(\sqrt{\beta_{1}} \theta_{1} \sin \psi_{41}+\sqrt{\beta_{2}} \theta_{2} \sin \psi_{42}\right) / \sin \psi_{43} \\
\sqrt{\beta_{4}} \theta_{4}=-\left(\sqrt{\beta_{1}} \theta_{1} \sin \psi_{31}+\sqrt{\beta_{2}} \theta_{2} \sin \psi_{32}\right) / \sin \psi_{43}
\end{array}\right.
$$

The orbit displacement inside of the region of the orbit bump can be obtained by applying the transfer matrix to the initial coordinates.

Extraction from the booster is also performed with a fourbump scheme using kicker correctors. The following map is used to calculate the kicker's transport map:

$$
\begin{aligned}
& \left(\begin{array}{l}
x \\
y \\
x^{\prime} \\
y^{\prime} \\
t \\
\delta \\
1
\end{array}\right)_{2}=\left(\begin{array}{ccccccc}
1 & 0 & 0 & 0 & 0 & 0 & 0 \\
0 & 1 & 0 & 0 & 0 & a 26 & a 27 \\
0 & 0 & 1 & 0 & 0 & 0 & 0 \\
0 & 0 & 0 & 1 & 0 & 0 & 0 \\
0 & 0 & 0 & 0 & 1 & 0 & 0 \\
0 & 0 & 0 & 0 & 0 & 1 & a 67 \\
0 & 0 & 0 & 0 & 0 & 0 & 1
\end{array}\right)\left(\begin{array}{c}
x \\
y \\
x^{\prime} \\
y^{\prime} \\
t \\
\delta \\
1
\end{array}\right)_{1} \\
& a 26=\frac{B y}{B r}=\frac{a 26^{*} a 67}{2}-a 26 \\
& a 67=-C E_{0}^{3}\left(\frac{1}{B r}\right) B y^{2}\left(\frac{1}{2 \pi l}\right)
\end{aligned}
$$

All the calculations presented above define a golden beam trajectory - the calculated trajectory of the beam.

\section{Programming}

All the calculations described above were written in Python language in order to plot the electron beam trajectories through the injection straight section. The code editing was done in a "Sublime Text 2" text editor and ran with iPython-2.7 and Python application on the Mac OS X Lion System. In order to generate the main graph of the three trajectories the following standard Python packages were used: NumPy, PyLab, Math, Matplotlib. The three-trajectory plot is shown in Fig. 4. The real and injected beam trajectories are given by BPM readings, while the golden trajectory is being calculated using element matrices and beta functions.

\section{Graphic User Interface (GUI)}

GUI is a type of user interface that allows an operator to interact with the program via graphical icons and visual indicators. In this project a GUI is made to allow an NSLS II operator to control the golden trajectory by varying the current that goes through the corrector magnets. Be varying the strength of the field an operator can control the distance between the stored beam and the injected beam. The two beams have to come close together at approximate the distance less then one millimeter in order to be kicked back into the storage beam vacuum chamber. In the future the GUI should allow the operator to model a situation where he could turn on and off any corrector along the line. 


\section{Acknowledgements}

Bill Wahl, Weiming Guo for valuable discussions an support

Yoshiteru Hidaka, Lingyun Yang, and Guimei Wang for IT support. This project was supported in part by the U.S. Department of Energy, Office of Science, Office of Workforce Development for Teachers and Scientists (WDTS) under the Science Undergraduate Laboratory Internships Program (SULI).

\section{References}

D. Padrazo, O. Singh, I. Pinayev, R. Fliller, T. Shaftan, B.

Kosciuk, R. Meier, Y. Hu, Brookhaven National Laboratory, Upton, NY 11973, USA "NSLS-II injector system diagnostics"

Lee, S Y. Accelerator Physics. Hackensack, NJ: World Scientific, 2007. Print.

Conte, M, and W W. MacKay. Physics of Particle Accelerators. River Edge, NJ: World Scientific, 1991. Print.

Wiedemann, H. Particle Accelerator Physics I. Germany: Springler, 1999. Print.

Berz, M. Introductory Beam Physics. 1999. Michigan State University. Web. 5 Aug. 2013. <http://bt.pa.msu.edu/cgibin/display.pl?name=bphy861>.

\section{Figures}

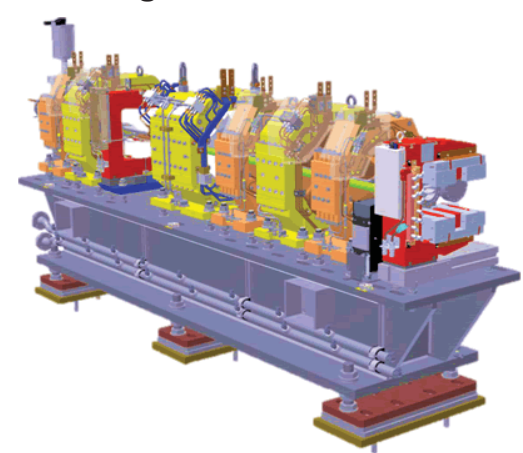

Fig.1. Rendering of girder supporting high-precision magnets: quadrupole, sextopoles, correctors, and beam position monitor devises (the drawing is created in an Autodesk Inventor software) (Source: www.bnl.gov)
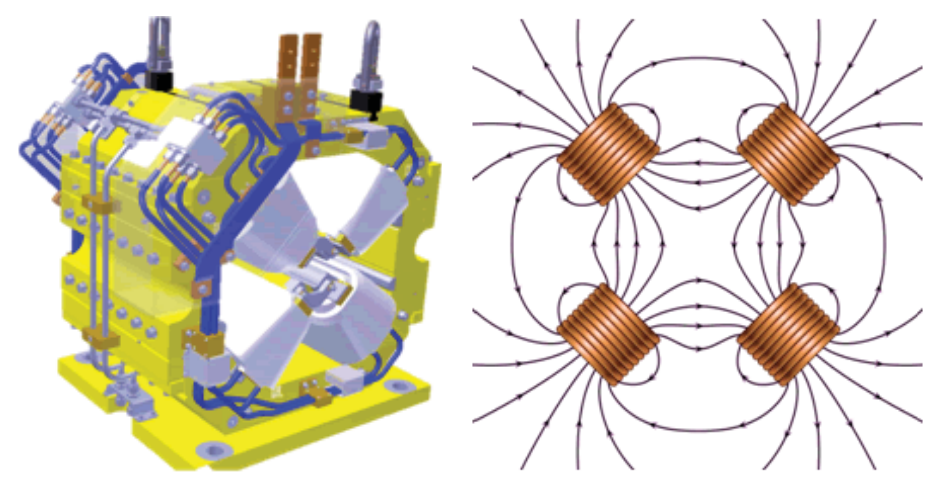

Fig.3. Rendering of storage ring quadrupole magnet (left) and magnetic field lines (right) in a quadrupole magnet showing zero effect in the center and increasing effect towards the sides (Source: www. bnl.gov)

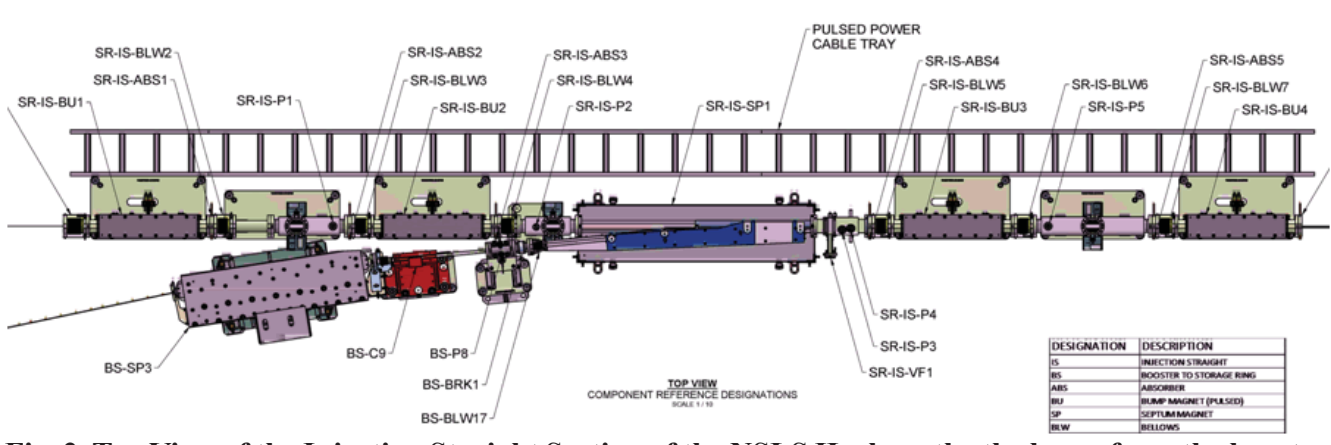

ring merges with the storage ring beam. (Source: BNL Photon Science Department)

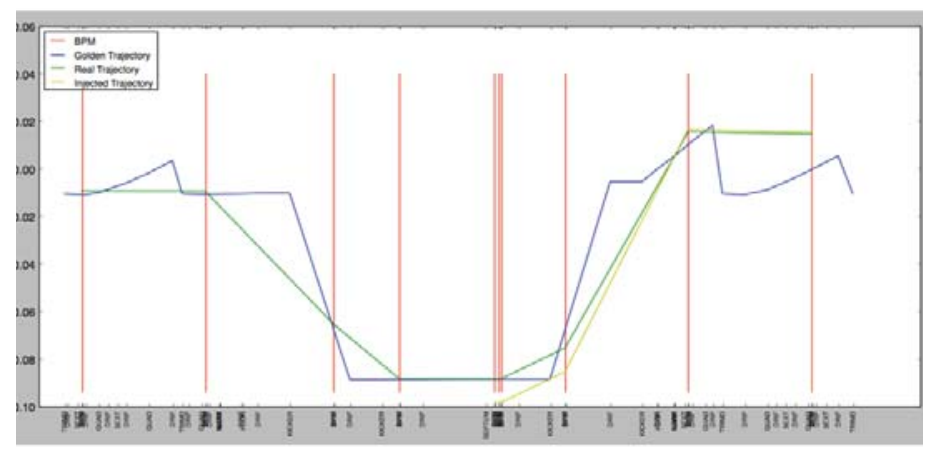

Fig.4. This plot shows three beam trajectories. The real and the injected trajectories are determined by the BPM readings, and the golden trajectory is calculated using elements transport maps and beta functions. 


\title{
Brookhaven atmospheric tracer sampler: Urban atmospheric dispersion study
}

\author{
Jessica Saintibert \\ Department of Biology, Tallahassee Community College, Tallahassee, FL 32304 \\ Adedoyin Akingba \\ Department of Biology, State University of New York at Potsdam, Potsdam NY, 13676 \\ Lezou Dali and Ilia Vardishvili \\ Department of Electrical Engineering, Stony Brook University, Stony Brook, NY 11790 \\ Marie Lamothe \\ Department of Engineering Science, Suffolk Community College, Selden, NY 11784 \\ Matthew B. Mayers \\ Department of Human Ecology, Cornell University, Ithaca NY, 14850-2824 \\ John Heiser \\ Environmental Sciences and Sustainable Technology, BNL, Upton NY
}

\begin{abstract}
The New York City Police Department requested that Brookhaven National Laboratory (BNL) develop an air flow survey for the Tristate Area. This Subway Surface Air- Flow Exchange project will produce a detailed map of air flow patterns between the surface and subway systems to assist against chemical terrorism on a large populated city. In this study, a chemical (Perflurocarbon (PFT)) will be dispersed on surface and below surface, and then it will imitate a chemical that can be either accidentally or intentionally released. The tracers being used are odorless, tasteless and non-radioactive, thus the population will not be affected in any way. Air samplers developed by BNL were used to record flow rates of PFTs. The city was divided into three sections labeled as Intensive Operation Period (IOP) 1, IOP 2 and IOP 3. These areas were surveyed during a period of three days, which were weather and wind based. The Brookhaven Atmospheric Trace Samplers (BATS) and Black Box Sampler (BBS) units were assembled by our specialized team in the laboratory from ground up for this specific study. The air sampling units were then distributed throughout the city to their respective IOP locations, and monitored by different groups. The data collected from this survey will provide the Department of Energy and Homeland Security with information for the New York Police Department's (NYPD) chemical counterterrorism unit. The information gathered will help the NYPD better understand how to protect and prepare the city if an incident were to occur. Assisting in this project will equipped me with a different perspective thus better preparing me for a life of doing scientific research in a professional setting in and outside the laboratory.
\end{abstract}

New York City is one the populated cities in the world; therefore it is more susceptible to terrorist attacks. Brookhaven National Laboratory (BNL) and the Department of Homeland Security has decided to conduct an air flow study, the Subway-Surface Air Flow Exchange (S-SAFE), in New York City to investigate air flow throughout its subway systems and surface locations. Although this study has been "conducted in subway systems in Boston, and Washington, D.C.,"1 this will be the largest dispersion study yet. The air will be sampled through air sampling devices known as Black Box Samplers (BBS) and Brookhaven Atmospheric Tracer Samplers (BATS). About 200 of these sampling devices are used during the study which disperse perflurocarbons at "select subway and street-level locations over three, non-consecutive days in July" 1 . These perflurocarbon tracers (PTFs) are harmless, odorless, and colorless gases which "are used in medical applications including eye surgeries and artificial breathing systems." This project is highly contingent upon the weather conditions; therefore, meteorologists are assisting BNL with monitoring the weather patterns throughout the week. The results of this project will provide the NYPD and the Department of Homeland Security with detailed statistics to execute a proper emergency response in the event a chemical toxin is released in New York City. Its sensitive nature requires that the data used in this experiment be held as confidential from the public.

To begin, as stated above, New York City is one of the most populated and busiest cities in the world which makes it more vulnerable to attacks. The S-SAFE project which took a couple of years to prepare for will help the New York Police Department and Homeland Security understands how air travels throughout the city with a chemical known as Perflurocarbon (PFT). The perflurocarbon when released into the air mimics a chemical that may be accidently or intentionally release above or below surface. However, first the equipment that would be used for the S-SAFE project had to be appropriately prepared and functioning properly. The two instruments mentioned earlier, the BATS and BBS air sampling units, were tested and modified in preparation for S-SAFE study including several other pieces that would benefit the project.

To continue, in assisting with the S-SAFE project on the Intensive Operation Period (IOP) days which were randomly selected according to the weather, more interns were hired to aid in the process. On the IOP days, the NYPD had specific locations where our teams picked up and, later, dropped off the BATS and BBS. As the day went along for each IOP day, each team logged onto the BATS Wi-Fi to check the flow rates with an increment that started off with 30 minutes and went up to an hour as the day ended. For the BBS air sampling unit which were mostly used below the surface on the subway, those teams had to check which tube was sampling by simply looking on top of the BBS. There a light would show which tube was on and, therefore, sampling. Each air 
sampling unit started the process starting at eight o'clock in the morning and ending at two o'clock in the afternoon as they were programmed to do. This procedure was done for each BATS and BBS air sampling unit that were at the exact locations chosen for each of the IOP days.

In other parts for the S-SAFE project, the several other tasks were completed including fixing the wires, checking CATS tubes inside the BATS, placing guard tubes in the BBS, which were used to hold the air contaminants, and making cable wires. We had to make sure that the wires were in the proper order and they were correctly trimmed. After we finished working with the wiring systems of the BATS, we were assigned to make wooden strips to hold the guard tubes and the Capillary Absorption Tubes (CATS) inside each BBS. One of the mentors prepared the many pieces of wood since we were not allowed to use the machines needed to complete that part of the job. Once he was done, we proceeded by putting some strips on them and numbering them according to each BBS number. We also drilled holes in several hundreds of caps and attached them in sets of two connectors. These caps that we attached together with connectors were used to connect the CATS tubes and the guard tubes. We were also assigned to charge the BATS to make sure that they were working properly on the selected IOP day. For the cable wires, several of them were made which aided in holding the BATS and BBS securely in places alongside bungee cords for extra support.

How is this data collection possible? One may ask. The BATS were built with the industry's latest technology. The brain behind the sampling units contains four microprocessors which enable the samplers to have a GPS location, be Wi-Fi enabled, and store the schedule of a specific program to collect the data. The circuit layout of the sampler contains a custom circuit board with through-hole and surface mount technology. The circuitry also contains four power shifter and other small components similar to capacitors, diodes, transistors, and resistors. The power shifters make it possible to switch between different valves of the BATS units; therefore, data can be collected. The switching system design of the units is built in a way that it is power conserving and environmentally efficient. They have a mass flow meter and pump controller, which resulted in accurate and precise flow rates over an operational range from 0 to $450 \mathrm{sccm}$. The perfluorocarbron was collected through the BATS using an absorbent called ambersorb. With this new technology, we are also able to synchronize new schedules and programs wirelessly and also download the data logging of the each individual sampler. Furthermore, to power the units, they are equipped with $24 \mathrm{~V}$ rechargeable batteries and also a small $3 \mathrm{~V}$ battery to power the GPS tracking. Everything on the BATS units is up-to-date technology.

The Big Black Box units function in a slightly different way. They are much older technology, the brain is much simplified circuitry and is not equipped with most of the tools that the BATS units have. Since the technology is older, it has less sufficient data and more troubleshooting problems. On the other hand, it still gathers useful data.

The Subway Surface Air Flow Exchange is, a \$3.4 million project funded by the Department of Homeland Security. This study is expected to help the New York Police Department and other agencies to refine their emergency evacuation plans. If a terrorist act were to result in the release of a dangerous gas that could harm citizens, the city's first responders must immediately know whether to shelter people, or in a case of evacuation, know what evacuation routes they should take. While the study focuses more on a potential terrorist attack; the results would also help with determining the dispersion of other inhalational gases such as smoke or fumes from an industrial accident or a chemical spill. These results will help ensure America's security and prosperity through environmental means.

\section{REFERENCES}

${ }^{1}$ BNL Newsroom | "New York City Police Department and Brookhaven Lab to Conduct Airflow Study In New York City Streets and Subways This Summer." BNL Newsroom | New York City Police Department and Brookhaven Lab to Conduct Airflow Study In New York City Streets and Subways This Summer. Brook Haven National Lab, 24 Apr. 2013. Web. 28 July 2013.

\section{ACKNOWLEDGEMENTS}

This project was supported in part by the U.S. Department of Energy, Office of Science, and Office of Workforce Development for Teachers and Scientists (WDTS) under the Community College Internships Program (CCI). 


\title{
Human machine interfaces for the Access Controls Security system Linear Accelerator upgrade
}

\author{
Fidel Salgado \\ Cañada Community College, Redwood City, CA 94061 \\ Jonathan Reich and Vincent Castillo \\ Collider-Accelerator Department, Brookhaven National Laboratory, Upton, NY 11973
}

\begin{abstract}
The Linear Accelerator (Linac) provides polarized protons for experiments at the Relativistic Heavy Ion Collider (RHIC) and (regular) protons to the Brookhaven Linac Isotope Producer (BLIP) to produce radioisotopes used in medical research. It also provides protons to the National Aeronautics and Space Administration (NASA) Space Radiation Laboratory (NSRL) for studying the effects of radiation on human beings for future travels in space. The Access Controls Security (ACS) system controls personnel safety around the collider-accelerator complex, and also maintains operating equipment within specified parameters. Up to now, ACS has been controlling Linac by using a relay-based system, which has been in operation for more than 40 years. This summer 2013 the move was made to upgrade the ACS Linac control system from a relay-based system to a Programmable Logic Controller (PLC) system. PLC systems provide greater flexibility with implementing logic, are more compact, and more cost effective. One of the greatest advantages of the PLC system is that it allows for Human Machine Interfaces (HMIs), which is an effective way for machine control and system monitoring in real time. My project for the summer is to build the HMIs for the Linac ACS system. With the help of Joel Vasquez, I have been able to learn the software FactoryTalk View Visual Studio the one used to build HMIs. I have learned how to create displays where you can have multiple indicators to show all the equipment in operation in Linac. The indicators show the different states of the gates, the various supplies that are on, radiation levels, and the statuses of other critical equipment. With the help of other ACS engineers, I have been able to acquire a better understanding of how the ACS system will work in Linac, and I have learned how the PLC communicates with HMIs. Creating user-friendly HMIs is very important in the ACS system because its primary mission is to assure personnel safety.
\end{abstract}

\section{Introduction}

The ACS system's primary mission is to assure personnel safety in the collider-accelerator complex. The ACS system accomplishes its mission by maintaining operating equipment within specified parameters and monitoring it in real time. The ACS system has monitored Linac for 40 years in a relay-based logic - a method of controlling equipment by using relays. However, relay logic systems have some disadvantages compared to PLCs. PLCs provide many advantages such as being cost effective, space efficient, and flexible with implementing logic. Additionally, HMIs ability to communicate easily with PLCs is a great advantage for ACS since HMIs are used for monitoring equipment in real time.

Linac's upgrade is not trivial, so a team project is needed. The PLC has to be communicating with all the operating equipment in
Linac such as gates, chipmunks, critical devices, hardware, crashes, and power supplies. The PLC also has to communicate with the HMIs which are set up in the Main Control Room (MCR) by using tags. In the HMIs, indicators are built with different states that show the equipment statuses. The project has been divided into smaller projects, and my part of the project is to create the user-friendly HMIs that communicate with the PLC through the tags assigned to the various devices.

\section{Progress}

In order to be able to create HMIs, I had to learn how to use the software the ACS system uses. The software to build the HMIs for Linac is FactoryTalk View [1]. By using the FactoryTalk View Site Edition user's guide [2] and help from engineer Joel Vasquez, I was able to design, create, and implement the application for Linac.

By reading the user guide manual for FactoryTalk View, I was able to build multiple displays, and navigate through them. FactoryTalk View is a very user-friendly software that lets you build different type of buttons such as navigation buttons, toggle buttons, and menu buttons. All of these capabilities of having different type of buttons are crucial for monitoring all the operating equipment in Linac. I also learned more about indicators. The indicators that you can build in FactoryTalk View can have multiple states, and each state indicates a different status of a device in Linac. For example, the chipmunks measure the level of radiation. If there is an excess of radiation in a zone in Linac the chipmunk will change the status of the PLC and this will show in the indicator built in the HMI. This allows easy monitoring of the radiation levels in all the zones of Linac. I also learned how to launch a Client in FactoryTalk View; this allows implementing the HMI in the MCR so the ACS group can use it and monitor Linac from the MCR.

In order to get a better understanding of how the HMIs will be implemented in Linac, Joel assigned me the task to get familiar with PLCs and how tags are connected to the HMIs. By using the PLC Basics - Allen-Bradley PLC [3], I was able to have a better understanding of how the PLC communicates with the different machines. First, it has to be understood that PLC simulates relay-based logic since is programmed in ladder logic - a programming language for people with a relay-based logic system background. The simulation is done by using bits $(0 \mathrm{~s}$ and $1 \mathrm{~s})$ that can be understood as de-energized and energized in the relay-based systems. The PLC will be connected to different operating devices such as an entrance gate, chipmunks, sweep-stations, crashes, and critical devices. The purpose of the PLC is to change the logic if any of these devices changes their state of operation. This provides a safety envelope to run Linac since it can only run if all devices are operating properly. After learning about PLCs, I was able to build a ladder logic program to simulate the operation of 
a washer machine, and show all the different states and processes in an HMI.

After getting familiar with FactoryTalk View and PLCs the design for my HMIs was discussed. The design was based on the HMIs for the Alternating Gradient Synchrotron (AGS). The AGS was just recently upgraded from a relay-based logic to PLC logic. The different schematics for all my displays such as color, font, indicators, and button sizes were all based on the AGS HMI displays to keep the two systems consistent. The challenging part of the design was to understand all the different devices that the ACS system wanted to monitor in real time. I was provided all the tags that will be implemented in Linac, and I had to learn and understand them so I can create multiple indicators that will represent those tags.

All of the display parameters for Linac such as font, indicator size, state colors, and navigation buttons were the same as those for the AGS displays. For the creation of all the displays, I was provided with two important documents. The first one was the Linac Input/Output definitions [4] which contains all the tags that will be implemented on the displays, and the second one was the Linac State Table [5] which is an overview of all the operations and devices that the ACS group will be responsible for monitoring and controlling in the Linac. With these two documents, I was able to start creating all the displays for the Linac HMI.

The first display I created named "1008 - LINAC Main Menu", Figure 1, Appendix A. This display is simply a navigation display. It lets you navigate through Linac HMI. The four navigations buttons are Operations, Access, Interruptions, and Maintenance. The display is called Main Menu since it is the first display seen when you access the Linac HMI. This display was straightforward to create since it is very similar to the AGS Main Menu display.

The second display I created was named "2000 - LINAC Access", Figure 2, Appendix A. This is a sub-menu navigation display. It lets you navigate throughout all of Linac gates $\_-\mathrm{Plug}$ Door gate and Tank1 gate. Linac has more gates but the ACS system is only interested in monitor these two since they are the only ones that let personnel access and exit the Linac. The display also has two indicators that show the current access mode of Linac - all the different access modes will be explained in detail in the Operations display. In this display there is also three more navigation buttons that simply lets you navigate throughout the Operations, Interruptions, and Main Menu displays. To be able to create this display, I had to know the Linac gates that must appear on this display.

The third and fourth displays were very similar since both are to monitor access gates of Linac. One display was named "2001 - LINAC Plug Door Access" and the other one "2002 - LINAC Tank1 Gate Access". The Plug Door display can be seen in Figure 1. The only differences between both displays are some of the labels and which camera they are connected to. However, all the functionality is the same. The purpose of this display is to monitor the sweep status of that part of Linac (sweep details will be explained in the Sweep display). It also monitors the current access mode of Linac and shows the state of the gate if it is locked/unlocked and open/closed. This display has also a button that allows personnel to exit or enter the gate without losing the sweep.

The fifth display named "8000 - LINAC Interruptions", Figure 3, Appendix A. This display is a sub-menu that allows you to navigate through multiple displays such as chipmunks, crashes, critical devices, gates, and sweeps.

The chipmunks display was named "8003 - Int-NMON LINAC" since NMON stands for Nuclear Monitor and Int stands for Interruptions. The chipmunk display can be seen in Figure 4, Appendix A. The display shows all the different states of the chipmunks which are devices that are used to monitor radiation. Therefore, if there is an excess of radiation in a particular zone of Linac, the indicator state would switch and the ACS group will be

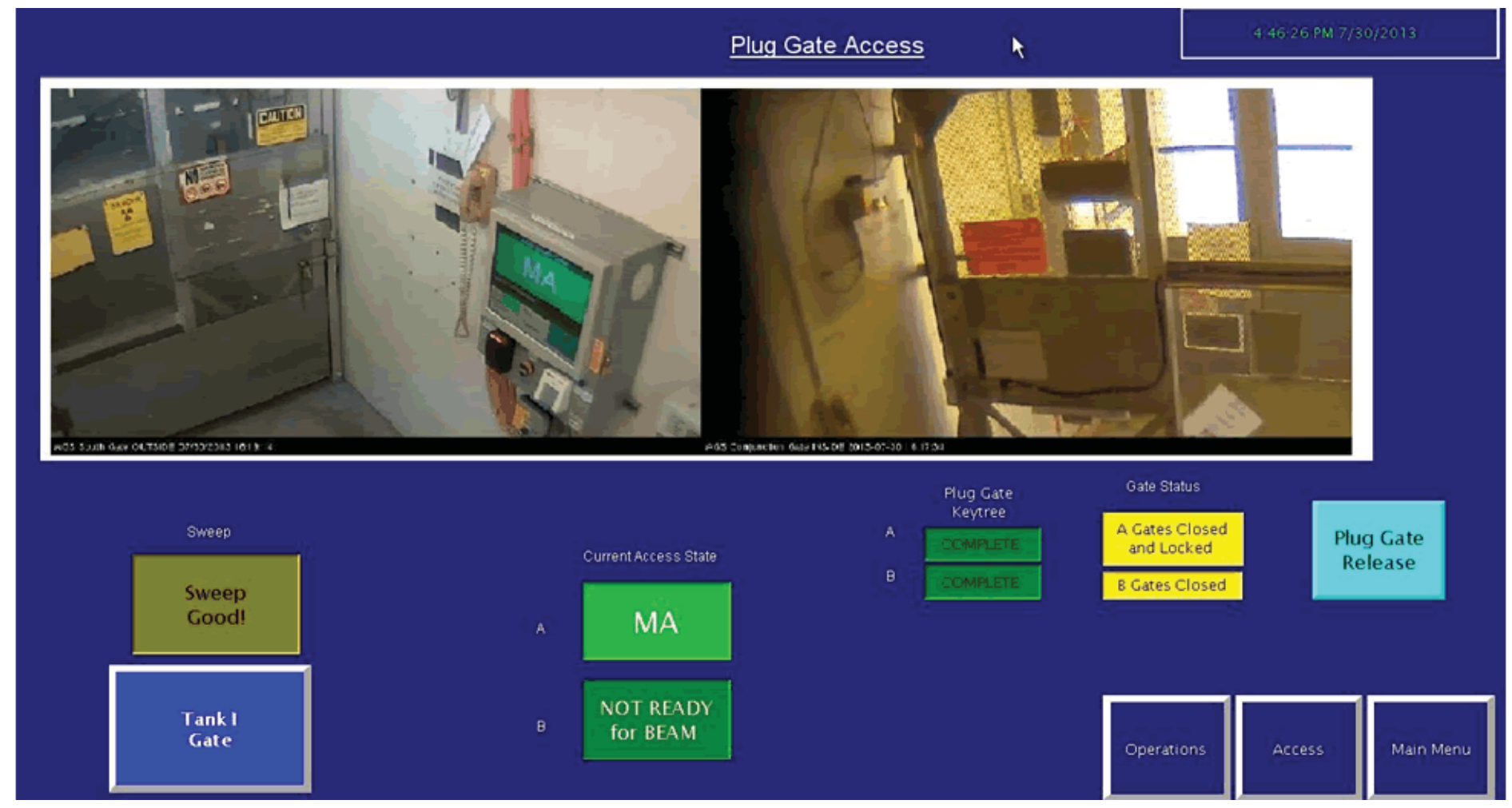

Figure 1 - Plug Gate Access Display 
able to monitor this and solve the problem. This is really important for radiation safety.

The crashes display was named "8004 - Int-Crash_LIANC", Figure 5, Appendix A. The crashes display also called the emergency stop display is used for emergency stops. If after a sweep there is still somebody inside Linac, they can "crash" it and Linac would stop running. However, this should never happen, but in case it ever happens the ACS group can monitor if there is or there is not a crash. In order to create this display I had to divide all the crash devices into the different Linac zones, for good readability. In order for Linac to run, the state of all indicators must be in the good condition.

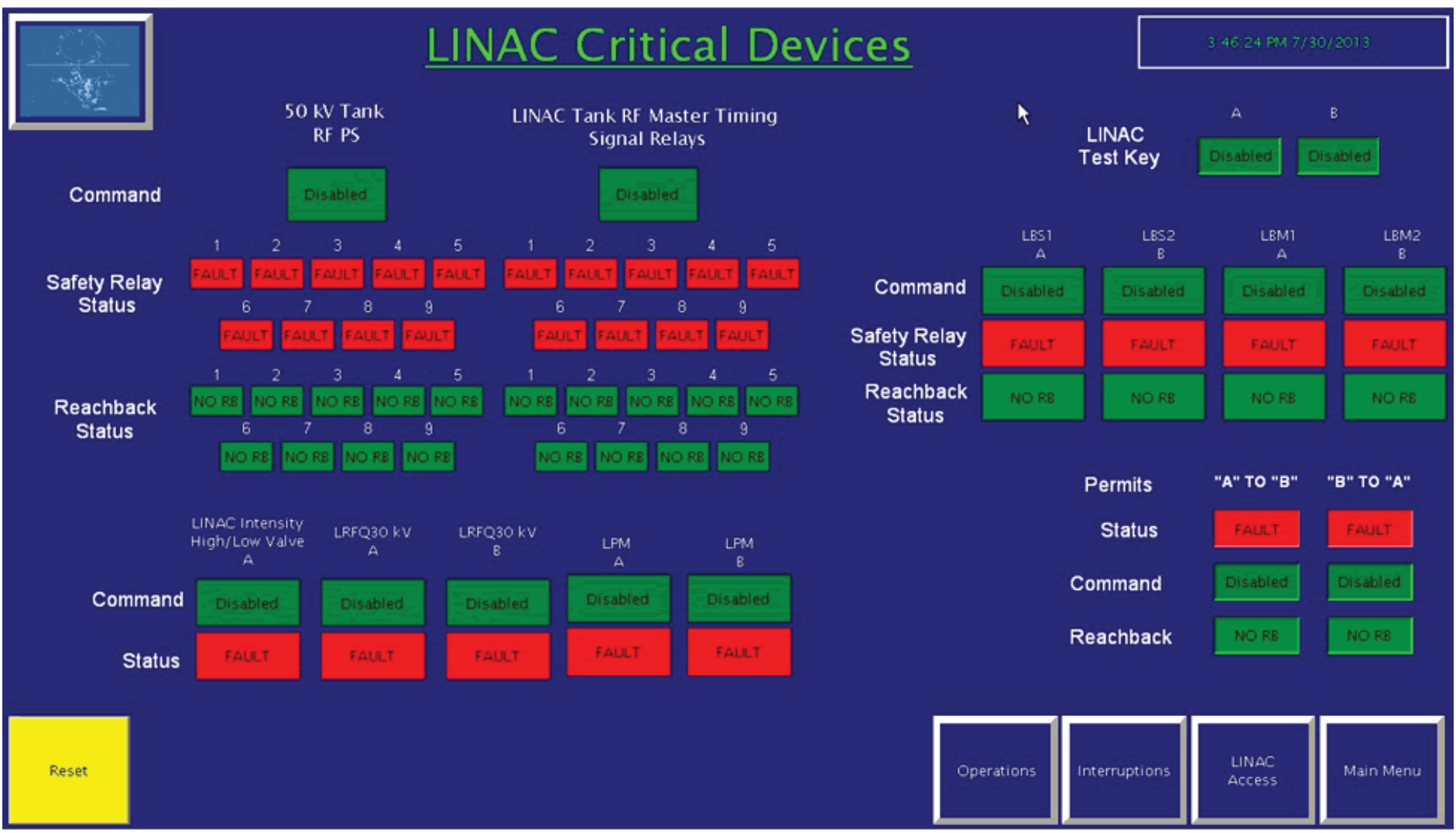

Figure 2 - Critical Devices Display

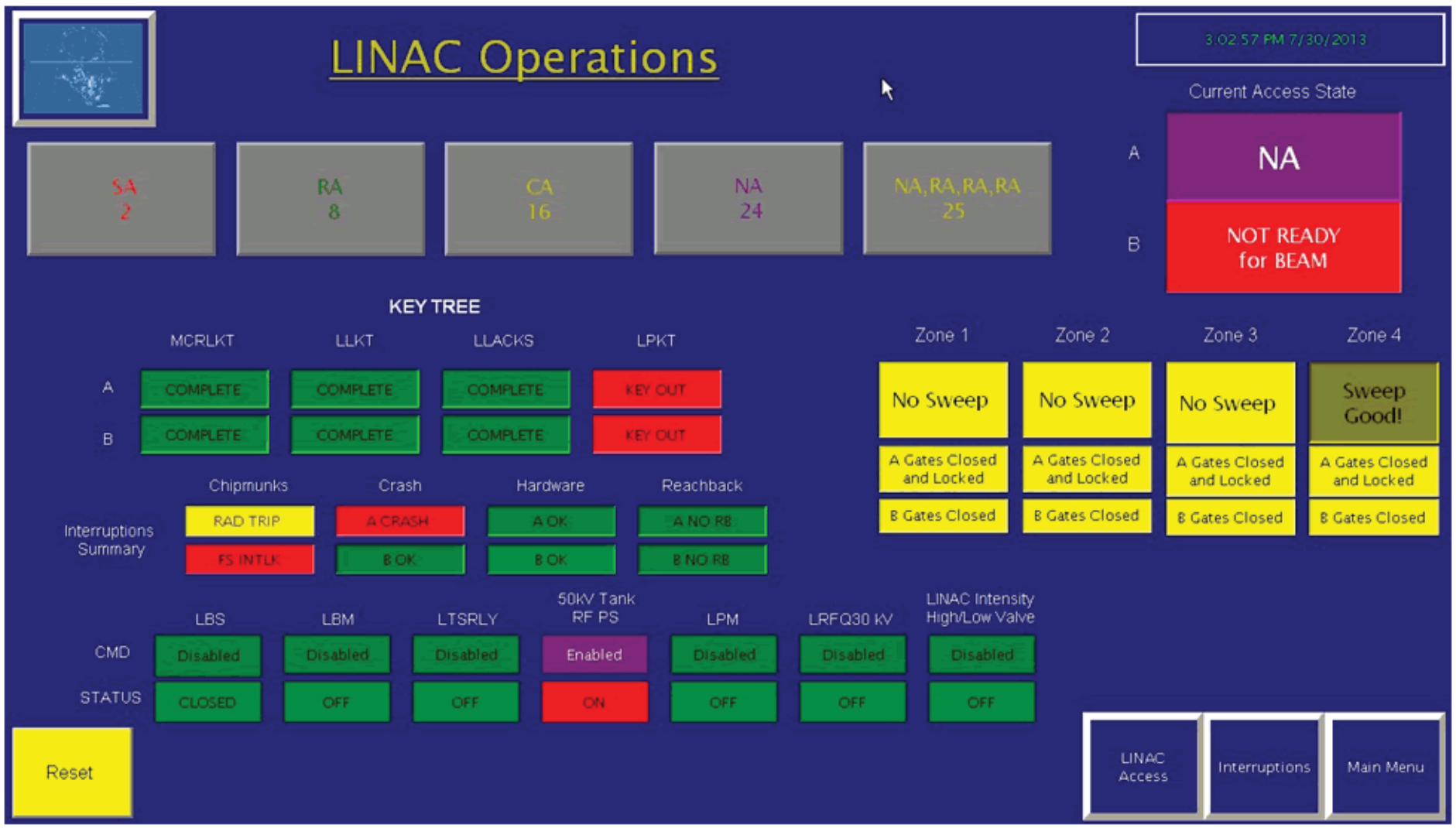

Figure 3 - Operations Display 
The critical devices display was named "8004 - Int-CritDevices_LINAC". The critical devices display was definitely the most challenging display of all the interruptions displays since it was very different than the AGS interruption display. However, with the help of ACS engineer Darren Hillis [6], I was able to understand the reason to monitor critical devices which have the basic function of stopping the beam if something wrong happens while Linac is operating. The State Table was also a big help for creating this display. This display shows all the different critical devices in Linac. ACS Engineer Susheng Xin is building the tags that will represent the critical devices and by talking to him, we designed the critical devices display and then I created it. The critical devices display can be seen in Figure 2.

The gates display was named "8008 - LINAC int-gates", Figure 6, Appendix A. This display is a summary of all the gates in Linac. It shows if a particular gate is open or closed and if it is locked or unlocked. All the gates are divided into different zones for smooth readability. For this display, I had to learn all of Linac's different zones, and where each gate was located. The highlighted gates are the only ones that let personnel access and exit the Linac (Plug Door and Tank1 gates). The rest of them are to access different zones in Linac.

The last display of interruptions is the sweeps display named "8009 - int-sweeps_LINAC", Figure 7, Appendix A. This display is to monitor sweeps. A sweep is done whenever Linac is going to run. Basically, a sweep is when authorized personnel goes through a particular Linac zone and checks if the zone is empty; if the zone is empty he turns a key that lets the PLC know and the area is shown as swept on the HMI. When all areas are swept, then Linac can run; this is a requirement for Linac to run. The indicators change state when a particular area is swept and this is how the ACS group monitors the sweeps.

Another display I created is the Maintenance display which is a sub-menu that allows access to all the different interruptions devices but also to the I/O Status displays. The maintenance display was named "8800 - LINAC Maintenance", Figure 8, Appendix A. The maintenance display also allows the operator to change the access mode to Maintenance Mode.

It is really important for the ACS group to monitor the $\mathrm{I} / \mathrm{O}$ Status display since it shows all the different statuses of all the PLC modules in Linac. There are two status displays, the A-Division Status and the B-Division Status. The A-Division Status can be seen in Figure 9, Appendix A, and the B-Division can be seen in Figure 10, Appendix A. All the modules are divided among different areas. These areas are Tank1, Plug Gate, MCR, and Caged area. There is also an overview indicator that if one of the modules stops working it changes status to warn the ACS that there is a faulty module in the system.

The last display I created, and probably the most challenging one was the Operation display. It was named "3000 - LINAC Operations", Figure 3. The operations display works as an overview display. It shows all the different key trees, the current access mode, chipmunks, crash, hardware, reach-backs, sweeps by zones, and critical devices. It also allows the operator to change the access modes of Linac. The access modes for Linac are Safe Access, Restricted Access, Control Access, No Access, and another mode that is a combination of No Access and Restricted Access. This display is extremely important for the ACS system because is an overview of the Linac system.

\section{Figure 3 - Operations Display}

\section{Impact on Laboratory or National Missions}

The motivation for this project is personnel safety. I worked in the ACS group and their primary mission is to control access to the entire Collider-Accelerator complex. By building Linac HMIs and upgrading the logic system from a relay-based system to a PLC system we will improve Linac access system. This improvement is very important because it allows the ACS group to monitor the Linac devices in real time and it improves considerably the safety-access to Linac.

\section{Future Work and Conclusions}

After the design and creation of the HMI for Linac, it was implemented in the MCR for testing. We made sure that all the indicators were not tagged, that all the navigation buttons were working, and that all the HMI displays were consistent in design with those of the AGS, RHIC, and NSRL HMI displays. I explained all the displays of the HMI to Joel Vasquez because he will connect the HMIs with the PLC by using tags. The tags for Linac are being build and coded by engineer Susheng Xin, with whom I have kept close communication so there would be no problems implementing the tags to the HMIs I built. Moreover, I helped Joel to implement cameras in the different Linac gates. These cameras will allow for smooth communication between the MCR and personnel entering and exiting the Linac gates.

\section{Acknowledgment}

I wish to thank everyone in the ACS group for all of their support and help throughout the summer: Group Leader Jonathan Reich, Heather Hartman, Darren Hillis, Joel Vasquez, Susheng Xin, Rich Conte, Walter Lamar, Dan Carrol, Frank Scheifele and my mentor Vincent Castillo.

This project was supported in part by the U.S. Department of Energy, Office of Science, Office of Workforce Development for Teachers and Scientists (WDTS) under the Community College Internships Program (CCI).

\section{References}

${ }^{1}$ Rockwell Automation Technologies Inc. FactoryTalk View. Computer software. Vers. 6.1. N.p., n.d. Web.

${ }^{2}$ Rockwell Automation Technologies Inc. FactoryTalk View Site Edition User's Guide. N.p.: n.p., n.d. PDF.

${ }^{3}$ Chisholm Institute. PLC Basics, Module Code NE130, Allen Bradley PLC. N.p.: n.p., n.d. PDF.

${ }^{4}$ Xin, Susheng. Linac Cable Run List. N.p.: n.p., n.d. XLS.

${ }^{5}$ Access Controls Security. LINAC ACS System State Tables, Document Number: D40-EO63. N.p.: n.p., n.d. DOC.

${ }^{6}$ Hillis, Darren. "Conversation with ACS Engineer." 


\section{Appendix A}

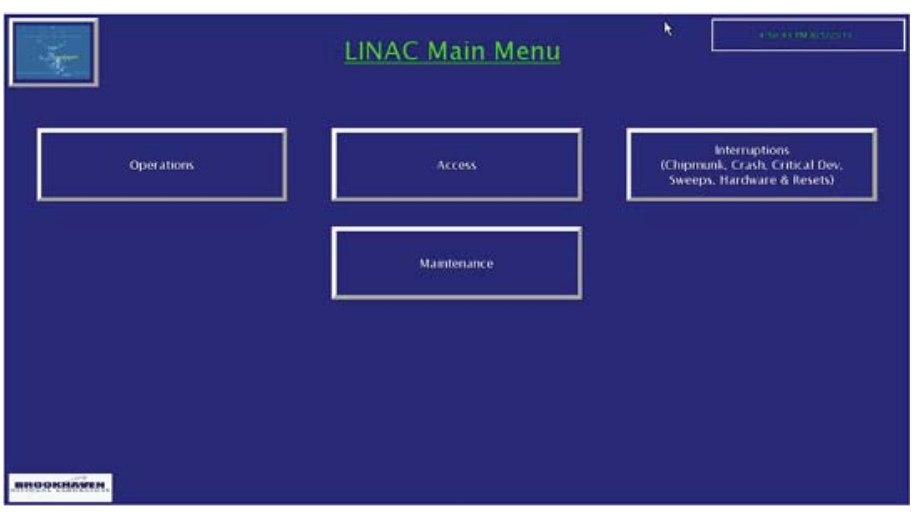

Figure 4 - Main Menu Display

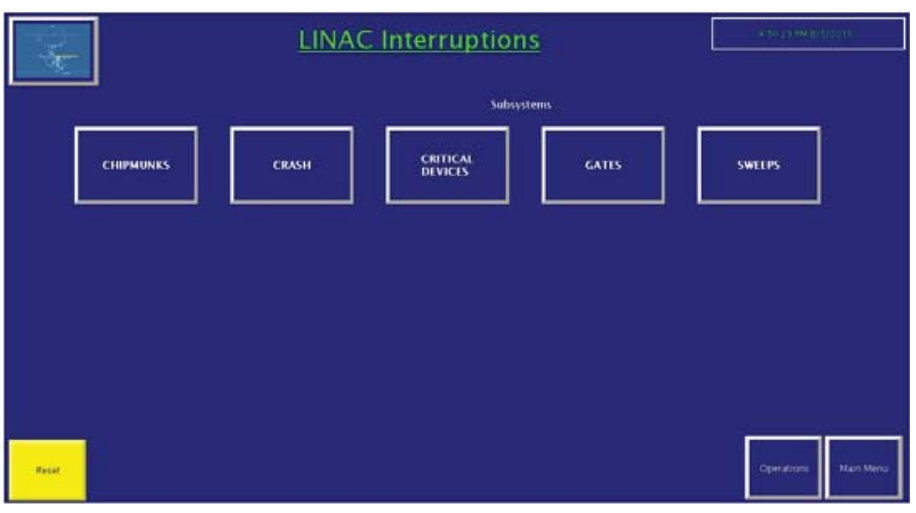

Figure 6 - Interruptions Display

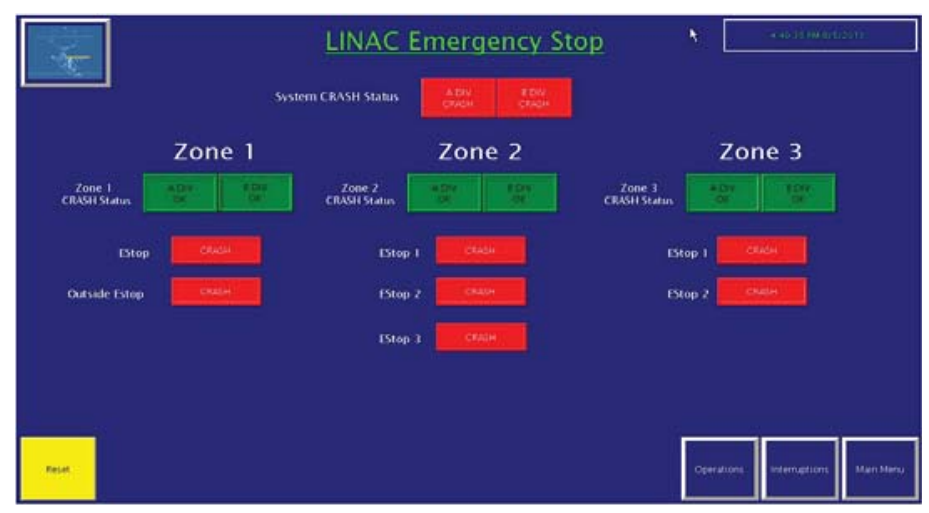

Figure 8 - Crash Display

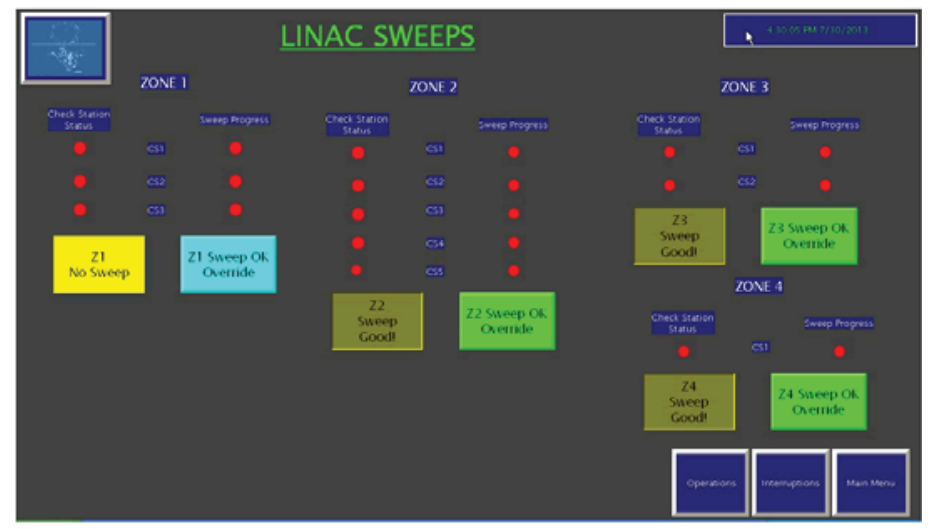

Figure 10 - Sweeps Display

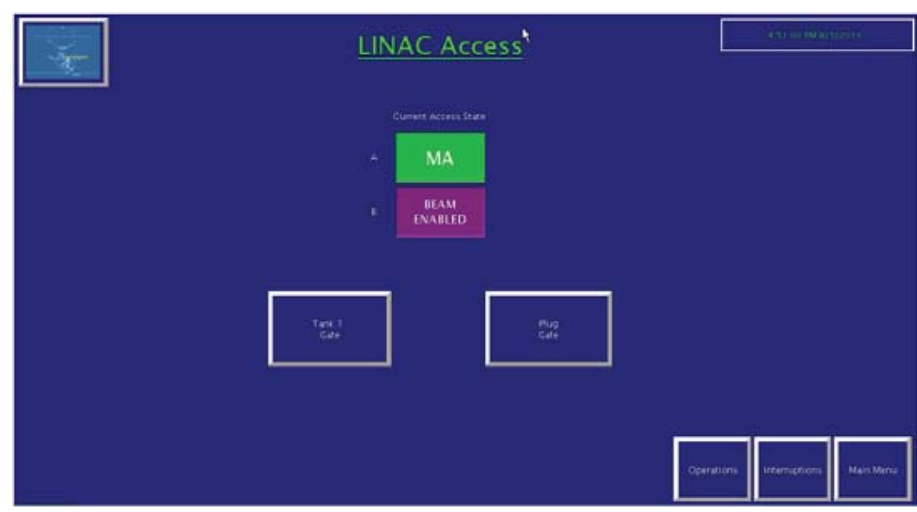

Figure 5 - Access Display

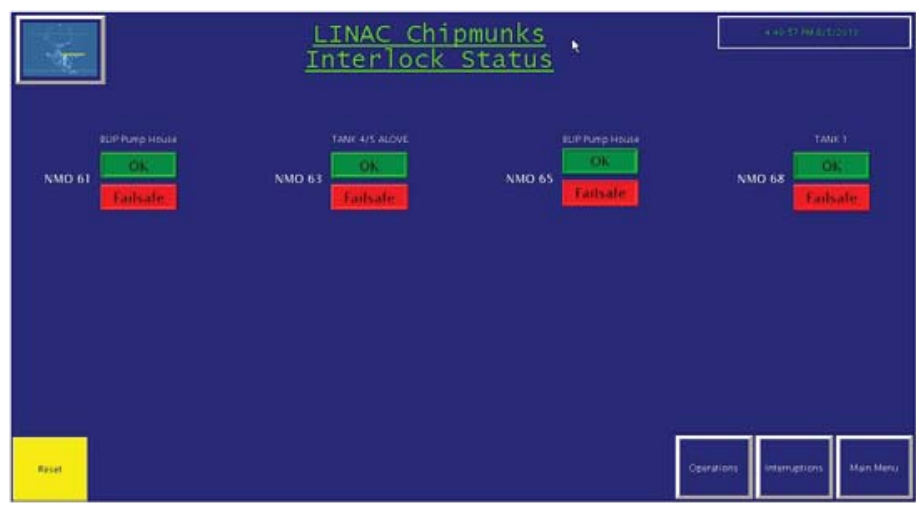

Figure 7 - Chipmunks Display

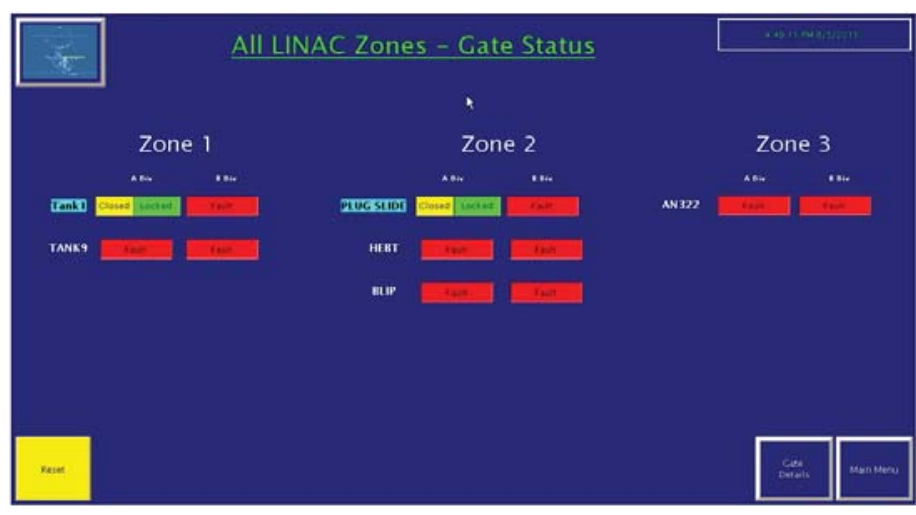

Figure 9 - Gate Status Display

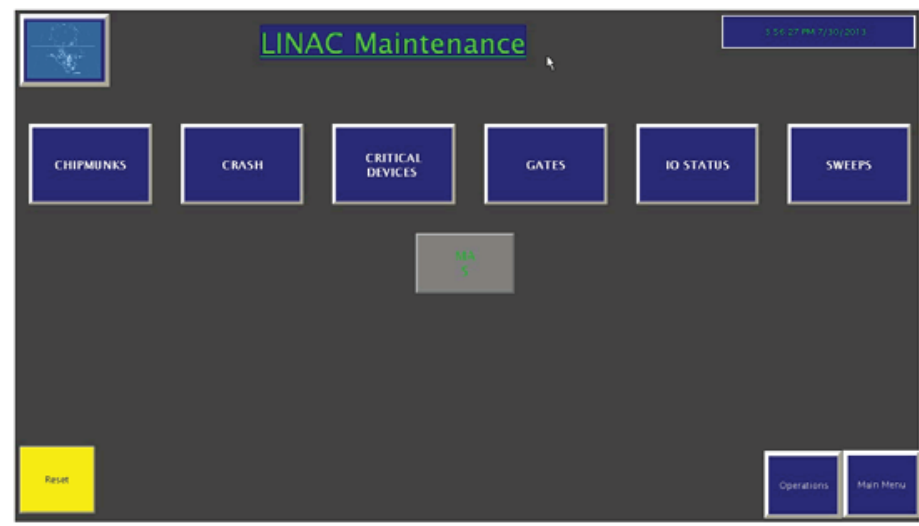

Figure 11 - Maintenance Display 


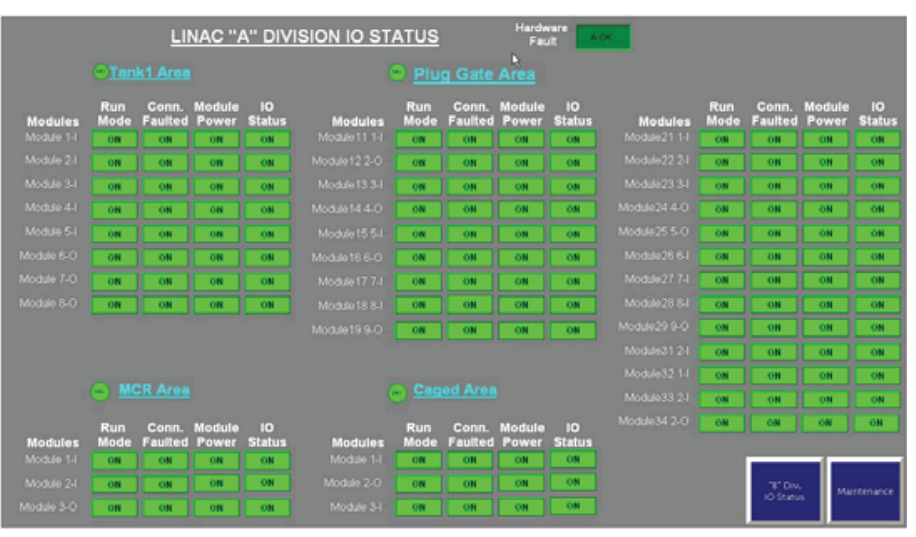

Figure 12 - A Division I/O Status Display

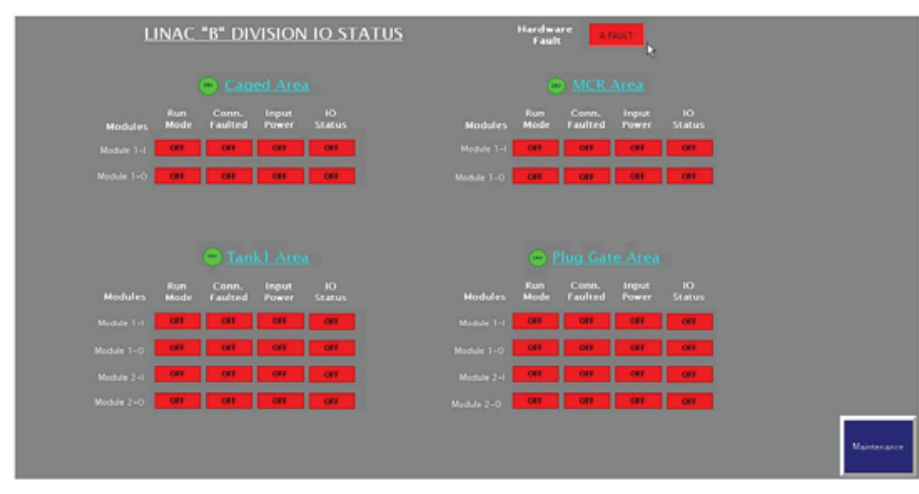

Figure 13 B Division I/O Status Display 


\title{
Digital multi-meter cable and powered magnet testing; creating datasets for tracking and monitoring one-wire sensors at the National Synchrotron Light Source II
}

\author{
Pavel Salnikov \\ College of Engineering and Applied Sciences, SUNY at Stony Brook, Stony Brook NY 11794 \\ Wing Louie \\ Photon Sciences, Brookhaven National Laboratory, Upton NY 11973
}

\begin{abstract}
Powered magnets are used to control electron beam at the National Synchrotron Light Source II (NSLS-II). Our job was to test parameters of the magnet operation, such as magnet temperature, inlet and outlet temperatures of the de-ionized cooling water, and the current drawn by each magnet. Power supplies current signals for the magnets are connected to a digital multi-meter (DMM) that located in each rack group. The DMM cables had to be checked for a proper resistance sequence, which was done by connecting a computer to DMM LAN port through Ethernet cable and running the test under LINUX operating system (OS). When NSLS-II becomes operational, the 1-Wire system is deployed to monitor parameters of sensors continuously, including temperature, humidity and AC current. Besides monitoring, the 1-Wire system can perform reset functions of the magnet power supplies; the reset function reboots the power supply to a known state. Another job for this summer was to record a dataset of the 1-Wire sensor ID numbers on an Excel spreadsheet for each of the power supply rack groups. To accomplish this, I had built a 1-wire adapter, which I was using to communicate with the sensors through two USB servers and the OneWireViewer software program on my work laptop. All of the sensors are 1-wire, meaning that power and data are delivered to these devices through one connection; the devices are daisy-chained, and earth ground serves as a return path for the signal. The entire NSLS-II system has over twenty thousand sensors. Monitoring the sensors connected to the power supplies is vital for successful operation of NSLS-II. Each sensor returns information on the parameters of the power supplies and interfaces, which can determine overcurrent and overheating faults. During this internship, I was introduced to new to me methods of communicating with sensors; I learned about NSLS-II magnet operation and testing. I gained experience working with LINUX OS and learned how to perform troubleshooting when a signal was not being transmitted.
\end{abstract}

The National Synchrotron Light Source II (NSLS-II) at BNL is in the installation, testing, and database-building phase of its existence. We were conducting some of the tests and data-gathering, required in preparation for a successful operation of NSLSII. These tests and data-gathering included powered magnet measurements, digital multi-meter (DMM) cable check, and building datasets of one-wire sensor serial numbers.

NSLS-II utilizes a high-energy, circular electron beam in its storage ring to produce synchrotron radiation; beamlines guide the $\mathrm{x}$-rays to experimental end stations for conducting scientific experiments. The circulating electron beam needs a high level of control and focus, which is to be accomplished through a multitude of magnets. In addition to the dipole bending magnets, which put electrons into circular path, there are 90 girders of quadrupole, sextupole, and corrector magnets in the storage ring. Quadrupoles and sextupoles will be used to focus and de-focus the electron beam.

These magnets need power supply current to produce necessary magnetic fields. The current supplied to them varies, depending on how much electron orbit needs to be adjusted. DC current creates a steady magnetic field, resulting into a constant orbit. Corrector magnets get AC current to counteract effects such as ground movements and vibrations to produce precise and stable orbit. The overall shape of the current waveform would possibly be a distorted sine wave, with frequency varying from sub $\mathrm{Hz}$ to a few hundred Hz. Maximum current supplied to the magnets can be 100 Amps or more in quadrupoles and sextupoles, while correctors get less than \pm 20 Amps.

Testing runs we conducted were focused on measuring the maximum current applied to the magnets, temperature rise of the magnets, and temperature rise of the cooling water in the magnets. Each girder gets cooling water from a supply pipe located below the magnets. The de-ionized water branches off into each individual magnet through an inlet and exits through an outlet pipe and goes to a main return manifold. In our testing runs we used a hand-held digital temperature probe to measure the temperatures of water inlet and outlet pipes, as close to each magnet as possible. Temperature of the magnets was measured by a thermal imaging infrared camera. We looked for the highest temperature reading observed on the camera for a magnet. Maximum current was measured with a current sensing clamp-on that attached to a hand-held digital multi-meter.

Each testing run we performed for a particular pentant of NSLS-II. There are five pentants with six cells each and three magnet girders per cell. Every girder consists of eight to nine magnets. The power supplies, located in cabinets on the floor above the magnets, were turned on and set to deliver various power settings on a testing run. We normally tested the magnets at 25 percent, 50 percent, and 100 percent of operational current. Several hours were given in the morning to let the magnets warm up. The measured values for each magnet were recorded into an Excel spreadsheet. While measuring, we were looking for any abnormal readings, such as magnet temperatures exceeding 45 to 50 degrees 
Celsius, with relatively low cooling water outlet temperatures. Such readings signified poor water delivery, circulation, and heat dissipation inside a magnet. These occurrences needed to undergo troubleshooting to determine the cause and to fix the problem.

A magnet girder is powered by its own power supply rack group, which has its own digital multi-meter (DMM) connected to every power supply in the rack group. Another test we performed was DMM cable check. It involved checking DMM cables to each power supply in a rack group for passing sequence resistance readings through the DMM. To conduct this test, the DMM LAN needed to be configured first: its IP address, gateway, and subnet needed to be set according to the cell number and rack group letter. Once LAN was configured, test program in LINUX computer could communicate with the DMM through Ethernet cable. DMM cable test was run on the computer by opening terminal window for starting The Experimental Physics and Industrial Control System (EPICS) Extensible Display Manager (EDM) and running the test for a rack group.

DMM cables are connected to a DMM on a Terminal Blocks Paddle Board, which is located on the back of the DMM case and connected to an Internal Channel Select Relay Board inside the DMM case. The EDM window on the computer showed the terminal numbers from the Terminal Blocks Paddle Board, as well as resistance values between high and low ends of the terminals. The DMM cables to power supplies occupy specific terminals on the Paddle Board. Single power supplies have three cables to a DMM, dual power supplies have six. Spreadsheets, used in testing of each rack group, show the allotted terminal groups for each power supply on the Paddle Board. DMM test plugs, separate for single and dual power supplies, with loopbacks were attached to the cable connectors to run the test. After several seconds, the terminals of the looped cables highlighted and the resistance values were displayed on EDM display. At the bottom of EDM it showed whether the test had passed. For a test to pass, all the plugged cable terminals must highlight and display proper values in a proper sequence. For single power supply cables the values are $1.1 \mathrm{k} \mathrm{ohms}, 2.0 \mathrm{k}$ ohms, $3.0 \mathrm{k}$ ohms in that order from the lowest number terminal to the highest. For dual power supply cables the values are $6.0 \mathrm{k}$ ohms, $7.0 \mathrm{k}$ ohms, $8.0 \mathrm{k}$ ohms, $10 \mathrm{k}$ ohms, 12 $\mathrm{k}$ ohms, and $14 \mathrm{k}$ ohms in that order.

When a test did not pass, it was time to troubleshoot the cause. Sometimes all the terminals for a cable group highlighted with the values but their order was not correct. In this case the wires needed to be switched into proper order on the Terminal Blocks Paddle Board. In other cases one or more cable values did not highlight with a value. When this happened, it could be a loss of continuity somewhere along the line from the DMM to the power supply cable end. So, we needed to test the continuity of the cable with a hand-held meter; if the cable turned out to be good and with the proper resistance reading, the problem could lie in the Terminal Blocks Paddle Board, or the Internal Channel Select Relay Board, or their connection pins, or the DMM itself. In the process of elimination, we isolated the problem location. In two such cases, we ended up replacing a Terminal Blocks Paddle Board with a new one to correct the problem. The problematic Paddle Boards were then sent to determine the cause of the malfunction, which could be something like a broken trace inside the printed circuit board (PCB).

Aside from magnet measurements and DMM cable checks, my tasks included building a 1-wire adapter, and using it and the
OneWireViewer software to plug into and record data from 1-wire sensors in power supply rack groups. 1-wire is a type of electronic transmission, in which a single contact serial interface is used for both power and communication. In such a way, all devices can be wired into a single serial chain, along which a master device can communicate with slave devices. Ground serves as a return path for a signal. As opposed to 2-wire and 3-wire communications, 1-wire does not require external power connections and minimizes interconnect for the systems where it is required. ${ }^{1}$ But when distances for signal transmission exceed fifty feet with ten or more 1-wire devices, 3-wire connection with separate power, data, and return is needed for efficient and reliable communication. Most of NSLS-II wiring is 3-wire due to the quantity of devices and long signal transmission between devices; typically, each string contains over sixty devices with cable length around three hundred feet.

NSLS-II employs an extensive system of 1-wire sensors for monitoring temperature, 3-phase AC current, humidity, and reset functions for the power supply rack groups. Each 1-wire sensor has a unique ID, in which the last two digits specify the device type. DS18B20 are temperature sensors - IDs ending with 28, DS2450 are current monitoring sensors - IDs ending with 20, DS2438 are humidity sensors - IDs ending with 26, DS2406 are reset sensors - IDs ending with 12. 1-wire IDs or registration numbers are in hexadecimal form; they can be used to track and monitor each sensor. Using the constructed DS1990A adapter with USB protocol and the installed OneWireViewer software, my job was to plug into the power supply sensors and to record their ID numbers into Excel spreadsheets, which were provided to me.

All of the sensors at NSLS-II have built-in redundancy: there are two devices connected in two separate chains for each sensor to provide backup if one device or a whole chain fails. The 1-wire adapter connects to two separate chains and the software displays them through two USB ports. The OneWireViewer software, in addition to providing a selected device description and registration ID, shows the parameters monitored by the device. For a temperature sensor it displays the temperature readings and constructs a real-time graph of the readings.

In addition to using OneWireViewer to obtain sensor IDs, most power supplies and power supply interfaces have 2-D bar codes, which can be scanned to obtain device IDs. Using a 2-D bar code scanner was sometimes necessary to obtain the numbers, since some power supplies are difficult to plug into with the 1 -wire reader due to obstructions and complicated wiring. The 2-D bar codes are also quicker to use: a quick scan reads devices on both chains. By knowing which sensors IDs belonged to, based on the ending digits, the sensor spreadsheet for a rack group with about 150 sensors could be filled relatively quickly, as compared to plugging into every device. But the bar codes are limited in their use: some codes do not store all the sensor information, others have ID recordings with missing or incorrect last digits, some devices do not have bar codes at all, while some codes do not respond to the scanner. But by combining the use of the 1-wire reader and the bar code scanner, all of the required data could be obtained in most rack groups.

The work conducted by us in NSLS-II is contributing to making certain successful operation of the National Synchrotron Light Source II. The magnet measurement and testing ensures that the magnets can operate properly and reliably to produce a beam. 
DMM cable testing ensures that a digital multi-meter will obtain proper readings from the magnet power supplies. And obtaining IDs from the 1-wire sensors at NSLS-II will provide the necessary information on the state of the power supplies. The sensor ID numbers will be extracted by the Control System Studio (CSS) software, which will enable operators to see the data received by the sensors in every cell and rack group of NSLS-II and will alert them of any problems, such as overheating of power supplies, so action can be taken to ensure smooth and safe operation of NSLSII.

\section{References}

1 "1-Wire Devices" - Maxim Integrated. 02 Aug. 2013 $<$ http://www.maximintegrated.com/products/1-wire/>. 


\title{
Phosphorylation in the phosphoenolpyruvate branch point: the extension of a dynamic model of Brassica napus seeds
}

\author{
Bradley T. Salvato \\ Mathematics, College of DuPage, Glen Ellyn, Illinois 60137 \\ Jordan O. Hay \\ Biosciences, Brookhaven National Laboratory, Upton NY 11973
}

Abstract

The phosphoenolpyruvate (PEP) branch point is an important metabolic pathway in plants because it is critical in the synthesis and elongation of fatty acids which are eventually converted to oil and, eventually, biofuels. A dynamic model of this pathway in Brassica napus embryos, which is currently being developed in silico, depends greatly on the kinetic properties of the enzymes that catalyze the reactions, and the rate equations that mathematically represent the velocities of each reaction. This project primarily focuses on modification and extension of the rate equations, specifically by accounting for phosphorylation. Prior to this project, the rate equations did not account for protein phosphorylation. Phosphorylation alters the kinetic properties of an enzyme, which may lead to differences in metabolite concentration and the rates of each reaction. Initially, I manually recreated the model from Python in Scilab's Xcos, a visual programming software which I used to modify the rate equations. Assumptions from previous research on enzyme kinetics guided all rate equation modifications. Accounting for phosphorylation of phosphoenolpyruvate carboxylase (PEPC) led to a significant increase in the magnitude of flux of PEPC and a mild increase in the proportion of flux on the PEPC side of the PEP branch point. The concentrations of aspartate and oxaloacetate also measurably increased while the concentration of PEP notably decreased with PEPC phosphorylation. The extension of the dynamic model serves to encourage more effective predictive analyses and hypotheses regarding the metabolism of Brassica napus plants, especially with regard to maximizing oil production. In attending this internship, I learned how to work within $X c o s$ as well as understand the biology background of the PEP branch point. I hope to apply these computational, mathematic, and biology-based skills to further math classes I have yet to take and to a renewed interest in plant biology.

\section{Introduction}

Phosphoenolpyruvate carboxylase (PEPC) is a crucial enzyme in plants, especially with regards to the tricarboxylic acid (TCA) cycle. PEPC plays a major role in the flux partitioning of the phosphoenolpyrvuate (PEP) branch point (Figure 1). It catalyzes the irreversible reaction of PEP in the presence of carbon dioxide $\left(\mathrm{CO}_{2}\right)$ and water $\left(\mathrm{H}_{2} \mathrm{O}\right)$ to yield oxaloacetate (OxA) and a phosphate group. $\mathrm{PEPC}$ is subject to allosteric inhibition by L-aspartate (Asp), L-glutamate (Glu), and L-malate [1]. In many $\mathrm{C}_{3}$, $\mathrm{C}_{4}$, and crassulacean acid metabolism (CAM) plants, PEPC is reversibly phosphorylated by a PEPC kinase (PEPCK) and dephosphorylated by a protein phosphatase type 2A (PP2A), which are each expressed in Brassica napus [2]. Phosphorylation describes the addition of a phosphate group to an enzyme, altering its kinetic properties. Phosphorylation renders PEPC less sensitive to allosteric inhibitors, more sensitive to activators, and gives it a higher affinity for PEP [3]. In essence, phosphorylation activates PEPC which may lead to increases in its total flux.

Although phosphorylation affects PEPC similarly in each plant type, PEPCK's effectors vary in each plant type. In CAM plants, PEPCK follows a circadian rhythm but depends on light in $\mathrm{C}_{4}$ plants and on light and nitrogen supply in $\mathrm{C}_{3}$ plants [3].

Computational models often attempt to reflect metabolic functions to predict hypotheses. Traditionally, a dynamic model of plant metabolism consists of a set of metabolites and reactions catalyzed by specific enzymes. The reactions catalyzed by each enzyme are represented by a rate equation that reflects how they are allosterically regulated. These assumptions and previous in vivo and in vitro studies on enzyme kinetics provide the framework for accounting for PEPC phosphorylation in silico. Therefore, the purpose of this project seeks to modify a dynamic metabolic model of the PEP branch point in Brassica napus seeds [4]. These modifications will provide a more effective predictive picture of the dynamic nature of metabolism.

\section{Materials and Methods}

The dynamic model reflects the PEP branch point and consists of nine reactions, six metabolites with fixed concentrations, and five state-dependent metabolites. A stochiometric matrix represents the production and consumption of each metabolite in their respective reactions. Each reaction has a rate of catalysis represented by their respective rate equations, and these rates depend on kinetic properties of each enzyme. Consequentially, the rate equations reflect these properties, and they account for allosteric regulation of the enzymes by the metabolites in the model. For example, as stated earlier, PEPC has an affinity for PEP and is subject to feedback inhibition by Glu and Asp. The rate equation for PEPC accounts for these regulations by dividing the concentrations of PEP, Glu, and Asp their respective affinity and inhibition constants. Because Glu and Asp inhibit PEPC activity, their inhibition expressions exist in the denominator of the rate equation (Figure 2). Each rate equation follows a similar construction, but their rationale is discussed elsewhere [4]. A representation of the metabolic pathway of the PEP branch point is displayed in Figure 1, and the names of enzymes and metabolites and their respective abbreviations are displayed in Table 1.

The model, implemented in Python, was manually imported in Xcos, a visual programming software offered in Scilab 5.4.1. Xcos consists of numerous mathematical blocks that provide the user with an alternative to Scilab's console [4]. Most blocks contain one or more inputs, one output, and a symbol that visually 
describes the block's function. Arrows pointing towards the symbol represent inputs while arrows pointing outward represent the output.

Using these blocks, we replicated this model to resemble the structure of the PEP branch point (Figure 3). To simplify the model, only the state-dependent metabolites were implemented into the schematics of the Xcos model. The other six metabolite concentrations (Glu, ATP, ADP, alKG, CO2, PG2) are assumed to remain constant, so their initial concentrations were assigned as their values. Each block labeled as a metabolite or enzyme represents a superblock, a tool that allows the user to condense a group of icons into one block. Within the metabolite superblocks, we used summation blocks to represent the stoichiometry. The sign on each input reflects whether the metabolite is produced or consumed in a given reaction. The production and consumption of the metabolite is initially expressed as a rate of production in terms of time $(\mathrm{mM} / \mathrm{min})$, so the summation block must be integrated with respect to time. The integral accounts for each metabolite's initial concentration and allows for the calculation of their state concentrations. Then, we labeled output blocks as the metabolites themselves to create variables representing their concentrations. To represent PEP, we used a summation block with four inputs because PEP is required in four reactions. The positive inputs represent the production of PEP in the forward reactions catalyzed by ENO and PPCK while the negative inputs represent PEP's consumption in the irreversible reactions catalyzed by PEPC and PK (Figure 4).

The metabolite superblocks connect to enzyme blocks that reflect the rate of each reaction as well as the flux through each enzyme. Each superblock labeled as an enzyme contains its rate equation. The superblocks for the enzymes include expression blocks that show the written form of the rate equations. The number of input blocks on an expression block depends on the number of variables in the rate equation. The state-dependent metabolites reflect the variables in the model prior to any equation modifications. The expression is then defined with an output block as the enzyme that represents its rate equation. Initially, we replicated the expressions as they were from the dynamic model.

To account for the effects of PEPC phosphorylation, we modified PEPC's rate equation. These modifications relied on previous studies that analyzed the enzyme kinetics of PEPC in Brassica napus and Arabidopsis thaliana. These studies suggested that, when phosphorylated, PEPC's sensitivity to Asp decreased 2.5-fold while its sensitivity to Glu decreased 4-fold and its affinity for PEP doubled [6, 7]. To represent these kinetic changes, we defined additional sensitivity and affinity constants to represent the phosphorylated enzyme's properties. The values assigned to the constants for Asp, Glu, and PEP represented the values of the original constants multiplied by $2.5,4$, and 2 respectively. The values of each constant can be seen in Table 2 . The new equation accounts for phosphorylation with a phosphorylation ratio (PR) and the addition of an identical expression with altered kinetic constants. Variables that deregulated and amplified PEPC activity, deregulated pyruvate kinase, and knocked out phosphoenolpyruvate carboxykinase (PPCK) were added. Changes in steady state concentrations of each metabolite and enzyme flux were analyzed to demonstrate the effects of PEPC phosphorylation on the model. The original and new rate equations for PEPC are shown in Figure 4.

\section{Results}

With increased PEPC phosphorylation, steady state concentrations varied mildly. With no deregulation, at 100\% phosphorylation, concentrations of Asp and OxA demonstrated 14.1\% increases at steady state, while PEP decreased by $10.9 \%$ (Figure 5). The relative flux on the PEPC/PPCK side of the branch also demonstrated a mild increase with phosphorylation, and the relative flux on the PK side decreased accordingly (Figure 6), despite a slight increase in PK's actual flux (Figure 7). This is consistent with a slight but notable increase in AcCoA and slight decrease in PYR. Phosphorylation also seems to increase the total amount of flux available in the model because ENO, the first reaction in the model, experiences a mild increase in flux. However, the flux through PEPC and PPCK both increase significantly, and this is reflected in the small changes in relative flux because PEPC and PPCK reflect a substrate cycle (Figure 7). Knocking out PPCK or increasing the enzyme activity of PEPC amplifies each effect due to PEPC phosphorylation greatly (Figure 5, Figure 7).

\section{Conclusion}

These findings suggest that PEPC phosphorylation has a measurable but important effect on the dynamic model. Strikingly, PK experiences a mild increase in flux with increased PEPC phosphorylation at normal activity. This leads to an increase in flux throughout the PK branch and mild increases in AcCoA levels as a result. As a crucial metabolite in fatty acid synthesis and elongation, this may be a pathway for greater yields of oil in Brassica napus embryos. Both phenomena are further amplified by knocking out PPCK. Therefore, the phosphorylation of PEPC combined with complete deactivation of PPCK may be an option for increasing oil production and should be investigated. Furthermore, the model only accounts for PEPC phosphorylation. Previous research has suggested that PPCK $[8,9,10]$, PK [11], and $\mathrm{PDH}[12,13]$ are also phosphorylated. Accounting for the phosphorylation status of these enzymes may alter or displace the effects demonstrated by PEPC phosphorylation, so these modifications should be analyzed.

\section{Acknowledgements}

I thank my mentor, Jordan Hay, for his guidance and kindness throughout the internship. I thank the Department of Energy, the Office of Science, and Brookhaven National Laboratory for allowing me the opportunity to engage in this project. I thank Jorg Schwender, Inga Hebbelmann, Alistair Rogers, and Dhiraj Naik for their professionalism and dedication to this project. I thank Ann Emrick and Denise Monteleone for their assistance in providing a comfortable workplace. Financial support to Jorg Schwender (PI) and Jordan Hay for quantitative analysis and modeling of central metabolism in plants is greatly acknowledged (Division of Chemical Sciences, Geosciences, and Biosciences, Office of Basic Energy Sciences, Field Work Proposal BO-133). This project was supported in part by the U.S. Department of Energy, Office of Science, Office of Workforce Development for Teachers and Scientists (WDTS) under the Science Undergraduate Laboratory Internships Program (SULI). 


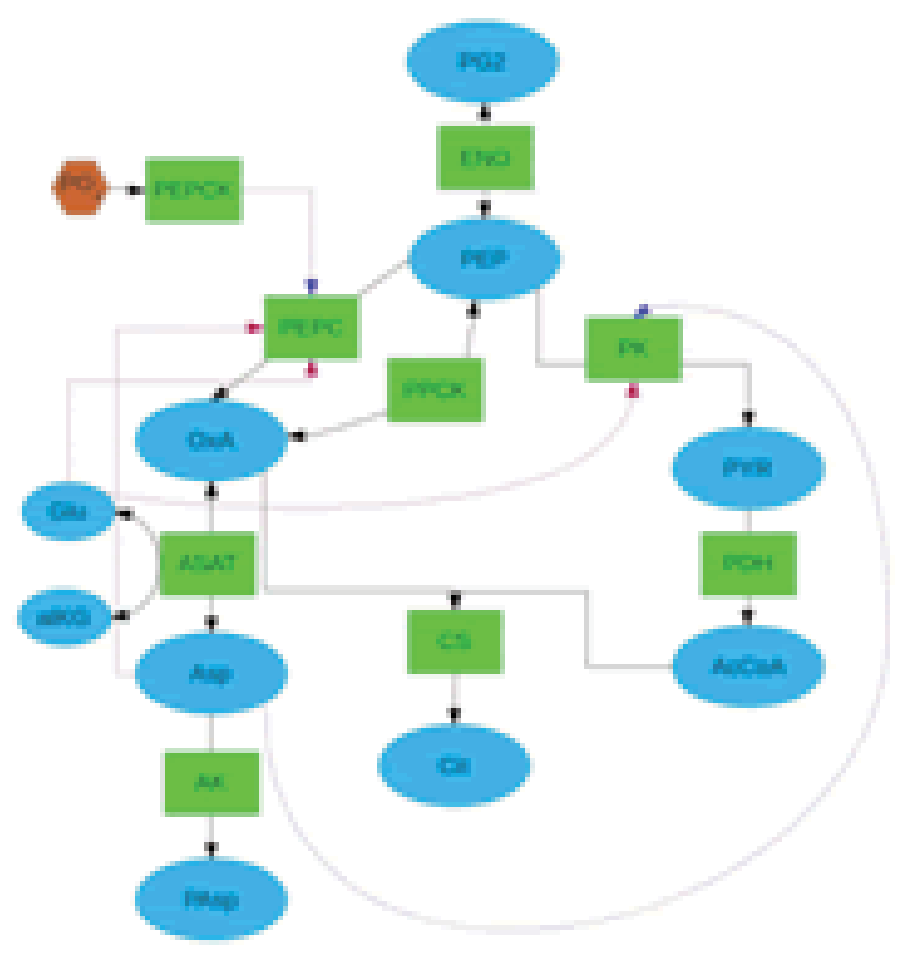

Figure 1. The Phosphoenolpyruvate (PEP) Branch Point. The PEP branch point is a complex metabolic network. PEPC, PPCK, and $P K$ all play pivotal roles in regulating the flux and synthesis of each metabolite. Some metabolites may serve as allosteric activators or inhibitors of a specific enzyme's activity. For example, aspartate and glutamate act as feedback inhibitors of PEPC. However, when PEPC is phosphorylated by PEPCK, its sensitivity to aspartate and glutamate decreases greatly, increasing its own flux, the flux of PPCK towards PEP, and slightly increasing PK's flux.

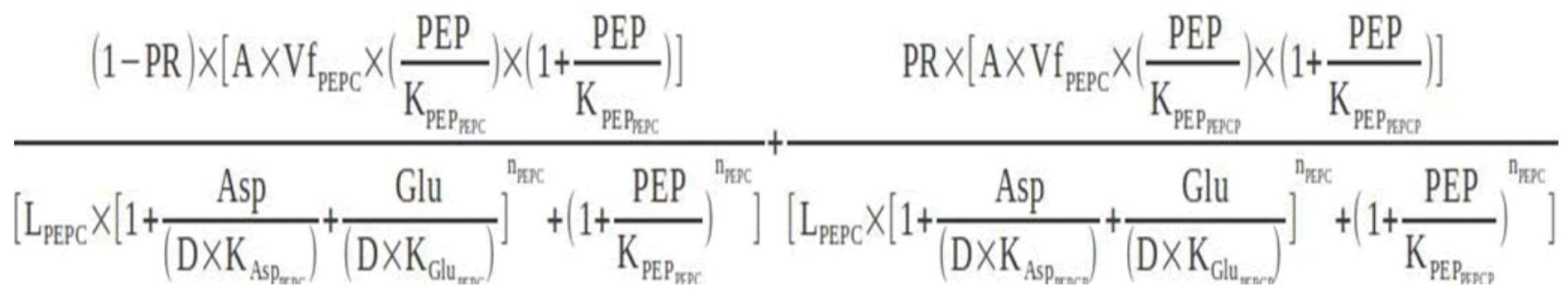

Figure 2. Rate Equation for PEPC after Modifications, where $A$ is a multiplier of PEPC activity level

$D$ is the level of deregulation $(1=$ no deregulation, $\infty=$ total deregulation $)$

PR is the "phosphorylation ratio", which reflects the proportion of the PEPC pool that is phosphorylated

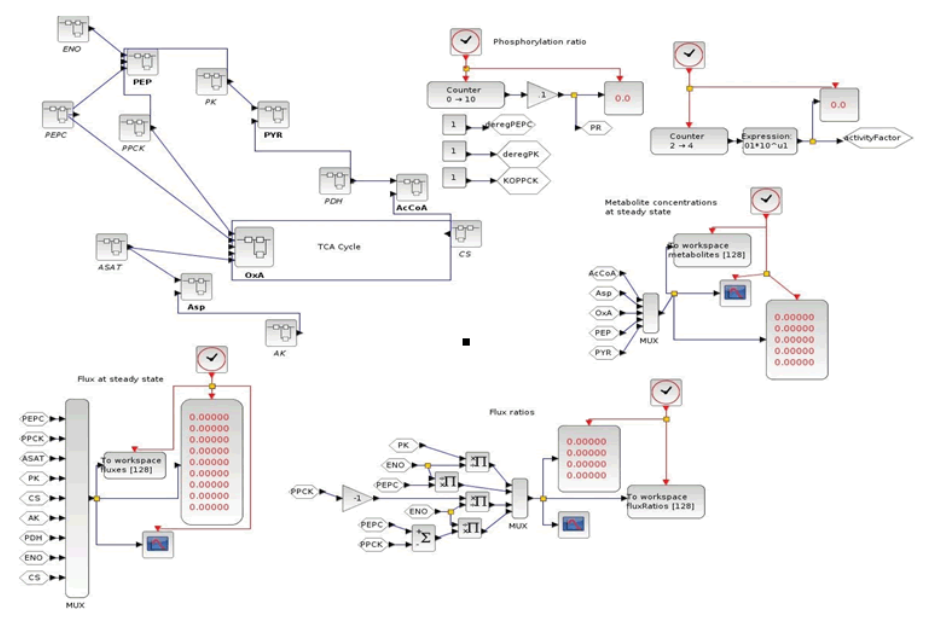

Figure 3. Dynamic Metabolic Model as Represented in Xcos Using Visual Programming

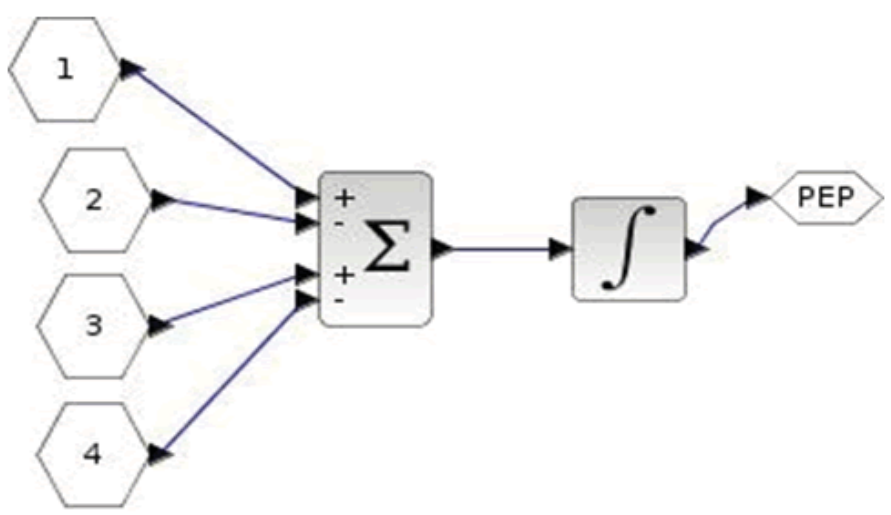

Figure 4. Stoichiometry of PEP in Xcos. These blocks make up the superblock labeled "PEP" in Figure 2. The summation block represents PEP's rate of accumulation, and integration is used to simulate PEP's concentration state. 


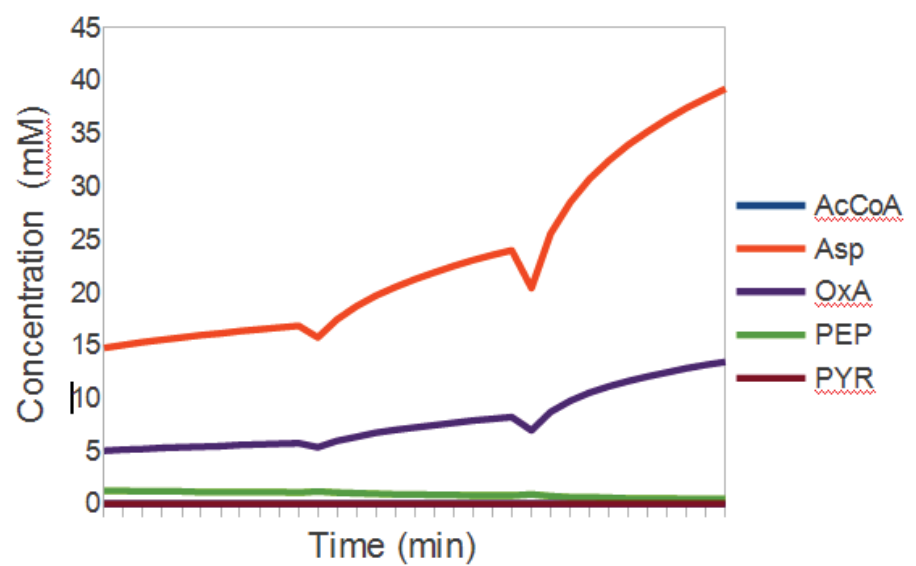

Figure 5. Steady State Metabolite Concentrations, Varying PEPC Phosphorylation and Activity

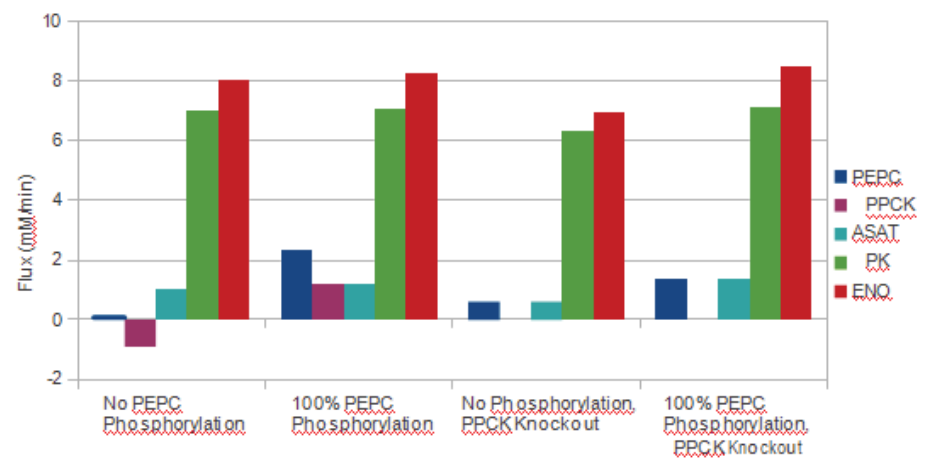

Figure 7. Flux Changes due to Varying PEPC Phosphorylation Status. 1x PEPC Activity.

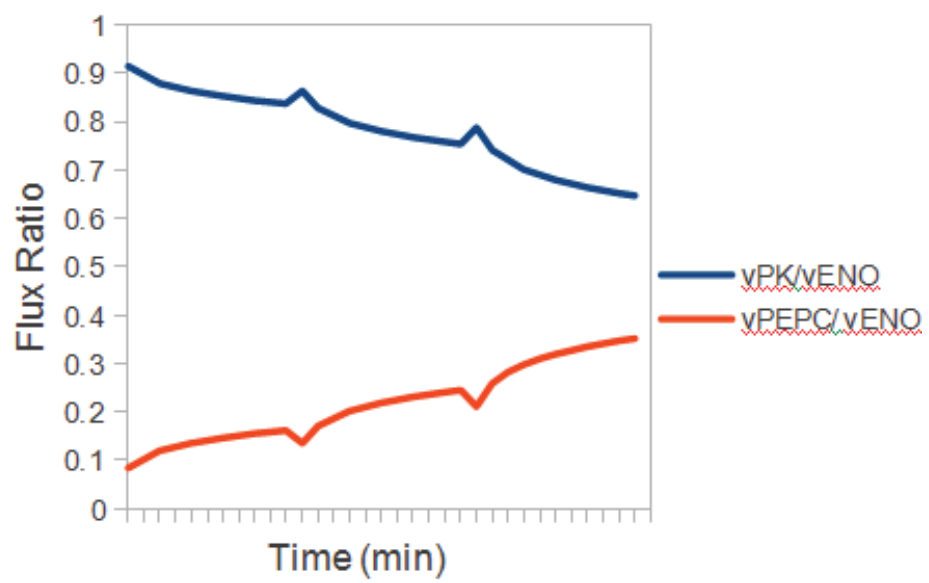

Figure 9. Relative Flux after Varying PEPC Phosphorylation and Activity, PPCK Knockout

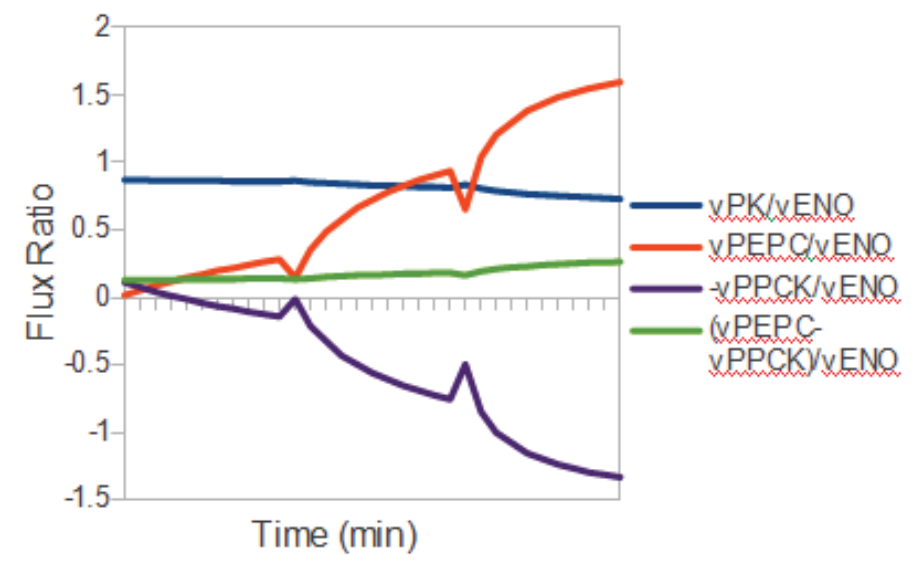

Figure 6. Relative Flux after Varying PEPC Phosphorylation and Activity

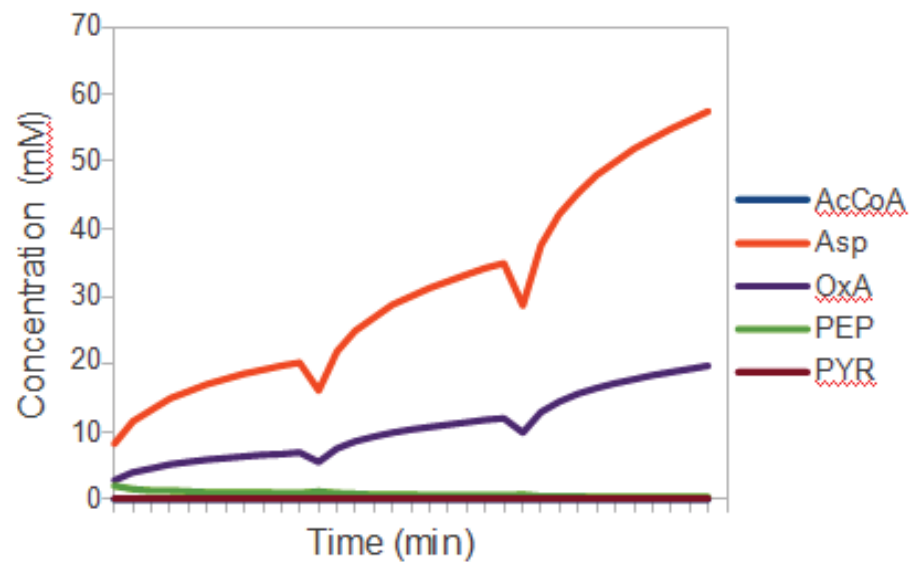

Figure 8. Steady State Metabolite Concentration, PPCK Knockout, Varying PEPC Phosphorylation and Activity. 
Table 1. Abbreviations for Enzymes and Metabolites

\begin{tabular}{|l|l|l|l|}
\hline \multicolumn{1}{|c|}{ Reaction Enzymes } & & \multicolumn{1}{c|}{ Metabolites } \\
\hline ENO & Phosphopyruvate Hydratase & PEP & Phosphoenolpyruvate \\
\hline PEPC & PEP Carboxylase & OxA & Oxaloacetate \\
\hline PPCK & PEP Carboxykinase & Glu & L-Glutamate \\
\hline ASAT & Aspartate Transaminase & Asp & L-Aspartate \\
AK & Aspartate Kinase & PYR & Pyruvate \\
\hline PK & Pyruvate Kinase & AcCoA & Acetyl Coenzyme A \\
\hline PDH & Pyruvate Dehydrogenase & alKG & 2-Oxoglutarate \\
\hline CS & Citrate Synthase & PG2 & 2-Phospho-D-glycerate \\
TCA & Tricarboxylic Acid Cycle & PAsp & 4-Phospho-L-aspartate \\
\hline PEPCK & PEP Carboxylase Kinase & Cit & Citrate \\
\hline
\end{tabular}

Table 2. Reconstructed kinetic constant differences due to phosphorylation of phosphoenolpyruvate carboxylase in Brassica napus (mM). In this context, phosphorylation is defined as the bonding of a phosphate to an enzyme by a specific kinase. Phosphoenolpyruvate carboxylase (PEPC) has been shown to phosphorylate in numerous $\mathrm{C}_{3}, \mathrm{C}_{4}$, and $\mathrm{CAM}$ plants, including Brassica napus, and this process significantly alters its kinetic properties. The K values represent PEPC's sensitivity to its allosteric regulators-a higher value represents a lower sensitivity to a specific regulator. For example, PEPC's sensitivity to inhibition by aspartate significantly decreases when phosphorylated, so the respective kinetic constant $\left(\mathrm{K}_{\mathrm{i}} \mathrm{Asp}\right)$ increases.

\begin{tabular}{|l|r|r|r|r|}
\hline & \multicolumn{1}{|c|}{ Km PEP } & \multicolumn{1}{c|}{ Ki Asp } & \multicolumn{1}{c|}{ Ki Glu } & Reference \\
\hline Phosphorylated & .1 & 3.75 & 22.5 & 6,7 \\
\hline Dephosphorylated & .2 & 1.5 & 5 & 1 \\
\hline
\end{tabular}

\section{References}

1. Moraes, Trevor F and Plaxton, William C. "Purification and Characterization of Phosphoenolpyruvate Carboxylase from Brassica Napus (Rapeseed) Suspension Cell Cultures and the Integration of Glycolysis with Nitrogen Assimilation." European Journal of Biochemistry 4476 (2000): 4465-4476.

2. Troncoso-Ponce, Manuel A et al. "Comparative Deep Transcriptional Profiling of Four Developing Oilseeds." The Plant Journal 68.6 (2011): 1014-27.

3. O'Leary, Brendan, Joonho Park, and William C Plaxton. "The Remarkable Diversity of Plant PEPC (phosphoenolpyruvate carboxylase): Recent Insights into the Physiological Functions and Post-translational Controls of Non-photosynthetic PEPCs." The Biochemical Journal 436.1 (2011): 15-34.

4. Hay, Jordan and Jörg Schwender. "Metabolic Network Reconstruction and Flux Variability Analysis of Storage Synthesis in Developing Oilseed Rape (Brassica napus L.) Embryos." The Plant Journal 67.3 (2011): 526-41.

5. Scilab Enterprises (2012). Scilab: Free and Open Source software for numerical computation (OS, Version 5.4.1) [Software]. Available from: http://www.scilab.org.

6. Gousset-Dupont, a. et al. "Metabolite and Post-translational Control of Phosphoenolpyruvate Carboxylase from Leaves and Mesophyll Cell Protoplasts of Arabidopsis Thaliana.” Plant Science 169.6 (2005): 1096-1101.
7. Gregory, Allison L, Hurley, Brenden A, Tran, Hue T, Valentine, Alexander J, She, Yi-Min, Knowles, Vicki L, and Plaxton, William C. "In Vivo Regulatory Phosphorylation of the Phosphoenolpyruvate Carboxylase AtPPC1 in Phosphate-starved Arabidopsis Thaliana." The Biochemical Journal 420.1 (2009): 57-65. 8. Leegood, Richard C, and Robert P Walker. "Regulation and Roles of Phosphoenolpyruvate Carboxykinase in Plants." Archives of Biochemistry and Biophysics 414.2 (2003): 204-210.

9. Walker, Robert P, Chen, Zhi-Hui, Acheson, Richard M, and Leegood, Richard C. "Effects of Phosphorylation on Phospho Enol Pyruvate Carboxykinase from the C4 Plant Guinea Grass 1.” Plant Physiology 128.January (2002).

10.Walker, Robert P, and Richard C Leegood. "Phosphorylation of Phosphoenolpyruvate Carboxykinase in Plants." Biochemical Journal 317 (1996): 653-658.

11.Tang, Guo-Qing et al. "A Novel C-terminal Proteolytic Processing of Cytosolic Pyruvate Kinase, Its Phosphorylation and Degradation by the Proteasome in Developing Soybean Seeds.” The Plant Journal 34.1 (2003): 77-93.

12.Tovar-Mendez, Alejandro, Miernyk, Jan A and Douglas D. Randall. "Regulation of Pyruvate Dehydrogenase Complex Activity in Plant Cells." European Journal of Biochemistry 270.6 (2003): 1043-1049.

13.Li, Rong-Jun et al. "Cloning and Functions Analysis of a Pyruvate Dehydrogenase Kinase in Brassica Napus." Plant Cell Reports 30.8 (2011): 1533-40. 


\title{
Linac early symptoms detection software
}

\author{
Kevin Sanders \\ St. Joseph's College, Patchogue, NY 11772 \\ Omar Gould \\ Brookhaven National Laboratory, Upton, NY 11973
}

\begin{abstract}
The $200 \mathrm{MeV}$ Linear Accelerator at Brookhaven National Laboratory is comprised of hundreds of devices that vary in degree of sensitivity of performance. A software program has been designed to monitor the behavior of the devices. The program detects abnormal behavior before it results in a degradation of beam or loss of beam time and accordingly notifies the Linac staff of the conditions detected. The Linac Early Symptoms Detection Software (LESDS) will benefit the production of beam at the Linac because it will enable the staff to diagnose potential problems and implement a remedy on a scheduled maintenance day to avoid a loss of beam time. The LESDS retrieves data that is collected by ADO's, or Accelerated Data Objects. The software is written in Java, importing a combination of standard Java API libraries and BNL/C-AD libraries, as well as swing and asynchronous threads.
\end{abstract}

\section{Introduction}

The Linac Early Symptoms Detection software is a unique software design at the Linac (Linear Accelerator). There is no method currently at the Linac to monitor the behavior of the devices concurrently to allow for correlations to be made between the behaviors of the devices and the performance of the beam. The software is looking to catch symptoms where a device's current might be a little outside of an acceptable range, but the device is still operational, because it may not be an immediate problem, but may cause failure over a long period of time. The software is also looking for any behavior where the measurement might rise or fall quickly but return to normal afterwards, because that behavior might also lead to a fault in that device.

This accelerator is comprised of many different hardware components, ranging from quadropole magnets to vacuum pumps. The LESDS uses data that is collected by the ADO pertaining to the device it is monitoring. Programs such as PET and LogView both display the same data, but a user would have to watch those programs manually to notice that a device isn't operating at the set point it was assigned. In addition, correlations between conditions and behaviors would be difficult to achieve using that method. The LESDS brings in the same set point and measurement data values as PET and $\log$ View, but instead of displaying the data, it compares the measurements to the set points, and issues a warning if a measurement is too far away from a set point. The LESDS keeps a log of each of these warnings, and these logs are organized by day.

\section{Methods and Materials}

To get an understanding of the devices that make up the Linac, it was necessary to obtain documentation of the Linac. In the first week of the internship, diagrams of the Lebt, Mebt, Linac, and Hebt sections were studied to get a better understanding of the devices that the LESDS would be dealing with. Multiple tours of different sections, such as the OPPIS (Optically pumped polarized ion source), H-, the RF Cavities, and EBIS, helped to give an idea of the positioning of devices and to understand the scale of the Linac. Determining which devices would be monitored was accomplished through meetings with Tom Flanagan, Tim Lehn, and Deepak Raparia. The LESDS is monitoring the quad magnets and RF data from the Linac tanks 1-9, vacuum pumps throughout the Linac, the Lebt Bending magnet 1 and the Hebt bending magnets 1 and 2, and $\mathrm{H}$ - and OPPIS devices.

The LESDS is written in the Eclipse IDE (Integrated Development Environment). The Collider-Accelerator Department uses Eclipse on Linux machines only. The software NoMachine was downloaded on a Windows computer to allow for access to the Eclipse IDE residing on a Linux computer. This was necessary because a Windows computer was the workstation for the project. NoMachine created a Gnome desktop and allowed for a remote connection through an available network to a Linux computer. Then, using the command prompt, access to the Eclipse IDE was obtained on the designated Linux computer.

The program that was very useful for obtaining data information was Pet; a data read back software that can also allow users to modify the ADO set points. Pet was used to figure out what the names of the data were in the databases in order to be able to call them into the LESDS. LogView is a program allows staff to select a time interval and view a graphical format of the data collected on certain devices. These graphs were used to determine what would be acceptable ranges for magnets and where the cutoff was for vacuums.

\section{Development}

There were two stages to the development of the LESDS: The algorithm and the GUI. The algorithm is relatively simple and for the most part uses a threshold of 5\% for the calculations. The challenge of writing this software was the GUI development. It is very hard without a strong understanding of graphic design and layout managers to get the GUI to look and behave like it is supposed to.

Using the Eclipse IDE was very helpful in writing this application. It can auto complete certain lines of code, and whenever I wasn't sure which method of an API class to call, a "." was typed, and Eclipse would give a list of all applicable methods that could be called.

Writing this software introduced the concept of threads. The swing thread was always something I was implementing in other software, but never realized that it was a thread. Writing asynchronous classes was the first usage of threads other than swing. It also introduced the dangers of threads. Using different threads can be great to get multiple things done at once, but threads can deadlock and kill the application. The problem arises when two 
threads or parts of a thread try to access the same information at the same time. This deadlock is similar to two overly polite people waiting for the other person to go through a doorway. Both threads deadlock because both wait for the other to finish, causing nothing to happen.

\section{Results}

There are classes corresponding to each type and location of each device. For instance, tank 1, tank 2, and tank 3 would represent the first three tanks of the linac section. These data classes are called into the Mainframe class, which is where the GUI (graphic user interface) is also created. It is in this class that the calculations to determine warnings are done. To determine if a magnet isn't operating correctly, the LESDS compares the measurement and the set point. If the measurement is over 5\% away from the set point, a warning is issued. Most vacuum pumps are compared to $1(10-5)$ Torr, and if their measurements are greater, a warning is issued.

In order to write the graphic portion of the LESDS, a combination of standard Java API classes were imported, such as JList, ArrayList, and JRadioButton, and BNL/CAD written classes, such as DecimalFixedField, MessageArea, Xframe, and GlobalFonts. The LESDS is currently written synchronously. This means that the program polls for all necessary data at once, and then processes the information. The button, "Poll Data," is the trigger for the data collection. When it is clicked, there is a poll for data, the button's text changes to "cancel," and a timer starts counting down. When the timer reaches zero, it polls for data again and resets the timer. The user can modify this timer by deleting what is in the text field next to the timer label and setting it to any integer between 10 and 60 seconds, and pressing the enter key. Synchronous data collection is the first step towards the completed product, which will incorporate asynchronous data collection.

There are two logs that are created by the LESDS, a log of all warnings that have occurred, and a log of "cleared" warnings. The software automatically detects the date and attaches it to the end of a predetermined name, such as "acklog," plus the date for the acknowledged or cleared warnings. The window that displays these filters the logs by the date that the log was entered, and there are two calendar buttons to select the date interval. Inside the logs, each entry has an associated time stamp for when it was put into the log, the user and group of the user who was logged in when it was cleared, and the name of the device along with comments.

The bottom of the main screen of the LESDS is the configuration panel. This panel displays a list of all of the devices, based on which radio buttons above are clicked, and allows the user to configure the percentage window of those devices. The default value is $5 \%$, but a user may find that it is too small or too large of a range for certain devices. Because vacuum device algorithm is not based on percentages, this panel only supports changing the percent range for magnet and RF devices.

\section{Conclusion}

The primary functions of my program, such as the logic of handling data and retrieving the data, were completed. However, there are components that are still being worked on. The most important aspect that needs to be added is the change from synchronous to asynchronous data collection. The issue with synchronous data collection is that it collects data at a certain time interval, so there is a time period where no data is being retrieved by the program. Therefore it may never warn the user of certain conditions, if those conditions occur only in between poll peri-

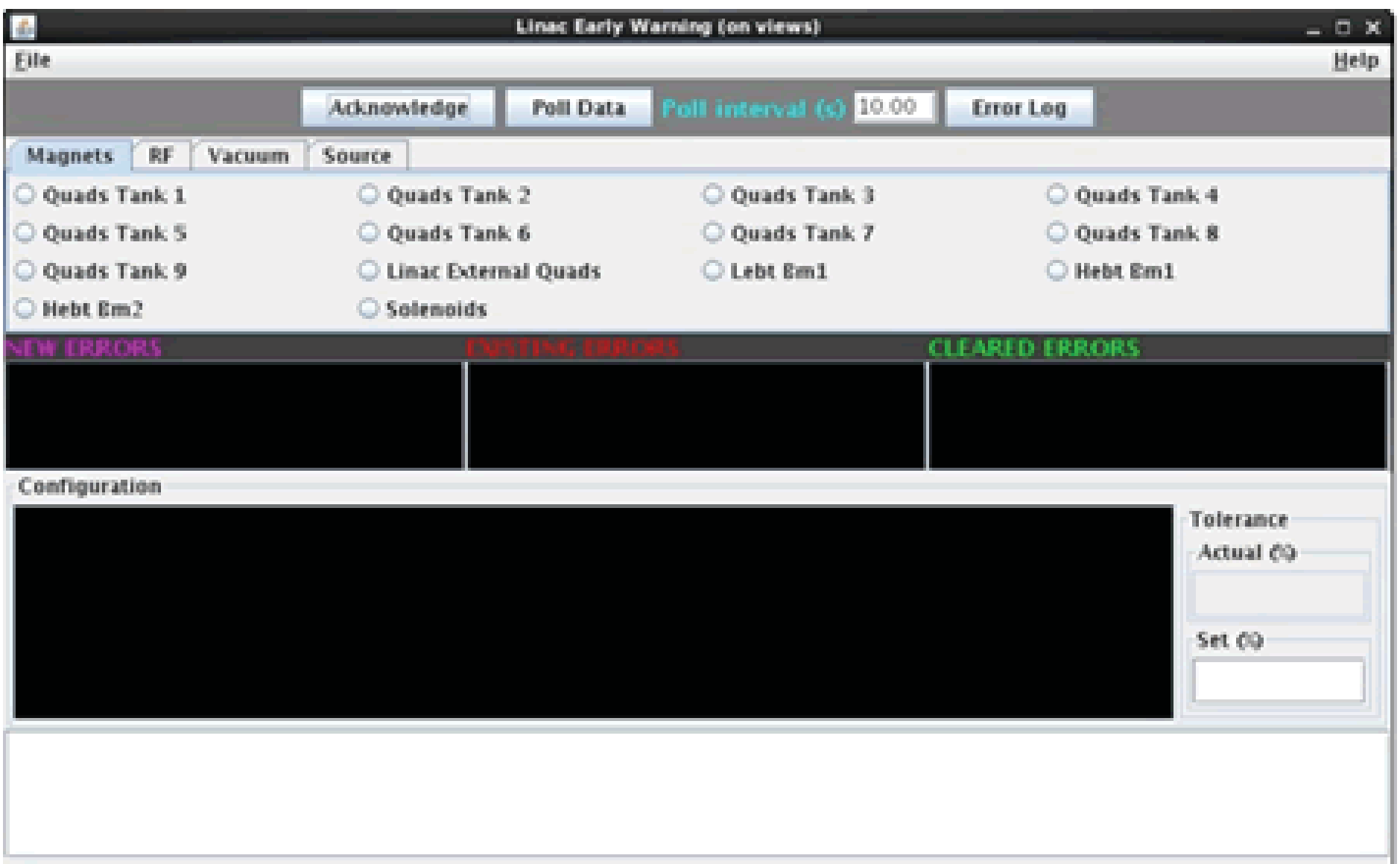

Main Screen of LESDS 
ods. The asynchronous data collection means that the program will operate on the data as soon as it is available. The data will not always be obtained in the same order. This greatly differs from the synchronous logic because the order in which data is polled is sequential. However, these differences are transparent to the user. Asynchronous logic might depend on the data names and types as opposed to the order.

As of this writing, the asynchronous operations are in the design stage. It is expected that it will allow for greater accuracy and reliability in software operations. It will be more accurate because the data will update every AGS cycle, or 4 seconds, instead of every 10 seconds. Synchronously, the system wouldn't be able to handle polling for data every 4 seconds because it is too much data to be processed. Asynchronous data polling sends small groups of data at a time, which puts less of a strain on data operations.

\section{Acknowledgements}

I would like to thank my mentor, Omar Gould, for his help and guidance throughout my internship. I would also like to thank Seth Nemesure, who helped me greatly with the writing of the software. I would also like to thank Deepak Raparia and the Linac Group Technical Staff for their help on my project.

This project was supported in part by the U.S. Department of Energy, Office of Science, Office of Workforce Development for Teachers and Scientists (WDTS) under the Science Undergraduate Laboratory Internships Program (SULI). 


\title{
Determining the optimum carbon stripping foil for use in cancer radiotherapy
}

\author{
Rachel Sandler \\ Department of Physics, University of Arizona, Tucson AZ 85721 \\ Nicholaos Tsoupas and Omar Gould \\ Collider-Accelerator Department, Brookhaven National Laboratory, Upton NY 11973
}

The synchrotron accelerates carbon ions up to $400 \mathrm{MeV} / \mathrm{u}$ for use in cancer radiotherapy. $\mathrm{C}^{5+}$ ions are injected into the synchrotron and focused into a tight beam during acceleration. Unlike protons, which can be extracted in a single turn and used for treatment, carbon ions must be extracted in partial bunches to prevent hazardous doses of radiation. In order to achieve this socalled turn-by-turn extraction, the carbon beam is passed partially through foil which strips the final electron from a portion of the beam. It is then possible to extract only this portion for use. Two programs, LISE, named for the beam line of super-stripped ions in Caen, France, and SRIM, Stopping and Range of Ions in Matter, were used to find that carbon foil $23 \mu \mathrm{m}$ thick creates the optimal stripping conditions.

\section{INTRODUCTION}

Ion beams have been a form of cancer treatment for more than seventy years. Currently, a majority of radiotherapy locations utilize protons beams, which scatter within human tissue to distribute energy across a large volume. This prevents the energy dose from exceeding the maximum which can be safely absorbed by the human body. However, this scattering also increases the likelihood of damaging the healthy tissues surrounding the tumor. Carbon ions do not scatter as much within the human body and thus can be focused onto a more precise region. Unfortunately, due to this lack of scattering, if a full beam of carbon ions were extracted for use the same energy dose would be supplied to a smaller volume of cells, with potentially hazardous results. To maintain optimal treatment conditions, the ion beam must be extracted in pieces which will each deliver a radiation dose lower than the tolerance thresholds. This partial extraction is performed by placing foil in the path of the beam as it is accelerated within a synchrotron. A fraction of the beam passes through the foil and each ion is stripped of its remaining electrons. These ions, which now have a greater charge than the rest of the beam, are pulled by magnets into a septum containing magnetic dipoles. The fraction is focused tightly and ejected from the synchrotron at the tumorous region. The remaining beam is accelerated to a higher energy before another fraction is stripped and ejected. Ions with higher energies will come to rest at deeper points in the tumor, thereby irradiating different regions. With this turn-by-turn extraction process, even large cancerous areas can be quickly treated with fewer side-effects than other radiation techniques. [1]

Simulation programs were performed with foils of varying metals and thicknesses to find the foil with the most desirable properties.

\section{THE SYNCHROTRON}

The synchrotron will allow for more sophisticated radiotherapy techniques, as it is able to accelerate heavy ions to high energies while maintaining the tight beam radius necessary for precise work. This will allow tumorous regions to be quickly treated with minimum damage to healthy tissues - an aspect which is unattainable with other methods.

\section{A. Ion Beam Source}

The ions for the $\mathrm{C}^{5+}$ beam will be created by focusing a laser on the surface of a solid carbon target. The surface atoms will heat up under the laser, which is controlled using mirrors and lenses,

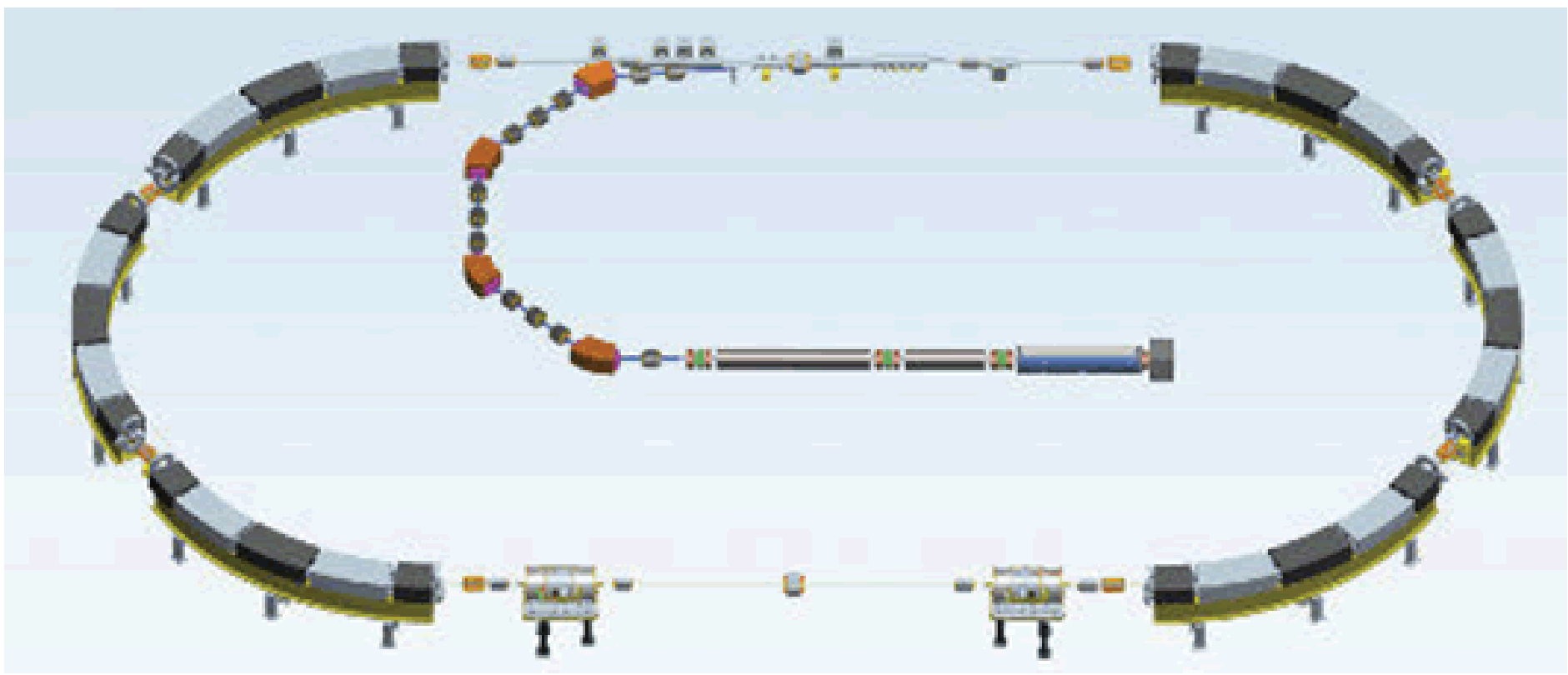

Figure 1 - A medical synchrotron [1] 
to create plasma. Different plasma temperatures allow for differently charged ions to be created as necessary. A high voltage cage traps the ions as they are created until a sufficient number has been collected and the entire bunch can be injected into the beam line by a septum magnet. [2]

\section{B. The Septum}

The beam is injected and extracted from the synchrotron by a septum magnet. A septum is created from two long sheets of current flowing in opposite directions. Each sheet creates a magnetic field on either side of the currents which runs parallel to the sheets and perpendicular to the currents. Between the sheets, the fields point in the same direction and combine to form a strong magnetic field. Outside of this space, the fields caused by the opposing currents point in opposite directions and cancel.

This creates a strong magnetic field within the septum which can push the beam into or out of the synchrotron following the magnetic aspect of the Lorentz Force Law:
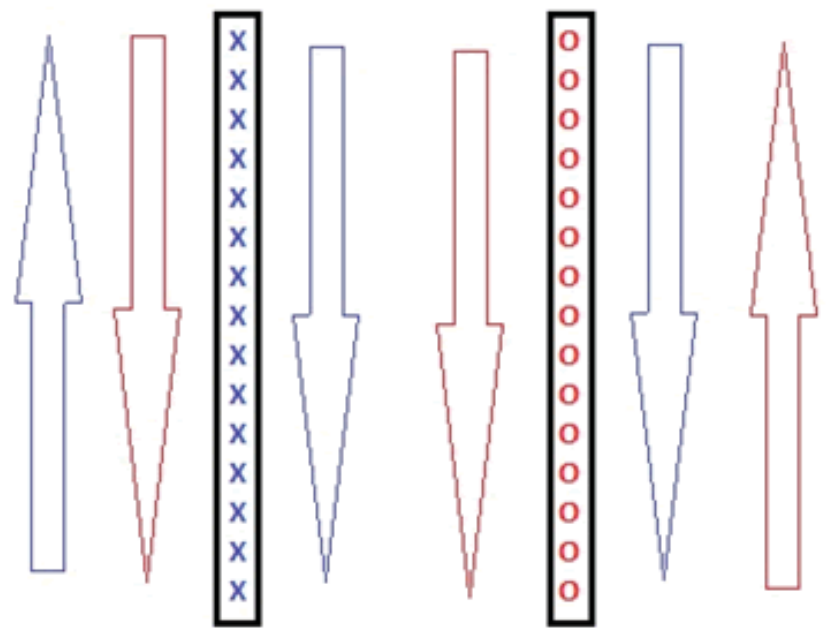

Figure 2 - The magnetic field of a septum magnet

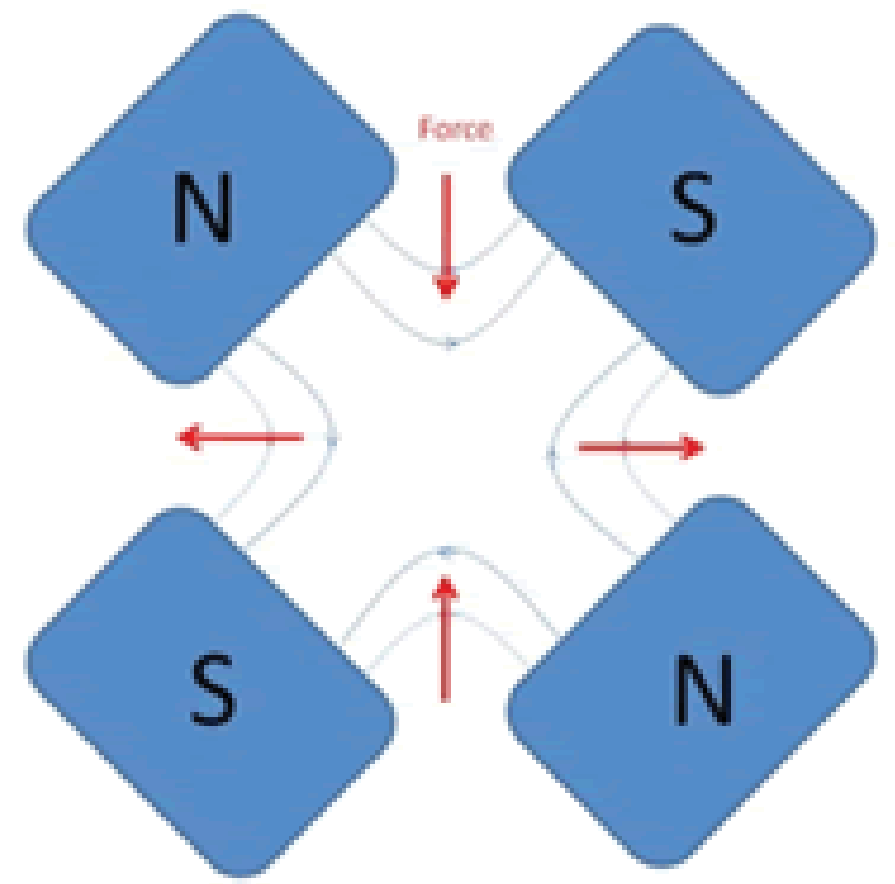

Figure 4 - The magnetic field of a quadrupole magnet [5]

$$
\vec{F}=q \vec{v} \times \vec{B}
$$

Outside of the septum, however, there is no magnetic field which might alter the path of the beam in the synchrotron. [3]

\section{Accelerating the lon Beam}

After the C5+ beam has been injected into the synchrotron, a radio frequency (RF) cavity is utilized to accelerate the beam to the necessary energy. A sinusoidal electric field is created within the cavity and carefully calibrated so that the revolution period of the beam bunch - the time required for the bunch to travel one complete lap around the synchrotron - is harmonic with the field. During each pass through the cavity, the bunch experiences the same electric field magnitude and thus the same force following the electric component of the Lorentz Force Law:

$$
\vec{F}=q \vec{E}
$$

This force on the charged particles increases the momentum of the bunch. Particles which stray slightly to the front the bunch and leave the harmonic frequency experience a smaller force from a smaller electric field magnitude. The opposite is true for particles which begin to lag behind. This focuses the bunch longitudinally. [4]

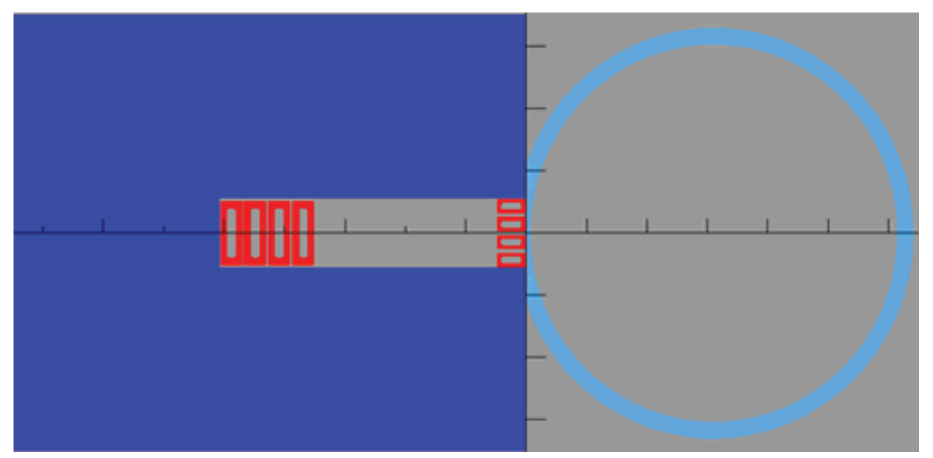

Figure 3 - A physical septum, with the sheets of current shown in red

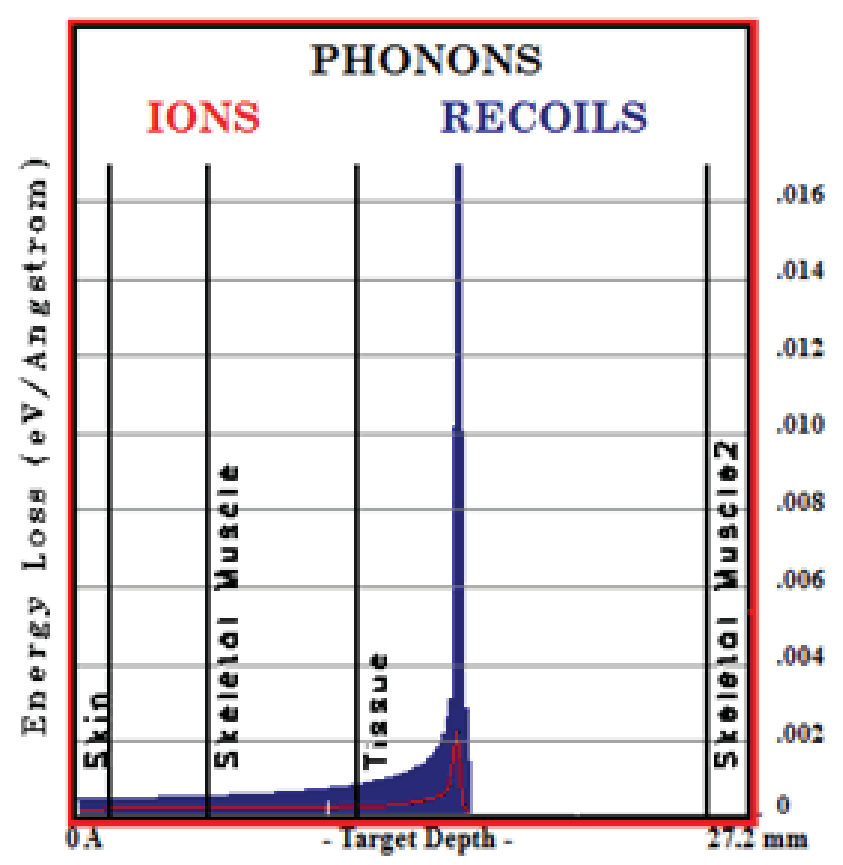

Figure 5 - Energy loss of the carbon beam within human tissue 


\section{Focusing the lon Beam}

Found throughout the synchrotron are quadrupole magnets, set into series of threes. The quadrupoles again utilize the magnetic portion of the Lorentz Force Law to focus the beam transversely.

The quadrupole magnets are set up at right angles to each other. When passing through the first and third quadrupoles, the beam is focused vertically, as show in the diagram above. When passing through the second quadrupole, the magnets are reversed to focus the beam in the horizontal direction. Overall, this compresses the bunch into a narrow beam with a minimal cross-section, preventing radiation from spreading over the patient's skin. [3]

\section{E. Single-Bunch Extraction}

When radiating with protons, single-bunch extraction techniques can be utilized. A bunch containing $7.5 \times 10^{7}$ protons is accelerated to an energy on the order of $206 \mathrm{MeV}$ - this is changed as necessary based on the tumor depth. At this point, the entire bunch of protons is allowed to enter the septum. After focusing, the beam enters tissue, depositing very little energy until it comes to a stop within the tumor.

This sudden deposit of energy is known as the Bragg curve. At this point, the bunch expands from its minimal volume to release 2.5 greys of radiation over a volume of 714.9 cubic millimeters. This dose is the limit imposed by radiation oncologists to prevent harmful effects. [6]

\section{F. Turn-by-Turn Extraction}

When treating cancer with carbon ions, however, calculations show that a pencil beam scatters over a volume of only 13.8 cubic millimeters. Thus, in order to keep the radiation dose at or below the 2.5 grey limit, only $4.5 \times 10^{4}$ ions can be in a bunch. Current acceleration techniques cannot accurately control such a small number of ions. Instead, a bunch containing a larger number of ions is accelerated and a fraction is pulled away with each revolution to enter the septum. This is accomplished with stripping foils. The original $\mathrm{C}^{5+}$ ions do not have a great enough charge to be sent into the septum and revolve freely around the synchrotron. With each revolution, a small section of the bunch is passed through a thin foil, which strips the final electron from each particle. The newly created $\mathrm{C}^{6+}$ ions have a low enough rigidity to be pulled into the septum, focused, and released into tissue. The smaller bunches allow the radiation dose to remain below the limit while the minimal expansion of carbon ions in tissue allows for more precise tumor treatment. [6]

\section{G. Foils}

An important aspect of creating a viable medical synchrotron is to select the optimum foil composition and thickness. The foil must successfully strip electrons without causing the beam to lose significant amounts of energy. The foil also must be thin enough that the beam does not spread out as it passes through. Thicker foils, while stronger and better at stripping, will be more likely to slow the particles or change their directions as each carbon ion will collide with more atoms in the foil. The foil must also have a long lifetime - if the carbon beam damages the foil to the point of needing replacement, the foil will not be useful.

\section{METHODS}

The program LISE++ was organized with a beam of $\mathrm{C}^{5+}$ atoms incident on carbon foil. The desired result after passing through the foil was set to be a beam of $\mathrm{C}^{6+}$ ions. The incident beam was given an initial energy ranging from $10 \mathrm{MeV} / \mathrm{u}$ to 400 $\mathrm{MeV} / \mathrm{u}$. For each energy level, the program was employed to find the optimum thickness of carbon foil to achieve the desired result. The thickness was then entered back into the simulator to find the percentage of the beam which was successfully stripped, the average energy lost by the beam as it passed through the foil, and the amount the beam spread due to differences in individual energy loss, better known as straggling energy. These simulations were all repeated for gold, tungsten, and beryllium foil.

The simulation data was then compiled into graphs which compared the values found for each of the three metals. Graphs were also used to compare the changes in optimal foil thickness at the energy of the incident beam increased.

After reviewing the graphs, it was decided to rerun the simulations with energies ranging from $75 \mathrm{MeV} / \mathrm{u}$ to $400 \mathrm{MeV} / \mathrm{u}$. It was also decided that the simulations would be run without changing the foil thickness to match the energy, as the foil within the synchrotron would not change in thickness. For each metal, the simulation was run twice: the first run utilized the optimum foil for a $100 \mathrm{MeV} / \mathrm{u}$ beam while the second utilized the optimum foil for a $400 \mathrm{MeV} / \mathrm{u}$ beam. Again, data was collected and compiled regarding beam stripping, energy loss, and straggling energy.

From this data, the optimum foil thickness was determined for each metal. This value was entered into a second program, known as SRIM, which simulates the paths ions follow within a target. It calculates energy release within the target material and finds the damage caused to the target. From this, inferences regarding foil lifetimes were made.

\section{RESULTS}

It was evident that the thickness of the foil did not affect the percentage of the beam stripped, only the composition. Foil composed of carbon had the most complete stripping, though the number of unstripped ions was negligible for all four metals. Further, the composition of the foil did not affect the energy lost by the beam. Thin foils caused less energy loss than the thicker foils. Scattering energy, however, was affected by both composition and thickness of foil. Thin foils had lower straggling energies than thick foils, while carbon foil had a lower scattering energy than gold, tungsten, or beryllium. There was no difference in the straggling energy of gold or tungsten foil while beryllium was between the two energies.

SRIM was run with a beam of carbon atoms incident upon a thin foil of each of the four metals. It was revealed that for carbon and beryllium foils, each ion in the incident beam displaced 3 target atoms, with each displacement causing damage to the foil. For gold and tungsten foils, each ion displaced 11 target atoms. It can be inferred that the carbon and beryllium foils will be usable for a greater number of bunches than the gold or tungsten. 


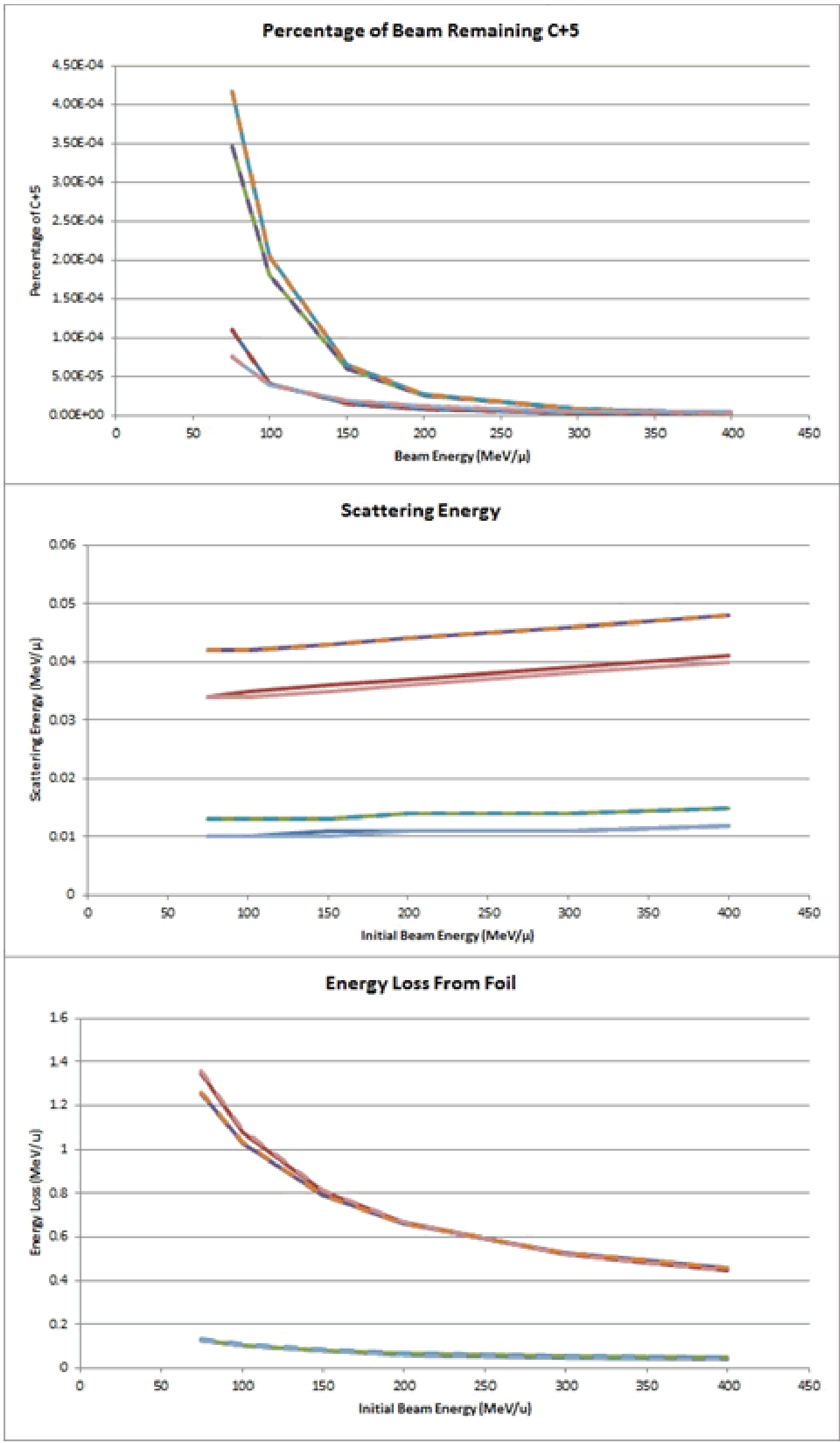

Figure 6 - Results from the LISE simulations 

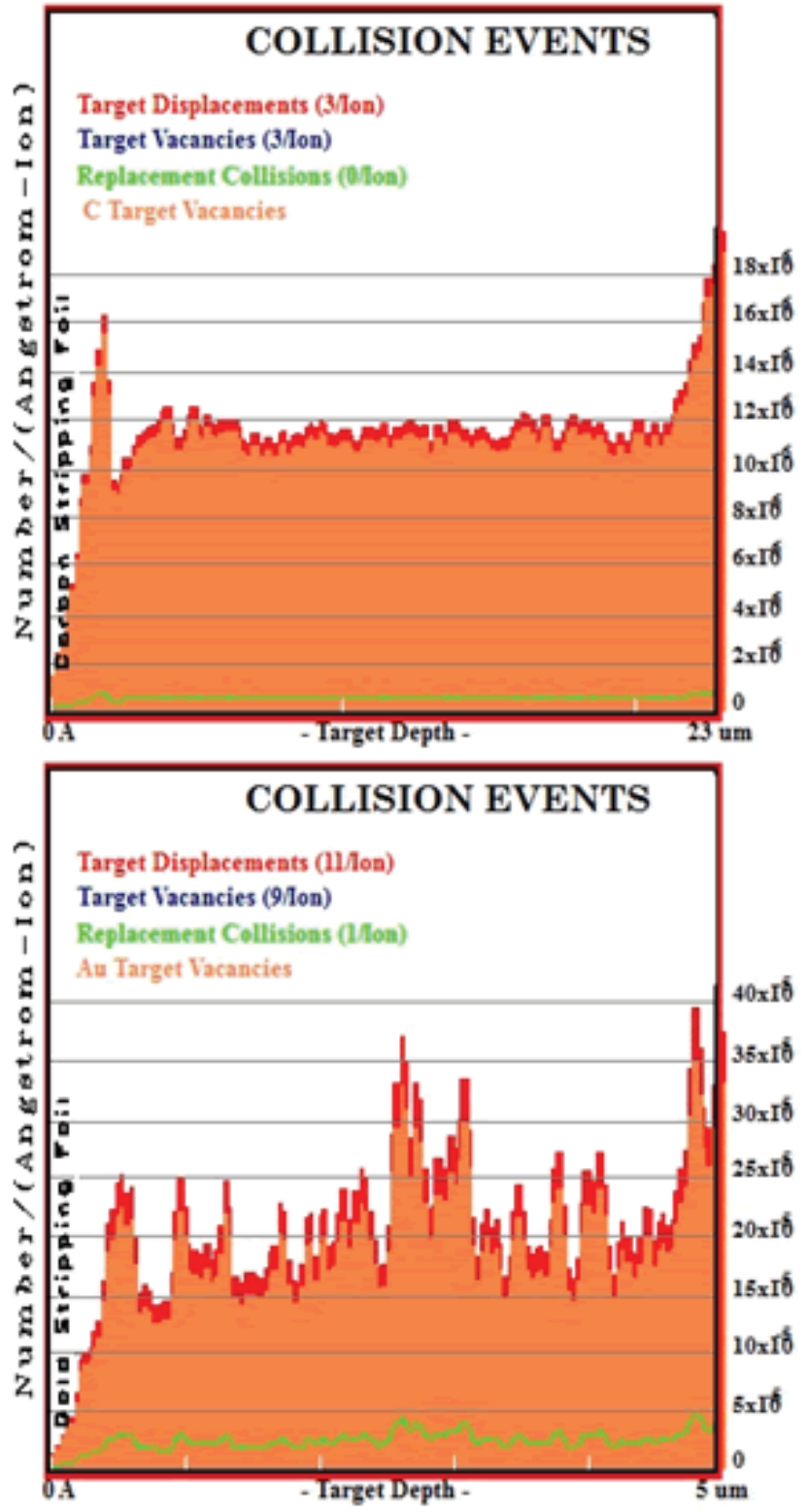
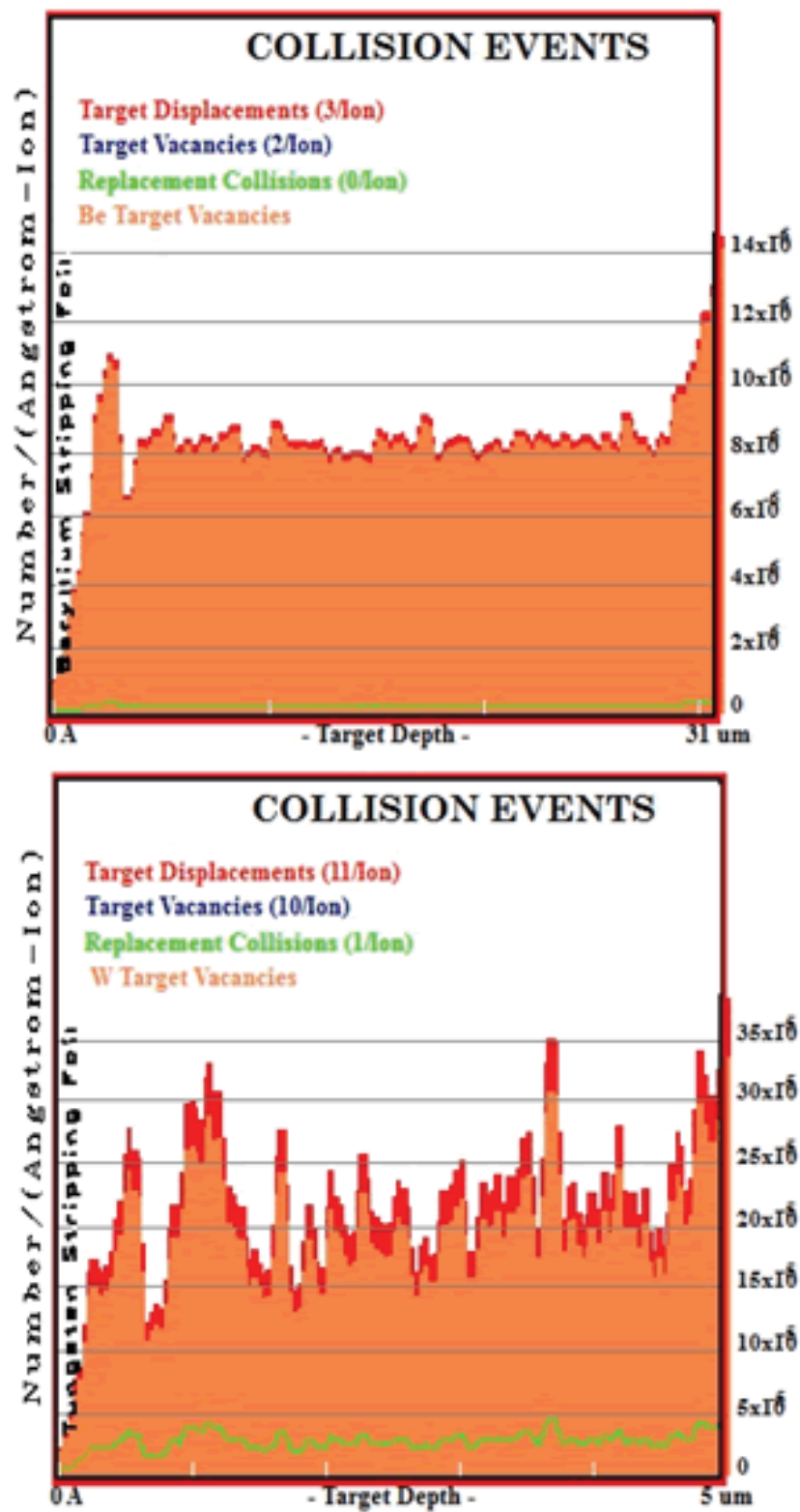

Figure 7 - Results from the SRIM simulations - clockwise from top left: Carbon, Beryllium, Tungsten, and Gold

\section{CONCLUSION}

Utilizing all four parameters, it was decided that carbon foil $23 \mu \mathrm{m}$ thick was the optimum foil of those tested, as it resulted in the greatest beam stripping, the least energy loss, and the lowest straggling energy while experiencing little interaction with the incident beam. With the same parameters $31 \mu \mathrm{m}$ beryllium foil was the second best, which stripped the beam more completely and experienced less beam interaction than the thin gold or tungsten foil while avoiding the energy loss and straggling caused by the thicker foils.

\section{ACKNOWLEDGEMENTS}

This project was supported in part by the US Department of Energy, Office of Science, Office of Workforce Development for Teachers and Scientists (WDTS) under the Science Undergraduate Laboratory Internships Program (SULI). It was made possible by Nicholaos Tsoupas and Omar Gould, who provided patient guidance, as well as Masahiro Okamura, Kevin Smith, and Nicholaos Tsoupas who provided invaluable information regarding the design of the medical synchrotron.

\section{WORKS CITED}

[1] N. Tsoupas, Interviewee, Turn-by-Turn Extraction of Carbon Ions from a Synchrotron. [Interview]. 7 June 2013.

[2] M. Okamura, Interviewee, Injector for iRCMS. [Interview]. 7 June 2013.

[3] N. Tsoupas, Interviewee, Magnets and Magnetic Fields. [Interview]. 8 July 2013.

[4] K. Smith, Interviewee, Ion Acceleration Within an RF Cavity. [Interview]. 14 June 2013.

[5] K. Kahle, "How Accelerators Work," European Coordination for Accelerator Research and Development, July 2006. [Online]. Available: http://eucard.web.cern.ch/. [Accessed June 2013].

[6] N. Tsoupas, Interviewee, Synchrotron Ion Bunch Extraction Methods. [Interview]. 12 June 2013. 


\title{
Differences between carbon and proton beam radiotherapy
}

\author{
Rachel Sandler \\ Department of Physics, University of Arizona, Tucson AZ 85721 \\ Nicholaos Tsoupas and Omar Gould \\ Collider-Accelerator Department, Brookhaven National Laboratory, Upton NY 11973
}

\begin{abstract}
Tumors found in deeper tissues are often treated with particle radiotherapy. Currently, protons are used most commonly. Carbon ions, however, are thought to scatter less within human tissue. It is also theoretically confirmed that carbon deposits a greater percentage of its energy in a smaller volume. Both of these differences would allow for less radiation of healthy tissue surrounding the tumor and the potential for a more effective treatment. In order to calculate these quantities for specific ion species, a program called SRIM, the Stopping and Range of Ions in Matter, was run with a human body target and an incident beam of carbon ions or protons. Data was gathered and analyzed regarding the scattering of ions within the human body and the radiation dose. It was found that protons have a greater radial range and deposit energy over a greater area in human tissue as compared to carbon ions.
\end{abstract}

\section{INTRODUCTION}

Proton radiotherapy is becoming a more and more common form of cancer treatment as doctors, biologists, and physicists learn more about radiation and its effects on cells, as well as how to safely control radioactive particles. More sophisticated particle accelerators have also assisted this rise in popularity as the energy of ions and the thickness of an ion beam becomes more controllable. Particles, unlike x-ray or gamma-ray radiation used in early attempts at radiotherapy, tunnel deep into tissue before depositing energy which makes them superior for treating deep tumors which may not be treatable through surgery. Particle radiation is also localized unlike chemotherapy which affects the entire body broadly, and thus causes fewer side effects throughout the body.

Protons, the most commonly used particles, unfortunately have a wide theoretical Bragg curve. The Bragg curve is the graph of energy loss as a function of target depth. A wide Bragg curve means a large amount of energy is deposited in tissue closer to the beginning of the proton's path - in this case, while surrounded by healthy tissue. Carbon has a narrower Bragg curve, depositing a larger percentage of its energy at the very end of its path - within the cancerous tissue being treated. This precision is akin to microsurgery and prevents side effects such as skin problems, as energy is released more exclusively into the deeper layers of tissue. [1]

Few real-life experiments have been conducted, so it is unknown exactly how this difference between the two Bragg curves affects the behavior of the different ions within the human body. There is also a question regarding the level of radiation deposited by carbon ions. Most doctors set an upper limit of 2.5 joules per kilogram (Gy) for a radiation dose. [2] The narrow width of the carbon Bragg curve, and the subsequently small volume into which energy is released, may lead to the energy per kilogram exceeding this dose. Little is also known about the multiple scattering of different ions within human tissue. Maintaining a tight, narrow beam is of the upmost importance in preventing extraneous radiation to healthy cells. A wide Bragg curve leads to the theory that protons will interact more with the atoms composing the tissues, allowing them to scatter in a wider pattern than carbon ions, which are heavier and will travel along a straight, narrow path unaffected by target atoms.

In order to investigate these differences between proton and carbon ion radiation, simulations were created using the program SRIM, the Stopping and Range of Ions in Matter. SRIM is a program designed to calculate the scattering and energy loss of atoms incident upon a target of specified composition and density. In this case, the target was a representation of the human body. Data was gathered regarding scattering and energy loss and compiled to compare the respective merits of carbon and proton radiation.

\begin{tabular}{|l|r|r|r|r|}
\hline \multicolumn{1}{|c|}{ Layer } & \multicolumn{2}{|c|}{ Skin } & \multicolumn{1}{c|}{ Adipose } & \multicolumn{1}{c|}{ Skeletal Muscle } \\
\hline Density (g/cm3) & 1.09 & 0.92 & 1.05 & 1.05 \\
\hline Percent Hydrogen & 0.62744 & 0.63482 & 0.63169 & 0.6387 \\
\hline Percent Carbon & 0.08237 & 0.28408 & 0.07432 & 0.06133 \\
\hline Percent Nitrogen & 0.01654 & 0.00306 & 0.01515 & 0.01061 \\
\hline Percent Oxygen & 0.27202 & 0.07775 & 0.27703 & 0.28826 \\
\hline Percent Chlorine & 0.00053 & 0.00018 & 00018 & 0.00035 \\
\hline Percent Sodium & 0.00054 & 0.00012 & 0.00058 & 0.00054 \\
\hline Percent Sulfur & 0.00039 & 0 & 0.00064 & 0 \\
\hline Percent Potassium & 0.00016 & 0 & 0.0004 & 0.00016 \\
\hline Percent Phosphorus & 0 & 0 & & 0 \\
\hline
\end{tabular}

Table 1 - The stoichiometric composition of different layers within the human body [3] 


\section{METHODS}

The SRIM program was organized with a human body target composed of skin, adipose (the tissue connecting skin to muscle and bone), skeletal muscle, and tissue as defined by the following densities and stoichiometric guidelines:

The target was composed of a $1.2 \mathrm{~mm}$ layer of skin followed by a $4 \mathrm{~mm}$ layer of adipose, a $6 \mathrm{~mm}$ layer of skeletal muscle, a 14 $\mathrm{mm}$ layer of tissue, and a $2 \mathrm{~mm}$ layer of skeletal muscle. In order to view the scattering of ions throughout multiple layers, it was assumed that the tumor being targeted by the therapy was found within the tissue layer.

The carbon atoms were given an initial energy of $75 \mathrm{MeV} / \mathrm{u}$ while the protons were given an initial energy of $50 \mathrm{MeV}$. Each simulation was run for a single bunch: $4.5^{*} 10^{5}$ carbon ions or $1.0^{*} 10^{6}$ protons. After the simulation was completed, the volume subjected to radiation was calculated from the lateral and radial scattering following the equation,

$$
V=L \pi R^{2} \text {, }
$$

where $V$ is the volume irradiated, $L$ is the lateral range in which the ions stopped, and $R$ is the radial scattering.

Next, the radiation dose was graphed using the equation

$$
D(d)=1.6 \times 10^{-8} \times L(d) \times F
$$

where $D(d)$ is the radiation dose as a function of target depth, $L(d)$ is the linear energy transfer as a function of target depth, and $F$ is the fluence, or atoms per centimeter squared of the beam. The linear energy transfer due to both the ion beam and the recoiling target atoms was found from the raw data provided by SRIM for two cases: ionization linear energy transfer and phonon linear energy transfer

$$
\begin{gathered}
L_{\text {ionization }}(d)=\left[L_{\text {iion }}(d)+L_{\text {irecoil }}(d)\right] \rho \times 10^{-14} \\
L_{\text {phonons }}(d)=\left[L_{\text {pion }}(d)+L_{\text {precoil }}(d)\right] \rho \times 10^{-14}
\end{gathered}
$$

where $\rho$ is the density of the material as given in Table 1 and $10^{-14}$ is a unit correction.

The radiation dose in Greys from both sources was graphed against target depth in millimeters. Finally, the total radiation
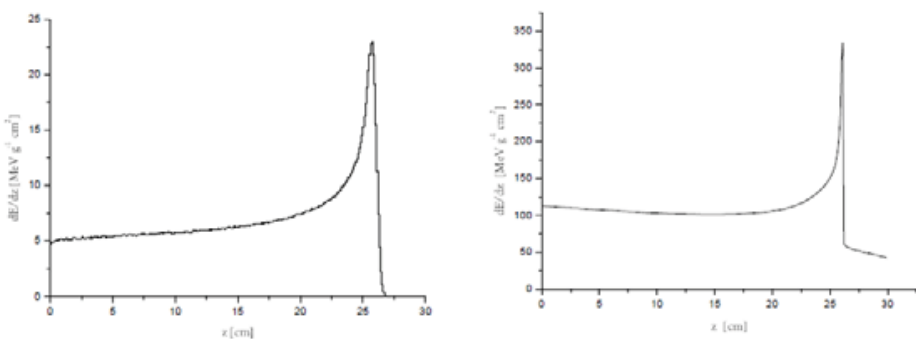

Figure 1 - The Bragg curves for proton (left) and carbon (right) in water [1]

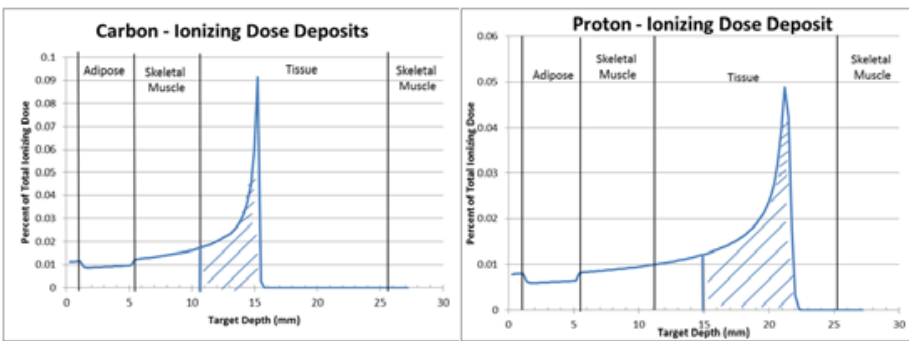

Figure 3 - The dose percentage as a function of target depth for carbon ions and protons in the human body dose was summed and the percentage of the dose deposited as a function of target depth was calculated and graphed. It was found that the linear energy transfer due to phonons - the excitation of electrons of atoms in the target - was negligible compared to the linear energy transfer due to ionization - the removal of electrons from atoms in the target - and so the total radiation dose was changed to the total radiation dose due to ionizations.

\section{RESULTS}

The protons scattered significantly more than the carbon ions, covering a volume of $2.24 * 10^{-1}$ cubic millimeters while the carbon ions only scattered over a volume of $2.32 * 10^{-3}$ cubic millimeters. The greater scattering range of the protons has the potential to damage healthy tissue surrounding the tumor being treated. The carbon maintained a tight configuration even after passing through layers of skin, adipose, and skeletal muscle while the protons began to scatter in different directions immediately after entering the skin layer.

The proton beam also had significantly greater energy straggling, depositing fifty percent of the total ionizing energy along a distance of 7.34 millimeters. This non-localized radiation prevents doctors from altering the energy of the beam during treatment to move the peak of the Bragg curve within the tumor. Attempting to move the Bragg peak to the surface of the tumor, for example, will cause a majority of the energy to be deposited before reaching the cancerous cells. Very little energy would enter the tumor in this case and the treatment would not be effective. Carbon ions, in comparison, deposited fifty percent of the total ionizing energy along a distance of 4.35 millimeters. This precision allows for better control over the location of the Bragg peak and more effective, efficient treatment sessions.

Finally, having twelve nucleons holding energy rather than a single proton allowed the carbon to provide a greater dose of radiation to the tumor while still remaining well below the $2.5 \mathrm{~Gy}$ maximum. Though the carbon atoms were only accelerated to an energy $25 \mathrm{MeV} / \mathrm{u}$ greater than the protons, they provided more
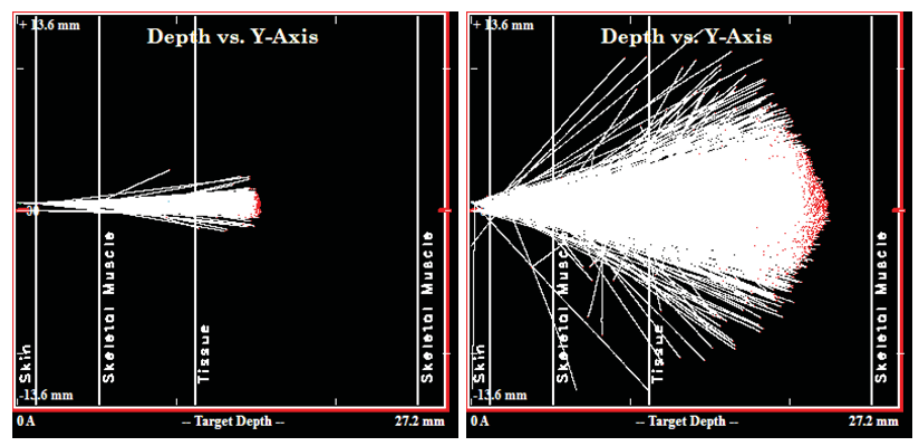

Figure 2 - The radial straggling for carbon ions (left) and protons (right) in the human body
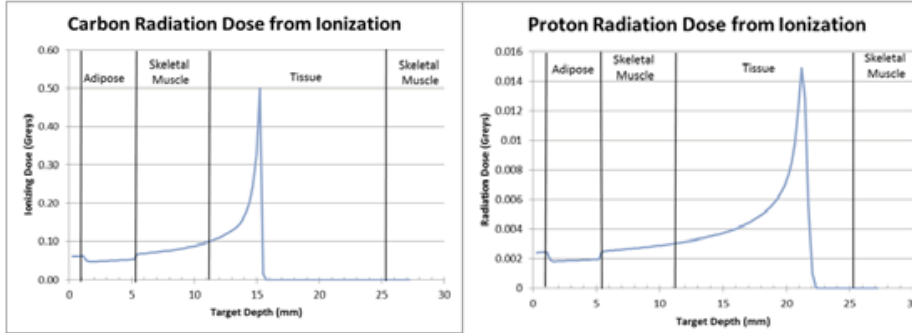

Figure 4 - The radiation dose as a function of target depth for carbon ions and protons in the human body 
than thirty times the radiation dose to the tumor. The peak dose from a single bunch of carbon ions was $0.5 \mathrm{~Gy}$ while the peak dose from a single bunch of protons was $0.015 \mathrm{~Gy}$ - an amount unlikely to affect the tumor significantly.

Far more bunches would be needed for complete treatment with protons, leading to a longer appointment and greater discomfort for the patient. Statistically, more bunches also leads to a greater likelihood of ions scattering widely within tissue, harming healthy cells far away from the tumor. Utilizing fewer bunches comprised of fewer atoms each allows for greater control and fewer errors, preventing mistakes and side effects from the treatment.

\section{CONCLUSION}

When treating tumors buried deep within the human body, it is important to damage as little tissue as possible to prevent side effects and patient discomfort. Carbon ions have a much steeper Bragg curve than protons, depositing a majority of their energy in a very small volume because each nucleon holds energy, leading to more efficient and effective treatment without surpassing the radiation dose limits currently set by doctors and biologists. Finally, carbon atoms scatter over a smaller range, leading fewer particles to enter healthy tissue and release energy into non-cancerous cells. All of these results reveal carbon ions as being a superior form of treating deep-tissue tumors.
It is, of course, still necessary to perform real-life experiments in order to confirm these computer simulated results. However, the simulations agree clearly with theoretical Bragg curves and the conclusions which had been extrapolated from theory regarding energy deposition and multiple scattering. Research is currently being performed at Brookhaven National Laboratory's Collider-Accelerator Department to design a medical synchrotron able to accelerate and strip carbon ions for use in cancer radiotherapy. Such a progression in technology could lead to a new era of cancer treatment and open the way for new medical knowledge.

\section{ACKNOWLEDGEMENTS}

This project was supported in part by the US Department of Energy, Office of Science, Office of Workforce Development for Teachers and Scientists (WDTS) under the Science Undergraduate Laboratory Internships Program (SULI). It was made possible by Nicholaos Tsoupas and Omar Gould, who provided patient guidance and the initial musings regarding the comparison of ion species irradiation.

\section{WORKS CITED}

[1] M. Granlund, "Influence of Multiple Scattering on the Dose Distributions of Ion Beams for Radiation Therapy," Royal Institute of Technology, Stockholm, 2001.

[2] N. Tsoupas, Interviewee, Turn-by-Turn Extraction of Carbon Ions from a Synchrotron. [Interview]. 7 June 2013.

[3] A. Mowlavi, M. Homaei and M. Alipoor, "SRIM Code Use for the Evaluation of Optimum Proton Energy Interval for Thyroid Cancer Therapy," African Physical Review, vol. 4, no. 13, pp. 107-111, 2010.

[4] J. Gomes and H. Shea, "Displacement damage effects in silicon MEMS at high proton doses," SPIE, vol. 7928, 2011.

[5] J. Ziegler, "srim.org," 2013. [Online]. 


\title{
Evaluation of DNA double-strand break repair kinetics in cells exposed to low dose charged particle irradiation and vorinostat treatment
}

\author{
Katherine Z. Sanidad \\ Stony Brook University, Stony Brook, NY 11790 \\ Alicia M. Johnson, Paula V. Bennett, Deborah J. Keszenman, James H. Jardine, and Paul F. Wilson \\ Biosciences Department, Brookhaven National Laboratory, Upton, NY 11973
}

\begin{abstract}
Ionizing radiation (IR) can cause several forms of DNA damage, the most critical being DNA double-strand breaks (DSBs). If incorrectly repaired or neglected, DSBs can generate potentially carcinogenic chromosomal aberrations. During radiotherapy, cells in surrounding normal tissues are exposed to lower doses of IR, and are at risk for secondary cancer induction. We examined how a histone deacetylase inhibitor, vorinostat (suberoylanilide hydroxamic acid, SAHA), impacts survival and DNA damage induction/repair kinetics in human cells exposed to low doses of cesium-137 $\gamma$-rays or $290 \mathrm{MeV} / \mathrm{n}$ carbon ions delivered at the NASA Space Radiation Laboratory (NSRL) at BNL. We show that SAHA-treated NFF28 fibroblasts are significantly more radiosensitive than dimethyl sulfoxide (DMSO)-treated control cells following $\gamma$-irradiation, but not after carbon ion irradiation. We evaluated DSB levels up to $24 \mathrm{~h}$ post-irradiation by monitoring co-localization of two DSB markers, $\gamma-\mathrm{H} 2 \mathrm{AX}$ and 53BP1, using immunocytochemistry techniques and microscopy. We show that SAHA-treated cells irradiated with carbon ions had lower DSB induction levels but interestingly showed delayed but more efficient repair (below background levels) in contrast to cells irradiated with $\gamma$-rays.
\end{abstract}

\section{INTRODUCTION}

During cancer radiotherapy, cells in the surrounding normal tissues can be exposed to lower doses of ionizing radiation (IR), which place them at a higher risk for secondary cancer induction. IR is a genotoxic agent that appears in different forms such as low linear energy transfer (LET) photons (X and gamma rays) and protons, and high LET "heavy" ions such as carbon ions. IR can cause several forms of DNA damage, the most critical being DNA double-strand breaks (DSBs). If DSBs are incorrectly repaired or neglected, they can generate potentially carcinogenic chromosomal aberrations. Previous studies have shown increased DSB levels observed in precancerous tissues [1]. To monitor DSBs, researchers have used the phosphorylated histone H2AX $(\gamma-\mathrm{H} 2 \mathrm{AX})$, a variant of the core histone H2A family, as a biomarker. H2AX molecules are phosphorylated by several DNA damage response protein kinases when DSBs are formed. This phosphorylation is one of the earliest events detected after exposure to a DNA damaging agent. Specific antibodies can be used against $\gamma$-H2AX molecules leading to immunochemical detection of $\gamma$-H2AX at Ser139 through immunofluorescence [2].

Another potential contribution to carcinogenesis is the disruption of the activities of histone acetyltransferases (HATs) and histone deacetylases (HDACs), which are responsible for the acetylation and deacetylation on lysine residues and affects the regulation of gene expression. Researchers have developed histone deacetylase inhibitors $\left(\mathrm{HDAC}_{\mathrm{i}}\right)$, chemotherapeutic agents used alone or as adjuvants with other cancer therapies that function to relax DNA packing around histones in cell nuclei. One particular inhibitor called vorinostat (SAHA), a hydroxamic acid multi-HDACi is currently used to treat cutaneous T-cell lymphoma. SAHA is responsible for blocking enzymatic activity by directly binding to catalytic sites of Class I and Class II HDACs and efficient at low nanomolar concentrations. When SAHA inhibits HDAC activity, it causes the activation of gene expression responsible for causing induction of differentiation or apoptosis, and may aid in the inhibition of tumor growth [3].

We examined how SAHA impacts survival and DNA damage induction/repair kinetics in human cells exposed to low doses of gamma rays in the BNL Biosciences Department or carbon ions from the NASA Space Radiation Laboratory (NSRL). We show that NFF28 neonatal foreskin fibroblasts treated with 10 $\mu \mathrm{M}$ SAHA are significantly more radiosensitive than cells treated with dimethyl sulfoxide (DMSO; drug vehicle control) following gamma irradiation but interestingly not carbon ion irradiation. To better understand this difference, we evaluated DSB levels in the first $24 \mathrm{~h}$ following irradiation by monitoring co-localization of two DSB markers, $(\gamma-\mathrm{H} 2 \mathrm{AX}$ and 53BP1) which form nuclear foci at DSB sites using immunocytochemistry techniques.

\section{MATERIALS AND METHODS \\ A. Cell culture and HDACi treatments}

Low-passage apparently normal primary human neonatal foreskin fibroblasts NFF28 were used. Cells were cultured in aMEM medium with $15 \%$ fetal bovine serum, $100 \mathrm{U} / \mathrm{ml}$ penicillin, $100 \mu \mathrm{g} / \mathrm{ml}$ streptomycin, vitamins, amino acids, and Gluta$\mathrm{MAX}^{\mathrm{TM}}-\mathrm{I}$ (GIBCO/Invitrogen) at $37^{\circ} \mathrm{C}$ in an incubator supplied with $95 \%$ air $/ 5 \% \mathrm{CO}_{2}$ mixture. They seeded in two-well microscope chamber slides at $\sim 30 \%$ density and grown for 5 days to full confluency. On day 2 , the medium was changed and replaced with $\alpha$ MEM medium with $0.2 \%$ fetal bovine serum, antimpitioncs, and GlutaMAX ${ }^{\mathrm{TM}}-\mathrm{I}$, and $25 \mathrm{mM} \mathrm{N}-2$ hydroxyethylpiperazine-N'-2 ethanesulfonic acid (HEPES). $5 \mu \mathrm{M}$ SAHA or $0.2 \%$ DMSO were added to slides $18 \mathrm{~h}$ prior to gamma ray irradiation and $10 \mu \mathrm{M}$ HDACi or $0.2 \%$ DMSO were added to the slides $18 \mathrm{~h}$ prior to carbon ion irradiation. Both SAHA concentrations radiosensitized cells to the same degree following gamma ray irradiation $(\sim 2-$ fold; see Fig. 3).

\section{B. Carbon ion and gamma cell irradiations}

$290 \mathrm{MeV} / \mathrm{n}$ carbon ion irradiations (LET=13 keV/ $\mu \mathrm{m})$ were performed at the NSRL and ${ }^{137} \mathrm{Cs} 662 \mathrm{keV}$ gamma ray irradiations $(\mathrm{LET}=0.8 \mathrm{keV} / \mu \mathrm{m})$ were performed using a J.L. Shepherd Mark I 
Model 68A irradiator in Building 490. Samples were immediately returned to the incubator and then fixed with $4 \%$ paraformaldehyde at 10, 30, 120, 360, and $1440 \mathrm{~min}$ and rinsed with PBS.

\section{DSB-associated $\mathrm{y}-\mathrm{H} 2 \mathrm{AX}$ pS139 and 53BP1 foci measurements immunofluorescence microscopy}

The chamber slides were fixed with $4 \%$ formaldehyde at room temperature for $15 \mathrm{~min}$, then permeabilized with $0.5 \%$ Triton X-100 on ice for $10 \mathrm{~min}$. Slides were blocked with in PBS with $1 \%$ BSA, $2 \%$ fetal bovine and goat sera, $0.1 \%$ Triton $\mathrm{X}-100$, and $0.05 \%$ Tween-20 for $30 \mathrm{~min}$ at room temperature. Slides were then incubated in a $37^{\circ} \mathrm{C}$ humid chamber for $30 \mathrm{~min}$ with primary monoclonal antibodies (anti- $\gamma-\mathrm{H} 2 \mathrm{AX}$ pS139 and 53BP1) diluted 1:400 in PBS with 1\% BSA. Slides were rinsed in PBS and incubated in a $37^{\circ} \mathrm{C}$ humid chamber for 30 min with Alexa Fluor ${ }^{\circledR} 488$ (green) and 594 (red) secondary antibodies (Molecular Probes/Invitrogen) diluted 1:500 in PBS. Slides were rinsed in PBS, treated with $3.7 \%$ formaldehyde, and mounted with Pro-Long Gold antifade reagent with $0.2 \mu \mathrm{g} / \mathrm{ml} \mathrm{4}$, 6-diamidino-2-phenylindole (DAPI; Molecular Probes/Invitrogen). Images were captured at 630X magnification using a Zeiss Axio Observer Z1 microscope equipped with the appropriate fluorescence filters and Zeiss imaging software as shown in Fig. 2B-C (150-200+ cells per treatment). Cells were scored by eye and nuclei with atypical size or morphology or those with very high foci counts (presumably S-phase cells) were not scored. Data analyses and graphs were created using Excel (Microsoft Corporation) software package.

\section{RESULTS}

53BP1 foci was adopted as a marker to verify the presence of $\gamma$-H2AX-associated DSBs based on the criteria that both large $\gamma$-H2AX foci and smaller $\gamma-\mathrm{H} 2 \mathrm{AX}$ foci with co-localizing 53BP1 foci are scored, as shown in Fig. 1B-C. Serving as controls for NFF28 cells irradiated by gamma rays, non-irradiated DMSO-treated and SAHA-treated cells had averages of 1.65 foci/nuclei and 2.03 foci/nuclei, respectively. These averages were lower than averages of controls for cells irradiated by carbon ions that were 1.79 foci/nuclei (DMSO-treated) and 2.70 foci/nuclei (SAHA-treated).

Differences in kinetics of peak induction and repair over 24 $\mathrm{h}$ among the NFF28 cell strains are documented in detail in Fig. 1A. DMSO-treated gamma-irradiated NFF28 cells were only analyzed at two time points (10 $\mathrm{min}$ and $1440 \mathrm{~min}$ ) so DSB repair kinetics could not be compared to other treatments in these experiments. Despite this, curves reveal that DSB foci peak $30 \mathrm{~min}$ post-irradiation for all other treatments as shown in Fig. 1A. They also show that the DMSO-treated and SAHA-treated NFF28 cells irradiated with gamma rays had higher DSB foci induction than cells irradiated with carbon ions. SAHA-treated carbon-irradiated NFF28 cells had the lowest foci induction of all the treatments and the slowest repair kinetics from $30 \mathrm{~min}$ to $120 \mathrm{~min}$. Interestingly, foci levels $24 \mathrm{~h}$ post-irradiation were reduced below background levels only after carbon ion irradiation.

\section{DISCUSSION}

NFF28 cells were shown to have DSB foci peaks at $30 \mathrm{~min}$ versus the initial $10 \mathrm{~min}$ time point. There is speculation that this may reflect slower damage recognition or initial repair compared to other apparently normal fibroblast strains [1]. Also, according to aforementioned averages of non-irradiated, drug-treated cells, it appears that the NFF28 control cells for carbon ion irradiation may have undergone extra stress compared to the controls for gamma irradiation. This may be due to mechanical stresses of being transferred a farther distance to NSRL compared to the distance to the Building 490.
A

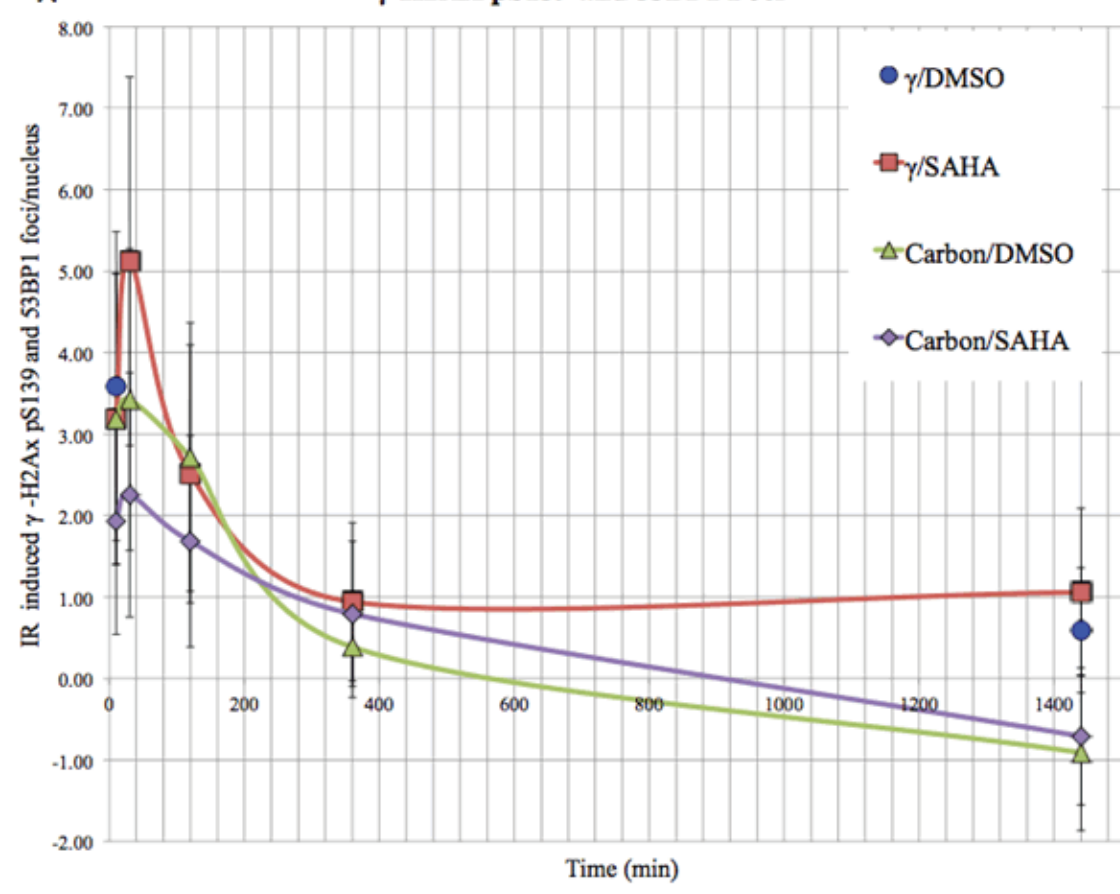

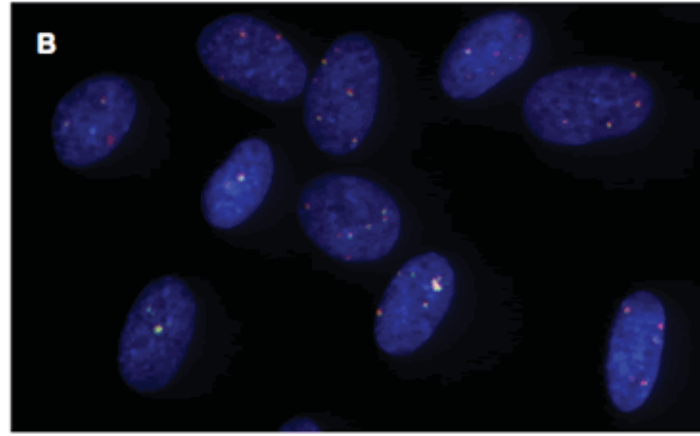

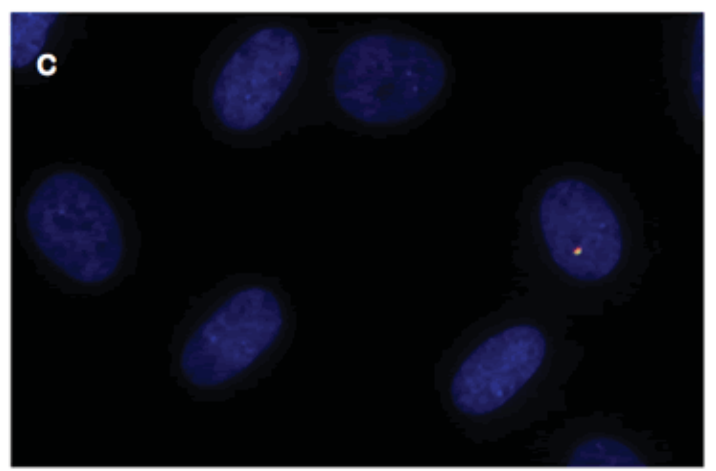

Figure 1. (A) NFF28 DSB-associated foci kinetics (mean \pm SEM) following $18 \mathrm{~h}$ treatment with SAHA or DMSO (drug vehicle control). Immunofluorescent images of co-localized $\gamma$-H2AX/53BP1 foci in SAHA-treated carbon-irradiated NFF28 nuclei at 30 min (B) and 1440 min (C) post-irradiation (green: $\gamma$-H2AX pS139; red: 53BP1; yellow: merge; blue: DAPI). 
Radiation cell survival curves of NFF28 fibroblasts treated with SAHA or DMSO after carbon ion irradiation or gamma irradiation are documented in Fig. 2. DSB induction/repair kinetics were evaluated to aid in our understanding of the patterns of these survival curves that show significantly decreased survival of SAHA-treated NFF28 cells compared to DMSO controls after gamma-irradiation as shown in Fig. 2B. Interestingly, these curves show significantly increased survival of SAHA-treated NFF28 cells (dashed line) compared to DMSO controls (solid line) after irradiation of carbon ions (Fig. 2A). The DSB induction/repair kinetics analyzed supported these survival analyses, i.e., SAHA-treated NFF28 cells after carbon irradiation had lower DSB induction levels than DMSO-treated cells. Such sparing following carbon ion irradiation was an unexpected result given the $\sim 2$-fold radiosensitization observed following gamma irradiation. These findings may limit the utility of HDACi for hadron radiotherapy.

Another interesting finding was that although SAHA-treated NFF28 cells after carbon irradiation had lower DSB induction levels, they appeared to have a delayed DSB repair compared to the other treated cells. Other researchers believe that the combination of SAHA and radiation helped prolong the expression of DNA repair proteins such as $\gamma-\mathrm{H} 2 \mathrm{AX}$ [4].

To summarize, per Fig. 2 above, radiation cell survival curves show that SAHA-treated NFF28 fibroblasts are significantly more radiosensitive than DMSO-treated cells following $\gamma$-irradiation, but the opposite occurred following carbon ion irradiation. Evaluation of $\gamma$-H2AX pS139 and 53BP1 foci kinetics show that SAHA-treated cells irradiated with carbon ions had lower DSB induction levels, showed delayed repair, and repaired DSB levels to below spontaneous untreated control cultures in contrast to cells irradiated with $\gamma$-rays. These experiments are also being conduct-

NFF28 $290 \mathrm{MeV} / \mathrm{n}$ Carbon Ion Survival

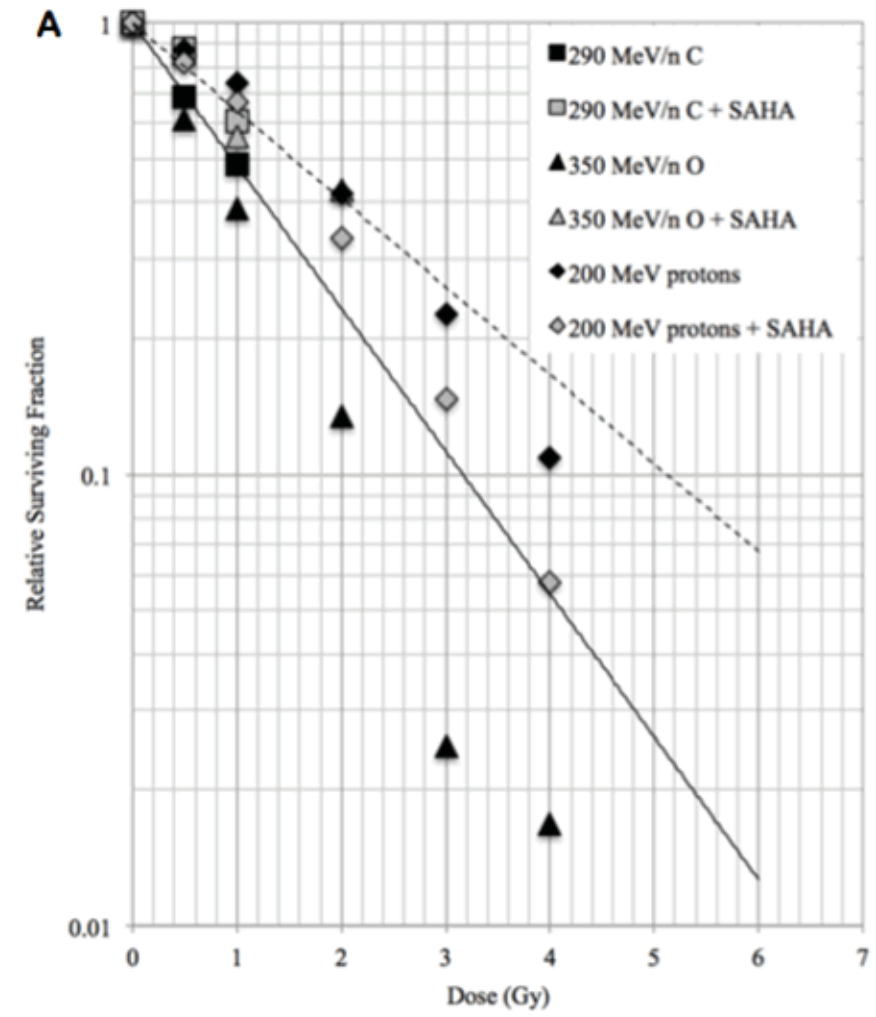

ed using U2OS osteosarcoma cells and U87 glioma cells treated with SAHA and two other related HDACi with similar chemical structures to SAHA (M344 and PTACH). These cell lines, along with NFF28, have been irradiated with protons, oxygen, helium, and carbon ions during the NSRL-13A spring campaign. Further experiments will be conducted during the NSRL-13C and NSRL$14 \mathrm{~A}$ campaigns to examine the relative effectiveness of these different ions for hadron radiotherapy and the utility of adjuvant HDACi treatments.

\section{ACKNOWLEDGEMENTS}

This project was supported by BNL LDRD 12-012 (PFW) and in part by the U.S. Department of Energy, Office of Science, Office of Workforce Development for Teachers and Scientists (WDTS) under the Science Undergraduate Laboratory Internships Program (SULI) (KZS). Thank you to the NSRL scientists for their assistance with beam delivery. I would like to also thank my mentor Dr. P. F. Wilson for all of his guidance, instruction, and encouragement during my SULI placement; A. M. Johnson for her encouragement and providing images for this project; $\mathrm{P}$. Bennett, D. Keszenman, and J. Jardine for their assistance; and A. Emerick, B. Daniels, L. Kolodiuk, L. Selva, S. Tafrov, and J. Trunk for their support.

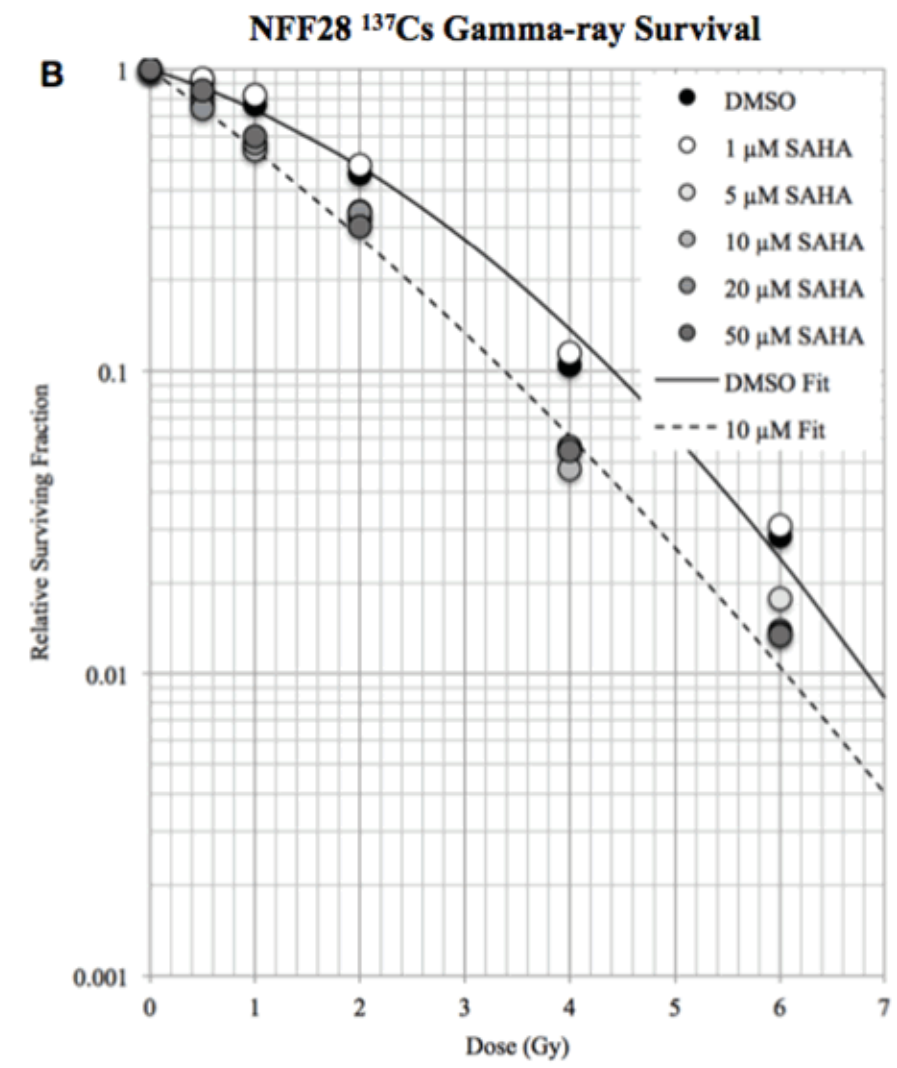

Figure 2. (A) Survival of NFF28 fibroblasts treated with SAHA (dashed line) or DMSO (solid line) after $290 \mathrm{MeV} / \mathrm{n}$ carbon ions. (B) Survival of NFF28 fibroblasts treated with SAHA or DMSO after ${ }^{137} \mathrm{Cs}$ gamma irradiation. 


\section{REFERENCES}

[1] P.F. Wilson, P.B. Nham, S.S. Urbin, J.M. Hinz, I.M. Jones, and L.H. Thompson. "Inter-individual variation in DNA double-strand break repair in human fibroblasts before and after exposure to low doses of ionizing radiation." Mutation Research 683, 91-97 (2010).

[2] A. Ivashkevich, C.E. Redon, A.J. Nakamura, R.F. Martin, and O. A. Martin. "Use of the $\gamma-\mathrm{H} 2 \mathrm{AX}$ assay to monitor DNA damage and repair in translational cancer research." Cancer Letters 327, 123-133 (2012).

[3] V.M. Richon, J. Garcia-Vargas, and J.S. Hardwick. "Development of vorinostat: Current applications and future perspectives for cancer therapy." Cancer Letters 280, 201-210 (2009).

[4] A. Munshi, T. Tanaka, M.L. Hobbs, S.L. Tucker, V.M. Richon, R.E. Mayb. "Vorinostat, a histone deacetylase inhibitor, enhances the response of human tumor cells to ionizing radiation through prolongation of $\gamma-\mathrm{H} 2 \mathrm{AX}$ foci." Molecular Cancer Therapeutics 5 (8), 1967-1974 (2006). 


\title{
Cobalt Molybdenum Nitride Nanosheet as an efficient catalyst for producing hydrogen from water
}

\author{
Andrea Sarmiento \\ Chemical \& Molecular Engineering, Stony Brook University, Stony Brook, NY 11794 \\ Wei-Fu Chen \\ Chemistry, Brookhaven National Laboratory, Upton NY 11973
}

\begin{abstract}
Energy and climate crises have motivated hydrogen fuel to be a sustainable solution to these challenges. The electrochemical process accounts as one of the most promising methods for generating hydrogen by using solar power to split water into hydrogen and oxygen. Currently the most effective catalyst for the hydrogen evolution reaction (HER) is platinum, but it is too expensive. However, the possible introduction of non-precious materials in an HER could become an effective strategy in renewable energy conversion systems that employ solar power. My project involves determining transition metal-based materials and optimizing their composition to improve the HER performance. Different ratios of cobalt and molybdenum were annealed at high temperatures with ammonia gas. Molybdenum nitride as an electrocatalyst was efficient in acidic solutions. By adding cobalt, we predicted an increased HER performance in neutral or basic solutions. Electrochemical measurements have been performed to analyze the HER activity in different $\mathrm{pH}$ solutions. We have found that cobalt molybdenum nitride is more active in alkaline solution than in acidic media. The catalyst that had a 2:1 and 1:1 ratio (Co:Mo) worked best in alkaline solutions. While in acidic solutions, the catalyst with ratio 1:2 (Co:Mo) worked best and as well as increasing annealing temperatures resulted in decreased over potentials. By working in the Chemistry Department at Brookhaven National Laboratory, I have learned about hydrogen technology, as well as how to operate a X-Ray Diffractometer, a Scanning Electron Microscope, and a Thermogravimetric Differential Thermal Analyzer. I have also gained confidence in executing and designing a research experiment independently.
\end{abstract}

\section{Introduction}

Currently the energy source comes mainly from fossil fuels, natural gas and coal. These forms of energies cause environmental issues and their reserves are finite. This crisis has motivated hydrogen fuel to become a sustainable solution to these challenges. Hydrogen is the most abundant element on Earth; it can be produced by various feedstocks such as water or biomass. 1 Hydrogen is non-toxic, not a pollutant and highly combustable.1 There are many ways to produce hydrogen, but the most promising method is the electrochemical way. Water electrolysis and photochemical hydrogen production are two specific way hydrogen can be produced. Water electrolysis uses electricity, and photolytic processes use solar power to split water into hydrogen and oxygen 1 . These methods virtually offer no pollution or toxic byproducts, and they operate at ambient temperatures. 1 However, the critical challenges that surface like a cost effective device and the low system efficiency. ${ }^{1}$

Currently the most active catalyst for the hydrogen evolution reaction (HER) is platinum. Effective HER catalysts have spe- cific properties, like high catalytic rates, high density of catalytic sites, good electrical connections and high stability in various $\mathrm{pH}$ conditions. Durable, cheaper and efficient electrocatalytic materials are being investigated and developed. Part of this research entails the integration of non-precious materials to catalyze the HER. These inexpensive materials should also be stable in acidic, neutral and alkaline media. To accomplish this cobalt and molybdenum are alloyed to help improve the bond strength between metals and hydrogen. Figure 1 shows a volcano curve of exchange current densities verse $\mathrm{H} 2$ bond strength. ${ }^{3}$ For the best HER activity, the binding strength between the catalyst and the hydrogen atom should be optimized; it should not be too strong or weak. If the interaction is too weak, the adsorption of proton on the catalyst will be no reaction; and if the interaction is too strong the catalyst gets blocked by the hydrogen. On the plot, molybdenum has a strong hydrogen bond strength; cobalt is located on the opposite side of the plot from molybdenum where it has a weaker bond strength. It has been reported that when alloying two metals with different $\mathrm{H}$-bond strength, could reach perfect bond strength like platinum or iridium.

\section{Experimental \\ A. Materials synthesis}

All of the chemical reagents used included Cobalt(II) nitrate, Ammonium molybdate tetrahydrate and carbon black. The starting martials of $\mathrm{Co}\left(\mathrm{NO}_{3}\right)_{2}$ and $\left(\mathrm{NH}_{4}\right)_{6} \mathrm{Mo}_{7} \mathrm{O}_{24} \cdot 4 \mathrm{H}_{2} \mathrm{O}$ with desired Co : Mo ratios (molar ratios: $1: 1,1: 2,2: 1$ ) was mixed with $400.00 \mathrm{mg}$ of carbon black (Vulcan XC-72R), all samples had a loading of $30 \%$. The samples were sonicated and allowed to dry overnight in an oven. The experiments were carried out in a sealed tubular reactor were the samples were loaded. These samples were heated with ammonia gas at three different temperatures $\left(700^{\circ} \mathrm{C}\right.$,

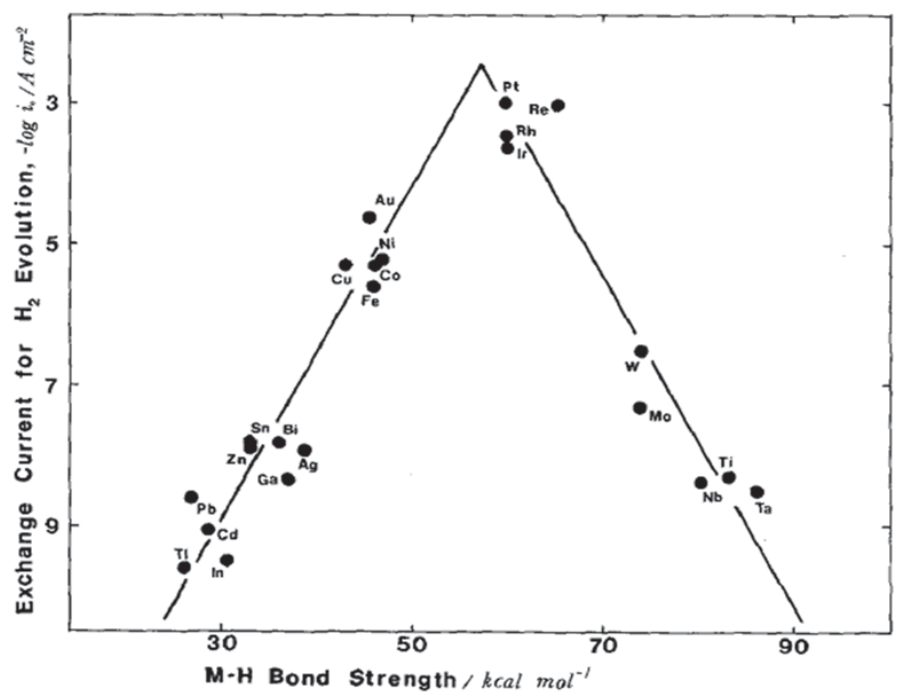

Figure 1. Exchange currents for electrolytic hydrogen evolution ${ }^{3}$ 
$800^{\circ} \mathrm{C}, 900^{\circ} \mathrm{C}$ ). A control was made which had a $1: 1$ molar ratio and it was annealed at $700^{\circ} \mathrm{C}$ under $8.5 \% \mathrm{H} 2 /$ Argon After 2 hours, the reactor was cooled naturally and the solid product was collected for further analysis.

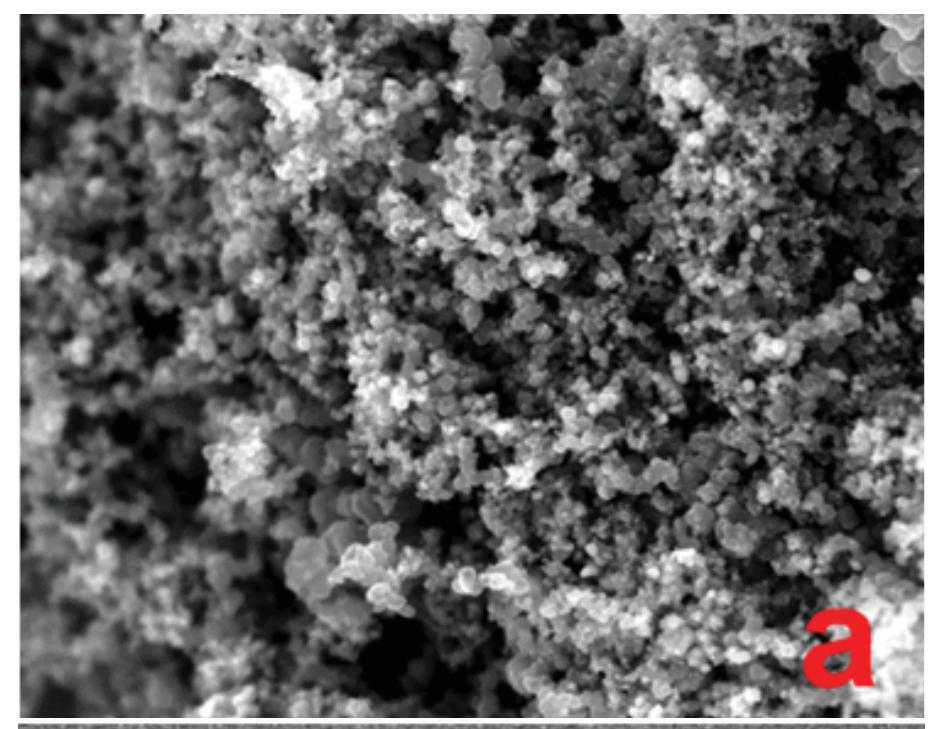

\section{Standing sheet stack}
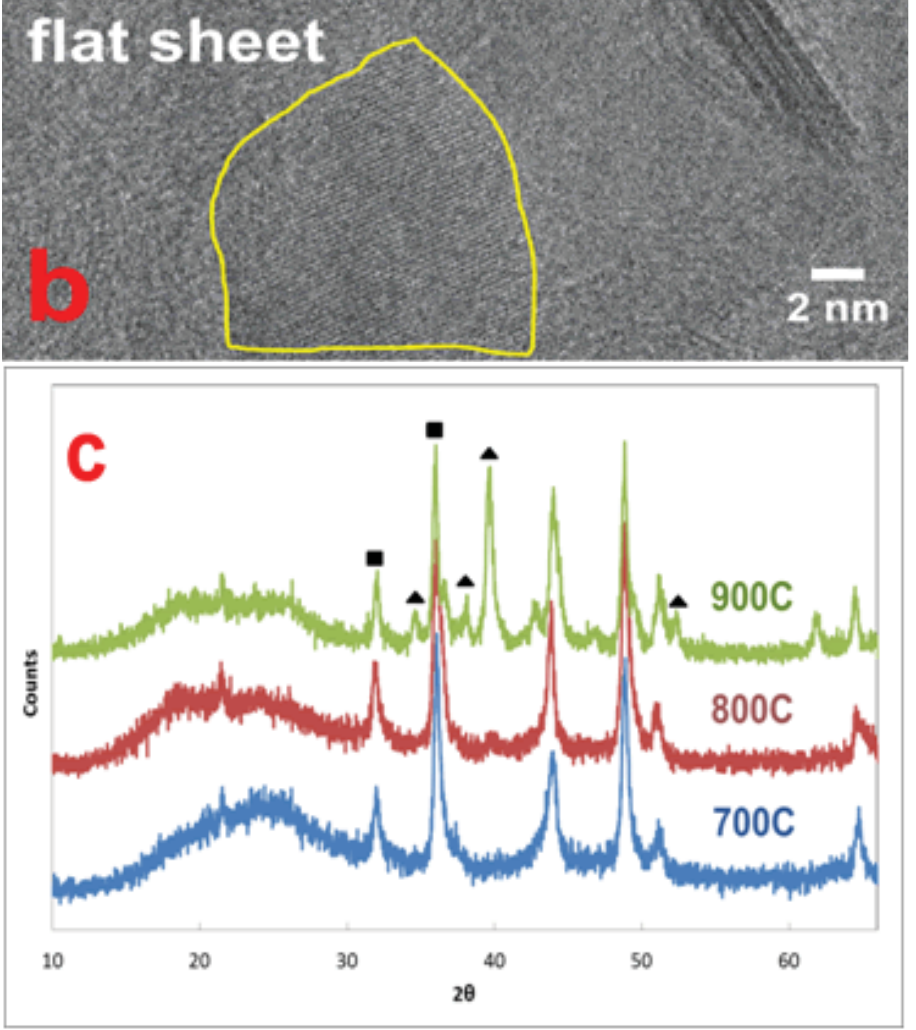

Figure 2a-c. Scanning electron micrographs (a) and transmission electron micrographs (b) and x-ray diffraction of Co-Mo nitride nanosheets.

\section{B. Material characterization}

Chemical compositions of the samples were performed using a Field Emission Scanning Electron Microscope (JEOL JSM7600F). Samples were prepared for TEM by making a solution of catalyst powders in ethanol, using a sonication bath. The solution was dropped onto a clean carbon-coated copper grid for TEM.

\section{Electrocatalytic activity tests}

The electrocatalytic activity of cobalt molybdenum nitride for hydrogen production was tested by cyclic voltammetry. The measurements were conducted in an electrochemical cell in $0.5 \mathrm{M}$ HClO4, 0.5 M Phosphate Buffer pH 7 and 1.0 M NaOH. About
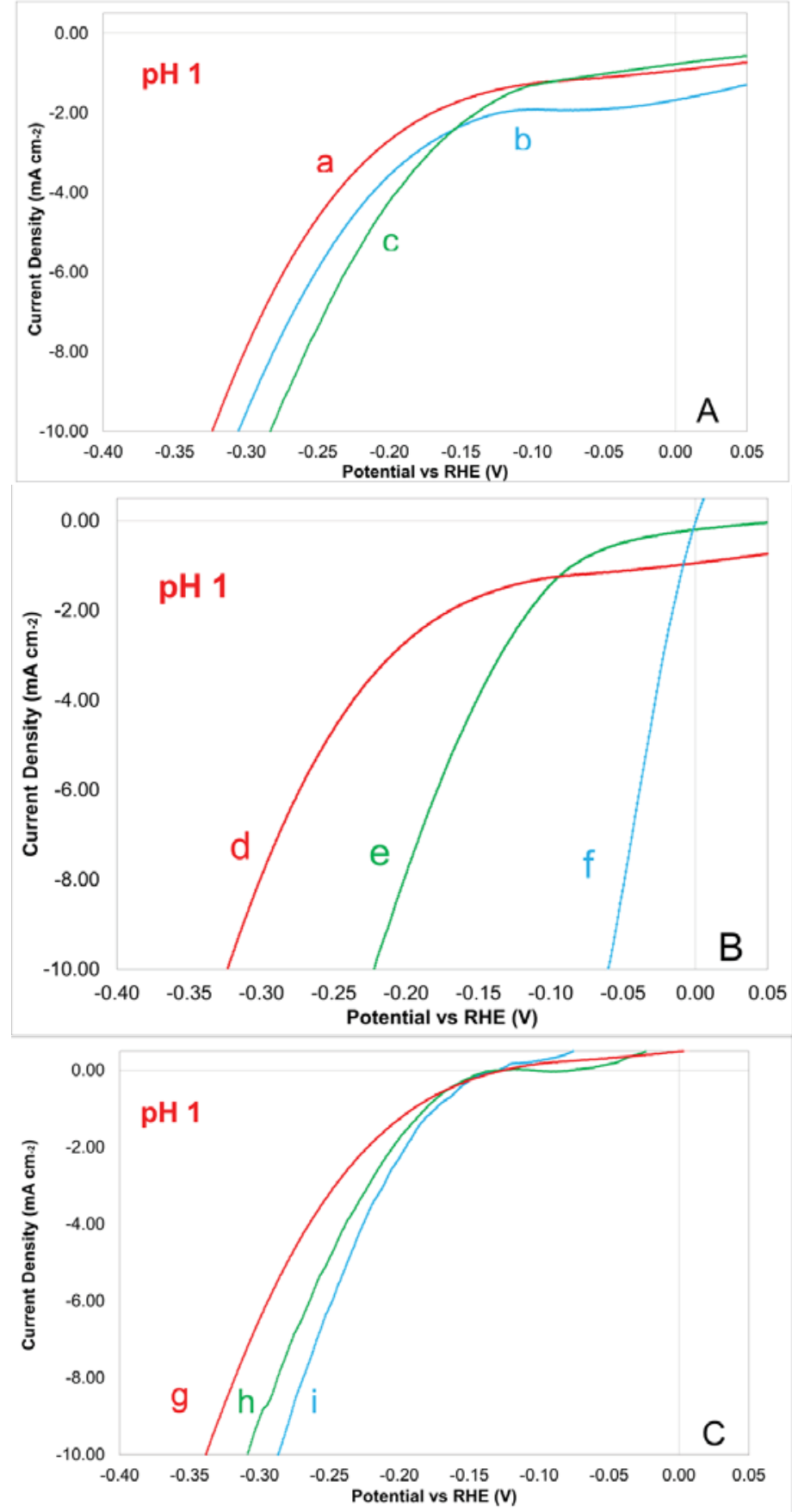

Figures 3A-C. Comparison of HER catalytic activities for various electrodes in $0.5 \mathrm{M} \mathrm{HClO4}$. The counter electrode was platinum foil, and the reference was $\mathrm{Ag} / \mathrm{AgCl}$. The data is labeled as followed: (a),(d),(g) $1: 1$ at $700^{\circ} \mathrm{C}$; (b) $1: 1$ at $800^{\circ} \mathrm{C}$; (c) $1: 1$ at $900^{\circ} \mathrm{C}$; (e) $1: 1$ control; (f) Pt/C; (h)(Co:Mo) 2:1; (i) (Co:Mo) 1:2. 
10 to $12 \mathrm{mg}$ of the solid sample collected after annealing was weighed out and mixed with 4 drops of $5 \%$ Nafrion. The electrode was made out of carbon paper and it was cut to dimensions of $1 \mathrm{~cm}$ by $3 \mathrm{~cm}$. A carbon electrode with dimension of $3 \mathrm{~cm}$ by $1 \mathrm{~cm}$ was used for the working electrode, consisting of a thin layer of the electrocatalyst. A platinum foil and an $\mathrm{Ag} / \mathrm{AgCl}$ electrode were used as the counter electrode and the reference electrode.

\section{Results and Discussion}

Figure 2 shows scanning electron microscope (SEM), transmission electron microscope (TEM) images and shows X-ray diffraction (XRD) of all three samples annealed at three different temperatures.
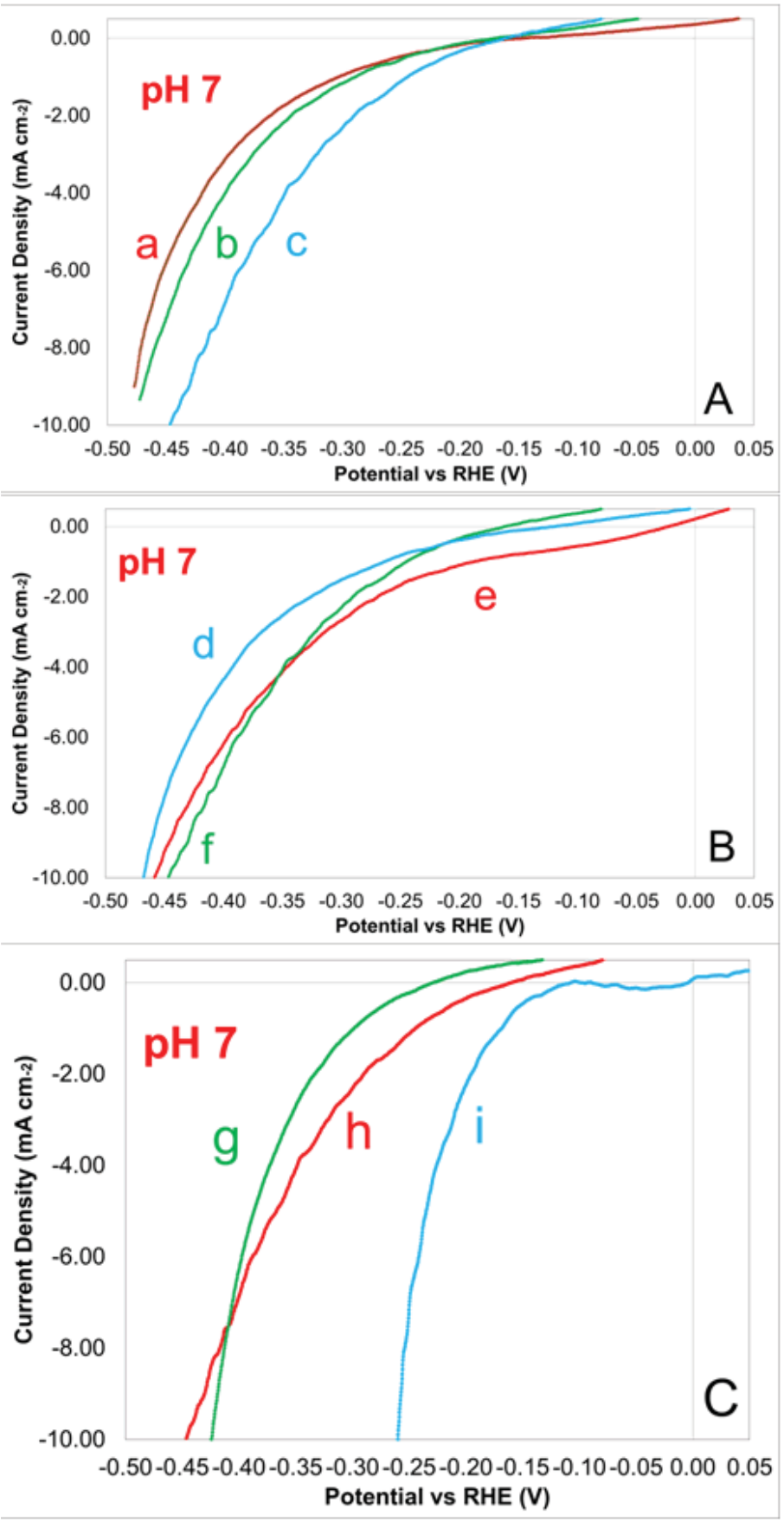

Figures 4A-C. Comparison of HER catalytic activities for various electrodes in 0.5 M Phosphate Buffer pH 7. The counter electrode was platinum foil, and the reference was $\mathrm{Ag} / \mathrm{AgCl}$. The data is labeled as followed: (a) $1: 1$ at $800^{\circ} \mathrm{C}$; (b) $1: 1$ at $900^{\circ} \mathrm{C}$; (c),(f),(h) $1: 1$ at $700^{\circ} \mathrm{C}$; (d) (Co:Mo) 1:2; (e) (Co:Mo) 2:1; (g) 1:1 control; (i) Pt/C.
The Co-Mo-N catalyst exhibited a porous morphology (Figure $2 \mathrm{a}$ ). The catalyst also exhibited formation of nanosheet stacks. Figure $2 \mathrm{~b}$ shows the distinction between standing and flat sheet stack, the average particle size ranged from 10 to $15 \mathrm{~nm}$. The XRD indicated two phases present in the 1:1 molar ratio annealed at $900^{\circ} \mathrm{C}$, peaks resembled $\mathrm{CoMo}_{4} \mathrm{~N}_{5}$ and $\mathrm{Mo}_{2} \mathrm{C}$ (Figure 2c). Samples annealed at $700^{\circ} \mathrm{C}$ and $800^{\circ} \mathrm{C}$ had peaks that resembled Co$\mathrm{Mo}_{4} \mathrm{~N}_{5}$.

The Co-Mo-N nanosheets were evaluated electrochemically for their activities toward the HER. Samples were made into a slurry, this was then deposited onto a clean carbon electrode and tested for electrocatalytic hydrogen evolution activity under acidic, neutral and alkaline conditions. The highest HER activity was observed during the last reduction step has been performed due to the tendency of the nanoparticles to form surface oxides in air.
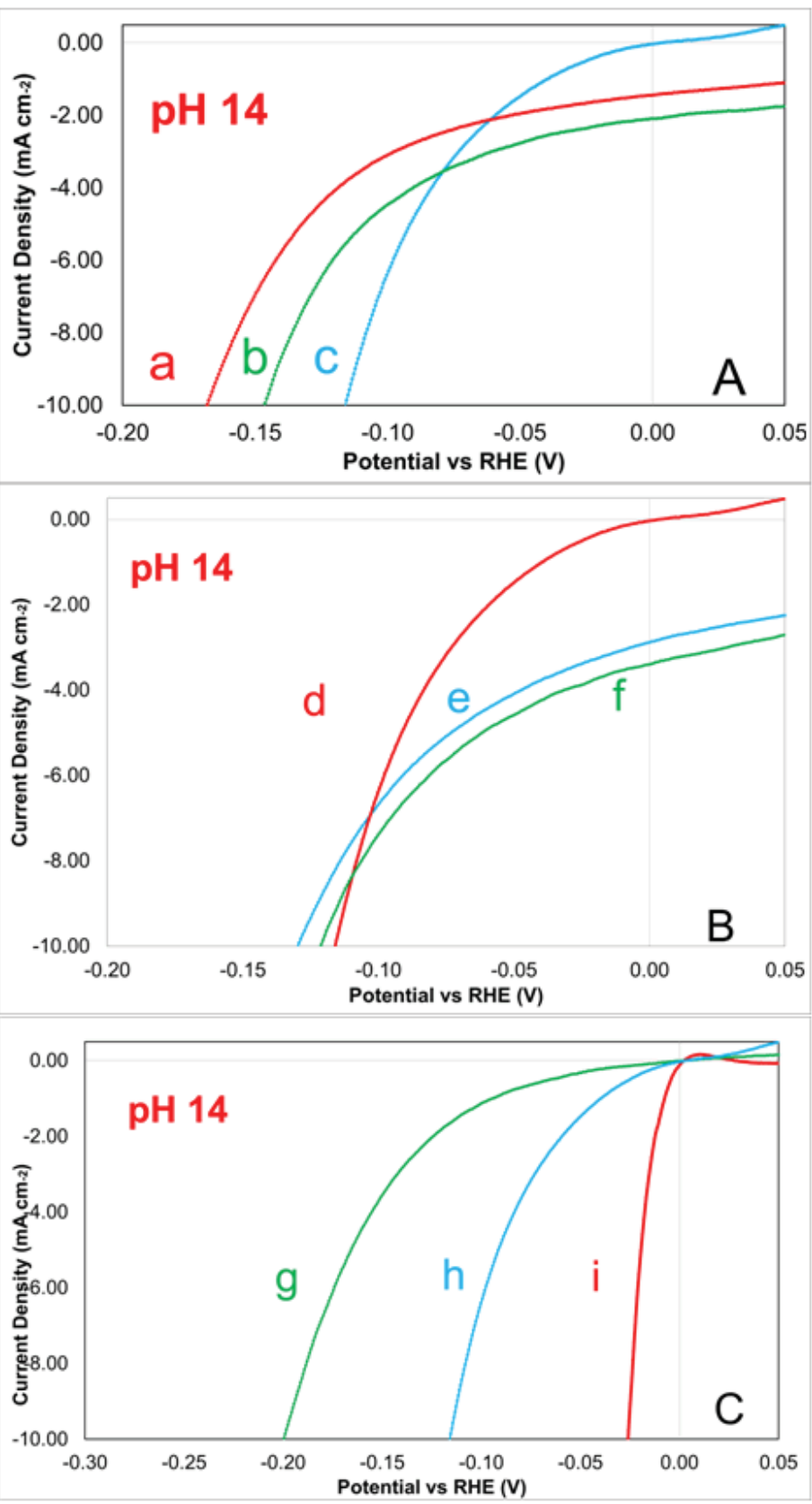

Figures 5A-C. Comparison of HER catalytic activities for various electrodes in 1.0 $\mathrm{M} \mathrm{NaOH}$. The counter electrode was platinum foil, and the reference was $\mathrm{Ag} / \mathrm{AgCl}$. The data is labeled as followed: (a) $1: 1$ at $800^{\circ} \mathrm{C}$; (b) $1: 1$ at $900^{\circ} \mathrm{C}$; (c),(d),(h) $1: 1$ at $700^{\circ} \mathrm{C}$; (e) (Co:Mo) 1:2; (f) (Co:Mo) 2:1; (g) 1:1 control; (i) Pt/C. 
Figure 3A shows HER activity under acidic media comparing samples annealed at three different temperatures. The data showed a monotonic trend, in which increasing the annealing temperature resulted in decreased overpotentials. The sample annealed at $900 \mathrm{oC}$ had the best activity with a potential of $280 \mathrm{mV}$ at $10 \mathrm{mAcm}-2(\eta)$. The sample of $1: 1$ molar ratio annealed at $700^{\circ} \mathrm{C}$ had a same potential in acidic media as the NiMoNx catalysts in $0.1 \mathrm{M} \mathrm{HClO}_{4}, 280 \mathrm{mV} .2$ This implies that adding cobalt does not decrease HER performance in acidic media, it further implies that its efficiency is up to par with NiMoNx, another catalysts that is also commonly used. ${ }^{2}$ HER activities for samples with different molar ratios were compared in figure $3 \mathrm{~B}$. The data showed that having a greater amount of molybdenum present in the sample resulted in lower overpotentials. The 1:2 (Co:Mo) molar ratio had the best HER activity with a potential of $290 \mathrm{mV}$ at $\eta$. In figure $3 \mathrm{C}$, the effects of nitridaion were observed. The control, annealed under $8.5 \% \mathrm{H} 2$ /Argon, has the highest activity with a potential of $220 \mathrm{mV}$ at $\eta$. These results support the notion that alloying Co into Mo and adding nitride does not increase the fundamental hydrogen evolution activity of Co metal.

Figure 4A shows HER activity under neutral media comparing samples annealed at three different temperatures. The data showed no particular trend; samples annealed at $800^{\circ} \mathrm{C}$ and $900^{\circ} \mathrm{C}$ had very similar HER activity and the sample annealed at $700^{\circ} \mathrm{C}$ had the highest HER performance with a potential of $450 \mathrm{mV}$ at $\eta$. HER activities for samples composed of different molar ratios are compared in figure 4B. The data shows that introducing more cobalt into the catalyst has a very minor effect on its activity in neutral media. In figure $4 \mathrm{C}$, the effects of nitridation were observed. Co-Mo nitride has a potential of $450 \mathrm{mV}$ which is more positive than $420 \mathrm{mV}$ of the Co-Mo control sample. The data shows that adding nitride greatly improves HER performance.

Figure 5A shows HER activity under basic media comparing samples annealed at three different temperatures. The data shows no particular trend between annealing temperatures and HER performance. The XRD for the sample annealing at $900^{\circ} \mathrm{C}$ showed to have $\mathrm{Mo}_{2} \mathrm{C}$, which has high activity in alkaline media; this explains why the sample annealed at $800^{\circ} \mathrm{C}, 170 \mathrm{mV}$ at $\eta$, had a more positive potential than the sample annealed at $900^{\circ} \mathrm{C}, 140$ $\mathrm{mV}$ at $\eta$. The best sample was annealed at $700^{\circ} \mathrm{C}$ with a potential of $110 \mathrm{mV}$ at $\eta$. HER activities for samples composed of different molar ratios are compared in figure 5B. The data showed that increasing the amount of cobalt in the catalyst had minor effects on its HER activity in alkaline media. The sample with the highest HER performance was the 1:1 molar ratio with a potential of 110 $\mathrm{mV}$ at $\eta$. In figure $5 \mathrm{C}$, the effects of nitridation were observed. The addition of nitride to the catalyst improves it's HER activity in alkaline media. The best sample with a potential of $110 \mathrm{mV}$ at $\eta$ was the 1:1 molar ratio.

\section{Conclusion}

In conclusion, Co-Mo nitride nanosheets with lengths of 10 to $15 \mathrm{~nm}$ can be readily prepared. Annealing temperatures and molar ratios were varied throughout samples to observe their effects on HER performance in acidic, neutral and alkaline media. All samples were annealed under nitride except for the control. Nitridation resulted in high HER activity in both acidic and neutral conditions. However, in alkaline media nitridation, low annealing temperature and a 1:1 molar ratio resulted in high HER activity.

\section{Acknowledgments}

This work was supported by the U.S. Depatment of Energy - Office of Science Education, BNL Technology Maturation Funding TM12-008 and the Aftificial Photosynthesis Group. It was also supported by the Ofice of Workshops Development for Teachers and Scientists (WDTS) under the Science Undergradutate Laboratory Internships Program (SULI). I would like to thank my mentor Wei-Fu Chen for his guidance in this project, as well as Jonathan Schneider for helpful discussion.

\section{References}

${ }^{1}$ Hydrogen Production. "Overview of Technology Options.". Freedom Car \& Fuel Partnership.

${ }^{2}$ Chen et al, "Highly Active, Durable and Nanostructured Molybdenum Carbide Electrocatalysts for Hydrogen Production" 2012, Submitted.

${ }^{3}$ S. Trasatti, J. Electroanal. Chem. 1972, 39, 163. 


\title{
Synthesis and characterization of Tungsten Carbide/ Nitride on Graphene as a Hydrogen evolution electrocatalyst
}

\author{
Jonathan Schneider \\ Chemical Engineering, Stony Brook University, Stony Brook, NY 11790 \\ Wei-Fu Chen \\ Chemistry, Brookhaven National Laboratory, Upton, NY 11973
}

\begin{abstract}
The production of hydrogen from polymer electrolyte membrane electrolyzers requires expensive platinum catalysts. To make this process more competitive, non-precious-metal catalysts are necessary. In this study, a series of graphene supported tungsten carbide/nitride nanocomposites were synthesized by in situ solid-state carburization of ammonium tungstate and aniline on graphene. Catalysts were prepared with different $[W]:[$ Aniline] ratios to study the effect of varying amounts of aniline. Accelerated deterioration tests showed that samples with aniline were more active and durable than those without aniline. After acceleration deterioration testing, the overpotential for a current density of $-10 \mathrm{~mA} \mathrm{~cm}^{-2}\left(\eta_{10}\right)$ was 330 $\mathrm{mV}$ for the sample without aniline. The best sample with aniline had a $\eta_{10}$ of $119 \mathrm{mV}$ after accelerated deterioration tests. The crystal structures analyzed by $\mathrm{X}$-ray diffraction showed the presence of $\mathrm{W}_{2} \mathrm{C}$ in the sample without aniline and both $W_{2} C$ and $W N$ in samples with aniline. This study shows that nitriding improves corrosion resistance for hydrogen evolution reaction (HER) catalysts and the synergy between carbide and nitride phases creates an active and durable catalyst. Tungsten carbide/nitride on graphene requires less energy than other non-noble metal catalysts for the HER.
\end{abstract}

\section{Introduction}

Environmental concern about $\mathrm{CO}_{2}$ emissions and fossil fuel depletion call for a clean and alternative energy source such as hydrogen [1]. However, current methods of producing hydrogen industrially, which include steam reforming and the water-gas shift reaction hurt the environment by emitting greenhouse gases [1]. Furthermore, those processes require methane, a hydrogen source that is not cheap and abundant. The alternative is polymer exchange membrane (PEM) electrolysis, which instead of methane uses water, the most abundant and cheapest hydrogen source. Electrolysis does not emit greenhouse gases. Furthermore, it has the potential to use the sun's energy to oxidize water, which would make for a successful renewable energy storage approach. The PEM electrolyzer produces $\mathrm{H}_{2}$ by splitting water into oxygen and hydrogen [1]. The focus of this project is on the cathodic half-reaction known as the hydrogen evolution reaction (HER): $2 \mathrm{H}^{+}+2 \mathrm{e}-\rightarrow \mathrm{H} 2$. Currently, platinum is used to catalyze this reaction under acidic conditions, however platinum is expensive and scarce. According to a 2012 DOE report, a major cost of PEM electrolyzers is platinum. Currently, the production cost of hydrogen from electrolyzers is $\$ 4-5 / \mathrm{kg}$ [2], but the DOE has set a goal for hydrogen production to cost between $\$ 2-3 / \mathrm{kg}$ (including production, delivery, and dispensing) in order to be cost-competitive with fossil fuels. Therefore, the main challenge to make PEM electrolysis more commercialized is to replace platinum with non-precious metal catalysts [3]. However, it remains difficult to find abundant and cheap catalysts, which are both as active and durable as platinum. To overcome this, non-precious metals are manipulated by optimizing metal-hydrogen bond strength [4-7], nanostructuring to increase the number of accessible reactive sites [8-10], and nitriding [9] to improve corrosion resistance in acid. Tungsten, a promising metal for HER, is one of the most abundant transition metals and is about 1,000 times cheaper than platinum [11]. Tungsten carbide has been studied as a hydrogen evolution catalyst as far back as the 70s by Armstrong and Bell, Nikolova et. al, and Zoltowski [12-14], and has more recently been studied by Kimmel et. al [15] Tungsten carbide is known for its stability in acidic media and is considered an active HER catalyst [3]. This research improves upon previous tungsten carbide catalysts by nanostructuring with graphene and nitriding.

\section{Method and Materials \\ A. Material Synthesis}

Graphene supported tungsten carbides/nitrides were prepared by carburization of XG Science graphene with ammonium tungstate $\left(\left(\mathrm{NH}_{4}\right)_{10} \mathrm{H}_{2}\left(\mathrm{~W}_{2} \mathrm{O}_{7}\right)_{6} \cdot 4 \mathrm{H}_{2} \mathrm{O}\right.$, Aldrich $)$ and aniline. The following is the procedure for preparing $30 \%$ loading (by mass) of tungsten carbide on graphene: $30 \mathrm{mg}$ AMT powder and $50 \mathrm{mg}$ graphene were mixed in water with varying amounts of aniline. The amount of aniline was varied to create ratios [W]:[Aniline] of $1: 0,1: 0.8,1: 2,1: 5$, and 1:10. After mixing, the slurry was dried at $80^{\circ} \mathrm{C}$. The mixture was then annealed in a Thermo Scientific quartz tube furnace at $900^{\circ} \mathrm{C}$ for $2 \mathrm{~h}$ under argon, and a black powder was obtained.

\section{B. Electrochemical Measurements}

Electrodes were made as follows: catalyst ink was prepared by mixing catalyst powder with a $5 \%$ nafion dispersion. Catalyst ink was brushed onto a $1 \mathrm{~cm}^{2}$ area of a $3 \mathrm{~cm} \mathrm{x} 1 \mathrm{~cm}$ carbon fiber electrode (Toray carbon paper). The electrolyte used for all electrochemical measurements was $0.1 \mathrm{M} \mathrm{HClO}_{4}$, prepared from Milli-Q water and Optima perchloric acid. $\mathrm{An} \mathrm{Ag} / \mathrm{AgCl}(3 \mathrm{M})$ electrode was used as the reference and a platinum foil was used as the counter electrode. The two-chamber electrochemical cell was purged with Argon gas before starting all electrochemical measurements. To test for changes in activity after accelerated deterioration testing of different samples, up to 10,000 cycles performed at a scan rate of $200 \mathrm{mV} \mathrm{s}^{-1}$ from 0 to $-800 \mathrm{mV}$ were preceded and followed by $2 \mathrm{mV} \mathrm{s}^{-1}$ scans. All voltammetry experiments were carried out using a BASi Epsilon electrochemical workstation at room temperature. All reported voltages are versus RHE and internal resistance corrected using the equation $\eta_{\text {corr }}=$ 
$\eta-j R_{s}$, where $R_{s}$ is the internal resistance of the electrochemical cell and $\eta$ is potential. To examine long-term stability in acidic media, chronopotentiometry was performed on the 1:0.8 sample with an applied current of $-10 \mathrm{~mA}$, sample interval of 60 seconds, and potential range of $\pm 1 \mathrm{~V}$.

\section{Results and discussion}

A. Morphology

JEOL SEM 7600F scanning electron microscopy was used to analyze surface morphology. Uniform particle size and graphene nanoplatelets are pictured in the figure 1 below.

\section{B. Characterization of Crystal Structures}

The X-Ray diffraction data in Figure 2 shows evidence that the amounts of tungsten carbide and tungsten nitride vary as different amounts of aniline are added. As more aniline is added, the amount of tungsten carbide decreases. For instance, the $\mathrm{X}$-ray diffraction showed eight $\mathrm{W} 2 \mathrm{C}$ peaks in the 1:0 sample at $2 \theta=34.14^{\circ}, 37.83^{\circ}, 39.34^{\circ}, 51.73^{\circ}, 61.22^{\circ}, 69.17^{\circ}, 72.56^{\circ}$, and $74.33^{\circ}$. Samples with more aniline, such as 1:2, 1:5, and 1:10 have less carbide peaks but show four WN peaks at $2 \theta=36.90^{\circ}$, $42.64^{\circ}, 61.80^{\circ}$, and $74.58^{\circ}$. Therefore, as the amount of aniline increases, tungsten nitride becomes the more predominant species. It is interesting to note that tungsten nitride is formed although the boiling point of aniline is around $184^{\circ} \mathrm{C}$. This may suggest a pi-pi interaction between the graphene and aniline preventing the aniline from evaporating. The crystal structure of the tungsten nitride is cubic and has crystal faces $111\left(2 \theta=36.90^{\circ}\right), 200$ $\left(2 \theta=42.64^{\circ}\right), 208\left(2 \theta=61.80^{\circ}\right)$, and $311\left(2 \theta=74.58^{\circ}\right)$ (JCPDSPDF 65-2898), while the crystal structure of the tungsten carbide is hexagonal and has crystal faces $100\left(2 \theta=34.14^{\circ}\right), 002$ $\left(2 \theta=37.77^{\circ}\right), 101\left(2 \theta=39.33^{\circ}\right), 102\left(2 \theta=51.73^{\circ}\right), 110\left(2 \theta=61.42^{\circ}\right)$, $103\left(2 \theta=69.25^{\circ}\right), 112\left(2 \theta=72.73^{\circ}\right)$, and $201\left(2 \theta=74.33^{\circ}\right)($ JCPDSPDF 35-0776). The grain sizes, calculated from the XRD data using the Debye-Scherer equation [16], further suggest a difference
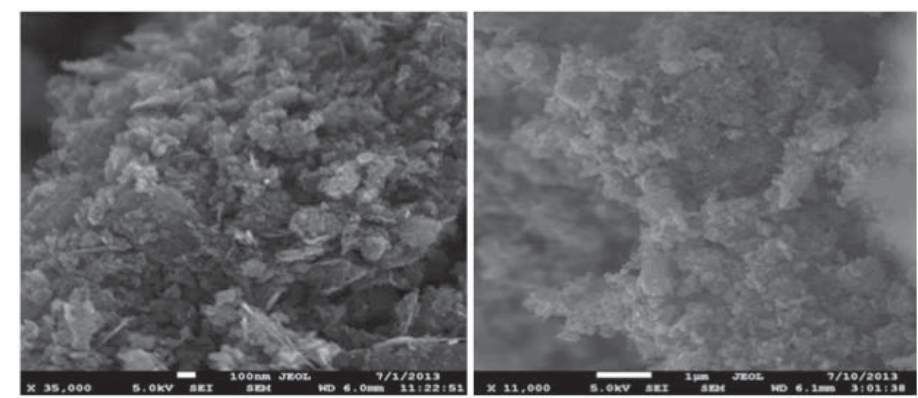

Figure 1- Scanning electron micrographs of 1:0 (L) and 1:0.8 (R).

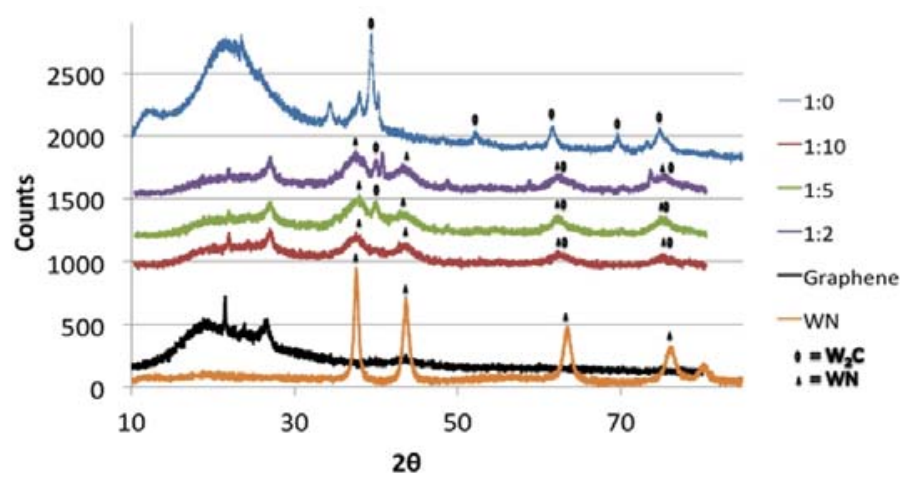

Figure 2 - X-Ray diffraction data for WN, Graphene, 1:10, 1:5, 1:2, and 1:0 in the amounts of carbide versus nitride as the amount of aniline varies. For example, the grain size of the $\mathrm{W} 2 \mathrm{C}$ phase in the 1:0 sample was $15 \mathrm{~nm}$. Grain sizes of the W2C phase in 1:2 and 1:5 samples were $11 \mathrm{~nm}$ and $8.36 \mathrm{~nm}$, respectively. While the grain size of the carbide decreases with increasing amounts of aniline, the grain size of the nitride increases. For instance, the grain sizes of tungsten nitride for 1:2, 1:5, and 1:10 samples were $2.9 \mathrm{~nm}, 3.4$ $\mathrm{nm}$, and $3.4 \mathrm{~nm}$ respectively. From this data, it is clear that as the amount of aniline increases, the carbide grain size decreases because there is more tungsten nitride than tungsten carbide. Thus, it can be assumed that the tungsten reacts preferentially with aniline to make tungsten nitride over the tungsten carbide. The grain sizes are based on the 111 peak of the WN phase and the 101 peak of the $\mathrm{W}_{2} \mathrm{C}$ phase.

\section{HER Activity}

Nanostructuring transition metals on carbon supports has been known to increase HER activity [9]. In figure 3A, we see that tungsten carbides nanostructured on graphene and carbon black are much more active than bulk tungsten carbide. This fact illustrates experimentally that nanostructuring improves HER activity,

Table 1. $\eta_{10}$ values ( $V$ vs RHE, Internal resistance corrected):

\begin{tabular}{|l|l|l|l|}
\hline $\begin{array}{l}\text { Catalyst } \\
\text { [W]:[Ani- } \\
\text { line }]\end{array}$ & $\begin{array}{l}\text { Before cy- } \\
\text { cling }\end{array}$ & $\begin{array}{l}\text { After 5,000 } \\
\text { cycles }\end{array}$ & $\begin{array}{l}\text { After 10,000 } \\
\text { cycles }\end{array}$ \\
\hline $1: 0$ & 0.189 & 0.330 & -- \\
\hline $1: 0.8$ & 0.169 & 0.180 & 0.230 \\
\hline $1: 2$ & 0.121 & 0.117 & 0.119 \\
\hline $1: 5$ & 0.133 & 0.122 & 0.120 \\
\hline $1: 10$ & 0.169 & 0.134 & 0.130 \\
\hline
\end{tabular}

A

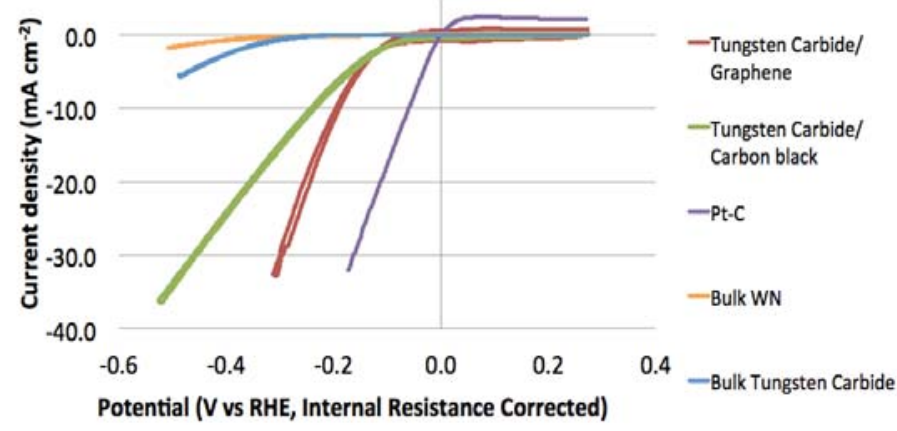

$\mathrm{B}$

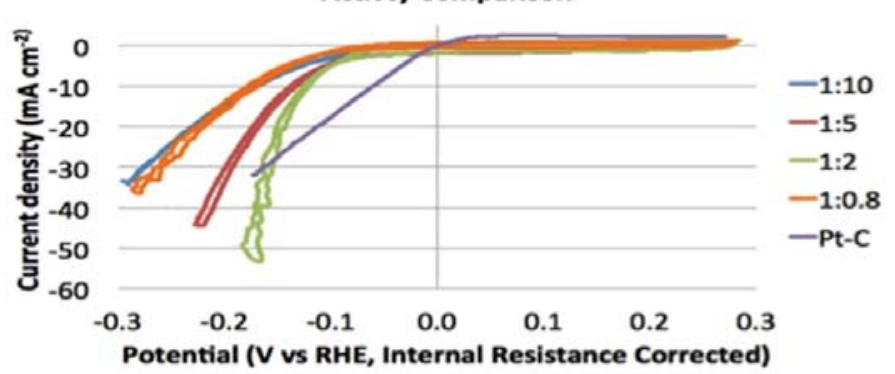

Figure 3 - Cyclic voltammetry scans taken at $2 \mathrm{mV} \mathrm{s}^{-1}$. Figure A contains curves comparing tungsten carbide/graphene, tungsten carbide/carbon black, tungsten nitride, tungsten carbide, and commercial platinum. Figure B compares tungsten carbide/nitride on graphene with varying amounts of aniline to commercial platinum. 
because it creates more accessible reactive sites. In fact, the recently developed MoSoy catalyst has markedly improved activity when supported on graphene [16]. Furthermore, nanostructuring $\mathrm{Mo}_{2} \mathrm{C}$ on carbon nanotubes lowers the $\eta_{10}$ value from $210 \mathrm{mV}$ (without nanotube) to $152 \mathrm{mV}$ (with nanotube) [17-18]. This data provides evidence that nanostructuring lowers the overpotential required for hydrogen evolution. Nanostructuring, especially on a surface like graphene, is known to provide for faster electron transport [16]. For these reasons, current research on HER catalysis involves nanostructuring metal complexes on carbon supports. In addition to nanostructuring, HER activity is affected by relative amounts of carbide and nitride. In figure 3B we see that HER activity increases as the ratio of [W]:[Aniline] changes from 1:10 to $1: 2$. Because both the amount of tungsten carbide on graphene and HER activity increases as less aniline is added, it can be assumed that tungsten carbide is the active species for hydrogen evolution. In the controlled experiment in Figure 3A, it can be seen that bulk tungsten carbide is more active for HER than bulk tungsten nitride, which further verifies the hypothesis that it is the carbide and not the nitride which contributes to the majority of the HER activity. Figure 3B shows that the 1:2 sample is the most active for HER. In fact, the HER activity of the 1:2 sample overtakes that of commercial platinum at higher overpotentials. For the 1:2 sample, the overpotential for a current density of $-10 \mathrm{~mA}$ $\mathrm{cm}^{-2}\left(\eta_{10}\right)$ is only $121 \mathrm{mV}$. On the other hand, the tungsten carbide catalyst reported by Bell, et al. had a current density of only $-8 \mathrm{~mA}$ $\mathrm{cm}^{-2}$ with a voltage as high as $200 \mathrm{mV}[12]$. WS2 nanosheets made by $\mathrm{Wu}$, et al. exhibited a lower $\eta_{10}$ of $150 \mathrm{mV}$ [10], but they are still not as active as $\mathrm{W}_{2} \mathrm{C}$ and $\mathrm{WN}$ on graphene. The tungsten carbide catalysts presented here are also more active than many modern molybdenum catalysts. For instance, compounds $\mathrm{MoS}_{3} /$ CNT, $\mathrm{MoS}_{2} / \mathrm{RGO}$, and $\mathrm{Mo}_{2} \mathrm{C} / \mathrm{CNT}$ exhibit $\eta_{10}$ values of 250,160 , and $152 \mathrm{mV}$, respectively [18-20]. From this data, it is clear that tungsten is more active than molybdenum for HER in acidic media, and that tungsten carbide/nitride on graphene is very active for HER compared to other catalysts in the literature.

\section{Durability}

Catalyst durability is the most important issue surrounding commercial hydrogen production. This is because many non-noble metals have poor corrosion stability [16]. To test durability, accelerated deterioration tests were conducted on the samples with different [W]:[Aniline] ratios. It was found that samples with aniline were more durable than those without aniline. As seen in figure 4A, the sample with no aniline possesses poor corrosion stability, because its activity decreases significantly after cycling. The $\eta_{10}$ value increases from $189 \mathrm{mV}$ to $330 \mathrm{mV}$ after only 5,000 cycles. On the other hand, the 1:0.8 sample in figure $4 \mathrm{~B}$ is more durable, however it is still not durable enough to be competitive with platinum, as its activity decreases markedly after 10,000 cycles: before cycling, the $\eta 10$ was $169 \mathrm{mV}$, but after 5,000 cycles it increased to $180 \mathrm{mV}$ and after 10,000 it was $230 \mathrm{mV}$. The $1: 2$ sample in figure $4 \mathrm{C}$ is much more active and durable than the 1:0.8. After cycling, the $\eta_{10}$ value decreased from $121 \mathrm{mV}$ to 117 $\mathrm{mV}$ and increased slightly to $119 \mathrm{mV}$ after 10,000 cycles. This data provides clear evidence that the 1:2 sample is very durable, because after 10,000 cycles, the $\eta_{10}$ value is lower than before cycling.

Figure 4 - Polarization curves before and after durability testing for varying amounts of aniline.

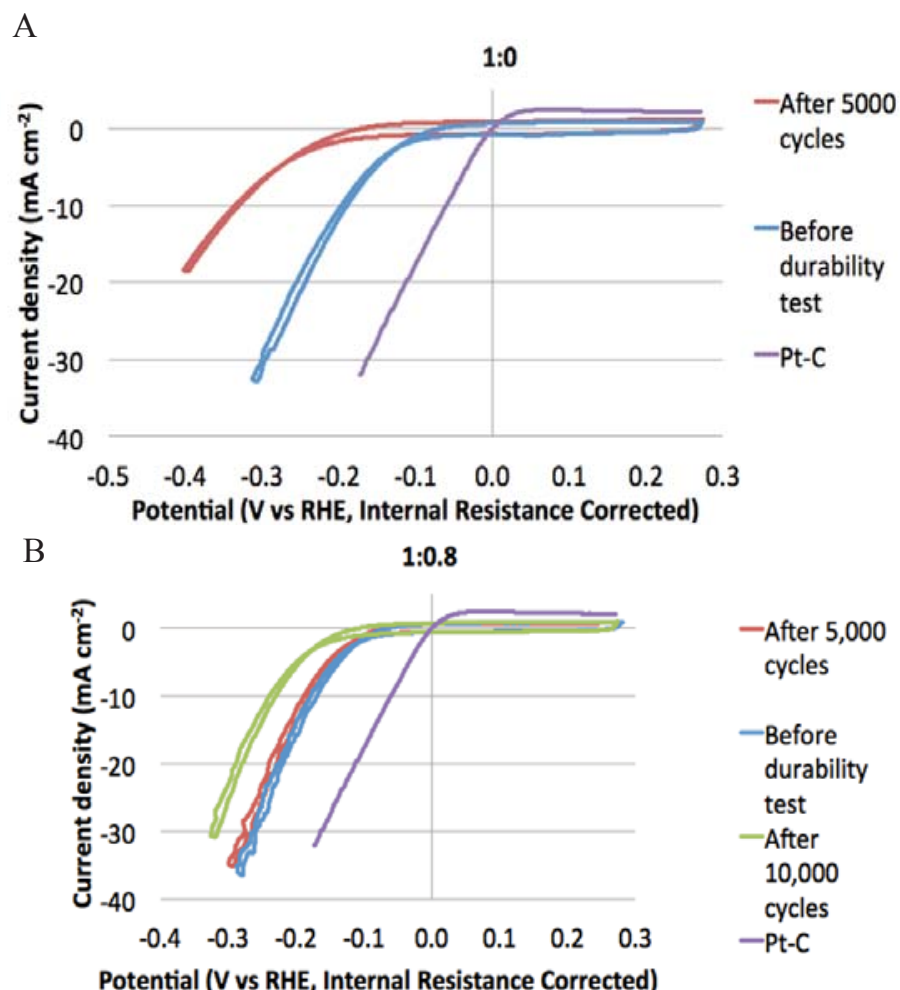

C

$1: 2$

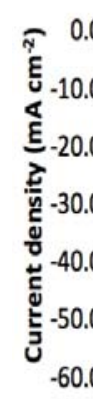

1

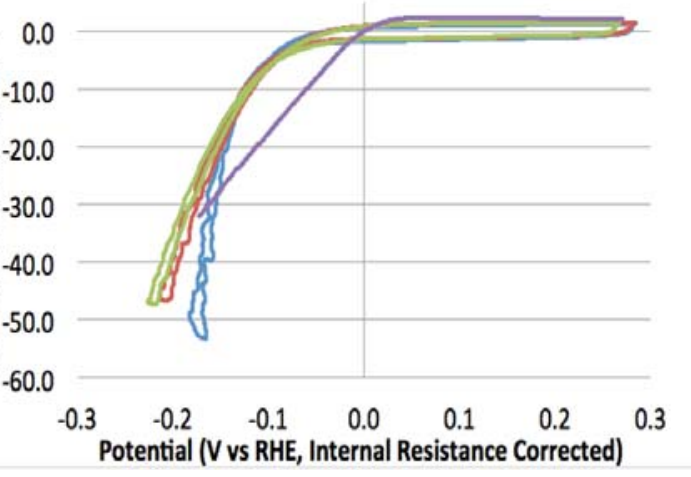

-Before durability test

-After 5,000 cycles

-After 10,000 cycles

- Pt-C

D $\quad 1: 5$

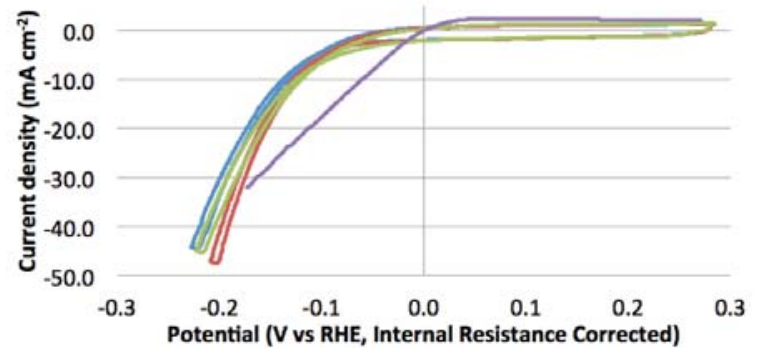

-Before durability test -After
5,000 cyles 10,000
cycles

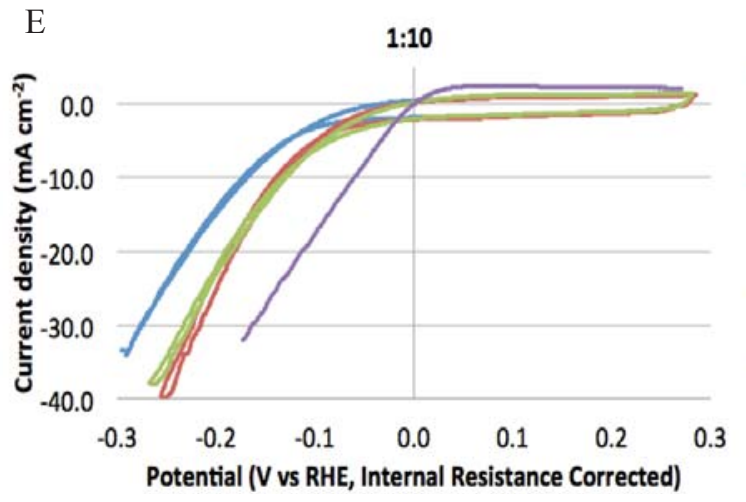

-Before durability test -After 5,000 cycles - After 10,000 cycles 
The 1:2, 1:5, and 1:10 samples did not decrease much in activity after 10,000 cycles, because the nitride contributes largely to corrosion stability. According to Chen et al. the $\mathrm{NiMoN}_{\mathrm{x}}$ catalyst is durable because nitrides exhibit a stabilizing effect [9]. Another compound, MoSoy, which also included nitride, was stable for five-hundred hours in an acidic medium [16]. On the other hand, nanostructured nickel phosphide without nitride, reported by Popczun, exhibits an increase in overpotential from 130 to 155 $\mathrm{mV}$ for a current density of $-20 \mathrm{~mA} \mathrm{~cm}^{-2}$ after only 500 cycles [21]. Such comparison of catalysts with nitride and without nitride indicates that those containing nitride are more durable. As seen in figure 4D, the 1:5 sample is more durable than 1:2. Before cycling, the 1:5 sample had a $\eta 10$ of $133 \mathrm{mV}$. After 5,000 cycles the $\eta_{10}$ improved to $122 \mathrm{mV}$ and further improved to $120 \mathrm{mV}$ after 10,000 cycles. The 1:5 sample is slightly more durable than $1: 2$, but it may also have more amorphous carbon. The amorphous carbon formed from the aniline during annealing could contribute to the activity of the 1:5 sample increasing after cycling. For instance, during cycling, hydrogen evolution may cause amorphous carbon to leave the electrode, activating it more over time. The 1:10 sample in figure 4E had an $\eta 10$ of $169 \mathrm{mV}$, and after 5,000 cycles this value decreased to $134 \mathrm{mV}$ and further decreased to $130 \mathrm{mV}$ after 10,000 cycles. Evidently, the 1:10 is less active than $1: 5$ and $1: 2$, because it has less tungsten carbide. From the aforementioned data, it can be concluded that aniline increases durability significantly. Because X-Ray diffraction shows tungsten nitride in samples with aniline and no tungsten nitride in the sample without aniline, it can be said that it is the tungsten nitride that contributes to the durability factor and not the tungsten carbide. Therefore, it is necessary to balance the amount of carbide with nitride to achieve the best activity and durability possible.

\section{E. Chronopotentiometry}

A chronopotentiometry at $-10 \mathrm{~mA}$ was conducted in $.1 \mathrm{M}$ $\mathrm{HClO}_{4}$ for 54 hours on the 1:0.8 sample [16]. Over the 54 hours, the $\eta_{10}$ increased from $168 \mathrm{mV}$ to $209 \mathrm{mV}$. Clearly, the 1:0.8 sample is not durable in acid over long time ranges. This test should be repeated for 500 hours with the 1:2 sample. Because the 1:2 sample was significantly more stable than 1:0.8 after 10,000 cycles, it should have better long-term results.

\section{Conclusion}

The solid-state reaction between ammonium tungstate, aniline, and graphene is an effective way of producing tungsten carbide and tungsten nitride on graphene. As seen from polarization curves before and after durability testing, nitriding improves catalyst durability significantly. However, it is necessary to optimize the ratio between carbide and nitride for the best catalytic activity and durability. This is because carbide is the active species for hydrogen evoluton while the nitride contributes to durability. From comparing the polarization curves, we see that the optimal ratio of [W]:[Aniline] is 1:2 because it is the most active after 10,000 cycles with an $\eta_{10}$ of $119 \mathrm{mV}$. Tungsten carbide/nitride on graphene is a promising HER catalyst.

\section{References}

Hydrogen production road map: Technology pathways to the future, FreedomCAR \& Fuel partnership. Hydrogen Production Technical Team, 2009.

J. Turner, G. Sverdrup, M. K. Mann, P.-C. Maness, B. Kroposki, M. Ghirardi, R. J. Evans and D. Blake, Int. J. Energy Res., 2008, 32, 379-407.

D. V. Esposito, S. T. Hunt, Y. C. Kimmel and J. G. Chen, J. Am. Chem. Soc., 2012, 134, 3025-3033.

J. G. Highfeld, E. Claude and K. Oguro, Electrochim. Acta, 1999, 44, 2805-2814.

J. R. McKone, E. L. Warren, M. J. Bierman, S. W. Boettcher, B. S. Brunschwig, N. S. Lewis and H. B. Gray, Energy Environ. Sci., 2011, 4, 3573-3583.

E. L. Warren, J. R. McKone, H. A. Atwater, H. B. Gray and N. S. Lewis, Energy Environ. Sci., 2012, 5, 96539661.

L. Birry and A. Lasia, J. Appl. Electrochem., 2004, 34, 735-749.

Y. Sun, H. Cheng, S. Gao, Z. Sun, Q. Liu, Q. Liu, F. Lei, T. Yao, J. He, S. Wei and Y. Xie, Angew. Chem. Int. Ed., 2012, 51, 8727-8731.

W.-F. Chen, K. Sasaki, C. Ma, A. I. Frenkel, N. Marinkovic, J. T. Muckerman, Y. Zhu and R. R. Adzic, Angew. Chem. Int. Ed., 2012, 51, 6131-6135.

Z. Z. Wu, B. Z. Fang, A. Bonakdarpour, A. K. Sun, D. P. Wilkinson and D. Z. Wang, Appl. Catal., B, 2012, 125, 59-66.

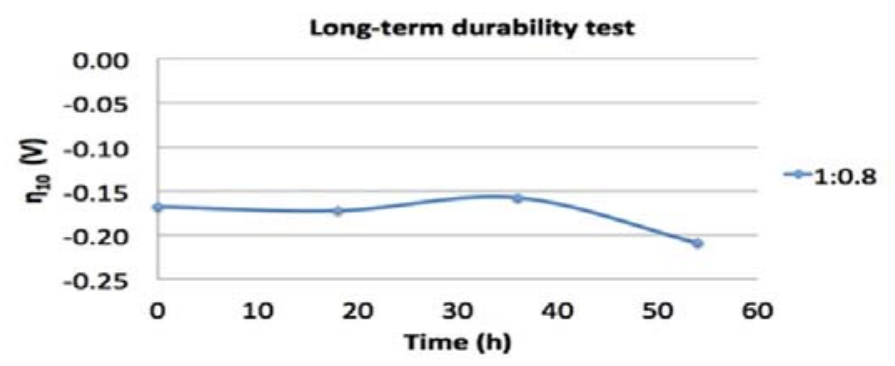

Figure 5 - Chronopotentiometry conducted for 54 hours at $-10 \mathrm{~mA}$ for 1:0.8 sample. 
"Platinum Prices and Platinum Price Charts." InvestmentMine. N.p., n.d. Web. 06 Aug. 2013.

R.D. Armstrong and M.F. Bell, Electrochimica Acta, 1978, 23, 1111-1115.

I. Nikolov, T. Vitanov, and V. Nikolova, Journal of Power Sources, 1979, 5, 197-206.

P. Zoltowski, Electrochimica Acta, 1979, 25, 1547-1554. Y. C. Kimmel, D. V. Esposito, R. W. Birkmire, J. G. Chen, International Journal of Hydrogen Energy, 2012, 37, 3019-3024.

W.-Fu. Chen, S. Iyer, S. Iyer, K. Sasaki, C.-H. Wang, Y. Zhu, J. T. Muckerman, E. Fujita, Energy Environ. Sci., 2013, 6, 1818-1826.

H. Vrubel and X. Hu, Angew. Chem. Int. Ed., 2012, 51, $12703-$ 12706.

W.-F. Chen, C. H. Wang, K. Sasaki, N. Marinkovic, W. Xu, J. T. Muckerman, Y. Zhu and R. R. Adzic, Energy Environ. Sci., 2013, 6, 943-951.

D. Merki, S. Fierro, H. Vrubel and X. L. Hu, Chem. Sci., 2011, 2, 1262-1267.

Y. Li, H. Wang, L. Xie, Y. Liang, G. Hong and H. Dai, J. Am. Chem. Soc., 2011, 133, 7296-7299.

E. J. Popczun, J. R. McKone, C. G. Read, A. J. Biacchi, A. M. Wiltrout, N. S. Lewis, R. E. Schaak, J. Am. Chem. Soc., 2013

\section{Acknowledgments}

This project was supported in part by the U.S. Department of Energy, Office of Science, Office of Workforce Development for Teachers and

Scientists (WDTS) under the Science Undergraduate Laboratory Internships Program (SULI) and by BNL Technology Maturation Funding

TM12-008. I thank my mentor, Dr. Wei-Fu Chen, and all the members of the artificial photosynthesis group at BNL for their guidance and support. 


\title{
Analysis of the perfluorocarbon tracer compounds used in the New York City Subway-Surface Airflow Exchange program using gas chromatography with electron-capture and thermal conductivity detection.
}

\author{
Mackenzie Shipley \\ Department of Chemistry, Hartwick College, Oneonta, NY 13820 \\ Richard Wilke and Thomas Watson \\ Environmental Science, Brookhaven National Laboratory, Upton, NY 11973
}

\begin{abstract}
This research project sought to quantify the composition of the Perfluorocarbon Tracer Compounds (PFTs) used by Brookhaven's Tracer Technology Group. These compounds are used to help identify underground leaks in utility company cable systems, evaluate air exchange in homes and commercial buildings, as well as provide input for modeling atmospheric transport. The main objective of this research project was to determine the composition of the PFT compounds using gas chromatography. By using a thermal conductivity detector to analyze which PFT peaks are present in each PFT, the percent composition of each sample was calculated. To confirm that the contaminants found in each PFT were, in fact, other PFT compounds, these samples were also analyzed using an electron capture detector. This research supports the Department of Energy's mission to increase understanding of global climate by quantifying atmospheric transport and dispersion, measuring building energy efficiency, and developing tools that can be used to support emergency response planning and operations. It will also support tracer research programs of the Brookhaven National Laboratory Tracer Technology Group. The data gathered in this research project will be used to calibrate the gas chromatograph as well as quantify PFT concentrations in samples taken from the S-SAFE project in New York City. S-SAFE is the largest urban dispersion airflow study ever performed in New York City. The purpose of this study is to better understand the risks posed by airborne contaminants for optimization of emergency response following any hazardous threats. I have learned how to develop analytical methods for analyzing PFTs, calculate the composition of the measured samples and solve mechanical and operational problems of gas chromatography. The opportunity to work at a government laboratory with professional scientists and equipment has given me and helped me develop many laboratory and data analysis skills that will be useful in my scientific career.
\end{abstract}

\section{Introduction}

Perfluorocarbon Tracer Compounds (PFTs) are hydrocarbons in which the hydrogen atoms have been replaced with fluorine atoms. Due to the strength of the fluorine-carbon bond, these compounds are very stable and inert. PFTs will persist in the atmosphere for thousands of years due to their stability and resistance to oxidation in the atmosphere. The only way in which these compounds can be destroyed is by photolysis in the mesosphere. The quantities of PFTs used in tracer programs are so small that their environmental effect has been found to be minimal (Watson et al. 2007).

\section{A. Uses of Perfluorocarbon Tracer Compounds}

These compounds are used for many purposes. Because PFTs are nonreactive and dissolve oxygen, they have been studied as blood substitutes and are used in eye surgery and diagnostic imaging. Previous tracer experiments include measuring rates of building infiltration, detecting gas leaks, and tracking mass movement in geological formations. Building heating, ventilation, and air conditioning (HVAC) performance tests have been executed using PFTs on large government buildings to test security systems and hazardous material mitigation equipment. PFTs are used to study transport in the atmosphere on local, regional and continental scales (Watson et al. 2007). These inert compounds are present in extremely low concentrations within the atmosphere and can be measured at levels as low as parts per quadrillion (10-15) using a gas chromatograph (GC) instrument with electron capture detector (ECD).

\section{B. Current Research Project}

The purpose of this research is to develop analytical methods using gas chromatography that will be used to measure the composition of the seven major PFTs used by Brookhaven National Laboratory's (BNL) Tracer Technology Group. The physical properties of the seven PFTs are given in Table 1 (Watson et al. 2007). Liquid PFTs are used to make the primary calibration standards. The purity of the PFTs must be determined so that the exact composition will be known. The composition data will be used to formulate standards that will be used to calibrate the gas chromatograph as well as quantify the concentration of PFTs present in air samples.

\section{Materials and Methods \\ A. Gas Chromatography}

The instruments used for analysis of the PFT compounds were a Hewlett-Packard (HP) 6890 gas chromatograph with a thermal conductivity detector (TCD) and a Varian 6000 gas chromatograph with an ECD. A $30 \mathrm{~m}$ carbograph capillary column with $0.53 \mathrm{~mm}$ diameter was used in the HP $6890 \mathrm{GC}$ while a $30 \mathrm{~m}$ carbograph capillary column with a $0.25 \mathrm{~mm}$ diameter was used in the Varian GC. Gas Chromatography is the distribution of a 
sample with unknown components between two phases within a capillary column. GC can be used to determine the composition of an unknown sample of gas by separating and measuring the quantity of each compound in the sample. Within the capillary column there is a stationary phase which is a large surface area of a specific compound that is coated on the inside of the column. In the case of this research project, the stationary phase is carbograph, a solid material made up of graphitized carbon and the column is known as a carbon layer open tubular column (CLOT). There is also the mobile phase made up of the carrier gas and the compounds of interest which passes over the stationary phase and through the column. The carrier gas that we used to serve as the source of constant flow that would carry the PFT sample through the carbograph column in the HP 6890 GC was helium. The carrier gas used in the Varian 6000 GC was a mixture of argon containing $10 \%$ methane. The solid stationary phase is coated throughout the inside of the capillary column where the mobile phase carrying the compounds of interest will interact with the stationary phase at different levels, causing separation to occur (McNair, Bonelli 1968). We used a capillary column because they have better separation and resolution which results in relatively easy chromatographic analyses (McNair, Bonelli 1968).

\section{B. Injection Method}

Due to the large difference in sensitivity of the two detectors being used in this project, two samples of each PFT were created. The first sample contained a specific PFT compound in air at several hundred to several thousand parts per million. The samples were injected directly into the inlet of the hp 6890 gas chromatograph where they traveled through the carbograph column and interacted with the stationary phase present on the inside of the column. The second sample of each PFT was created using a 1:1000 dilution of the first sample in ultra high purity (UHP) nitrogen. $10 \mu \mathrm{L}$ quantities of these samples were injected onto capillary action tubes (CATS) that were then loaded into metal racks that hold these tubes and form a seal using O-rings. Once the seal is formed, $1 \%$ hydrogen gas in nitrogen flows through the tubes. Each tube is individually heated which causes the PFT sample within the tube to desorb and travel into the Varian 6000 $\mathrm{GC}$ where it is analyzed. The analysis results in a chromatogram that displays the signal $(\mathrm{mV})$ vs. time (min) resulting from the PFT sample. The signal for each unique component is displayed as a peak; the larger the peak height and peak area are, the higher the concentration of the compound that is present within the sam- ple. Depending upon the level of interaction that occurs between each component of the sample and the stationary phase; there will be different retention times for each individual component of the sample. The most important factor when attempting to improve chromatograms and data is the retention time of the peak(s). If the retention time of one peak is identical to the retention time of another, then it will be virtually impossible to determine which peaks belong to which component of the sample. Therefore, the developed methods for analyzing the composition of each PFT sample must have unique retention times for each component of the sample in order for it to be analyzed properly. The order of elution of each PFT is based on the molecular weight of the PFT as well as the vapor pressure of the compound; the lightest PFTs elute the fastest while the heavier PFTs elute later.

\section{Thermal Conductivity Detector}

One of the detectors used to analyze each sample was a thermal conductivity detector (TCD). Using a single filament, this detector analyzes samples based on the difference in the thermal conductivity as the different components of the sample pass through the GC. The TCD filament has a constant current pass through it which causes the temperature to rise and when there is pure helium carrier gas flowing, the temperature of the filament is constant (McNair, Bonelli 1968). We chose helium as the carrier gas because it is readily available; it has the highest thermal conductivity next to hydrogen, and it would not reduce the oxide coating of the filament, which could result in inconsistent response from the detector (Hyver, Sandra 1989). The filament's temperature will change when a component of the sample passes through the filament. This is due to the fact that there is a difference in the thermal conductivity of the carrier gas and the sample component which causes a corresponding change in the electrical resistance of the filament. The change in resistance is measured by a Wheatstone bridge circuit which generates an output signal and in turn, a sample peak is created (McNair, Bonelli 1968). Differences in thermal conductivity of various gases are based on the speed at which the gas molecules can diffuse from the TCD filament (McNair, Bonelli 1968). The TCD is able to analyze individual components of a sample In order for the column to operate at maximum efficiency; the flow of the make-up gas through the column must be operated at its optimum flow rate. The detector performs best when the sum of the column flow and the makeup gas is around $5.00 \mathrm{~mL} / \mathrm{min}$. Such flow rates eliminate peak broadening and ensure that proper interaction occurs between the sample and the stationary phase.

\begin{tabular}{|l|l|l|l|l|}
\hline \multicolumn{1}{|c|}{ Acronym } & \multicolumn{1}{|c|}{ Chemical Name } & \multicolumn{1}{c|}{ Formula } & Molecular Weight $\left(\mathrm{g} \mathrm{mol}^{-1}\right)$ & Boiling Point $\left({ }^{\circ} \mathbf{C}\right)$ \\
\hline PDCB & perfluorodimethylcyclobutane & $\mathrm{C}_{6} \mathrm{~F}_{12}$ & 300 & 45.0 \\
\hline PMCP & perfluoromethylcyclopentane & $\mathrm{C}_{6} \mathrm{~F}_{12}$ & 300 & 48.1 \\
\hline PMCH & perfluoromethylcyclohexane & $\mathrm{C}_{7} \mathrm{~F}_{14}$ & 350 & 76.0 \\
\hline m-PDCH & perfluoro-1,2-dimethylcyclohexane & $\mathrm{C}_{8} \mathrm{~F}_{16}$ & 400 & 102.0 \\
\hline o-PDCH & perfluoro-1,3-dimethylcyclohexane & $\mathrm{C}_{8} \mathrm{~F}_{16}$ & 400 & 102.0 \\
\hline p-PDCH & perfluoro-1,4-dimethylcyclohexane & $\mathrm{C}_{8} \mathrm{~F}_{16}$ & 400 & 102.0 \\
\hline i-PPCH & perfluoroisopropylcyclohexane & $\mathrm{C}_{9} \mathrm{~F}_{18}$ & 450 & 130.0 \\
\hline PTCH & perfluorotrimethylcyclohexane & $\mathrm{C}_{9} \mathrm{~F}_{18}$ & 450 & 125.0 \\
\hline
\end{tabular}

Table 1. PFTs used as tracers by BNL Tracer Technology Group; acronyms, IUPAC names, chemical formulas, molecular weights, and boiling points. 


\section{Electron Capture Detector}

An electron capture detector was used in the Varian $6000 \mathrm{GC}$ to analyze the different PFT samples. This detector works by detecting highly electronegative species present in the sample, causing a signal to be emitted in the form of a peak in the chromatogram. Typically, the species that are detected at the highest level are electronegative species that are in organic molecules (Grob 1985). This detector is designed so that there are two electrodes with a radiation source present in-between them with carrier gas flowing throughout the system (Grob 1985). In the case of the Varian $6000 \mathrm{GC}$ used in this experiment, the carrier gas used is argon containing $10 \%$ methane and the radiation source is Nickel-63. Argon with methane is used as the carrier gas because the methane reduces the concentration of argon which is only somewhat stable. Methane also promotes thermal equilibrium of the electrons (Grob 1985). 63Ni which emits $\beta$ rays, is used as the radiation source because it is a great radiation source for ECD chromatography because it has a longer cell distance $(8.0 \mathrm{~mm})$, high maximum temperature $\left(400^{\circ} \mathrm{C}\right.$ ), and is highly durable (Grob 1985). When an electronegative compound passes through the detector, it essentially "captures" an electron; and a negatively charged species is formed (Grob 1985). The change in the signal is detected when the background current decreases as the negatively charged species is formed. This phenomenon is best detected when the electrode potential is low (Grob 1985).

The electron capture detector's response factor is influenced by many components such as temperature, the electronegativity of the species being analyzed, the presence of contaminants, and the energy of the electrons (Grob 1985).

\section{E. Developing an Analytical Method}

The chromatograms that are created for each PFT sample from the detectors determine whether or not the individual components are successfully separated from one another. A chromatogram that has good separation of all of the sample's components will have narrow peaks that are sharp and symmetrical. Factors that influence the level of separation between peaks are column diameter, column length, type of stationary phase, type of carrier gas, and column temperature (Hyver and Sandra 1989). A chromatogram with broad peaks can be improved by changing the conditions in the column. The most significant adjustable parameters are the flow rate of the carrier gas through the column and oven temperature. The higher the heat of the oven; the less interaction that the PFT sample will have with the stationary phase, which results in shorter retention times for the components within the sample. There will be more interaction between the PFT sample and the stationary phase at low oven temperatures, resulting in longer retention times. The faster the carrier gas flows through the column, the less interaction the sample has with the stationary phase. If the flow rate of the carrier gas is slowed instead, there will be more interaction between the sample and the stationary phase.

\section{F. Oven Temperature Program}

In order to separate all seven of the major PFT samples that were tested, an analytical method had to be developed. Using the HP 6890 gas chromatograph oven, a unique oven program was created that separated all seven PFTs within fifteen minutes. For the first four minutes, the oven held at a low temperature of $30^{\circ} \mathrm{C}$; such a low temperature allowed for the sample to react more with the stationary phase in the beginning of the run. Having the lighter PFTs-PDCB 1, PDCB 2, and PMCP-react more with the stationary phase improved their separation from each other. PDCB and PMCP typically elute right on top of each other and it is difficult to integrate the two peaks separately. Next, the oven increased at a rate of $25^{\circ} \mathrm{C} / \mathrm{min}$ to reach $65^{\circ} \mathrm{C}$ where the temperature was held for two minutes. Increasing the temperature quickly helps eliminate dead time in the chromatogram, in which no peaks are eluting. Finally, the oven ramped again at $25^{\circ} \mathrm{C} / \mathrm{min}$ to reach a final temperature of $90^{\circ} \mathrm{C}$ that was held constant for the final seven minutes of the run. Having such a high oven temperature near the middle and end of the run caused there to be less interaction between the sample and the stationary phase within the column. Less interaction means that the heavier PFT compounds which take much longer to elute- $\mathrm{PDCH}$ isomers, $\mathrm{iPPCH}$ and $\mathrm{PTCH}-$ are able to move through the column faster and in turn, elute sooner. Initially, PTCH's six isomers were taking longer than twenty minutes to elute. Also, there was a large gap in the chromatogram between the PDCH isomers, the iPPCH peak and the PTCH isomers. Dramatically increasing the temperature of the oven caused all of these PFTs and their isomers to elute much sooner and in turn, the dead time was reduced.

\section{G. Intermediate Standards}

There were seven samples containing the major PFT compounds that were analyzed; one sample vial of each PFT contained about $1.0 \mathrm{~mL}$ of the liquid PFT in about $6.0 \mathrm{~mL}$ of air. Samples taken from the PFT vials were injected directly into the back inlet of the HP 6890 GC. To ensure that enough data was present to analyze, six injections from each sample vial were made. The samples of PFT that were injected into the Varian 6000 GC needed to be diluted before injection. $500.0 \mu \mathrm{L}$ of the PFT vials were injected into a sample bag containing $500 \mathrm{~mL}$ UHP nitrogen. This first sample bag was used to create a 1:1000 dilution by injecting $500 \mu \mathrm{L}$ from the first sample bag into a second sample bag that contained $500 \mathrm{~mL}$ of UHP nitrogen. To analyze these sample bags, a $10 \mu \mathrm{L}$ sample of each bag was injected onto two CATS tubes (duplicates). Then, the data for each sample - specifically retention time $(\mathrm{min})$ and peak area $(\mathrm{mVs})$ - was combined and averaged. All of the minor peaks present in each chromatogram were also combined individually and averaged. The standard deviation and standard deviation of the mean were found for each component and then the percent composition of each PFT was determined using the calculated averages from the collected data.

\section{H. Calculations}

Percent Composition $(\%)=(($ major peak area average $) /$ (major peak area average + averages of minor peak areas) $) \times 100$

$$
\text { Standard Deviation of Mean }\left(\partial \overline{A_{m}}\right)=\frac{\sigma \mathrm{A}_{m}}{\sqrt{n}}
$$

where $\sigma \mathrm{A}_{\mathrm{m}}$ is the standard deviation of the peak areas of the major PFT in the sample and $\mathrm{n}$ is the number of trials performed for that specific PFT.

\section{Results and Discussion}

From the collected data, it was found that the composition of many of the PFT intermediate samples was not very pure; a lot of the intermediate standards had many other PFTs present in them (Appendix, Tables 3-17). However, the relative standard devia- 
tion calculations for each PFT demonstrate that the five trials performed yielded fairly consistent results. In Table 2 is a summary of the calculated composition for each PFT intermediate sample.

The calculated compositions of each of the eight measured intermediate standards were not as pure as expected. For instance, iPPCH (Tables 15-16 and Figure 7) yielded a composition of approximately $89 \%$. With such a low composition and high levels of impurities, the consequences of using such a PFT as a primary standard could be negative. Many impurities and other PFT peaks present in these intermediate standard samples presents the problem of not being able to create an accurate primary calibration standard with these results. If the developed primary standard has such high levels of impurities it will be difficult to use it as a baseline to compare other samples of unknown concentration to.

Another interesting result was the data collected for both samples of PMCH (Tables 9-12 and Figures 4-5). One problem with the data was that the compositions of both samples were not consistent within the five trials performed and they were also inconsistent with one another; although the two sample bags have different concentrations of $\mathrm{PMCH}$, their composition should be identical. Another concern with the $\mathrm{PMCH}$ data was that the impurities found in the PMCH 1 sample were not the same impurities found in the PMCH 2 sample. PDCB 2 was found in both samples but PMCP was only present in the PMCH 1 sample. These problems could be due to contamination of the syringe used to inject the samples into the gas chromatograph or another issue such as a dirty sample bag or poor peak integration. In future work of this type, more sample injections should be performed to acquire more precise data.

Now that the purity of the individual PFTs has been determined, the next step of this research project is to measure the response of the analytical instrument to the tracers as well as quantify the detection limits of each PFT. The ultimate goal of this project is to develop a gas phase, primary calibration standard using the analyzed compounds. The primary calibration standard will be used as a baseline to compare air samples containing unknown concentrations of PFTs and determine the amount of each PFT present in these air samples.

\section{Acknowledgements}

I would like to acknowledge the Department of Energy for their funding and support of the SULI Program at BNL as well as the funding of my internship. I would like to thank Brookhaven National Laboratory and the Office of Educational Programs for hosting me and providing an exceptional research atmosphere. I also thank the Tracer Technology Group at BNL for their knowledgeable insight and help they provided me during my internship. I would especially like to thank my mentor Dr. Tom Watson and scientist Rick Wilke for their continuous support and constructive criticism throughout the entire research project. This project was supported in part by the U.S. Department of Energy, Office of Science, Office of Workforce Development for Teachers and Scientists (WDTS) under the Science Undergraduate Internships Program (SULI).

\section{References}

Atkins, P.W. Physical Chemistry. P.W. Atkins 1978. 141. Hewlett-Packard. HP 6890 Series IV Plus Operating Manuals. Agilent Technologies 2000.

Hyver, K. J., Sandra, P. High Resolution Gas Chromatography. Third Edition. Hewlett-Packard Co. 1989, 1-15, 4-7.

McNair, H.M.; Bonelli, E.J. Basic Gas Chromatography. Varian Aerograph 1968. 1, 9, 29, 91-94, 236, 239.

Watson, T. B., Heiser, J.; Kalb, P.; Wilke, R. The atmospheric background of Perfluorocarbon compounds used as tracers. Environ. Sci. and Technol. 2007, 41, 6909.

Watson, T. B., Sullivan, T. Feasibility of a Perfluorocarbon tracer based network to support Monitoring, Verification, and Accounting of Sequestered CO2. Environ. Sci. Technol. 2012, 46, 1692-1699 


\begin{tabular}{|c|c|c|c|c|c|c|c|c|c|c|}
\hline \multicolumn{2}{|c|}{ Major PFT } & \multicolumn{9}{|c|}{ Minor Component } \\
\hline & & PDCB 1 & PDCB 2 & PMCP & PMCH 1 & PMCH 2 & oc-PDCH & mt-PDCH & ot-PDCH & mc-PDCH \\
\hline PDCB 1 & $100 \pm 2.36 \%$ & - & - & - & - & - & - & - & - & - \\
\hline PDCB 2 & $100 \pm 1.83 \%$ & - & - & - & - & - & - & - & - & - \\
\hline PMCP & $98.92 \pm 0.05 \%$ & - & - & - & - & $0.11 \pm 0.05 \%$ & $0.47 \pm 0.05 \%$ & $0.04 \pm 0.05 \%$ & $0.39 \pm 0.05 \%$ & $0.07 \pm 0.05$ \\
\hline PMCH 1 & $91.42 \pm 0.34 \%$ & - & $8.16 \pm 0.34 \%$ & $0.42 \pm 0.34 \%$ & - & - & - & - & - & - \\
\hline PMCH 2 & $82.66 \pm 1.90 \%$ & - & $17.34 \pm 1.90 \%$ & - & - & - & - & - & - & - \\
\hline oc-PDCH & $42.00 \pm 0.27 \%$ & - & - & - & - & $6.49 \pm 0.27 \%$ & - & - & - & - \\
\hline mt-PDCH & $5.55 \pm 0.27 \%$ & - & - & - & - & $6.49 \pm 0.27 \%$ & - & - & - & - \\
\hline ot-PDCH & $38.88 \pm 0.27 \%$ & - & - & - & - & $6.49 \pm 0.27 \%$ & - & - & - & - \\
\hline mc-PDCH & $7.08 \pm 0.27 \%$ & - & - & - & - & $6.49 \pm 0.27 \%$ & - & - & - & - \\
\hline iPPCH & $89.14 \pm 0.45 \%$ & - & - & - & - & - & - & $3.02 \pm 0.45 \%$ & $1.78 \pm 0.45 \%$ & $1.14 \pm 0.45^{\circ}$ \\
\hline PTCH-1 & $55.64 \pm 0.34 \%$ & - & - & - & - & - & - & $3.59 \pm 0.34 \%$ & - & $4.06 \pm 0.34^{\circ}$ \\
\hline PTCH-2 & $18.35 \pm 0.34 \%$ & - & - & - & - & - & - & $3.59 \pm 0.34 \%$ & - & $4.06 \pm 0.34^{\circ}$ \\
\hline РTCH-3 & $4.11 \pm 0.34 \%$ & - & - & - & - & - & - & $3.59 \pm 0.34 \%$ & - & $4.06 \pm 0.34^{\circ}$ \\
\hline PTCH-4 & $5.36 \pm 0.34 \%$ & - & - & - & - & - & - & $3.59 \pm 0.34 \%$ & - & $4.06 \pm 0.34^{\circ}$ \\
\hline PTCH-5 & $5.11 \pm 0.34 \%$ & - & - & - & - & - & - & $3.59 \pm 0.34 \%$ & - & $4.06 \pm 0.34^{\circ}$ \\
\hline PTCH-6 & $3.77 \pm 0.34 \%$ & - & - & - & - & - & - & $3.59 \pm 0.34 \%$ & - & $4.06 \pm 0.34^{\circ}$ \\
\hline
\end{tabular}

Table 2. Summary of composition results for each PFT intermediate standard.

\section{Appendix 1}

\begin{tabular}{|c|c|c|}
\hline Injection \# & $\begin{array}{c}\text { Percent Com- } \\
\text { position PDCB 1 }\end{array}$ & $\begin{array}{c}\text { Percent Com- } \\
\text { position Impurities }\end{array}$ \\
\hline 1 & $100 \%$ & $0 \%$ \\
\hline 2 & $100 \%$ & $0 \%$ \\
\hline 3 & $100 \%$ & $0 \%$ \\
\hline 4 & $100 \%$ & $0 \%$ \\
\hline 5 & $100 \%$ & $0 \%$ \\
\hline
\end{tabular}

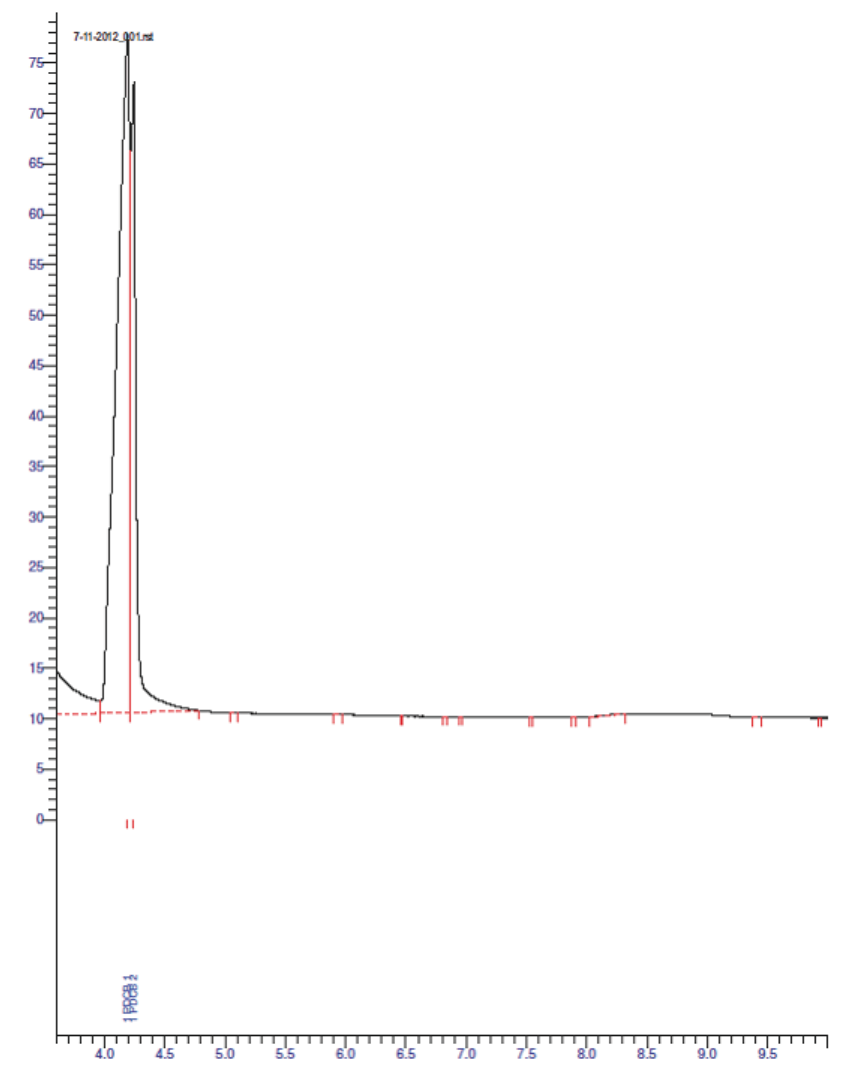

Figure 1. Chromatogram of intermediate standard PDCB 1 isomers using developed analytical method.

Table 3. Percent composition of PFT intermediate standard PDCB 1.

\begin{tabular}{|l|l|l|l|l|}
\hline Mean Peak Area & $\begin{array}{l}\text { Standard Deviation } \\
\text { (Major Peak Area) }\end{array}$ & $\begin{array}{l}\text { Relative Std Dev } \\
\text { (Peak Area) }\end{array}$ & Standard Deviation of Mean & Error of \% Comp \\
\hline $7.3 \times 10^{5}$ & $0.4 \times 10^{5}$ & $5 \%$ & $0.2 \times 10^{5}$ & 0 \\
\hline
\end{tabular}

Table 4. Mean and standard deviation data for PDCB 1 intermediate standard. 


\begin{tabular}{|l|l|l|l|l|l|l|l|}
\hline & iPPCH & PTCH-1 & PTCH-2 & PTCH-3 & PTCH-4 & PTCH-5 & PTCH-6 \\
\hline & - & - & - & - & - & - & - \\
\hline & - & - & - & - & - & - & - \\
\hline$\%$ & - & - & - & - & - & - & - \\
\hline & - & - & - & - & - & - & - \\
\hline & - & - & - & - & - & - & - \\
\hline & - & - & - & - & - & - & - \\
\hline & - & - & - & - & - & - & - \\
\hline & - & - & - & - & - & - & - \\
\hline & - & - & - & - & - & - & - \\
\hline 0 & - & $0.41 \pm 0.45 \%$ & - & - & - & - & $4.51 \pm 0.45 \%$ \\
\hline 0 & - & - & - & - & - & - & - \\
\hline 0 & - & - & - & - & - & - & - \\
\hline 0 & - & - & - & - & - & - & - \\
\hline 0 & - & - & - & - & - & - & - \\
\hline 0 & - & - & - & - & - & - & - \\
\hline 0 & - & - & - & - & - & - & - \\
\hline
\end{tabular}

\begin{tabular}{|c|c|c|}
\hline Injection \# & $\begin{array}{c}\text { Percent Com- } \\
\text { position PDCB 2 }\end{array}$ & $\begin{array}{r}\text { Percent Com- } \\
\text { position Impurities }\end{array}$ \\
\hline 1 & $100 \%$ & $0 \%$ \\
\hline 2 & $100 \%$ & $0 \%$ \\
\hline 3 & $100 \%$ & $0 \%$ \\
\hline 4 & $100 \%$ & $0 \%$ \\
\hline 5 & $100 \%$ & $0 \%$ \\
\hline
\end{tabular}

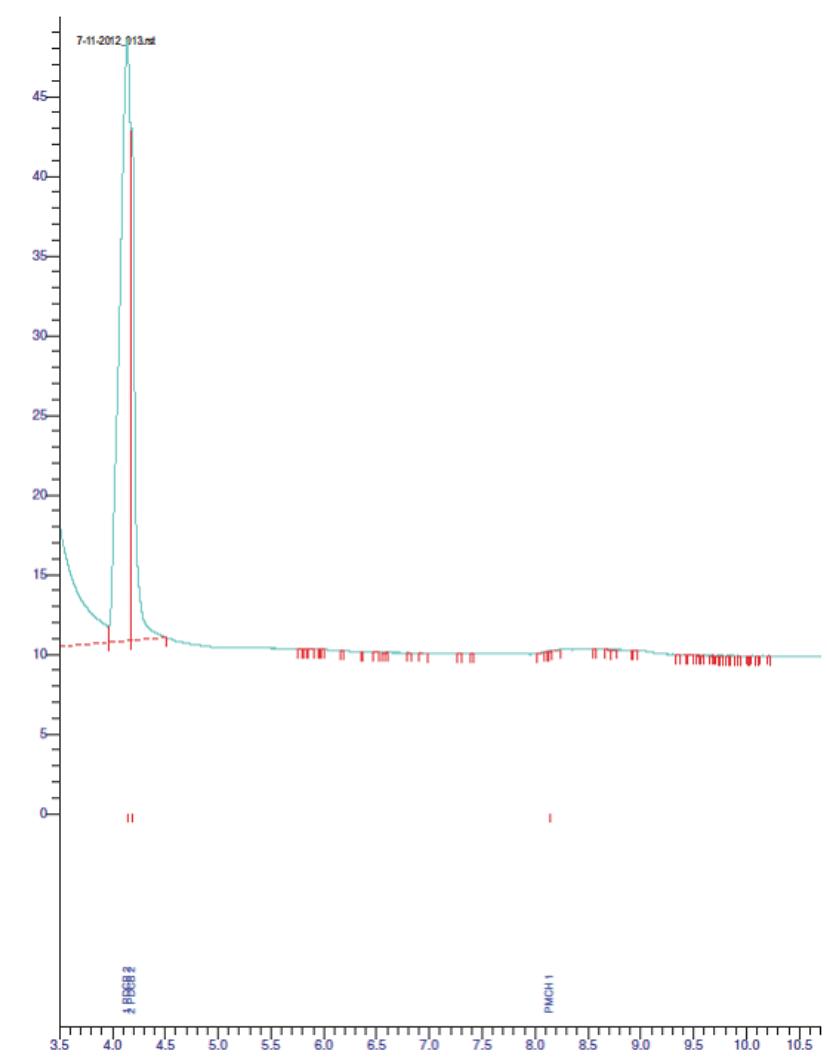

Figure 2. Chromatogram of intermediate standard PDCB 2 isomers using developed analytical method.

Table 5. Percent composition of PFT intermediate standard PDCB 2.

\begin{tabular}{|l|l|l|l|l|}
\hline $\begin{array}{l}\text { Mean Peak } \\
\text { Area }\end{array}$ & $\begin{array}{l}\text { Standard } \\
\text { Deviation } \\
\text { (Major } \\
\text { Peak } \\
\text { Area) }\end{array}$ & $\begin{array}{l}\text { Relative } \\
\text { Std Dev } \\
\text { (Peak } \\
\text { Area) }\end{array}$ & $\begin{array}{l}\text { Standard } \\
\text { Deviation } \\
\text { of Mean }\end{array}$ & $\begin{array}{l}\text { Error } \\
\text { of \% } \\
\text { Comp }\end{array}$ \\
\hline $3.5 \times 10^{5}$ & $0.1 \times 10^{5}$ & $4 \%$ & $0.06 \times 10^{5}$ & 0 \\
\hline
\end{tabular}

Table 6. Mean and standard deviation data for PDCB 2 intermediate standard.

\begin{tabular}{|c|l|l|l|l|l|l|}
\hline Injection \# & $\begin{array}{l}\text { \% Comp } \\
\text { PMCP }\end{array}$ & $\begin{array}{l}\text { \% Comp } \\
\text { PMCH 2* }\end{array}$ & $\begin{array}{l}\text { \% Comp } \\
\text { oc-PDCH }\end{array}$ & $\begin{array}{l}\text { \% Comp } \\
\text { mc-PDCH }\end{array}$ & $\begin{array}{l}\text { \% Comp } \\
\text { ot-PDCH }\end{array}$ & $\begin{array}{l}\text { \% Comp } \\
\text { mt-PDCH }\end{array}$ \\
\hline 1 & $98.98 \%$ & $0.08 \%$ & $0.48 \%$ & $0.03 \%$ & $0.39 \%$ & $0.06 \%$ \\
\hline 2 & $98.96 \%$ & $0.10 \%$ & $0.47 \%$ & $0.03 \%$ & $0.39 \%$ & $0.05 \%$ \\
\hline 3 & $99.10 \%$ & $0 \%$ & $0.45 \%$ & $0 \%$ & $0.37 \%$ & $0.08 \%$ \\
\hline 4 & $98.96 \%$ & $0 \%$ & $0.46 \%$ & $0.06 \%$ & $0.44 \%$ & $0.07 \%$ \\
\hline 5 & $98.89 \%$ & $0.13 \%$ & $0.48 \%$ & $0.04 \%$ & $0.38 \%$ & $0.09 \%$ \\
\hline
\end{tabular}

Table 7. Percent composition of PFT intermediate standard PMCP.

\begin{tabular}{|l|l|l|l|l|}
\hline $\begin{array}{l}\text { Mean (Major Peak } \\
\text { Area) }\end{array}$ & $\begin{array}{l}\text { Standard Deviation } \\
\text { (Major Peak Area) }\end{array}$ & $\begin{array}{l}\text { Standard Deviation } \\
\text { Mean (Major Peak) }\end{array}$ & $\begin{array}{l}\text { Relative Std Dev } \\
\text { (Peak Area) }\end{array}$ & Error \\
\hline $7.1 \times 10^{5}$ & $0.7 \times 10^{5}$ & $0.3 \times 10^{5}$ & $10 \%$ & $3.4 \times 10^{2}$ \\
\hline
\end{tabular}

Table 8. Mean and standard deviation data for PMCP intermediate standard. 


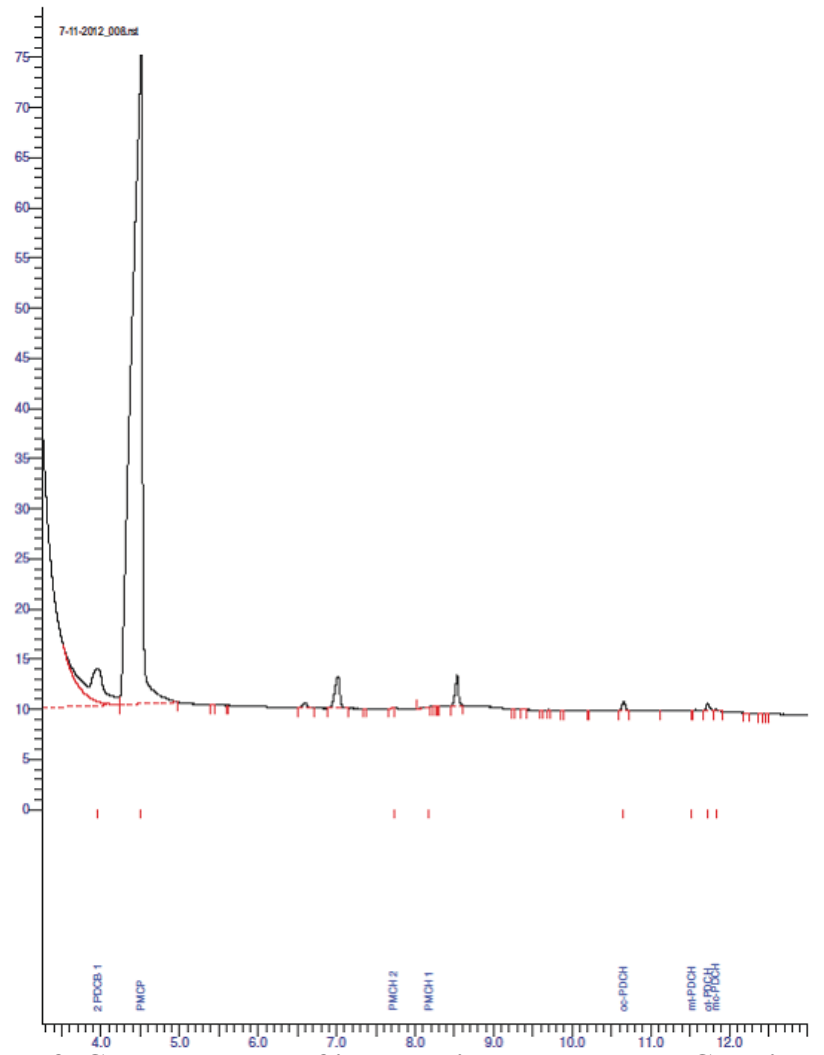

Figure 3. Chromatogram of intermediate standard PMCP using developed analytical method.

\begin{tabular}{|l|l|l|l|}
\hline Injection \# & $\begin{array}{l}\text { \% Comp } \\
\text { PMCH 1 }\end{array}$ & $\begin{array}{l}\text { \% Comp } \\
\text { PDCB 2 }\end{array}$ & $\begin{array}{l}\text { \% Comp } \\
\text { PMCP }\end{array}$ \\
\hline 1 & $92.34 \%$ & $7.66 \%$ & $0 \%$ \\
\hline 2 & $89.97 \%$ & $9.41 \%$ & $0.62 \%$ \\
\hline 3 & $90.29 \%$ & $9.14 \%$ & $0.57 \%$ \\
\hline 4 & $92.47 \%$ & $7.28 \%$ & $0.24 \%$ \\
\hline 5 & $92.43 \%$ & $7.37 \%$ & $0.20 \%$ \\
\hline
\end{tabular}

Table 9. Percent composition of PFT intermediate standard PMCH 1.

\begin{tabular}{|l|l|l|l|l|}
\hline $\begin{array}{l}\text { Mean } \\
\text { (Major } \\
\text { Peak } \\
\text { Area })\end{array}$ & $\begin{array}{l}\text { Standard } \\
\text { Deviation } \\
\text { (Major } \\
\text { Peak } \\
\text { Area) }\end{array}$ & $\begin{array}{l}\text { Relative } \\
\text { Std Dev } \\
\text { (Peak } \\
\text { Area) }\end{array}$ & $\begin{array}{l}\text { Standard } \\
\text { Deviation } \\
\text { of Mean }\end{array}$ & Error \\
\hline $5.6 \times 10^{5}$ & $0.6 \times 10^{5}$ & $10 \%$ & $0.3 \times 10^{5}$ & $0.03 \times 10^{5}$ \\
\hline
\end{tabular}

Table 10. Mean and standard deviation data for PMCH 1 intermediate standard.

\begin{tabular}{|l|l|l|}
\hline Injection \# & \% Comp PMCH 2 & \% Comp PDCB 2 \\
\hline 1 & $81.66 \%$ & $18.34 \%$ \\
\hline 2 & $80.70 \%$ & $19.30 \%$ \\
\hline 3 & $86.58 \%$ & $13.42 \%$ \\
\hline 4 & $86.61 \%$ & $13.39 \%$ \\
\hline 5 & $78.21 \%$ & $21.79 \%$ \\
\hline
\end{tabular}

Table 11. Percent composition of PFT intermediate standard PMCH 2.

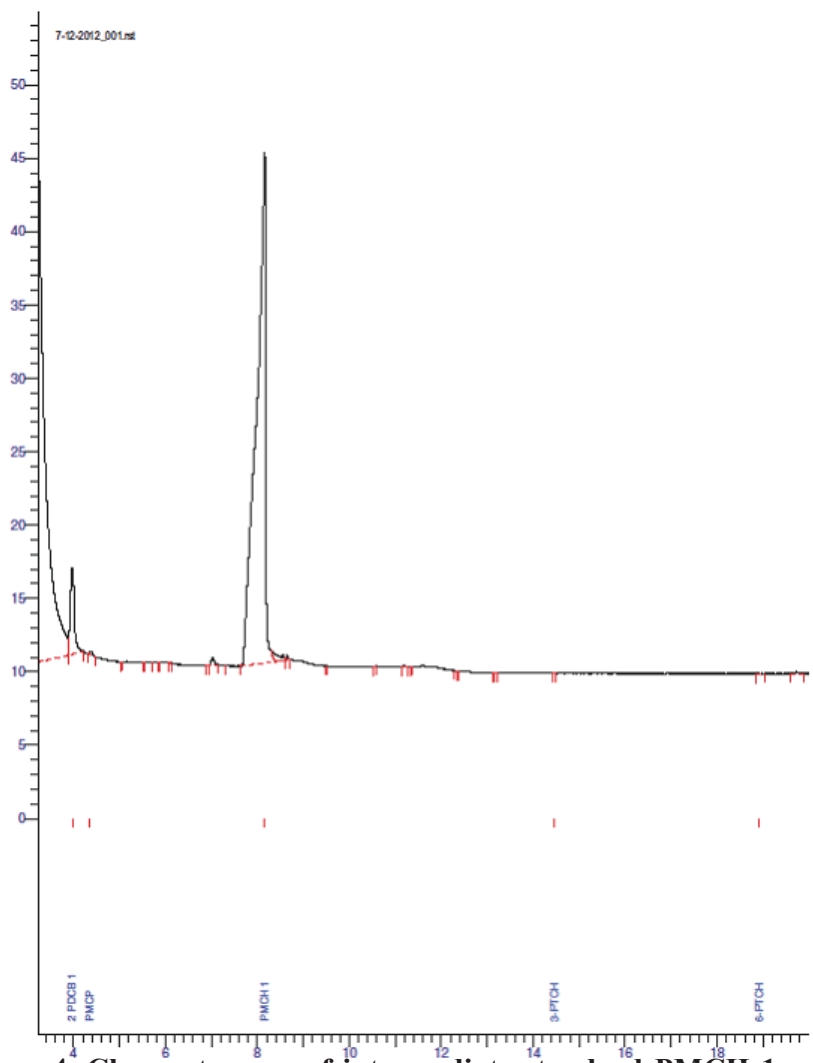

Figure 4. Chromatogram of intermediate standard PMCH 1 using developed analytical method.

\begin{tabular}{|l|l|l|l|l|}
\hline $\begin{array}{l}\text { Mean } \\
\text { (Major } \\
\text { Peak } \\
\text { Area })\end{array}$ & $\begin{array}{l}\text { Standard } \\
\text { Deviation } \\
\text { (Major } \\
\text { Peak } \\
\text { Area) }\end{array}$ & $\begin{array}{l}\text { Relative } \\
\text { Std Dev } \\
\text { (Major } \\
\text { Peak } \\
\text { Area) }\end{array}$ & $\begin{array}{l}\text { Standard } \\
\text { Deviation } \\
\text { of Mean }\end{array}$ & Error \\
\hline $0.98 \times 105$ & $0.03 \times 10^{5}$ & $3 \%$ & $0.01 \times 10^{5}$ & $0.02 \times 10^{5}$ \\
\hline
\end{tabular}

Table 12. Mean and standard deviation data for PMCH 2 intermediate standard.

\begin{tabular}{|l|l|l|l|l|l|}
\hline $\begin{array}{l}\text { Injec- } \\
\text { tion \# }\end{array}$ & $\begin{array}{l}\% \\
\text { Comp } \\
\text { oc- } \\
\text { PDCH }\end{array}$ & $\begin{array}{l}\% \\
\text { Comp } \\
\text { mt- } \\
\text { PDCH }\end{array}$ & $\begin{array}{l}\% \\
\text { Comp } \\
\text { ot- } \\
\text { PDCH }\end{array}$ & $\begin{array}{l}\% \\
\text { Comp } \\
\text { mc- } \\
\text { PDCH }\end{array}$ & $\begin{array}{l}\% \\
\text { Comp } \\
\text { PMCH } \\
2\end{array}$ \\
\hline 1 & $41.90 \%$ & $5.55 \%$ & $38.88 \%$ & $7.21 \%$ & $6.46 \%$ \\
\hline 2 & $41.94 \%$ & $5.70 \%$ & $39.04 \%$ & $6.91 \%$ & $6.41 \%$ \\
\hline 3 & $41.95 \%$ & $5.47 \%$ & $38.85 \%$ & $7.15 \%$ & $6.58 \%$ \\
\hline 4 & $42.44 \%$ & $5.41 \%$ & $38.90 \%$ & $6.89 \%$ & $6.36 \%$ \\
\hline 5 & $41.75 \%$ & $5.62 \%$ & $38.76 \%$ & $7.22 \%$ & $6.65 \%$ \\
\hline
\end{tabular}

Table 13. Percent composition of PFT intermediate standard o-PDCH. 


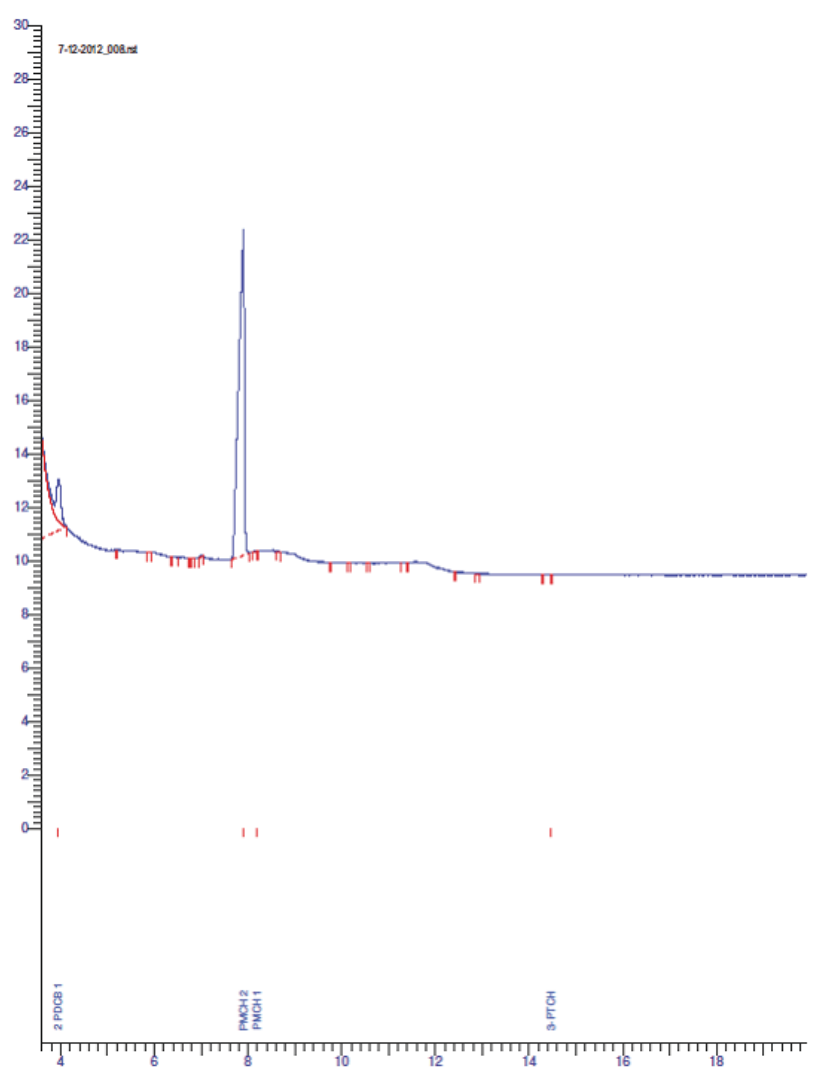

Figure 5. Chromatogram of intermediate standard PMCH 2 using developed analytical method.

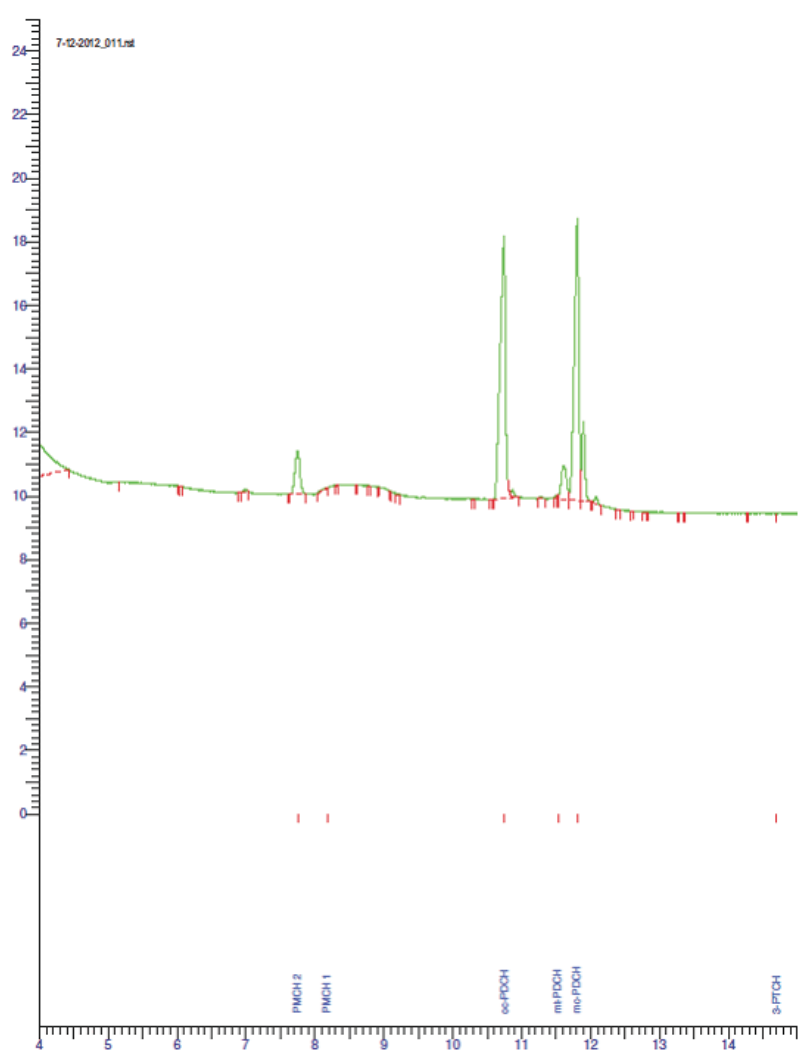

Figure 6. Chromatogram of intermediate standard o-PDCH using developed analytical method.

\begin{tabular}{|l|l|l|l|l|l|}
\hline o-PDCH Isomer & $\begin{array}{l}\text { Mean (Major } \\
\text { Peak Area) }\end{array}$ & $\begin{array}{l}\text { Standard Deviation } \\
\text { (Major Peak Area) }\end{array}$ & $\begin{array}{l}\text { Relative Std Dev } \\
\text { (Major Peak Area) }\end{array}$ & $\begin{array}{l}\text { Standard Deviation of } \\
\text { Mean }\end{array}$ & Error \\
\hline oc-PDCH & $0.4 \times 10^{5}$ & $0.02 \times 10^{5}$ & $4.7 \%$ & $0.009 \times 10^{5}$ & $0.003 \times 10^{5}$ \\
\hline mt-PDCH & $0.06 \times 10^{5}$ & $0.001 \times 10^{5}$ & $2.3 \%$ & $6.0 \times 10^{1}$ & $0.003 \times 10^{5}$ \\
\hline ot-PDCH & $0.4 \times 10^{5}$ & $0.02 \times 10^{5}$ & $4.1 \%$ & $0.008 \times 10^{5}$ & $0.003 \times 10^{5}$ \\
\hline mc-PDCH & $0.08 \times 10^{5}$ & $0.003 \times 10^{5}$ & $4.1 \%$ & $0.001 \times 10^{5}$ & $0.003 \times 10^{5}$ \\
\hline
\end{tabular}

Table 14. Mean and standard deviation data for 0 -PDCH intermediate standard.

\begin{tabular}{|r|r|r|r|r|r|r|}
\hline Injection \# & \% Comp iPPCH & \% Comp mc-PDCH & $\begin{array}{l}\text { \% Comp } \\
\text { ot-PDCH }\end{array}$ & $\begin{array}{l}\text { \% Comp mt- } \\
\text { PDCH Comp }\end{array}$ & $\begin{array}{l}\text { \% Comp } \\
\text { PTCH 1 }\end{array}$ \\
\hline 1 & $89.25 \%$ & $3.03 \%$ & $1.77 \%$ & $1.11 \%$ & $0.37 \%$ & $4.46 \%$ \\
\hline 2 & $89.41 \%$ & $3.10 \%$ & $1.80 \%$ & $1.12 \%$ & $0 \%$ & $4.58 \%$ \\
\hline 3 & $89.52 \%$ & $3.00 \%$ & $1.79 \%$ & $1.20 \%$ & $0 \%$ & $4.49 \%$ \\
\hline 4 & $89.66 \%$ & $3.02 \%$ & $1.79 \%$ & $0.97 \%$ & $0 \%$ & $4.38 \%$ \\
\hline 5 & $89.36 \%$ & $3.00 \%$ & $1.77 \%$ & $1.15 \%$ & $0 \%$ & $4.73 \%$ \\
\hline
\end{tabular}

Table 15. Percent composition of PFT intermediate standard iPPCH.

\begin{tabular}{|l|l|l|l|l|}
\hline $\begin{array}{l}\text { Mean (Major Peak } \\
\text { Area) }\end{array}$ & $\begin{array}{l}\text { Standard Deviation } \\
\text { (Major Peak Area) }\end{array}$ & $\begin{array}{l}\text { Relative Std Dev } \\
\text { (Peak Area) }\end{array}$ & $\begin{array}{l}\text { Standard Deviation of } \\
\text { Mean }\end{array}$ & Error \\
\hline $1.5 \times 10^{5}$ & $0.2 \times 10^{5}$ & $12 \%$ & $0.8 \times 10^{5}$ & $0.008 \times 10^{5}$ \\
\hline
\end{tabular}

Table 16. Mean and standard deviation data for iPPCH intermediate standard. 


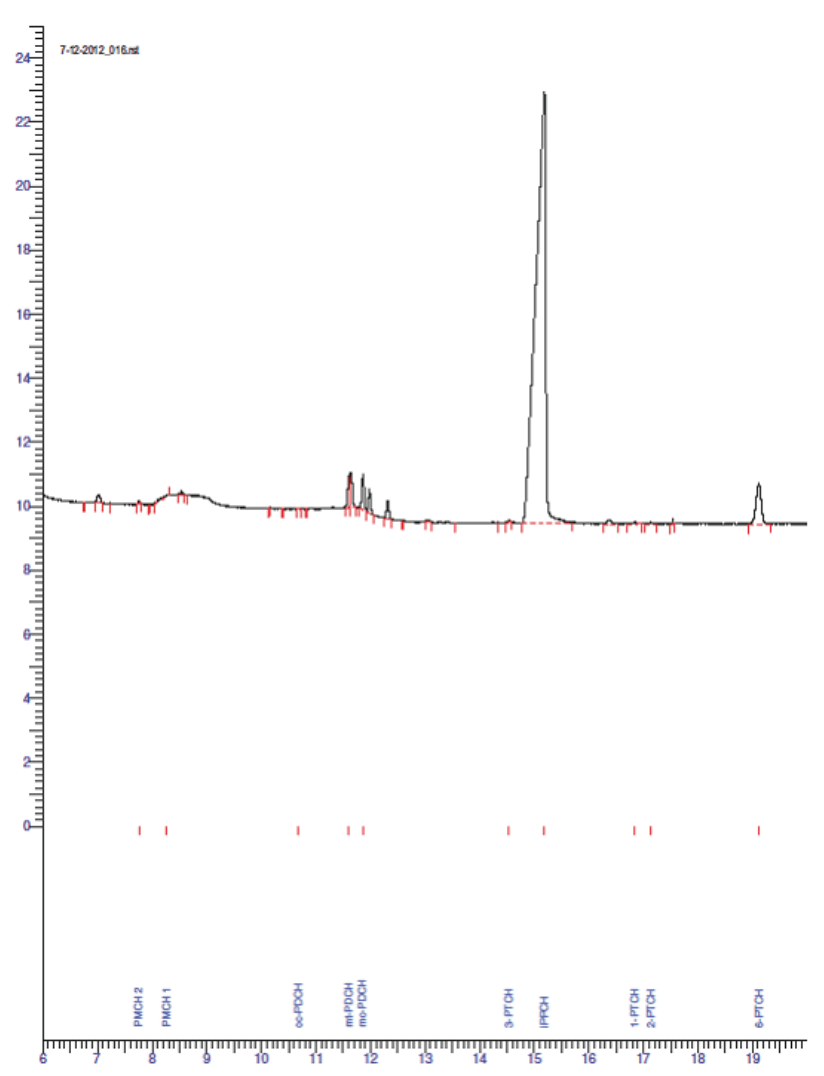

Figure 7. Chromatogram of intermediate standard iPPCH using developed analytical method.

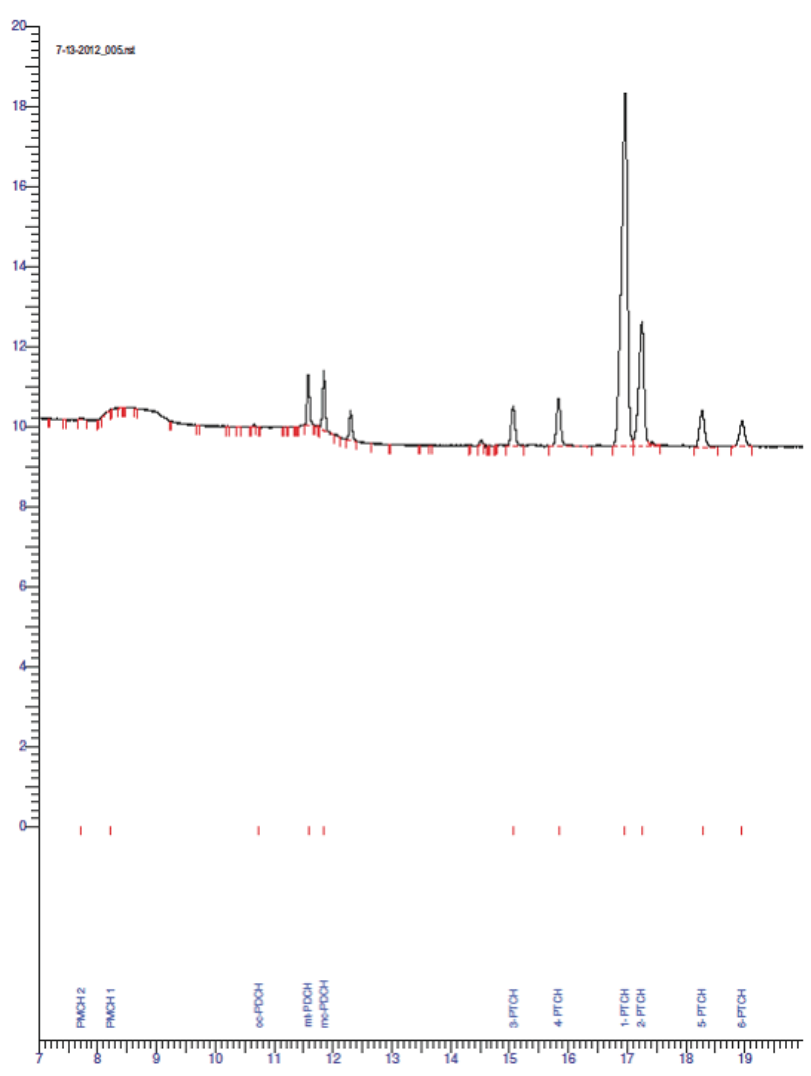

Figure 8. Chromatogram of intermediate standard PTCH using developed analytical method.

\begin{tabular}{|c|l|l|l|l|l|l|l|l|l|}
\hline $\begin{array}{c}\text { Injection } \\
\#\end{array}$ & $\begin{array}{l}\text { Total } \\
\text { Peak Area } \\
\text { PTCH } \\
(\mu \mathrm{Vs})\end{array}$ & $\begin{array}{l}\% \text { Comp } \\
\text { PTCH-1 }\end{array}$ & $\begin{array}{l}\% \text { Comp } \\
\text { PTCH-2 }\end{array}$ & $\begin{array}{l}\% \text { Comp } \\
\text { PTCH-3 }\end{array}$ & $\begin{array}{l}\% \text { Comp } \\
\text { PTCH-4 }\end{array}$ & $\begin{array}{l}\% \text { Comp } \\
\text { PTCH-5 }\end{array}$ & $\begin{array}{l}\% \text { Comp } \\
\text { PTCH-6 }\end{array}$ & $\begin{array}{l}\% \text { Comp } \\
\text { mt-PDCH }\end{array}$ & $\begin{array}{l}\% \text { Comp mc- } \\
\text { PDCH }\end{array}$ \\
\hline 1 & $1.1 \times 105$ & $55.1 \%$ & $18.6 \%$ & $4.0 \%$ & $5.0 \%$ & $6.0 \%$ & $3.7 \%$ & $3.5 \%$ & $4.0 \%$ \\
\hline 2 & $1.0 \times 105$ & $56.0 \%$ & $17.6 \%$ & $4.1 \%$ & $5.9 \%$ & $4.9 \%$ & $3.6 \%$ & $3.6 \%$ & $4.2 \%$ \\
\hline 3 & $0.95 \times 105$ & $56.6 \%$ & $17.2 \%$ & $4.2 \%$ & $5.3 \%$ & $4.9 \%$ & $3.9 \%$ & $3.7 \%$ & $4.3 \%$ \\
\hline 4 & $0.98 \times 105$ & $55.4 \%$ & $19.1 \%$ & $4.2 \%$ & $5.1 \%$ & $5.0 \%$ & $3.8 \%$ & $3.5 \%$ & $4.0 \%$ \\
\hline 5 & $1.1 \times 105$ & $55.2 \%$ & $19.2 \%$ & $4.0 \%$ & $5.5 \%$ & $4.7 \%$ & $3.9 \%$ & $3.6 \%$ & $3.9 \%$ \\
\hline
\end{tabular}

Table 17. Percent composition of PFT intermediate standard PTCH.

\begin{tabular}{|l|l|l|l|l|l|}
\hline PTCH Isomer & $\begin{array}{l}\text { Mean (Major } \\
\text { Peak Area) }\end{array}$ & $\begin{array}{l}\text { Standard Deviation } \\
\text { (Major Peak Area) }\end{array}$ & $\begin{array}{l}\text { Relative Std Dev } \\
\text { (Major Peak Area) }\end{array}$ & $\begin{array}{l}\text { Standard Devia- } \\
\text { tion of Mean }\end{array}$ & Error \\
\hline PTCH 1 & $0.6 \times 10^{5}$ & $0.03 \times 10^{5}$ & $5.2 \%$ & $0.01 \times 10^{5}$ & $0.004 \times 10^{5}$ \\
\hline PTCH 2 & $0.2 \times 10^{5}$ & $0.02 \times 10^{5}$ & $9.5 \%$ & $0.009 \times 10^{5}$ & $0.004 \times 10^{5}$ \\
\hline PTCH 3 & $0.05 \times 10^{5}$ & $0.002 \times 10^{5}$ & $3.8 \%$ & $0.0008 \times 10^{5}$ & $0.004 \times 10^{5}$ \\
\hline PTCH 4 & $0.06 \times 10^{5}$ & $0.006 \times 10^{5}$ & $9.5 \%$ & $0.003 \times 10^{5}$ & $0.004 \times 10^{5}$ \\
\hline PTCH 5 & $0.06 \times 10^{5}$ & $0.007 \times 10^{5}$ & $13.1 \%$ & $0.003 \times 10^{5}$ & $0.004 \times 10^{5}$ \\
\hline PTCH 6 & $0.04 \times 10^{5}$ & $0.003 \times 10^{5}$ & $6.5 \%$ & $0.001 \times 10^{5}$ & $0.004 \times 10^{5}$ \\
\hline
\end{tabular}

Table 18. Mean and standard deviation data for PTCH intermediate standard. 


\title{
Android application for Olog, a logbook service for accelerator operations
}

\author{
Michael Skinner \\ Marist College, Poughkeepsie, NY 12601 \\ Howard Robinson \\ Brookhaven National Laboratory, Upton, NY 11973
}

During my appointment with the SULI program this summer I wrote an application for the Android mobile device platform to support the research being performed at the National Synchrotron Light Sources. Its interface is written in Android XML and its software is written in Java. The purpose of this application is to retrieve and display a shared set of logs that are written and read by the Synchrotron's operators and users. This should make relevant information instantly accessible to large networks of researchers. The logs are stored in an SQL database and distributed using an on-site Glassfish V3 server with an HTTP based REST interface. These logs are user-created and provide a way to track and share information like work status and research progress and may have associated file or image attachments. Logs are separated into non-exclusive groups referred to as logbooks. The program provides an interface to select a logbook of interest and displays a list of associated $\operatorname{logs}$ in a "Username $>>$ Description..." format, tailoring its width to the size of the device screen and displaying the most recent entries first. Selecting an individual log from this list provides a detailed view with the description's full text along with all other relevant information. The application also provides a search feature for the logs, allowing for a keyword-based search through all available log descriptions and the content of their associated attachments, and displaying the results in the previously described list/detail view format. I have learned a great deal from my experience working with android devices and android software. It has provided me with access to a new platform for software design, as well as experience working with servers and databases.

The diagrams on either side are the activity layouts for each screen size. On the left is the layout designated for tablet devices, and on the right is the layout designated for cellular phones. Each panel is referred to as an activity, and controls the contents of the screen while it is visible. Activities that spawn other activities and become hidden behind them are added to a 'back stack' and can be retrieved by using the back-button, which destroys the currently visible activity and renders the first one on the stack.

The lists and detailed views are broken into interchangeable screen parts referred to as fragments. Fragments are the core of a design method intended to provide function variation across differing screen sizes. Note how the contents of both the tablet and cellular layouts are exactly the same. Each is hosted within a fragment object which can be created, added, removed, replaced, but never exchanged. The tablet version presents two of these fragments side-by-side to increase information visibility. The hand-held version presents only a single fragment, which must be navigated from to reach the next, due to its visual real-estate limitations.

This application also contains a search function for searching for logs based on their contents. Accessed by pressing on the magnifying glass icon in the program's action bar, a small search query box allows for non-obtrusive search entries, and creates a new activity when submitted. This activity presents search results in the same manner which it presents the contents of an entire logbook, in a list of individual logs with a limit of 20 per page, navigable through the use of either the swipe gesture or the action bar page control buttons.

Implemented using the Action Bar Sherlock support packages to allow for backwards compatibility with versions as old as 1.7, the action bar provides unique navigation and application functions which were placed in the 'menu' hardware button in older versions of the android operating system. This allows for the more necessary or more repetitive functions to be easily accessible to the user at any time. The in-bar search query portion allows a sleek handling of accepting search queries, without covering the entire screen with a bloated pop up, or redirecting the user immediately to a new activity. The page control buttons house a function which may be repeated several times in quick succession, and would be cumbersome to require a second click to reach each time. The icons used are part of the standard android release, and are meant to be standardized for various application functions based on their images to facilitate consistent user experiences and ease of understanding.

This application utilizes the swipe gesture to provide an alternative to the 'previous' and 'next' page buttons, Swiping left or right across the screen over a list of logs will trigger the same action as the next and previous buttons would, respectively, as if flipping the page of a book. Also, on the cellular layout, when viewing a single $\log$ in the detailed view, swiping left or right will progress to the next or previous log in the designated logbook. This is intended to prevent the application from requiring the user to navigate backward and forward between the list and detailed views when viewing logs in quick succession.

The application is compatible with all versions of the Android operating system after release 7 . This is expected to reach at least $95 \%$ of the android devices currently in use. It manages to achieve this while maintaining many of the features not released until later versions, such as the Action Bar, by implementing support packages like Action Bar Sherlock. These mechanisms allow it to maintain the same look and feel of navigating a new application on the newer devices, while keeping with the original design goal of accessibility to users.

This project was supported in part by the U.S. Department of Energy, Office of Science, Office of Workforce Development for Teachers and Scientists (WDTS) under the Science Undergraduate Laboratory Internships Program (SULI). 


\title{
Urban Atmospheric Tracer Sampling: Samples throughout NYC
}

\author{
Nicholas Smith \\ Engineering, Tallahassee Community College, Tallahassee, FL 32333 \\ John Heiser and Rick Wilke \\ Environmental Sciences, Brookhaven National Laboratory, Upton NY 11973
}

\begin{abstract}
As an economic cornerstone, New York City has to be wary of constant terrorism acts that would hope to slow its economic growth, but through the New York Police Department (NYPD) anti-terrorism unit's funding of the S-SAFE (Subway-Surface Air Flow Experiment) project these fears can be lessened. The S-SAFE project is the release of non-toxic purflourocarbons (PFT) into the subway system to see how gases would disperse through the city. In order to read the amounts of PFTs in the air, we used 200 sampler units that captured the density of PFT's in the air samples. When the three days of surveys had been completed, we would have over $\mathbf{7 2 0 0}$ samples waiting to be analyzed using a gas chromatograph. The gas chromatograph ran the samples to see the parts per trillion of the PFTs that were present in the atmosphere after the release of the agent. Based upon the amounts of the PFT in the air in these samples, the NYPD can determine where the most affected areas of the city will be if an attack were ever to occur.
\end{abstract}

The goal of this experiment through Brookhaven National Laboratory is to ensure not only the economic security of the U.S., but also our national security. I have been working specifically with the gas chromatograph in order for it to be prepared to run the samples. I cleaned out each sample tube in preparation for it to be used in the experiment. Mechanical Engineering directly coincides with the work I am doing with the Gas Chromatograph. The electron trap on the gas chromatograph is designed with a particular circumference and material to allow the flow of the air samples through to the detector; which has prepared me for the materials portion of my degree.

The Subway-Surface Air Flow Experiment (S-SAFE) had many parts and processes throughout that needed to be completed in order to have viable results. The first part was the Capillary Absorption Tracer Samplers (CATS) which had to be cleaned from all outside contaminants through use of bake out trays. Another of elements in the process were the Brookhaven Air Tracer Samplers (BATS) that had to be loaded with clean CATS, and sealed correctly in order to not cut off any air flow to the tubes during the experiment. The BATS then had to be programmed correctly to take samples at the specific time for the correct duration. The units were then transported into the city and deployed to over 600 location during the 3 days of testing.

After the conclusion of the testing, the BATS were unloaded of their CATS, and the CATS were then run on the Gas Chromatograph (GC). The GC determines the density of PFT in the air samples. These densities can then be compared to the amount released and see how quickly the gas dissipated and how long it took to get to other parts of the city.

A critical role was that of the CATS and how the air was thrust through them during the experiment. Because of the amber- sorb inside of them, they were effective in the pickup of the PFTs.

"Ambersorb, ${ }^{\circledR}$ a line of carbonaceous resins developed and patented by Rohm and Haas (Neely, 1997 and Maroldo et al., 1989), is created from the partial pyrolysis of macroporous sulfonated styrene-divinylbenzene ion-exchange resin (Parker, 1992)..... All of the resins come in the form of spherical beads with diameters of approximately $1 \mathrm{~mm}$ or less....The flexible polymeric structure of synthetic sorbents, compared to the rigid structure of GAC, allows resins to be regenerable through steam stripping or microwave irradiation. When resin beads are heated using steam, for example, the polymeric matrix relaxes or loosens and the pores widen. As the pores widen, they become accessible to steam, which can then re-solubilize formerly adsorbed organic compounds. In addition, heating the resin and widening the resin pores can volatilize pockets of condensed organic compounds in micropores." (Flores 1999)

To prepare them for this step, they had to be decontaminated from any previous use. The only way to clean the ambersorb from the inside of the CATS tube here was to bake out the tubes at $400^{\circ} \mathrm{C}$ while flowing pure nitrogen through all of the tubes on the rack. The natural liquid in the air caused steam to be a byproduct of the super heating, and because of this allowed for the spherical beads to be cleaned of all outside contaminants except for the nitrogen being cycled through. Nitrogen was selected as the gas to be used to during cleaning because it has a relatively low reactivity. Once finished the tubes were capped and placed in trays waiting to be loaded into the samplers.

The BATS units were then opened and the mounting brackets were unscrewed from the wall of the unit. The mounting bracket was raised, and used CATS were replaced with new baked out CATS. Once the baked out CATS were in place the bracket was lowered until all the tubes were nestled inside the O-rings and screwed into place. The BATS units were then fully loaded with samples and ready to be programmed. The programming was started by turning on the processor inside the unit and enabling the WIFI. Once the WIFI had been enabled, the computers would then connect and program a start time and end time for all the units to cycle through their twelve tubes. It would also schedule the duration for each tube; the flow meter which cycled air through, and the internal clock of the machines. The use of WIFI allowed a task that would take about 5 minutes per unit and made it a task that takes about a minute. A savings of countless man hours.

Once all the BATS were programmed they were deployed to Manhattan for the Intensive Operational Periods (IOP). There were three IOPs over a three week period. The IOPs were based upon the weather patterns that were similar to the project models. The IOPs moved from the northeast to the southwest through each day. Each IOP had 200 samplers deployed taking 12 samples each over the six hour period after the release. After each IOP the sampling units were unloaded and reloaded with fresh CATS tubes 
for the next IOP. We collected 2400 samples during each IOP, and transported them back to the lab for analysis in the GC.

"Gas Chromatography is a means to separate different aspects of a substance from another one" (Gas Chromatography n.d.). A GC analyzes the density of the PFT in the air sample taken and can be used to find the dissipation rate of the gas into the atmosphere if the initial amount released is known. Key to this process is that there be a background sample. The GC had several complications that had to be fixed before the results could be run, and the main issue was with the Electron Capture Detector (ECD).

"The ECD uses a radioactive Beta emitter (electrons) to ionize some of the carrier gas and produce a current between a biased pair of electrodes. When organic molecules that contain electronegative functional groups, such as halogens, phosphorous, and nitro groups pass by the detector, they capture some of the electrons and reduce the current measured between the electrodes." (University of Adelaide, Australia n.d.)

The part of the ECD that was causing issues was the main fluorouracil trap, due to poor contacts it was not showing that the electronegative molecules were being absorbed, so the detector could not read any of the samples. This caused current to be increased to the trap until it would eventually burn out and would register all the samples run as blanks.

Once the ECD was correctly regulated, then the samples were read through the GC and they appeared as a graph of the substances in the air. The GC could not identify what substance was represented by each peak in the graph, so it was important to write into the program the ability to differentiate which substance was which PFT. The samples ran for 15 minutes on the GC and the PFTs with the least mass were seen first; then the PFTs with the most mass were seen last. This happened because they would have to be heated to a certain temperature to get the larger PFTs to react with the beta particles, and that would happen at the latter stages of the 15 minute period. Seen below is an actual Chromatogram from the testing periods.

The Chromatogram is then processed, and all the peaks and lows are converted into numerical form and transferred to an Excel worksheet. In Excel, through macros and other features, the samples are turned into a chart as a parabolic function, showing the degradation of the PFT over time in the air. The parabolic functions show the critical points (in time or in location?) that were the most affected, and the areas that even after a substantial amount of time will have amounts of the tracer still intact. Dependent upon how negative the slope of the function is, is how we can determine the length of time required to say that the tracer is in the air. This same set of data shows that depending on how

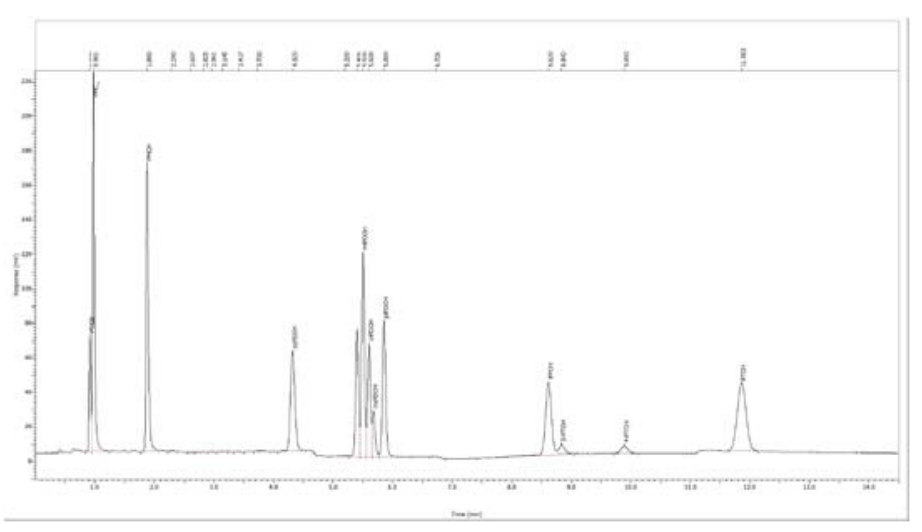

positive the slope is after the release of the first sample, it can be determined whether the area is a critical point.

The graph above shows some interesting and unanticipated results. Please note that due to security concerns, some of the facts such as the type of PFT; which subway tracks this was taken at; and where the release points were, have had to be omitted. The expected outcome was to see one PFT and in reality two PFTs reached this one subway station. It is not known why this happened, but through joint efforts of Rick Wilke and Dr. Tom Watson it has been stipulated that it could possibly be that the trains acted as pistons in an engine. The PFT bunches up in a certain area then the train forces it through the tunnel to all the other stops. Through this experiment many different aspects of the subway's air flow have been uncovered that were unknown before.

The project was directly funded by the NYPD antiterrorism unit as a means of preparing against an act of terrorism in the form of an airborne toxin release in New York City. Due to the severity of our results they will not be published or released to anyone other than the NYPD antiterrorism unit and a few select participants. These few individuals outside of the NYPD will be allowed to analyze the results. They will be the people who run all the samples, and compare those with the original background samples. The goal of this project was to deliver a preventative strike against any acts of terrorism and it was successfully achieved during the summer of 2013.

\section{Works Cited}

Flores, Amparo. 1999. "Synthetic Resin Sorbents." National Water Research Institute. Accessed August 3, 2013. http://www.nwri-usa.org/pdfs/TTChapter5Resins.pdf.

n.d. Gas Chromatography. Accessed August 1, 2013. http:// www.gaschromatography.org/usesofgaschromatography. html.

University of Adelaide, Australia. n.d. Electron Capture Detector. Accessed July 31, 2013. http://www.chemistry. adelaide.edu.au/external/soc-rel/content/ecd.htm.

PFT Time Series at Sta "R" IOP 3 July 92013

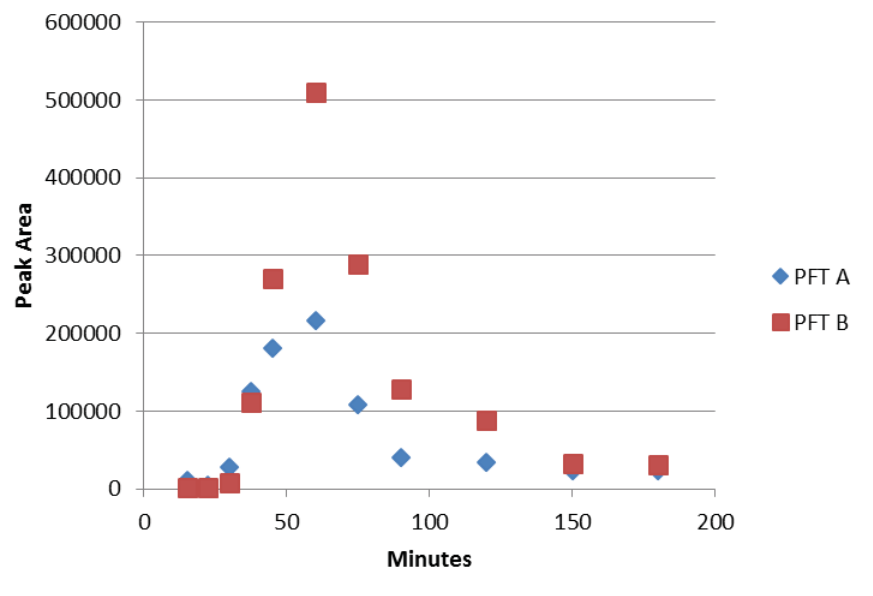




\title{
From massive magnets to molten glass: My summer reporting on science at Brookhaven National Laboratory
}

\author{
Nicholas St. Fleur \\ Cornell University, Ithaca, NY 14850 \\ Karen Walsh \\ Brookhaven National Laboratory, Upton, NY 11973
}

- Education News $\mid$ Summer Students Get Tips on Telling the Stories ... Center for Functional Nanomaterials) and Mei Bai (Collider-Accelerator Department), and science writers Karen McNulty Walsh and Nicholas St. Fleur (himself an www.bnl.gov/education/news/news.asp? $\mathrm{a}=4147 \& \mathrm{t}=$ today $-23 \mathrm{k}$

- BNL Newsroom | Brookhaven Researchers Use Weekends to Teach Math ... By Nicholas St. Fleur | September 10, 2013 printer icon Print. Brookhaven Researchers Use Weekends to Teach Math and Physics at SchoolNova. ... www.bnl.gov/newsroom/news. php? $\mathrm{a}=24271-41 \mathrm{k}$

- BNL Newsroom | Shedding New Light on the 'Electron Highways' of ... Contacts: Peter Genzer, (631) 344-3174 | Written by Nicholas St. Fleur printer icon Print. Shedding New Light on the 'Electron Highways' of Organic Solar Cells. ... www.bnl.gov/ newsroom/news.php? $\mathrm{a}=11567$ - 34k

- BNL Newsroom | Scientists Set Off for Alaska to Study Climate ... By Nicholas St. Fleur | July 11, 2013 printer icon Print. Scientists Set Off for Alaska to Study Climate Change. The Arctic Circle. ... www.bnl.gov/newsroom/news.php?a=24128 - 41k

- BNL Newsroom | Scientists Use X-rays to Connect Mantle Chemistry ... By Nicholas St. Fleur | June 25, 2013 printer icon Print. Scientists Use X-rays to Connect Mantle Chemistry with Carbon Cycle. Study ... www.bnl.gov/newsroom/news.php?a=24076 $41 \mathrm{k}$

- BNL | Center for Functional Nanomaterials (CFN) | Shedding New ... Contacts: Peter Genzer, (631) 344-3174 | Written by Nicholas St. Fleur printer icon Print. Shedding New Light on the 'Electron Highways' of Organic Solar Cells. ... www.bnl.gov/CFN/ news/news.php?a $=11567-36 \mathrm{k}$

- Photon Sciences News | Scientists Use X-rays to Connect Mantle ... By Nicholas St. Fleur | June 25, 2013 printer icon Print. Scientists Use X-rays to Connect Mantle Chemistry with Carbon Cycle. Study ... www.bnl.gov/ps/news/news.php?a=24076 - 37k

- BNL | Center for Functional Nanomaterials (CFN) | Shedding New ... Contacts: Peter Genzer, (631) 344-3174 | Written by Nicholas St. Fleur printer icon Print. Shedding New Light on the 'Electron Highways' of Organic Solar Cells. ... www.bnl.gov/cfn/news/ news.php? $\mathrm{a}=11567$ - 36k

- Photon Sciences News | Scientists Use X-rays to Connect Mantle ... By Nicholas St. Fleur | June 25, 2013 printer icon Print. Scientists Use X-rays to Connect Mantle Chemistry with Carbon Cycle. Study ... www.bnl.gov/ps/news/news.php?a=24076 - 37k

- BNL Newsroom | Summing Up a Summer of Fun and Science for Students ... By Nicholas St. Fleur | August 28, 2013 printer icon Print. Summing Up a Summer of Fun and Science for Students at Brookhaven Lab. ... www.bnl.gov/newsroom/news. php? $=24231-42 k$

- BNL Newsroom | The Big Move: A Last Look at the Muon g-2 Ring’s ... By Nicholas St. Fleur |August 8, 2013 printer icon Print. The Big Move: A Last Look at the Muon g-2 Ring's Departure from Brookhaven. ... www.bnl.gov/newsroom/news.php?a=24180 $-43 \mathrm{k}$ 


\title{
Electrochemical and spectrophotometric studies of conjugated polymers
}

\author{
Hosts: Drs. John Miller and Steven Feldberg \\ Mentor: Alvin A. Holder \\ Interns: Ms. Altramiese Tippie and Ms. Tiera Corey
}

\begin{abstract}
Conjugated polymers are materials of interest for alternative energy applicators as active materials for solar cells because they are more easily processed and cheaper than the more commonly used silicon. In this study, we have collected and contrasted the cyclic voltammetry (CV) results for a conjugated polymer called poly(3-decylthiophene-2,5-diyl) (P3DT) a molecule of nearly similar reduction potential, fluoranthene (FLA), and a second polymer, poly[(9,9-di-n-octylfluorenyl-2,7-diyl)-alt-(benzo[2,1,3]thiadiazol-4,8-diyl) (F8BT). The $\mathrm{CV}$ for each species was acquired in tetrahydrofuran (THF) with tetrabutylammonium hexafluorophosphate (TBAPF ${ }_{6}$ ) $(0.10 \mathrm{M})$ as the supporting electrolyte in the presence of an $\mathrm{Ag} / \mathrm{Ag}^{+}$reference electrode and glassy carbon as the working electrode. The $E_{1 / 2}$ values for the FLA/FLA' and P3DT/P3DT redox couples were found to be $-\mathbf{2 . 5 0} \mathrm{V}$, while the value for the F8BT/F8BT redox couple was found to be $-2.14 \mathrm{~V}$. The diffusion coefficient for the $\mathrm{Fc}^{0 /+}$ redox couple in the presence of glassy carbon working electrode was determined as $2.1 \mathrm{x}$ $10^{-5} \mathrm{~cm}^{2} \mathrm{~s}^{-1}$ in the presence of the supporting electrolyte, 0.10 $\mathrm{M}$ tetrabutylammonium hexafluorophosphate and $\mathrm{a} \mathbf{A g} / \mathbf{A g}^{+}$ reference electrode. Spectrophotometric studies were carried out using the Laser Electron Accelerator Facility (LEAF). The $K_{\mathrm{eq}}$ value obtained for an electrolyte environment of $0.1 \mathrm{M}$ $\mathrm{TBAPF}_{6}$ was $34.7 \pm 5.5$, for which a $\Delta \mathrm{G}$ of $\mathbf{- 0 . 1} \mathrm{eV}$ was determined.
\end{abstract}

\section{Background}

In recent years, energy-related issues have received considerable attention concerning the rising costs of fossils fuels and the growing emission global greenhouse gases. ${ }^{1}$ There is an urgent need to develop clean and renewable energy technologies in order to circumvent these effects. The largest potential source of renewable energy is the solar energy incident on the Earth's surface. ${ }^{2}$ Solar cells are devices which convert solar energy directly into electricity, and the most common material used for solar cells is silicon, ${ }^{3}$ but today, the most widely studied organic solar cells use conjugated polymers due to their low cost and high processability. ${ }^{4-6}$

Polymer-based solar cells have attracted considerable attention as a promising long-term solution for clean, renewable energy due to advantages including simple and low cost fabrication, flexibility and lightweight. ${ }^{7}$ The utilization of the photovoltaic effect to generate electricity from solar energy has been hailed as an appealing solution to our growing need for clean, abundant, and renewable energy sources, and to our desire for protecting the environment. Over the last five years, organic photovoltaic devices have emerged as a new competitor to silicon-based solar cells.

For many years, chemists have been trying to understand the nature of charge distribution, delocalization, and charge carriers in conjugated polymers. 8 As such, the horizons for delocalization broadened with the advent of conjugated polymers, where in these molecules the concept of a bare charge carrier does not fully account for experimental observations. Instead, charges and excitons in conjugated molecules are thought to form polarons:8 quasiparticles composed of charges and their accompanying polarization field. Polarons are the essential charge carriers in solar cells, sensors, and electronic devices, and their natures have impact on charge separation as well as transport. ${ }^{8}$

Experiments $^{9-11}$ and theory ${ }^{10-13}$ on oligomers of definite lengths can yield rich information about what polarons are like for both charges and excitons. ${ }^{8}$ To understand how these species participate in electron-transfer reactions, (both charge separation and recombination) and how they move within and between polymer chains, there remains a need for a deeper understanding of the nature of polarons. Experiments and theory as by Miller and co-workers have sought to seek answers to questions such as: ${ }^{8}$ (1) What are the delocalization lengths of electrons and singlet excitons?, (2) When two electrons are introduced into an oligomer, how do they coexist in Fn chains of varied lengths?, (3) What are the natures of the visible, NIR, and "neutral" absorption bands?, (4) What are the energies (redox potentials) to inject electrons?, and (5) How well do computational methods describe the behaviors of the polarons, bipolarons, and related species? A central goal of the research as by Miller and co-workers ${ }^{8}$ was to gain a better understanding, including a pictorial sense, of what polarons are like.

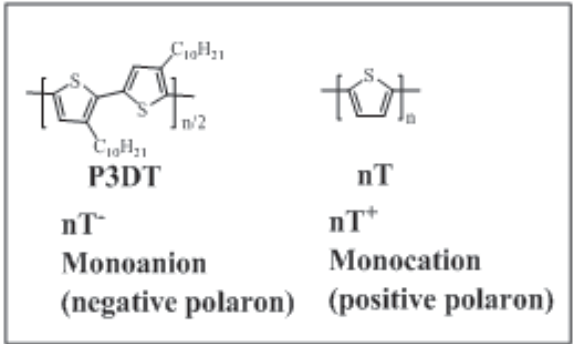

Recently, in a study as reported by Takeda and Miller, ${ }^{14}$ UV-visible absorption spectra of anions and cations of poly(3-decylthiophene) (P3DT) in solution were identified as single polarons. Pulse radiolysis of P3DT in THF determined the spatial extent of one negative polaron to be $\sim 11.5$ thiophene units by observing transient absorption of $\mathrm{P}^{2} \mathrm{DT}^{-}{ }^{-}$radical anions, which are principally free ions, at $850 \mathrm{~nm}$ with $\varepsilon=(7.25 \pm 0.47) \times 104 \mathrm{M}^{-1}$ $\mathrm{cm}^{-1}$ and bleaching of the neutral absorption band at $450 \mathrm{~nm}$. A $\mathrm{P}^{-1} \mathrm{DT}^{-}$radical anion was formed in a combination of diffusive reactions and fast "step" processes. Similarly, a positive polaron of P3DT was estimated to delocalize over $\sim 8.7$ thiophene units by pulse radiolysis in chloroform. Chemical reduction by sodium and oxidation by $\mathrm{FeCl}_{3}$ injected multiple charges into a single P3DT chain, while showing absorption spectra in the early stages of reaction resembling those observed by pulse radiolysis. Take- 
da and Miller ${ }^{14}$ concluded that the results indicated that multiple polarons exist in a single chain of P3DT before coalescing into bipolarons or transforming into other forms of polaron.

The main obstacles to practical applications of conductive polymers are the relatively poor environmental stability (mainly due to their oxidation by oxidation by oxygen and Joule heating) and their short lifetime when electrochemically cycled between oxidation states. Thus the development of new high-performance conducting polymers that possess high chemical, electrochemical, and thermal stability remains a challenge. Various methodologies have been proposed to overcome these problems, including the use of electrolytes (example, ionic liquids) ${ }^{15}$ nanoparticles, and the preparation of new high-performance conductive polymers by designing new monomers and/or controlling the effective $\pi$-conjugation of the polymer backbone. In this proposal, we will use our experience to use electrochemical and spectrophotometric studies on conjugated polymers in order to gather data so that we can design and synthesize more efficient and environmental stable conjugated polymers (with collaborators) that can exhibit the desired properties such as sunlight absorbers and light emitters.

The theory behind rotation disk electrochemistry (RDE):

Rotating disk electrochemistry (RDE) is a technique in which diffusional current is measured in a solution relative to an applied potential, using a rotating working electrode in contrast with a stationary working electrode as used in CV. The working electrode used for rotating disk electrochemistry contains a polished disc which is enclosed by an insulating sheath. The electrode is rotated along the axis perpendicular to the disc during measurement. This rotation provides a well-defined solution flow pattern in the form of a convection path which flows perpendicularly to the working electrode. ${ }^{16}$ This motion provides a steady flow of the detected species to the electrode surface and minimizes the effects of diffusion on transport measurements, making it a steady state measurement. ${ }^{17}$ The Levich current, $\mathrm{I}_{\mathrm{L}}$, being produced by the reduction or oxidation of the electroactive species can be described by the Levich equation:

$$
\mathrm{I}_{\mathrm{L}}=0.62 n \mathrm{~F} A \mathrm{CD}^{2 / 3} v^{-1 / 6} \omega^{1 / 2}
$$

where $\mathrm{n}$ is the number of electrons being transferred per molecule, F is Faraday's constant $\left(96,487 \mathrm{C} \mathrm{mol}^{-1}\right), \mathrm{A}$ is the electrode surface area $\left(\mathrm{cm}^{2}\right), \mathrm{C}$ is the analyte concentration $\left(\mathrm{mmol} \mathrm{cm}^{-3}\right), \mathrm{D}$ is the analyte diffusion coefficient $\left(\mathrm{cm}^{2} \mathrm{~s}^{-1}\right), \mathrm{v}$ is the kinematic viscosity of the solution $\left(\mathrm{cm}^{2} \mathrm{~s}^{-1}\right)$, and $\omega$ is the angular velocity of the rotating disk $\left(\mathrm{s}^{-1}\right)$.

\section{A. The LEAF}

LEAF is a facility equipped with an apparatus capable of performing picosecond and nanosecond-resolution pulse radiolysis. Radiolysis is a pump-probe technique whereby a sample (in our case a solution) is "pumped" with high energy radiation (in our case a $\sim 5 \mathrm{ps}, \sim 20 \mathrm{nC}$ electron pulse) and probed to observe the time-dependent quantitative change of some property of the system (in our case optical absorbance) corresponding with the radiolysis products and their reactions.

In LEAF, THF solutions of polythiophene (p3DT), fluoranthene (FLA), and binary solutions of the two were prepared in sets according to electrolyte content. The set of solutions of primary interest to this study involves the solutions containing $0.1 \mathrm{M}$ $\mathrm{TBAPF}_{6}$ as this was the supporting electrolyte for the CV exper- iments with which the LEAF data is meant to serve as a control. Whereas in the $\mathrm{CV}$ experiments, the number of electrons involved in the reduction process is not certain, in the LEAF experiments for this study, only one-electron reduction occurs. This is because radiolysis of the THF solution results in a byproduct of $\sim 1 \mu \mathrm{M}$ free electrons following ionization of THF molecules, which rapidly solvate and then react at diffusion-controlled rates with solutes (i.e. fluoranthene or polythiophene). In the case of the binary solutions, the concentration of p3DT was fixed at $20 \mu \mathrm{M}$ per chain while fluoranthene was varied in concentration in 5 concentrations ranging from $0.25 \mathrm{mM}$ to $10 \mathrm{mM}$, ensuring that fluoranthene molecules will capture virtually all of the electrons initially, preceding an equilibrium reaction modeled by scheme (1).

Fluoranthene (FLA) and p3DT have very similar reduction potentials, and therefore an equilibrium reaction of the form represented in Scheme 1 should be observable using LEAF and the equilibrium constant of the reaction, $\mathrm{K}_{\mathrm{eq}}$, can be calculated from equation

$$
K_{e q}=\frac{k_{f}}{k_{r}}=\frac{\left[F L A^{\cdot-}\right][p 3 D T]}{[F L A]\left[p 3 D T^{\cdot-}\right]}
$$

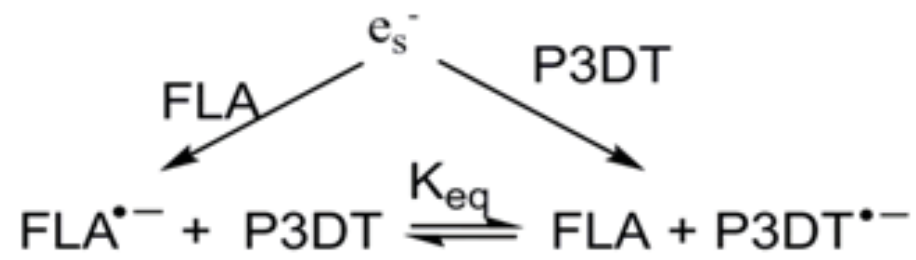

Scheme 1. The reaction scheme for LEAF experiments involving THF solutions containing fluoranthene (FLA) and polythiophene (P3DT), where [FLA] $>$ [P3DT]

In electrochemistry, the quantity in which we are most interested is E, the potential of the system. We can relate this idea of work done in electrochemistry to the thermodynamic concept of work, free energy, through the equation:

$$
\Delta \mathrm{G}=-\mathrm{q} E=-\mathrm{nFE}
$$

where "q" is the charge moved through some potential "E", $\mathrm{n}$ is the number of moles of electrons transferred in a cell reaction (a.k.a. half reaction), and " $F$ " is the Faraday constant - the number of coloumbs per mole of electrons $\left(9.648 \times 10^{4} \mathrm{C} \mathrm{mol}^{-1}\right)$. Also,

$$
\Delta \mathrm{G}=-\mathrm{RT} \ln \mathrm{K}_{\mathrm{eq}}
$$

where $\mathrm{R}$ is the universal gas constant $\left(8.314 \mathrm{~J} \mathrm{~K}^{-1} \mathrm{~mol}^{-1}\right)$, $\mathrm{T}$ is temperature, and $\mathrm{K}$ is the equilibrium constant.

The radiolysis data was used in conjunction with cyclic voltammetric data to try to learn about how many electrons are injected into (or removed from) a polymer chain during reduction (or oxidation) at the working electrode during the acquisition of a CV. In order to observe the reaction, we ideally chose a wavelength for measurement where (if possible) the ratio of extinction coefficients for the two species is $>>$ or $<<1$ (depending on which extinction coefficient is in the numerator).

If only one species absorbs at a given wavelength, its concentration can be easily determined by use of Beer Lambert's Law:

$$
\mathrm{A}=\varepsilon \mathrm{cl}
$$

where $\varepsilon=$ the molar extinction coefficient, $\mathrm{c}=$ the concentration, and $1=$ the path length of the light being absorbed.

The absorbances that we are collecting in LEAF are those of radiolysis products. In this project, for each solution, we wish to observe the effect of salt concentration on the equilibrium con 
stant. The solutions were titrated with varying concentrations of $\mathrm{TBAPF}_{6}$ and the reaction in equation (1) will proceed with different rate constants, $\mathrm{k}_{\mathrm{f}}$ and $\mathrm{k}_{\mathrm{b}}$, and hence a shifting equilibrium constant.

\section{Hypotheses and research objectives and goals}

The main hypotheses are as follows: (i) a conjugated polymer will undergo multiple electron reduction during reduction in a $\mathrm{CV}$ scan, (ii) CVs of conjugated polymers are broad and featureless when acquired in non-aqueous media and (iii) the idea that the one-electron oxidation (or reduction) creates single polarons, and that the CVs for polymers and oligomers will comprise one- and multi-electron processes.

The main goal of the project is to gain an insight into multi-electron redox processes of conjugated polymers. No, with accurate one-electron potentials and well-characterized CVs in hand we hope to determine a quantitative relationship. We hope to create a mathematical model the CVs, and possibly DPVs, in order to obtain a series of potentials and to create a "map" giving potential versus number of holes (or electrons) injected into a single polymer or oligomers. The model will include least-squares fitting and digital modeling with DigiSIM electrochemical modeling programs.

We hope that the series of measurements we propose will also enable us to determine diffusion coefficients of the oligomers and polymers to be measured, and to delineate their effects on the currents in the CVs.

\section{Experimental:}

\section{A. Materials and methods}

Analytical or reagent grade chemicals were used throughout this study. Poly(3-decylthiophene-2,5-diyl) (P3DT) polymer was obtained from Rieke Metals, Inc. Poly[(9,9-di-n-octylfluorenyl-2,7-diyl)-alt-(benzo[2,1,3]thiadiazol-4,8-diyl) (F8BT) polymer, fluoranthene (FLA), tetrabutylammonium hexafluorophosphate, and tetrahydrofuran were obtained from Sigma Aldrich.

\section{B. Cyclic voltammetry:}

Cyclic voltammetry (CV) on P3DT, fluoranthene and ferrocene were carried out using a CHI Instruments Electrochemical Analyzer. Electrolyte solutions were prepared as 0.1 M TBAPF6 in THF in an argon-saturated glovebox, just prior to use. Analyte solutions of fluoranthene and ferrocene were prepared by directly adding the analytes to electrolyte solution in a Pyrex measurement cell after collecting $\mathrm{CV}$ measurements of the electrolyte solution. The P3DT solutions which were prepared by adding the polymer to electrolyte solution in vials were heated; then soni- cated. The solutions were prepared to achieve a volume of $10 \mathrm{ml}$. After cooling, molecular sieve was added to the vials to dry the solutions. Each CV measurement collected for a solution was preceded by purging with argon for 15 minutes. A glassy carbon working electrode $(3 \mathrm{~mm})$ was polished with $0.05 \mu \mathrm{m}$ alumina (Baikalox), rinsed with THF and carefully wiped and dried with kim wipes before use. A Pt wire and a $\mathrm{Ag} / \mathrm{Ag}^{+}(10 \mathrm{mM} \mathrm{AgNO}$ in $0.1 \mathrm{M} \mathrm{TBAPF}{ }_{6} / \mathrm{THF}$ ) were used as a auxiliary and a reference electrode, respectively. The instrumental resistance compensation was turned off. For calibration of uncompensated resistances, the $\mathrm{CVs}$ of ferrocene were collected at varied concentrations.

\section{Rotation disk electrochemistry}

Cyclic voltammagrams were acquired for P3DT using a rotating disk electrode (RDE) at various rotation rates using a Pine Instrument Company MSRX Speed Control rotator and a CHI Instruments Electrochemical Analyzer. A $2.5 \mathrm{mM}$ per repeat unit concentration of P3DT was prepared by adding the polymer to a $0.1 \mathrm{M}$ TBAPF6 solution in vials which were then heated and sonicated to fully dissolve the polymer. After cooling, $3 \AA$ molecular sieves were added to the vials to dry the solutions. The solution was prepared to achieve a total volume of $45 \mathrm{ml}$. CVs were acquired after purging the solution with argon for 40 minutes.

\section{The LEAF experiments}

Radiolysis data in LEAF were acquired with a $4 \mathrm{GHz}, 20$ GS s-1 Lecroy Waverunner 640Zi Transient Digitizer. Electrolyte solutions of TBAPF6 and TBABF4 at various concentrations containing P3DT and/or fluoranthene were prepared in $0.5 \mathrm{~cm}$ stopcock-sealed optical cells while under argon atmosphere in a glovebox. Data was collected on a $20 \mu$ s time scale and at a gain setting of 5 or $10 \mathrm{mV} /$ division.

\section{Results and discussion:}

A. The molecules used in this study:

The molecules used in this study are shown below: FLA FBBT P3DT

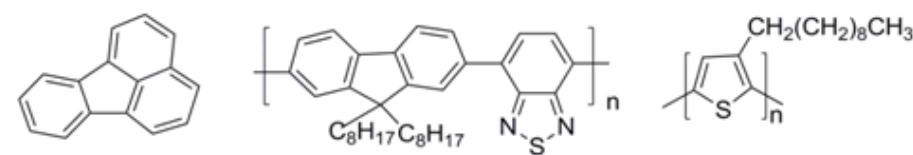

FLA $=$ fluoranthene, F8BT $=$ poly $[(9,9-$ di-n-octylfluorenyl-2,7-diyl)-alt-(benzo[2,1,3]thiadiazol-4,8-diyl, and P3DT = poly(3-decylthiophene)

\begin{tabular}{|r|r|r|r|r|r|}
\hline [ferrocene]/mM & \multicolumn{1}{|c|}{$\mathbf{i}_{\mathbf{p a}} / \boldsymbol{\mu} \mathbf{A}$} & $\mathbf{1 0 6} \mathbf{D} / \mathbf{c m}^{\mathbf{2}} \mathbf{~ s}^{\mathbf{1}}$ & $\mathbf{E}_{\mathbf{p a}} / \mathbf{V}$ & $\mathbf{E}_{\mathbf{p c}} / \mathbf{V}$ & $\mathbf{E}_{1 / 2} / \mathbf{V}$ \\
\hline 1.0 & -27.7 & 21 & -0.04 & -0.28 & -0.16 \\
\hline 2.0 & -46.9 & 15 & 0.02 & -0.32 & -0.15 \\
\hline 3.99 & -77.0 & 10 & 0.11 & -0.39 & -0.14 \\
\hline 5.99 & -98.0 & 7.2 & 0.20 & -0.43 & -0.12 \\
\hline 7.98 & -116.0 & 5.7 & 0.30 & -0.43 & -0.08 \\
\hline
\end{tabular}

Table 1. Tabulation of the calculations for the diffusion coefficient. Solvent $=$ THF, working electrode $=$ glassy carbon, auxiliary electrode $=$ Pt wire, reference electrode $=\mathbf{A g} / \mathbf{A g}^{+}$, Diameter of the working electrode $=3.0 \mathrm{~mm}$, area of the working electrode $=0.071 \mathrm{~cm}^{2}$, and supporting electrolyte $=0.10 \mathrm{M}$ tetrabutylammonium hexafluorophosphate. Scan rate $=100 \mathrm{mV} \mathrm{s}-1$. Temperature $=20.0^{\circ} \mathrm{C}=293.15 \mathrm{~K}$. 


\section{B. Cyclic voltammetry}

\section{Ferrocene:}

CVs were acquired for ferrocene in THF with 0.1 M TBAPF6 as a supporting electrolyte. Figure 1 shows the CVs for ferrocene at various concentrations. The ipa values were found to increase linearly with concentration of ferrocene, but with a constant of proportionality that is less than 1 . The values of $\mathrm{i}_{\mathrm{pa}}, \mathrm{E}_{\mathrm{pa}}, \mathrm{i}_{\mathrm{pc}}$, and $\mathrm{E}_{\mathrm{pc}}$ along with the calculated diffusion coefficients for each concentration of ferrocene are provided in Table 1.

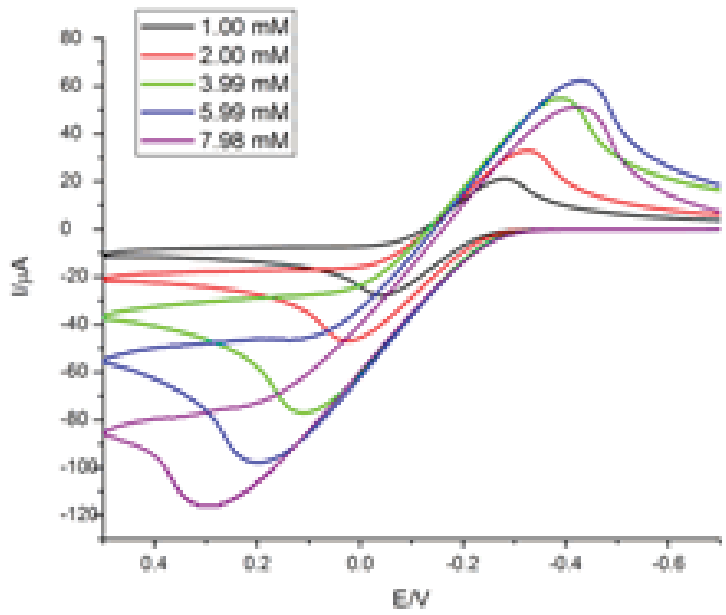

Figure 1. Cyclic voltammagrams of various concentrations of ferrocene in THF. Supporting electrolyte $=0.10 \mathrm{M}$ tetrabutylammonium hexafluorophosphate, working electrode $=$ glassy carbon, auxiliary electrode $=\mathrm{Pt}$ wire, reference electrode $=\mathbf{A g} / \mathbf{A g}^{+}$, and scan rate $=$ $100 \mathrm{mV} \mathrm{s}^{-1}$

It has been proposed that the oxidation of ferrocene, $\left[\mathrm{Fe}\left(\mathrm{C}_{5} \mathrm{H}_{5}\right)_{2}\right]$ to the ferrocenium cation, $\left[\mathrm{Fe}\left(\mathrm{C}_{2} \mathrm{H}_{5}\right)_{2}\right]^{+}$is a standard one-electron transfer reversible process with an incredibly fast rate of electron transfer. For this reason, it has been useful as a standard reference with a well-known redox potential of $+0.64 \mathrm{~V}$ versus standard hydrogen electrode (SHE)18 Therefore, it is expected that values of ipa should be linear with the concentration of ferrocene, which they are but with a proportionality constant that is less than one due to uncompensated resistance.

Ferrocene also serves as a good calibration standard from which uncompensated resistance can be determined since both uncompensated resistances and slow heterogeneous electron transfer are contributing factors to concentration-dependent separation between forward and reverse current peak potentials.

Diffusion coefficients (D) and $\mathrm{E}_{1 / 2}$ values of $2 \mathrm{mM}$ ferrocene as obtained by Tsierkezos 18 in various non-aqueous solvents were compared with the one calculated in this study using THF. It should be noted that the values obtained by Tsierkezos for $\mathrm{D}$, increases according to solvent in the following order: DMSO $<$ NMK $<$ DMF $<$ PEN $<$ DCM $<$ CAN $<$ ACE (see Table 2). This order suggests inverse dependence on absolute viscosity with the $\mathrm{D}$ values as predicted by the Stokes-Einstein equation:

\section{$\mathrm{D}=\mathrm{kBT} / 6 \pi \mathrm{c} \eta \mathrm{r}_{\mathrm{h}}$}

where $\mathrm{k}$ is Boltzmann's constant, $\mathrm{T}$ is temperature in Kelvin, $r_{h}$ is the effective radius of the diffusing species, $\eta$ is absolute viscosity of the solvent medium, and $\mathrm{c}$ is a constant. According to the same equation, the value of D for ferrocene in THF is expected to be between that of dichloromethane and pentanone, and according to our calculation of D in THF, our result finds agreement with that expectation.

\section{P3DT and FLA \\ 1. P3DT}

CVs were acquired for P3DT in THF. Figure 2 shows a typical CV in THF with 0.1 M TBAPF6 for P3DT with a starting potential sufficiently positive to observe formation of $\mathrm{p} 3 \mathrm{DT}^{*+}$, while figure 3 shows the overlay of the CVs for $4.0 \mathrm{mM}$ fluoranthene and $4.0 \mathrm{mM}$ per repeat unit (PRU) of P3DT. A reversible redox reaction is observed, with an $\mathrm{E}_{1 / 2}$ of $-2.5 \mathrm{~V}$ for the redox couple. The fact that $\mathrm{E}_{\mathrm{pa}}$ is smaller in magnitude than Epc may indicate that the reduced P3DTn - (where ' $n$ ' is the unknown number of electrons added during reduction) is not fully stable.

According to the Randles-Sevcik equation

$i_{p}=0.4463 n \mathrm{FAC}(n \mathrm{FvD} / \mathrm{RT})^{1 / 2}$,

where $i_{p}=$ current maximum in amps

$n=$ number of electrons transferred in the redox event for a reversible process
$\mathrm{A}=$ electrode area in $\mathrm{cm} 2$
$\mathrm{F}=$ the Faraday Constant in $\mathrm{C}$ mol-1
$\mathrm{D}=$ diffusion coefficient in $\mathrm{cm} 2 \mathrm{~s}-1$
$\mathrm{C}=$ concentration in $\mathrm{mol} \mathrm{cm}-3$
$v=$ scan rate in $\mathrm{V} \mathrm{s}-1$

\begin{tabular}{|c|c|c|}
\hline Solvent & $105 \mathrm{D} / \mathrm{cm}^{2} \mathrm{~s}^{-1}$ & $\mathrm{E}_{1 / 2} / \mathrm{V}(\mathrm{vs} \mathrm{Ag} / \mathrm{AgCl})$ \\
\hline Acetonitrile & $2.24 \pm 0.06$ & $\mathrm{~N} / \mathrm{A}$ \\
\hline Acetone & $2.76 \pm 0.07$ & 1.020 \\
\hline N-methylformamide & $0.54 \pm 0.01$ & N/A \\
\hline $\mathrm{N}, \mathrm{N}$-dimethylformamide & $0.95 \pm 0.02$ & 0.951 \\
\hline N,N-dimethylacetamide & $0.76 \pm 0.02$ & N/A \\
\hline 3-pentanone & $1.41 \pm 0.04$ & $\mathrm{~N} / \mathrm{A}$ \\
\hline Dimethyl sulfoxide & $0.44 \pm 0.01$ & 0.869 \\
\hline Dichloromethane & $1.67 \pm 0.05$ & 1.125 \\
\hline THF & 2.1 & $-0.15^{\mathrm{a}}$ \\
\hline
\end{tabular}

Table 2. Diffusion coefficients and $\mathrm{E}_{1 / 2}$ values for $2 \mathrm{mM}$ ferrocene solutions in various solvents (collected for $2 \mathrm{mM}$ ferrocene solutions at 298.15 K).

${ }^{\text {a}}$ From our work with a $\mathrm{Ag} / \mathrm{Ag}+$ reference electrode and $2.0 \mathrm{mM}$ ferrocene at $20^{\circ} \mathrm{C}(298.15 \mathrm{~K})$. 
The $\mathrm{E}_{1 / 2}$ values for the redox couples of both FLA/FLA ${ }^{-}$and $\mathrm{P} 3 \mathrm{DT} / \mathrm{P} \mathrm{DT}^{-}$were determined to be $-2.50 \mathrm{~V}$ versus a $\mathrm{Ag} / \mathrm{Ag}^{+}$reference electrode, but the ip values of fluoranthene are significantly larger than those of P3DT at $4.0 \mathrm{mM}$ fluoranthene and $4.0 \mathrm{mM}$ (PRU) P3DT. The CVs for both were collected under identical experimental conditions, therefore the only variables affecting the ip were the diffusion coefficients and the number of electrons transferred during the redox event. Due to the fact that P3DT is so much larger than fluoranthene, the diffusion coefficient of P3DT is an order of magnitude smaller. Additionally, because an excess electron on P3DT occupies approximately 11 monomer units whereas each molecule of fluoranthene is capable of accepting an electron, $n_{\mathrm{P} 3 \mathrm{DT}} \leq(1 / 11) * n_{\mathrm{FLA}}$. Due to these two factors, the ipc of the $4.0 \mathrm{mM}$ P3DT is expected to be substantially smaller than the $i_{p c}$ of the equivalent molecular concentration of fluoranthene. Comparison of the $i_{p c}$ values of p3DT at $4.0 \mathrm{mM}$ with other concentrations of P3DT shows a linear relationship. A graph corresponding with the CVs of P3DT at various concentrations is shown in Figure 4.

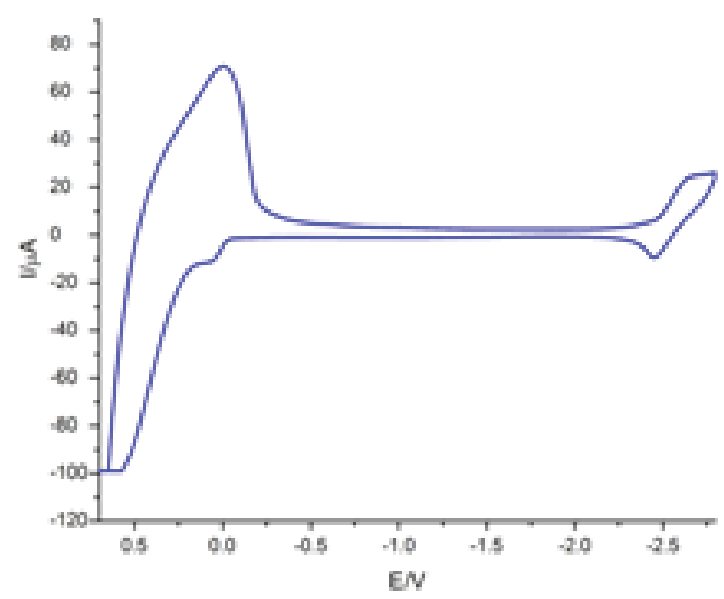

Figure 2. A CV of P3DT mM (PRU) in THF. Supporting electrolyte $=0.10 \mathrm{M}$ tetrabutylammonium hexafluorophosphate, working electrode $=$ glassy carbon, auxiliary electrode $=\mathrm{Pt}$ wire, reference electrode $=\mathbf{A g} / \mathbf{A g}^{+}$, and scan rate $=100 \mathrm{mV} \mathrm{s}{ }^{-1}$

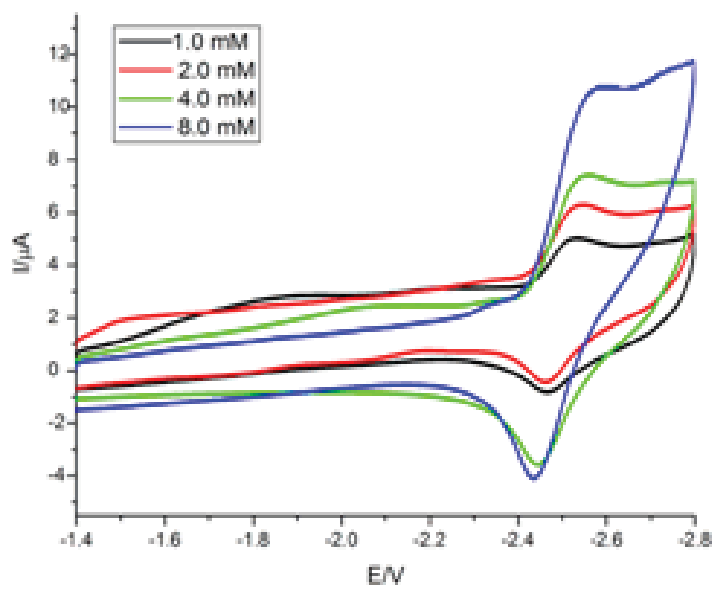

Figure 4. CVs of various concentrations of P3DT in THF are shown. Supporting electrolyte $=0.10 \mathrm{M}$ tetrabutylammonium hexafluorophosphate, working electrode $=$ glassy carbon, auxiliary electrode $=$ $\mathrm{Pt}$ wire, reference electrode $=\mathbf{A g} / \mathbf{A g}^{+}$, and scan rate $=100 \mathrm{mV} \mathrm{s}^{-1}$
The Randles-Sevcik equation was used to calculate theoretical values for DP3DT by assuming values of $\mathrm{n}$ and assuming a concentration of $21.7 \mu \mathrm{M}$ (or $8 \mathrm{mM}$ PRU). The results are tabulated in Table 3. Work is in progress with modeling via Igor and DigiSim in order to determine the diffusion coefficient and the number of electrons for the P3DT/P3DT redox couple of $-2.50 \mathrm{~V}$.

\begin{tabular}{|l|l|l|l|}
\hline$[\mathrm{P} 3 \mathrm{DT}] / \mu \mathrm{M}$ & $\mathrm{n}$ & $\mathrm{D} / \mathrm{cm}^{2} \mathrm{~s}^{-1}$ & $i_{p c} / \mu \mathrm{A}$ \\
\hline 21.7 & 1 & $3.43 \times 10^{-3}$ & 7.17 \\
\hline 21.7 & 10 & $2.94 \times 10^{-6}$ & 7.17 \\
\hline 21.7 & 20 & $3.68 \times 10^{-7}$ & 7.17 \\
\hline 21.7 & 40 & $4.60 \times 10^{-8}$ & 7.17 \\
\hline
\end{tabular}

Table 3. Tabulated diffusion coefficient values calculated for assumed values of $n$. ip = peak current, $A ; a=0.071 \mathrm{~cm}^{2} ; F=9.65 \times 104 \mathrm{C}$ $\mathrm{mol}^{-1} ; \mathbf{c}=21.7 \times 10^{-9} \mathrm{~mol} \mathrm{~cm}^{-3} ; \mathbf{T}=298.15 \mathrm{~K} ; v=0.1 \mathrm{~V} \mathrm{~s}^{-1} ; \mathbf{R}=8.314 \mathrm{~J}$ $\mathrm{mol}^{-1} \mathrm{~K}^{-1}$; and $\mathrm{D}=$ diffusion coefficient, $\mathrm{cm} 2 \mathrm{~s}^{-1}$.

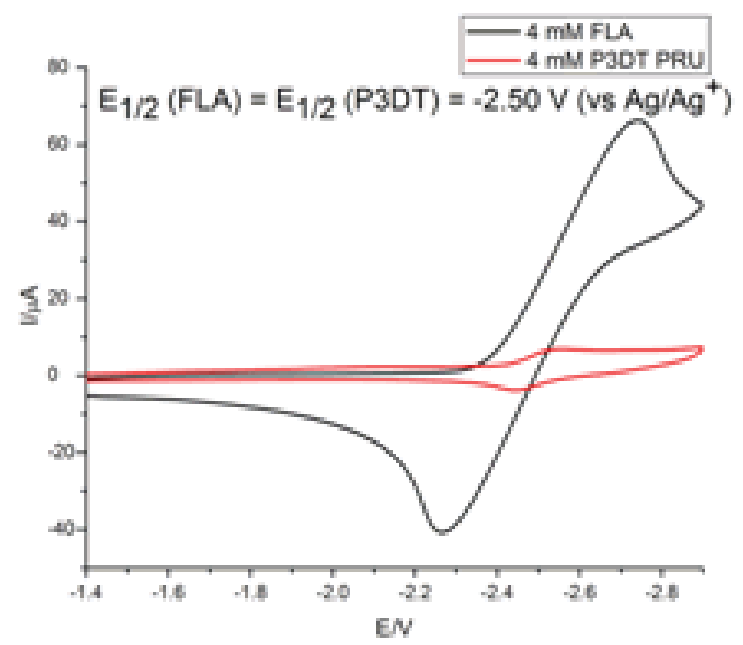

Figure 3. A CV of $4.0 \mathrm{mM}$ fluoranthene and $4.0 \mathrm{mM}$ P3DT PRU in THF. Supporting electrolyte $=0.10 \mathrm{M}$ tetrabutylammonium hexafluorophosphate, working electrode $=$ glassy carbon, auxiliary electrode $=\mathrm{Pt}$ wire, reference electrode $=\mathrm{Ag} / \mathrm{Ag}+$, and scan rate $=100 \mathrm{mV} \mathrm{s}^{-1}$

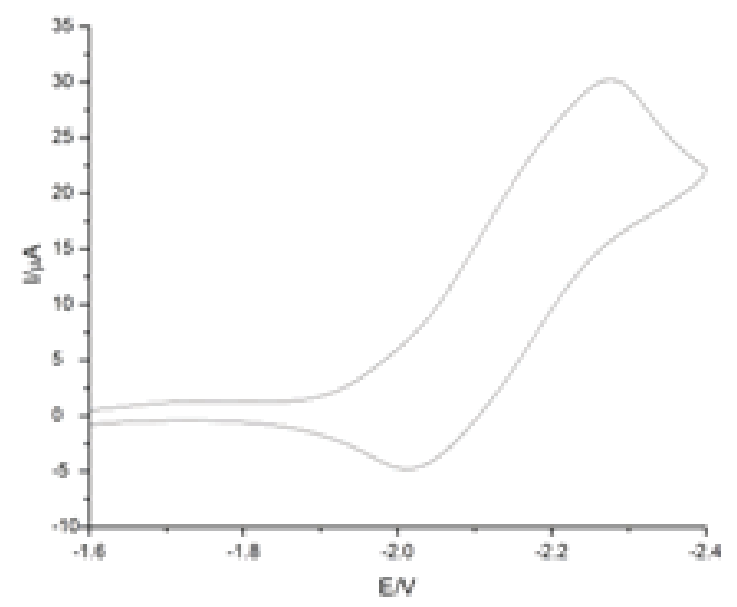

Figure 5. A CV of a 5.0 mM RPU F8BT solution in THF. Supporting electrolyte $=0.10 \mathrm{M}$ tetrabutylammonium hexafluorophosphate, working electrode $=$ glassy carbon, auxiliary electrode $=$ Pt wire, reference electrode $=\mathrm{Ag} / \mathrm{Ag}^{+}$, and scan rate $=100 \mathrm{mV} \mathrm{s}^{-1}$ 
A CV was acquired for $1.09 \mathrm{mM}$ fluoranthene in THF from which several parameters were calculated. The diffusion coefficient was calculated as $6.14 \times 10-6 \mathrm{~cm}^{2} \mathrm{~s}^{-1}$ for the reversible $\mathrm{CV}$ where the $\mathrm{E}_{1 / 2}\left(\mathrm{FLA} / \mathrm{FLA}^{-}\right)=-2.53 \mathrm{~V}$ versus $\mathrm{Ag} / \mathrm{Ag}^{+}$. Interestingly, with a $4.0 \mathrm{mM}$ FLA, the $\mathrm{E}_{1 / 2}$ value was calculated as $-2.50 \mathrm{~V}$ for the FLA/FLA- redox couple.

A literature reference for the reduction potential for fluoranthene has not yet been identified for comparison.

\section{A. F8BT}

The $\mathrm{E}^{1 / 2}$ value for the F8BT/F8BT- redox couple was determined as $-2.14 \mathrm{~V}$ (see Figure 5). The CV shows great reversibility and broad waves somewhat similar to those observed in CVs of P3DT as reported by Dong et al.19 Interesting enough, the authors did not report the type of working electrode used in this study, but they carried out the study with films on the working electrode in acetonitrile/ $\mathrm{CH}_{2} \mathrm{Cl}_{2}$.

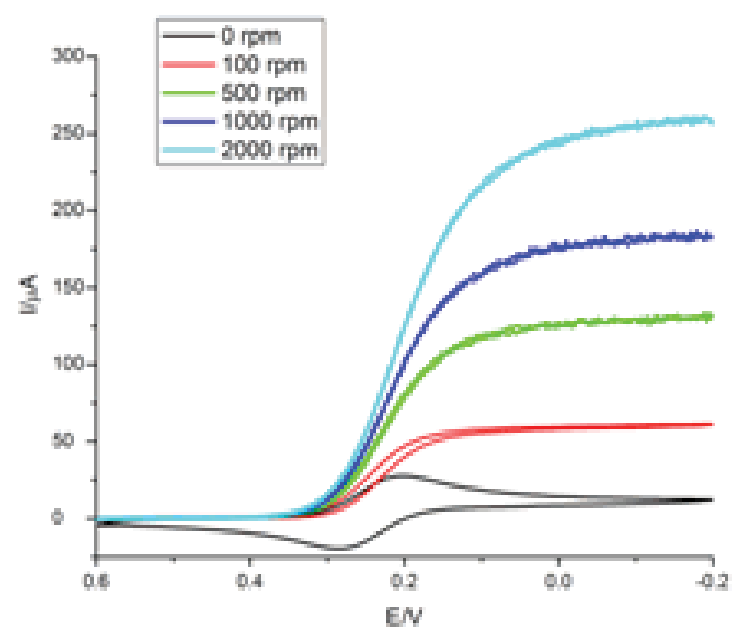

Figure 6. A CV of a $1.9 \mathrm{mM}\left[\mathrm{K}_{3} \mathrm{Fe}(\mathrm{CN})_{6}\right]$ in THF. Scan rate $=10 \mathrm{mV}$ $\mathbf{s}^{-1}$, working electrode $=$ glassy carbon, auxiliary electrode $=\mathbf{P t}$ wire, reference electrode $=\mathrm{Ag} / \mathrm{AgCl}$, and supporting electrolyte $=1.0 \mathrm{M}$ $\mathrm{KCl}$. Area of the working electrode $0.07065 \mathrm{~cm}^{2}$, and diameter $=$ $5 \mathrm{~mm}$.

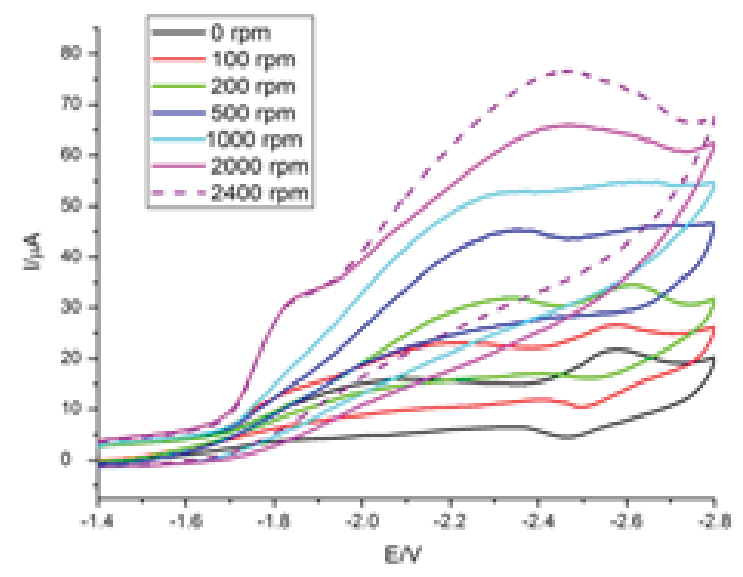

Figure 7: An overlay of rotating disk electrode voltammagrams for a $2.5 \mathrm{mM}$ (PRU) solution of P3DT in THF. Working electrode = glassy carbon, auxiliary electrode $=\mathbf{P t}$ wire, reference electrode $=\mathbf{A g} / \mathbf{A g}^{+}$, supporting electrolyte $=0.10 \mathrm{M}$ tetrabutylammonium hexafluorophosphate and scan rate $=50 \mathrm{mV} \mathrm{s}^{-1}$

\section{B. Cyclic voltammetry using an RDE:}

Potassium ferricyanide in an aqueous solution at $1.9 \mathrm{mM}$ served as a reference material for testing the voltage-dependent current response for the RDE measurement system. The supporting electrolyte used was $1.0 \mathrm{M} \mathrm{KCl}$. Figure 6 shows the CVs of potassium ferricyanide at several rotation rates. The resulting voltammagrams for potassium ferricyanide have negligible hysteresis as expected due to the measurement being steady state. The Levich currents, produced at the various rotation rates, allow for the diffusion coefficient of an analyte being accurately determined from the Levich equation due to the precision of the current measurements.

In contrast with the results of the potassium ferricyanide solution, the RDE for the P3DT solution at $2.5 \mathrm{mM}$ (PRU) shown in Figure 7, contains hysteresis that increases rather than decreases with electrode rotation rate. While this result is not yet understood, one possibility considered is that of a change in the distribution spread of the number of electrons per p3DT chain. Another important difference in the RDE voltammagrams of potassium fericyanide and P3DT is the observation of a current plateau in the former, and an increasing current "hill" which continues to increase with more negative potentials. This seems to suggest that P3DT is capable of being further reduced as the potential is pushed even more negative while potassium fericyanide levels after being reduced with one electron.

\section{The LEAF study}

The absorbance transients used for these calculations were measured at $1629 \mathrm{~nm}$ where only P3DT*- absorbs. This facilitated the calculation of $\left[\mathrm{P}^{2} \mathrm{DT}^{*}\right] /\left[\mathrm{FLA}^{*}\right]$ at each concentration of fluoranthene via comparison of the absorbance at equilibrium with the absorbance when fluoranthene is not present. The Keq values were obtained for each fluoranthene concentration and $\Delta \mathrm{G}$ values were calculated from the average of the $\mathrm{K}_{\text {eq }}$ values, with an accompanying standard deviation. For the $0.1 \mathrm{M}$ TBAPF6 electrolyte environment, the average $\mathrm{K}_{\text {eq }}$ was determined as $34.7 \pm 5.5$, and $\Delta \mathrm{G}$ as $-0.1 \mathrm{eV}$.

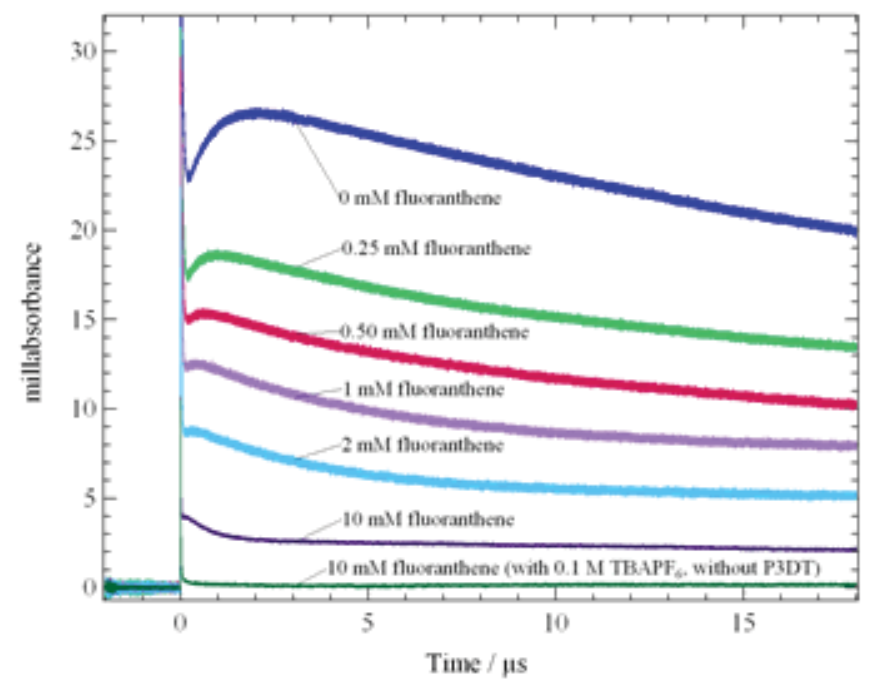

Figure 8. The radiolysis absorbance transients for a series of $20 \mu \mathrm{M}$ (per polymer) P3DT $(n=369)$ solutions containing varied concentrations of fluoranthene, collected at $1629 \mathrm{~nm}$ where only P3DT"- absorbs. 
A $\Delta \mathrm{G}$ of $-0.1 \mathrm{eV}$ corresponds to a potential difference of -0.1 $\mathrm{V}$ which would indicate that the $\mathrm{E}_{1 / 2}$ of the one electron reduced p3DT should be formed at $-2.60 \mathrm{~V}$ rather than the value of -2.50 $\mathrm{V}$ determined for $\mathrm{p}^{\mathrm{D}} \mathrm{T}^{\mathrm{n}-\boldsymbol{-}}$ from the $\mathrm{CV}$. This result along with the calculation of " $n$ " using a reasonable estimated value for the DP3DT in the Randles-Sevcik equation and known values for concentration and ipc of P3DT, suggest that " $n$ " is indeed more than one and perhaps close to 20 .

In addition to the radiolysis measurements performed on binary solutions of fluoranthene and P3DT in $0.1 \mathrm{M} \mathrm{TBAPF}_{6}$, measurements were collected for binary solutions in $0 \mathrm{M} \mathrm{TBAPF}_{6}$ and $0.6 \mathrm{mM} \mathrm{TBAPF}_{6}$. An interesting finding is that the value for the free energy is nearly the same in $0.6 \mathrm{mM}$ as in $0.1 \mathrm{M} \mathrm{TBAPF}_{6}$, while the value of the magnitude of the free energy has increased for the solution containing no electrolyte. This implies that at 0.6 $\mathrm{mM} \mathrm{TBAPF}{ }_{6}$ there is sufficient ion pairing for both FLA $^{-}$and $\mathrm{P}^{2} \mathrm{DT}^{\circ-}$ to make the equilibrium conditions nearly the same. If most of the $\mathrm{FLA}^{-}$were ion paired with $\mathrm{TBA}^{+}$cations and most P3DT"- were not paired, then it is conceivable that the forward reaction rate would decrease substantially, leading to a $\Delta \mathrm{G}$ of even greater magnitude than observed for the case where neither anion is paired when no electrolyte is present. As the cation-paired FLA" $^{*}$ is expected to have a smaller dissociation constant than P3DT*-, there may exist a concentration of electrolyte less than 0.6 $\mathrm{mM}$, where ion pairing is dominant only for FLA" and a further study is underway to test this hypothesis.

\begin{tabular}{|l|r|r|}
\hline Polymer used & \multicolumn{1}{|c|}{$\left[\right.$ TBAPF $\left._{6}\right] / \mathbf{M}$} & \multicolumn{1}{|c|}{$\Delta \mathbf{G} / \mathbf{e V}$} \\
\hline P3DT $(n=170)$ & 0.10 & -0.09 \\
\hline P3DT $(n=170)$ & 0.00 & -0.15 \\
\hline P3DT $(n=170)$ & 0.0006 & -0.10 \\
\hline P3DT $(n=369)$ & 0.10 & -0.10 \\
\hline
\end{tabular}

Table 4. Calculated $\Delta G$ values for binary solutions of P3DT and fluoranthene under various electrolyte conditions.

In the future, further studies will be carried out in order to prove that P3DT conducts a multiple electron redox process which will make it a good replacement for silicon in solar cells. We would like to strive forward into achieving one of our challenging goals which is the acquisition of thermodynamic and one-electron potentials for electrons and holes in the kind of solid film environment in which the polymers would actually be used. Acquiring CVs via film in order to learn more about the polymer's holes and energies of the electrons seems to be of interest as well.

\section{References}

(1) Gratzel, M. Chem. Lett. 2005, 34, 8-13.

(2) Kamat, P. V. J. Phys. Chem. C 2007, 111, 2834-2860.

(3) Shah, A.; Torres, P.; Tscharner, R.; Wyrsch, N.; Keppner, H. Science 1999, 285, 692-698.

(4) Chen, J.-T.; Hsu, C.-S. Polym. Chem. 2011, 2, 2707-2722.

(5) Guenes, S.; Neugebauer, H.; Sariciftci, N. S. Chem. Rev. 2007, 107, 1324-1338.

(6) Thompson, B. C.; Frechet, J. M. J. Angew. Chem., Int. Ed. 2008, 47, 58-77.

(7) Boudreault, P.-L. T.; Najari, A.; Leclerc, M. Chem. Mater. 2011, 23, 456-469.

(8) Zaikowski, L.; Kaur, P.; Gelfond, C.; Selvaggio, E.; Asaoka, S.; Wu, Q.; Chen, H.-C.; Takeda, N.; Cook, A. R.; Yang, A.; Rosanelli, J.; Miller, J. R. J. Am. Chem. Soc. 2012, 134, 10852-10863.

(9) Hill, M. G.; Penneau, J. F.; Zinger, B.; Mann, K. R.; Miller, L. L. Chem. Mater. 1992, 4, 1106-13.

(10) Zade, S. S.; Zamoshchik, N.; Bendikov, M. Acc. Chem. Res. 2011, 44, 14-24.

(11) Fratiloiu, S.; Grozema, F. C.; Koizumi, Y.; Seki, S.; Saeki, A.; Tagawa, S.; Dudek, S. P.; Siebbeles, L. D. A. J. Phys. Chem. B 2006, 110, 5984-5993.

(12) Zade, S. S.; Bendikov, M. J. Phys. Chem. B 2006, 110, 15839-15846.

(13) Jansson, E.; Jha, P. C.; Aagren, H. Chem. Phys. 2007, 336, 91-98.

(14) Takeda, N.; Miller, J. R. J. Phys. Chem. B 2012, 116, 14715-14723.

(15) Lu, W.; Fadeev, A. G.; Qi, B.; Smela, E.; Mattes, B. R.; Ding, J.; Spinks, G. M.; Mazurkiewicz, J.; Zhou, D.; Wallace, G. G.; MacFarlane, D. R.; Forsyth, S. A.; Forsyth, M. Science 2002, 297, 983-987.

(16) Bard, A. J.; Faulkner, L. R. Electrochemical methods : fundamentals and applications; 2nd ed.; Wiley: New York, 2001.

(17) Burgess, K. S. D.; Kable, J. W.; Justice, J. B. Electroanalysis 1999, 11, 337-343.

(18) Tsierkezos, N. G. J. Solution Chem. 2007, 36, 289.

(19) Dong, W.; Xue, S.; Lu, P.; Deng, J.; Zhao, D.; Gu, C.; Ma, Y. J. Polymer Sci. Part A: Polymer Chemistry 2011, 49, 4549-. 


\title{
Growth of tropical cumulus congestus clouds
}

\author{
Katherine Towey \\ Department of Atmospheric and Environmental Sciences, University at Albany, Albany, NY 12222 \\ Michael Jensen and Tami Toto \\ Environmental Sciences Department, Brookhaven National Laboratory, Upton, NY 11973
}

\begin{abstract}
Cumulus congestus is an important mode of tropical convection. The Atmospheric Radiation Measurement (ARM) site at Nauru Island is one of three sites in the Tropical Western Pacific (TWP) region being used to study the growth of these clouds. By using ground-based observations, including millimeter cloud radar, the cloud top vertical velocity values that determine the life cycle stage of the cumulus congestus were obtained. The snapshot observations captured by the radar portrayed the diurnal cycle of cloud growth. Terminal cumulus congestus will have lost buoyancy and ceased growth, while transient cumulus congestus will have continued to ascend to greater altitude and gained buoyancy. From this analysis, it was determined that only $8 \%$ of the 50 cases from $1999-2002$ exhibited upward vertical velocity at the cloud top.
\end{abstract}

\section{Introduction}

The Department of Energy (DOE) Atmospheric Radiation Measurement climate research facility at Nauru Island was established in November 1998 and was chosen due to its proximity to the eastern edge of the Pacific warm pool under La Niña conditions, which has a profound impact on weather patterns in the Pacific ["TWP Nauru Site"]. At the Nauru site, three main modes of tropical convection are often observed - shallow trade cumulus whose cloud tops reach somewhere between $2-4$ kilometers; deep convective cloud systems whose towering cloud tops ascend to 12 kilometers or more; and cumulus congestus whose cloud tops top out around the melting level [Johnson et al., 1999]. Cumulus congestus is a unique diabatic heat source and has an important role in the local atmospheric energy balance [Luo et al., 2009]. These clouds reflect solar energy and also emit and absorb energy between the Earth's surface and outer space. At the Nauru site, a vertically pointing millimeter cloud radar observes clouds as they advect over the site, offering a "snapshot" of the cloud at a single stage in its life cycle.

As an important mode of tropical convection, understanding the life cycle phase of cumulus congestus clouds has important implications on the local atmospheric energy balance. In particular, the impact of dry air entrainment has a significant impact on the cloud life cycle. Entrainment occurs when dry environmental air mixes in with cloudy air. Dry air in the mid and upper levels of the atmosphere is one of the most important limiting factors of cloud growth [Jensen and Del Genio, 2006]. Cloud growth can be limited by either dry air entrainment above the boundary layer or from a loss in buoyancy as air parcels encounter weak stability near the freezing level [Jensen and Del Genio, 2006]. One of the most notable differences between transient (still growing) and terminal (done growing) cumulus congestus clouds is the thermodynamic structure of the cloud. For transient cumulus congestus, the cloud top temperature is warmer than the environmental temperature, allowing the cloud top to remain pos- itively buoyant (upward vertical velocity) and ascend to the level of neutral buoyancy [Luo et al., 2009]. The convective updraft associated with a terminal cumulus congestus cloud is accompanied by a significant amount of dry air entrainment during ascent, which ultimately ends up prohibiting further growth from occurring [Luo et al., 2009]. In this case, the cloud top temperature would be equal to the environmental temperature and the parcel at the cloud top would have neutral or negative buoyancy (zero or downward vertical velocity) [Luo et al., 2009].

50 cases of cumulus congestus clouds from 1999 to 2002 at Nauru Island were analyzed. These cumulus congestus clouds had cloud base heights below 1 kilometer and cloud top heights between 3 and 9 kilometers [Jensen and Del Genio, 2006]. Mid-level cumulus congestus cloud characterizations include cloud base heights near their lifted condensation level (LCL), cloud top heights near their freezing level, a lack of hydrometeors, and measurable precipitation reaching the ground [Jensen and Del Genio, 2006]. With some of the cases, however, reflectivity values associated with the hydrometeors near the cloud top were contributing to the mean Doppler velocity observed at the cloud top. This needed to be accounted for when setting the parameters to be plotted in the code.

The role of dry air entrainment in the creation of cumulus congestus presents a problem for general circulation models whose cumulus parameterizations tend to underestimate the occurrence of all but deep convective clouds [Jensen and Del Genio, 2006]. Previous studies determined based on analysis of one year of data that approximately $30-40 \%$ of the cumulus congestus observed by the snapshot observations were in transient mode [Luo et al., 2009].

\section{Objective}

Understanding the life cycle phase of the cumulus congestus clouds has important implications on retrieved entrainment rate estimates. The relationship between the life cycle phase of the cumulus congestus cloud and the amount of entrainment occurring will allow for further study in understanding the thermodynamic structure of the cumulus congestus clouds. The snapshot observations captured by the radar do not portray the full vertical growth of the cumulus congestus clouds - they only capture the height of the cloud at that precise moment in time. It is assumed that growth at the cloud top does not cease at the snapshot. As a result, a significant number of cumulus congestus cases would be in transient mode.

\section{Methodology}

The data used was obtained from the millimeter wavelength cloud radar, which probes the extent and composition of the cumulus congestus clouds and whose main purpose is to determine cloud bases and tops ["Instrument: Millimeter Wavelength Cloud Radar (MMCR)"]. It is a zenith-pointing radar that 
reports radar reflectivity of the atmosphere up to 20 kilometers and possesses a Doppler capability that will allow the measurement of cloud constituent vertical velocities ["Instrument: Millimeter Wavelength Cloud Radar (MMCR)"]. Using Interactive Data Language (IDL), plots of both radar reflectivity and mean Doppler velocity were made to qualitatively analyze the direction of growth in the cumulus congestus cases. In order to obtain the air velocity of the cloud top, the fall velocity associated with hydrometeors had to be removed from the observed mean Doppler velocity. The contribution of the hydrometeors in the original code skewed the results of the true mean doppler velocity of the cloud top by showing a positive mean doppler velocity value indicating downward vertical velocity when there was a negative mean doppler velocity indicating upward vertical velocity. The code was adjusted to not include the fall velocity associated with the high reflectivity values of the falling hydrometeors. Each case was then further analyzed quantitatively through plotting profiles of the minimum, maximum and median air velocity values for a 1-kilometer deep rectangular area near cloud top, with the area's width being determined on a case-by-case basis.

\section{Discussion \& Results}

Fifty cases of cumulus congestus from $1999-2002$ at Nauru Island in the tropical western pacific were analyzed. The top 1 kilometer of the cumulus congestus cloud was extracted and analyzed using the mean profile of the cloud top.

The cumulus congestus cases in terminal mode possess pos-
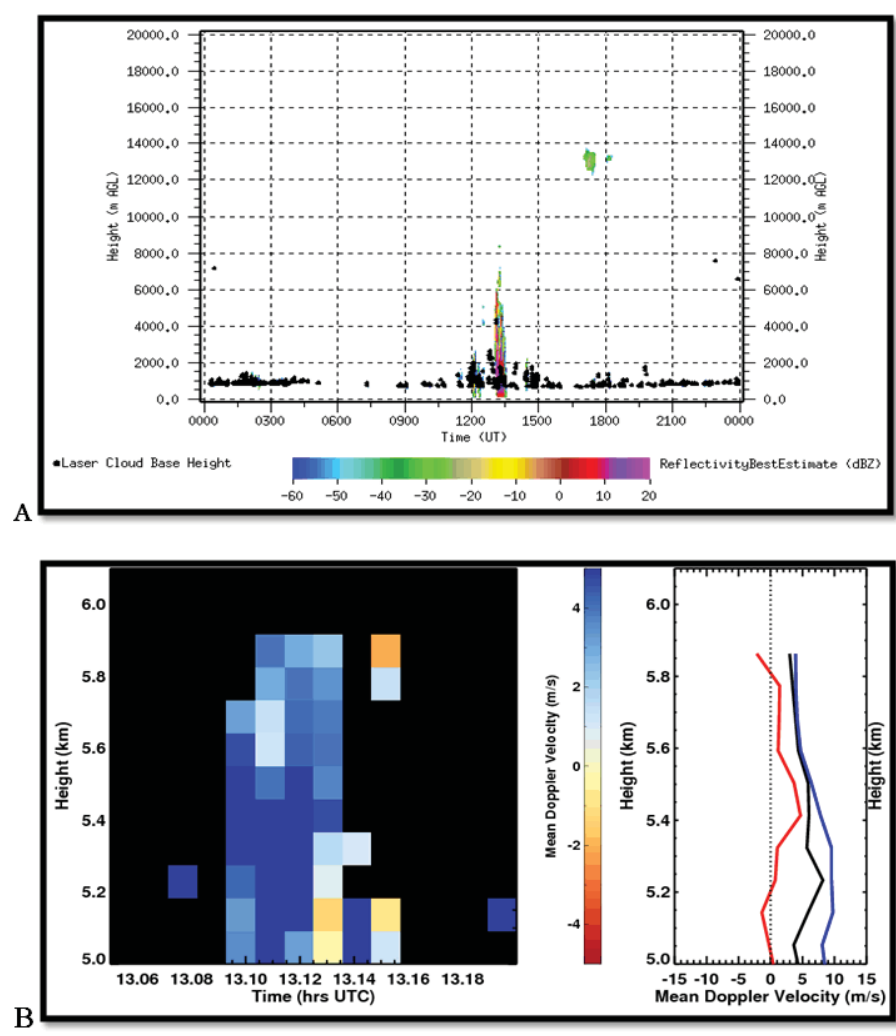

Figure 1 - Cumulus congestus cloud in terminal mode on September 8, 2000 at Nauru. (A) Radar reflectivity from MMCR shows reflectivity associated with cumulus congestus cloud. (B) Mean doppler velocity plotted for top 1 kilometer of cloud on right with mean profile of top 1 kilometer including minimum (red), median (black) and maximum (blue) values for given cloud top. In this case, the mean Doppler velocity of this cloud top is 5.15 meters per second $(\mathrm{m} / \mathrm{s})$. itive mean Doppler velocity values, shown by blue coloring in Figure 1. Positive mean Doppler velocity values are indicative of downward vertical velocity. This motion means that cloud growth has ceased and has lost buoyancy, thus prompting the weakening of the cumulus congestus cloud. This is what a vast majority of the fifty cases of cumulus congestus clouds at Nauru exhibited.

The cumulus congestus cases in transient mode possess negative mean Doppler velocity values, shown by red, orange and yellow coloring in Figure 2. Negative mean Doppler velocity values are indicative of upward vertical velocity. This motion means that the cloud is continuing to ascend to greater heights and is maintaining or gaining positive buoyancy. This is what was expected to occur in a significant amount of the cumulus congestus cases, but only occurred in a minority of them.

After taking the top 1-kilometer of the cumulus congestus cloud and analyzing the mean profile of the mean Doppler velocity, 4 cases were found to be in transient mode, while the remaining 46 were in terminal mode.

\section{Conclusion}

The fall velocity associated with the hydrometeors had a profound impact on the mean Doppler velocity near the cloud top and needed to be excluded from the total velocity. The high radar reflectivity values linked to the hydrometeors skewed the original results and the code had to be adjusted to account for downward vertical velocity associated with the falling precipitation, mainly drizzle. From this analysis, it is shown that only $8 \%$ of the 50 cas-
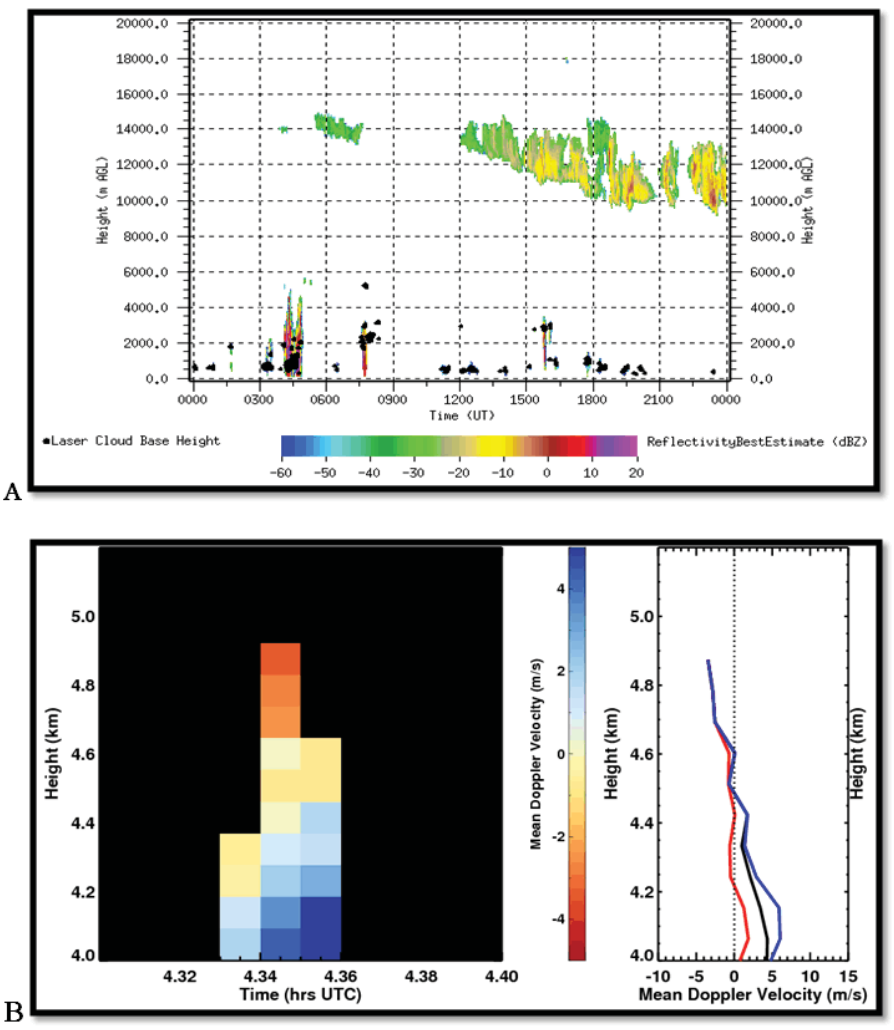

Figure 2 - Cumulus congestus cloud in transient mode on January 22, 2000 at Nauru. (A) Radar reflectivity from MMCR shows reflectivity associated with cumulus congestus cloud. (B) Mean doppler velocity plotted for top 1 kilometer of cloud on right with mean profile of top 1 kilometer including minimum (red), median (black) and maximum (blue) values for given cloud top. In this case, the mean Doppler velocity value is $\mathbf{- 0 . 5 7}$ meters per second $(\mathrm{m} / \mathrm{s})$. 
es looked at show cumulus congestus clouds in transient mode. The negative mean Doppler velocities associated with growing cloud top heights are representative of this. As only 4 of the 50 cases were found to be in transient mode, this data does not lend support to the proposed hypothesis that a significant amount of cumulus congestus clouds would be in transient mode. Further study on this topic can be done using cases at the two other ARM TWP sites at Darwin, Australia and Manus Island.

\section{Acknowledgements}

This project was supported in part by the U.S. Department of Energy, Office of Science, and Office of Workforce Development for Teachers and Students (WDTS) under the Science Undergraduate Laboratory Internships Program (SULI). I would also like to thank Brookhaven National Laboratory and the Office of Educational Programs.

\section{References}

1. "Instrument: Millimeter Wavelength Cloud Radar (MMCR)" ARM Climate

Facility $<$ http://www.arm.gov/instruments/mmcr $>$.

2. Jensen, M.P. and A.D. Del Genio, 2006: Factors limiting convective cloud top

height at the ARM Nauru Island climate research facility. J.Climate, 19, 2105-

2117

3. Johnson, R. H., T. M. Rickenbach, S. A. Rutledge, P. E. Ciesielski and W. H.

Shubert, 1999: Trimodal Characteristics of Tropical

Convection. J. Climate, 12, 2397-2418.

4. Luo, Z., Y. Liu, G.L. Stephens and R.H. Johnson, 2009:

Terminal versus

transient cumulus congestus; A CloudSat perspective.

Geophys. Res. Lett., 36, L05808, doi: 10.1029/2008GL036927

5. “TWP Nauru Site.” ARM Climate Facility $<$ http://www.arm.gov/sites/twp/C2>. 


\title{
Value engineering report: Brookhaven National Laboratory Alternatives to existing strontium-90 groundwater treatment system
}

\author{
Rebecca Winter \\ Earth and Environmental Engineering, Columbia University, New York, NY 10027 \\ Diane Rocco \\ Environmental Protection Division, Brookhaven National Laboratory, Upton, NY 11973
}

\section{Forward}

This Value Engineering (VE) report documents the results of the VE study on the strontium-90 groundwater treatment system in the Environmental Protection Division at the Brookhaven National Laboratory (BNL) Site in Upton, New York. Diane Rocco of the Environmental Protection Division coordinated the study. The study was conducted by Rebecca Winter, Brookhaven National Laboratory Summer Undergraduate Laboratory Internship (SULI) intern and recent Columbia University graduate.

The study was conducted on-site in the Environmental Protection Division Building 860 from June 3 through August 9, 2013. The objectives of the summer study were to develop improvement recommendations and a cost analysis for the strontium- 90 groundwater treatment systems, located in Buildings 855 and 670 .

The study included four meetings on June 10, June 17, June 19 , and July 22 with a team composed of DOE and BSA site personnel. The team members and other involved personnel are listed in the Acknowledgements section of this report. During the meetings, the study team brainstormed ideas and discussed potential solutions. Rebecca Winter pursued the team's recommendations and analyzed the costs and benefits of each option under the guidance of Diane Rocco.

\section{Executive Summary}

Radioactive strontium-90 ( $\mathrm{Sr}-90)$ is removed from groundwater onsite at BNL by pumping the water through a series of tanks containing a type of natural sand media called clinoptilolite zeolite. The structure of clinoptilolite causes the material to bond with radioactive strontium, removing it from water. When the clinoptilolite has become saturated with $\mathrm{Sr}-90$, the tanks, along with the sand media inside, are sent for disposal as low-level radioactive waste. This changeout process occurs three to four times annually, resulting in the disposal of 18 to 24 tanks every year. The steel tanks are heavy and expensive, so avoiding or reducing the transportation and disposal of these tanks every year by reusing the tanks would make this process more cost effective. The objective of this value engineering study is to reduce cost and waste while maintaining safe and effective removal of Sr-90 from contaminated groundwater through clinoptilolite filtration that adheres to all state and federal regulations, as well as DOE-specific requirements.

This study focuses on comparing the current method with alternatives that may provide less costly options for clinoptilolite disposal. The first alternative evaluated is the purchase of a less expensive vessel than the one currently being used and to continue to run the system with no change in process. The second option considered is to reuse the tanks by vacuuming the saturated sand media out of the tank into a separate disposal container so the tank can be refilled with new media and used again. The third involves sluicing the saturated media from a reusable and sluiceable tank into a disposal container before refilling the tank with new sand media. In this method, the clinoptilolite would be mixed with water and pumped out of the tank in a slurry through hoses. Additional options were also considered and are described in the report but were ultimately determined to be unfeasible or not cost effective. Each option was analyzed for its benefits and drawbacks and the costs of each were carefully considered. The recommended option to save the most money while allowing for a convenient and manageable process was found to be tank reuse of the Siemens PV1000ST tank by vacuuming. Based on vendor quotes, labor assumptions, and a three-year tank life, this option could save an estimated $\$ 78,000$ per year over the current method.

\section{Baseline System Overview}

There are two strontium filtration systems, one in building 855 and one in building 760. Each building has a 3 by 3 array of vessels, with three parallel rows of three vessels in each building. The Building 855 system processes about 60 gallons per minute (gpm) of water, $20 \mathrm{gpm}$ in each parallel row of vessels. Changeouts in this system occur every three to four months, resulting in the disposal of 18 to 24 tanks annually. This system will be the focus of the study and the basis for all cost projections. The second system, in building 670 , is on a pulse pumping schedule and alternates every month at $10 \mathrm{gpm}$ and $22 \mathrm{gpm}$. This system is changed out once every three years, with each changeout resulting in the disposal of 6 tanks. This system could be converted to a reusable tank method, as well, however these savings are not accounted for in the calculations.

The tanks that are currently used are custom made by Siemens. The tank drawing is shown in Appendix A, Document 1. The 350-gallon carbon steel tank has flat bottom with forklift pockets. It has a top inlet with an interior PVC distribution system, and an interior PVC catchment system at the bottom leading to a side outlet. There is also a top vent, a manway for inserting the media, and lifting lugs.

These tanks are shipped on flatbeds in orders of one dozen tanks to a local contractor. The clinoptilolite zeolite media is shipped to the same location from a mining company located in Nevada. The local vendor fills the tanks with media and does an initial backflush of the system. The backflush entails pumping water through the outlet of the tank at approximately 10 or 15 gpm and allowing water and fine zeolite particles to run out the top manway until the water runs clear. Six filled and backflushed tanks are sent to BNL when the system is ready for a changeout. 
BNL then performs a secondary backflush of each vessel prior to use. For each changeout, the leading two rows of tanks are replaced, a total of six tanks. The final row is moved to the lead row and the six new tanks become the final two rows of the system.

The system runs until breakthrough is detected on the last row of three tanks. At this point, the lead row and center row are again removed. Prior to disconnecting the tanks, the tanks and lines are flushed with air to remove as much of the water as possible. The six removed tanks are then drained of any remaining water in the bottom of the tanks through a drain in the bottom of each tank. The tanks are then left to sit for several weeks to allow the water to drain to the bottom from the pore spaces in the clinoptilolite, and the water is then drained out again. The tanks are then tilted at a 5-degree angle and are left to sit until the remaining water is removed from the tank a few more times. The entire de-watering process takes approximately 4 to 8 weeks. The liquid removed from the tanks is pumped back into the groundwater treatment system. Absorbent is added to the tanks, and the tanks are shipped for disposal with the media and the absorbent inside.

\section{System Options}

\section{Introduction}

A number of options for running the system were considered as a replacement to the current method of operating the system. These alternatives are described in detail in the following sections. Many of these options show promise to reduce the cost of running the system.

\section{A. Option \#1 - Tank Replacement 1. Overview}

In this option, less expensive tanks with the same functionality would directly replace the current tanks. These tanks could be made from steel, as they are now, fiberglass, or any other durable material. Manufacturing cost of the tanks would be the primary concern for this option, as this is the only change to the current process. All other aspects of the process, with its associated costs and labor requirements, would be unchanged. Additional savings may be realized by having BNL technicians fill the tanks with the media onsite, rather than the outside contractor.

\section{Tanks}

One tank offering promise for cost savings is the Calgon Protect-TW36, shown in Appendix A, Document 2. This tank is nearly identical to the current Siemens tank and costs only $\$ 4500$ with a freight delivery cost of $\$ 2000$ for 24 tanks, and potential additional savings for a bulk order.

A second tank is the Tigg CL-1000, shown in Appendix A, Document 3. This tank can also be manufactured for $\$ 4500$, however its dimensions are taller and narrower than our current tanks and it has a dish bottom. The taller dimensions may be a concern for forklifting and for ensuring that the pipe connections can reach from the bottom of one tank to the top of the next. However, the narrower design and rounded bottom may be more efficient in the use of zeolite media, since it is possible that the water funnels through the center more consistently than it reaches the outside perimeter of the current tank. This new design could potentially result in a longer time between changeouts, but this benefit would need to be verified by a true comparison test to justify accounting for it in the cost calculations. Since the tank is completely saturat- ed with water in operation, it is possible that the effect is minimal or nonexistent.

Fiberglass tanks were considered, as well, however all of the fiberglass options considered that could withstand the design pressure of 75 psi would cost more than the current Siemens tanks. One such tank, made by Siemens, is depicted in Appendix A, Document 6 and would cost $\$ 6535$ per unit. This polyglass tank will be discussed further in option \#3.

\section{B. Option \#2 - Tank Reuse by Vacuuming 1. Overview}

In this option, a similar or identical tank to the current Siemens tank would be used but it would not be disposed of along with the media. Instead, the media would be vacuumed out and into a waste disposal container. The tank could then be filled with new media and reused through multiple changeouts. A major benefit of this reuse method is that the tank would not require many additional features. In fact, the current Siemens tank could be used for vacuuming. However, as with every reuse method considered, the process would need to be altered to reflect the new changeout procedure and the residual contamination in the tank from its previous use.

\section{Tanks}

The current Siemens tanks could be used effectively with this system. A high-powered vacuum, potentially along with an air knife or power sprayer, could be used to remove the media from the tank through the top manway. If it is practical to vacuum from the current tanks, they could simply be reused until they eventually needed to be replaced due to age or damage. At this point, either a new shipment of the current tanks could be ordered or a different tank that is better equipped for easy vacuuming could be used going forward.

Of all the vessels considered, the best suited for vacuuming is the $\$ 6905$ Siemens PV1000ST steel tank, shown in Appendix A, Document 4. Its dual manways would provide easy access for vacuuming. Once the media is removed to the level below the side manway, the side could be opened in order to remove the remaining media more easily. Additionally, the dish bottom with the bottom drain could be useful for power washing the bottom and sides of the tank when most of the media has already been removed, as the remaining clinoptilolite would drain out this bottom hole. If vacuuming the current vessel proves to be somewhat burdensome, the remaining flat-bottom vessels could be used as before until they are discarded, and this vessel could replace them.

\section{Process}

When breakthrough occurs and the media is ready to be changed out, the tank would be dewatered as usual. Then the hatch would be opened and a high-pressure vacuum would be used to remove the material from the tank. This could be done in conjunction with an air knife or power sprayer to loosen the sand and speed up the vacuuming process. Any small amounts of water generated could be removed and flowed back through the system after the changeout. Absorbent would then be added to the sand media for disposal.

The cleaned vessels could be filled with new clinoptilolite media and backwashed. As a precaution in case the backflush water picks up trace amounts of Sr-90 from the vessel, the backflush 
water would be collected in a tank at the time of backflushing and tested for contamination. If contamination is found, this backflush water must be run through the system, adding labor time and radiological controls costs to the process. While the additional labor required in this process was quantified in the calculations as accurately as possible with the given information, a true test of this system has not been performed and would be necessary to verify all labor assumptions made in the calculations.

\section{Experimental Trial}

A trial was performed of the vacuuming process with backwashed, non-radioactive media in a clean tank. The vacuuming process was performed using a pneumatic shop-vac equipped with a HEPA filter. Two technicians spent approximately 45 minutes vacuuming one filled tank into six steel drums. Due to the size of the HEPA filter inside, the drums could only be about twothirds filled before they needed to be changed out of the system. The sand in the drums was then combined so the media from one tank could fit into $51 / 2$ drums. It is recommended that a plenum attachment be ordered or constructed that allows the HEPA filter to be above the height of the drum. This would ensure that the drums could be filled completely with media. Another observation from performing the trial was the inability to use lined cardboard cubic yard boxes or IBCs as disposal containers since they would be incapable of withstanding the pressure of the vacuum and would be crushed during the vacuum process.

After the vacuuming trial, the wet sand was put back into the tank using a hopper. The steel drums were dumped into the hopper, which was then forklifted above the tank and opened to allow the sand to exit the hopper. This process took approximately one hour. Due to the moisture in the sand, the sand occasionally became stuck in the hopper, but moving the hopper on the forklift remedied the problem. In the actual process, dry sand would be used to fill the tanks so this would not be a significant issue.

\section{Option \#3 - Tank Reuse by Sluicing 1. Overview}

In this option, a new reusable tank would be used, but instead of vacuuming the material out of the vessel during changeouts, pressurized water would be used to slurry the clinoptilolite from the vessels.

\section{Tanks}

It is important for a sluicing method that the vessel has a rounded bottom and wide sluicing connections on top and bottom. The dual manway tank that was discussed in option \#2 and shown in Appendix A, Document 4 would be useful for sluicing. The tank has a rounded bottom and sluicing connections closed with a ball valve. Additionally, if it is determined that sluicing is too time-consuming or ineffective, then a vacuuming approach could be taken with the same tank.

The Tigg custom CL tank, shown in Appendix A, Document 5 , may be the most long lasting tank considered. The $\$ 8980$ steel vessel is lined with a vinyl ester coating instead of the standard epoxy, making it much more resistant to rust. This tank, like the temporary Tigg tank in Document 3 , is taller and narrower than our current tanks, so it is less suitable for vacuuming.

Finally, the Siemens polyglass tank, shown in Appendix A, Document 6, is also suitable for sluicing. While it is too expensive to be considered for a disposable application, at $\$ 6535$ it is the least expensive sluiceable tank option considered. Polyglass tanks present a unique advantage for this application. The tank is not welded together, so quality assurance concerns such as ensuring that certified welders are used and needing to re-weld areas of the tank that are sealed insufficiently do not apply to these tanks. Additionally, since it cannot rust, the lifespan may be longer than the epoxy-coated steel tanks. However, there is a concern for the structural strength of polyglass tanks compared to steel vessels, so it is also possible that these tanks will have shorter lifetimes than steel tanks. Polyglass tanks also do not have a large manway, so vacuuming is not possible and the filling and backwashing process may be more cumbersome.

\section{Process}

When the media is ready to be changed out, two hoses are connected to the tank, on top and bottom. The ball valve at the bottom would be opened and water would be pumped into the tank through the bottom, loosening and fluidizing the sand. The compressor is then connected to the top hose to pressurize the vessel and push out the water and clinoptilolite into a disposal container. The water and sand mixture would then be left to settle, and the water would be drained out and put back into the system for treatment. The sand would be mixed with absorbent and disposed in the waste container.

\section{Other Options Considered 1. Turn-key Contractor}

In this option, a contractor would take full or partial control over the system. A major company in groundwater management was contacted and requested to provide a budgetary estimate for equipment and procedures to accomplish the Sr-90 removal. The budgetary estimate provided by the contractor yields a system cost of $\$ 242,000$ to $\$ 252,000$ annually. The budgetary estimate is only intended to give a general framework for the associated costs, and many assumptions were made in the calculations. The quote does not include labor, and it is assumed in calculations that BNL employees would run the system at a labor cost equal to that of the sluicing option, as a sluicing approach is used in the contractor's quote. This cost also does not include many considerations such sampling, maintenance, repairs, alarm responses, integration of the control system with the current BNL system, mobilization, and shipping. The costs presented for either purchase or rental of equipment were significantly higher than other options, and therefore this option was discarded.

\section{System Pressure Reduction}

In this option, less expensive tanks designed for a lower system pressure would replace the current tanks. Pressure relief valves and automated pressure sensors would be introduced in the system in order to ensure that the pressure would not reach above a predetermined set point, likely between 20 and 30 psi. When this pressure was reached, the pumps would be shut off and the water would be diverted to the open-top plastic holding vessel. The new tanks could be made from steel, polyglass, or any other durable material and would only need to be rated just above the pressure designated by the relief valves. The estimated one-time cost of installing the entire pressure relief setup is $\$ 10,000$, however pressure switches already in the system could potentially be adjusted instead of being replaced, yielding a smaller initial cost. Aside from this initial installation, the process, along with its as- 
sociated costs and labor requirements, would be unchanged. This option was abandoned based on several industry sources' claims that low-pressure fiberglass should not be used in applications of pressures higher than approximately 3 psi. Steel tanks designed for the lower pressure were not significantly cheaper than the higher pressure tanks.

\section{Alternate Sand Media}

Synthetic alternatives to clinoptilolite zeolite were considered in order to increase the amount of time between changeouts. It was ultimately determined that clinoptilolite is an efficient material for removing Sr-90 and is far less expensive than synthetic alternatives.

\section{Reuse by Dumping}

One proposed idea involved removing the sand media from reusable tanks by turning the tanks upside-down and dumping the media out and into a disposal bin. This would have required a tank with strong forklift supports capable of holding the inverted tank, a safe and reliable process for inverting the tanks, and a tank with a large enough opening to allow the sand media to be removed easily in the inverted position. The possibility of a completely removable-top design was discussed with manufacturers, and they stated that their experience indicated potential for leakage over time.

\section{Non-radioactive Waste Disposal}

Due to the low levels of Sr-90 being treated, one proposed idea was to dispose of the used sand media in a Subtitle D Landfill. However, the New York Model City RCRA permit states:

Any wastes containing trace levels of radioactive material that reads slightly above background may not be land disposed without NYSDEC approval. Wastes with higher levels of radioactivity are prohibited from land disposal.

Since the Sr-90 is present in the saturated media of concentrations of approximately $165 \mathrm{pCi} / \mathrm{g}$, which is above the standard soil clean up goal of $15 \mathrm{pCi} / \mathrm{g}, \mathrm{BNL}$ would need to appeal for State approval, which was considered undesirable.

\section{Analysis and Recommendations \\ A. Discussion}

Table 1 below shows the full process from changeout to changeout including all associated annual system costs. The chart compares the current system to each of the three major alternative options discussed. The burdened average annual cost in contracts and labor for each individual step is shown in bold at the top of the process step box. The last row shows all of the major tank choices associated with that option, with the burdened price per tank listed in bold. The total yearly cost for each option is listed below the tank name.

Since a trial of the alternate systems has not yet been performed, the BNL labor estimates are based on assumptions that are explained in more detail below Table 1 . The prices for disposable tank options assume 20 tanks are disposed per year, and the reusable tank options assume a 5-year lifetime of the reusable tanks, except the custom Tigg tank, which assumes a 10-year lifetime due to its lining. The two tanks produced by Tigg do not include shipping costs to send the new tanks to BNL.

The most cost-effective process investigated is a vacuuming process with a Siemens dual-manway steel tank. This process would cost $\$ 182,736$ in the first year, because 9 tanks and a vacuum must be purchased, and the backwash will be supervised. The average annual cost of running this system, with the cost of tanks and the vacuum spread out over their 5 year lifespan, is $\$ 58,448$ plus $\$ 54,806$ per cycle in Brookhaven-employed labor, or a total of $\$ 113,254$ per year, with Brookhaven labor accounting for $48 \%$ of the cost. The current system costs $\$ 191,335$ per year, of which $4.2 \%$ is Brookhaven labor.

The option involving the least change to the current process would involve purchasing the less expensive Calgon tanks and replacing the contractor who is currently filling and backflushing the tanks with Brookhaven-employed technicians. With these two small changes, the total cost of running the system in the same way it is currently run, with disposal of tanks after each cycle, is $\$ 159,765$ per year, compared to the current $\$ 191,335$ per year.

Table 2 shows each option and its associated first-year and average annual cost. The first year costs of the reusable tank options assume all 9 tanks are purchased and an $\$ 8000$ high-powered vacuum is purchased for the vacuuming options. The vacuuming option with the current Siemens tank does not assume 9 new tanks are purchased in the first year, since the current Siemens tanks could be vacuumed out. Only the sluicing option with the Tigg custom tank is more expensive in the first year than the current system, but the return on investment for this tank is just over one year. The rest of the options require a small enough initial investment that money is saved within the first year of operation.

\section{B. Recommendations}

It is recommended that the processes for one or more of these options be tested in order to validate labor assumptions and adjust the labor costs accordingly. Based on those results, it may be possible to alter the existing process for removal of Sr-90 from groundwater at BNL and realize significant cost savings. Based on vendor quotes and labor assumptions in this study, the vacuuming option would provide the most cost savings. If this proves to be true, purchase of the Siemens dual-manway tank is recommended for easier vacuuming of sand. 
Table 1. Process Comparison of Major Options

\begin{tabular}{|c|c|c|c|c|c|}
\hline$\frac{O}{\Delta}$ & Current & \multicolumn{2}{|c|}{$\begin{array}{l}\text { Option\#1: } \\
\text { Tank Replacement }\end{array}$} & $\begin{array}{l}\text { Option \#2: } \\
\text { Vacuuming Reuse }\end{array}$ & $\begin{array}{l}\text { Option \#3: } \\
\text { Sluicing Reuse }\end{array}$ \\
\hline 1 & $\begin{array}{l}\text { See Last Row } \\
\text { Tank purchased and } \\
\text { shipped to contractor }\end{array}$ & \multicolumn{2}{|c|}{$\begin{array}{l}\text { See Last Row } \\
\text { Tank purchased and } \\
\text { shipped to contractor/BNL }\end{array}$} & $\begin{array}{l}\text { See Last Row } \\
\text { Tank purchased and } \\
\text { shipped to BNL }\end{array}$ & $\begin{array}{l}\text { See Last Row } \\
\text { Tank purchased and } \\
\text { shipped to BNL }\end{array}$ \\
\hline 2 & $\begin{array}{l}\$ 20,054 \text { b } \\
\text { (2000 Ibs/tank) } \\
\text { Clinoptilolite } \\
\text { purchased and } \\
\text { shipped to contractor }\end{array}$ & \multicolumn{2}{|c|}{$\begin{array}{l}\mathbf{\$ 2 0 , 0 5 4} \text { b } \\
\text { (2000 Ibs/tank) } \\
\text { Clinoptilolite purchased } \\
\text { and shipped to } \\
\text { BNL/contractor }\end{array}$} & $\begin{array}{l}\$ 20,054 \text { b } \\
\text { (2000 Ibs/tank) } \\
\text { Clinoptilolite purchased } \\
\text { and shipped to BNL }\end{array}$ & $\begin{array}{l}\$ 20,054 \text { b } \\
(2000 \text { Ibs/tank) } \\
\text { Clinoptilolite purchased } \\
\text { and shipped to BNL }\end{array}$ \\
\hline 3 & $\begin{array}{l}\mathbf{\$ 2 1 , 4 2 8} \\
\text { Contractor fills and } \\
\text { stores tank }\end{array}$ & $\begin{array}{l}\mathbf{\$ 2 1 , 4 2 8} \\
\text { Contractor } \\
\text { fills and } \\
\text { stores tank }\end{array}$ & $\begin{array}{l}\$ 6258 \text { lbr }{ }^{c} \\
(2 \text { techs } 1 \text { day } / 6) \\
\text { BNL fills and } \\
\text { stores tank }\end{array}$ & $\begin{array}{l}\text { \$12,516 labor }{ }^{d} \\
\text { (2 techs 2day/6) } \\
\text { BNL fills and stores tank }\end{array}$ & $\begin{array}{l}\text { \$12,516 labor }{ }^{d} \\
\text { (2 techs 2day/6) } \\
\text { BNL fills and stores tank }\end{array}$ \\
\hline 4 & $\begin{array}{l}\text { \$2381 } \\
\text { Contractor ships tank } \\
\text { to BNL }\end{array}$ & $\begin{array}{l}\mathbf{\$ 2 3 8 1} \\
\text { Contractor } \\
\text { ships tank } \\
\text { to BNL }\end{array}$ & N/A & N/A & N/A \\
\hline 5 & \multirow[t]{2}{*}{$\begin{array}{l}\$ 4000 \text { labor } \\
\text { BNL does final } \\
\text { backwash }\end{array}$} & \multirow[t]{2}{*}{$\begin{array}{l}\$ 4000 \\
\text { labor } \\
\text { BNL does } \\
\text { final } \\
\text { backwash }\end{array}$} & \multirow[t]{2}{*}{$\begin{array}{l}\$ 12,516 \\
\text { labor e } \\
\text { (2techs 2day/6) } \\
\text { BNL fully } \\
\text { backwashes } \\
\text { tank }\end{array}$} & $\begin{array}{l}\$ 22,516 \text { labor } 1^{\text {st }} \text { yr, } \\
\text { then } \$ 14,516 \\
\left(2 \text { techs } 2 \text { day/6 }+2 \text { day/6 șupxss. } 1^{\text {f }}\right. \\
\text { year }+6 \text { hr } / 6 \text { RadCon) } \\
\text { BNL fully backwashes } \\
\text { tank into container }\end{array}$ & $\begin{array}{l}\$ 22,516 \text { labor } 1^{\text {st }} \text { yr, } \\
\text { then } \$ 14,516^{f} \\
\left(2 \text { techs } 2 \text { day/6 }+2 \text { day/6 șupxsr. } 1^{\text {a }}\right. \\
\text { year }+6 \text { hr/6 RadCon) } \\
\text { BNL fully backwashes } \\
\text { tank into container }\end{array}$ \\
\hline 6 & & & & $\begin{array}{l}\text { \$7258 labor } 9 \\
\text { (2 techs } 1 \text { day } / 6+3 \mathrm{hr} / 6 \text { RadCon) } \\
\text { BNL flows backwash } \\
\text { water back thru system }\end{array}$ & $\begin{array}{l}\text { \$7258 labor } 9 \\
\text { (2 techs 1day/6 + 3hr/6 RadCon) } \\
\text { BNL flows backwash } \\
\text { water back thru system }\end{array}$ \\
\hline 7 & N/A & \multicolumn{2}{|c|}{ N/A } & $\begin{array}{l}\$ 12,497+\$ 14,516 / \mathrm{lbr}^{\mathrm{h}} \\
\text { (\$94 X6drums/tank + 2 techs } \\
\text { 2day/6 + radcon 6hr/6) } \\
\text { Vacuum to remove } \\
\text { sand, clean tank }\end{array}$ & $\begin{array}{l}\text { \$12,187 +\$21,774 lbr i } \\
\text { (\$275 X218C s/tank + 2 techs } \\
\text { 3days/6 + radcon 9hr/6) } \\
\text { Sluicing method to } \\
\text { remove sand, clean tank }\end{array}$ \\
\hline 8 & $\begin{array}{l}\text { \$4000 labor } \\
\text { Drain water from tank } \\
\text { and flow back } \\
\text { through system }\end{array}$ & \multicolumn{2}{|c|}{$\begin{array}{l}\$ 4000 \text { labor } \\
\text { Drain water from tank and } \\
\text { flow back through system }\end{array}$} & $\begin{array}{l}\$ 6000 \text { labor } \text { j }^{\mathrm{S}} \\
\text { (\$200/tank current + radsen } 1 \mathrm{hr} \text { ) } \\
\text { Drain water from tank } \\
\text { and flow back through } \\
\text { system }\end{array}$ & $\begin{array}{l}\text { \$10,000 labor } \\
\text { (\$400/tank 2Xcurrent + radson 1hr) } \\
\text { Decant liquid and flow } \\
\text { sluiced water back } \\
\text { through system }\end{array}$ \\
\hline 9 & $\begin{array}{l}15,385^{\prime} \\
\text { (13/truck) } \\
\text { BNL ships tank \& } \\
\text { sand for disposal }\end{array}$ & \multicolumn{2}{|c|}{$\begin{array}{l}\mathbf{\$ 1 5 , 3 8 5} \\
\text { (13 per truck) } \\
\text { BNL ships tank \& sand for } \\
\text { disposal }\end{array}$} & $\begin{array}{l}\$ 10,526 \mathrm{~m} \\
\text { (19 per truck) } \\
\text { BNL ships sand in boxes } \\
\text { for disposal }\end{array}$ & $\begin{array}{l}10,526 \mathrm{~m} \\
\text { (19 per truck) } \\
\text { BNL ships sand in } \\
\text { containers for disposal }\end{array}$ \\
\hline$\stackrel{\sqrt[6]{5}}{\circ}$ & $\begin{array}{l}\text { \$6204" Current } \\
\$ 183,335+\$ 8000 \text { labor } \\
=\$ 191,335 / \text { yr }^{\circ}\end{array}$ & \begin{tabular}{|l|} 
\$5078" \\
Calgon: \\
$\$ 160,800$ \\
$+\$ 8000$ labor \\
$=\$ 168,800 / y r^{\circ}$ \\
$\$ 4986^{\mathrm{n}} \mathrm{Tigg:}^{\mathrm{r}}$ \\
$\mathbf{\$ 1 5 8 , 9 6 0}$ \\
$+\$ 8000$ labor \\
$=\$ 166,960 / \mathrm{yr}^{\circ}$
\end{tabular} & 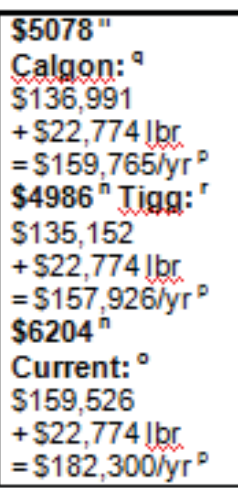 & 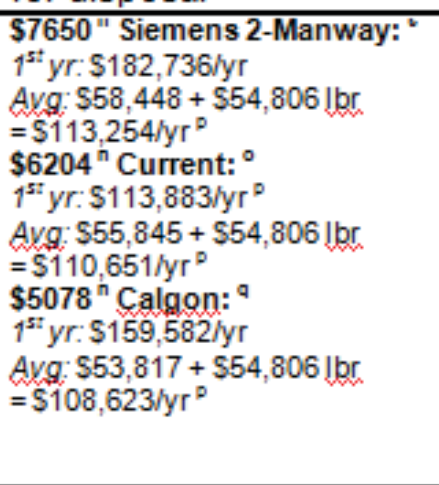 & 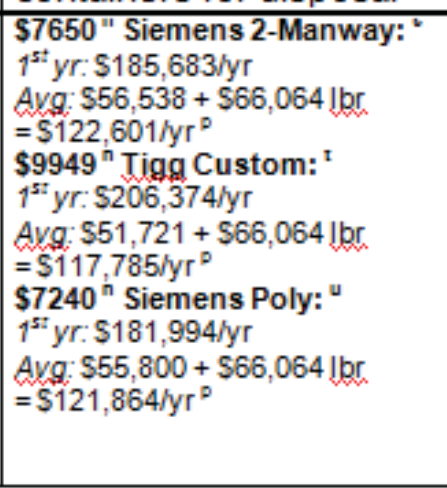 \\
\hline
\end{tabular}




\section{Notes and Assumptions from Table 1}

a. All prices are per year unless otherwise noted. 20 cycles per year are assumed for all estimates. Burdening factors are 1.11 for purchases over $\$ 25,000$ and 1.19 for services and leases. Technician (tech) rate with recharge is $\$ 117.34 / \mathrm{hr}$, supervisor rate is assumed to be $\$ 150 / \mathrm{hr}$, and Radiological Control (RadCon) specialist rate is assumed to be $\$ 100 / \mathrm{hr}$.

b. $2000 \mathrm{lbs}$ of clinoptilolite media are purchased at an unburdened cost of $\$ 905$ per tank per changeout cycle (20 changed out tanks per year assumed).

c. Estimated labor cost based on two technicians spending 1 day ( 8 hours) to fill six new tanks at the tech pay rate.

d. Estimated labor cost based on two technicians spending 2 days (16 hours) to fill six cleaned tanks at the tech pay rate.

e. Estimated labor cost based on two technicians spending 16 hours to fully backwash six new tanks at the tech pay rate.

f. Estimated labor cost based on two technicians spending 16 hours with 16 hours of supervision and 6 hours of RadCon supervision to fully backwash six cleaned tanks and collect all potentially contaminated water from the backwash. Supervision would only be required for the first year.

g. Estimated labor cost based on two technicians spending 8 hours with 3 hours of RadCon supervision to test the collected backwash water and flow the water back through the system.

h. Disposal container cost based on $\$ 94$ (plus burdening factor) steel drums and assuming the media from one tank would fill six disposal containers. Cost of one high-powered vacuum estimated at $\$ 8000$ with a 5-year lifespan. Estimated labor cost based on two technicians spending 16 hours to fully vacuum the media from six saturated tanks and completely clean the six tanks with 6 hours of supervision by a RadCon specialist. This labor estimate is a conservative estimate based on the vacuuming trial performed.

i. Disposal container cost based on two $\$ 275$ (plus burdening factor) Intermediate Bulk Containers (IBCs) and assuming the media from one tank would fill two disposal containers. Estimated labor cost based on two technicians spending 24 hours to fully sluice the media from six saturated tank and completely clean the six tanks with 9 hours of supervision by a RadCon specialist.

j. Assumed labor cost of draining water from tank and flowing it back through the system is equal to current labor costs of \$200 per tank per changeout, with 20 tanks changed out annually, plus 1 hour of supervision by a RadCon specialist.

$\mathrm{k}$. Assumed labor cost of decanting sluice water from tank and flowing it back through the system based on double the current labor costs of $\$ 200$ per tank per changeout, with 20 tanks changed out annually, plus 1 hour of supervision by a RadCon specialist. Increased labor costs are due to the increased quantity of water and the additional time required to allow the media to settle in the container.

1. Based on current disposal practices.

$\mathrm{m}$. Based on current disposal practices and relative weight calculations.

n. Burdened price per tank (not annual cost).

o. Current Siemens steel tank, shown in Appendix A, Document 1.

p. Total cost of entire process per year. For reusable options, the assumed lifetime of the tank is 5 years, except the Tigg custom tank has a 10-year assumed lifetime. It is assumed that 20 tanks are changed out per year for all calculations.

q. Calgon Protect-TW36 steel tank, shown in Appendix A, Document 2. The price is based on $\$ 4500 /$ tank unburdened vendor quote plus $\$ 2000$ shipping cost for 24 tanks.

r. Tigg CL-1000 steel tank, shown in Appendix A, Document 3.

s. Siemens dual-manway PV1000ST steel tank, shown in Appendix A, Document 4.

t. Tigg Custom CL steel tank, shown in Appendix A, Document 5.

u. Siemens PG1100 polyglass tank, shown in Appendix A, Document 6.

Table 2. Total System Cost Comparison

\begin{tabular}{|l|l|r|r|r|l|}
\hline \multicolumn{1}{|c|}{ Option } & \multicolumn{1}{|c|}{ Tank } & $\begin{array}{c}\text { Money Spent } \\
\text { in Year 1 }\end{array}$ & \multicolumn{1}{c|}{$\begin{array}{c}\text { Avg Annual } \\
\text { Thereafter }\end{array}$} & \multicolumn{1}{c|}{ ROI } & \multicolumn{1}{c|}{ Comments } \\
\hline Current & Siemens Current Tank & $\$ 191,335$ & $\$ 191,335$ & 0 & Current System \\
\hline Replace w/ Contractor & Calgon Tank & $\$ 168,800$ & $\$ 168,800$ & 0 & \\
\hline Replace w/ Contractor & Tigg CL1000 Tank & $\$ 166,960$ & $\$ 166,960$ & 0 & \\
\hline Replace w/o Contractor & Calgon Tank & $\$ 159,765$ & $\$ 159,765$ & 0 & \\
\hline Replace w/o Contractor & Tigg CL1000 Tank & $\$ 157,926$ & $\$ 157,926$ & 0 & \\
\hline Replace w/o Contractor & Siemens Current Tank & $\$ 182,300$ & $\$ 182,300$ & 0 & \\
\hline Vacuum & Siemens Dual Manway Tank & $\$ 182,736$ & $\$ 113,254$ & $<1$ & \\
\hline Vacuum & Siemens Current Tank & $\$ 113,883$ & $\$ 110,651$ & $<1$ & \\
\hline Vacuum & Calgon Tank & $\$ 159,582$ & $\$ 108,623$ & $<1$ & \\
\hline Sluice & Siemens Dual Manway Tank & $\$ 185,683$ & $\$ 122,601$ & $<1$ & \\
\hline Sluice & Tigg Custom Tank & $\$ 206,374$ & $\$ 117,785$ & 1.2 & ROI is just above \\
& & & & & one year \\
\hline Sluice & Siemens Polyglass Tank & $\$ 181,994$ & $\$ 121,864$ & $<1$ & \\
\hline
\end{tabular}




\section{References}

1. Environmental Management Directorate. "Strontium-90 BGRR/ WCF/ PFS Groundwater Treatment System." Brookhaven National Laboratory and J.R. Holzmacher P.E., LLC Consulting Engineers. Operation And Maintenance Manual U.S. Department Of Energy.

2. Robinson, S. M. "The Development Of A Zeolite System For Upgrade Of The Process Waste Treatment Plant." Chemical Technology Division, Oak Ridge National Laboratory.

3. Dorsch, W.R. "Groundwater Remediation Program At Brookhaven National Laboratory Upton, New York" Environmental and Waste Management Services Division, Brookhaven National Laboratory. Fourteenth Conference on the Geology of Long Island and Metropolitan New York: SUNY at Stony Brook. April 14, 2007.

4. Rocco, D. F. "Standardized Waste Profile Sheet and RCRA Technical Basis Document: Sr-90 Vessel Waste Stream." Waste Management, Brookhaven National Laboratory. January 2011.

\section{Appendix A. Tank Information}

The following are drawings, quotes, and specification sheets of all tanks being considered for this system. The documents in this appendix are as follows:

Document 1. Current Siemens Tank Drawing 18

Document 2. Calgon Protect TW Tank Specifications 19

Document 3. Tigg CL-1000 Steel Tank Drawing 23

Document 4. Siemens PV1000ST Steel Tank Drawing 24

Document 5. Tigg Custom CL Tank Drawing 25

Document 6. Siemens PG1100 Polyglass Tank Specifications and Drawing

\section{Acknowledgements}

I would like to give a special thanks to Diane Rocco for all of her knowledge and guidance on this project. I would also like to thank the rest of the Value Study Team for their help and insight. This project was supported in part by the U.S. Department of Energy, Office of Science, Office of Workforce Development for Teachers and Scientists (WDTS) under the Science Undergraduate Laboratory Internships Program (SULI).

\section{Value Study Team Members}

NAME DISCIPLINE

Diane Rocco (TL)

William Dorsch

George Goode

Eric Kramer

Terri Kneitel

Brian McCaffrey

Edmund Pavlak

Ronald Prwivo, Jr

Vincent Racaniello

Jason Remien

Stanley Sakry

Steven Stein

Glen Todzia

$\mathrm{TL}=$ Team Leader

VE Facilitator

Rebecca Winter
Operations Manager, Waste Management

Manager, Groundwater Protection Group

Assistant Laboratory Director, ESH

Groundwater Protection Field Engineer

DOE-BHSO Waste Management Manager

Facility Complex Engineer

Waste Management Representative

Operations Supervisor, Waste Management

Environmental Project Manager

Interim Division Manager, Environmental Protection

Facility Project Manager

Quality Assurance Specialist

Support Services Manager, Waste Management

Environmental Engineer 


\title{
Categorization and Statistical Analysis of Fast Solar Transients
}

\author{
Jovani Withers, and Raul Armendariz \\ Engineering, Queensborough Community College, Bayside NY, 11364 \\ Xiaoyu Wang, and Meng Yue \\ Engineering, Brookhaven National Laboratory, Upton NY, 11973
}

\begin{abstract}
This project set out to develop, tune, and verify the parameter settings of a MATLAB script to allow for the capturing and enumeration of fast cloud transients (FCT) over the solar panels of Long Island Solar Farm (LISF). Since the cloud movement is difficult to predict, its impact on the solar irradiance variations has to be modeled statistically terms of the irradiance magnitude (W/m2) changes. An FCT across a solar farm may cause a severe drop in the irradiance level, and ultimately affecting the output power generated from the solar farm, which can negatively influence the operation of the associated power systems. One remedy to fix the power variations is to integrate a Battery Energy Storage System (BESS) with a solar generation system; which would act as a buffer system to smooth out the power fluctuations from the solar generation system as well as provide some other ancillary service, such as grid inertial response improvement. The objective of this study is to quantify the statistics of the occurrence of FCTs in terms of their initial irradiance value, lasting time duration, and magnitude drop (modeled by random variables RV1 3), which can be used for a BESS capacity specification method in a probabilistic approach. A MATLAB script was developed to extract the varying irradiance patterns caused by FCTs, then categorize the variation patterns using different parameter settings. Results showed that by properly setting the values of the three parameters in the algorithm, the developed method can accurately capture the fast transients located in the following four categories, dropping 1) above $200 \mathrm{~W} / \mathrm{m} 2$, 2) between 150 and $200 \mathrm{~W} / \mathrm{m} 2,3$ ) between 100 and $150 \mathrm{~W} / \mathrm{m} 2$, and 4) between 50 and $100 \mathrm{~W} / \mathrm{m} 2$, all within 10 seconds. These results will facilitate the future statistical analysis of the FCTs in terms of the above three random variables, RV1 3.
\end{abstract}

\section{Introduction}

Solar energy is definitely not one of the newest topics for technology in our current techno-social era. Starting from 7th century B.C., people used magnifying classes to focus sunlight onto ants, burning them in the process 1 , which is quite comical to be honest. Fast forwarding literally 2573 years later, an inventor by the name of Willoughby Smith finds out the photoconductivity of selenium1, and ten years from that find, Charles Fritts describes the first solar cells from selenium wafers. Of course there have been many enhancements to the idea of solar panels since then. However, let's take a look at our current power system. Normally they involve large turbines and generators in order to provide inertia to the electric grid2. Inertia is a property of matter by which it remains at rest or in uniform motion in the same straight line unless acted upon by some external force3. In this case, the inertia provided by the turbines and/or generators is used to produce usable electricity. This system, although popular, is typically uncontrollable if a large disturbance occurs, distorting the system frequency; The slowness of the turbine governor control is also a factor for making the current energy producing system uncontrollable during a large disturbance2, which could be either a strong thunderstorm, a mechanical problem, or a multitude of issues. Instead of this outdated system, it can be replaced with a system that generates no inertia. In other words, a Solar Generation System(SGS). The concerns with integrating a solar generation system is the fact that no inertia is created, which leads to a concern with grid frequency. A remedy to this concern is to integrate a Battery Energy Storage System (BESS) with a SGS. A notable fact about BESS' $s$ is that they possess response speeds that are superior to conventional generators and can improve the grid inertial response2. Although integrating a BESS is a great idea for grid response, the problem with integrating one is cost. In order to optimize inertial response, there has to be a BESS of a desired capacity2. This is important because there is a correlation between size of BESS and cost; as the size of the BESS increases, the cost increases as well. How do we find this proper capacity? This is possible by making a probabilistic risk assessment. An assessment on what? For this project, a MATLAB script will be used to examine how cloud transients, particularly fast cloud transients, affect irradiance levels from SGS's.

\section{The MATLAB Program and Parameters}

As was previously stated, a MATLAB script will be used to examine how fast cloud transient affected irradiance levels from SGS's. Over time, and of the ten weeks, enhancements were made to the script. However, three settings remained the same throughout the developments. Although it did take a while to gather the most proper parameters for analysis, there was a lot of trial and error that did occur. At first, the script had to be understood in terms of what it does, and how it does that. From the first MATLAB script, what was learned was that essentially the scripts that would be worked on will provide transient data, as well as show transients that fit parameters that were set. At the time, the parameters were completely unknown, but getting the same results that Xiaoyu Wang and Meng Yue had was surprisingly simple. At the time, only 46 transients were captured. This number wasn't good enough analytically for performing probabilistic risk assessment yet. The next step taken was to understand different portions of the script on an in-depth level. What were understood then were why the histograms had their $\mathrm{x}$ labels only known as "Case Number of Cloud Transients", as well as what the parameters were that needed to be adjusted. These parameters were known as, at first, "TolStepNum", "NextDev", and "ConInc".

To explain these parameters more, let's start with "TolStepNum". Mainly it's referred to as Total Step Number (TSN). What this parameter does is set the number of points after a data point will be examined in accordance with the starting data point. For example, let's examine TSN parameter numbers 1 and 3; Should 
the parameter be set to 1 , MATLAB will examine the first data point with the second data point, then the second data point with the third data point, and so on and so forth. With a TSN parameter of 3, the MATLAB analysis will take a similar approach as it did with the parameter of 1 ; However, with a parameter of 3 , MATLAB will take the first data point and analyze it with the second, third and fourth data points, then the program will take the second data and analyze it with the third, fourth, and fifth data points, and so on and so forth. The next parameter to explain is "NextDev", and it's referred to as Next Deviation (ND). The purpose of this parameter is to set a lower limit of irradiance drop per number of data points provided by TSN. For example, let's take two different parameter sets: one with $\mathrm{TSN}=1$, and $\mathrm{ND}=20$, and another with $\mathrm{TSN}=3$, and $\mathrm{ND}=50$. With the first parameter set, MATLAB will check every data point by data point, and check if there's an irradiance drop of $20 \mathrm{~W} / \mathrm{m} 2$ from one data point to another. With the second parameter set, MATLAB will examine the first data point with the second, third and fourth data points, and check if there's an irradiance drop of $50 \mathrm{~W} / \mathrm{m} 2$ between the first and second data points, or the first and third data points, or the first and fourth data points. The last important parameter of the fast cloud transient seeking MATLAB script is "ConInc"; Also referred to as Counter Increase(CI), and it has two separate purposes. One of this parameters' purposes is to allow MATLAB to loop a certain number of times, as long as the parameter condition ND is met within TSN. For example, let's just use one parameter set, TSN and ND should be clear by now. The parameter set to be analyzed will be: $\mathrm{TSN}=1, \mathrm{ND}=10, \mathrm{CI}=10$. With this parameter set, MATLAB will examine data from point to point, and see if there is a deviation of $10 \mathrm{~W} / \mathrm{m} 2$ between each point. This time however, if the irradiance drop between one data point to another is not met, then MATLAB will restart the counter to zero, then resume the program and start analyzing data from the data point right after the data point that was previously examined, and run the script based on the parameters set, and so on and so forth, as long as the parameter ND is not met within TSN. If ND is met within TSN, then MATLAB checks next data point in accordance with TSN, and checks for an irradiance deviation greater than or equal to the value set for ND, in this case greater than or equal to $10 \mathrm{~W} / \mathrm{m} 2$. There are two situations that can occur now, which theoretically is the second purpose of CI: MATLAB successfully loops the program as many times as the value set for $\mathrm{CI}$, in this case 10 , or MATLAB successfully loops less than 10 times then fails. If the program loops successfully 10 times, or rather loops as many times as is set for CI, then MATLAB will record those data points and their irradiance values at each point, which results in a transient being captured. For the second CI situation, even if the script successfully loops a few times, if ND is consistently met nine times in a row, but fails the tenth, unfortunately MATLAB will throw out that transient data, but will restart the counter increase variable to 0 and start anew at the data point that starts afterward. By tuning these parameters, what happens?

\section{Results}

Although finding proper parameters for accurate results were completely trial and error at first, over time, and recently with the help of Xiaoyu Wang, parameters were being found. For example, here's some transient data that will be explained from November 2011:

\begin{tabular}{|l|r|r|}
\hline \multicolumn{1}{|c}{ Effects of Parameters on Transients } \\
\begin{tabular}{|l|r|r|} 
Parameters \\
(TSN.ND.CI)
\end{tabular} & $\begin{array}{c}\text { Number of } \\
\text { Transients }\end{array}$ & $\begin{array}{c}\text { Number of } \\
\text { Transients with } \\
\text { an Irradiance } \\
\text { Drop of 200 W/ } \\
\text { m2 or over }\end{array}$ \\
\hline 1.20 .10 & 9 & 9 \\
\hline 1.30 .10 & 5 & 5 \\
\hline 1.40 .10 & 4 & 4 \\
\hline 1.50 .10 & 4 & 4 \\
\hline & & 64 \\
\hline 2.20 .10 & 93 & 64 \\
\hline 2.30 .10 & 85 & 60 \\
\hline 2.40 .10 & 77 & 53 \\
\hline 2.50 .10 & 69 & 75 \\
\hline & & 72 \\
\hline 3.20 .10 & 139 & 72 \\
\hline 3.30 .10 & 125 & \\
\hline 3.40 .10 & 114 & \\
\hline 3.50 .10 & 116 & \\
\hline
\end{tabular}

According to my findings, as TSN increased, the number of transients increase by a lot. However, if we make ND bigger, the number of transients decrease because as we increase it, MATLAB will sift through data to find larger and larger transients. $\mathrm{TSN}=1$ is selected because it provided the most accurate results, hence why all parameters for analysis have a TSN of 1 . The goal was to find which choice of the three parameters resulted in detecting the highest number of the type cloud transients of interest, for example those that lasted a short duration, but have a large magnitude of $200 \mathrm{~W} / \mathrm{m} 2$. By reducing ND, it was definitely possible to gather more transients, more specifically, with irradiance drops of $200 \mathrm{~W} / \mathrm{m} 2$ or more, which was the very first transient categorical group to be found. To show the results of the parameters that did fit the specifications that were set as was previously stated when selecting a proper value for TSN, here's another chart to display found results: 
Results of Parameters on 18 days (November 2011)

\begin{tabular}{|l|r|r|r|}
\hline $\begin{array}{l}\text { Category \& } \\
\text { TSI.ND.CI }\end{array}$ & $\begin{array}{c}\text { Total } \\
\text { Number of } \\
\text { Transients }\end{array}$ & $\begin{array}{c}\text { Transients } \\
\text { above } \\
\text { Categorical } \\
\text { value }\end{array}$ & $\begin{array}{c}\text { Transients } \\
\text { Below } \\
\text { Categorical } \\
\text { Value }\end{array}$ \\
\hline $\begin{array}{l}\text { "Transients } \\
\text { Wove 50 }\end{array}$ & & & \\
W/m2" \& & & & \\
1.3 .10 & 220 & 216 & \\
\hline $\begin{array}{l}\text { "Transients } \\
\text { above 100 }\end{array}$ & & & \\
$\begin{array}{l}\text { W/m2"\& } \\
1.5 .10\end{array}$ & & & \\
\hline "Transients & & & \\
above 150 & & & \\
W/m2"\& & & & \\
1.7 .10 & & & \\
\hline "Transients & & & \\
above 200 & & & \\
W/m2"\& & & & \\
1.9 .10 & & & \\
\hline
\end{tabular}

Although the found parameters for ND ominously run a pattern, they result in the most accurate results per parameter, or rather per category. Even applying the same parameters to that of May 2012 yielded accurate results:

Results of Parameters on 16 days (May 2012)

\begin{tabular}{|l|r|r|r|}
\hline $\begin{array}{l}\text { Category \& } \\
\text { TSI.ND.CI }\end{array}$ & $\begin{array}{c}\text { Total } \\
\text { Number of } \\
\text { Transients }\end{array}$ & $\begin{array}{c}\text { Transients } \\
\text { above } \\
\text { Categorical } \\
\text { value }\end{array}$ & $\begin{array}{c}\text { Transients } \\
\text { Below } \\
\text { Categorical } \\
\text { Value }\end{array}$ \\
\hline $\begin{array}{l}\text { "Transients } \\
\text { above 50 }\end{array}$ & & & \\
W/m2" \& & & & \\
1.3 .10 & 302 & 296 & \\
\hline "Transients & & & \\
above 100 & & & \\
W/m2"\& & & & \\
1.5 .10 & 175 & & \\
\hline "Transients & & & \\
above 150 & & & \\
W/m2"\& & & & \\
1.7 .10 & 124 & & \\
\hline "Transients & & & \\
above 200 & & & \\
W/m2"\& & & & \\
1.9 .10 & & & \\
\hline
\end{tabular}

However, this analysis is still undergoing and we are deploying the developed algorithms to a larger data set, say 1 year's irradiance data collected from LISF. Results from both analyses will be generated and presented in future reports.

\section{Conclusion}

Unfortunately at this time, the capacity of the BESS is still unknown. However, with the acquiring of the highly accurate parameters, there is no doubt that the size of the BESS may be found soon. However, It was determined that by tuning the software parameters fast cloud transients of approximately 10 seconds in duration can be accurately detected: settings of TSN $=1, \mathrm{CI}=10$, and ND $=9,7,5$, and 3 accurately detects such FCTs of magnitude $>200 \mathrm{~W} / \mathrm{m} 2,>150 \mathrm{~W} / \mathrm{m} 2 ;>100 \mathrm{~W} / \mathrm{m} 2$, and $>50 \mathrm{~W} / \mathrm{m} 2$ accordingly.

\section{Acknowledgements}

First of all, This project was supported in part by the U.S. Department of Energy, Office of Science, Office of Workforce Development for Teachers and Scientists (WDTS) under the Visiting Faculty Program (VFP). I'd also like to thank my family, friends, and for urging me to go through with this internship, and keep going until the end of it. I'd also like to thank Raul Armendariz for noticing my hard-working nature and inviting me for the VFP program. I'd also like to thank Noel Blackburn and Cindi Biancarosa for being very supportive prior to my first day here at Brookhaven National Laboratory. I'd like to thank Xiaoyu Wang and Meng Yue for being the best supporting mentors ever, and I'd like to work for them again, perhaps without the need for making a poster and paper. Also, and finally, I'd like to thank Susan Lattuca, Ralph Garappolo, and entire Housing Department, Jim (BNL Cashier), and the janitor that works in building 130 for making my experience here a lot more enjoyable.

\section{References}

1. U.S. Department of Energy, The History of Solar, http:// www1.eere.energy.gov/solar/pdfs/solar_timeline.pdf, (Washington, DC, 2002)

2. Yue, Meng and Xiaoyu Wang, A Probabilistic Approach to Sizing Energy Storage System Capacity for Improving Grid Inertia Response under High Solar Penetration (Upton, NY, 2013)

3. Merriam-Webster, inertia, http://www.merriam-webster.com/ dictionary/inertia (Web, 2013) 


\title{
Bio-oil material compatibility
}

\author{
Michael Worek \\ University of Illinois Urbana-Champaign, Urbana, Illinois 61801 \\ Thomas Butcher \\ Department of Sustainable Energy Technologies, Brookhaven National Laboratory, Upton NY 11973
}

\begin{abstract}
Pyrolysis oil is an alternative source of renewable and sustainable energy and has been brought to the forefront as a replacement for heating oil in the Northeastern United States [Energetics Incorperated 2,59 (2012)]. Pyrolysis oil is also known to have detrimental properties that fossil fuels do not have such as, high oxygen content, increased viscosity, and increased acidity. The purpose of this study is to perform an assessment of the compatibility of upgraded pyrolysis oil in existing systems. The authors will accomplish this through two avenues of study. First, components from existing systems will be immersed in simulated pyrolysis oils of differing acid contents, made from a hydrocarbon fuel and an organic acid, at conditions that are common in oil fired boilers. Second, the authors will test the performance of a novel, thermally conductive polymer heat exchanger (PHX) affixed to a condensing oil fired boiler which will test both the performance and the integrity of the material in a corrosive environment. It will be shown that the acid content of the fuel does have an appreciable effect on the state of the materials under certain conditions. The authors will also show that the performance of the thermally conductive polymer heat exchanger is more efficient than standard polymers heat exchangers such as ones made from polypropylene and the integrity will stand under corrosive conditions thus making this material a viable material for corrosive environments.
\end{abstract}

\section{Introduction}

The U.S. Northeast is the largest consumer of heating oil in the United States. Of the 120 million barrels of oil that is used for heating the 5.75 million households in the U.S. northeast, approximately 80 million of those barrels are imported. This makes the heating oil market and the oil prices in the region dependent upon external factors [1]. To solve this dependency on foreign oil and external factors, the Department of Energy (DOE) is engaging in the introduction of domestic sustainable energy sources into existing oil burning systems. To ensure this shift of fuel consumption happens with as few setbacks as possible, the foreseeable problems of the chosen source of fuel must be accessed. A source of fuel that is accessible to a vast majority of the Northeast's population is wood and wood based pyrolysis oil. Unlike other renewable sources of energy like wind, solar, or hydropower, bio-oils such as pyrolysis oil will require the least amount of changes in infrastructure and will be attainable at a moment's notice. However these fuels pose challenges when used with existing systems. Pyrolysis oils are based from organic sources so they contain organic chemicals and properties that are incompatible with many modern systems. Such properties and chemicals include, high viscosity levels, debris from the wood that it was derived from, and high acetic acid contents. Arguably the biggest complication that arises from all of these is the acidity of the fuel and the corrosion that results from it. This issue can be fixed with refining the oil, however this process is not without cost and it raises an economical question of how refined does pyrolysis oil have to be so that the acidity doesn't affect present oil burning systems? Because of this there are two aspects that must be addressed; what acid content is allowable in order to store fuels without incident and what combustion processes are affected and how can they be fixed.

\section{Methods}

To do this, two investigations were designed to answer these questions. The first investigation was to measure the effects of simulated pyrolysis oils, which are made from a hydrocarbon fuel such as diesel and an organic acid commonly found in pyrolysis oils such as acetic acid. To do this common boiler materials were submerged in simulated pyrolysis oils of varying acidity. The materials tested consisted of yellow-metal-based spray nozzles that are used to inject oil into the combustion chamber of a conventional boiler. The reason behind using yellow metal like brass and copper is, of the materials used in boilers corrosion is known to affect yellow metals significantly more than stainless steel. Stainless steel in fact, it is known to be corrosion resistant which would invalidate the experiment [2]. To test these yellow metal nozzles, four samples of simulated pyrolysis oil were created with varying acid contents and the nozzles were submerged in the solution for a period of five days at an elevated temperature

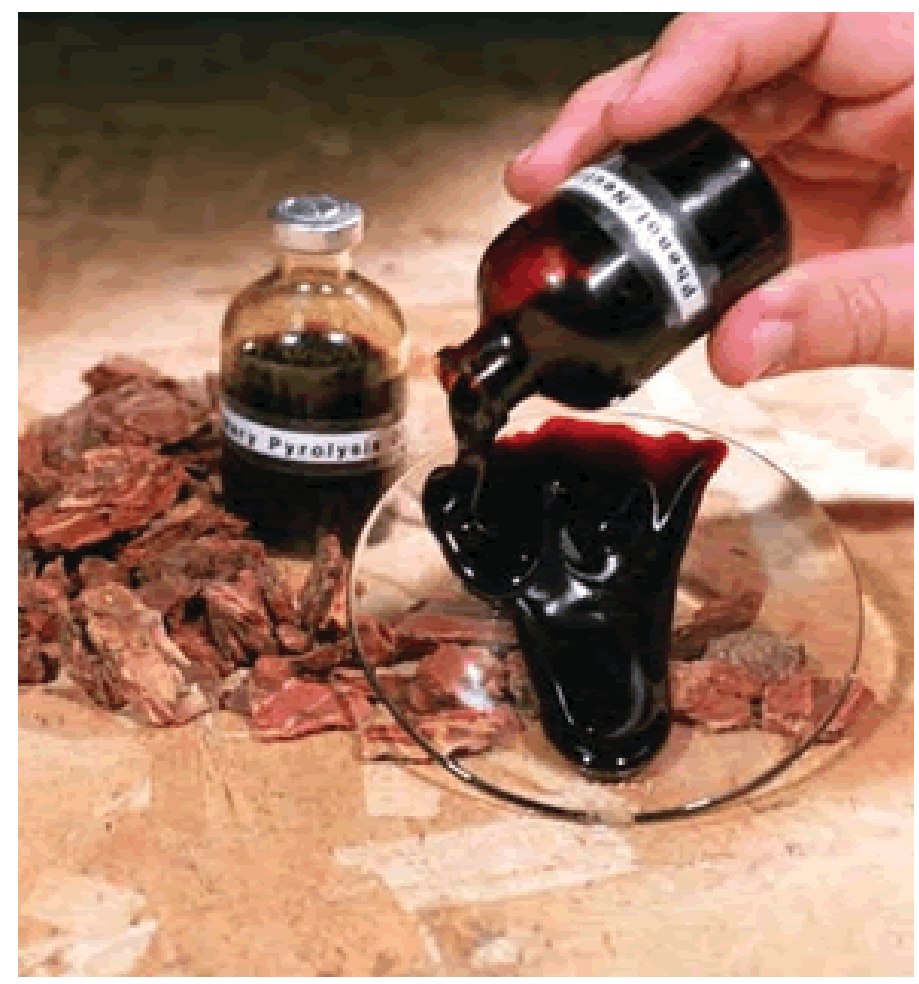

Fig. 1 Non-upgraded pyrolysis oil and wood chips Source: Technical Information Exchange on Pyrolysis Oil 2012 
of $170{ }^{\circ} \mathrm{F}$, after which the nozzles were removed to be examined. From past experiments in this line of research at Brookhaven National Laboratory (BNL) it was determined that when samples are left in solutions of varying acid content at room temperature the materials showed minimal to no corrosion or distress. Also nozzles are the highest temperature part of an oil-fired boiler system thus testing these materials at equally high temperatures simulates their operating conditions. It was for this reason that the samples were placed at an elevated temperature. The author's decision to use simulated pyrolysis oil was a result of the availability of partially refined pyrolysis oil. Refining pyrolysis oil to a specific acid content is very challenging and is not available in small batches.

The decided acid content used per sample was based in terms of acid number. A chemical's acid number is the amount in milligrams of a base, namely potassium hydroxide $(\mathrm{KOH})$, necessary to neutralize one gram of a chemical substance. The Total acid number (TAN) is a common measure of the acidity of fuels. For example the maximum TAN value of biodiesel specified by the American Society for Testing and Materials is $0.50 \mathrm{mg}$ of $\mathrm{KOH} / \mathrm{g}$ [3]. From this information the authors derived a simple equation to calculate the amount of acetic acid and diesel fuel necessary to create the acid numbers $0,2,4$, and 20 , where a TAN of 0 means

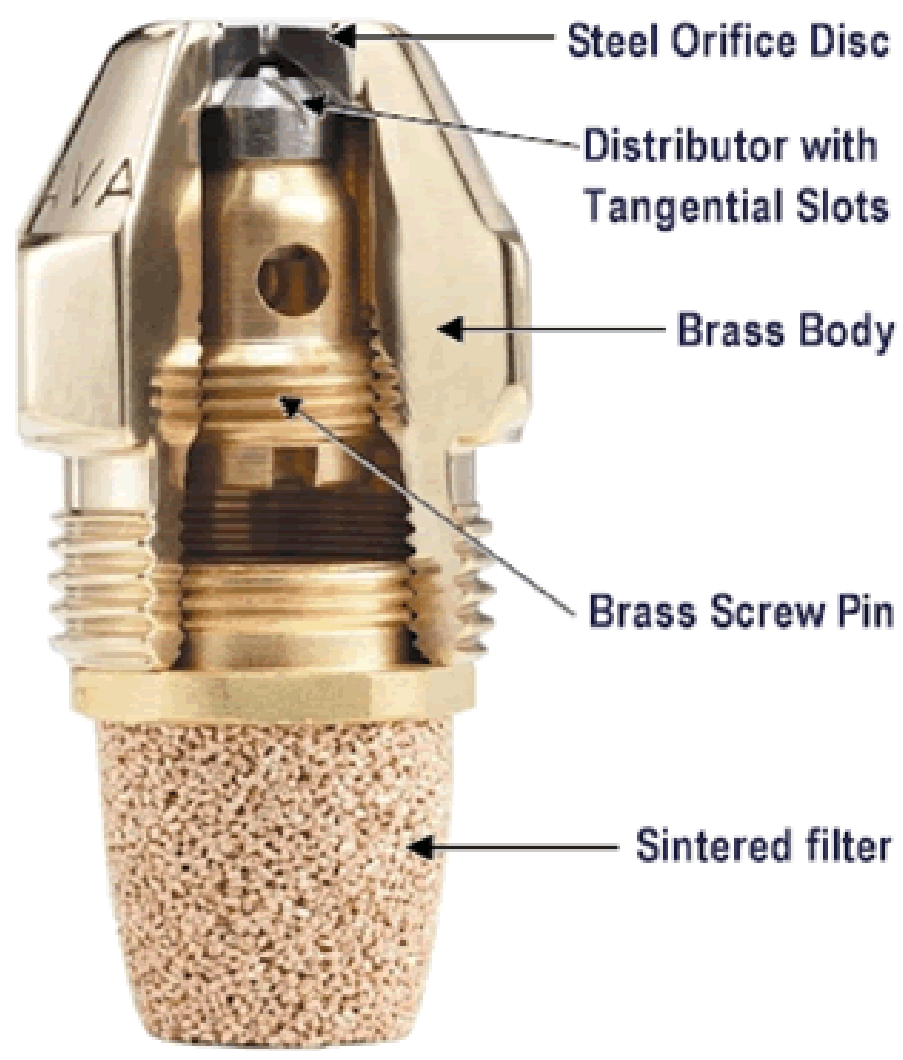

Fig. 2 Yellow metal boiler nozzle Source: http://www.delavaninc.com/oilburner.htm pure diesel fuel; this can be seen in Fig. 3. Once the amount of acid and fuel required to achieve a set TAN was calculated, the densities of each substance were used to calculate the volumes of acid and fuel needed. The four solutions were made and left to sit in an oven for five days at $170^{\circ} \mathrm{F}$.

At the end of the five days the samples were taken out and inspected. What was found was shocking but enlightening. The slightly green tint that had started to form just before the samples were put into the oven (Fig 4) had adhered onto the nozzles and all the solutions' color with the exception of the TAN 0 sample became drastically darker (Fig. 5). Also, the volume of each of the samples decreased proportionately to how much acid was added indicating that the acetic acid evaporated from the solution while being stored at $170{ }^{\circ} \mathrm{F}$. These results were not expected but they are helpful in determining what acetic acid does yellow metals under extreme conditions. From the clear accumulation of the green film in the solutions before the samples were put in the oven and the apparent density layer found in the samples (Fig. 4). It may be concluded that the solution did not remain at the TAN that was intended. To test this a titration of the sample that originally contained a TAN of 2 was preformed. What was found was that the sample had a TAN of about 10 . This increase in TAN is unexplained at the moment however a reason for this could be related to the lack of miscibility of the diesel and the acetic acid due to the acid's aversion to alkanes, which the diesel could have contained. This lack of miscibility would also explain the corrosion patterns on the nozzles. At varying locations there appeared lines corresponding to how the nozzles were placed into the samples where the effects of corrosion visibly changed. Also, the increase in the TAN could be explained by the degradation of the diesel fuel. When diesel rancidifies the triglycerides that are held in the oil-fats break down into fatty acids and glycerols.

The second experiment to address the question of material compatibility was focused on the end products of boilers, the exhaust. Exhaust was focused on because of its potential to create a corrosive environment that can be problematic in high efficiency condensing boilers. Condensing boilers use heat exchangers in to take heat from their exhaust gas and transfer it to their incoming water supply thus increasing efficiency. When any type of fuel is burned the exhaust gas contains byproducts that weren't burned during combustion. These byproducts often contain corrosive agents such as sulfuric acid in gaseous form and in the case of pyrolysis oil, may contain acetic acid in gaseous form. This makes the exhaust gas of boilers one of the most critical components for maintaining efficiency and function. The investigation that will run will test a novel conductive polymer that was made specially for BNL by Cool Polymers ${ }^{\circledR}$ (Fig 7).

Certain materials transfer heat very well and polymers generally aren't one of them. Materials such as copper are the most effective for transferring heat, however copper is not practical in corrosive environments while at high temperature as shown previ-

$$
\begin{aligned}
& \text { AcidNumber }=\left[\frac{m g K O H}{g S o l}\right] \times \frac{g}{10^{3} m g} \times \frac{m o l K O H}{56.11 g} \times \frac{\text { olC }_{2} \mathrm{H}_{4} \mathrm{O}_{2}}{m o l K O H} \times \frac{60.05 g}{m_{2} C_{2} \mathrm{H}_{4} \mathrm{O}_{2}}=\frac{g \mathrm{C}_{2} \mathrm{H}_{4} \mathrm{O}_{2}}{g S o l} \\
& A N \times .001069=\frac{g \mathrm{C}_{2} \mathrm{H}_{4} \mathrm{O}_{2}}{g \mathrm{Sol}}
\end{aligned}
$$

Fig. 3 Acid number calculations. Sources: Thomas Butcher, Michael Worek, and Andrew Salamone BNL 2013 
ously. This is where polymers excel. They are moldable, relatively cheap, and very corrosion resistant [4]. Ceramics are another branch of materials that tend to have a high thermal conductivity; unfortunately they are expensive which makes them uneconomical. However thermally conductive polymer offers the advantage of high thermal conductivity seen in metals and/or ceramics along with the corrosion resistance and processing ease seen in polymers. When we received the thermally conductive plastic heat exchanger tubing, there was need to design and build the frame that would maximize heat transfer and keep the design rigid without restricting airflow. From previous work at BNL it was decided that the coil would be wound in a double wall formation (Fig. $8 \&$ 9) with 1-millimeter spacing between adjacent tubes. This

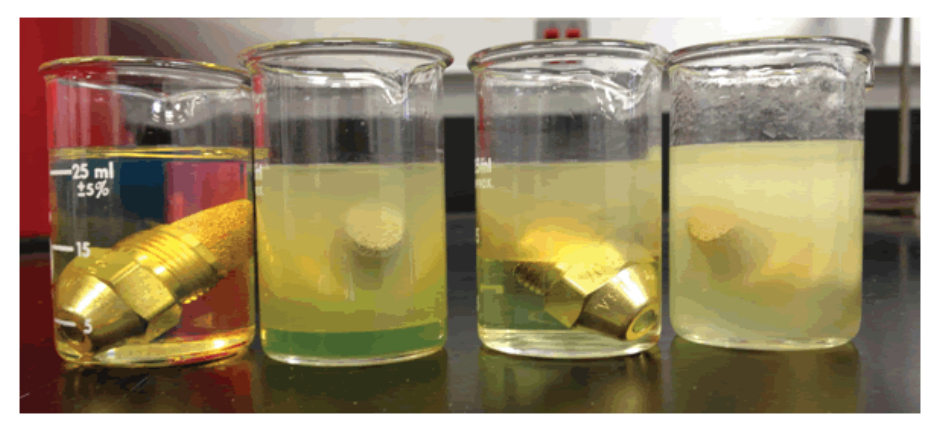

Fig. 4 Simulated pyrolysis oil pre-oven (from left: TAN 0, 2, 4, 20) Source: Andrew Salamone, Michael Worek BNL 2013

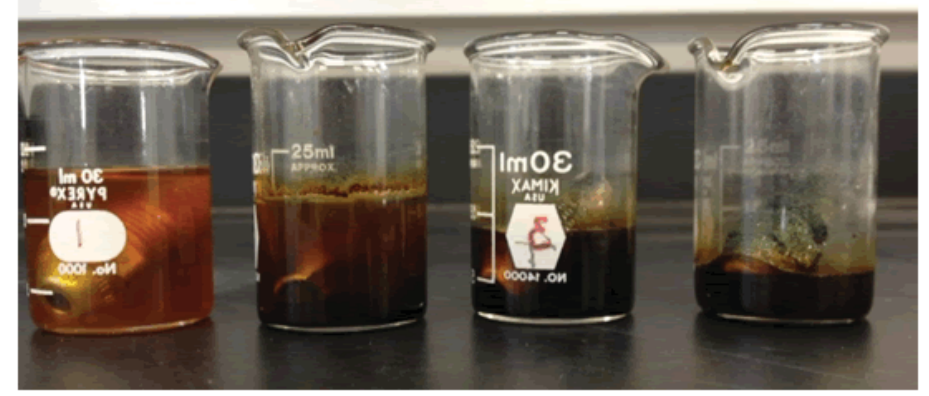

Fig.5 Simulated pyrolysis oil post-oven (from left: TAN 0, 2, 4, 20) Source: Andrew Salamone, Michael Worek BNL 2013

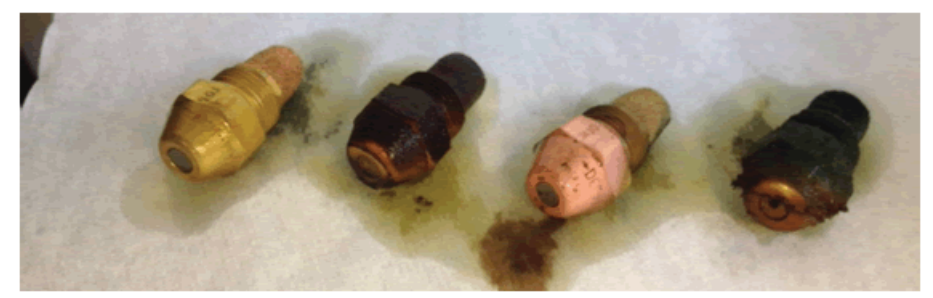

Fig. 6 Yellow metal nozzles post-oven (from left: TAN 0, 2, 4, 20) Source: Andrew Salamone, Michael Worek BNL 2013

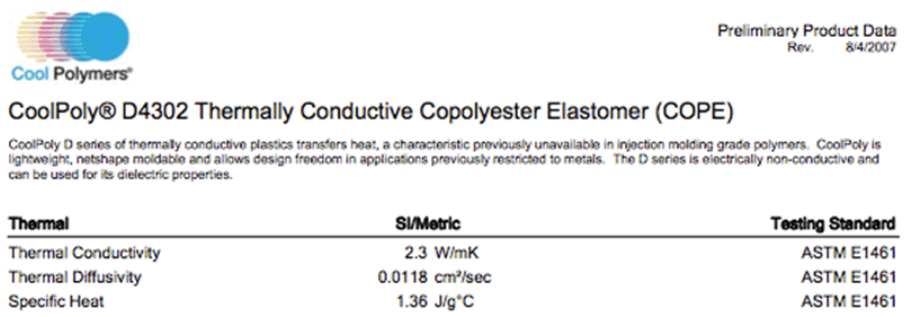

Fig. 7 Thermal conductivity of PHX Source: Cool Polymers ${ }^{\circledR}$ will support turbulent airflow across the surface of the thermally conductive polymer. Also the increase in surface are that can be achieved through spacing the tubing closely together will further aid heat transfer. The challenge with creating the frame design for the heat exchanger was the parts must be designed with high levels of precision, down to a fraction of a millimeter. Once a support piece was manufactured from the computer aided design (CAD) drawings the part was tested to make sure that the coils would stay rigid and maintain a 1-millimeter spacing. The part preformed well during testing keeping the tubes equidistant from one another at 1 millimeter. Further fabricating and assembly are being performed to make the final pieces of the plastic heat exchanger (PHX) but from what has been shown in previous experiments outcomes such as performance and efficiency look positive.
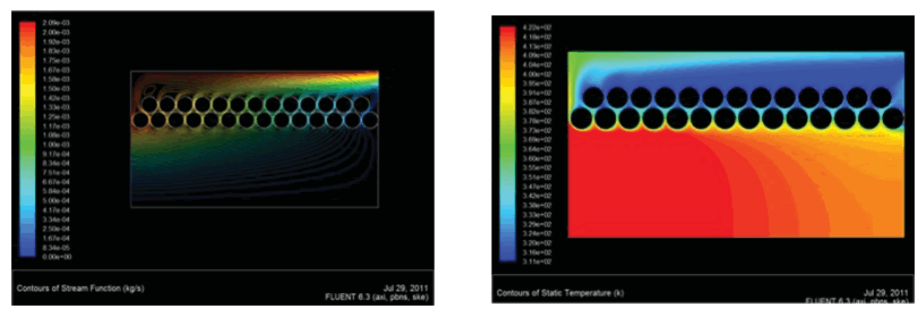

Fig. 8 (left). Flow diagram for double walled PHX Source: Tom Butcher BNL 2011

Fig. 9 (right). Temp contour for double walled PHX Source: Tom Butcher BNL 2011

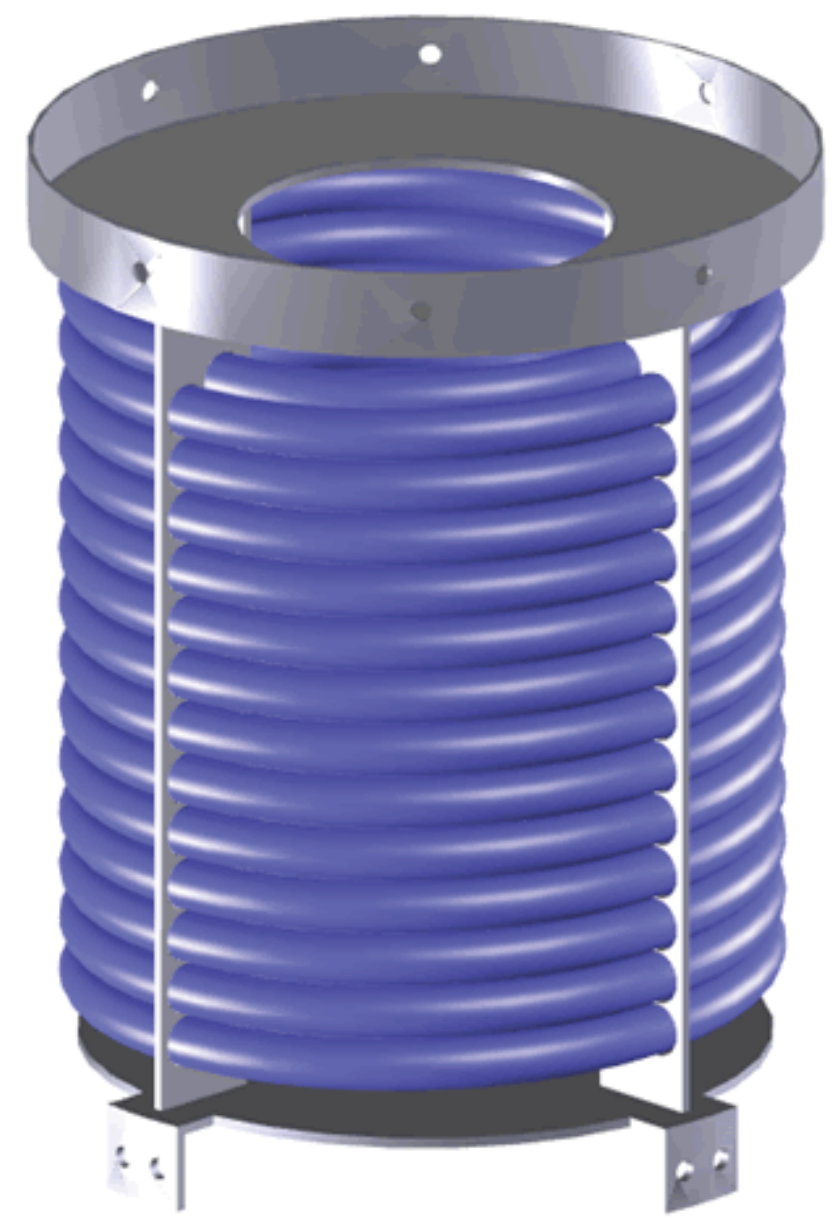

Fig. 10 CAD PHX model

Source: Michael Worek BNL 


\section{Conclusion}

The two investigations prepared both answer the overarching question in different ways. The investigation of the TAN of fuels showed that acetic acid does indeed affect metals such as yellow metals and operating at elevated temperatures can be devastating on boiler materials. Also the polymer heat exchanger solves the problem of corrosive environments through the use of materials that can withstand the effects of harmful conditions without sacrificing efficiency. All progress taken from this point onward must reflect these findings and hold them in contention when new ideas arise. Whether that is the development of a better refining method for pyrolysis oils or the manufacturing of boilers specifically built to run on pyrolysis oil these conclusions are applicable to future study. With regards to the continuation of experiments that were explained, tests must be run to verify the efficiency and heat transfer capabilities of this novel thermally conductive PHX. Also further analysis of the simulated pyrolysis oil must be performed in order to fully test that limits of modern boiler materials. The idea of renewable and sustainable energy sources, not only for the U.S. northeast but also for the whole of the United States of America, must be invested in for the sake of future generations both domestic and foreign and their security.

\section{References}

[1] Energetics Incorporated. "Potential for a Renewable Heating Oil Substitution Fuel in New England." Technical Information Exchange on Pyrolysis Oil (2012): 1-59. Print.

[2] Wissmiller, Derek. "Pyrolysis oil combustion characteristics and exhaust emissions in a swirl-stabilized flame." Iowa State University Graduate Theses and Dissertations (2009): n. pag. Print.

[3] Huali, Wang, et al. "Total Acid Number Determination of Biodiesel and Biodiesel Blends." Journal of the American Oil Chemical Society 85 (2008): 1083-86. Print.

[4] Reay, D. A. "The Use of Polymer in Heat Exchangers." Heat Recovery Systems \& CHP 9.3 (1989): 209-16. Print.

\section{Acknowledgments}

This project was supported in part by the U.S. Department of Energy, Office of Science, and Office of Workforce Development for Teachers and Scientists (WDTS) under the Science Undergraduate Laboratory Internships Program (SULI). I would also like to thank my mentor, Tom Butcher and collaborators, Andrew Salamone, Rebecca Trojanowski, and Christopher Brown. 


\title{
A study of muon injection efficiency improvements involving the electrostatic focusing quadrupoles in the Muon g-2 Experiment
}

\author{
David Wu \\ Dartmouth College, Hanover, NH 03755 \\ William Morse, Vladimir Tishchenko, and Yannis Semertzidis \\ Brookhaven National Laboratory, Upton, NY 11973
}

The muon beam entering the storage ring must go through the first quadrupole $\left(\mathrm{Q}_{1}\right)$ outer electrode. The muons crossing the outer electrode multiple Coulomb scatter and lose energy, resulting in an estimated $40 \%$ reduction in the number of stored muons [1]. A proposed improvement on the number of stored muons is to reposition the $\mathrm{Q}_{1}$ outer electrode horizontally. In this note, we address how far, radially outward, to move the outer electrode and improved quadrupole standoff designs.

\section{Quadrupole description}

The quadrupoles used to focus the beam occupy two vacuum chambers. The first quadrupole is separated into a half quadrupole $\left(Q_{1}\right.$ half $)$, located upstream, which is $13^{\circ}$ azimuth, and a full quadrupole $\left(\mathrm{Q}_{1}\right.$ full), located downstream, which is $26^{\circ}$ azimuth. There is a gap of $4^{\circ}$ azimuth between the half and full quadrupole.

The inflector is centered $+77 \mathrm{~mm}$ from the center of the storage region, and the muon beam must cross through the $\mathrm{Q}_{1}$ outer electrode, located $+50 \mathrm{~mm}$ from the center of the storage region, to enter into the ring. This is seen in figure 1.

\section{Beam simulation}

We calculate the phase space of the muon beam from the end of the inflector until the kicker region, centered approximately 11 meters downstream. The horizontal and vertical position of a muon as a function of distance downstream from the inflector are given by [2]

$$
\begin{aligned}
& x=\frac{R_{0}}{1-n} \frac{p-p_{0}}{p}+A_{x} \cos \left(v_{x} \frac{s}{R_{0}}+\varphi_{x}\right) \\
& y=A_{y} \cos \left(v_{y} \frac{s}{R_{0}}+\varphi_{y}\right)
\end{aligned}
$$

where $\mathrm{R}_{0}$ is the central orbit radius, $\mathrm{p}_{0}$ is the magic momentum, $\mathrm{p}$ is momentum at the end of the inflector $(\mathrm{s}=0)$, and $\mathrm{s}$ is the distance around the ring in azimuth. $\mathrm{A}_{x}$ is the amplitude of the betatron oscillation about the equilibrium orbit, $\mathrm{n}$ is the field index, and $\varphi_{x}$ is the phase of the betatron oscillation [2]. The tunes are $v_{x}=\sqrt{1-n}$ and $v_{y}=\sqrt{n}$.

Before the beam crosses the $\mathrm{Q}_{1}$ outer electrode into the storage region, it does not experience focusing from the quadrupoles, so $\mathrm{n}=0$, and equations $(1,2)$ simplify to:

$$
\begin{gathered}
x=R_{0} \frac{p-p_{0}}{p_{0}}+A_{x} \cos \left(\frac{s}{R_{0}}+\varphi_{x}\right) \\
y=y_{0}+y^{\prime} s
\end{gathered}
$$

The derivatives are:

$$
x^{\prime}=\frac{d x}{d s}=-\frac{A_{x}}{R_{0}} \sin \left(\frac{s}{R_{0}}+\varphi_{x}\right)
$$

$$
y^{\prime}=\frac{d y}{d s}
$$

At $\mathrm{s}=0$, we can solve for $\varphi_{\mathrm{x}}$ and $\mathrm{A}_{\mathrm{x}}$ in vacuum

$$
\begin{aligned}
& \varphi_{x}=\tan ^{-1} \frac{-x^{\prime} R_{0}}{x-R_{0} \frac{p-p_{0}}{p_{0}}} \\
& A_{x}=\frac{x-R_{0} \frac{p-p_{0}}{p_{0}}}{\cos \left(\varphi_{x}\right)}
\end{aligned}
$$

where $\mathrm{x}, \mathrm{x}$, and $\mathrm{p}$ are values at $\mathrm{s}=0$, given in a E821 simulation data file by Hugh Brown [3]. Using $\mathrm{A}_{x}$ and $\varphi_{x}$, we can calculate the muon horizontal position by equation 3 , and vertical position by equation 4 , as a function of $s$, the distance from the end of the inflector.

In figure 2(a) we can see the horizontal and vertical beam phase space at $\mathrm{s}=0$. The ellipses show the storage ring acceptances. The beam at the end of the inflector $(\mathrm{s}=0)$ is located $+77 \mathrm{~mm}$ horizontally from the storage ring center [2]. After a quarter of a turn $(\mathrm{s}=11.2 \mathrm{~m})$ the horizontal distribution has rotated 90 degrees to below the storage ring accepted $x$ ' and is then kicked into the storage ring acceptance [2], as seen in figure 2(b). In figure 2(b) we can see that the beam has been cut. We will discuss the various beam cuts in the next section.

\section{Beam cuts}

We only consider muons that are storable, i.e., within the stor-

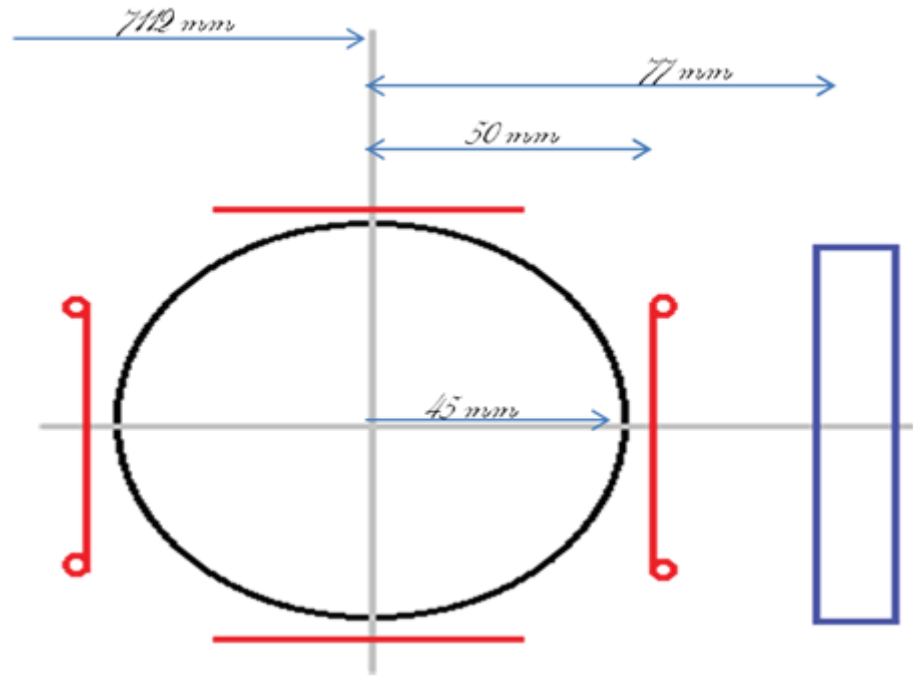

Figure 1. Cross-section of the beam including the horizontal positions of the beam channel (blue), quadrupoles (red), and storage region (black). The ring center is to the left. Not drawn to scale. 
age ring admittance assuming a perfect kick. We must examine the beam downstream of the first quadrupole to see which muons are worth considering, i.e., storable. We cut from consideration muons that do not fall within $\pm 0.25 \%$ of the magic momentum [2]. Then we fit the distribution of muon momentum to the E821 stored muon momentum distribution given in figure 20 of [2]. The result of these momentum cuts are seen in figure 3.

Next we cut the muons that hit the outer kicker plate positioned at $\mathrm{x}=50 \mathrm{~mm}$. Since the kicker plate is thick, most of the muons that hit the plate will be lost. This cuts $26 \%$ of injected muons for the E821 kicker and 35\% injected muons for the E989 kicker geometry . Finally, we cut muons that are not accepted into the storage ring circular aperture. The storage ring acceptance is defined by the maximum accepted horizontal and vertical positions in the $45 \mathrm{~mm}$ radius storage volume [2]

$$
A_{x s}^{2}+A_{y s}^{2}<(45 m m)^{2}(9)
$$

where $\mathrm{A}_{x s}$ and $\mathrm{A}_{y s}$ are the storage ring betatron amplitudes.

In the storage ring, we use equations $(1,2)$ with the E821 n $=0.14$ [2], and $\mathrm{E} 989 \mathrm{n}=0.18$. We solve for $\mathrm{A}_{x s}$ and $\mathrm{A}_{y s}$ at the $\mathrm{s}$ at which the muon crosses into the quadrupole field.

$$
x^{\prime}=\frac{d x}{d s}=-\frac{A_{x s} v_{x}}{R_{0}} \sin \left(v_{x} \frac{s}{R_{0}}+\varphi_{x}\right)(10)
$$
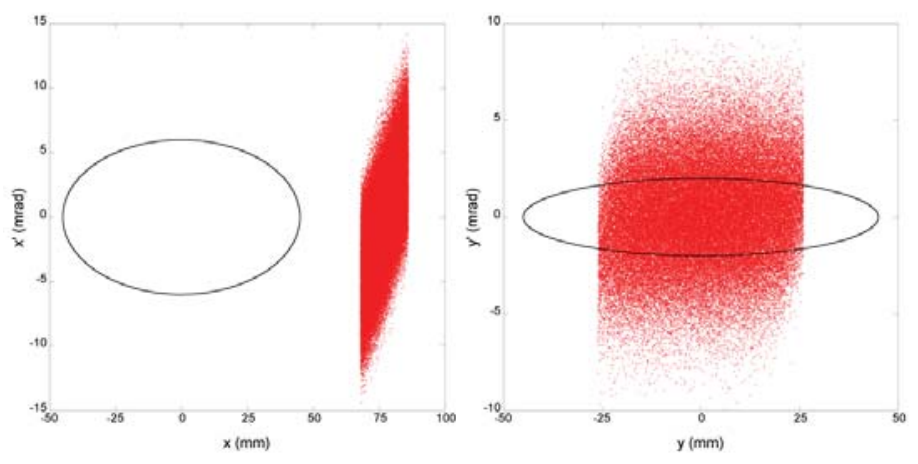

Figure 2(a). The horizontal (left) and vertical (right) phase space for the muon beam at $s=0$, as the beam leaves the inflector. The horizontal beam phase is displaced by $77 \mathrm{~mm}$ to the right due to the inflector position in relation to the storage ring center. The black ellipses represent the storage ring acceptance.
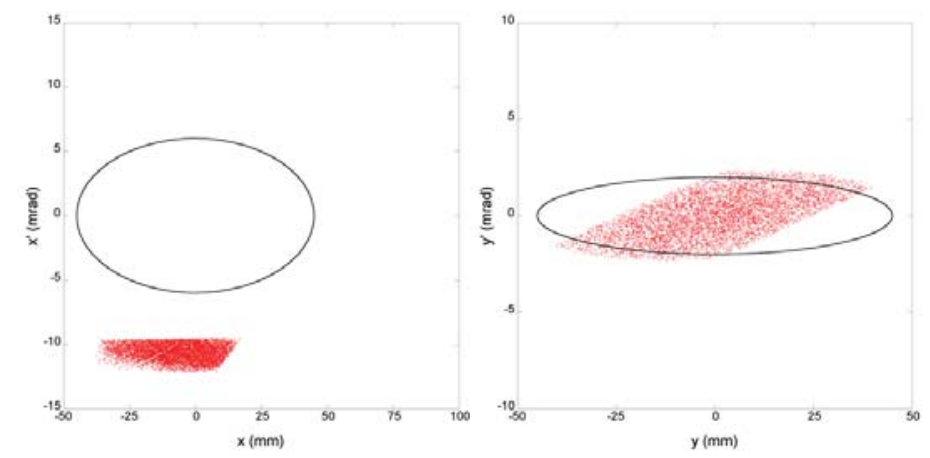

Figure 2(b). The horizontal (left) and vertical (right) phase space for the muon beam at a quarter of a turn, $s=11 \mathrm{~m}$, as the beam is about to be kicked in $x$ ' into the ring acceptance. We see that the beam has rotated 90 degrees in the horizontal plane. The beam is cut because we only consider storable muons.

$$
\begin{aligned}
& y^{\prime}=\frac{d y}{d s}=-\frac{A_{y s} v_{y}}{R_{0}} \sin \left(v_{y} \frac{s}{R_{0}}+\varphi_{y}\right)(11) \\
& \varphi_{x s}=\left(\tan ^{-1} \frac{-x^{\prime} R_{0}}{\left(x-\frac{R_{0}}{1-n} \frac{d p}{p}\right) v_{x}}\right)-v_{x} \frac{s}{R_{0}}(12) \\
& A_{x s}=\frac{x-\frac{R_{0}}{1-n} \frac{d p}{p}}{\cos \left(v_{x} \frac{s}{R_{0}}+\varphi_{x s}\right)}(13) \\
& \varphi_{y s}=\left(\tan ^{-1} \frac{-y^{\prime} R_{0}}{y v_{y}}\right)-v_{y} \frac{s}{R_{0}}(14) \\
& A_{y s}=\frac{\cos \left(v_{y} \frac{s}{R_{0}}+\varphi_{y s}\right)(15)}{(15)}
\end{aligned}
$$

where $\mathrm{p}, \mathrm{x}, \mathrm{x}^{\prime}, \mathrm{y}$, and $\mathrm{y}^{\prime}$ in equations (12-15) are values at the $\mathrm{s}$ at which the muon crosses into the quadrupole field. This cuts $60 \%$ of injected muons.

After these cuts, $8.9 \%$ of injected muons are storable, assuming the E821 kicker geometry and $\mathrm{n}=0.14$ (8.3\% for E989 kicker and $\mathrm{n}=0.18$ ), with a perfect kick. Although muons lose energy and scatter when they hit the outer $\mathrm{Q}_{1}$ electrode and its support, we do not factor this effect into our storable muon calculation. Our study concerns the number of muons that go through the electrode and support, and since energy loss and scattering only affects the beam after it goes through these structures, we do not include this in our simulation.

\section{Electric field outside $Q_{1}$ outer electrode and inflector tilt}

Between the outer $Q_{1}$ electrode and the vacuum chamber there exists an electric field due to the potential difference between the negatively charged outer electrode and grounded vacuum chamber. We simplify this field to an electric dipole field, which decreases the radius of curvature of the muons to 7.102 meters in the region between the outer electrode and vacuum chamber. From the inflector exit to the start of $Q_{1}$ half, in the gap between quadrupole sections, and inside the quadrupole region,

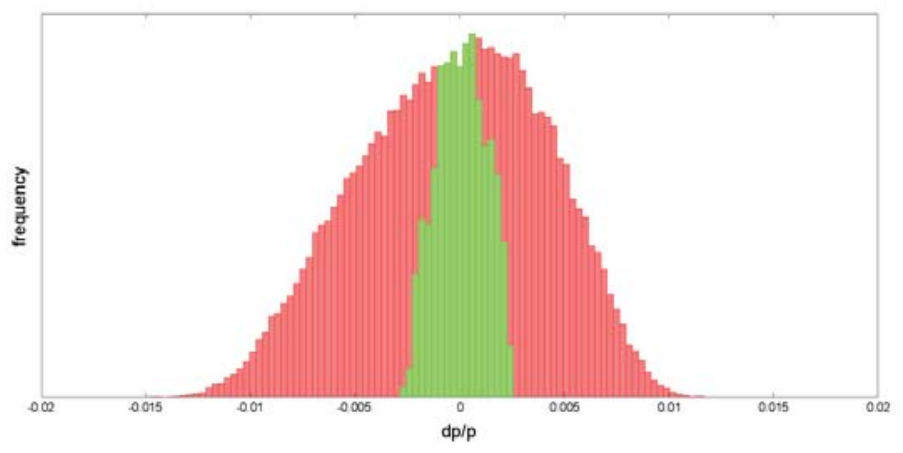

Figure 3. Red is the momentum distribution of Hugh Brown's muons at the inflector exit. Green is the momentum distribution of this simulation after applying the momentum cuts discussed, matching the stored $\mathrm{dp} / \mathrm{p}$ of $[2] .72 \%$ of muons do not meet the green momentum distribution. 
the radius of curvature is 7.112 meters.

$$
\begin{aligned}
& \frac{d p}{d t}=e(\vec{v} \times \vec{B}+\vec{E})=e\left(\vec{v} \times \vec{B}\left(1+\frac{\vec{E}}{\vec{v} \times \vec{B}}\right)\right) \\
& \vec{E} \approx \frac{\text { voltage }}{\text { distance }}=\frac{24 \mathrm{kV}}{0.04 \mathrm{~m}}=0.6 \vec{M} \mathrm{~V} / \mathrm{m}
\end{aligned}
$$

Between the outer electrode and vacuum chamber,

$$
\begin{aligned}
& \vec{v} \times \vec{B}_{0}=1.45 \mathrm{~T} \cdot 300 \cdot 10^{6} \mathrm{~m} / \mathrm{s}=435 \mathrm{MV} / \mathrm{m} \\
& \vec{v} \times \vec{B}_{E}\left(1+\frac{\vec{E}}{\vec{v} \times \vec{B}_{0}}\right)=435(1+0.00138)=435.6 \mathrm{MV} / \mathrm{m} \\
& \vec{B}_{E}=\frac{435.6 \mathrm{MV} / \mathrm{m}}{v}=\frac{435.6 \mathrm{MV} / \mathrm{m}}{300 \cdot 10^{6} \mathrm{~m} / \mathrm{s}}=1.452 \mathrm{~T} \\
& p\left(\frac{G e V}{c}\right)=0.3 B(T) R(\mathrm{~m}) \\
& R_{0}=\frac{3.094 \mathrm{GeV} / \mathrm{c}}{1.45 \mathrm{~T} \cdot 0.3}=7.112 \mathrm{~m} \\
& R_{E}=\frac{3.094 \mathrm{GeV} / \mathrm{c}}{1.452 \mathrm{~T} \cdot 0.3}=7.102 \mathrm{~m}
\end{aligned}
$$

We also studied the effect of tilting the inflector in x' on the number of storable muons. A negative tilt (-1 mrad) resulted in $2 \%$ less muons hitting both the E821 kicker and E989 kicker, which slightly increased the number of muons storable. A positive tilt $(+1 \mathrm{mrad})$ resulted in $2 \%$ more muon hitting both types of kickers and slightly decreased the number of muons storable.

\section{Simulation validity}

The checks on the accuracy of the simulation are as follows. The injection efficiency after the cuts simulated is $8.9 \%$, which is close to the $7.5 \%$ estimation given in [4] for an ideal kick. The phase space plots simulated show that the phase space agrees with the experimental 90 degree rotation after a quarter of a turn. Finally, we can see in figure 4 that the beam at $\mathrm{s}=11$ fits the storage ring acceptance. In this simulation, we assume uniform magnetic field, a perfect kicker field, and smooth betatron oscillation approximations. Further GEANT4 simulations should verify these results.

\section{Calculated results}
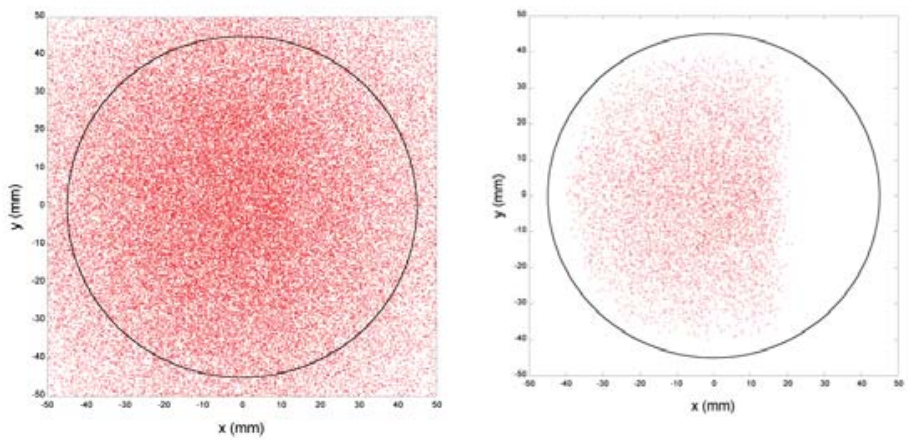

Figure 4. Left is a plot of the beam position without cuts and right is a plot of the beam position with cuts at $s=11 \mathrm{~m}$. The black circle is the storage ring aperture with radius $45 \mathrm{~mm}$.
After the beam leaves the inflector, which is positioned radially outward from the storage ring center, it swings in horizontally, going through the $\mathrm{Q}_{1}$ outer electrode and enters the center of the kicker. This is seen in figure 2. The muon beam horizontal distribution as a function of $\mathrm{s}$, distance downstream from the inflector exit, is shown in figure 5.

For this figure, muons that hit the outer kicker plate at $\mathrm{s}=7.7$ $\mathrm{m}$ are not included, nor are muons that have a vertical absolute position greater than $23.5 \mathrm{~mm}$ (the height of the outer electrode) at the point of crossing the electrode.

If the $\mathrm{Q}_{1}$ outer electrode is left at $\mathrm{x}=50 \mathrm{~mm}$, then we see that all these muons will hit the plate and the particles will scatter and lose energy [4]. The proposed solution is to move the electrode out radially. In figure 6 we see the distribution of $\mathrm{s}$ that muons cross the location of the $\mathrm{Q}_{1}$ outer electrode. We see that few muons cross $\mathrm{x}=50 \mathrm{~mm}$ before $3.9 \mathrm{~m}$ so we propose only moving $\mathrm{Q}_{1}$ full (as opposed to moving both $\mathrm{Q}_{1}$ full and $\mathrm{Q}_{1}$ half) out radially. From figure 5 we can also see that we would have to move $Q_{1}$ half out to $\mathrm{x}=85 \mathrm{~mm}$ for all muons to miss the plate while $\mathrm{Q}_{1}$ full can be moved out to only about $\mathrm{x}=70 \mathrm{~mm}$ for all muons to miss the plate.

We calculate that the maximum horizontal position of muons hitting $Q_{1}$ full is $72 \mathrm{~mm}$. This can also be seen in figure 5. If we move the $Q_{1}$ full outer electrode to $x>72 \mathrm{~mm}$, only muons that go through the $\mathrm{Q}_{1}$ half outer electrode will scatter or lose energy. With the $Q_{1}$ full outer electrode at $x=70 \mathrm{~mm}$ and the $Q_{1}$ half outer electrode at $x=50 \mathrm{~mm}$, we calculate that $13 \%$ of storable muons will hit the $\mathrm{Q}_{1}$ outer electrode (half + full). This is a 6-fold improvement from the calculated $78 \%$ that hit the $\mathrm{Q}_{1}$ outer electrode in E821.

The $22 \%$ of muons that don't hit the outer electrode in E821 miss the electrode vertically, that is, their vertical position absolute value was greater than $23.5 \mathrm{~mm}$ (the height of the electrode) at the point at which they crossed the outer electrode horizontally.

There is a small difference if we factor in the different operating field indices. For $\mathrm{n}=0.14$, the percent storable muons that hit $\mathrm{Q}_{1}$ are $13.4 \%$ with the new geometry and $78.2 \%$ with the old geometry. For $\mathrm{n}=0.18$, the percentages are $13.0 \%$ with the new geometry and $77.3 \%$ with the old geometry. We use $78 \%$ because E821 operated at $\mathrm{n}=0.14$. A inflector tilt of $-1 \mathrm{mrad}$ in $\mathrm{x}$ ' resulted in a $1 \%$ increase in storable muons that hit the $Q_{1}$ outer electrode. Figure 7 shows the muon fraction that hit the $\mathrm{Q}_{1}$ outer electrode (half + full) as a function of the $\mathrm{Q}_{1}$ full outer electrode horizontal position.
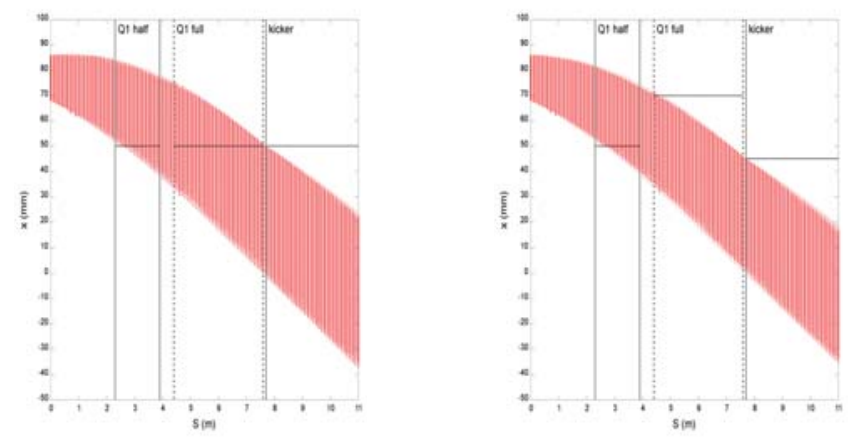

Figure 5. Beam horizontal position distribution as a function of distance from the end of the inflector. $Q_{1}$ half is located between $s=2.3$ $\mathrm{m}$ and $3.9 \mathrm{~m}, Q_{1}$ full is located between $\mathrm{s}=4.4 \mathrm{~m}$ and $7.6 \mathrm{~m}$, the kicker starts at $s=7.7 \mathrm{~m}$. Left: E821 $Q_{1}$ position, E821 kicker, $\mathbf{n}=\mathbf{0 . 1 4}$. Right: proposed $Q_{1}$ position, E989 kicker, and $n=0.18$. 


\section{Electrode Standoffs}

Here we also study the number of muons that go through the $\mathrm{Q}_{1}$ standoffs of varying design, thickness, and number. Three designs are considered: the E821 design, a thin horizontal standoff design, and a thin vertical standoff design, shown in figure 8. Figure 9 shows the percent storable muons that hit the two proposed designs as a function of thickness. The three graphs differ in their $\mathrm{Q}_{1}$ outer plate position and kicker type.

The percent storable muons hitting the E821 standoff geometry is $31 \%$ for $Q_{1}$ full $x=50 \mathrm{~mm}$ and $27 \%$ for $x=75 \mathrm{~mm}$. There are three standoffs per half quadrupole in the E821 design, with $10 \mathrm{~mm}$ thickness. We propose a thin vertical standoff design to minimize muon energy loss and scattering. With three standoffs per half-quadrupole and a thickness of $4 \mathrm{~mm}$, only $8 \%$ of storable muons hit the standoffs for the proposed $\mathrm{Q}_{1}$ outer electrode horizontal location.

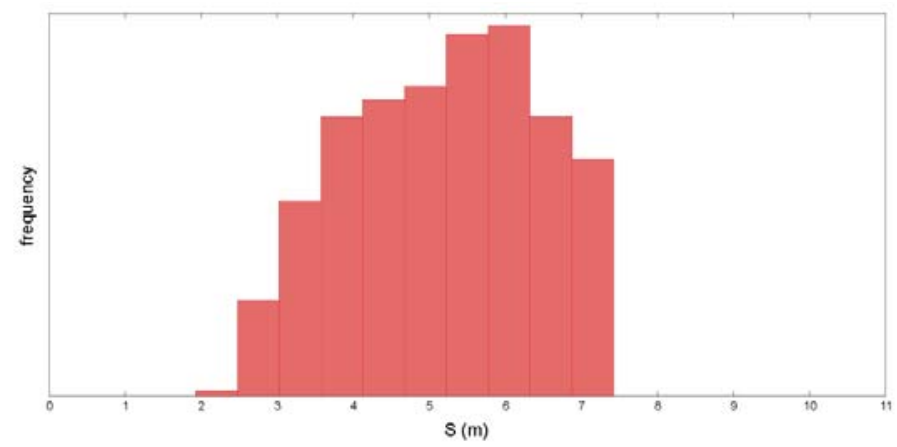

Figure 6. Distribution of distance after the end of the inflector, s, at which the beam crosses the horizontal position of the outer $Q_{1}$ plate, $x=50 \mathrm{~mm}$. Few muons cross the outer plate of $Q_{1}$ half $(2.3 \mathrm{~m} \leq \mathrm{s} \leq$ $3.9 \mathrm{~m})$.

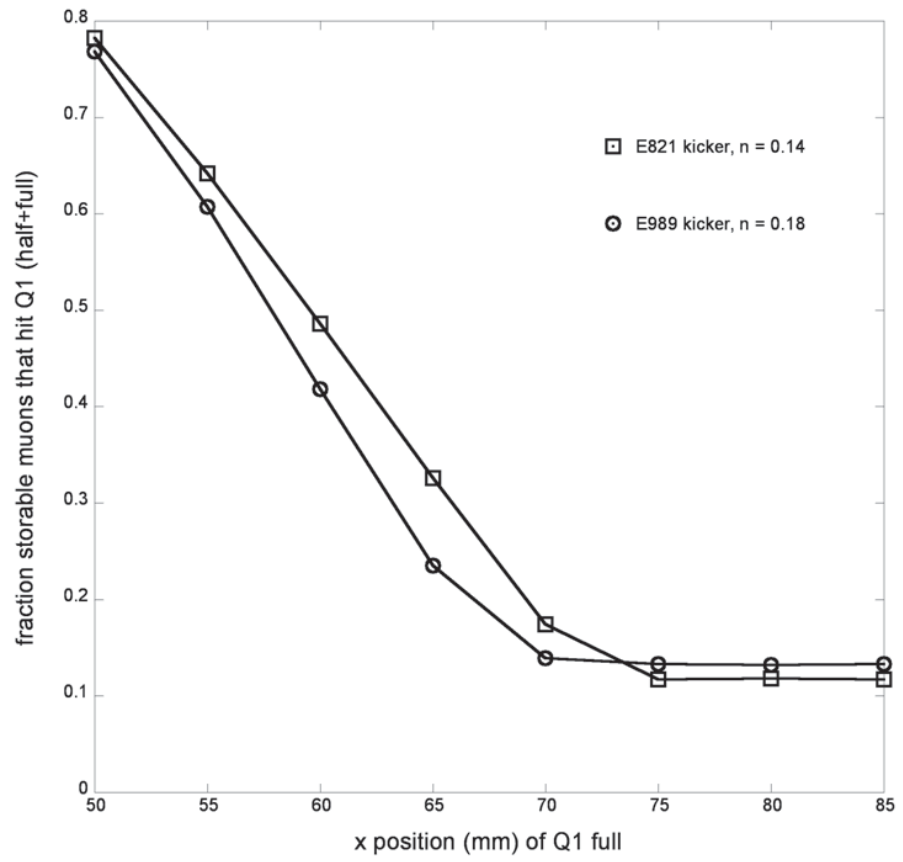

Figure 7. Fraction of storable muons that hit $Q_{1}$ outer (half + full) as a function of the horizontal position of $Q_{1}$ full. The percent that hit the outer electrode does not change as we move the plate from $x=$ $85 \mathrm{~mm}$ to $x=75 \mathrm{~mm}$ (E821 kicker) or from $x=85 \mathrm{~mm}$ to $x=70 \mathrm{~mm}$ (E989 kicker). Changing the field index from 0.14 to 0.18 changes the plot by a negligible amount

\section{Conclusions}

We find that moving the $\mathrm{Q}_{1}$ full outer plate out to $70 \mathrm{~mm}$ horizontally, assuming the E989 kicker and $\mathrm{n}=0.18$, will result in a factor of 6 increase in the muons that do not hit the $Q_{1}$ outer plate and scatter in E989. We plan to leave the $\mathrm{Q}_{1}$ half outer plate at $\mathrm{x}$ $=50 \mathrm{~mm}$. We also propose a vertical standoff design to maximize the number of muons that will be storable in the E989 experiment.

\section{References}

[1] Chapter 14. E989 CDR

[2] G.W. Bennett, et al., Phys. Rev. D Lett. 89 (2002) 101804

[3] Chapter 6. E821 CDR

[4] E. Efstathiadis et al., Nucl. Inst. Meth. A 496, 8 (2003).

[5] Y. K. Semertzidis et al., Nucl. Inst. Meth. A 503, (2003).

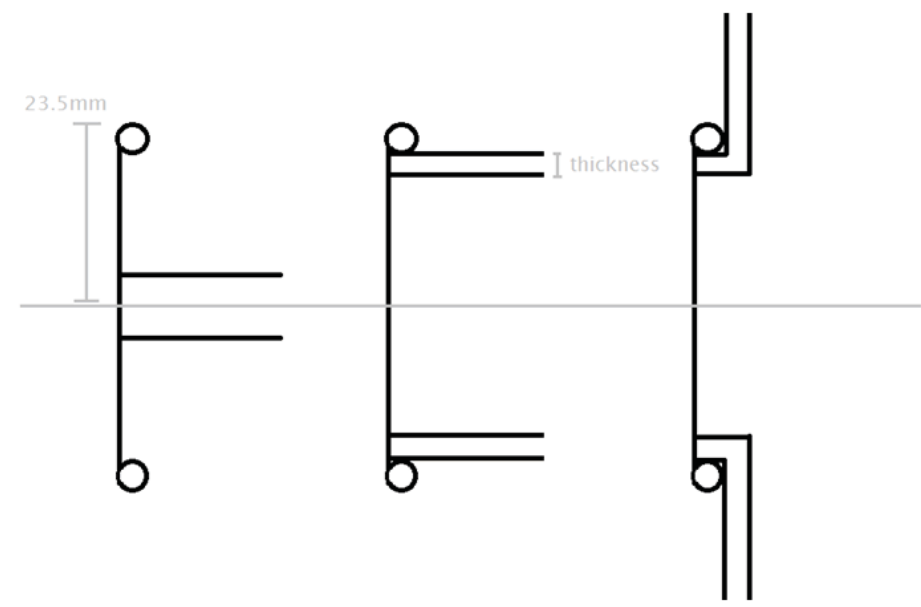

Figure 8. Three standoff designs. The left is the E821 design, measuring $10 \mathrm{~mm}$ thick. The middle is a horizontal standoff design, and the right is a vertical standoff design, both with varying thicknesses.
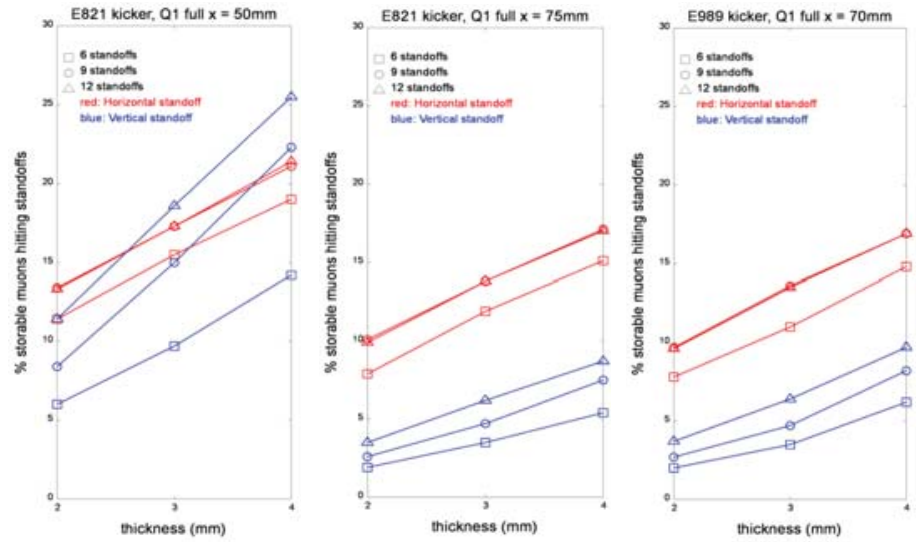

Figure 9. Percent storable muons hitting the standoffs as a function of thickness. The first graph is with the E821 kicker and no change in Q_1 full outer plate position. The second is with the E821 kicker and Q_1 full outer plate at $x=75 \mathrm{~mm}$. The decrease in percent storable muons hitting the standoffs from the first to second graph is due to the decrease in number of muons hitting the standoffs for $Q_{-} 1$ full. The last graph is with the proposed E989 kicker and proposed Q_1 outer plate $x$ position of $x=70 \mathrm{~mm}$. The first two graphs assume $n=$ 0.14 , the last, $n=0.18$. 


\title{
Phase shift full bridge power converter analysis and test fixture design
}

\author{
Man Zhang \\ Engineering Department, Suffolk County Community College, Selden NY 11784 \\ Chaofeng Mi \\ Collider-Accelerator Department, Brookhaven National Laboratory, Upton NY 11973
}

\begin{abstract}
One major type of power supply used in the Relativistic Heavy Ion Collider (RHIC) is a bipolar power supply which provides current at either +/-150A or $+/-300$ A to specific magnets. This type of power supply contains a switching converter that feeds the linear output stage. The converter modules of the power supplies convert 208 A three phase AC input to a DC output. The converter output voltage varies based on required output voltage and current to the external load. We analyzed the converter design and, furthermore, designed a fixture to test and understand the performance of the converter. The converter module is simulated as a part of the original power supply converting three phase $\mathrm{AC}$ input to $\mathrm{DC}$ voltage. The full $\mathrm{H}$ bridge circuit in the converter is controlled by a Pulse Width Modulation (PWM) controller integrated circuit. This paper will describe the above analysis and design.
\end{abstract}

Certain magnets in RHIC such as dipole and quadruple utilize DC current to produce a steady, unidirectional magnetic field. The power supply studied in this project provides power to such magnets.

The converter in the power supply design performs the function of converting AC voltage to DC. The converter takes threephase AC power and rectifies it to DC unregulated voltage. This

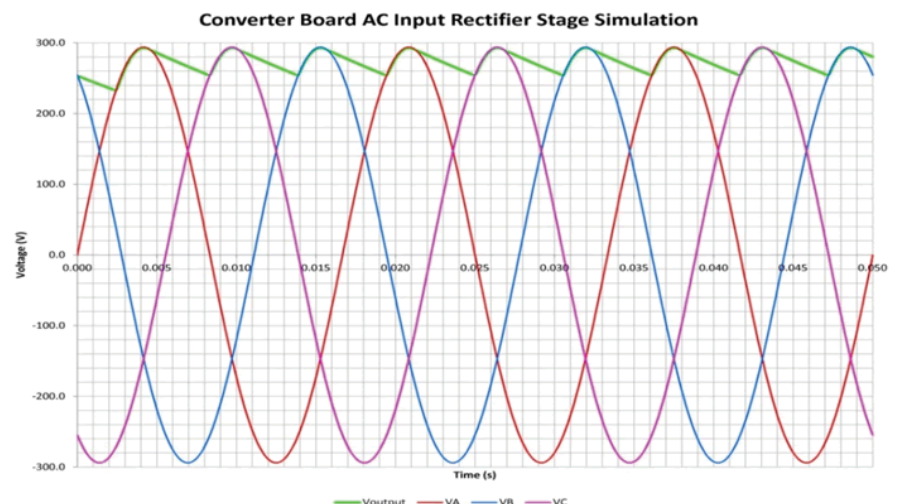

Figure 1: converter board AC input rectifier stage simulation waveform voltage is used for the H-bridge in the next stage. In an H-bridge circuit, the power is transferred through a diagonal pair of MOSFETs. The output power of $\mathrm{H}$-bridge is coupled out through a high power switching transformer and the rectifier, L-C filter and output storage capacitor banks for linear output stage to use.

As shown in figure 1 and 2, 208V, the $60 \mathrm{~Hz} 3$ phase input with 120 degree shift between each line enters the converter via port J501. And then the Electromagnetic interference (EMI) filter screens the input noise and passes the power to the rectifier circuit via port J502A. The two pairs of full bridge rectifiers consist of 8 diodes convert AC power to $290 \mathrm{~V} \mathrm{DC}$, as shown in figure 1 . The full bridge transfer power on both half-cycles and the output ripple are multiples of the input frequency. Figure 5 is the simulated output waveform of one full bridge rectifier. The average voltage is below the peak value. The two full bridge rectifiers are more efficient compare to one pair full bridge rectifier.

The filtered DC voltage is then applied to the H-bridge. A conventional H-bridge drives the diagonal switches together which alternately place the transformer primary across the input supply for some period of time. The on/off of the switches are controlled by the Pulse Width Modulation (PWM) controller. Figure 7 shows the waveforms of a conventional full bridge PWM. The diagonal pairs Q1 and Q3, or Q2 and Q4 are driven together. When switch-

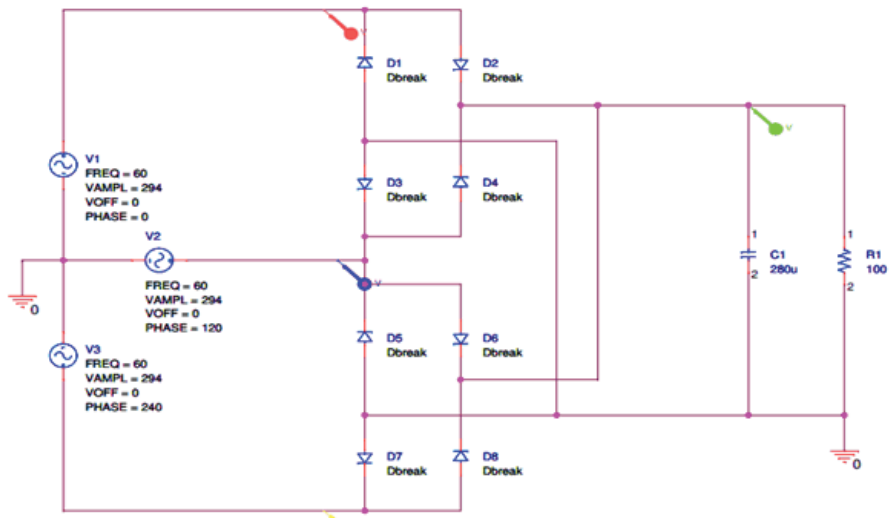

Figure 2: converter board AC input rectifier stage simulation block diagram

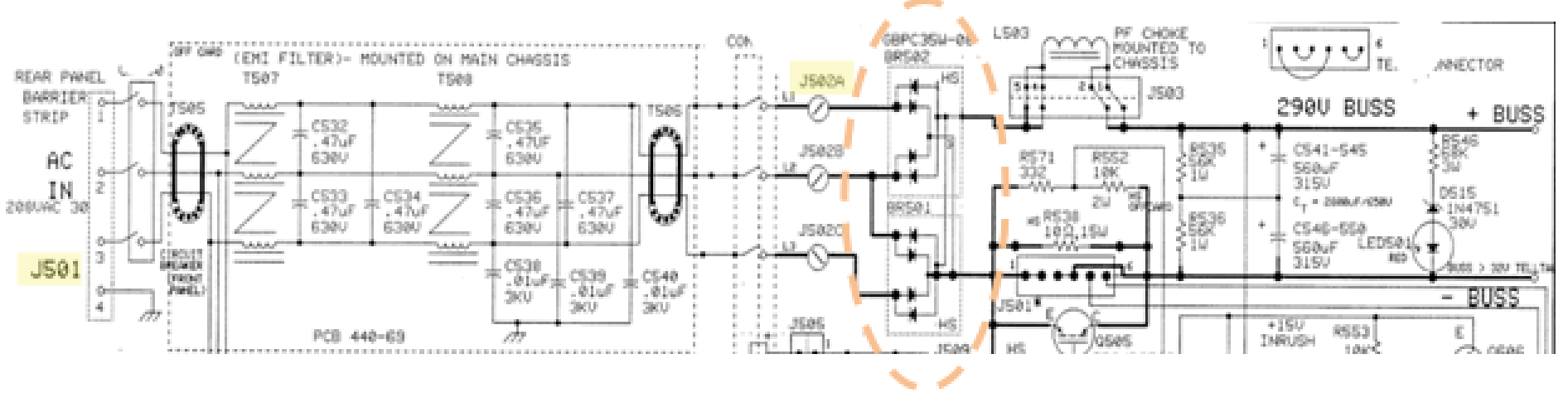

Figure 3: converter schematic 1 
es Q1 and Q3 are on and the other components are off (figure 8a), power derived from the input rectifier and slugs of energy from Cin flows through Q1, the primary winding of the transformer Tp and Q3. A voltage Vin is developed across the primary winding of transformer. A similar voltage Vin* $\mathrm{N}$ is developed across the secondary winding of the transformer $(\mathrm{N}$ is the ratio of the transformer primary winding and secondary winding.) The secondary voltage causes rectifiers D5 and D8 to conduct current. When Q2 and $\mathrm{Q} \neg 4$ are on (figure $8 \mathrm{c}$ ), power flows through Q2, TP and Q4 counterclockwise, a voltage -Vin is developed across Tp. The induced secondary voltage causes rectifiers D6 and D8 to conduct current. Notice that the voltages across transformer primary and secondary reverse direction while the switches alternates, the polarity of the voltage at the load never change due to the rectifier circuit D5-8.

Unique to the UC3875 PWM controller which controls the ON time of the $\mathrm{H}$ bridge switches, a phase shift switching is introduced to the commands. Compare to the conventional full bridge,

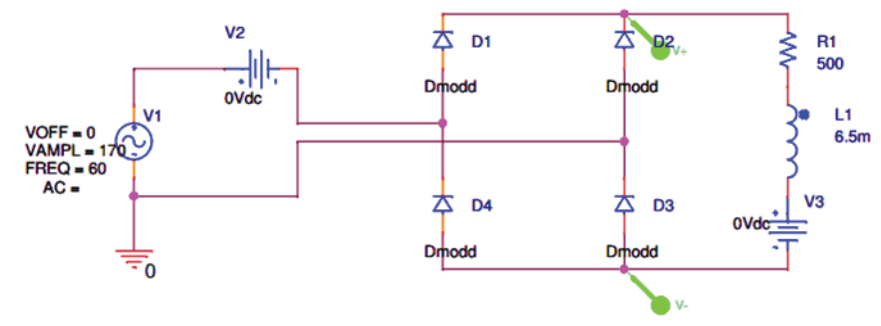

Figure 4: 4 diode full bridge rectifier simulation block diagram

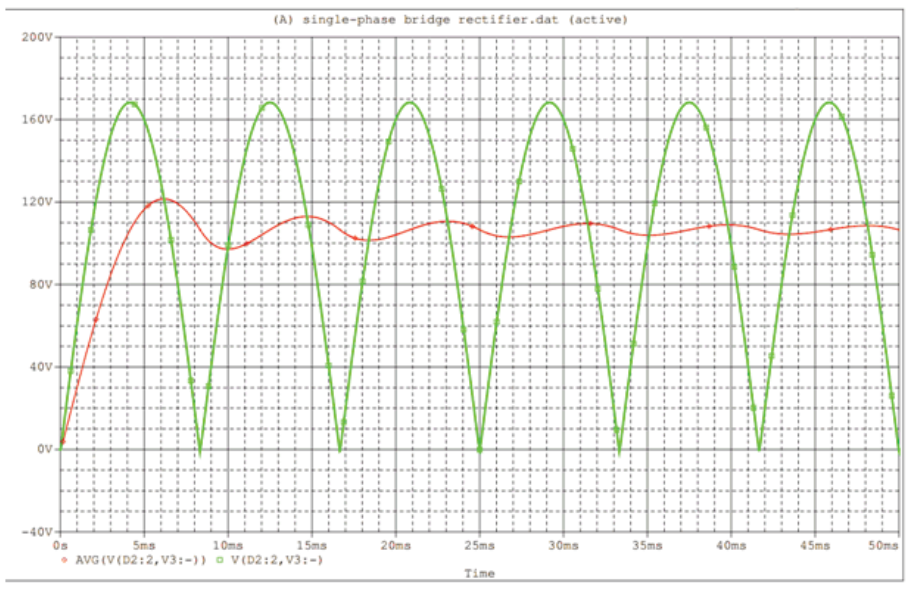

Figure 5: 4 diode full bridge rectifier simulation waveform

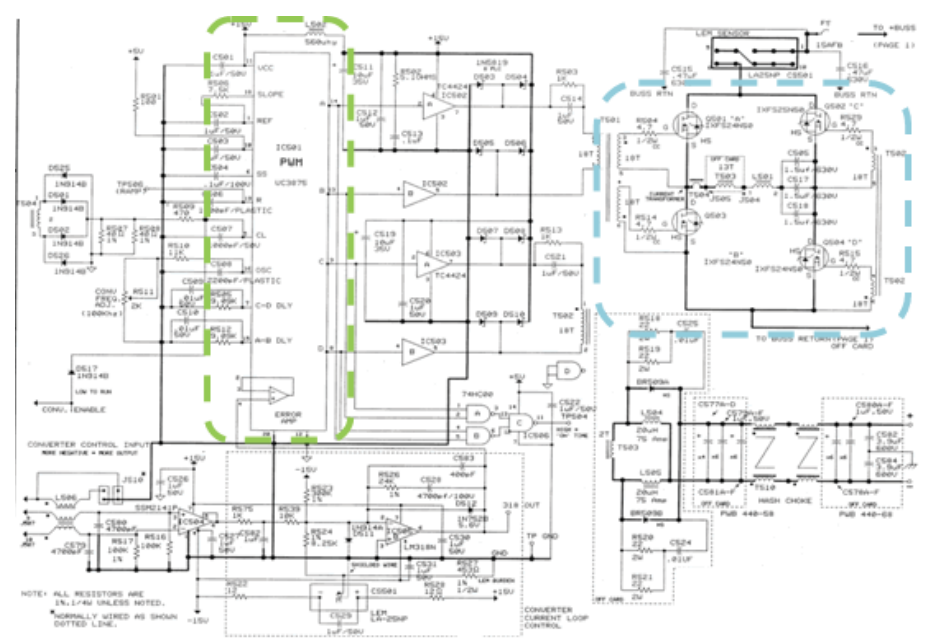

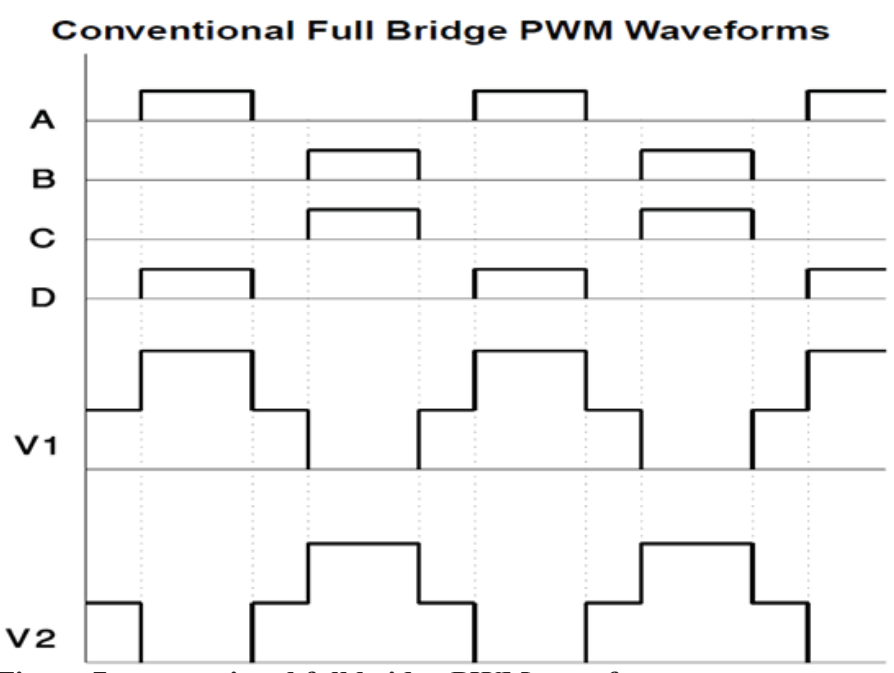

Figure 7: conventional full bridge PWM waveforms

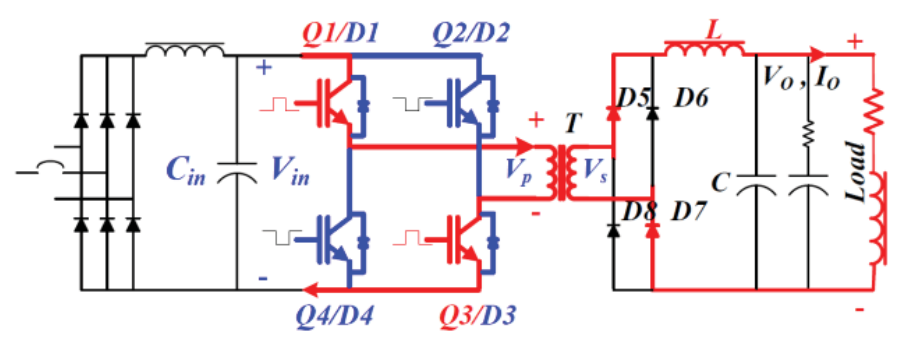

Figure 8a: Q1, Q3=ON

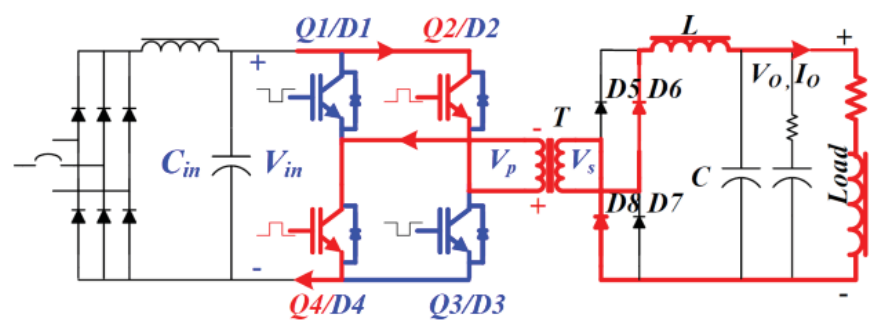

Figure 8b: Q2, Q4=ON

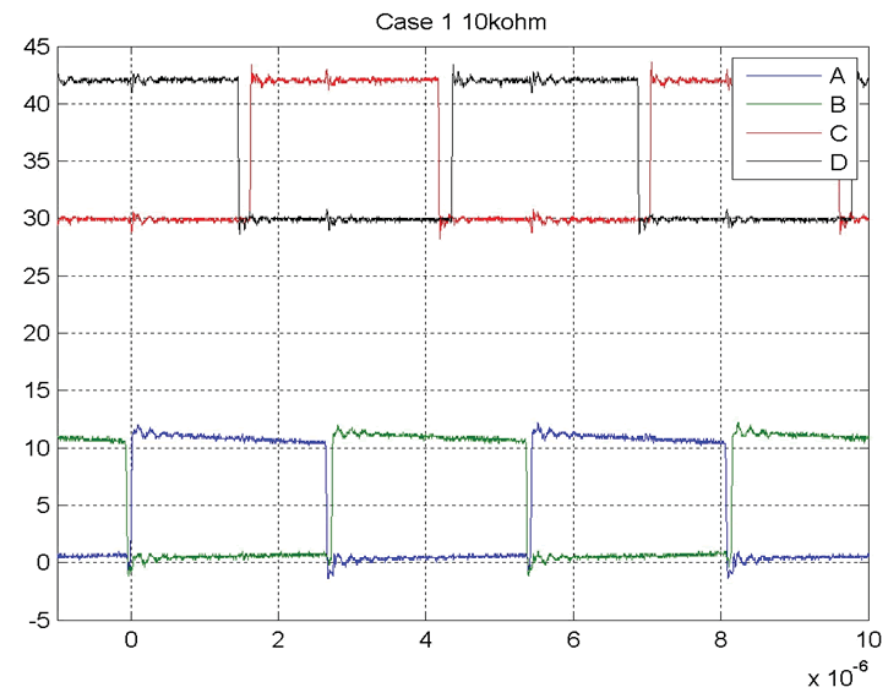

Figure 6: converter schematic 2 


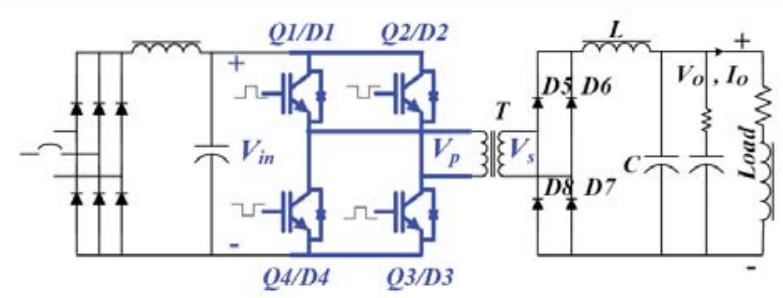

Figure 9a: $Q 1, Q 2=O N$

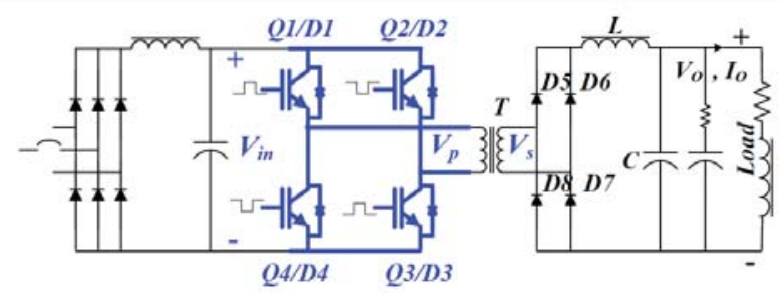

Figure 9b:Q3, Q4=ON

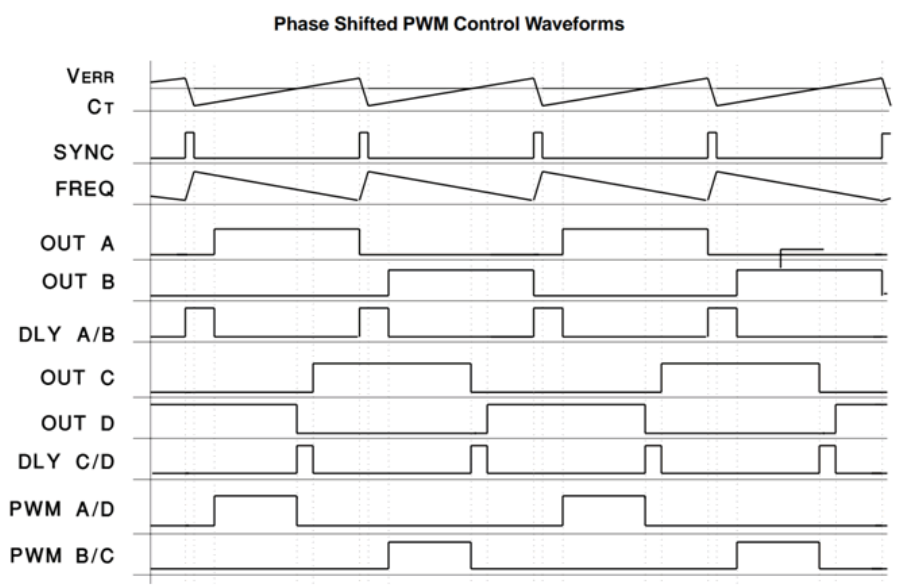

Figure 10: phase shifted PWM control waveforms

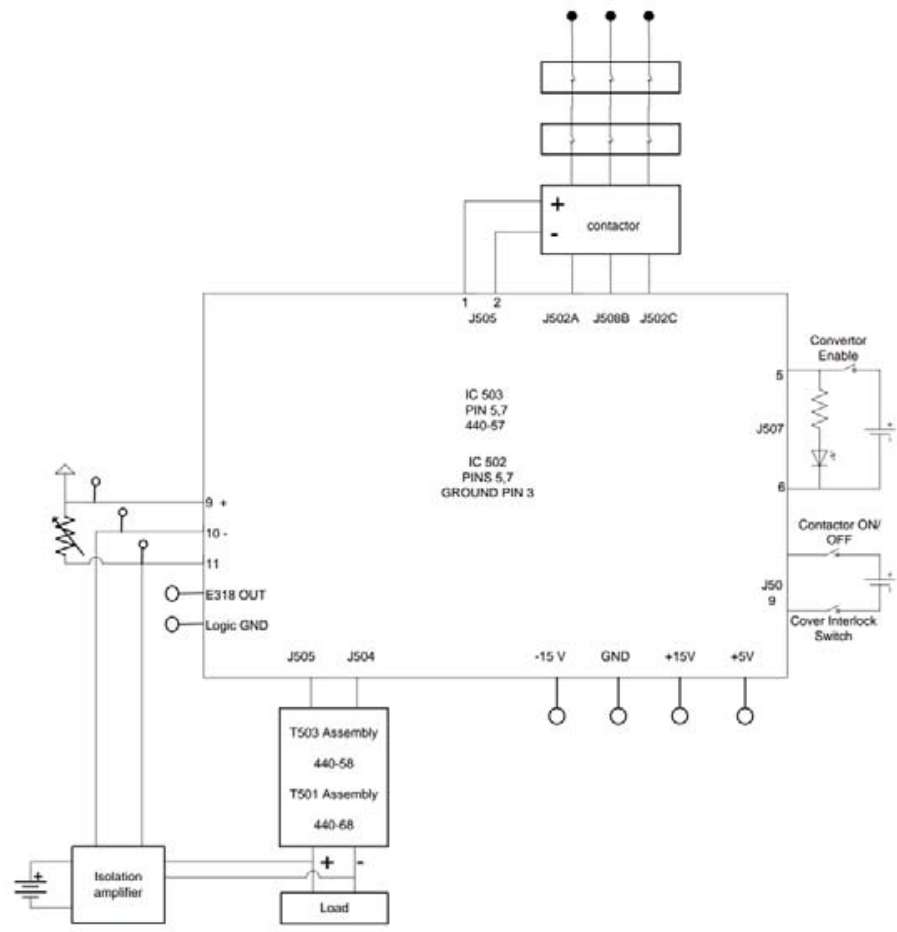

Figure 11:test fixture block diagram which open or close the diagonal switches at the same time, the phase shift switching add a deliberate delay between their turn-on commands; therefore, not just the diagonal pairs, but also the two upper or lower switches can be turn-on at the same time. In this mode, when the transformer primary is essentially short-circuited and the current in the closed loop decreases to zero, the switches can be turned off in this state enabling zero current switching which reduces switch noise.

\section{A. Interval 1: Q1, Q3=ON}

The primary current IP is being conducted through Q1 TP and Q3 initially. A voltage Vin is developed across the primary winding of transformer. A similar voltage Vin* $\mathrm{N}$ is developed across the secondary winding of the transformer.(see figure 8a)

\section{B. Interval 2: Q1, Q2=ON}

Instantly the switch Q3 is turned off and Q2 is turned on by the PWM controller. The current freewheels through Q1-TP-Q2 loop. During the transition, the current in the loop has decreased from Ip to zero. (See figure 9a.)

\section{Interval 3: Q2, Q4=ON}

Q4 is turned on and Q1 is turned off while the current is zero thus lowering conduction losses. Current flows through Q2, TP and Q4 counterclockwise, a voltage -Vin is developed across Tp (see figure 9b.)

\section{Interval 4: Q3, Q4=ON}

Q2 is turned off and Q3 is turned on by the PWM controller. The current free wheels through Q3-TP-Q4 loop. During the transition the current in the loop has decreased from Ip to zero (see figure 9a.) as one transition cycle has been completed. Switch Q4 will be turned off and Q1 will be turned-on next to start a new cycle.

The switching operation will eventually result a square wave across the transformer secondary at maximum $1 \mathrm{MHZ}$ frequency. The full bridge at the output rectifiers the square wave to DC voltage.

Power is only transferred to the output section during the $\mathrm{ON}$ times or the overlapping time of the two diagonal switches. The $\mathrm{ON}$ time is controlled by the programmable time delay function of the PWM controller. By adjusting the ON time, the supply only

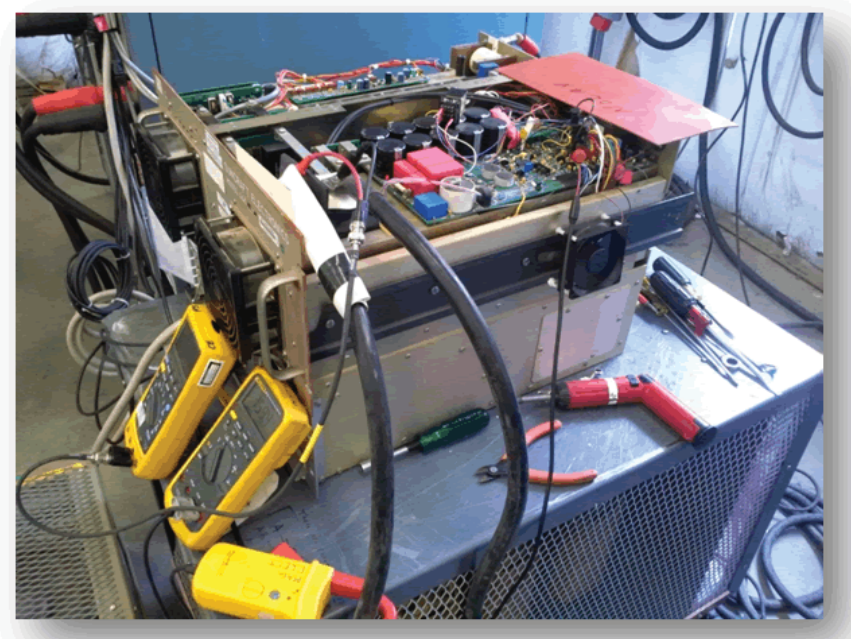

Figure 12: test setup 
transfer power to the load in the required amount and therefore improves the power usage efficiency.

In order to help the user to understand the performance of the converter, as well as trouble shoot the converter when there is a problem, a test fixture is designed to serve such purpose. The test fixture provides access to the isolated converter without any other parts of the power supply.

The test fixture consists of input, output, and interface. The $208 \mathrm{~V} 3$ phase input goes through the contactor to the convertor board via point J502A, J508B and J502C. The output is delivered to the assembly and the load via port J505 and J504. The contactor $\mathrm{ON} / \mathrm{OFF}$ at port J509 is a $15 \mathrm{~V}$ interlock switch controls on and off of the contactor. The power is delivered to the converter board only if the contactor is on, which secures the safety of the user. Port 9, 10, 11 is the control interface for the converter circuitry. The external potentiometer at port 11 generates the command signal to drive the converter. Port 10 is the feedback voltage from isolation amplifier, which is a measurement of the output voltage across the load. Because the power supply itself is floating during operation, an isolation amplifier is necessary to isolates the user interface and the output power stage.

A number of test point are brought out to the front panel allow the user to access the signals easily.

\section{Conclusion}

The operation of the full-bridge phase shifted converter was analyzed and its test fixture designed. The test fixture is under construction. Measurements were taken in a test set up similar to the test fixture. (See figure 12 for test setup.) A converter board inside of actual power supply was connected to a $5 \mathrm{~m} \Omega$ load. As shown in table 1, as we adjust the external potentiometer to increase the set point from 0 to 7.0, the power delivered to the load increases from $84 \mathrm{w}$ to $1.4 \mathrm{kw}$.

\section{Reference}

1. Bellomo, Paul, and Fernando Rafael. Accelerator Power Electronics Engineering. N.p.: n.p., 2012. Print.

2. Bill Andeycak, "Phase shifted, zero voltage transition design considerations and the UC3875 PWM controller", Unitrode Application note U-136A

3. SCE150A service manual

4. Rashid, Muhammad H. SPICE for Power Electronics and Electric Power. 2nd ed. N.p.: Taylor\&Francis, 2006. Print.

5. UC3875/6/7/8 Phase Shift Resonant Controller, Unitrode Production from Texas Instruments

6. UCC3895EVM-001 Configuring The UCC3895 For Direct Control Driven Synchronous Re (Rev. B), 02 Dec 2008

\section{Acknowledgement}

This analysis and fixture design was conducted in association with Brookhaven National Laboratory. I appreciate my mentor Chaofeng Mi for his generosity and guidance during the summer internship. I would express my gratitude to Carl Schultheiss for his professionalism. Further I wish to thanks Donald Bruno, Thomas Nolan, Jon Sandberg for their hospitality and continuous support. Finally, I would also like to thank the U.S. Department of Energy, Office of Educational Program and the SURP program for allowing me the opportunity to participate in this magnificent internship program. 


\title{
Regulation of triacylglycerol biosynthesis and storage in plant vegetative tissues
}

\author{
Elizabeth Zipf \\ Tulane University, New Orleans, LA 70118) \\ Jillian Fan and Changcheng Xu \\ Brookhaven National Laboratory, Upton, NY 11973
}

In plants, triacylglycerol (TAG, oil) is stored in a unique subcellular organelle known as oil droplets, which consist of a central matrix of TAG coated by a phospholipid membrane embedded with proteins called oleosins. In past years, plant oils have been explored as a solution for the world's growing food demand. More recently, plant oils have been utilized as feedstocks for the production of biodiesel and industrial chemicals. Despite its importance, very little is known about how TAG is synthesized and stored in the vegetative tissues of plants. The objective of this project was to 1) develop a genetic screen for mutants defective in oil biosynthesis and storage in leaves; 2) isolate mutants accumulating oil in leaves; and 3) characterize the putative mutants at biochemical and genetic levels. To achieve these goals, oleosin-green fluorescent protein (GFP) was overproduced in vegetative tissues of the model plant Arabidopsis to visualize the oil droplets. Then, seeds of homozygous transgenic plants carrying the oleosin-GFP transgene were mutagenized. The mutagenized seeds were sowed on soil and allowed to self-pollinate, producing M2 seeds. The leaves from individual M2 plants were examined using fluorescence microscopy. Individual plants with abnormal size and number of oil droplets. To characterize the putative mutants, leaf oil content and lipid composition of the adult putative mutant plants were analyzed by thin-layer chromatography. This screening method identified approximately twenty mutant candidates. If these mutations are indeed novel, they will help lay a solid foundation for future studies aimed at understanding the regulation of oil biosynthesis and storage in vegetative tissues at the molecular genetic level. In addition, the results could potentially have important implications strategies to develop new crops as renewable bioenergy sources.

\section{INTRODUCTION}

Plant oils, or triacylglycerols (TAGs), play a crucial role not only as a food source for the worlds growing population, but also as feedstocks for biofuel and industrial chemicals. As the world pushes forward to develop new sources of renewable energy, TAGs serve as a definite option. They are one of the most energy-rich sources found in nature. Other biofuel research has focused on hemicellulose and lignin from plant cell walls because these materials are the most abundant form of terrestrial biomass. However, TAGs are a superior option because TAGs produce more energy content per kilogram, do not require fermentation for conversion to liquid fuel, and are more compatible with more efficient diesel engines. ${ }^{1}$ Production and expansion of TAG for biofuel has been limited due to competition with food, crop yield, and availability of suitable land. ${ }^{2}$

A strategy to increase TAG production is to increase the amount of oil found in vegetative tissue. Naturally, TAG is found in seeds as an energy reserve for germination and postgerminative growth. ${ }^{3}$ Most plant tissues have the ability to synthesize TAG, although the amount of TAG found in non-seed tissues is insignif- icant. ${ }^{1}$ TAG is stored in unique structures called oil bodies, which have a TAG core surrounded by a phospholipid membrane embedded with specific proteins called oleosins. Oleosins are known to be responsible for keeping oil bodies from coalescence, as well as the size of the oil body. Oil bodies can form clusters, but always remain as individual units within the cluster. When the Oleosin gene is over expressed, it has been seen that the amount of oil in vegetative tissue increases.

This project used the model plant Arabidopsis, specifically Columbia 2 (Col2), to overexpress the Oleosin gene. The transgenic plant was then mutagenized to provide DNA mutations, which would alter oil synthesis. Past research has identified transcription factors including LEC1, LEC2, and WRI-1, which are known to control the expression of genes involved in the synthesis of oil.4 This project seeks to find additional genes or transcription factors that play a role in TAG synthesis. In order to do so, we have developed a genetic screen for mutants defective in oil biosynthesis and storage in leaves. The screen identifies putative mutants that will be further analyzed at the genetic and biochemical levels. If novel mutations are found, it will contribute to the understanding of the regulation of oil biosynthesis and storage in vegetative tissues at the molecular genetic level.

\section{METHODS AND MATERIALS \\ A. Transgenic plant, Col2Ole1}

To produce the transgenic plant, Col2Ole1, T-DNA mediated gene transfer was utilized. The T-DNA cassette consisted of a $35 \mathrm{~s}$ promoter and the Oleosin gene fused with GFP (Fig. 1). This recombinant T-DNA was than transformed into Agrobacterium tumefaciens to be introduced into the model plant Arabidopsis, specifically ecotype Columbia 2 (Col2). Antibiotic selection was used to determine plant cells that successfully integrated the T-DNA. From this procedure, there were approximately thirty

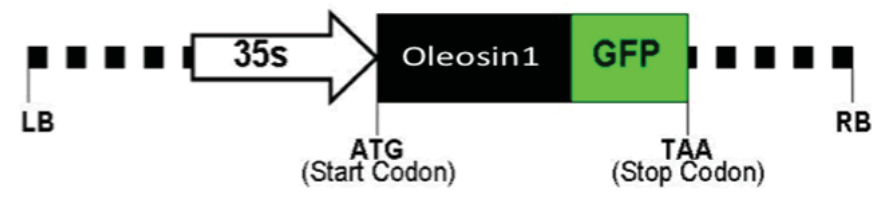

Figure 1: T-DNA cassette to overexpress the Oleosin1 gene

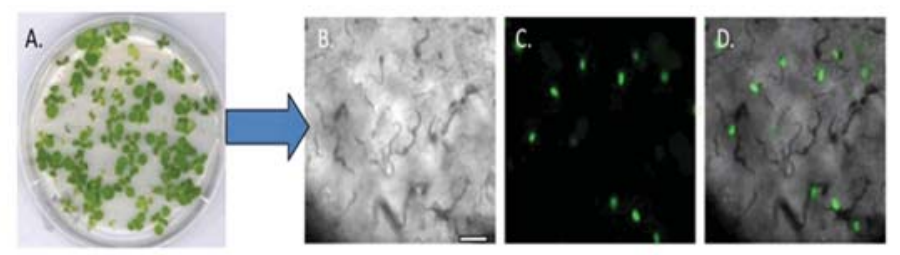

Figure 2: Mutant screening: A.) EMS treated col2ole1 growing in agar-solidified petri dishes B.) Bright field view of leaf C.) Fluorescent microscope view (GFP filter) D.) Overlay of bright field and fluorescent view. Scale bars $=10 \mu \mathrm{m}$ 
lines of transformants obtained. Each T1 line was examined using fluorescent microscopy and TLC analysis to screen for the line with the strongest GFP signal and most abundant amount of oil. T2 seeds harvested from the chosen T1 line were plated on half MS plates containing antibiotics. The T2 plants that survived were transferred to soil. T3 seeds were harvested from the T2 plants individually, and antibiotic selection was performed once again to obtain the homozygous transgenic Col2Ole1 plants

\section{B. EMS mutagenesis of col2ole1}

The chemical mutagen, EMS, was applied to the Col2Ole1 seeds (M1 population) to induce gene mutations. The M1 seeds were planted in soil and allowed to self-pollinate. Seeds were then harvested (M2 seeds) and pooled into approximately twenty-five groups. The M2 plants were the mutant population utilized for screening.

\section{Mutant screening}

\section{Preliminary mutant screening}

M2 seeds were sterilized with bleach and sowed on half MS agar-solidified plates. Leaves that were approximately three weeks old were examined under the fluorescent microscope (Zeiss microscope). Plants with dramatically more or less oil were transferred to soil (candidate mutant population).

\section{Candidate mutant TLC}

When leaves were large enough, samples were obtained from mature leaves of candidate mutants. Lipid extraction was performed using a lipid extraction solution (methanol, chloroform, formic acid) and buffer solution (potassium chloride). Silica-coated TLC plates were baked at 120 degrees for at least twenty minutes prior to loading. Sixty microliters of lipid extract were loaded for each sample. Plates were then placed in a TLC tank containing hexane, diethyl ether, and acetic acid (70:30:1). After the TLC was finished running, the plates were dried, sprayed with sulfuric acid, and baked until bands were visible. Candidate mutant oil bands were compared to each other, as well as to a control sample (Col2oOe1). Those that had a dramatic amount of oil were noted.

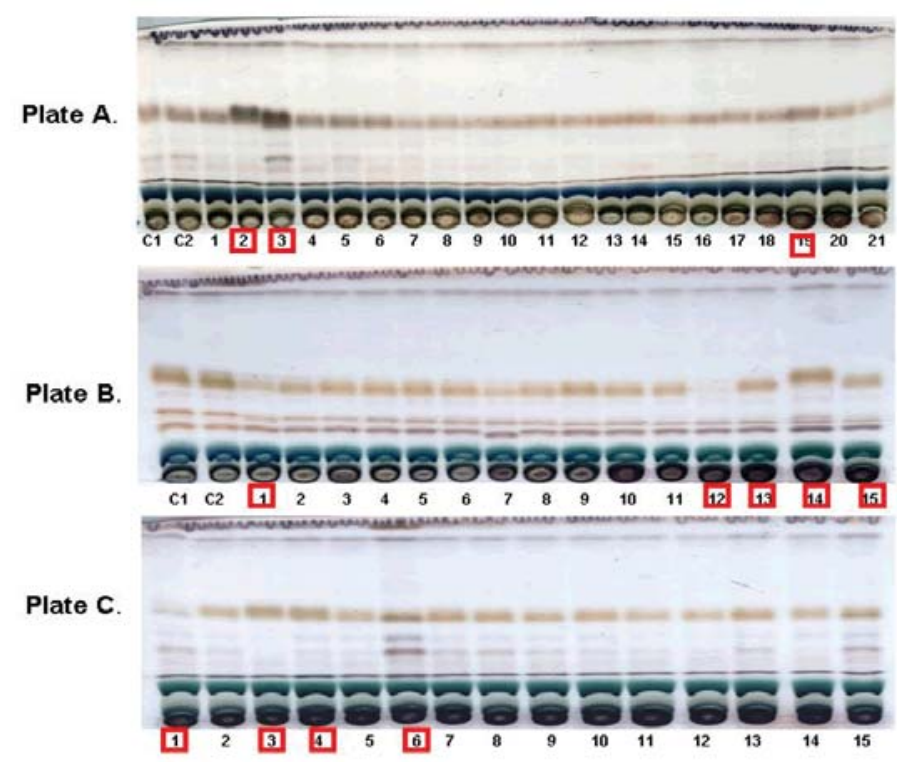

Figure 3: Candidate mutant TLC results for M1 pool.

\section{Candidate mutants}

Mutant candidates identified by TLC were further examined. Samples of young, mature, and old leaves were studied. Candidates were imaged at magnifications of 20, 40, and 60X. Candidates were then backcrossed with the background plant (Co120le1) to reduce additional mutations.

III. Results

A. Preliminary mutant screening

Two pools of M2 plants were screened thoroughly. Each pool contained approximately 1,000 EMS treated Col2Ole1 plants (Fig. 2). Plants with a dramatic difference in oil accumulation compared to the control (Col2Ole1) were kept for further screening. Approximately 45 candidate mutants were chosen per pool and transferred to soil. Often, the smaller plants exhibited a large amount of oil, but were not able to grow in the soil. To avoid the issue of smaller plants dying, they were first transferred to a fresh MS plate to allow additional time to grow. Then, the smaller plants were examined for oil, and transferred to soil if a dramatic oil body phenotype was seen.

\section{B. Candidate mutant TLC}

Figure 3 shows the TLC plate results for the M1 pool. The candidate mutants kept for further screening were noted by red square boxes. Candidates A-2 and A-3 (Plate A, samples 1 and 3) displayed drastic oil content compared to both the control (Co12Ole1) and all other putative mutants. Meanwhile, candidates B-12 and C-1 exhibited almost no oil. Other candidates such as A-19, B-1, B-13, B-14, B-15, C-3, C-4, and C-6 displayed a phenotype that was not as dramatic, but still differed from the control samples. The same process was repeated for the M2 population.

\section{Candidate mutants}

Candidate mutants were further examined with the fluorescent microscope. Oil body morphology, abundance, and distribution varied across mutants. Therefore, candidates were classified according to oil body characteristics. As shown in Figure 4, Candidate A-2 contained oil bodies that were large, round, and spaced closer than the wild type oil bodies. Candidate A-3, had a less uniform distribution. Oil bodies were scattered, closer together, and contained a mixture of smaller and larger oil bodies (Fig. 4- c,f,i).
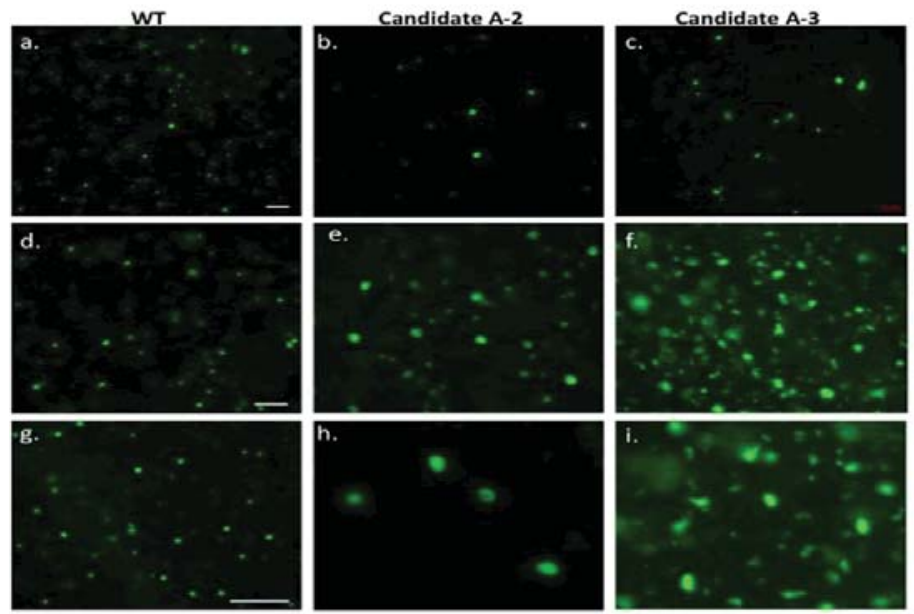

Figure 4: Images of Wild Type, candidate A-2 (TLC Plate A, sample 2), Candidate A-3 (Plate A, sample 3); a.-c.) 20X comparison; d.-f.) 40X comparison; g.-i.) $63 \mathrm{X}$ comparison. scale bars $=10 \mu \mathrm{m}$ 
Additional candidate mutants displayed similar characteristics to the more dramatic mutants A-2 and A-3 in that they exhibited larger oil body clusters and different patterns of distribution. The different mutants were grouped accordingly. Classifying the candidates according to similarities in morphology, abundance, and distribution may suggest a common mutation among the candidate mutants.

\section{DISCUSSION}

Approximately 1,000 individual plants were examined from each pool. Over twenty candidate mutants were obtained from the two screened pools (2,000 plants). A few specific candidates showed an extremely dramatic phenotype. By using a system of classifying the candidates according to abundance, morphology, and distribution, a better preliminary understanding of the different phenotypes can be acquired. This classification system could be improved by quantifying the oil present in each sample. In addition, the mutants were compared to transgenic plants that are already known for synthesizing more oil such as tgd1. If an individual phenotype reflected the known gene mutation, the candidate was still kept for further genetic screening.

The next step for candidate mutant analysis is to backcross the candidates with the wild type (Col2) and Col2Ole. TLC and fluorescent microscopy will be performed to confirm that the candidate indeed has a dramatic oil body phenotype. Once confirmed, further biochemical and genetic analysis will be conducted to identify the gene mutation that is responsible for this phenotype. If it is discovered that mutation identified is indeed a novel mutation, it will contribute to a greater understanding of the regulation of oil biosynthesis and storage in vegetative tissues at the molecular genetic level.

\section{Conclusion}

The screening method developed to identify mutants with abnormal amounts of oil in vegetative tissues is effective. Mutant candidates were identified, and further screening will reveal if a novel gene mutation has been discovered.

\section{Acknowledgements}

I would like to sincerely thank my mentors Jilian Fan and Changcheng $\mathrm{Xu}$ for their knowledge and support throughout this entire internship program. I would also like to thank the Brookhaven National Laboratory, the OEP, and the DOE for giving me the opportunity to participate in this educational and inspirational experience.

\section{Works Cited}

[1.] Ohlrogge, John, and Kent Chapman. Expanding the contribution of plant oils as biofuels: The seeds of green energy. The Biochemical Society: 2011.

[2.] Slocombe, Steve, Jo Cornah, Helen Pinfield-Wells. Oil accumulation in leaves directed by modification of fatty acid breakdown and lipid synthesis pathways. Plant Biotechnology Journal: 2009.

[3.] Hsieh, Kai, Anthony Huang. Endoplasmic reticulum, oleosins, and oils in seeds and tapetum cells. American Society of Plant Biologists: 2004.

[4.] Dyer, John, Robert Mullen, and Kent Chapman. Oil in biomass: a step-change for bioenergy. <www.aocs.org/ membership/freecover> 


\section{Appendix}

This appendix lists those reports which were completed, but not included in the preceding compendium. They were withheld because their contents were not available for one or more of the following reasons: in prepartion for publication in a peer-reviewed journal, incomplete data, preliminary data for a longitidunal study, or other reasons.

\begin{tabular}{|c|c|c|}
\hline Name & Mentor & Title \\
\hline Carla Alberto & Suzanne Smith & $\begin{array}{l}\text { Development of quality control and assurance methods for radioiso- } \\
\text { topes produced at Brookhaven National Laboratory }\end{array}$ \\
\hline Timothy Alston & Lisa Miller & $\begin{array}{l}\text { Fourier Transform Infrared Spectroscopy imaging and biochemical } \\
\text { quantification of } P \text {. fluorescens Pf5 quorum sensing induced extracellu- } \\
\text { lar polymeric substances. }\end{array}$ \\
\hline Melissa Anthony & Andrew Gifford & Unavailable \\
\hline Christopher Awad & Lisa Miller & $\begin{array}{l}\text { Improving biomass production: Imaging the diffusion of nutrients and } \\
\text { signaling molecules in the Rhizosphere }\end{array}$ \\
\hline Brian Bergh & Karl Kusche & Design of a high-power $\mathrm{CO}_{2}$ laser transport system \\
\hline Aaron Blevins & Michael Mapes & Unavailable \\
\hline Matthew Bossart & Matthew Eisaman & $\begin{array}{l}\text { Reconstruction of the refractive index profiles of thin-film photovolta- } \\
\text { ics }\end{array}$ \\
\hline Ryan Brito & Nicholas Brown & Simulating novel nuclear fuels with enhanced safety features \\
\hline Alissa Bruno & Abhijit Karve & $\begin{array}{l}\text { Use of }{ }^{11} \mathrm{CO}_{2} \text { to measure photosynthate allocation and transport speeds } \\
\text { in grain and sweet sorghum }\end{array}$ \\
\hline Entebbe Butler & William Russell & Unavailable \\
\hline Kasey Campbell & James Dickerson & $\begin{array}{l}\text { Electrophoretic deposition and post-electrophoretic deposition as a } \\
\text { means to fabricate carbon nanotube thin films }\end{array}$ \\
\hline Michelle Cerniglia & Vivian Stojanoff & Effects of external fields on protein crystallization \\
\hline Vivian Chan & Stojanoff Vivian & Unavailable \\
\hline Richie Chen & Masafumi Fukuto & Unavailable \\
\hline William Dell'Isola & Jennifer Higbie & $\begin{array}{l}\text { Evaluating small mammal diversity within the Long Island Solar Farm, } \\
\text { Upton, New York. }\end{array}$ \\
\hline Ariel Dowdy & Ralph James & Unavailable \\
\hline Aleksander Durumeric & James Wishart & Preparation and purification of ionic liquids \\
\hline Daniel Ericson & Alexei Soares & Unavailable \\
\hline Eric Evangelou & Plyush Joshi & Unavailable \\
\hline Bryan Fallier & Jorg Kewisch & Unavailable \\
\hline Thomas Fasano & Tim Green & $\begin{array}{l}\text { Cesium-137 levels in white-tailed deer at Brookhaven National Labo- } \\
\text { ratory }\end{array}$ \\
\hline Miriam Figueroa & Jun Wang & $\begin{array}{l}\text { 3D nanoscale morphological analysis of conductive adhesive materials } \\
\text { using x-ray nano-tomography }\end{array}$ \\
\hline Molly Foley & Tim Green & $\begin{array}{l}\text { Gestation and nest site selection impacts on eastern box turtle home } \\
\text { range }\end{array}$ \\
\hline Joseph Garlow & Javier Pulecio & Unavailable \\
\hline Kristen Glass & Jennifer Higbie & $\begin{array}{l}\text { Shedding light on Herpetofauna: the state of Herpetofauna populations } \\
\text { within the Long Island Solar Farm at Brookhaven National Laboratory }\end{array}$ \\
\hline Zachary Gotlib & Suzanne Smith & $\begin{array}{l}\text { Separation of no carrier added cobalt- } 57 \text { for use in calibration of nucle- } \\
\text { ar medicine imaging devices }\end{array}$ \\
\hline Justin Gray & Aleksey Bolotnikov & Post-growth annealing of Cadmium Manganese Telluride crystals \\
\hline
\end{tabular}




\begin{tabular}{|c|c|c|}
\hline Nicholas Hannah & Aleksey Bolotnikov & Relation of extended defects to $\mathrm{CdZnTe}$ detector performance \\
\hline Kendall Harris & Jennifer Higbie & $\begin{array}{l}\text { Evaluating small mammal diversity within the Long Island Solar Farm } \\
\text { at Brookhaven National Laboratory Upton, New York. }\end{array}$ \\
\hline Sumar Hayan & Qin Liu & Unavailable \\
\hline Maegan Hearney & Xiaohong Yu & Analysis of transformed seeds that produce cyclopropane fatty acid \\
\hline John Hirdt & David Brown & Data mining the EXFOR database \\
\hline Gregory Jones Jr. & James Dickerson & $\begin{array}{l}\text { The effectiveness of using a mixture of cadmium selenide/zinc sul- } \\
\text { fide nanoparticles suspended in hexane to assemble a fully deposited } \\
\text { heterogeneous film }\end{array}$ \\
\hline Emma Kast & Benjamin Babst & Analyzing sucrose uptake in sorghum with $14 \mathrm{C}$ sucrose radiotracer \\
\hline Awa Keinde & Andrew Gifford & Unavailable \\
\hline Alexander Koenig & Scott Smith & Unavailable \\
\hline Kristina Krylova & Ivan Kotov & $\begin{array}{l}\text { Charge coupled device traps identification using a pocket pumping } \\
\text { technique }\end{array}$ \\
\hline Daniel Kurtz & David Grills & Electrochemical studies of processes in artificial photosynthesis \\
\hline Erica Lai & Charles Black & $\begin{array}{l}\text { Understanding the self-assembled phases of blended block copolymer } \\
\text { thin film nanostructures }\end{array}$ \\
\hline Mikalie Lai & Oleg Gang & Finite-sized clusters of nanoparticles assembled with DNA structures \\
\hline Pui Ching Lan & Feifei Li & Unavailable \\
\hline Mark Lange & Eric Dooryhee & $\begin{array}{l}\text { PyXDA: designing analysis software for the new X-ray Powder Dif- } \\
\text { fraction beamline }\end{array}$ \\
\hline Alyssa Liguori & Tim Green & Unavailable \\
\hline James McClintock & Vivian Stojanoff & $\begin{array}{l}\text { CrySis: a neural network based software for high-throughput protein } \\
\text { crystallography }\end{array}$ \\
\hline Diana McGrath & Tim Green & $\begin{array}{l}\text { Gestation and nest site selection impacts on eastern box turtle home } \\
\text { range }\end{array}$ \\
\hline Barbara Mello & David Grills & $\begin{array}{l}\text { Comparison of two difunctional photocatalysts for solar-driven } \mathrm{CO} 2 \\
\text { conversion }\end{array}$ \\
\hline Eric Metodiev & Yannis Semertzidis & Unavailable \\
\hline Amanda Muraca & Tatiana Pyatina & Unavailable \\
\hline Francesca Nedvin & Kenneth White & Unavailable \\
\hline Alexander Newman & Dario Arena & Unavailable \\
\hline Adel Njeim & Vivian Stojanoff & Development of sensors for synchrotron beamlines \\
\hline Maja Oblepias & James Dickerson & $\begin{array}{l}\text { Formation of heterostructured } \mathrm{CdSe} / \mathrm{ZnS} \text { core/shell nanoparticle thin } \\
\text { films via electrophoretic deposition }\end{array}$ \\
\hline Steven Oliveri & James Wishart & Physical characterization of dialkylphosphate ionic liquids \\
\hline David Peters & Mike Gaffney & Unavailable \\
\hline Julia Petrullo & Benjamin Babst & $\begin{array}{l}\text { Investigating the role of potassium in phloem transport using Zea mays } \\
\text { sut1 mutants }\end{array}$ \\
\hline Gina Polizzo & Alexei Soares & Unavailable \\
\hline Charles Prochaska & Oleg Gang & Chiroptical activity of nanoparticle-immobilized proteins \\
\hline Eric Puma & Matthew Eisaman & $\begin{array}{l}\text { Deposition of ultra-thin semiconductor nanocrystal films for use in } \\
\text { novel photovoltaics }\end{array}$ \\
\hline Hayley Reed & Suzanne Smith & $\begin{array}{l}\text { A new microspectrophotometric assay for the detection of metal ions } \\
\text { in radioisotopic solutions }\end{array}$ \\
\hline Eric Ruhoy & Vincent DeAndrade & Unavailable \\
\hline Michelle Rusek & Joe Tuozzolo & Unavailable \\
\hline Valerie Sabatasso & Antonio Checco & Impacts of water droplets on nanopatterned hydrophobic surfaces \\
\hline
\end{tabular}




\begin{tabular}{|c|c|c|}
\hline Luis Sagastume & Oleg Eyser & Calibration of silicon strip detectors in a Polarimeter at RHIC \\
\hline Patrick Saitta & Esther Takeuchi & $\begin{array}{l}\text { Investigation of dialkoxyaryl compounds as redox shuttles to protect } \\
\text { batteries }\end{array}$ \\
\hline Claire Saunders & Kin Yip & $\begin{array}{l}\text { Simulating soil activation in radiation hotspots of accelerators and } \\
\text { colliders }\end{array}$ \\
\hline Heather Savage & Carl Shultheiss & $\begin{array}{l}\text { Test setup for the Relativistic Heavy Ion Collider current or voltage } \\
\text { regulator cards }\end{array}$ \\
\hline Alexander Scalia & Alexei Soares & Unavailable \\
\hline Sam Schraer & Dmytro Nykypanchuk & $\begin{array}{l}\text { A versatile metal nanowire synthesis through carbon nanotube templat- } \\
\text { ing }\end{array}$ \\
\hline Anthony Sementilli & Abhijit Karve & $\begin{array}{l}\text { Use of positron emission tomography and gene expression analysis to } \\
\text { study sugar accumulation in Sorghum bicolor }\end{array}$ \\
\hline Maria Sirenko & Lisa Miller & $\begin{array}{l}\text { Determining metal ion concentrations and the oxidation state of copper } \\
\text { in Alzheimer's Disease mouse model amyloid plaques }\end{array}$ \\
\hline Phil Smith & Kevin Yager & Investigating the electrical properties of doped graphene \\
\hline Marie Smulczeski & Helio Takai & Unavailable \\
\hline Kylen Solvik & Suzanne Smith & $\begin{array}{l}\text { Production of high purity } \mathrm{Cu}-67 \text { using } 40-45 \mathrm{MeV} \text { protons at Brookha- } \\
\text { ven National Laboratory }\end{array}$ \\
\hline Zachary Sorrell & Terry Sullivan & Unavailable \\
\hline Taylor Stiegler & Scott Bronson & Scientific communication: a neglected necessity \\
\hline Andrew Sung & Benjamin Babst & $\begin{array}{l}\text { Auxin signaling is necessary for gravitropic stem bending in tall grass- } \\
\text { es: does auxin biosynthesis contribute? }\end{array}$ \\
\hline Greg Swirson & Terry Sullivan & Unavailable \\
\hline Ben Tuttle & Ray Conley & Unavailable \\
\hline Joseph Valenti & Jun Wang & $\begin{array}{l}\text { Development of microchemical cells for in-situ catalysis studies using } \\
\text { full-field x-ray nanotomography }\end{array}$ \\
\hline Brittany Wienclaw & Benjamin Babst & The role of sugar signaling in apical dominance \\
\hline Emily Yang & Matthew Eisaman & High-resolution spatial mapping of thin-film photovoltaic cells \\
\hline James Young & Robert McGraw & $\begin{array}{l}\text { Application of linear programming in determining atmospheric liquid } \\
\text { water content }\end{array}$ \\
\hline Suhua Zeng & Tim Green & $\begin{array}{l}\text { Monitoring the bird population at Brookhaven National Laboratory by } \\
\text { using point count bird surveys }\end{array}$ \\
\hline Nicole Zmich & James Wishart & $\begin{array}{l}\text { Synthesis of electron-donating ionic liquids based on 4-(dimethylami- } \\
\text { no)pyridine }\end{array}$ \\
\hline Joseph Zoino & Paul Freimuth & Misfolded protein toxicity in bacteria \\
\hline
\end{tabular}




\section{List of interns, their colleges or univerisities, and location in this compendium}

\begin{tabular}{|c|c|c|}
\hline Intern & College / University & Page \\
\hline Adedoyin Akingba & SUNY Potsdam & 9,182 \\
\hline Carla Alberto & Suffolk County Community College & 270 \\
\hline Timothy Alston & Claflin University & 270 \\
\hline Melissa Anthony & Medgar Evers City University of New York & 270 \\
\hline Neil Aram & SUNY Binghamton & 11 \\
\hline Christopher Awad & Fordham University & 270 \\
\hline Catherine Ayuso & Forida University & 14 \\
\hline Adam Batley & (No school affiliation) & 21 \\
\hline Shannon Baxter & SUNY Geneseo & 23 \\
\hline Brian Bergh & University at Buffalo & 270 \\
\hline Ricky Bhola & CUNY Hostos Community College & 26 \\
\hline Marie Blatnik & Cleveland University & 29 \\
\hline Aaron Blevins & Penn State University & 270 \\
\hline Kevin Bogaert & Georgia Institute of Technology & 35 \\
\hline Matthew Bossart & Dartmouth College & 270 \\
\hline Jessica Bristol & Eastern Connecticut State University & 39 \\
\hline Ryan Brito & Texas A\&M University & 270 \\
\hline Alissa Bruno & St.Joseph's College & 270 \\
\hline Entebbe Butler & Suffolk County Community College & 270 \\
\hline Kasey Campbell & Lincoln University & 270 \\
\hline Michelle Cerniglia & Binghamton University & 270 \\
\hline Trevor Chan & University of Alabama in Huntsville & 44 \\
\hline Richie Chen & Stony Brook University & 270 \\
\hline David Cinquegrani & University of Michigan & 47 \\
\hline Tiera Corey & The University of Southern Mississippi & 50,235 \\
\hline Lezou Dali & SUNY Stony Brook & 9,182 \\
\hline Benjamin Daniels & Rensselaer Polytechnic Institute & 57 \\
\hline Ryan Davella & University of Delaware & 61 \\
\hline Kyle Davis & Delaware University & 65 \\
\hline William Dell'Isola & SUNY- ESF & 270 \\
\hline Olivia Dickens & Howard University & 67 \\
\hline Kahlil Dixon & Howard University & 70 \\
\hline Ariel Dowdy & Alabama A\&M University & 270 \\
\hline Aleksander Durumeric & University of Iowa & 270 \\
\hline Alison Egbon & SUNY Stony Brook & 72 \\
\hline Daniel Ericson & Buffalo University & 270 \\
\hline Eric Evangelou & Rochester Institute of Technology & 270 \\
\hline Bryan Fallier & Binghamton University & 270 \\
\hline Thomas Fasano & SUNY Stony Brook & 270 \\
\hline Miriam Figueroa & University of Puerto Rico, Mayagüez Campus & 270 \\
\hline
\end{tabular}




\begin{tabular}{|c|c|c|}
\hline Intern & College / University & Page \\
\hline Molly Foley & University of Wisconsin - Stevens Point & 270 \\
\hline Michael Galazka & Housatonic Community College & 75 \\
\hline Joseph Garlow & Stony Brook University & 270 \\
\hline Kristen Glass & Siena College & 270 \\
\hline Kenneth Goodman & Long Island University & 81 \\
\hline Michelle Gostic & Cornell University & 84 \\
\hline Zachary Gotlib & Kutztown University & 270 \\
\hline Justin Gray & Alabama University & 270 \\
\hline Joshua Greenough & SUNY Oneonta & 86 \\
\hline Nathan Gubser & University of the Virgin Islands & 88 \\
\hline Christopher Guerrero & Fordham University & 90 \\
\hline Nicholas Hannah & Arizona State University & 271 \\
\hline Kendall Harris & Sweet Briar College & 271 \\
\hline Sumar Hayan & SUNY Stony Brook & 271 \\
\hline Maegan Hearney & Swarthmore College & 271 \\
\hline IIana Heckler & SUNY New Paltz & 92 \\
\hline Lauren Herrera & Stony Brook University & 95 \\
\hline John Hirdt & St. Joseph's College & 271 \\
\hline Daniel Hirsh & City College of New York & 98 \\
\hline Richard Houanche & University at Albany & 102 \\
\hline Julia Huang & Duke University & 104 \\
\hline Mark Jensen & Suffolk County Community College & 108 \\
\hline Ankit Johari & Stony Brook University & 113 \\
\hline Gregory Jones Jr. & Delaware State University & 271 \\
\hline Mark Kamuda & University of Illinois & 115 \\
\hline Emma Kast & Hofstra University & 271 \\
\hline Awa Keinde & Hofstra University & 271 \\
\hline Alexander Koenig & Binghamton University & 271 \\
\hline Lucia Kolodiuk & Stony Brook University & 118 \\
\hline Kevin Korol & Suny Maritime & 122 \\
\hline Kristina Krylova & University at Buffalo - SUNY & 271 \\
\hline Daniel Kurtz & Oakland University & 271 \\
\hline Erica Lai & Massachusetts Institute of Technology & 271 \\
\hline Mikalie Lai & Stony Brook University & 271 \\
\hline Johanne Lamothe & Suffolk CCC & 9,182 \\
\hline Pui Ching Lan & University of California, Berkeley & 271 \\
\hline Sarah Langan & Keystone College & 124 \\
\hline Mark Lange & South Plains College & 271 \\
\hline Nathaniel Lashley-Colthirst & Howard & 130 \\
\hline Raynold Lewis & Stony Brook University & 132 \\
\hline Alyssa Liguori & Dowling College & 271 \\
\hline Danielle Mallon & University at Albany, SUNY & 135 \\
\hline Mauro Mancilla & Tallahassee Community College & 139 \\
\hline Matthew Mayers & Cornell University & 9,182 \\
\hline
\end{tabular}




\begin{tabular}{|c|c|c|}
\hline Intern & College / University & Page \\
\hline James McClintock & Cornell University & 271 \\
\hline Diana McGrath & SUNY Geneseo & 271 \\
\hline Barbara Mello & University of Massachusetts Boston & 271 \\
\hline Eric Metodiev & Harvard University & 271 \\
\hline Aaron Mohammed & Texas A\&M University & 142 \\
\hline Brittany Morgan & Binghamton University & 147 \\
\hline Amanda Muraca & William Paterson University & 271 \\
\hline Francesca Nedvin & Binghamton University & 271 \\
\hline Alexander Newman & Morgan State University & 271 \\
\hline Adel Njeim & City College of New York - CUNY & 271 \\
\hline Maja Oblepias & University of California - Berkeley & 271 \\
\hline Scott Oldham & Michigan State University & 150 \\
\hline Steven Oliveri & Queensborough CC & 271 \\
\hline Naa Adokaley Pappoe & City College Of New York & 154 \\
\hline Divas Persaud & City College of New York & 159 \\
\hline David Peters & Hofstra University & 271 \\
\hline Julia Petrullo & University at Buffalo (SUNY) & 271 \\
\hline Gina Polizzo & St. Joseph's College & 271 \\
\hline Kevin Powell & Florida A\&M University & 162 \\
\hline Lucas Price & West Virginia University & 165 \\
\hline Charles Prochaska & St.Joseph CC & 271 \\
\hline Eric Puma & Pomona CC & 271 \\
\hline Hayley Reed & Muhlenberg College & 271 \\
\hline Brandon Rhymer & University of the Virgin Islands & 170 \\
\hline Jonathan Rolland & University of Illinois & 172 \\
\hline Mark Romanelli & Cornell University & 176 \\
\hline Evgeniya Rubin & The City College of New York & 179 \\
\hline Eric Ruhoy & St. Joseph's College & 271 \\
\hline Michelle Rusek & Penn State University & 271 \\
\hline Valerie Sabatasso & SUNY Stony Brook & 271 \\
\hline Luis Sagastume & Florida University & 272 \\
\hline Jessica Saintibert & Tallahassee CC & 9,182 \\
\hline Patrick Saitta & University at Buffalo & 272 \\
\hline Fidel Salgado & Canada College & 184 \\
\hline Pavel Salnikov & Stony Brook University & 190 \\
\hline Bradley Salvato & College of DuPage & 193 \\
\hline Kevin Sanders & St.Joseph's College & 198 \\
\hline Rachel Sandler & University of Arizona & 201,206 \\
\hline Katherine Z. Sanidad & Stony Brook University & 118,209 \\
\hline Andrea Sarmiento & Stony Brook University & 213 \\
\hline Claire Saunders & Duquesne University & 272 \\
\hline Heather Savage & Stony Brook University & 272 \\
\hline Alexander Scalia & Binghamton University & 272 \\
\hline Jonathan Schneider & Stony Brook University & 272 \\
\hline Sam Schraer & Cornell University & 272 \\
\hline
\end{tabular}




\begin{tabular}{|l|l|r|}
\hline \multicolumn{1}{|c|}{ Intern } & \multicolumn{1}{|c|}{ College / University } \\
\hline Anthony Sementilli & St. Joseph's College & 272 \\
\hline Mackenzie Shipley & Hartwick College & 222 \\
\hline Maria Sirenko & Cornell University & 272 \\
\hline Michael Skinner & Marist College & 23 \\
\hline Nicholas Smith & Tallahassee CC & 232 \\
\hline Phil Smith & Columbia University & 272 \\
\hline Marie Smulczeski & Suffolk CC & 272 \\
\hline Kylen Solvik & Haveford College & 272 \\
\hline Zachary Sorrell & Messiah College & 272 \\
\hline Nicholas St.Fleur & Cornell & 234 \\
\hline Taylor Stiegler & Boston College & 272 \\
\hline Andrew Sung & Stony Brook University & 272 \\
\hline Greg Swirson & Stony Brook University & 272 \\
\hline Altramiese Tippie & Mountain View College & 50,235 \\
\hline Katherine Towey & SUNY Albany & 242 \\
\hline Ben Tuttle & Cedarville University & 272 \\
\hline Joseph Valenti & SUNY Farmingdale State College & 272 \\
\hline Ilia Vardishvili & Stony Brook University & 9,182 \\
\hline Brittany Wienclaw & University of New Haven & 272 \\
\hline Rebecca Winter & Columbia University & 345 \\
\hline Jovani Withers & Queensborough CC & 252 \\
\hline Michael Worek & University of Illinois Urbana-Champaign & 255 \\
\hline David Wu & Dartmouth College & 259 \\
\hline Emily Yang & Pomona College & 272 \\
\hline James Young & St. Joseph's College & 272 \\
\hline Suhua Zeng & New York City College of Technology & 272 \\
\hline Man Zhang & Suffolk County Community College & 263 \\
\hline Elizabeth Zipf & Tulane University & 267 \\
\hline Nicole Zmich & Stony Brook University & 272 \\
\hline Joseph Zoino & Syracuse University & \\
\hline
\end{tabular}

